Doctoral Thesis

\title{
Electronic Structure of Quantum Dots: Response to the Environment and Externally Applied Fields
}

\author{
Author: \\ Carlos Segarra Ortí \\ ADVISORS: \\ Josep Planelles Fuster \\ Juan Ignacio Climente Plasencia
}

June 2016

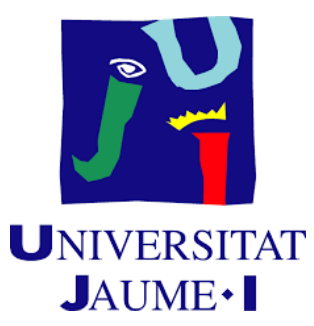

Departament de Química Física i Analítica

Universitat Jaume I

Castelló de la Plana, Spain 

Als meus pares 

Els Doctors Josep Planelles Fuster i Juan Ignacio Climente Plasencia, del Departament de Química Física i Analítica de la Universitat Jaume I,

\section{CERTIFIQUEN:}

Que la memòria presentada pel llicenciat Carlos Segarra Ortí amb títol "Electronic Structure of Quantum Dots: Response to the Environment and Externally Applied Fields" ha estat realitzada sota la nostra direcció i constitueix la Tesi Doctoral de l'esmentat llicenciat. Autoritzem la presentació d'aquesta mitjançant el present escrit.

Castelló de la Plana, juny de 2016 



\section{Agraïments}

La present Tesi doctoral és el resultat final de quatre anys de treball i esforç en què he tingut la sort de comptar amb el suport de companys, familiars i amics que han compartit amb mi aquesta enriquidora experiència. Sens dubte, el treball que ací es mostra no haguera sigut possible sense la seua ajuda.

En primer lloc, voldria agrair a Josep Planelles i Juan Ignacio Climente el haver-me oferit la possibilitat de realitzar aquest projecte de Tesi sota la seua supervisió. Durant tot el temps que he tingut el plaer de treballar amb ells han mostrat un gran interès i una dedicació plena en proporcionar-me la millor formació investigadora tenint sempre present el que seria millor per al meu futur. De la mateixa manera, el meu agraïment és extensiu a la resta de membres del grup de Química Quàntica Fernando Rajadell, José Luis Movilla, Miquel Royo i Ana Ballester que d'una manera o altra han contribuït a la realització d'aquesta Tesi. En especial vull destacar a Ana $\mathrm{B}$, amb qui vaig tindre la sort de compartir laboratori durant els primers anys de doctorat i que prompte es va convertir en una molt bona amiga.

D'altra banda, voldria mostrar també la meua gratitud als amics que han estat al meu costat en el dia a dia durant aquests anys i que han compartit amb mi molts bons moments dintre i fora de la universitat. En concret vull destacar a Ana M, David, Erica, Neus, Paula i, com no, a chéritounet Loucou. A més, cal mencionar a tots els membres del Departament de Química Física i Analítica, especialment a Merche per la seva predisposició a ajudar-me amb tota la paperassa i els tràmits burocràtics. Done també les gràcies a Sergio E. Ulloa per donar-me l'oportunitat de realitzar una estada breu en el seu grup de recerca en la Universitat d'Ohio (Estats Units), així com a tots els membres del grup per la seua acollida.

Finalment, voldria agrair de cor als meus pares el suport i la confiança incondicionals que m'han donat durant tota la meua vida. Perquè són els principals responsables de que haja arribat fins ací, els dedique aquesta Tesi. 



\section{Acronyms}

1D one-dimensional

2D two-dimensional

3D three-dimensional

AB Aharonov-Bohm

CB conduction band

ch crystal-field split hole

DQD double quantum dot

DSOI Dresselhaus SOI

e-h electron-hole

EFA envelope function approximation

EMA effective mass approximation

hh heavy-hole

LED light emitting diode

lh light-hole

PL photoluminescence

QD quantum dot

QR quantum ring

RSOI Rashba SOI

so split-off 
SOI spin-orbit interaction

TMDC transition metal dichalcogenide

VB valence band

WZ wurtzite

ZB zinc-blende 


\section{Contents}

\begin{tabular}{lll}
\hline & Introduction & 1
\end{tabular}

\begin{tabular}{|llr}
2 & Theoretical framework & 9
\end{tabular}

2.1 The k.p method . . . . . . . . . . . . . . . . . . . 10

2.1 .1 General formulation of the k.p method . . . . . . . 10

2.1 .2 Envelope function approximation . . . . . . . . . . . . 12

2.1 .3 Hamiltonians for zinc-blende structures . . . . . . . . 15

2.1 .4 Hamiltonians for wurtzite structures . . . . . . . . . 17

2.1.5 Hamiltonians for polytypes . . . . . . . . . . . . . . 19

2.2 Externally applied fields . . . . . . . . . . . . . . . 21

$2.2 .1 \quad$ Electric field $\ldots \ldots \ldots \ldots$. . . . . . . . . . . . 21

2.2 .2 Magnetic field . . . . . . . . . . . . . . . . . . . . . . . . . . . 21

2.3 Spin-orbit interaction (SOI) $\ldots \ldots \ldots \ldots \ldots$

2.3 .1 Dresselhaus SOI . . . . . . . . . . . . . . . . 24

2.3 .2 Rashba SOI . . . . . . . . . . . . . . . 25

2.4 Strain and polarization fields . . . . . . . . . . . 25

$2.4 .1 \quad$ Strain . . . . . . . . . . . . . . 26

$2.4 .2 \quad$ Piezoelectric polarization . . . . . . . . . . . 28

$2.4 .3 \quad \mathrm{k} \cdot \mathrm{p}$ Hamiltonians including strain and polarization fields 29

3 Magnetic field effects in semiconductor structures 31

3.1 Magnetic-field modulation of the hole ground state in cubic

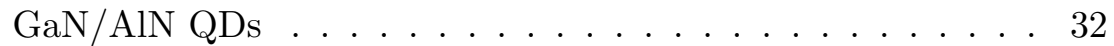

3.1 .1 Effect of the aspect ratio. . . . . . . . . . . . . 34

3.1 .2 Magnetic field modulation . . . . . . . . . . . . . . 36

3.2 Aharonov-Bohm effect in systems with hexagonal symmetry . 37

$3.2 .1 \quad$ AB effect in hexagonal quantum rings . . . . . . . 38

3.2.2 AB magnetoconductance oscillations and electron gas transitions in hexagonal core-shell nanowires . . . . . 43

\begin{tabular}{|lll}
4 & Spin-orbit-induced spin relaxation in semiconductor QDs & 53
\end{tabular} 
4.1 Theoretical formalism for the calculation of phonon-induced spin relaxation rates $\ldots \ldots \ldots \ldots \ldots \ldots 5$

$4.2 \quad$ Spin relaxation in 3D spheroidal QDs $\ldots \ldots \ldots \ldots$

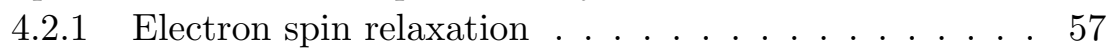

4.2 .2 Hole spin relaxation . . . . . . . . . . . . . . . . 61

4.3 Electron spin-relaxation anisotropy in [001] and [111] grown

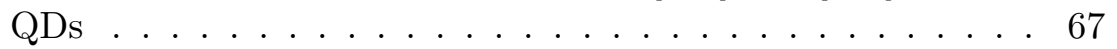

4.3 .1 Effect of the QD geometry . . . . . . . . . . 70

4.3 .2 In-plane rotation of the QD $\ldots \ldots \ldots \ldots$

$4.3 .3 \quad[111]$ grown QDs $\ldots \ldots \ldots \ldots . \ldots . \ldots 74$

4.4 Hole spin relaxation in InAs/GaAs quantum dot molecules . 75

\begin{tabular}{|lll}
\hline 4.5 & Control of electron spin-orbit anisotropy in pyramidal QDs \\
\hline
\end{tabular}

$4.5 .1 \quad$ Angular dependence of the SOI . . . . . . . . . . 82

4.5 .2 Effect of the QD composition and height. . . . . . . . . 83

4.6 Summary . . . . . . . . . . . . . . . . . . . . 84

\begin{tabular}{|llll}
5 & Strain and piezoelectricity in wurtzite and polytype QDs & $\mathbf{8 7}$
\end{tabular}

5.1 Piezoelectric control of exciton wave function in wurtzite QDs 88

$5.1 .1 \quad$ Spheroidal dot-in-dot systems . . . . . . . . . 90

5.1.2 Other CdSe/CdS core-shell structures . . . . . . . . . 96

$5.2 \quad$ Spontaneous polarization in GaAs polytype QDs $\ldots . . .996$

$5.2 .1 \quad$ Electrons in GaAs WZ/ZB/WZ polytype QDs . . . . 100

5.2 .2 Holes in GaAs ZB/WZ/ZB polytype QDs . . . . . . . 102

5.2.3 Excitons in GaAs WZ/ZB/WZ and ZB/WZ/ZB polytype structures . . . . . . . . . . . . 104

$6 \quad$ Edge states in monolayer $\mathrm{MoS}_{2}$ nanostructures 109

6.1 Effective Hamiltonian $\ldots \ldots \ldots \ldots$

6.2 Results and discussion . . . . . . . . . . . . . . . . . . 111

$6.2 .1 \mathrm{MoS}_{2}$ nanoribbons $\ldots \ldots \ldots \ldots 111$

$6.2 .2 \quad \mathrm{MoS}_{2}$ triangular QDs . . . . . . . . . . . 116

\begin{tabular}{lll}
\hline 7 & Conclusions & 119
\end{tabular}

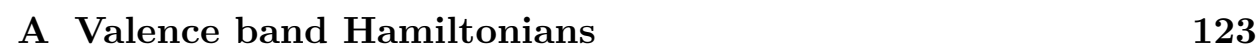

A.1 Zinc-blende crystal structure . . . . . . . . . . . . . . . 123

A.1.1 Four-band Luttinger-Kohn Hamiltonian . . . . . . . . 123

A.1.2 Six-band Burt-Foreman Hamiltonian . . . . . . . . . . 124

A.1.3 Six-band Burt-Foreman Hamiltonian in cylindrical co-

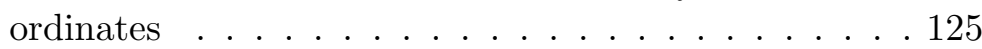

A.2 Wurtzite crystal structure . . . . . . . . . . . . . . . 129

A.3 \begin{tabular}{l} 
Aolytypes $\ldots \ldots \ldots \ldots \ldots$ \\
\hline
\end{tabular} 
A.3.1 Constant-mass Hamiltonian . . . . . . . . . . . . 130

A.3.2 Variable mass Hamiltonian . . . . . . . . . . . . . . 131

B Spin-orbit Hamiltonians in matrix form 133

B.1 Dresselhaus SOI . . . . . . . . . . . . . . . . . . . 133

B.1.1 Conduction band DSOI Hamiltonian . . . . . . . . . . 133

B.1.2 Valence band DSOI Hamiltonian . . . . . . . . . . . . 134

B.2 Rashba SOI . . . . . . . . . . . . . . . . . . . . . 135

B.2.1 Conduction band RSOI Hamiltonian . . . . . . . . . . 135

\begin{tabular}{ll}
\hline C Strain Hamiltonians & 137
\end{tabular}

C.1 Zinc-blende QDs . . . . . . . . . . . . . . . . . . . 137

C.1.1 Conduction band . . . . . . . . . . . . . . . . . . 137

C.1.2 Valence band . . . . . . . . . . . . . . . . . . 137

C.2 Wurtzite QDs . . . . . . . . . . . . . . . . . . . . . 138

C.2.1 Conduction band . . . . . . . . . . . . . . . . . 138

C.2.2 Valence band . . . . . . . . . . . . . . . . . . . . . 139

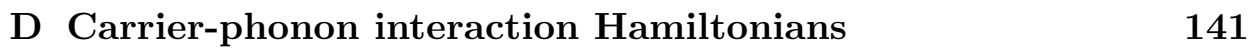

D.1 Piezoelectric potential . . . . . . . . . . . . . . . . . . . 142

D.2 Deformation potential . . . . . . . . . . . . . . . . 143

D.2.1 Conduction band . . . . . . . . . . . . . . . . . . . 143

D.2.2 Valence band . . . . . . . . . . . . . . . . . . . 143

$\begin{array}{ll}\text { Resum } & 145\end{array}$

\begin{tabular}{ll}
\hline Publications & 153
\end{tabular}

\begin{tabular}{ll}
\hline Bibliography & 289
\end{tabular} 



\section{СнАРтеR 1}

\section{Introduction}

Over the last decades, big efforts have been dedicated to the fabrication of smaller and smaller technological devices. This miniaturization process has led to structures with sizes in the nanometer scale (from few nanometers to few hundred nanometers). A clear example is the size evolution of the silicon transistors that integrate computer chips, decreasing from few micrometers to around $10 \mathrm{~nm}$ in the last 50 years. The investigation of such small systems has given rise to a relatively new area of research called nanotechnology, which is remarkably multidisciplinary and of current interest. When charge carriers (electrons and holes) are confined into systems with such length scale, of the order of their de Broglie wavelength, they start obeying the laws of quantum mechanics and a classical approach no longer holds. This fact is a natural limitation for the traditional methods of device fabrication, but it also offers fascinating novel physical properties that makes these structures promising candidates for future applications in medicine, electronics, solar cells and batteries, among others. [1]

In particular, nanoelectronic devices formed by low-dimensional semiconductor nanostructures have been intensively investigated and various types of nanostructures have been developed. Based on the number of dimensions in which the carriers are confined, these can be classified into quantum dots (QDs) (confined in all three spatial dimensions), quantum wires (confined in two dimensions) and quantum wells (confined in only one dimension). Each one of them presents different features, but this Thesis will mainly focus on the study of zero-dimensional QDs. As a result of the quantum confinement in these systems, their energy states form a discrete energy spectrum, similar to that of atoms. Due to this analogous behavior, QDs are also known as artificial atoms. 2. However, both systems 
present important differences. Electrons in an atom are subject to the attractive centrosymmetric Coulomb potential of the nuclei, while electrons in a $\mathrm{QD}$ move freely inside the available space defined by the confining potential. Additionally, the population of electrons inside a QD can be controlled from zero up to tens or even hundreds, and this is not possible working with atoms. This experimental tunability is an enormous advantage for QDs compared to atoms, since it offers the possibility to modify their electronic and optical properties through changes in size, shape and composition. For example, the color of the light emitted by CdSe QDs can be controlled by simply changing their size. 3. Bigger dots (radius of 5-6 nm) emit longer wavelengths like red, while smaller dots (radius of 2-3 nm) emit shorter wavelengths like green. This high tunability of properties is the reason why QDs are suitable for a wide range of applications such as photovoltaic devices, 4] biosensors, [5] quantum computation, 6] light emitting diodes (LEDs), [7] lasers, display technologies, [8, 9] etc.

Since the synthesis of the first quasi-zero-dimensional QDs in the late 1980s, the growth techniques have been greatly improved and nowadays it is possible to obtain high-quality semiconductor QDs of many shapes, sizes and materials. [10, 11] There is a wide variety of techniques for their production but, for the sake of brevity, only the three main approaches will be briefly discussed here. The first method of obtaining QDs was reported by Reed et al. [12] in 1986, who produced square QDs with a side length of $250 \mathrm{~nm}$ by means of lithographic techniques. Starting from a structure of quantum wells, where carriers are confined in one direction, small columns are etched and, thus, their motion is further restricted in the in-plane direction. The main disadvantage of the etching technique is the defect formation and contamination of the dots. A variant that circumvents this problem is to laterally confine the carriers by patterning several electrodes over the surface of the quantum well. 13 15 The QDs fabricated this way are flat and can be created with almost arbitrary lateral shape. Their diameter is of the order of 10-100 nm. Another synthesis technique is the growth of self-organized QDs by the Stranski-Krastanov method.[16, 17] This process consists in the epitaxial crystallization of one material on top of a layer of another one (usually referred to as wetting layer) with significantly different lattice constant. The first deposited monolayers are highly strained and, when a critical thickness is exceeded, a breakdown takes place. This originates randomly distributed three-dimensional (3D) islands of regular shape and size, usually known as self-assembled QDs, Their shape is typically pyramids, truncated pyramids, flat lenses or rings with heights of the order of $25 \mathrm{~nm}$ and base widths of $20 \mathrm{~nm}$. The biggest advantage of this method is its simplicity and the absence of edge defects. Lastly, it is also possible 
to fabricate QDS as colloidal semiconductor nanocrystals in glass dielectric matrices. [18] The first example of this method was carried out by Ekimov et al. [19], who created $\mathrm{CuCl}$ microcrystals in a solution of silicate glass. Colloidal QDs are nearly spherical in shape with small radii in the range of 1.2-10 nm. Such QDs can be further covered by a layer of another semiconductor material to form core-shell heterostructures that present altered properties in comparison to the uncovered ones. In light of the above, it is clear that the production method followed strongly determines the size and shape of the dots and, in turn, their properties. As for the materials used, typical dots are made of binary compounds of common zinc-blende (ZB) or wurtzite (WZ) semiconductors (PbS, PbSe, CdS, CdSe, InAs, GaAs, InP, GaN, InN and AlN) and their ternary and quaternary alloys. It is worth noting that QDs of some of these materials (e.g., GaAs and GaN) have been successfully synthesized in both crystal phases. Additionally, recent works have reported the fabrication of polytype QDS in which both $\mathrm{ZB}$ and WZ structures coexist within the same system. 20, 21] Moreover, since the discovery of graphene, 22 purely two-dimensional (2D) materials have been intensively studied and QDs made of graphene[23-25] and other related materials such as monoloyer transition metal dichalcogenides (TMDCs) 26 28] have also been fabricated. As can be seen, the diversity of semiconductor QDs is very rich and it is still growing.

In order to use QDs in real devices, a good understanding of their properties is needed from both experimental and theoretical points of view. As mentioned above, the optical and electronic properties of these structures are mainly governed by their shape, size and composition, i.e. by quantum confinement effects. Nevertheless, other factors that are intrinsic to the growth process, such as defects, impurities or crystal deformations to name a few, may also play an important role in their final performances. Therefore, it is crucial to identify and understand the phenomena that are relevant for each individual case under study, which will depend on the particular system and the application of interest. Then, based on this knowledge, QDs could be designed in order to enhance or diminish specific features. In addition to this, equally important is to have mechanisms to externally control and manipulate the system behavior in a reversible way. This is commonly done by switching on and off or changing the orientation of external electric and magnetic fields. Consequently, the effects of these fields in the conduction band (CB) and valence band (VB) of QDs must be also studied.

The aim of this Thesis is to theoretically investigate the electronic structure of semiconductor nanostructures. To this end, computational models are built to properly describe the $\mathrm{CB}$ and the $\mathrm{VB}$ of nanoscopic systems 
subject to various relevant phenomena. Particularly, we focus on QDs of different shape, dimensions, and composition to explore their behavior under external fields and interactions with the environment. Typical QDs are embedded into or grown on top of a different material, so that the lattice mismatch at the interface originates strain and this strain, in turn, gives rise to piezoelectricity. Here, the influence of these effects on the $\mathrm{CB}$ and $\mathrm{VB}$ electronic structure is explored, paying special attention to the role of the crystal phase, namely $\overline{\mathrm{ZB}}$, $\mathrm{WZ}$ or polytype crystal structure. Furthermore, the relaxation of the spin degree of freedom confined in QDS is also studied. Such spin scattering is mediated by the coupling of the spin states with acoustic phonons of the surrounding medium via the deformation and the piezoelectric potential mechanisms. For the relaxation to take place, the states involved in the transition need some spin admixture, which is produced by the spin-orbit interaction (SOI) in our case. By including all relevant sources of spin mixing (Rashba SOI (RSOI), Dresselhaus SOI (DSOI), and the coupling of light-hole (lh) and heavy-hole (hh) subbands) in a fully 3D model, it is shown that $\mathrm{SOI}$ is strongly anisotropic, which also translates into anisotropic spin relaxation. Additionally, the behavior of electrons and holes under externally applied fields is also investigated, focusing on the possibility of inducing ground state transitions and the emergence of the Aharonov-Bohm (AB) effect in quantum rings (QRs) as a consequence of their doubly-connected topology. Another topology-related effect is also analyzed in monolayer $\mathrm{MoS}_{2}$, a truly 2D system in which edge states are formed owing to the marginal topological character of the $\mathrm{MoS}_{2}$ material.

In the succeeding chapters we present the theoretical methods and the main findings of this Ph.D. Thesis together with a reasoned interpretation of the results. The remainder of this dissertation is structured as follows:

In chapter 2 we introduce the theoretical formalism used to model the electronic structure of the $\mathrm{CB}$ and the $\mathrm{VB}$ of semiconductor nanostructures 11 To be specific, the description of electrons and holes in such systems is carried out by means of the $\mathrm{k} \cdot \mathrm{p}$ method within the effective mass approximation (EMA) and the envelope function approximation (EFA), Briefly, it consists in a semi-empirical continuum model based on perturbation theory that provides good estimates of the low-energy properties at a relatively low computational cost. The Hamiltonians employed to investigate structures made of ZB, WZ, and mixed crystal phases (polytypes) are presented. Besides being computationally low demanding, $\mathrm{k} \cdot \mathrm{p}$ methods are also ad-

\footnotetext{
${ }^{1}$ We note that the details of very specific simulations will be given in the corresponding chapters.
} 
vantageous because they allow to take into account many phenomena by simply supplementing the base Hamiltonians with appropriate extra terms. In this respect, the basic aspects and the explicit Hamiltonians describing such phenomena are exposed, namely external electric and magnetic fields, [SOI] strain, and piezoelectricity. For the latter two, the corresponding fields are calculated using the continuum theory of elasticity.

Chapter 3 is dedicated to study the effects of applying an external magnetic field in two different systems. First, the electronic structure of the $\mathrm{VB}$ in axially-symmetric GaN/AlN cubic QDs is investigated. A positiondependent six-band Hamiltonian in cylindrical coordinates is derived to explore the hole spin purity and the possibility of modulating the energy spectrum via magnetic fields to cause inversions of the ground state. In this way, optical properties such as light polarization could be easily tuned. Second, the response of nanostructures in the multi-particle regime pierced by axial magnetic fields is analyzed. In particular, the system considered is a flat hexagonal QR defined as the cross-section of a multishell nanowire. Remarkable signatures of the discrete geometry symmetry and of the correlation are found in the $\mathrm{AB}$ oscillation patterns, which allow to justify observations reported in recent magnetoconductance experiments.

In chapter 4 we describe the physics of the spin of carriers confined in zero-dimensional structures with various shapes, dimensions and crystallographic orientations. Special attention is paid to the $\mathrm{SOI}$ and its role in the spin relaxation of electrons and holes. Both RSOI and DSOI effects are taken into account in a fully $3 \mathrm{D}$ model, going beyond the commonly employed quasi-2D simplified description in which cubic DSOI terms are disregarded. Indeed, the importance of including all three spatial dimensions is confirmed in self-assembled dots and core-shell nanocrystals which are clearly not flat. Also, the high anisotropic character of the spin relaxation is shown by varying the aspect ratio of the QDs and by rotating the orientation of external magnetic fields. Such anisotropy leads to substantial spin relaxation suppressions, offering the possibility to obtain long-lived spins. Furthermore, for the $\mathrm{VB}$ the geometry regime at which the different sources of spin mixing, i.e. SOI or $\mathrm{hh}$ hh coupling, prevail is identified. In addition, the intrinsic anisotropy of RSOI and DSOI is also demonstrated by studying the magnitude of the spin anticrossings in the energy spectra. All results are discussed in terms of the symmetry of the SOI Hamiltonians.

Chapter 5 deals with the influence of the environment on the properties of semiconductor QDs, Specifically, we account for the strain and piezoelectricity produced by the lattice mismatch between dot and surrounding materials. Interestingly, despite strain and piezoelectric fields can be 
undesirable for some applications, they offer the opportunity to fabricate strain-engineered QDs with improved performance. 29, 30] Two systems with different crystal structure are considered, core-shell [WZ nanocrystals and polytype QDs, in order to assess the role of the crystal phase in these phenomena. It is known that the generated piezoelectric fields are usually weak in $\mathrm{ZB}$ structures, but they turn out to be crucial in $\mathrm{WZ}$ and even more in polytype systems, where spontaneous polarization is found to predominate. It is shown that the resulting polarization fields strongly affect the electron-hole (e-h) spatial separation, thus enabling a substantial exciton lifetime tunability.

Lastly, in chapter 6 we investigate atomically thin structures. In particular, the electronic structure of monolayer $\mathrm{MoS}_{2}$ nanoribbons and QDs is analyzed. In such systems, states spatially localized near the edges and with energies lying in the band gap emerge, which play an important role in transport properties. The origin of these edge states is related to the marginal topological character of the system Hamiltonian.

The contents of the present report are based on the publications in which the author has contributed during the last four years. All of them have been published in international peer-reviewed journals. A copy of the works listed below can be found at the end of the present doctoral Thesis.

1. C. Segarra, J. I. Climente, and J. Planelles, Valence band mixing of cubic GaN/AlN quantum dots, J. Phys.: Condens. Matter 24, 115801 (2012)

2. J. Planelles, J. I. Climente, and C. Segarra, Electron spin relaxation in 3 Q quantum dots: geometrical suppression of Dresselhaus and Rashba spin-orbit interaction, J. Phys. Chem. C 116, 25143 (2012)

3. J. I. Climente, C. Segarra, and J. Planelles, Spin-orbit-induced hole spin relaxation in InAs and GaAs quantum dots, New J. Phys. 15, 093009 (2013)

4. A. Ballester, C. Segarra, A. Bertoni, and J. Planelles, Suppression of the Aharonov-Bohm effect in hexagonal quantum rings, EPL 104, 67004 (2013)

5. M. Royo, C. Segarra, A. Bertoni, G. Goldoni, and J. Planelles, AharonovBohm oscillations and electron gas transitions in hexagonal core-shell nanowires with an axial magnetic field, Phys. Rev. B 91, 115440 (2015)

6. C. Segarra, J. Planelles, and J. I. Climente, Control of electron spinorbit anisotropy in pyramidal InAs quantum dots, Physica E 66, 234 (2015)

7. C. Segarra, J. Planelles, J. I. Climente, and F. Rajadell, Anisotropy 
of spin-orbit induced electron spin relaxation in [001] and [111] grown GaAs quantum dots, New J. Phys. 17, 033014 (2015)

8. C. Segarra, J. I. Climente, F. Rajadell, and J. Planelles, Hole spin relaxation in InAs/GaAs quantum dot molecules, J. Phys.: Condens. Matter 27, 415301 (2015)

9. C. Segarra, J. Planelles, and S. E. Ulloa, Edge states in dichalcogenide nanoribbons and triangular quantum dots, Phys. Rev. B 93, 085312 (2016)

10. J. I. Climente, C. Segarra, F. Rajadell, and J. Planelles, Electrons, holes, and excitons in GaAs polytype quantum dots, J. Appl. Phys. 119, 125705 (2016)

11. C. Segarra, J. I. Climente, A. Polovitsyn, F. Rajadell, I. Moreels, and J. Planelles, Piezoelectric control of the exciton wave function in colloidal CdSe/CdS nanocrystals, J. Phys. Chem. Lett. 7, 2182 (2016) 



\section{снАРтвR 2}

\section{Theoretical framework}

The aim of this chapter is to provide the theoretical background of the methods used throughout this Thesis for the calculation of the QD electronic structure. A brief discussion of the general aspects of the methods is presented here and whoever interested in further details is invited to consult dedicated books. 31 33] Nevertheless, when extra information is required for understanding a specific topic, this will be given in the corresponding chapter.

The models typically employed to study QDs can be classified into two categories: atomistic and continuum models. Atomistic models, e.g. empirical pseudopotential and tight-binding methods, take all atoms of the crystal and their interactions explicitly into account in order to describe the behavior of the system. These models are generally considered more accurate because they are based on more fundamental principles, but have the disadvantage of being computationally expensive (typical QDs are composed by $10^{3}-10^{6}$ atoms) and the results are often hard to interpret. On the other hand, continuum models treat the systems as an ensemble of material domains whose properties are those of the bulk, thus ignoring the microscopic details. Although less accurate, these simpler models yield good estimates of the low-energy properties, offering more intuitive and computationally less demanding results.

Particularly, the approach taken for this Ph.D. project is the k.p method in the framework of the EMA and EFA. In spite of its simplicity, this continuum model has been successfully used to capture the main electronic and optical features of QDs at a reasonable computational cost. Additionally, it allows the implementation of phenomena such as externally applied fields or strain in a straightforward way. 


\section{$2.1 \quad$ The k·p method}

The $\mathrm{k} \cdot \mathrm{p}$ method was originally developed in the 1950s for the calculation of the band structure of bulk semiconductors and adapted to study heterostructures subsequently. It is a perturbative method that takes advantage of the crystal symmetries to predict the band structure as a function of only a few empirical parameters, which are obtained from experiments or ab initio calculations.

\subsubsection{General formulation of the $k \cdot p$ method}

An electron moving in a crystal, i.e. in the periodic potential of the atomic nuclei, is governed by the following Schrödinger equation including spinorbit:

$$
\left(\frac{\mathbf{p}^{2}}{2 m_{0}}+\frac{\hbar}{4 m_{0}^{2} c^{2}} \mathbf{p} \cdot\left(\boldsymbol{\sigma} \times \nabla V_{c r}(\mathbf{r})\right)+V_{c r}(\mathbf{r})\right) \psi(\mathbf{r})=E \psi(\mathbf{r}),
$$

where $V_{c r}(\mathbf{r})=V_{c r}(\mathbf{r}+\mathbf{R})$ is the periodic potential, $\mathbf{p}=-i \hbar \nabla, m_{0}$ is the free electron mass, $c$ is the velocity of light in vacuum and $\boldsymbol{\sigma}$ stands for the vector of Pauli spin matrices ${ }^{1}$ Taking into account Bloch's theorem, the wave function of a particle in a periodic potential can be written as the product of a plane wave, $e^{i \mathbf{k r}}$, and a periodic function with the same periodicity as the potential, $u_{n k}(\mathbf{r})=u_{n k}(\mathbf{r}+\mathbf{R})$. After substituting $\psi_{n k}(\mathbf{r})=e^{i \mathbf{k r}} u_{n k}(\mathbf{r})$ into (2.1) and left multiplying by $e^{-i \mathbf{k r}}$ one obtains

$$
\left[\frac{\mathbf{p}^{2}}{2 m_{0}}+\frac{\hbar^{2} k^{2}}{2 m_{0}}+\frac{\hbar}{4 m_{0}^{2} c^{2}} \mathbf{p} \cdot\left(\boldsymbol{\sigma} \times \nabla V_{c r}\right)+\frac{\hbar}{m_{0}} \mathbf{k} \cdot \boldsymbol{\pi}+V_{c r}\right] u_{n k}=E_{n k} u_{n k}
$$

with

$$
\boldsymbol{\pi}=\mathbf{p}+\frac{\hbar}{4 m_{0}^{2} c^{2}}\left(\boldsymbol{\sigma} \times \nabla V_{c r}\right) .
$$

The second term of $\boldsymbol{\pi}$, coming from the spin-orbit effect, has a small contribution and will be disregarded hereafter, so that $\boldsymbol{\pi}=\mathbf{p}$.

Equation $(2.2)$ is the basic formulation of the $\mathrm{k} \cdot \mathrm{p}$ method, whose name comes from the appearance of the $\mathbf{k} \cdot \mathbf{p}$ factor ${ }^{2}$. It can be solved for a fixed wave vector $\mathbf{k}=\mathbf{k}_{0}$, yielding a complete and orthonormal set of eigenfunctions $u_{n k_{0}}$. For simplicity, $\mathbf{k}=0$ is usually taken at the band extrema (the

\footnotetext{
1 The components of $\boldsymbol{\sigma}$ are $\sigma_{x}=\left(\begin{array}{ll}0 & 1 \\ 1 & 0\end{array}\right), \sigma_{y}=\left(\begin{array}{cc}0 & -i \\ i & 0\end{array}\right)$, and $\sigma_{z}=\left(\begin{array}{cc}1 & 0 \\ 0 & -1\end{array}\right)$.

${ }^{2}$ Note that $\mathbf{k}$ is a vector consisting of three real numbers with dimensions of inverse length, while $\mathbf{p}$ is a vector of operators.
} 
$\Gamma$ point in common semiconductors). Then, the band dispersion at finite $\mathbf{k}$ can be calculated by means of perturbation theory. To this end, equation 2.2 is rewritten as the sum of the Hamiltonian for $\mathbf{k}=0, H_{0}$, plus the k-dependent terms as a perturbation, $H_{k}^{\prime}$.

$$
(\underbrace{\frac{\mathbf{p}^{2}}{2 m_{0}}+\frac{\hbar}{4 m_{0}^{2} c^{2}} \mathbf{p} \cdot\left(\boldsymbol{\sigma} \times \nabla V_{c r}\right)+V_{c r}}_{H_{0}}+\underbrace{\frac{\hbar^{2} k^{2}}{2 m_{0}}+\frac{\hbar}{m_{0}} \mathbf{k} \cdot \mathbf{p}}_{H_{k}^{\prime}}) u_{n k}=E_{n k} u_{n k}
$$

In practice, only a limited number of functions can be taken as basis set. As a result, the validity of the results is restricted to a small area in the vicinity of the Brillouin zone center. Nevertheless, the physics of semiconductors is mostly governed by the carriers in the extrema of the various energy bands and, thus, the $\mathrm{k} \cdot \mathrm{p}$ method suffices to capture their main properties.

The choice of the bands included in the model depends on how isolated the bands of interest are. Let us consider a situation where the investigated band is far from the other bands. In such a case, $u_{n k}$ is mainly determined by $u_{n 0}$ and a basis set consisting of only this function can be used. This is true, as will become clear below, for the $\mathrm{CB}$ of most semiconductors. Applying second order non-degenerate perturbation theory to (2.4), the expressions for $E_{n k}$ and $u_{n k}$ are obtained

$$
E_{n k}=E_{n 0}+\frac{\hbar^{2} k^{2}}{2 m_{0}}+\frac{\hbar^{2}}{m_{0}^{2}} \sum_{n^{\prime} \neq n} \frac{\left|\left\langle u_{n 0}|\mathbf{k} \cdot \mathbf{p}| u_{n^{\prime} 0}\right\rangle\right|^{2}}{E_{n 0}-E_{n^{\prime} 0}}
$$

and

$$
u_{n k}=u_{n 0}+\frac{\hbar}{m_{0}} \sum_{n^{\prime} \neq n} \frac{\left\langle u_{n 0}|\mathbf{k} \cdot \mathbf{p}| u_{n^{\prime} 0}\right\rangle}{E_{n 0}-E_{n^{\prime} 0}} u_{n^{\prime} 0} .
$$

Here, $E_{n 0}$ and the so-called optical matrix elements $P_{n n^{\prime}}=\left\langle u_{n 0}|\mathbf{k} \cdot \mathbf{p}| u_{n^{\prime} 0}\right\rangle$ are unknown parameters that need to be inferred from experiments. Equation 2.5 can be rewritten as

$$
E_{n k}=E_{n 0}+\frac{\hbar^{2} k^{2}}{2 m^{*}}
$$

where $m^{*}$ is known as the effective mass of the band. This modified mass arises from the coupling of the considered band with other neighboring bands via the $\mathbf{k} \cdot \mathbf{p}$ term and has the following form:

$$
\frac{1}{m^{*}}=\frac{1}{m_{0}}+\frac{2}{m_{0}^{2} k^{2}} \sum_{n^{\prime} \neq n} \frac{\left|\left\langle u_{n 0}|\mathbf{k} \cdot \mathbf{p}| u_{n^{\prime} 0}\right\rangle\right|^{2}}{E_{n 0}-E_{n^{\prime} 0}} .
$$


The values of the effective masses are deduced from experimental data and are tabulated for most materials. A comparison between equation (2.7) and the Hamiltonian of a free electron show that both expressions are identical except for the mass. Then, the motion of an electron in a crystal can be seen as the motion of a free electron whose mass has been modified by the action of the periodic potential. This one-band model showing a quadratic dispersion relation is also known as EMA and, despite its simplicity, it has been extensively used in literature with surprisingly good results in the description of the $\mathrm{CB}$.

Contrary to the previous example, when studying the $\mathrm{VB}$ the bands of interest are commonly close in energy to other bands and they cannot be treated independently. In such a case, multiband models are necessary and the quasi-degenerate perturbation theory proposed by Löwdin [34] should be used. The $\mathbf{k} \cdot \mathbf{p}$ interaction between the few adjacent bands is explicitly taken into account, while the contribution from remote bands is introduced using the Löwdin perturbation theory. This approach results in a N-dimensional Hamiltonian containing additional terms of higher order in $\mathbf{k}$, with $\mathrm{N}$ being the number of bands included.

The separation between bands strongly depends on the material and its crystal structure. So, the model employed has to be selected based on the characteristics of the system under investigation. In sections 2.1.3 and 2.1.4 some typical cases will be discussed for $\mathrm{ZB}$ and $\mathrm{WZ}$ semiconductors. In general, single-band models for the $\mathrm{CB}$ and four- or six-band models for the $\mathrm{VB}$ are enough to obtain satisfactory results for most low-energy properties.

\subsubsection{Envelope function approximation}

The development of epitaxial growth techniques in the 1970s led to the fabrication of the first heterostructures with atomically sharp interfaces. In such systems, the carriers are confined due to the band misalignment of the constituting materials, as represented in figure 2.1 .

The breaking of the translational invariance at the interface prevents the use of the k.p model in these nanostructures. Several alternative theories have been suggested to overcome this problem but, among them, the ones based on the EFA are especially relevant. Following Bastard's formalism, 35. the EFA assumes the interface to be abrupt, defect-free and without interdiffusion effects, so that each domain can be taken as a perfect bulk

material. In addition, the materials are assumed to be perfectly matched and to present the same crystal structure. The latter allows one to take 


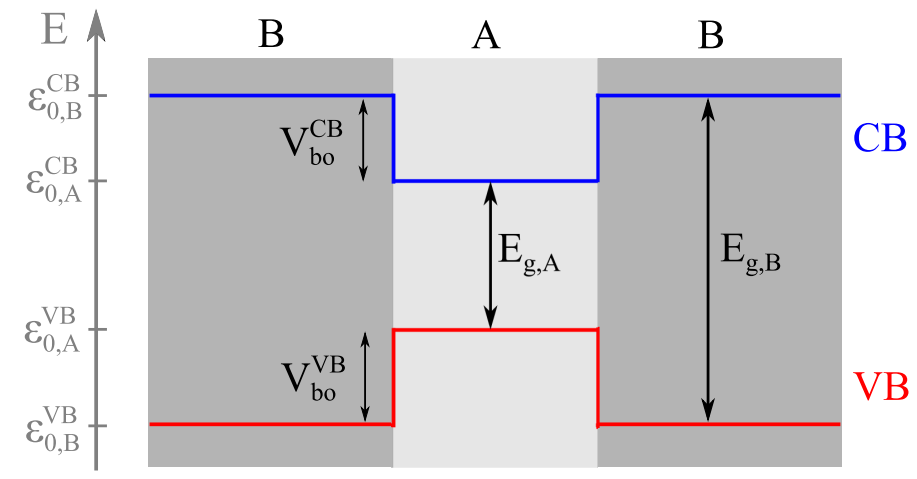

Figure 2.1: Sketch of the band edge profile along the $\mathrm{z}$ direction in a direct-gap heterostructure formed by two materials $\mathrm{A}$ and $\mathrm{B}$. Bandedge energies $\left(\varepsilon_{0, A}^{C B}, \varepsilon_{0, B}^{C B}, \varepsilon_{0, A}^{V B}\right.$ and $\left.\varepsilon_{0, B}^{V B}\right)$, band gaps $\left(E_{g, A}\right.$ and $\left.E_{g, B}\right)$ and band offsets $\left(V_{b o}^{C B}\right.$ and $\left.V_{b o}^{V B}\right)$ are indicated for both $\mathrm{CB}$ and $\mathrm{VB}$

the periodic parts of the Bloch functions to be the same in both materials, $u_{n k}^{A}=u_{n k}^{B}=u_{n k}$. Then, the wave function can be expanded as

$$
\psi_{n k}(\mathbf{r})=\sum_{n} f_{n}^{(A, B)}(\mathbf{r}) u_{n k_{0}}(\mathbf{r})
$$

where $f_{n}^{(A, B)}(\mathbf{r})$ is $f_{n}^{A}(\mathbf{r})$ or $f_{n}^{B}(\mathbf{r})$ depending on the region. This function varies slowly at the scale of the unit cell and it is usually referred to as envelope function.

For simplicity, a basis consisting of one band is used hereafter. Similarly to the derivation of the $\mathrm{k} \cdot \mathrm{p}$ method in the preceding section, equation (2.9) can be substituted into the Schrödinger equation (2.1) without spin-orbit and, after some algebraic manipulation ${ }^{3}$, one gets

$$
\left[-\frac{\hbar^{2}}{2 m_{0}} \nabla^{2}+\varepsilon_{0, A}+V_{b o}(\mathbf{r})\right] f_{n}^{(A, B)}(\mathbf{r})=E f_{n}^{(A, B)}(\mathbf{r}) .
$$

Here, $\varepsilon_{0, A}$ is the band energy of material $\mathrm{A}$ at $\mathbf{k}=0$ and $V_{b o}(\mathbf{r})$ is a step-like function that takes $V_{b o}(\mathbf{r} \in A)=0$ in layer $\mathrm{A}$ and $V_{b o}(\mathbf{r} \in B)=V_{b o}^{(C B, V B)}$ in layer B. Equation 2.10 is the second-order differential equation that governs the spatial behavior of the envelope function. This equation is solved after taking into account the appropriate boundary conditions. Typically, infinite barriers $(V=\infty)$ at the outer edges and the continuity of the wave function derivative at the interface are imposed.

\footnotetext{
${ }^{3}$ The details of the derivation have been omitted for brevity, but can be easily found in books, e.g. see chapter 3 of Bastard's book. 35.
} 
Just as the $\mathrm{k} \cdot \mathrm{p}$ method, this model can be further improved by perturbatively including the interaction with remote bands through the effective mass and by using several bands as basis in a multiband model. A comparison between both methods shows that the EFA Hamiltonian can be obtained from the $\mathrm{k} \cdot \mathrm{p}$ one by setting $\hbar \mathbf{k} \rightarrow-i \hbar \boldsymbol{\nabla}$ and adding a few terms. Hence, both models depend on the same set of parameters $P_{n n^{\prime}}$. Consequently, due to the close similarity, EFA Hamiltonians are also known as $\mathrm{k} \cdot \mathrm{p}-\mathrm{EFA}$ models.

The-one band model presented above is the simplest description of a heterostructure in which the presence of two materials is only taken into account through the band offset. Nevertheless, in such systems the effective mass $m^{*}$ and the other band parameters become position dependent, so that $\mathbf{k}$ and $m^{*}$ do not commute. This fact complicates the choice of the boundary condition at the interface. In fact, there has been much debate on the topic and several effective mass matching conditions have been proposed 4

The simplest model is the one based on BenDaniel-Duke boundary condition. [36] It considers a single parabolic and isotropic band and obtains a new Hamiltonian by changing the order of the differential operators in (2.10):

$$
-\frac{\hbar^{2}}{2 m^{*}} \nabla^{2} \text { is replaced by } \quad-\frac{\hbar^{2}}{2} \boldsymbol{\nabla} \frac{1}{m^{*}(\mathbf{r})} \boldsymbol{\nabla} \text {. }
$$

The new symmetrized Hamiltonian ensures the hermiticity and, thus, solutions with real eigenvalues and orthogonal eigenfunctions. The boundary condition is the continuity of $f_{n}(\mathbf{r})$ and $\frac{1}{m^{*}(\mathbf{r})} \frac{d f_{n}}{d \mathbf{r}}$ at the interface. It is interesting to notice that the effective mass mismatch leads to a discontinuity in the derivative of the envelope function at the interface.

Similarly, the operator symmetrization approach has also been widely used in the description of holes in heterostructures. In such a case, a generic matrix element of a $\mathrm{VB}$ multiband model

$$
\mathcal{H}=\sum_{i j} \mathcal{H}_{i j}^{(2)} k_{i} k_{j}+\sum_{i} \mathcal{H}_{i}^{(1)} k_{i}+\mathcal{H}^{(0)}
$$

is rewritten for a variable mass system as

$$
\mathcal{H}=\sum_{i j} k_{i} \mathcal{H}_{i j}^{(2)} k_{j}+\sum_{i}\left(\mathcal{H}_{i}^{(1)} k_{i}+k_{i} \mathcal{H}_{i}^{(1)}\right)+\mathcal{H}^{(0)}
$$

This reordering of the operators is, however, not strictly correct and may produce unrealistic results in some cases. As a consequence, in this Thesis

\footnotetext{
${ }^{4}$ A detailed discussion can be found in chapter 12 of Voon's book 31
} 
(a)

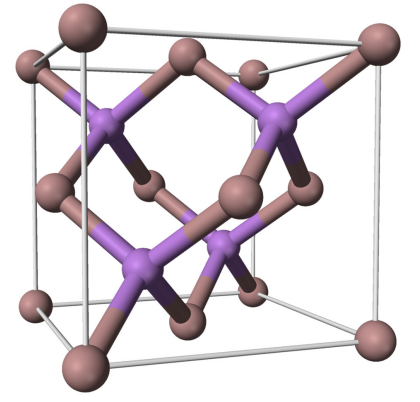

(b)

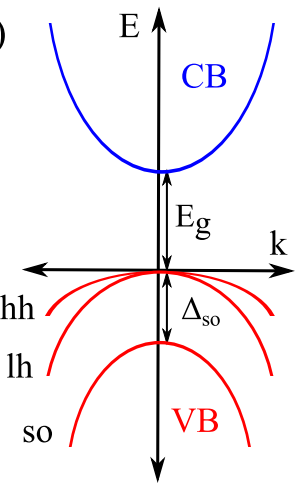

Figure 2.2: (a) Unit cell of ZB GaAs. Arsenic atoms are depicted in gray and gallium atoms in purple. (b) Schematic band dispersion of a $\mathrm{ZB}$ structure. The principal bands and their energy separations at the $\Gamma$ point are indicated. For finite $\mathbf{k}$ the valence band is split in three subbands: hh, Ih and split-off (so).

we follow the so-called Burt-Foreman model. Burt followed a completely different approach compared to Bastard's formalism. Instead of proposing an heuristic Hamiltonian and then search for valid solutions, he derived an exact envelope function theory from first-principles by first establishing constraints to the envelope function. 37-39] Later, Foreman used Burt's theory to derive an explicit multiband Hamiltonian and showed that this model gives reasonable results in particular cases where a symmetrized version of a conventional $\mathrm{k} \cdot \mathrm{p}$ model, namely the Luttinger-Kohn model, leads to nonphysical solutions. [40, 41]

\subsubsection{Hamiltonians for zinc-blende structures}

$\mathrm{ZB}$ is, together with $\mathrm{WZ}$, one of the most common crystal structures in which binary semiconductors are grown. Examples are GaAs, InAs, CdTe and AlSb, to name a few. The crystal lattice consists of a face-centered cubic array of anions with cations occupying one half of the tetrahedral holes as figure 2.2(a) illustrates. This structure lacks inversion symmetry and corresponds to one of the piezoelectric crystal classes. Piezoelectric effects in QDs will be discussed in section 2.4.2.

Figure 2.2(b) shows the typical band dispersion for direct band gap ZB materials around the $\Gamma$ point. Only four bands (eight with the spin degree of freedom) are depicted because other remote bands are far in energy and have negligible influence. 
On one hand, the band gap $E_{g}$ of the semiconductors studied in this Thesis is relatively large and allows the theoretical description of the $\mathrm{CB}$ in terms of a single-band model. The Hamiltonian reads

$$
H=-\frac{\hbar^{2}}{2} \nabla \frac{1}{m^{*}(\mathbf{r})} \boldsymbol{\nabla}+V(\mathbf{r})
$$

where a position-dependent effective mass $m^{*}(\mathbf{r})$ is assumed and $V(\mathbf{r})$ stands for the confining potential.

On the other hand, a multiband model is necessary to study the top of the $\mathrm{VB}$. In the absence of spin-orbit the three valence subbands are degenerate, but spin-orbit lifts this degeneracy even for $\mathbf{k}=0$. As shown in Fig. 2.2 (b), the so subband becomes separated from the other two by the spin-orbit splitting $\Delta_{s o}$. Then, depending on the magnitude of $\Delta_{s o}$, a fouror six-band model should be employed.

From a microscopic point of view, the electronic bands are formed due to the hybridization of the valence s- and p-orbitals. In fact, the $\mathrm{CB}$ and the $\mathrm{VB}$ are mainly made of s- and p-orbitals, respectively. Thus, the most simple set of unperturbed basis functions are the Bloch functions: $|S \uparrow\rangle$, $|X \uparrow\rangle,|Y \uparrow\rangle,|Z \uparrow\rangle,|S \downarrow\rangle,|X \downarrow\rangle,|Y \downarrow\rangle$ and $|Z \downarrow\rangle$. However, it is more convenient to use a basis set made of a linear combination of the above functions that is adapted to the total angular momentum. This new basis set i. 5

$$
\begin{aligned}
& \left|\frac{3}{2},+\frac{3}{2}\right\rangle=\frac{1}{\sqrt{2}}|(X+i Y) \uparrow\rangle, \\
& \left|\frac{3}{2},+\frac{1}{2}\right\rangle=\frac{1}{\sqrt{6}}|(X+i Y) \downarrow\rangle-\sqrt{\frac{2}{3}}|Z \uparrow\rangle, \\
& \left|\frac{3}{2},-\frac{1}{2}\right\rangle=-\frac{1}{\sqrt{6}}|(X-i Y) \uparrow\rangle-\sqrt{\frac{2}{3}}|Z \downarrow\rangle, \\
& \left|\frac{3}{2},-\frac{3}{2}\right\rangle=\frac{1}{\sqrt{2}}|(X-i Y) \downarrow\rangle, \\
& \left|\frac{1}{2},+\frac{1}{2}\right\rangle=\frac{1}{\sqrt{3}}|(X+i Y) \downarrow\rangle+\frac{1}{\sqrt{3}}|Z \uparrow\rangle, \\
& \left|\frac{1}{2},-\frac{1}{2}\right\rangle=-\frac{1}{\sqrt{3}}|(X-i Y) \uparrow\rangle+\frac{1}{\sqrt{3}}|Z \downarrow\rangle .
\end{aligned}
$$

\footnotetext{
${ }^{5}$ The definition of the basis set is not unique and Hamiltonians reported in literature may differ in the phase factors used. Here, we follow the standard basis generated with the angular momentum ladder operators. This is that employed in Voon's book. 31.
} 
(a)

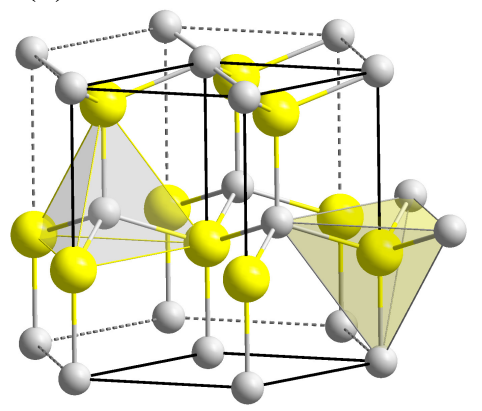

(b)

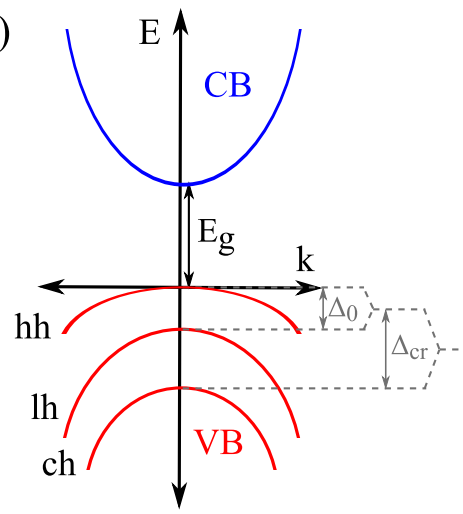

Figure 2.3: (a) Hexagonal structure of a WZ crystal, e.g. GaN. Gallium atoms are depicted in gray and nitrogen atoms in yellow. (b) Drawing of the band dispersion of a typical WZ semiconductor with $\Delta_{c r}>\Delta_{0}$. Band gap $E_{g}$, crystal-field splitting $\Delta_{c r}$ and spin-orbit splitting $\Delta_{0}$ are indicated.

In this basis, the total angular momentum and it's projection $\mathrm{J}_{z}$ become diagonal in matrix representation.

Throughout the present dissertation, various multiband models are used to investigate the valence band of QDs. The choice of a particular method is based on the material, specifically the value of $\Delta_{s o}$, and the particular type of QD studied. In appendix A, all Hamiltonians used are collected together for ease of reference. The four-band Luttinger-Kohn Hamiltonian for constant mass calculations is given in appendix section A.1, and the six-band variable-mass Burt-Foreman one in section A.2.

\subsubsection{Hamiltonians for wurtzite structures}

WZ is the other typical crystal structure for binary semiconductors. Among the compounds that can take the WZ structure are CdS, CdSe, GaN, AlN, etc. It is constructed from two interpenetrating hexagonal-close-packed lattices, as represented in figure 2.3(a). WZ and ZB crystals are quite similar since their structure differ only in the second-nearest neighbors. Nevertheless, this difference causes WZ to have lower symmetry and this, in turn, results in two additional features compared to $\mathrm{ZB}$ materials: the emergence of the crystal-field splitting $\Delta_{c r}$ and spontaneous polarization (pyroelectricity). The latter will be accounted for in section 2.4.2. 
Figure 2.3(b) displays the band structure of a common WZ semiconductor. Similarly to $\mathrm{ZB}$, four spinless bands are taken into account to study the properties of WZ QDs. However, this selection is not as clear as for ZB because the separation of the $\mathrm{CB}$ lowest in energy from other conduction bands is rather small for some WZ materials. 42 In spite of this, a singleband parabolic Hamiltonian is used in the present Thesis due to the lack of effective mass parameters describing such a coupling.

With respect to the $\mathrm{VB}$, it can be seen that, unlike $\mathrm{ZB}$, the three (spin doubly degenerate) valence bands are split at $\mathbf{k}=0$. In the absence of spin-orbit coupling, the hexagonal crystal field $\left(\Delta_{c r}\right)$ splits the p-like bands into two degenerate subbands and the crystal-field split hole (ch) subband. With the inclusion of spin-orbit coupling $\left(\Delta_{0}\right)$, the degenerate subband is further split into the hh and the $\mathrm{lh}$ subbands. In some works, the hh, $\mathrm{h}$ and $\mathrm{ch}$ subbands are also referred to as A, B and C subbands, respectively. It is interesting to notice that the order of the $\mathrm{lh}$ and $\mathrm{ch}$ shown in figure 2.3(b) can be altered depending on the values of $\Delta_{c r}$ and $\Delta_{0}$. Because of this, a six-band model is commonly used in WZ simulations.

Contrary to $\mathrm{ZB}$, the lower symmetry of WZ does not allow to find a basis set that diagonalizes the Hamiltonian at $\mathbf{k}=0$. The basis of Bloch functions considered here is 43 .

$$
\begin{aligned}
& \left|u_{1}\right\rangle=-\frac{1}{\sqrt{2}}|(X+i Y) \uparrow\rangle, \\
& \left|u_{2}\right\rangle=\frac{1}{\sqrt{2}}|(X-i Y) \uparrow\rangle, \\
& \left|u_{3}\right\rangle=|Z \uparrow\rangle, \\
& \left|u_{4}\right\rangle=\frac{1}{\sqrt{2}}|(X-i Y) \downarrow\rangle, \\
& \left|u_{5}\right\rangle=-\frac{1}{\sqrt{2}}|(X+i Y) \downarrow\rangle, \\
& \left|u_{6}\right\rangle=|Z \downarrow\rangle .
\end{aligned}
$$

Various authors have reported six-band models to study the WZ[VB, 32, 43, 44] In this Thesis, a position-dependent Hamiltonian derived following Burt-Foreman operator ordering is used. [45] The matrix representation can be found in appendix A.2. This Hamiltonian depends on 6 mass parameters $A_{1-6}$ and three energy splittings $\Delta_{1-3}$, with $\Delta_{1}=\Delta_{c r}$, and $\Delta_{2}$ and $\Delta_{3}$ being the spin-orbit matrix elements $\left(\Delta_{2}=\Delta_{3}=\Delta_{\text {so }} / 3\right.$ in the so-called quasi-cubic approximation). 


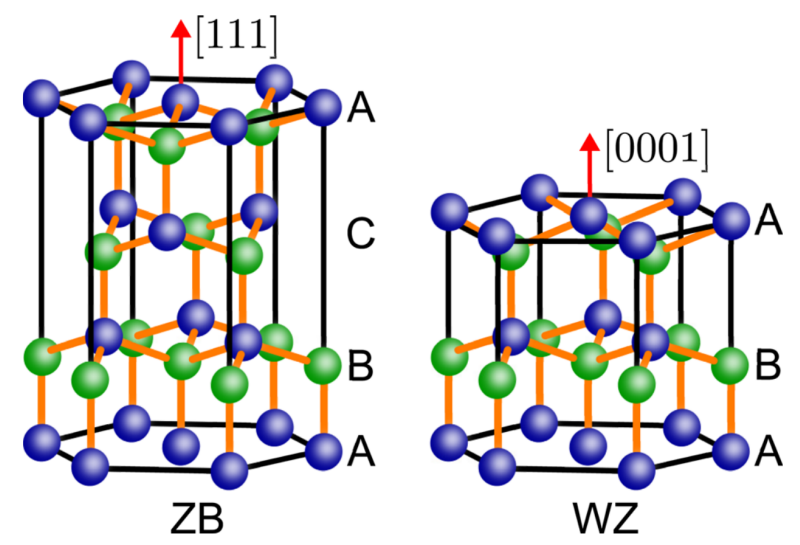

Figure 2.4: Crystal structures and their stacking sequence for (left) $\mathrm{ZB}$ in the [111] direction and (right) WZ in the [0001] direction. Reprinted with permission from [46]. Copyright 2012, AIP Publishing LLC.

\subsubsection{Hamiltonians for polytypes}

As mentioned above, $\mathrm{WZ}$ and $\mathrm{ZB}$ crystals present close similarities. This becomes evident when considering the $\mathrm{ZB}$ structure in the [111] direction and comparing it to $\mathrm{WZ}$ [0001]. In figure 2.4, it can be seen that both structures only differ in the stacking order of the layers: ABCABC for $\mathrm{ZB}$ while $\mathrm{ABABAB}$ for $\mathrm{WZ}$. As a result, polytypical nanostructures consisting of $\mathrm{ZB}$ [111] and WZ [0001] phases of the same material have been successfully fabricated. [20, 21] These systems present typical characteristics of heterostructures formed by different materials because the band gap and the parameters also depend on the crystalline phase.

The theoretical study of these systems requires a model able to describe both crystal structures simultaneously. For the $\mathrm{CB}$, this can be done by simply considering a different effective mass for each region in a positiondependent one-band Hamiltonian. For the $\mathrm{VB}$, instead, this may seem a complicate task in view of the six-band Hamiltonians proposed for both structures in appendix A. However, Bir and Pikus realized in their book (see page 328 in [32]) that a transformation of the ZB Hamiltonian to the appropriate coordinate system ${ }^{6}$ yields a new Hamiltonian which is similar

\footnotetext{
${ }^{6}$ In the new coordinate system, the $z^{\prime}$-axis is along [111] direction and $x^{\prime}$ - and $y^{\prime}$-axis are along $[11 \overline{2}]$ and [110] directions, respectively. To perform this transformation, the Hamiltonian is first rotated $45^{\circ}$ along the $z$-axis and then $54.7^{\circ}$ along the new $y^{\prime}$-axis. This rotation procedure is well described in [47.
} 
to the WZ one. This opens the possibility of constructing a general Hamiltonian for the whole system and then particularize it to the structure of each region by considering the pertinent parameters.

In order to compare both Hamiltonians systematically the Bloch basis functions of lower symmetry should be used, i.e. the basis set of WZ given in 2.16. In this basis, one gets a Hamiltonian for $\mathrm{ZB}$ that is formally identical to the standard WZ one, equation A.4 , but now two extra terms emerge:

$$
\begin{aligned}
\Delta K & =2 \sqrt{2} \frac{\hbar^{2}}{2 m_{0}} A_{z} k_{-} k_{z}, \\
\Delta H & =\frac{\hbar^{2}}{2 m_{0}} A_{z} k_{-}^{2} .
\end{aligned}
$$

These terms are zero for $\mathrm{WZ}\left(A_{z}=0\right)$ and allow the $\mathrm{ZB}$ Hamiltonian to regain the original isotropic symmetry. In addition to this, the following relations arise connecting the mass parameters and energy splittings of both structures:

$$
\begin{aligned}
& \Delta_{1}=0, \\
& \Delta_{2}=\Delta_{3}=\Delta_{s o} / 3, \\
& A_{1}=-\gamma_{1}-4 \gamma_{3}, \\
& A_{2}=-\gamma_{1}+2 \gamma 3, \\
& A_{3}=6 \gamma_{3}, \\
& A_{4}=-3 \gamma_{3}, \\
& A_{5}=-\gamma_{2}-2 \gamma_{3}, \\
& A_{6}=-\sqrt{2}\left(2 \gamma_{2}+\gamma_{3}\right), \\
& A_{z}=\gamma_{2}-\gamma_{3} .
\end{aligned}
$$

Taking into account (2.18) reduces the number of independent parameters from 9 in WZ to 4 in $\overline{\mathrm{ZB}}$, as expected from symmetry considerations.

The full six-band Hamiltonian derived to study polytypes can be consulted in appendix A.3.1. It is worth noting that all diagonal elements are over stabilized by $\Delta_{s o} / 3$ when using $\mathrm{ZB}$ parameters. Thus, equation A.3.1 must be corrected by subtracting this amount in the $\mathrm{ZB}$ region.

For simplicity, the above discussion has considered a situation with constant mass. Nevertheless, since the parameters in the two phases are different, it is more appropriate to use a position-dependent Hamiltonian and, thus, this is the model employed in all the polytype calculations of this Thesis. Starting from the WZ variable-mass Hamiltonian, equation (A.4), and 
following the same procedure as before, a position-dependent Hamiltonian for polytype systems is constructed. See appendix A.3.2 to consult its full matrix form. As expected from Foreman [40], the resulting Hamiltonian presents some extra coefficients compared to A.5.

\section{$2.2 \quad$ Externally applied fields}

Particle energy levels are modified in the presence of external fields. This paves the way for manipulating the properties of QDs and, thus, controlling devices by external means.

\subsubsection{Electric field}

An external homogeneous electric field pulls electrons and holes towards opposite directions, leading to lower e-h overlaps and the suppression of exciton recombination processes. In addition, it is also responsible for the quantumconfined Stark effect, which generates a redshift of the emitted/absorbed light.

Accounting for static electric fields $\mathbf{F}$ into $\mathrm{k} \cdot \mathrm{p}$ Hamiltonians is straightforward. An extra potential energy $V_{F}$ needs to be added to the confining potential of the heterostructure

$$
V_{F}(\mathbf{r})=-e \mathbf{F} \cdot \mathbf{r}
$$

where $e$ is the particle charge, $e=-1$ for electrons and $e=1$ for holes.

It is worth stressing that the presence of electric fields may also give rise to other phenomena in nanostructures, e.g. the Rashba SOI (see section 2.3.2.

\subsubsection{Magnetic field}

The application of magnetic fields to QDs originates shifts in the energy spectrum and lifts of spin degeneracies. The latter phenomenon is known as Zeeman effect and is the magnetic field analogous of the Stark effect.

The standard way of including a magnetic field $\mathbf{B}$ in the $\mathrm{k} \cdot \mathrm{p}-\mathrm{EFA}$ formalism is via minimal coupling, i.e. by replacing the canonical momentum $\mathbf{p}$ by the kinetic momentum $-i \hbar \boldsymbol{\nabla}-e \mathbf{A}$ and adding the Zeeman term to the 
Hamiltonian. Here, $e$ is the particle charge and $\mathbf{A}$ is the vector potential defining the magnetic field $\mathbf{B}=\boldsymbol{\nabla} \times \mathbf{A}$. The choice of the vector potential is not unique and it is common practice to use a different version based on the symmetry of the problem.

In a one-band isotropic model describing the $\mathrm{CB}$ and with positiondependent effective mass, the resulting Hamiltonian is as follows:

$$
H=\frac{1}{2}(-i \hbar \boldsymbol{\nabla}-e \mathbf{A}) \frac{1}{m^{*}(\mathbf{r})}(-i \hbar \boldsymbol{\nabla}-e \mathbf{A})+V(\mathbf{r})+\frac{g^{*}}{2} \mu_{B} \boldsymbol{\sigma} \cdot \mathbf{B}
$$

The last term in (2.20) is the Zeeman splitting, where $g^{*}$ is the effective Landé g-factor, $\mu_{B}=\frac{|e| \hbar}{2 m_{0}}$ the Bohr magneton and $\boldsymbol{\sigma}$ is a vector whose components are the Pauli matrices.

As for the magnetic field implementation in multiband models, the minimal coupling approach first used by Luttinger [48] has been widely employed in literature, providing satisfactory results for many experimental evidences. However, this model has been unable to describe some particular observations, such as the photoluminescence (PL) magnetoresonances in QRs under axial magnetic fields. 49] In this regard, recent works have proposed a new approach which outperforms the Luttinger approximation. [50, 51] It consists in performing the same replacement, $\mathbf{p} \rightarrow-i \hbar \boldsymbol{\nabla}-e \mathbf{A}$, but now prior to applying the EFA. The Hamiltonian obtained, unlike the Luttinger model, has no off-diagonal terms depending on $\mathbf{B}$, thus reducing the coupling between hole subbands.

We particularize this approach for a $\mathrm{ZB}$ constant mass system under an axial magnetic field $\mathbf{B}=\left(0,0, B_{0}\right)$ defined by the vector potential $\mathbf{A}=$ $\frac{B_{0}}{2}(-y, x, 0)$. In this specific case, the six-band Hamiltonian supplementing the zero-field one A.2 , presents the following diagonal elements:

$$
\begin{aligned}
& H_{11}^{B}=-\left(\gamma_{1}+\gamma_{2}\right)\left[\frac{B_{0}^{2}\left(x^{2}+y^{2}\right)}{8}+\frac{B_{0}}{2}\left(x p_{y}-y p_{x}\right)\right]-\frac{3}{2} \kappa \mu_{B} B_{0} \\
& H_{22}^{B}=-\left(\gamma_{1}-\gamma_{2}\right)\left[\frac{B_{0}^{2}\left(x^{2}+y^{2}\right)}{8}+\frac{B_{0}}{2}\left(x p_{y}-y p_{x}\right)\right]-\frac{1}{2} \kappa \mu_{B} B_{0} \\
& H_{33}^{B}=-\left(\gamma_{1}-\gamma_{2}\right)\left[\frac{B_{0}^{2}\left(x^{2}+y^{2}\right)}{8}+\frac{B_{0}}{2}\left(x p_{y}-y p_{x}\right)\right]+\frac{1}{2} \kappa \mu_{B} B_{0} \\
& H_{44}^{B}=-\left(\gamma_{1}+\gamma_{2}\right)\left[\frac{B_{0}^{2}\left(x^{2}+y^{2}\right)}{8}+\frac{B_{0}}{2}\left(x p_{y}-y p_{x}\right)\right]+\frac{3}{2} \kappa \mu_{B} B_{0} \\
& H_{55}^{B}=-\gamma_{1}\left[\frac{B_{0}^{2}\left(x^{2}+y^{2}\right)}{8}+\frac{B_{0}}{2}\left(x p_{y}-y p_{x}\right)\right]-\frac{1}{2} \kappa^{\prime} \mu_{B} B_{0}
\end{aligned}
$$




$$
H_{66}^{B}=-\gamma_{1}\left[\frac{B_{0}^{2}\left(x^{2}+y^{2}\right)}{8}+\frac{B_{0}}{2}\left(x p_{y}-y p_{x}\right)\right]+\frac{1}{2} \kappa^{\prime} \mu_{B} B_{0}
$$

with $\kappa$ and $\kappa^{\prime}$ standing for the hole effective g-factors and $\mu_{B}=\frac{|e| \hbar}{2 m_{0}}$.

As can be seen in (2.20) and (2.21), the Zeeman energy splitting is mainly determined by the value of the effective g-factor. In bulk systems, the $\mathrm{SOI}$ causes the $\mathrm{g}$-factor to deviate from the bare electron value $g \approx 2$ and effective g-factors $g^{*}$ are inferred experimentally. In QD simulations it is common to use these bulk effective g-factors, although some works have pointed out the quenching of the SOI-induced deviation in QDs due to confinement.[52] In this respect, van Bree et al. [53] suggest to disregard the contribution from remote bands and simply consider the bare Landé g-factors: $g^{*}=2, \kappa=4 / 3$ and $\kappa^{\prime}=2 / 3$.

\section{$2.3 \quad$ Spin-orbit interaction (SOI)}

In this section, we will present a theoretical description of the SOI effects on the $\mathrm{CB}$ and the $\mathrm{VB}$ of $\mathrm{ZB}$ materials. The $\mathrm{SOI}$ in $\mathrm{WZ}$ semiconductors is omitted here since in the present Thesis the spin dynamics of these crystal structures is not investigated.

In atomic physics, the $\mathrm{SOI}$ is a well-known phenomenon originating from the coupling of the electron spin to its orbital momentum via the electric field generated by the nuclei. Similarly, the SOI in solids comes from the interaction between the spin and the average electric field of the lattice nuclei. The most relevant effect of $\mathrm{SOI}$ on the band structure of semiconductors is the degeneracy breaking of the three topmost $\mathrm{VB}$ subbands. In particular, in cubic semiconductors such as $\mathrm{ZB}$ or diamond crystal structures this causes the energy separation at the center of the Brillouin zone of the so band from the $\mathrm{lh}$ and $\mathrm{hh}$ ones, which remain degenerate. In addition to this, $\mathrm{SOI}$ is also responsible for the spin splitting of the bands in materials lacking inversion symmetry, e.g. ZB semiconductors, even in the absence of a magnetic field. As a result, the $\mathrm{CB}$ and the three $\mathrm{VB}$ are no longer doubly spin-degenerate. The latter effect is, however, relatively small and it does not significantly affect most electronic properties, thus justifying not including it in many studies. Nonetheless, it may play an important role when investigating the properties of the spin degree of freedom, [54 56] such as the spin dynamics we will deal with in chapter 4 .

The models discussed in the previous sections take into account the band splitting of the $\mathrm{VB}$ through the $\mathrm{SOI}$ term in $H_{0}$, equation (2.4), but 
ignore the spin-orbit-induced spin splitting. In order to include this effect, the Hamiltonians need to be supplemented by extra terms coming from the SOI contribution in equation 2.3 that was initially disregarded. The expressions of these additional terms can be obtained by using both the theory of invariants or perturbation theory up to third or fourth order. For the sake of brevity, their derivation will not be presented here, but we invite the interested reader to consult Winkler's book [33] for a detailed presentation.

Next, we will briefly discuss the origin and introduce the Hamiltonians of the two main spin-orbit sources of spin splitting in $\mathrm{ZB}$ materials: DSOI and RSOI.

\subsubsection{Dresselhaus SOI}

The DSOI is an intrinsic property of some materials resulting from the absence of an inversion center in its crystal structure. [57] In such a case, the microscopic electric fields generated by the lattice atoms do not cancel each other, thus originating a net contribution to the SOI. This phenomenon is also known as bulk inversion asymmetry (BIA).

For the $\mathrm{CB}$, there are no terms up to second order in $k$, so that the cubic contributions are the lowest-order terms that characterize DSOI. The corresponding Hamiltonian for electrons reads: 33 ]

$$
H_{B I A}^{C B}=b_{41}^{C B}\left[\sigma_{x} k_{x}\left(k_{z}^{2}-k_{y}^{2}\right)+\sigma_{y} k_{y}\left(k_{x}^{2}-k_{z}^{2}\right)+\sigma_{z} k_{z}\left(k_{y}^{2}-k_{x}^{2}\right)\right],
$$

where $b_{41}^{C B}$ is a material-dependent parameter.

On the other hand, the contribution of DSOI in the $\mathrm{VB}$ includes linearand third-order-in- $k$ terms and is given by: 33

$$
\begin{aligned}
H_{B I A}^{V B} & =\frac{2}{\sqrt{3}} C_{k}\left[k_{x}\left\{J_{x}, J_{y}^{2}-J_{z}^{2}\right\}+\mathrm{cp}\right] \\
& +b_{41}^{V B}\left[\left\{k_{x}, k_{y}^{2}-k_{z}^{2}\right\} J_{x}+\mathrm{cp}\right] \\
& +b_{42}\left[\left\{k_{x}, k_{y}^{2}-k_{z}^{2}\right\} J_{x}^{3}+\mathrm{cp}\right] \\
& +b_{51}\left[\left\{k_{x}, k_{y}^{2}+k_{z}^{2}\right\}\left\{J_{x}, J_{y}^{2}-J_{z}^{2}\right\}+\mathrm{cp}\right] \\
& +b_{52}\left[k_{x}^{3}\left\{J_{x}, J_{y}^{2}-J_{z}^{2}\right\}+\mathrm{cp}\right],
\end{aligned}
$$

with $C_{k}, b_{41}^{V B}, b_{42}, b_{51}$ and $b_{52}$ being material-dependent coefficients, cp standing for cyclic permutations of the preceding terms, and $\{A, B\}=$ $\frac{1}{2}(A B+B A)$.

The matrix form of Hamiltonians (2.22) and (2.23) can be found in appendix B.1. 


\subsubsection{Rashba SOI}

Besides the bulk inversion asymmetry, a spin splitting can also be produced by the structure asymmetry generated by the confining potential of the heterostructure itself and/or an externally applied electric field. [58. Consequently, this effect is also referred to as structure inversion asymmetry (SIA). Unlike DSOI, RSOI is a combined effect of the microscopic electric fields of the nuclei and the macroscopic external field felt by the system. Both of them must be present in order to have RSOI

The Hamiltonian for the $\mathrm{CB}$ is linear in $k$ and presents the following form:

$$
H_{S I A}^{C B}=r_{41} \boldsymbol{\sigma} \cdot(\mathbf{k} \times \mathbf{F}) .
$$

Here, $r_{41}$ is a material-specific prefactor and $\mathbf{F}$ is an external electric field. Equation (2.24) points out that the magnitude of RSOI is proportional to both $r_{41}$, which is determined by the microscopic details of the lattice, and the macroscopic field. This fact is very important because it allows to tune the strength of this effect by changing the intensity of $\mathbf{F}$. The explicit matrix representation of 2.24 is given in appendix B.2.

As for the $\mathrm{VB}$, the RSOI contribution has been disregarded in all simulations included in this dissertation since it is less efficient than DSOI for moderate electric fields. This has been checked by carrying out a series of preliminary calculations for the particular systems investigated.

\subsection{Strain and polarization fields}

Heterostructures composed of various semiconductors, e.g. self-assembled QDs and core-shell nanocrystals, may present crystal deformations at the heterointerface originating from the lattice mismatch of the constituent materials. The resulting displacement of the lattice nuclei from their original equilibrium positions generates strain fields which, in turn, produce changes in the band structure of the system. Additionally, in non-centrosymmetric crystal structures such as $\mathrm{ZB}$ and $\mathrm{WZ}$, these strain fields lead to a piezoelectric polarization that also affects the $\mathrm{QD}$ electronic and optical properties.

In this section, the theoretical framework for the calculation of strain and polarization fields is presented for both $\mathrm{ZB}$ and $\mathrm{WZ}$ structures. Next, we explain how these effects are accounted for within $\mathrm{k} \cdot \mathrm{p}$ formalism. 


\subsubsection{Strain}

Strain fields can be calculated using the continuum theory of elasticity established by Cauchy and Poisson in the 1820s. [59] The strain tensor $\epsilon_{i j}(\mathbf{r})$ arising from the displacement field $\mathbf{u}(\mathbf{r})$ is defined by

$$
\epsilon_{i j}(\mathbf{r})=\frac{1}{2}\left(\frac{\partial u_{i}(\mathbf{r})}{\partial x_{j}}+\frac{\partial u_{j}(\mathbf{r})}{\partial x_{i}}\right) .
$$

This strain can be related to the stress forces by using the generalized Hooke's law

$$
\sigma_{i j}(\mathbf{r})=C_{i j k l} \epsilon_{k l}(\mathbf{r})
$$

where $\sigma_{i j}$ denotes the stress tensor and $C_{i j k l}$ is the four-rank stiffness tensor 7 The number of independent constants in $C_{i j k l}$ is determined by the symmetry of the crystal structure. The volumetric elastic energy of the system is formulated as a function of these tensors as follows: 60]

$$
U=\frac{1}{2} \sigma_{i j} \epsilon_{i j}=\frac{1}{2} C_{i j k l} \epsilon_{i j} \epsilon_{k l} .
$$

In practice, the system is initially considered as the matrix material not strained and the QD compressed/expanded by an initial strain that is estimated from the lattice constants of the materials. Then, the system is allowed to relax to the equilibrium state and the strain and displacement fields are calculated by minimizing the elastic energy.

\section{Strain in [001]-grown ZB structures}

In cubic materials as $\mathrm{ZB}$, strain is isotropic and the initial strain of the $\mathrm{QD}$ is calculated as

$$
\epsilon_{x x}^{0}=\epsilon_{y y}^{0}=\epsilon_{z z}^{0}=\left(\frac{a_{Q D}-a_{m}}{a_{m}}\right)
$$

with $a_{m}$ and $a_{Q D}$ denoting the lattice parameter of the matrix and the QD materials, respectively. Here, $\epsilon^{0}>0$ indicates expansion and $\epsilon^{0}<0$ compression of the QD.

Due to the high symmetry of cubic crystals only three elastic constants are independent. The stiffness tensor in Voigt notation $\left(C_{x x x x}=C_{11}\right.$,

\footnotetext{
${ }^{7}$ Note that there is an implied sum over repeated indices (Einstein summation notation).
} 
$C_{x x y y}=C_{12}$, and $\left.C_{x y x y}=C_{44}\right)$ is as follows: 61]

$$
C_{Z B}=\left(\begin{array}{cccccc}
C_{11} & C_{12} & C_{12} & 0 & 0 & 0 \\
C_{12} & C_{11} & C_{12} & 0 & 0 & 0 \\
C_{12} & C_{12} & C_{11} & 0 & 0 & 0 \\
0 & 0 & 0 & C_{44} & 0 & 0 \\
0 & 0 & 0 & 0 & C_{44} & 0 \\
0 & 0 & 0 & 0 & 0 & C_{44}
\end{array}\right) .
$$

Substituting 2.29 into 2.27 one obtains the expression to compute the strain energy of cubic structures. It reads

$$
\begin{aligned}
U_{Z B}= & \frac{1}{2}\left[C_{11}\left(\epsilon_{x x}^{2}+\epsilon_{y y}^{2}+\epsilon_{z z}^{2}\right)+2 C_{12}\left(\epsilon_{x x} \epsilon_{y y}+\epsilon_{x x} \epsilon_{z z}+\epsilon_{y y} \epsilon_{z z}\right)\right. \\
& \left.+4 C_{44}\left(\epsilon_{x y}^{2}+\epsilon_{x z}^{2}+\epsilon_{y z}^{2}\right)\right] .
\end{aligned}
$$

\section{Strain in [0001]-grown WZ structures}

WZ crystal structure is anisotropic and the unit cell is defined by two lattice constants: one in the $z$ direction $(c)$ and the other in the in-plane direction (a). Therefore, the initial strain will also depend on the direction, being

$$
\epsilon_{x x}^{0}=\epsilon_{y y}^{0}=\left(\frac{a_{Q D}-a_{m}}{a_{m}}\right) \quad \text { and } \quad \epsilon_{z z}^{0}=\left(\frac{c_{Q D}-c_{m}}{c_{m}}\right),
$$

where $a_{Q D}$ and $c_{Q D}$ are the lattice parameters of the QD, and $a_{m}$ and $c_{m}$ are the ones of the matrix material.

WZ structures have lower symmetry and five different constants are required to define the stiffness tensor 61]

$$
C_{W Z}=\left(\begin{array}{cccccc}
C_{11} & C_{12} & C_{13} & 0 & 0 & 0 \\
C_{12} & C_{11} & C_{13} & 0 & 0 & 0 \\
C_{13} & C_{13} & C_{33} & 0 & 0 & 0 \\
0 & 0 & 0 & C_{44} & 0 & 0 \\
0 & 0 & 0 & 0 & C_{44} & 0 \\
0 & 0 & 0 & 0 & 0 & C_{66}
\end{array}\right)
$$

Here, $C_{x x x x}=C_{11}, C_{x x y y}=C_{12}, C_{z z z z}=C_{33}, C_{x x z z}=C_{13}, C_{x y x y}=C_{44}$, and $C_{66}=\frac{1}{2}\left(C_{11}-C_{12}\right)$. 
Finally, the elastic energy in strained WZ systems is given by

$$
\begin{aligned}
U_{W Z}= & \frac{1}{2}\left[C_{11}\left(\epsilon_{x x}^{2}+\epsilon_{y y}^{2}\right)+C_{33} \epsilon_{z z}^{2}+2 C_{12} \epsilon_{x x} \epsilon_{y y}+2 C_{13} \epsilon_{z z}\left(\epsilon_{x x}+\epsilon_{y y}\right)\right. \\
& \left.+4 C_{44}\left(\epsilon_{x z}^{2}+\epsilon_{y z}^{2}\right)+2\left(C_{11}-C_{12}\right) \epsilon_{x y}^{2}\right] .
\end{aligned}
$$

\subsubsection{Piezoelectric polarization}

The application of an external strain causes the displacement of the charged atomic nuclei from their original positions in the crystal. In semiconductor materials lacking a center of inversion this displacement produces an electric polarization. The magnitude of such polarization is, neglecting higher-order contributions, proportional to the strain field as:

$$
P_{i}(\mathbf{r})=e_{i j k} \epsilon_{j k}(\mathbf{r}),
$$

with $e_{i j k}$ being the piezoelectric tensor.

The charge density $\rho(\mathbf{r})$ arising from the polarization $\mathbf{P}(\mathbf{r})$ is given by

$$
\rho(\mathbf{r})=-\nabla \cdot \mathbf{P}(\mathbf{r})
$$

Then, the corresponding electrostatic potential $\phi_{p z}(\mathbf{r})$ generated by $\rho(\mathbf{r})$ is obtained by solving Poisson's equation

$$
\varepsilon_{0} \nabla\left[\varepsilon_{r}(\mathbf{r}) \cdot \nabla \phi_{p z}(\mathbf{r})\right]=-4 \pi \rho(\mathbf{r}),
$$

where $\varepsilon_{0}$ is the dielectric constant in vacuum and $\varepsilon_{r}$ is the material-dependent dielectric tensor.

\section{Piezoelectric polarization in [001]-grown $\mathrm{ZB}$ structures}

For ZB crystals, only one independent coefficient does not vanish in the piezoelectric tensor $e_{i j k}$. It reads

$$
e_{Z B}=\left(\begin{array}{cccccc}
0 & 0 & 0 & e_{14} & 0 & 0 \\
0 & 0 & 0 & 0 & e_{14} & 0 \\
0 & 0 & 0 & 0 & 0 & e_{14}
\end{array}\right)
$$

and the resulting polarization after applying equation 2.34 is

$$
\mathbf{P}(\mathbf{r})=e_{14}\left(\begin{array}{c}
\epsilon_{y z} \\
\epsilon_{x z} \\
\epsilon_{x y}
\end{array}\right) \text {. }
$$




\section{Piezoelectric polarization in [0001]-grown WZ structures}

The piezoelectric tensor for WZ semiconductors depends on three nonvanishing coefficients:

$$
e_{W Z}=\left(\begin{array}{cccccc}
0 & 0 & 0 & 0 & e_{15} & 0 \\
0 & 0 & 0 & e_{15} & 0 & 0 \\
e_{31} & e_{31} & e_{33} & 0 & 0 & 0
\end{array}\right)
$$

In contrast to cubic ZB systems, WZ materials present an additional contribution to the total polarization, the so-called spontaneous polarization or pyroelectricity. It is present even without strain and originates from the inversion symmetry breaking along the $c$ axis in the WZ lattice. 62 The spontaneous polarization in WZ systems is a constant vector in the $z$ direction, $\mathbf{P}_{s p}=\left(0,0, P_{s p}\right)$, where $P_{s p}$ is material dependent. Therefore, the total polarization $\mathbf{P}(\mathbf{r})$ coming from both contributions is given by

$$
\mathbf{P}(\mathbf{r})=\mathbf{P}_{p z}(\mathbf{r})+\mathbf{P}_{s p}(\mathbf{r})=\left(\begin{array}{c}
e_{15} \epsilon_{x z} \\
e_{15} \epsilon_{y z} \\
e_{31}\left(\epsilon_{x x}+\epsilon_{y y}\right)+e_{33} \epsilon_{z z}+P_{s p}
\end{array}\right)
$$

\subsection{3 k·p Hamiltonians including strain and polarization fields}

As stated before, strain and polarization fields modify the energy band structure and, thus, these effects need to be incorporated into the $\mathrm{k} \cdot \mathrm{p}$ models discussed in previous sections.

With this aim in mind, we follow the approach taken by Bir and Pikus [32] who used group theory to calculate the strain effects on the band structure by employing deformation potentials. 8 Since the strain considered here is small, it may be treated as a perturbation. The additional Hamiltonian accounting for the strain contributions is derived up to first-order perturbation theory.

The resulting strain Hamiltonians $H_{\epsilon}$ using this procedure have the same form as their k.p Hamiltonians counterparts, but replacing $k_{i} k_{j}$ by $\epsilon_{i j}$ and the corresponding mass parameters by deformation potentials. This can be understood taking into account that the strain tensor is symmetric and, thus, its transformation properties are identical to $k_{i} k_{j}$. The explicit form

\footnotetext{
${ }^{8}$ Deformation potential theory was originally formulated by Bardeen and Shockley 63 and then generalized by Herring and Vogt 64.
} 
of the $\mathrm{CB}$ and $\mathrm{VB}$ strain Hamiltonians for both $\mathrm{ZB}$ and $\mathrm{WZ}$ structures are collected in appendix C.

On the other hand, the implementation of the piezoelectric potential $\phi_{p z}(\mathbf{r})$ generated by the charge polarization is straightforward as it enters the Hamiltonian as a diagonal term.

$$
H_{p z}=e \phi_{p z}(\mathbf{r}) \mathcal{I},
$$

with $\mathcal{I}$ denoting the identity matrix and $e$ the particle charge. 


\section{Magnetic field effects in semiconductor structures}

Zero-dimensional semiconductor nanostructures are systems with appealing optical and electronic properties for many applications. In most semiconductors, these properties are governed by the band-edge energies at the $\Gamma$ point. The application of external fields modifies the system band structure, thus offering an easy way to manipulate the QD features.

In general, particles in QDs have lighter effective masses and are subject to weaker confinements compared to atoms. As a consequence, the effect of a magnetic field in these systems is much stronger and may exceed the confinement energies, resulting in the emergence of new effects not present in atoms for the magnetic field intensities accessible in the laboratory. This opens the possibility of externally controlling QDs by means of magnetic fields. To that end, this topic has been the subject of intense research during the last decades. [10, 65]

Furthermore, magnetic fields are also responsible for the manifestation of the AB effect, which was predicted by Aharonov and Bohm [66] in 1959. They showed that, contrary to classical mechanics, charged particles are affected by potentials even in the regions where all fields vanish. This was soon confirmed in the laboratory by interference experiments. 67] In the 1980s, the progress on the fabrication and detection techniques allowed the observation of such phenomenon also in nanoscale ring structures, thus raising anew the old $\mathrm{AB}$ effect. These nanostructures are doubly-connected quantum systems, usually called QRs, that show distinct properties compared to QDs, a singly-connected structure. Since then, much effort has been dedicated to understand the implications of the $\mathrm{AB}$ effect in QRs, 68, 69. 
This chapter reviews three papers ${ }^{1}$ focusing on the behavior of two different systems under an externally applied magnetic field. First, the electronic structure of GaN/AlN QDs with ZB crystal structure and its dependence on the magnetic field is studied. In particular, we pay special attention to factors influencing the spin mixing of the hole states and the circumstances that may lead to ground state transitions. The second half of this chapter deals with the $\mathrm{AB}$ effect in hexagonal core-shell systems. The $\mathrm{AB}$ periodic oscillations of the electron energy spectrum for single- and few-electron hexagonal QRs are investigated. We compare the results with the well-known case or circular QRs to emphasize the consequences of the symmetry lowering of the confinement potential.

\subsection{Magnetic-field modulation of the hole ground state in cubic GaN/AIN QDs}

GaN/AlN QDs present good properties for optoelectronic applications owing to the direct wide band gap of GaN and AlN (3.5 and 6.25 eV, [70] respectively) that has led to successfully use them in blue lasers and LEDs, [71, 72 . Furthermore, these structures show strong particle confinement due to their large band offsets and large effective masses, and also weak SOI. 73 . The former allows to use them at high temperatures, while the latter makes them promising candidates for spintronic applications.

Nitrides semiconductors are commonly grown in WZ phase, but under certain conditions cubic ZB GaN/AlN QDs can also be fabricated. The symmetry of hexagonal crystals originates strong piezoelectric and spontaneous polarization fields of several $\mathrm{MV} / \mathrm{cm}$ in $\mathrm{WZ}$ heterostructures, [73] which heavily quenches the spin relaxation times. However, these built-in fields are negligible in $\mathrm{ZB}$ systems and much longer relaxation times are expected. Indeed, Lagarde et al. [74] studied the exciton spin dynamics of self-assembled GaN/AlN ZB QDs and showed that the linear polarization persists up to room temperature and the spin relaxation times (exceeding $10 \mathrm{~ns}$ ) are two or three orders of magnitude longer than in WZ phase.

Both optical polarization and exciton spin dynamics are governed by the $\mathrm{VB}$ mixing. [43, 75, 76] In GaN, the admixture between the topmost valence subbands is expected to be important since the $\mathrm{SOI}$ is weak (the spin-orbit splitting $\Delta_{0}$ is only $17 \mathrm{meV}$ [70]) and the so subband is close in

\footnotetext{
${ }^{1}$ The full version of them can be found in pages 155,163 and 169 of the present dissertation.
} 
3.1. Magnetic-field modulation of the hole ground state in cubic GaN/AlN QDs

energy to the $\mathrm{lh}$ and hh ones. Nevertheless, confinement and magnetic fields are known to modify the band edge energies, so that $\mathrm{VB}$ mixing in QDs is surely also affected by these factors and calculations are required to assess their influence.

The system investigated is a self-assembled cubic GaN/AlN QD with cylindrical shape. The dependence of the $\mathrm{VB}$ mixing on the $\mathrm{QD}$ size and on an axial magnetic field are studied. In order to do that, taking into account that the mass parameters of GaN and AlN are quite different and their spinorbit splitting $\Delta_{0}$ small, a six-band position-dependent Hamiltonian is used. The explicit form of this Hamiltonian can be found in appendix A.1.2. Since the system studied has axial symmetry, such Hamiltonian can be simplified by using cylindrical coordinates instead of Cartesian ones. In addition, the axial approximation $\tilde{\gamma}=\frac{1}{2}\left(\gamma_{2}+\gamma_{3}\right)$ [77, 78, is applied, so that the Hamiltonian becomes cylindrically symmetric and the problem can be reduced to two-dimensions by analytically integrating the angular coordinate. In axially symmetric systems the total angular momentum, $F_{z}=m_{z}+J_{z}$, is well defined and the states can be labeled by their $F_{z}$. Here, $m_{z}$ and $J_{z}$ are the envelope and Bloch angular momentum, respectively. The resulting Hamiltonian $H_{B F}^{Z B}\left(F_{z}\right)$ in cylindrical coordinates is shown in appendix A.1.3.

Additionally, an uniform magnetic field applied along the [001] direction, $\mathbf{B}=\left(0,0, B_{0}\right)$, is included by carrying out the replacement of the canonical momentum by the kinetic one before applying the EFA, following reference [50]. Such magnetic field is described by the vector potential in the symmetric gauge $\mathbf{A}=\frac{B_{0}}{2}(-y, x, 0)$. The total Hamiltonian reads

$$
H\left(F_{z}\right)=H_{B F}^{Z B}+H^{B}+V(\rho, z) \mathcal{I}
$$

with $\rho$ being the radius coordinate and $\mathcal{I}$ the identity matrix. $H^{B}$ is the Hamiltonian including the magnetic field contributions. It has the following nonzero elements:

$$
\begin{aligned}
& H_{11}^{B}=-\left(\gamma_{1}+\gamma_{2}\right)\left[\frac{B_{0}^{2} \rho^{2}}{8}+\frac{B_{0}\left(F_{z}-\frac{1}{2}\right)}{2}\right] \\
& H_{22}^{B}=-\left(\gamma_{1}-\gamma_{2}\right)\left[\frac{B_{0}^{2} \rho^{2}}{8}+\frac{B_{0}\left(F_{z}-\frac{1}{6}\right)}{2}\right] \\
& H_{33}^{B}=-\left(\gamma_{1}-\gamma_{2}\right)\left[\frac{B_{0}^{2} \rho^{2}}{8}+\frac{B_{0}\left(F_{z}+\frac{1}{6}\right)}{2}\right] \\
& H_{44}^{B}=-\left(\gamma_{1}+\gamma_{2}\right)\left[\frac{B_{0}^{2} \rho^{2}}{8}+\frac{B_{0}\left(F_{z}+\frac{1}{2}\right)}{2}\right] \\
& H_{55}^{B}=-\gamma_{1}\left[\frac{B_{0}^{2} \rho^{2}}{8}+\frac{B_{0}\left(F_{z}+\frac{1}{6}\right)}{2}\right]
\end{aligned}
$$



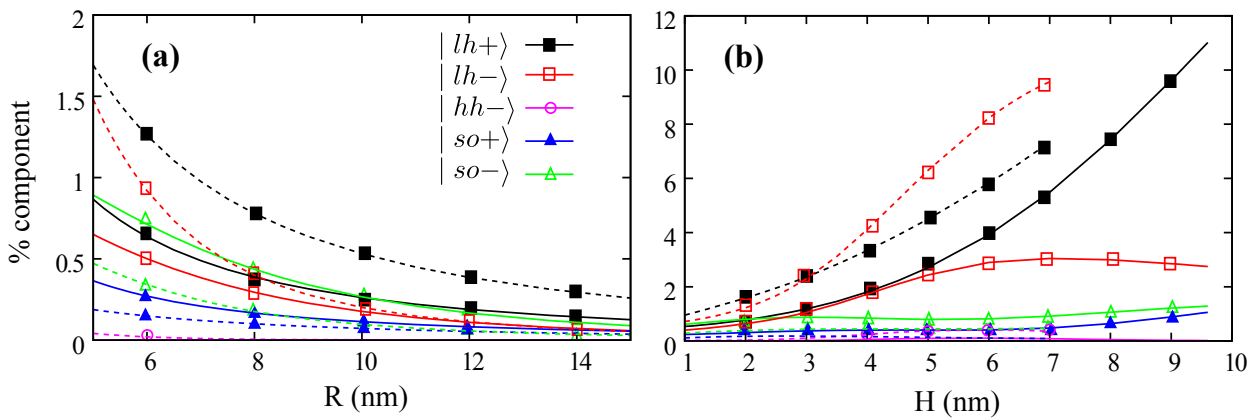

Figure 3.1: Composition of the minor hole subbands as a function of (a) the $\mathrm{QD}$ radius (fixed height $H=1.5 \mathrm{~nm}$ ) and (b) the $\mathrm{QD}$ height (fixed radius $R=6 \mathrm{~nm}$ ). In both panels, solid lines correspond to GaN/AlN QDs and dashed lines correspond to InGaAs/GaAs QDs.

$$
\begin{aligned}
& H_{66}^{B}=-\gamma_{1}\left[\frac{B_{0}^{2} \rho^{2}}{8}+\frac{B_{0}\left(F_{z}-\frac{1}{6}\right)}{2}\right] \\
& H_{25}^{B}=H_{52}^{B}=-\gamma_{2} \frac{B_{0}}{3} \\
& H_{36}^{B}=H_{63}^{B}=\gamma_{2} \frac{B_{0}}{3}
\end{aligned}
$$

Please note that $(3.2)$ differ from 2.21 because the spin Zeeman splitting is disregarded here.

All simulations are carried out by numerically integrating (3.1). Both GaN/AlN and InGaAs/GaAs QDs are considered for comparison and the corresponding material parameters can be consulted in the published paper (see page 155).

\subsubsection{Effect of the aspect ratio}

First, we perform calculations for both materials varying the aspect ratio of the dots. Preliminary calculations for a typical-size QD $(R=6 \mathrm{~nm}$ and $H=1.5 \mathrm{~nm}[74,79])$ show that the hole ground state has $F_{z}= \pm 3 / 2$ symmetry and a major contribution of the $|h h+\rangle$ component. In order to analyze the $\mathrm{VB}$ mixing, the relative weight of each component within the spinor is calculated as $c_{i}=\frac{\left\langle f^{(i)} \mid f^{(i)}\right\rangle}{\sum_{j=1}^{6}\left\langle f^{(j)} \mid f^{(j)}\right\rangle}$, and the results for the minor components represented in figure 3.1. Solid lines are used for GaN/AlN QDs and dashed lines for InGaAs/GaAs QDs. 
3.1. Magnetic-field modulation of the hole ground state in cubic GaN/AlN QDs

Table 3.1: Effective masses of hh, lh and so (times $\left.m_{0}\right)$

\begin{tabular}{lccccc}
\hline & $m_{h h}^{z}$ & $m_{l h}^{z}$ & $m_{h h}^{\perp}$ & $m_{l h}^{\perp}$ & $m_{s o}$ \\
\hline GaN & 0.85 & 0.24 & 0.29 & 0.52 & 0.37 \\
InGaAs & 0.38 & 0.05 & 0.07 & 0.15 & 0.09 \\
\hline
\end{tabular}

Two series of calculations are carried out: one varying the QD radius and the other varying the $\mathrm{QD}$ height. On one hand, figure 3.1 (a) shows the results for variable radius and fixed height $H=1.5 \mathrm{~nm}$. It can be seen that the weight of the minor components decreases with $R$ for both materials. On the other hand, the variation of the minor components contribution as a function of the $\mathrm{QD}$ height $(R=6 \mathrm{~nm})$ is depicted in figure 3.1 (b). The behavior is now opposite and the weight increases with $H$.

These results can be understood taking into account the effective masses of the bands along the $z$ ([001] axis) and the lateral directions. The concrete values are summarized in table 3.1. In QDs with vertical confinement much stronger than the horizontal one, such as the ones considered in figure 3.1(a) and figure 3.1(b) at smaller $H$, the lateral confinement can be disregarded. Since the kinetic energy in the $z$ direction is larger for the $\mathrm{ln}$ and so bands $\left(m_{h h}^{z}>m_{l h}^{z}\right)$, their energy separation from the hh ground state increases for smaller $H$ and their coupling weakens. Consequently, Ih and so components are less important the smaller the aspect ratio $(\mathrm{H} / 2 \mathrm{R})$ is, as shown in figure 3.1(a). Contrarily, as $H$ increases the lateral confinement becomes more important and the weight of the $\operatorname{lh}$ band raises $\left(m_{h h}^{\perp}<m_{l h}^{\perp}\right)$. In fact, in high enough QDs a ground state transition from $F_{z}= \pm 3 / 2$ symmetry to $F_{z}= \pm 1 / 2$ symmetry takes place. This is the situation in figure 3.1(b), where the curves have been truncated at the transition points. Now the ground state has dominant $\mathrm{th}$ character and can be used to emit strongly linearly polarized light. 80 .

It is also worth stressing the overall high spin purity obtained for both materials. This can be attributed to the fact that $|h h+\rangle$ is the only component of the $F_{z}=3 / 2$ ground state whose envelope function has angular momentum $m_{z}=0$, what stabilizes this component. In addition, a direct comparison between both materials in figure 3.1 reveals the smaller spin admixture for GaN/AlN. This high spin purity is in contrast to the initial predictions based on the much heavier effective masses and the smaller bulk spin-orbit splitting of GaN. It is, however, consistent with the long spin relaxation times observed in reference [74]. This surprising behavior can be explained considering the coupling terms in Hamiltonian A.3. For example, many of these terms are proportional to $\tilde{\gamma}$ and this parameter is 
much smaller in GaN, $\tilde{\gamma}_{G a N}=0.925$, than in InGaAs, $\tilde{\gamma}_{I n G a A s}=4.51$, thus justifying the smaller $\mathrm{VB}$ mixing.

\subsubsection{Magnetic field modulation}

Typical QDs have an aspect ratio of about 1/8 [79] and present a ground state with $F_{z}= \pm 3 / 2$ symmetry that is relatively far in energy from other states. This fact, together with the large effective masses of GaN, impedes the manipulation of the electronic structure via magnetic fields. Nevertheless, the energy of the $\mathrm{hh}$ and $\mathrm{hh}$ bands is similar in dots with aspect ratio close to 1 , so controlling the character of the hole ground state by using moderate magnetic fields should be possible in such dots.

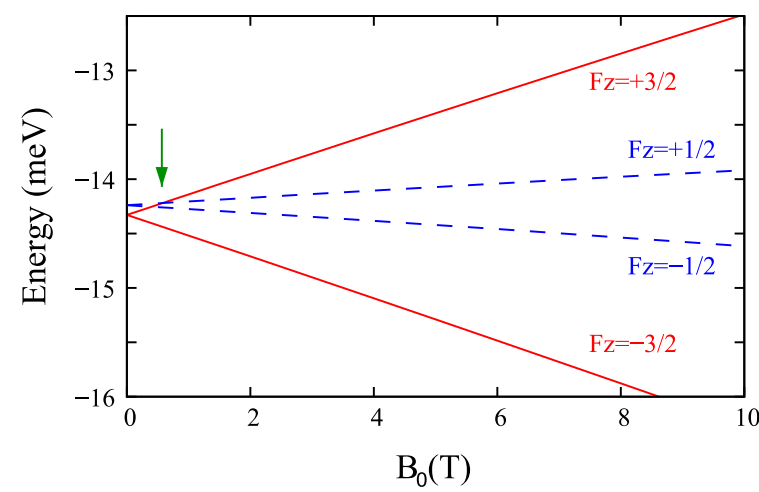

Figure 3.2: Magnetic-field-induced energy splitting of the lowestlying hole states in a GaN/AlN QD with aspect ratio $\approx 1$. The arrow indicates the ground state transition point, $B_{0} \approx 0.6 \mathrm{~T}$.

Figure 3.2 illustrates the orbital Zeeman splitting of the topmost VB states in a GaN/AIN QD with aspect ratio approximately 1. Blue dashed lines correspond to $F_{z}= \pm 1 / 2$ states and red solid lines correspond to $F_{z}= \pm 3 / 2$ states, which present a major contribution of $|l h\rangle$ and $|h h\rangle$ components, respectively. In the absence of a magnetic field the states are degenerate, but for finite $B_{0}$ the degeneracy is broken and the states split. The magnitude of the splitting is proportional to the coefficients of the linear-in-B terms in equation (3.2): $\left(\gamma_{1}+\gamma_{2}\right) / 2$ for $|h h \pm\rangle$ and $\left(\gamma_{1}-\right.$ $\left.\gamma_{2}\right) / 6$ for $|l h \pm\rangle$. Then, the splitting of $|h h+\rangle$ is larger and the ground state undergoes a transition from $F_{z}=+1 / 2$ to $F_{z}=+3 / 2$ at $B_{0} \approx 0.6 \mathrm{~T}^{2}$. As

\footnotetext{
${ }^{2}$ Note that hole states have negative energies.
} 
a consequence, because $F_{z}=+1 / 2$ and $F_{z}=+3 / 2$ yield different optical polarizations, these results show that external magnetic fields can be used to modify the optical response of GaN/AlN QDs.

\subsection{Aharonov-Bohm effect in systems with hexag- onal symmetry}

Nanowires are one-dimensional (1D) nanostructures in which carriers are confined laterally but move freely along the growth direction. Most nanowires made of III-V semiconductors present an hexagonal section when their diameter is less than $400 \mathrm{~nm}$. 81, 85] Taking this structure as starting point, coremultishell nanowires can be obtained after a few overcoating processes. [86, 87. Here, the electron motion can be further restricted in the radial direction by choosing the appropriate material sequence. Figure 3.3 shows an example of a multishell nanowire cross section in which a potential well is created by the misalignment of the $\mathrm{CBS}$ of the constituent materials. The electrons in the nanowire are then confined into a hexagonal prismatic tube surrounding the core. In addition, confinement in the growth direction can also be generated by cutting the nanowires or by modulating the materials in this direction, thus yielding hexagonal flat QRs. 88, 89.

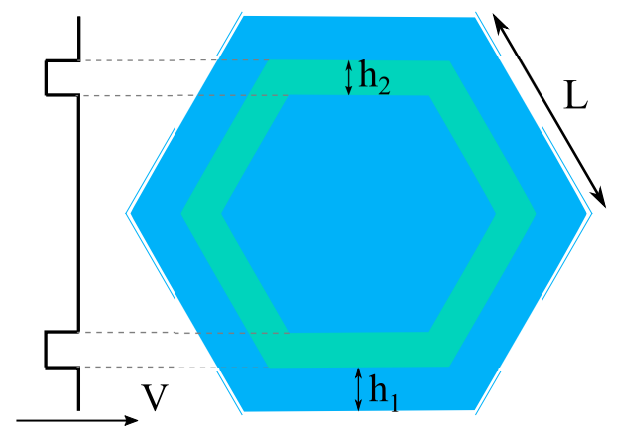

Figure 3.3: Drawing of a core-multishell nanowire cross section. Free electrons are confined into the hexagonal ring (green region). The band-edge profile showing the square-well-type confinement potential is indicated, as well as some geometry parameters.

Recent publications have shown that electrons in hexagonal QRs are not homogeneously distributed over the entire structure. They are mainly localized at the corners of the ring, giving rise to quasi-1D channels in the nanowires. [90, 91] Furthermore, in multi-particle systems the degree of localization is further enhanced as the number of electrons increases up to 
six. 92 This is a consequence of many-particle interactions, demonstrating the important role of correlation in these systems. The charge inhomogeneous localization constitutes a remarkable difference in comparison to circularly-symmetric systems which, in turn, may also result in distinct behavior and properties.

It is well established that when a magnetic field is axially applied to a ring or a tubular system the $\mathrm{AB}$ effect emerges. It manifests itself in the electronic spectra, magnetization, optical and transport properties of QRs. 69] For instance, the single-electron energy spectrum of a circular QRs shows an integer and periodic $\mathrm{AB}$ oscillation pattern. [93, 94] In nanowires, $\mathrm{AB}$-like oscillations have also been observed in magnetotransport experiments performed on radial heterostructures. 95 97] Most theoretical works studying $\mathrm{AB}$-related effects have considered systems with axial symmetry, but little is known about the implications of a symmetry lowering.

The aim of this section is to investigate the response of correlated hexagonal structures to an external axial magnetic field. In particular, we study $\mathrm{AB}$-derived properties, e.g. ground-state energy and magnetoconductance oscillations, in single- and few-electron hexagonal QRs and core-multishell nanowires. The results are compared with the ones of their circular counterparts to explore the role of the hexagonal symmetry.

\subsection{1 $\mathrm{AB}$ effect in hexagonal quantum rings}

We first consider a flat hexagonal QR similar to that represented in figure 3.3 with $L=66.5 \mathrm{~nm}, h_{1}=13.5 \mathrm{~nm}$ and $h_{2}=6.8 \mathrm{~nm}$. The materials are GaAs for the ring and AlAs for the core and the outer shell. All parameters, namely effective masses, $\mathrm{CB}$ offset and dielectric constants, are taken from reference [92. We carry out calculations of the low-energy spectrum for $N$ interacting electrons, from $N=1$ up to $N=7$, in the low-density regime.

\section{Single-particle energy spectrum}

The position-dependent effective Hamiltonian describing a single electron under an external magnetic field reads

$$
H_{s p}=\frac{1}{2}(\mathbf{p}+\mathbf{A}) \frac{1}{m^{*}(\mathbf{r})}(\mathbf{p}+\mathbf{A})+V(\mathbf{r})
$$

where $m^{*}$ is the isotropic effective mass and $\mathbf{A}=\frac{B}{2}(-y, x, 0)$ is the vector potential defining the magnetic field along the axial direction. Equation 
(3.3) is numerically solved following the finite element method over a uniform, triangular mesh. A grid with the same symmetry of the system is used to guarantee high accuracy, specially in the description of the boundary conditions, and to avoid artificial asymmetries in the discretization.

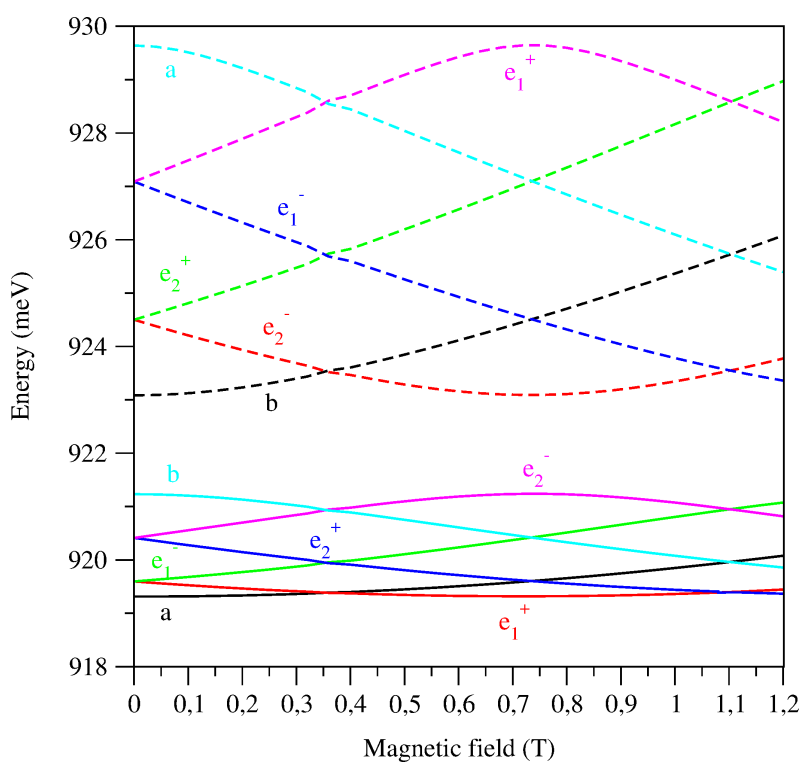

Figure 3.4: Single-electron energy spectrum as a function of the magnetic field intensity. The states are labeled and presented in different line styles according to the $C_{6}$ symmetry group.

Figure 3.4 shows the energy of the twelve lowest-lying states as a function of the magnetic field. Similarly to circular QRs, regular $\mathrm{AB}$ oscillations of the ground state energy are observed. However, for the hexagonal QRs the states are organized in groups of 6 orbitals each, separated by an energy gap of $\approx 2 \mathrm{meV}$. This is in clear contrast to the case of circular rings where all states form a single ensemble. The different behavior of the two structures can be justified from symmetry considerations. On one hand, systems with circular symmetry have an infinite number of irreducible representations (irreps), so that all states are associated to different irreps and can cross. On the other hand, orbitals in hexagonal QRs are associated to the six different irreps of the $C_{6}$ symmetry group. The states with different symmetry can cross while the states with the same symmetry anticross. As a result, groups of 6 orbitals with a different irrep each form a shell within which they cross, but that is spit from other shells. The states in figure 3.4 are labeled with their associated irreps to illustrate this. 


\section{Multi-particle energy spectra}

Next, we examine the effect of populating the system with a few interacting electrons. To this purpose, a full configuration interaction (FCI) approach is used. 98] FCI is a variational method that takes into account all possible Slater determinants out of the one-electron basis set chosen. Then, an approximate solution for the multi-particle problem is obtained by exactly solving the Hamiltonian within this basis set. The many-electron Hamiltonian is as follows

$$
H_{m p}=\sum_{i \sigma} \epsilon_{i} e_{i \sigma}^{\dagger} e_{i \sigma}+\frac{1}{2} \sum_{i j k l} \sum_{\sigma \sigma^{\prime}} U_{i j k l} e_{i \sigma}^{\dagger} e_{j \sigma^{\prime}}^{\dagger} e_{k \sigma^{\prime}} e_{j \sigma}
$$

with $e_{i \sigma}\left(e_{i \sigma}^{\dagger}\right)$ being the annihilation (creation) operator for an electron in the state $i$ and spin $\sigma$. The few-electron states are obtained by exactly diagonalizing equation (3.4) using 24 single-particle spin-orbitals as basis, i.e. two shells of six orbitals.

In correlated systems the period and amplitude of the energy oscillations decrease with the electron population. 99 101] The oscillation period scales as $1 / N$ and, hence, this phenomenon is known as the fractional AB effect.102] In figure 3.5 the energy spectra for a QR populated with up to seven electrons are displayed. The energies are taken relative to the ground-state energy at zero magnetic field (see the horizontal red line in the graphs). We observe regular oscillations for both $N=2$ and $N=3$, the period of which is in perfect agreement with the fractional $\mathrm{AB}$ effect. In fact, the first crossing of the ground state in the single-particle case (figure 3.4 is at $B \approx 0.4 \mathrm{~T}$, and is reduced to $B \approx 0.2 \mathrm{~T}$ and $B \approx 0.13 \mathrm{~T}$ for $N=2$ and $N=3$, respectively. For $N=4, N=5$ and $N=7$ the oscillations are not regular and the period clearly deviates from the behavior expected considering the fractional $\mathrm{AB}$ effect. The results for $N=6$ deserve especial consideration since the ground state does not cross with other excited states in the range of magnetic fields under study. The $\mathrm{AB}$ effect is, thus, completely suppressed.

The above results can also be seen, and perhaps more clearly, in figure 3.6 where the corresponding magnetization, $M=\partial E / \partial B$, is represented. Here, the magnetization for different $N$ has been offset for clarity. Figure 3.6 illustrates the loss of regularity in the oscillations period as $N$ increases as well as the flat magnetization profile for $N=6$.

The above-mentioned deviations in the oscillation period for larger $N$ have already been found in previous calculations. [99, 101] This behavior 

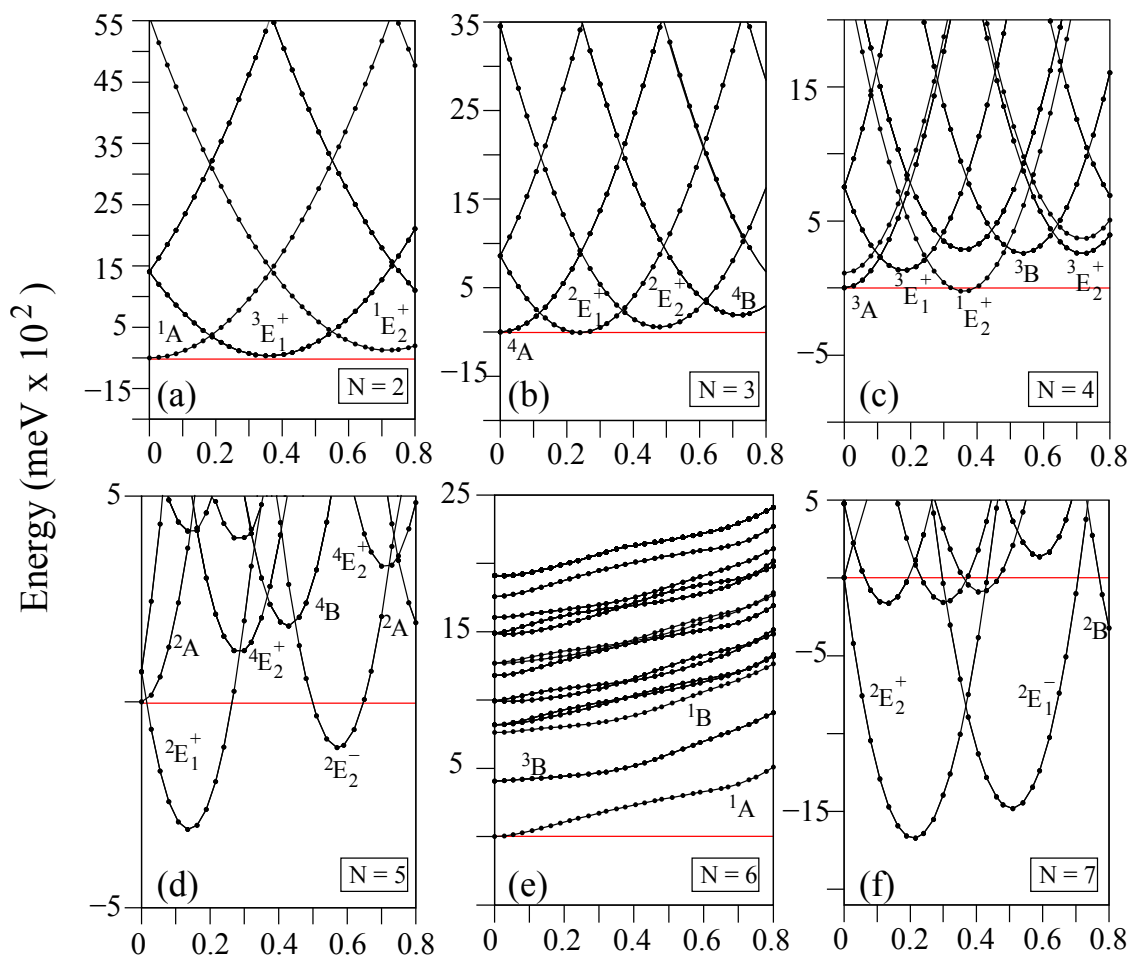

Magnetic field (T)

Figure 3.5: Energy of the lowest-lying states vs. the magnetic field intensity for systems containing from $N=2$ up to $N=7$ interacting electrons. The energy values are relative to the ground state energy at $B=0$, indicated by a horizontal red line. The states are labeled according to the $C_{6}$ symmetry group and spin multiplicity.

can be justified in the framework of the empirical Hubbard model.103 In this model it was concluded that the fractional $\mathrm{AB}$ oscillations emerge only for small values of $\alpha=N t / U L$, where $t$ is the tunneling integral, $U$ is the repulsion integral and $L$ is the number of sites along the QR where the states are localized. The value of $\alpha$ is inversely proportional to the number of electrons. Therefore, a low-density regime is needed to observe the fractional $\mathrm{AB}$ oscillations, explaining the deviations for larger $N$.

Conversely, the suppression of the $\mathrm{AB}$ effect in multi-particle systems has not been reported in literature. To understand this result, we repeat the calculations for $N=6$ but introducing a scaling factor $f$ to the electronelectron integrals. In this way we are able to determine the role of Coulomb interactions. Four series of calculations are carried out for $f=0,0.1,0.2$ 


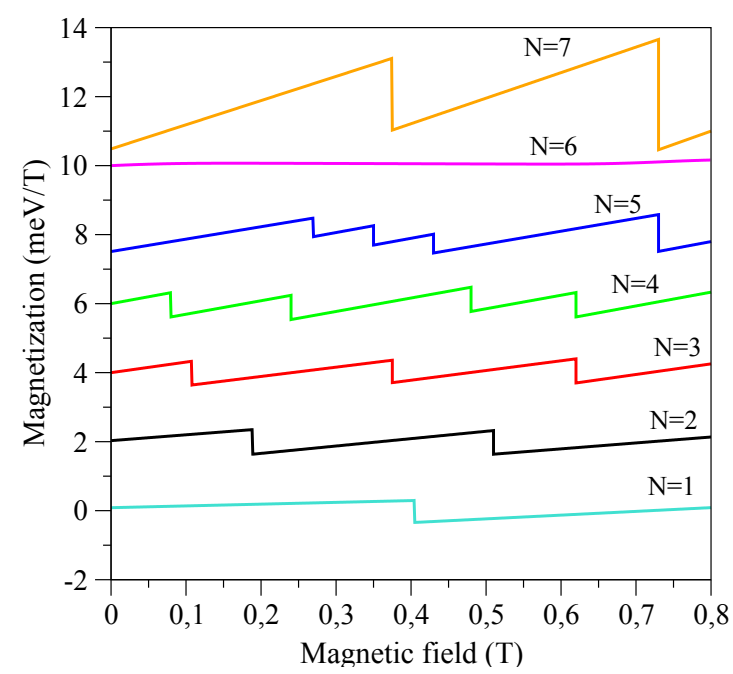

Figure 3.6: Magnetization profiles for a $N$-electron hexagonal QR with $N$ varying from 1 to 7 . For the sake of clarity, the results for each $N$ have been offset by $2 \mathrm{meV} / \mathrm{T}$.

and 0.5 , and the results are summarized in figure 3.7 . For a non-interacting system, figure 3.7(a), two different states with the same total symmetry and spin, ${ }^{1} A$, cross at $B \approx 0.4 \mathrm{~T}$. The configurations of these states are $a^{2}\left(e_{1}^{+}\right)^{2}\left(e_{1}^{-}\right)^{2}$ and $\left(e_{1}^{+}\right)^{2} a^{2}\left(e_{2}^{+}\right)^{2}{ }^{3}$ When the Coulomb interaction is activated the states with ${ }^{1} A$ symmetry anticross. The magnitude of the anticrossing increases with $f$ as can be easily seen by comparing panels (b), (c) and (d) of figure 3.7, thus causing the suppression of the $\mathrm{AB}$ oscillations.

It is worth mentioning that calculations for a three times smaller hexagonal QR have also been performed. The results obtained (not shown) reveal that the $\mathrm{AB}$ suppression is no longer present. This is because in this density regime the anticrossing is not big enough and the ${ }^{3} B$ state (see figure 3.5 cross the ${ }^{1} A$ ground state, originating a non-flat magnetization profile.

In summary, the $\mathrm{AB}$ suppression found for a hexagonal $\mathrm{QR}$ populated by six electrons is a symmetry-related effect that emerges in the highcorrelation, low-density regime as a consequence of an anticrossing between the ground state and an excited state with the same symmetry.

\footnotetext{
${ }^{3}$ We use the standard Schoenflies notation with lower- and upper-case letters referring to the symmetry of orbitals and $N$-electron states, respectively.
} 

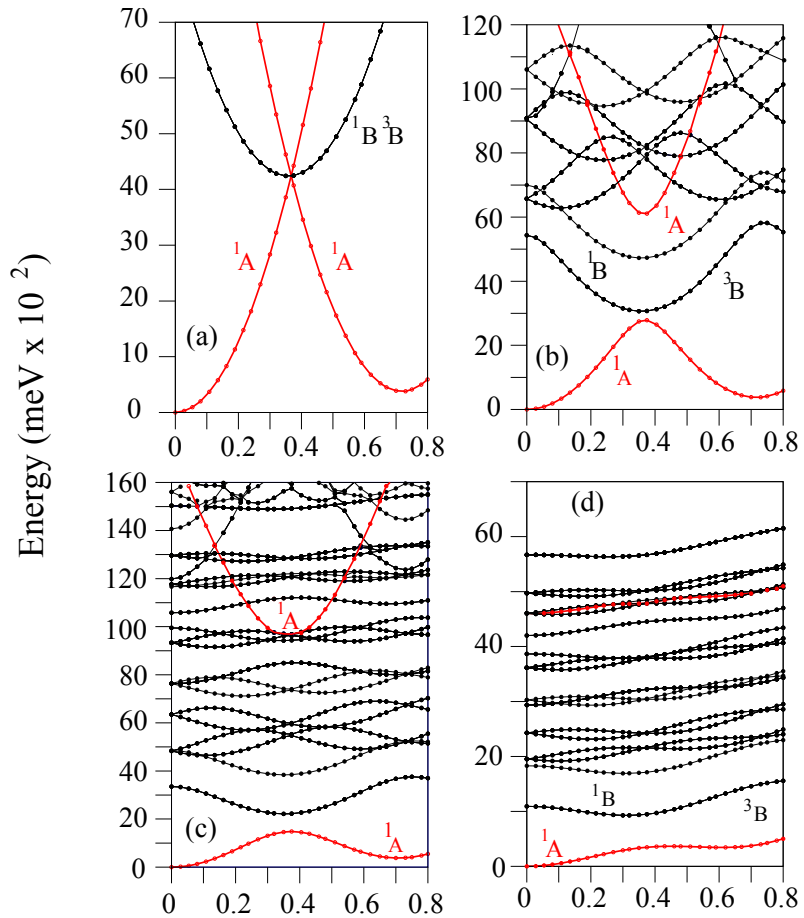

Magnetic field (T)

Figure 3.7: Same as figure 3.5(e) but for different weight of the electron-electron interaction integrals. This weight is modulated via an scaling factor $f$, which takes $f=0,0.1,0.2$ and 0.5 in panels (a), (b), (c) and (d), respectively. The total symmetry of the main states is indicated and the ${ }^{1} A$ ones are shown in red to improve their visibility.

\subsubsection{AB magnetoconductance oscillations and electron gas transitions in hexagonal core-shell nanowires}

In this section, we investigate the electronic states and magnetoconductance of a core-shell hexagonal nanowire pierced by an external magnetic field along the growth direction. The nanowire considered is infinitely long and its cross section has the same form as figure 3.3. Here, it is composed by a GaAs core with a minimal diameter of $100 \mathrm{~nm}$, a InAs shell with thickness of $25 \mathrm{~nm}$, and an external 30-nm-thick capping layer of $\mathrm{SiO}_{2}$.

Simulations are carried out within the spin-density-functional theory following an iterative procedure. Although the system is $3 \mathrm{D}$, the translational invariance along the $z$ direction allows one to write the wave function as 
$\Psi(x, y, z)=e^{i k z} \phi(x, y)$. If one further assumes the motion of the electrons in the longitudinal and transverse directions to be decoupled, the problem reduces from 3D to 2D. The effective Kohn-Sham Hamiltonian reads:

$$
H=H_{s p}(\mathbf{r})+V_{Z}^{\sigma}(\mathbf{r})+V_{H}(\mathbf{r})+V_{X C}^{\sigma}(\mathbf{r}),
$$

where $H_{s p}$ is the single-particle Hamiltonian under an external magnetic field presented in equation (3.3). $V_{Z}^{\sigma}(\mathbf{r})$ is the Zeeman splitting term, $V_{H}(\mathbf{r})$ is the Hartree potential energy and $V_{X C}^{\sigma}(\mathbf{r})$ is the exchange-correlation potential. Finally, $\sigma=\uparrow, \downarrow$ denotes the spin index of the electrons.

The Zeeman term is given by

$$
V_{Z}^{\sigma}(\mathbf{r})=g^{*} \mu_{B} B \eta_{\sigma}
$$

with $g^{*}$ standing for the effective g-factor, $\mu_{B}$ for the Bohr magneton and $\eta_{\sigma}=+1 / 2(-1 / 2)$ for $\sigma=\uparrow(\downarrow)$.

The Hartree potential energy is calculated as $V_{H}(\mathbf{r})=-e \phi(\mathbf{r})$ after obtaining the electrostatic potential $\phi(\mathbf{r})$ by means of the Poisson equation

$$
\nabla \varepsilon_{r}(\mathbf{r}) \nabla \phi(\mathbf{r})=\frac{e}{\varepsilon_{0}}\left[\rho(\mathbf{r})-\rho_{D}(\mathbf{r})\right] .
$$

Here, $\rho(\mathbf{r})$ is the total free-electron charge density calculated from the KohnSham eigenstates of equation (3.5). $\rho_{D}(\mathbf{r})$ corresponds to the density of donors and $\varepsilon_{r}$ to the material-dependent dielectric constant.

The last term in (3.5), the exchange-correlation potential $V_{X C}^{\sigma}(\mathbf{r})$, is defined as the functional derivative within the local-spin-density approximation (LSDA). The correlation functional used in all calculations is the one proposed by Perdew and Wang [104].

First, equation (3.5) is numerically integrated by using a finite-element scheme, and taking $V_{H}=0$ and $V_{X C}^{\sigma}=0$. From the spin eigenstates obtained we calculate the free-electron charge density and, using this density, the associated values of $V_{H}$ and $V_{X C}^{\sigma}$. Such potentials are incorporated in equation (3.5) and the process is started over. These steps are repeated iteratively until the convergence criteria are achieved.

In the subsequent sections, two device configurations are investigated: gate-all-around and back-gate. The gate-all-around configuration consists in an electrode surrounding the entire structure, so that the energy can be modulated while preserving the hexagonal symmetry of the system. This type of gate is simulated by forcing the electrostatic potential, $\phi(\mathbf{r})$, in the Poisson equation (3.7) to be that of the gate voltage $V_{g}$ at all nanowire edges. 
For a back-gate configuration we assume two flat electrons sandwiching the nanowire. This situation is simulated by defining the electrostatic potential to be zero at the boundary corresponding to one electrode and $V_{g}$ at the other. Unlike the all-around-gate case, the hexagonal symmetry is broken in this configuration.

For the simulations of the present section we consider the GaAs core to be doped with a homogeneous density of donors $\rho_{D}=5 \times 10^{15} \mathrm{~cm}^{-3}$. The Fermi energy $E_{F}$ is taken $75 \mathrm{meV}$ above the InAs $\mathrm{CB}$ edge and the temperature is set to $T=1.8 \mathrm{~K}$. All material parameters can be consulted in table I of the published article (page 169).

\section{Low-magnetic-field regime}

First, we study the electronic structure of the hexagonal core-shell nanowire for low magnetic fields and zero gate voltage, $V_{g}=0$. The results (not shown) are qualitatively the same as for the hexagonal QR of the previous section. That is, the electron density is mainly localized at the corners of the InAs shell, and the states in the energy spectrum are organized in groups of six, presenting $\mathrm{AB}$ oscillations as the magnetic field intensity increases, same as figure 3.4. Nevertheless, now the spin degree of freedom is taken into account and the Zeeman splitting included in the simulations. As a consequence, the spin-degenerate states at $B=0$ split for finite fields and two magnetic spin-subbands are formed: one with spin-up ( $\uparrow$-MSS) and the other with spin-down ( $\downarrow$-MSS). Each one of them is composed by six states of the same spin and show $\mathrm{AB}$ oscillations.

Next, we explore the result of applying a gate-all-around voltage to tune the Fermi energy of the system. To this end, the total magnetoconductance $G$ is calculated for various, both negative and positive, voltages. The value of $G$ is obtained using the linear-response Landauer formula ${ }^{4}$

$$
G_{\sigma}=\frac{e^{2}}{h} \sum_{n} \int_{B_{n, \sigma}}-\frac{\partial f\left(E-E_{F}, T\right)}{\partial E} d E
$$

where $f$ is the Fermi occupation function, with $E_{F}$ and $T$ being the Fermi energy and the temperature, respectively. The integral in equation $(3.8)$ is performed along each energy spin-subband $B_{n, \sigma}$. The magnetoconductance results obtained are shown in figure 3.8. Typical oscillations arising from

\footnotetext{
${ }^{4}$ This formula assumes a fully ballistic regime which is not exactly the experimental regime in InAs nanowires. However, this simplified formula can be used to get correct qualitative results.
} 


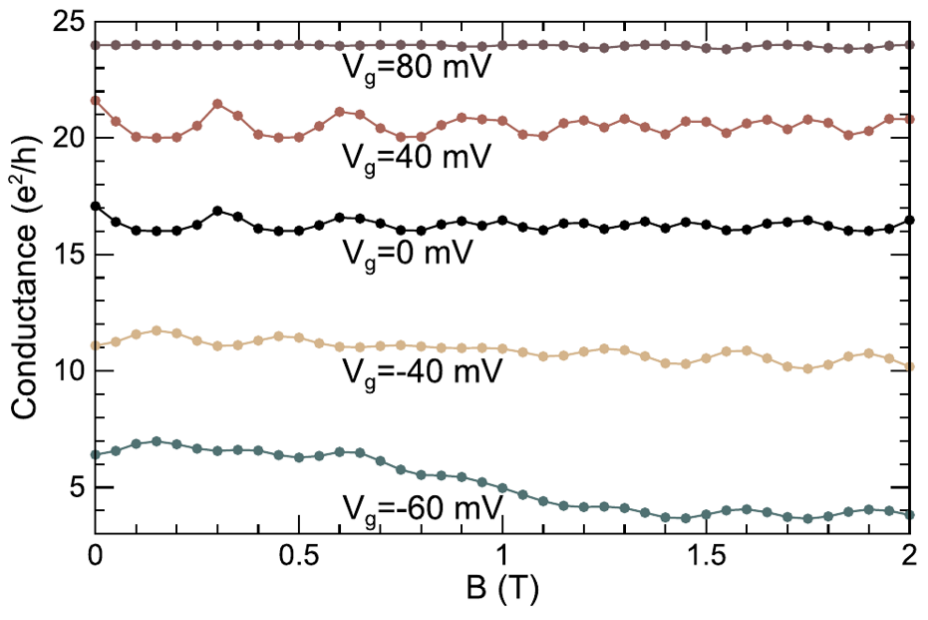

Figure 3.8: Total magnetoconductance for five gate-all-around voltages: $V_{g}=80 \mathrm{mV}, V_{g}=40 \mathrm{mV}, V_{g}=0 \mathrm{mV}, V_{g}=-40 \mathrm{mV}$ and $V_{g}=-60 \mathrm{mV}$.

the $\mathrm{AB}$ effect are observed for most $V_{g}$ but are absent, for instance, at $V_{g}=80 \mathrm{mV}$, where the conductance is completely flat.

In order to get insight into this singular behavior, in figure 3.9 we represent the energy spectrum vs. the magnetic field for $V_{g}=-60 \mathrm{mV}$, panel (a), and $V_{g}=80 \mathrm{mV}$, panel (b). By comparing both spectra, it can be seen that the gate voltage affects the width of the MSSs as well as the gaps separating them. For $V_{g}>0$ the electron density is more localized at the corners of the InAs ring, see inset in figure 3.9(b). This is due to the larger electronelectron interaction, which is also responsible for the larger gaps between MSSs. Contrarily, for $V_{g}<0$ the electron density is more delocalized, see inset in figure 3.9 (a), and the gaps become smaller or even disappear. In addition to this, figure 3.9 (b) also explains the flat magnetoconductance observed for $V_{g}=80 \mathrm{mV}$ in figure 3.8 . The position of $E_{F}$ exactly coincides with the energy gap between the second and third group of MSSs, so that it does not cross any MSS. As a result, the number of conducting channels is constant and so is the conductance profile. Since energy gaps between subbands increase with gate voltage, flat magnetoconductance profiles are more likely to be found at large positive $V_{g}$.

In light of the above results, one would expect to find flat magnetoconductances when sweeping $V_{g}$ in transport experiments on hexagonal nanowires. However, typical profiles show flux periodic oscillations, [96, 97] 

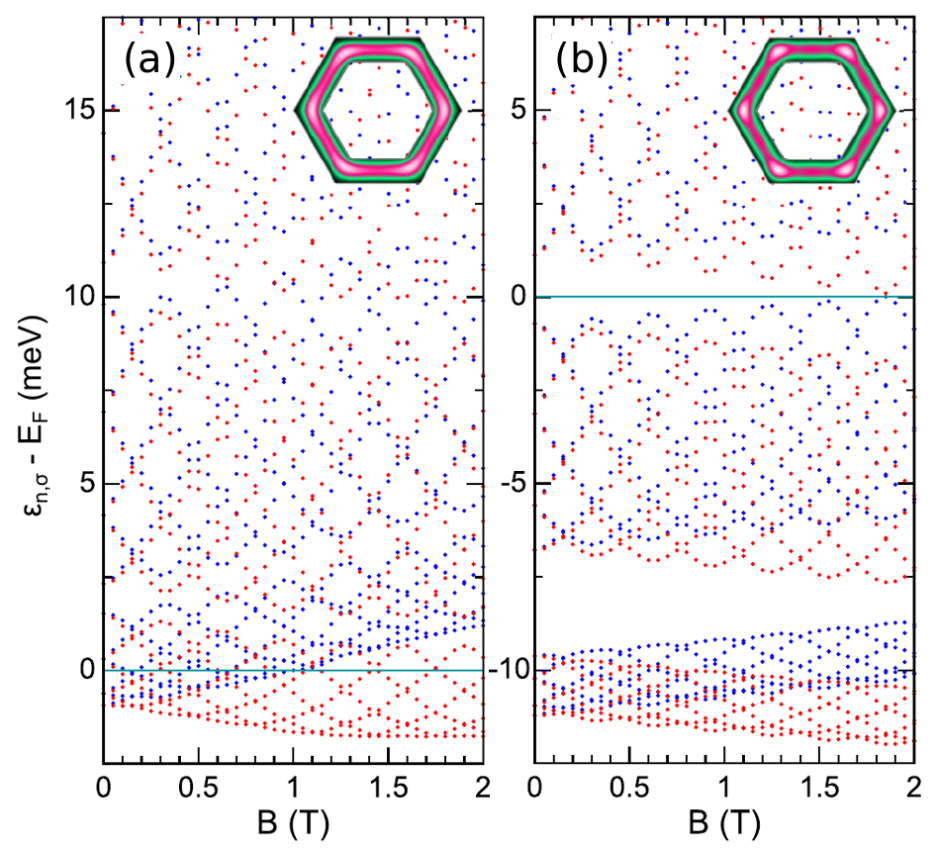

Figure 3.9: Energy spectrum as a function of the magnetic field for (a) $V_{g}=-60 \mathrm{mV}$ and (b) $V_{g}=80 \mathrm{mV}$. Red and blue dots correspond to $\downarrow$-MSS and $\uparrow$-MSS, respectively. The horizontal black line represents the Fermi energy $E_{F}$. Insets in panels (a) and (b) show the electron density distribution at $B=0$ for the nanowire cross section.

and a situation with constant $G$ has never been observed experimentally. This may be due to the fact that the most common device configuration for manipulating the electron density is to use a back-gate, instead of a gate-all-around. The main difference between both configurations is the breaking of the hexagonal symmetry when a back-gate is used. We know from the previous section that the separation of the states in groups in the energy spectrum is a direct consequence of the hexagonal symmetry of the system. Therefore, a back-gate device is expected to present no significant gaps and a situation with constant $G$ would not be possible, justifying the lack of flat profiles in experiments. Nonetheless, a back-gate voltage could also destroy the doubly-connected topology that originates the $\mathrm{AB}$ effect. In a such a case, $\mathrm{AB}$ oscillations should not emerge and one wonders why they are observed at all.

In order to understand the origin of the oscillations, we carry out the same calculations as in figures 3.8 and 3.9 , but for a back-gate device. The total magnetoconductance profiles in figure 3.10 evidence that flux oscilla- 


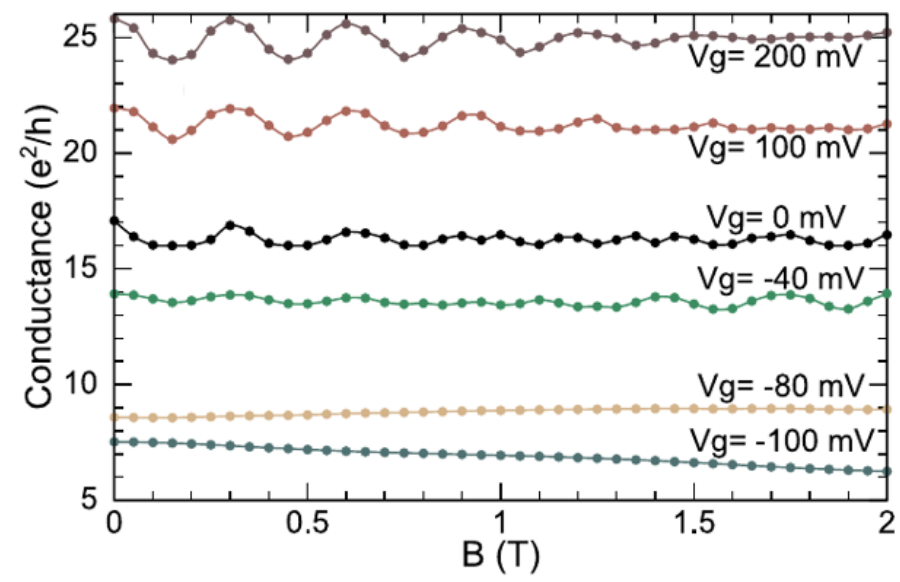

Figure 3.10: Same as figure 3.8 but for a back-gate configuration and different $V_{g}$.

tions take place for positive or slightly negative voltages but are absent for large enough negative values, $V_{g}=-80 \mathrm{mV}$ and $V_{g}=-100 \mathrm{mV}$. Again, this behavior can be explained looking at the energy spectra in figure 3.11 . To illustrate both situations, we have chosen two $V_{g}$ with different sign, $V_{g}=-80 \mathrm{mV}$ in figure 3.11(a) and $V_{g}=200 \mathrm{mV}$ in figure 3.11(b). As shown in the insets, the back-gate voltage clearly reshapes the electron density distribution, which is pushed towards the top (bottom) half of the cross section for negative (positive) $V_{g}$. A comparison between both insets show that the doubly-connected topology is more robust for $V_{g}>0$ since the electron density is more delocalized in panel (b) even though the voltage is stronger. As for the MSSs, for both $V_{g}$ we see that states lower and higher in energy behave very differently. Lowest-lying states are more affected by the back-gate voltage and exhibit a quasi-linear dispersion with $B$, i.e. the doubly-connected topology is completely broken, while typical $\mathrm{AB}$ oscillations are present in more excited states. For $V_{g}=-80 \mathrm{mV}$, the Fermi energy is in the region of states with linear dispersion and, thus, only states without doubly-connected topology are occupied. This justifies the flat magnetoconductance in figure 3.10 and the strongly localized electron density distribution in the inset of figure 3.11(a). In contrast, in 3.11(b) several states with doubly-connected topology are occupied, the electron density is more delocalized and the $\mathrm{AB}$-like magnetoconductance oscillations persist. The latter is indeed the usual regime in magnetotransport experiments, [96, 97] what explains why only oscillating profiles have been reported in literature. 

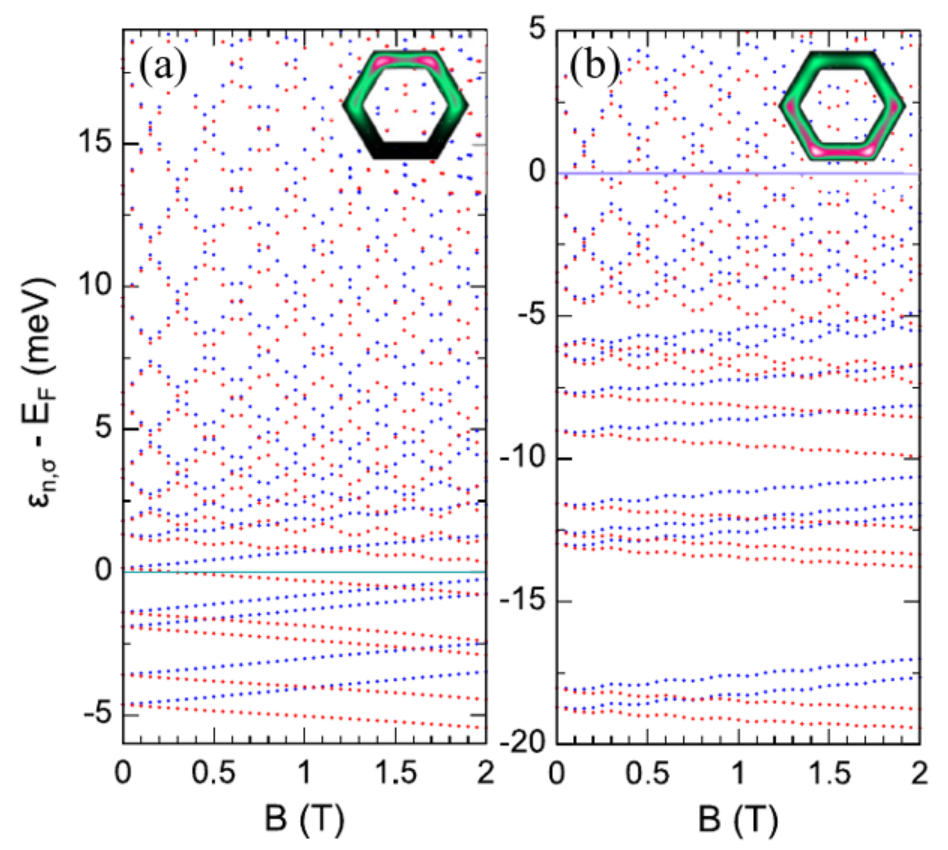

Figure 3.11: Same as figure 3.9 but for a back-gate device, and voltages (a) $V_{g}=-80 \mathrm{mV}$ and (b) $V_{g}=200 \mathrm{mV}$.

\section{High-magnetic-field regime}

We next study the high-magnetic-field regime for the same hexagonal nanowire system. All simulations are performed considering the absence of external gates, i.e. $V_{g}=0$. Figure 3.12 displays the MSSs up to $B=20 \mathrm{~T}$, where complete electron depletion occurs, and also the total electron density at selected fields. By comparing figures 3.12 (b)-3.12(f), it is clear that as $B$ increases the electron density undergoes a transition from being localized at the corners, panels (b) and (c), to a distribution with maximum density at the center of the facets, panels (e) and (f). This change in the electron density positioning is caused by the parabolic magnetic confinement. At low magnetic fields the confining potential originated from the materials band offset dominates, but at big enough fields the magnetic confinement becomes more important. The larger $B$ is, the more the electrons are pushed to lower radius, i.e. at the center of the facets, giving rise to a corner-to-facet transition.

A signature of the aforementioned transition can be identified in the energy spectrum shown in figure 3.12(a). At zero magnetic field, the states are 

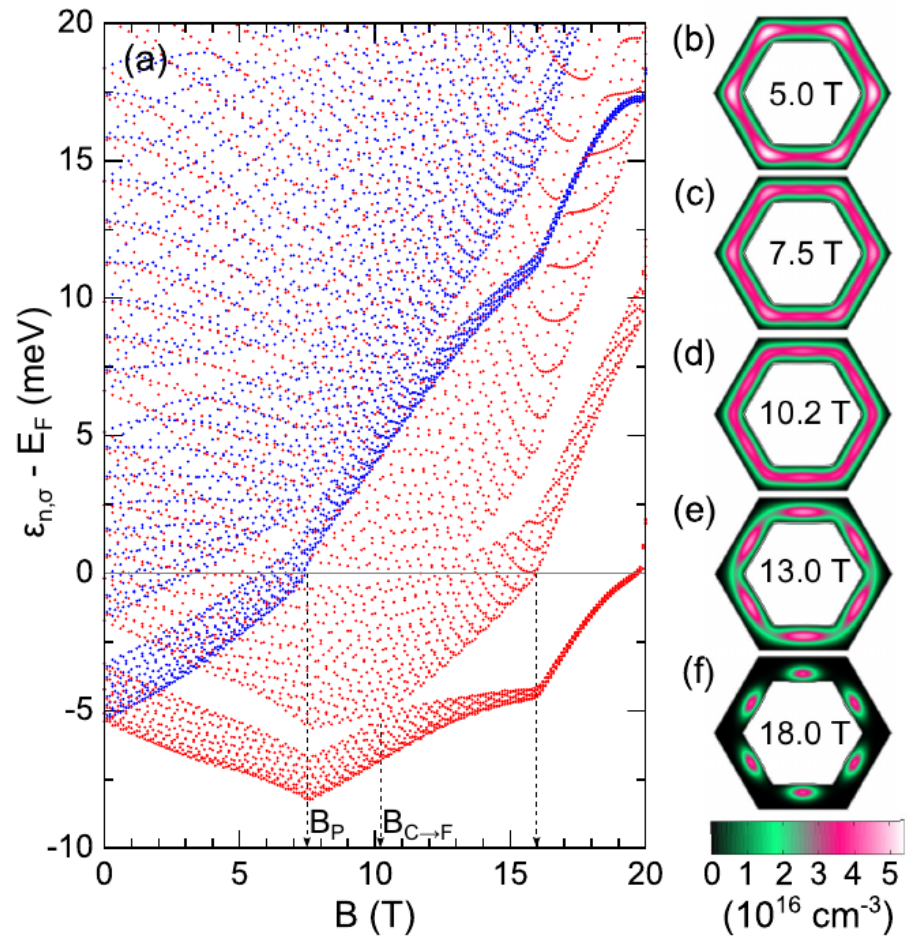

Figure 3.12: (a) MSSs for magnetic fields up to $B=20 \mathrm{~T}$, where the complete electron depletion takes place. Dashed vertical lines are added to indicate the fields at which spin or charge transitions occur. (b)-(f) Electron density distribution at selected magnetic-field intensities.

organized in spin-degenerate MSSs. The lowest-lying MSS is composed by states whose electron density is localized at the corners, while the states of the second MSS have their electron density mostly localized at the system facets for orthogonality. As the magnetic field increases, the $\downarrow$-MSSs are stabilized and the $\uparrow$-MSSs destabilized due to the Zeeman effect. Besides this spin-splitting, we also see that the first two $\downarrow$-MSSs get closer in energy for larger $B$ and eventually overlap at $B_{C \rightarrow F} \approx 10.2 \mathrm{~T}$. At this field, the electron density is equally distributed over the entire InAs ring and, therefore, $B_{C \rightarrow F} \approx 10.2 \mathrm{~T}$ can be identified as the transition point. For $B>B_{C \rightarrow F}$ the $\downarrow$-MSSs cross, and the lowest-lying states are now mainly localized at the facets of the hexagon. The same behavior is observed for the spin-up subbands, but they are already depopulated at this magnetic field and do not affect the electron density distribution. 

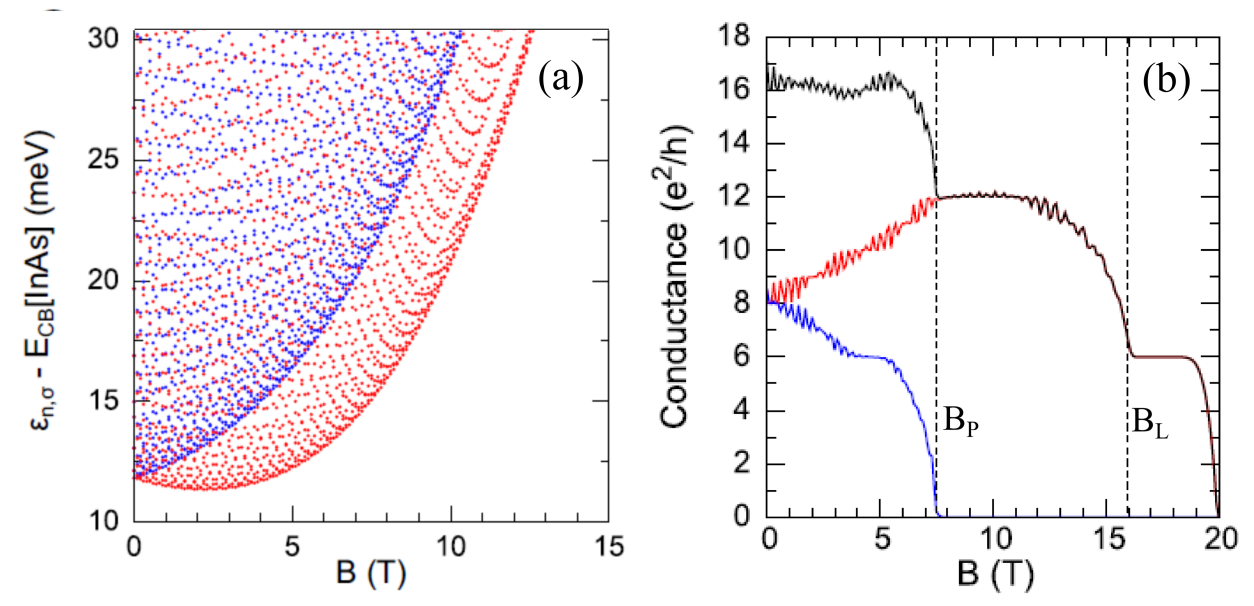

Figure 3.13: (a) Non-interacting MSSs with respect to the InAs $\mathrm{CB}$ edge. Both spin-up (blue) and spin-down (red) subbands are shown. (b) Total magnetoconductance $G=G_{\downarrow}+G_{\uparrow}$ (black) and spinprojected magnetoconductances $G_{\downarrow}$ (red) and $G_{\uparrow}$ (blue) as a function of the magnetic field $B$.

Figure 3.12 (a) exhibits two additional transition points in the energy spectrum. They are characterized by noticeable changes in the slope of the MSSs and are indicated with dashed vertical lines in the graph. The first transition is found at $B_{P} \approx 7.5 \mathrm{~T}$, where the slope of the lowest-lying MSS changes sign from negative to positive. If one looks at figure 3.12(a) carefully, it can be observed that the transition coincides with the complete depletion of the last $\uparrow$-MSS with charge. Consequently, the system becomes completely polarized and $B_{P}$ represents a transition to a ferromagnetic state. At fields higher than $B_{C \rightarrow F}$, the MSSs rearrange and tend to form Landau-like bands. When the magnetic field reaches $B_{L} \approx 16 \mathrm{~T}$, a second transition appears as an abrupt change in the subband slope. At this point, the first-excited $\downarrow$-MSS is fully depleted and only one subband remains below the Fermi energy. Finally, this subband becomes completely depopulated for magnetic fields higher than $B=20 \mathrm{~T}$.

In order to asses the role of many-electron contributions we also carry out simulations using a non-interacting model, i.e. by setting $v_{H}=0$ and $v_{X C}^{\sigma}=0$ in Hamiltonian (3.5). The obtained energy spectrum, see figure 3.13(a), shows smooth MSSs with no visible changes in slope. The absence of abrupt transitions in this non-interacting system demonstrates their multi-particle origin. Moreover, additional calculations neglecting only the exchange-correlation potential $v_{X C}^{\sigma}=0$ (not shown) present the same 
energy spectrum as figure 3.12(a). This indicates the minor role of this term, being the Hartree potential energy the term responsible for the presence of transitions.

Lastly, we explore the signatures of these spin and charge transitions in magnetoconductance experiments. The results are summarized in figure 3.13 (b). Here, the total magnetoconductance curve exhibits a clear step-like form. At low magnetic fields, we observe regular oscillations as predicted previously for the low-magnetic-field regime. As the magnetic field is increased, the oscillations persist up to $B \approx 6 \mathrm{~T}$, but close to the first transition point the conductance is strongly reduced. The value of $G$ drops from $G \approx 16 \mathrm{e}^{2} / \mathrm{h}$ to $G \approx 12 \mathrm{e}^{2} / \mathrm{h}$ at this point, and then remains constant with minimal oscillations up to $B \approx 12 \mathrm{~T}$. This plateau is generated because $E_{F}$ is located between the second and third $\downarrow$-MSS, so there are no crossings. At $B>12 \mathrm{~T}$, the conductance starts oscillating again as $E_{F}$ merges the second $\downarrow$-MSS, and $G$ experiences a progressive reduction until the second transition is reached. After this point we find another plateau for the same reason as before, that eventually drops to zero when the $\mathrm{CB}$ gets completely depleted.

Summing up, in the high-magnetic-field regime we have found various field-induced transitions, which can be related to the complete depletion of excited subbands. Such transitions can be identified in magnetoconductance experiments by an step-like behavior. 


\section{CHAPTER 4}

\section{Spin-orbit-induced spin relaxation in semiconductor}

QDs

Advances in the fabrication techniques over the last few decades have enabled the isolation and control of individual spins in solid-state systems. [54] This has opened the possibility of developing a new generation of devices that exploit the spin of the electron rather than its charge, giving rise to new fields in condensed matter physics such as spin-based electronics (spintronics) and quantum computing. [6, 55, 105, These spin-based devices are of great interest for future applications due to the predicted improved properties compared to the conventional electronic ones. For instance, some of their advantages would be the increased data processing speed, decreased electric power consumption, and increased integration densities. [6]

The electron spin degree of freedom is a natural two-level system in which information can be encoded through a particular spin orientation (either up or down). This stored information can then be carried over space in transport processes since spins are attached to electrons. Furthermore, the spin orientation is known to survive for a relatively long time (of the order of nanoseconds), offering the opportunity to store and manipulate phase coherence over length and time scales much longer than in typical charge-based devices. All this makes the spin degree of freedom particularly attractive and several novel devices have been proposed.[105] For example, some spin-valves and magnetoresistive random-access memories (MRAM) are already commercially available, but many other technologies are still under development. Among the latter, two of them deserve a special men- 
54 Chapter 4. Spin-orbit-induced spin relaxation in semiconductor QDs

tion: the spin field-effect transistor proposed by Datta and Das [106] and the spin quantum bit (qubit) proposed by Loss and DiVincenzo [107. On one hand, a spin-based transistor is one of the leading candidates to substitute the traditional silicon ones when the length limit of $7 \mathrm{~nm}$ is reached in 4 or 5 years. These new transistors are believed to improve the energy efficiency of the current ones. On the other hand, the fabrication of a quantum computer has been subject to intense research over the last years since it is expected to be much faster due to the direct use of quantum superposition and entanglement. Many different systems are being pursued for physically implementing a quantum computer, e.g. trapped ions, photons, superconductor junctions, and QDs. In particular, we focus on spin qubits as they are promising candidates that fulfill all the requirements needed for quantum computing. [108] However, despite important advances have been achieved, the fabrication of these spin-based devices is still not possible. To this end, a deeper understanding of the fundamental spin physics and the coupling with the environment is necessary.

One of the greatest challenges in using the spin degree of freedom in real applications is controlling or removing quantum decoherence. 109, 110, In a solid, the electron spin is not completely decoupled from other degrees of freedom, thus limiting its lifetime to be finite. Fortunately, spins in QDs exhibit longer lifetimes than in delocalized systems since quantum confinement suppresses the main bulk decoherence mechanisms. [111, 112] The two main spin relaxation channels in III-V ZB semiconductor QDs are the hyperfine interaction and the SOI, [54, 56] The former takes place as a result of the coupling with the spin bath constituted by the spins of the nuclei. This mechanism is dominant when the energy separation between spin states is small, i.e. at relatively weak magnetic fields. Additionally, the hyperfine interaction mechanism is further diminished in the $\mathrm{VB}$ because of the p-like nature of the hole orbitals. On the other hand, for moderate and strong fields, when the energy splitting exceeds the nuclear magnetic field, the phonon-assisted relaxation due to SOI prevails. The magnetic field regime we are interested in corresponds to the second case, so only the SOI-induced spin relaxation is considered here. As already discussed in section 2.3 , in semiconductors without inversion symmetry such as $\mathrm{ZB}$ and WZ structures, $\mathrm{SOI}$ in the $\mathrm{CB}$ is originated from bulk inversion asymmetry (DSOI) or structural inversion asymmetry (RSOI). Besides these two interactions, in the $\mathrm{VB}$ one has to consider the additional coupling between hh and $\mathrm{lh}$ subbands, which also results in spin mixing.

DSOI and RSOI Hamiltonians have different symmetries and present an anisotropic character. 33] Consequently, SOI-related effects are strongly affected by structural anisotropies, different crystallographic orientations as 
4.1. Theoretical formalism for the calculation of phonon-induced spin relaxation rates

well as the directions along which external fields are applied. This can be exploited to externally control and manipulate the spin degree of freedom. Most previous theoretical works dealing with $\mathrm{SOI}$-induced spin relaxation have considered quasi-2D systems where the lateral confinement has been modeled by a parabolic potential. Nevertheless, current synthetic methods are able to produce 3D QDs routinely and, thus, accounting for the 3D nature of SOI becomes essential to understand their properties.

In this chapter we present an overview of the results of five published works that deal with spin-orbit-related properties of single spins confined in ZB semiconductor QDs. A copy of these articles can be found at pages 179, 183, 193, 205 and 227 of the present dissertation. In particular, we focus on the role of three-dimensionality in the $\mathrm{QD}$ spin dynamics, paying special attention to the anisotropic behavior of SOI. First, the basic aspects of the theoretical procedure employed for computing the spin relaxation time are presented. Using this model, the spin dynamics of electrons and holes in spheroidal QDs under external fields is investigated. The study of this simple system allows one to understand the dependence of the spin relaxation on the QD geometry, laying the basis for the investigation of more complex systems where the 3D nature of the structures may be important, namely cuboidal QDs grown along different crystal directions, quantum dot molecules, and pyramidal QDs.

\subsection{Theoretical formalism for the calculation of phonon-induced spin relaxation rates}

In this section we present the expressions employed to estimate the spin relaxation rate of electrons and holes confined in $\mathrm{ZB}$ semiconductor QDs.

Any spin relaxation process needs both a source of spin admixture and a source of energy relaxation in order to take place. As stated above, the spin mixing in these systems is produced by SOI. The corresponding Hamiltonians are given in section 2.3. As for the source of energy relaxation, the dominant mechanism is determined by the transition energy $\Delta E_{f i}$. We study the spin relaxation between Zeeman-split sublevels of lowest energy, i.e. the ground and the first-excited state. For moderate magnetic fields, the energy splitting of the two states is of the order or few meV. It is known that in transitions of this energy range the main scattering mechanism is mediated by the interaction of the carriers spin with the phonon bath.

Phonons are originated in the quantization of lattice vibrations and can be classified into acoustic and optical phonons. Since we restrict to low 
56 Chapter 4. Spin-orbit-induced spin relaxation in semiconductor QDs

energies, short-wave optical phonons cannot participate in the spin relaxation processes investigated, so only acoustic phonons are included in our calculations. In addition, for low $\Delta E_{f i}$ the linear dispersion regime holds, $E_{\lambda q}=\hbar \omega_{q \lambda}=\hbar c_{\lambda} q$, where $c_{\lambda}$ is the phonon velocity of the longitudinal $(\lambda=l)$ or two transversal $\left(\lambda=t_{1}, t_{2}\right)$ acoustic phonon modes and $q$ is the phonon wave vector.

Vibrations of the bulk lattice (phonons) produce small displacements of the atoms from their equilibrium positions. These deviations lead to small shifts in the energy bands and also to the origin of additional electric fields that are responsible for the scattering processes. For ZB crystal structures, the two relevant scattering mechanisms at low temperature are the deformation potential and the piezoelectric potential.[113] Thus, the carrier-phonon Hamiltonian is as follows

$$
H_{c-p h}^{\lambda}=e \phi_{p z}^{\lambda} \mathcal{I}+H_{d p}^{\lambda},
$$

where $e$ is the particle charge, $\mathcal{I}$ is the identity matrix, and $\phi_{p z}^{\lambda}$ and $H_{d p}^{\lambda}$ denote the piezoelectric and the deformation potential terms, respectively. To compute the spin relaxation, Hamiltonian (4.1) needs to be written in terms of the normal modes of vibration. The derivation and complete expressions of $\phi_{p z}^{\lambda}$ and $H_{d p}^{\lambda}$ for both $\mathrm{CB}$ and $\mathrm{VB}$ are given in appendix $\mathrm{D}$.

The transition rate between the initial occupied state $\left(\left|\Psi_{i}\right\rangle\right)$ and the final unoccupied state $\left(\left|\Psi_{f}\right\rangle\right)$, mediated by the carrier-phonon interaction, is calculated within time-dependent first-order perturbation theory, specifically the Fermi's golden rule:

$$
\frac{1}{T_{1}}=\frac{2 \pi}{\hbar} \sum_{\lambda, \mathbf{q}}\left|\left\langle\Psi_{f}\left|H_{c-p h}^{\lambda}\right| \Psi_{i}\right\rangle\right|^{2} \delta\left(\Delta E_{f i}+E_{\lambda q}\right) .
$$

Here, $H_{c-p h}^{\lambda}$ is the carrier-phonon coupling Hamiltonian (4.1), $E_{\lambda q}=\hbar c_{\lambda} q$, and $\Delta E_{f i}=E_{f}-E_{i}$. The sum is done over all directions of wave vector $\mathbf{q}$ and all possible decay channels. We assume bulk phonons, which is an appropriate model for embedded QDs. Calculations are carried out at zero temperature for the sake of simplicity, so that phonon absorption and multiphonon processes are negligible. 114

\subsection{Spin relaxation in 3D spheroidal QDs}

We start investigating the spin relaxation rates between Zeeman-split sublevels in spheroidal QDs. This $3 \mathrm{D}$ system allows to easily tailor the QD 
shape in order to assess the effect of confinement on the $\mathrm{SOI}$ and, by extension, on the spin dynamics. Quantum confinement is known to influence the orbital motion of carriers which, in turn, affects its spin through SOI. Therefore, it is expected that the spin physics could be controlled by growing structures with specific geometries.

\subsubsection{Electron spin relaxation}

We first study the spin relaxation due to single-phonon emission in the $\mathrm{CB}$. The spheroidal QDs are modeled using parabolic confinement potentials and are subject to an axial magnetic field defined by the vector potential in the symmetric gauge, $\mathbf{A}=\frac{B}{2}(-y, x, 0)$, and an electric field $\mathbf{F}$ with arbitrary direction. The electron Hamiltonian reads

$$
H=\sum_{j=x, y, z} H_{H O}(j)-e \mathbf{F} \cdot \mathbf{r}+\frac{1}{2} g^{*} B \sigma_{z}+H_{S O I},
$$

where $e=-1$ is the electron charge and $H_{H O}(j)$ is the harmonic oscillator Hamiltonian

$$
H_{H O}(j)=\frac{1}{2 m^{*}}\left(k_{j}-e A_{j}\right)^{2}+\frac{1}{2} m^{*} \omega_{j}^{2} j^{2},
$$

with $\omega_{j}$ standing for the frequency of the confining parabola and $k_{j}=$ $-i \hbar d / d j$. The second term in equation 4.3 represents the electric field potential, equation (2.19). The third term accounts for the Zeeman splitting resulting from a magnetic field along the $z$ direction (see section 2.2.2 for details). The last term is the SOI Hamiltonian $H_{S O I}=H_{B I A}^{C B}+H_{S I A}^{V B}$. Here, $H_{B I A}^{C B}$ denotes the DSOI term, equation 2.22, and $H_{S I A}^{V B}$ the RSOI term, equation 2.24.

Hamiltonian 4.3 is solved by rewriting all derivatives and coordinates in terms of harmonic oscillator ladder operators and then projecting it onto a basis formed by oscillator eigenstates $\left|\nu_{x}, \nu_{y}, \nu_{z}\right\rangle$. The spin relaxation is computed by means of the Fermi's golden rule as explained in the previous section. In particular, we consider $\operatorname{In}(\mathrm{Ga}) \mathrm{As} \mathrm{QDs}$ in all calculations. The specific values of the corresponding parameters can be consulted in the published work, see page 183 of this Thesis.

The effect of the SOI in the electron spin dynamics is studied for each mechanism individually, namely DSOI and RSOI. The results are presented below. 
58 Chapter 4. Spin-orbit-induced spin relaxation in semiconductor QDs

\section{Dresselhaus SOI}

Figure 4.1(a) summarizes the results obtained for the spin relaxation rate as a function of the vertical confinement. Calculations are performed at $B=1 \mathrm{~T}$ and $\mathbf{F}=0$, considering various in-plane confinements $\left(\omega_{\perp}=\right.$ $\left.\omega_{x}=\omega_{y}\right)$ ranging from $\hbar \omega_{\perp}=5 \mathrm{meV}$ to $\hbar \omega_{\perp}=40 \mathrm{meV}$. Previous works in literature have reported an increasing dependence of $1 / T_{1}$ with $\hbar \omega_{z}$ for quasi-2D systems. [33, 54] Indeed, this is the behavior we find for the QD with weakest lateral confinement, $\hbar \omega_{\perp}=5 \mathrm{meV}$, which can also be seen as quasi-2D $\left(\omega_{z}>\omega_{\perp}\right)$. However, we observe from the other curves in figure 4.1. (a) that for non-flat systems the behavior is richer. In all cases, the spin relaxation rate becomes minimum for $\omega_{\perp}=\omega_{z}$ and rapidly increases as the QD geometry deviates from a perfect sphere. In other words, the spin relaxation is suppressed when the system has spherical symmetry and it is enhanced when the symmetry is lowered. These results stress the importance of three-dimensionality when investigating the spin dynamics of QDs,

In order to understand the origin of the minimum in the spin relaxation curves, we calculate the degree of spin admixing of the states involved in the transition. Other factors, such as the density of phonons, are not important since they are independent of $\omega_{z}$ and remain constant during each calculation series. The spin purity of the ground state (solid line) is plotted in figure 4.1(b). We see that it becomes maximum for a spherical QD and decreases in prolate and oblate structures. This fact indicates that a minimum value of $1 / T_{1}$ originates from a situation with maximum spin purity, i.e. where $\mathrm{SOI}$ is more hindered. Consequently, the dependence between spin relaxation rate and QD shape lies in the form of the SOI Hamiltonians for different system symmetries.

The spin admixture is determined by the $\sigma_{x}$ and $\sigma_{y}$ terms in $H_{B I A}^{C B}$, equation 2.22, since $\sigma_{z}$ is diagonal in spin-space and does not flip spins. The mixing Hamiltonian is then approximated as

$$
H_{B I A}^{m i x} \approx b_{41}^{C B}\left[p_{x}\left(\left\langle p_{y}^{2}\right\rangle-\left\langle p_{z}^{2}\right\rangle\right) \sigma_{x}+p_{y}\left(\left\langle p_{z}^{2}\right\rangle-\left\langle p_{x}^{2}\right\rangle\right) \sigma_{y}\right] .
$$

For a spherical QD we have $\left\langle k_{x}^{2}\right\rangle=\left\langle k_{y}^{2}\right\rangle=\left\langle k_{z}^{2}\right\rangle$. In the absence of a magnetic field the kinetic and canonical momenta coincide, $\left\langle k_{j}^{2}\right\rangle=\left\langle p_{j}^{2}\right\rangle$, but for finite $B$ this is not longer true since $p_{j}=k_{j}-e A_{j}$. In spite of this, in the limit of small fields, $B \rightarrow 0$, we can consider that $\left\langle p_{\perp}^{2}\right\rangle \approx\left\langle p_{z}^{2}\right\rangle$, so that equation 4.5 tends to vanish, thus justifying the profound minimum found in figure 4.1 (a) when $\omega_{z}=\omega_{\perp}$. 

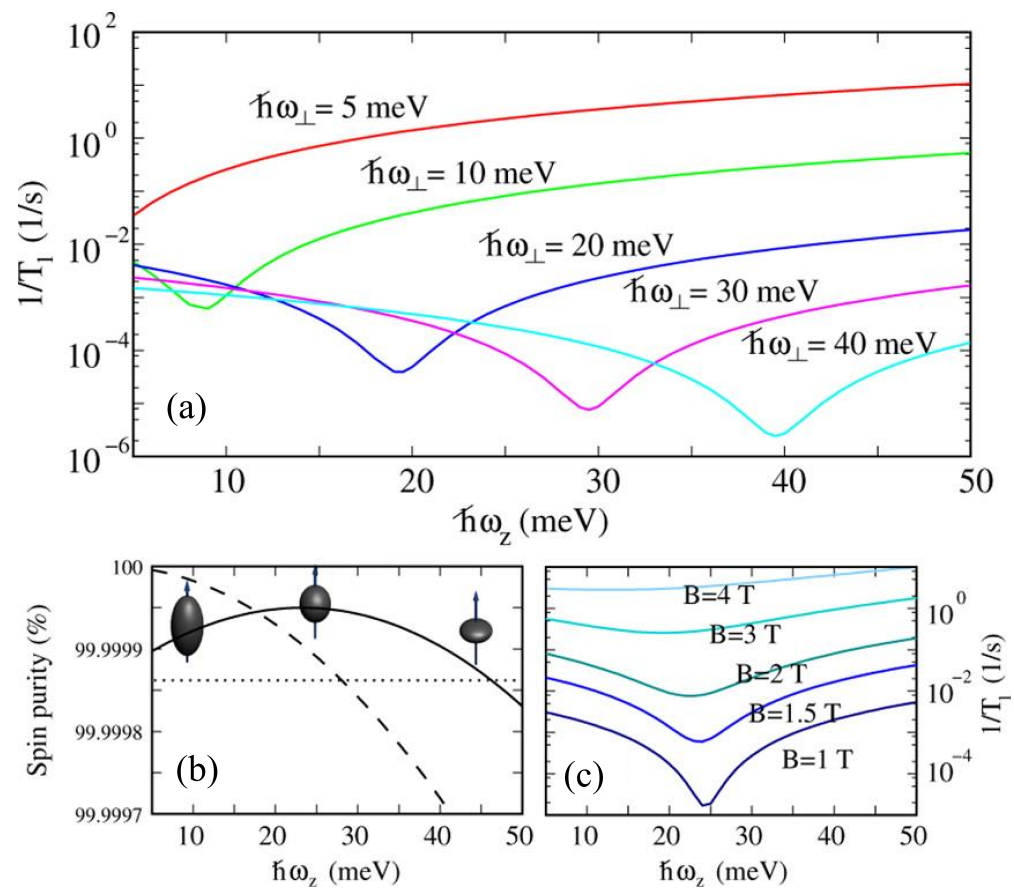

Figure 4.1: (a) Spin relaxation rate as a function of the vertical confinement $\hbar \omega_{z}$ with only DSOI included. Results for various in-plane confinements $\hbar \omega_{\perp}$ are presented. (b) Spin purity of the ground state for a QD with $\hbar \omega_{\perp}=25 \mathrm{meV}$. Three levels of calculation have been considered: exact result (solid line), linear approximation (dashed line), and in-plane cubic approximation (dotted line). Insets show schematic drawings illustrating QDs with different vertical confinement. (c) $1 / T_{1}$ vs. vertical confinement for increasing magnetic field and $\hbar \omega_{\perp}=25 \mathrm{meV}$.

The above approximation, $\left\langle k_{j}^{2}\right\rangle \approx\left\langle p_{j}^{2}\right\rangle$, is only valid for relatively small magnetic fields. As $B$ increases the canonical momentum $\left\langle p^{2}\right\rangle$ becomes more anisotropic, so that the suppression of the spin admixture is progressively reduced. This is exactly the behavior observed in figure 4.1(c), where the $1 / T_{1}$ minimum is gradually removed for increasing magnetic fields.

For the sake of completeness, we repeat the spin purity calculations for other commonly used approximations. [54] For oblate structures in the limit of $\left\langle k_{z}^{2}\right\rangle \ll\left\langle k_{\perp}^{2}\right\rangle$, equation 4.5 reduces to $H_{B I A}^{m i x} \approx b_{41}^{C B}\left\langle p_{z}^{2}\right\rangle\left(p_{y} \sigma_{y}-p_{x} \sigma_{x}\right)$ (linear approximation). The dashed line in figure 4.1(b) shows that this approximation provides a qualitatively correct estimate of the spin mixing for oblate QDs, albeit systematically overestimated. In the limit of quasi-1D 
prolate QDs, $\left\langle k_{z}^{2}\right\rangle \gg\left\langle k_{\perp}^{2}\right\rangle$, we have $H_{B I A}^{m i x} \approx b_{41}^{C B}\left\langle p_{\perp}^{2}\right\rangle\left(p_{x} \sigma_{x}-p_{y} \sigma_{y}\right)$ (cubic approximation). As can be seen in figure 4.1(b), the spin mixing does not depend on $\omega_{z}$ in this approximation (dotted line), hence the calculated spin purity is constant and mostly underestimated. In light of these results, we can state that the interplay between $3 \mathrm{D}$ degrees of freedom is crucial and the full DSOI Hamiltonian must be considered.

\section{Rashba SOI}

We next investigate the spin relaxation rate in QDs with RSOI. In particular, we pay special attention to the quantum confinement anisotropy and the direction of the external electric field $\mathbf{F}$ as mechanisms to control the RSOI strength and, in turn, the spin dynamics in QDs. In this respect, previous publications have pointed out the possibility of modulating the RSOI in quasi-2D systems due to the confinement anisotropy.[115]117] Here, we extend the study to 3D QDs.

Figure 4.2(a) shows a contour plot of $1 / T_{1}$ as a function of the vertical confinement $\omega_{z}$ and the polar angle $\theta$ of the electric field orientation. All calculations are carried out for QDS with in-plane confinement $\hbar \omega_{\perp}=50 \mathrm{meV}$ and under an axial magnetic field $B=5 \mathrm{~T}$. In general, one can see that the maximum (minimum) spin relaxation is found when $\mathbf{F}$ points in the direction of strongest (weakest) confinement. To improve the readability of this plot and help extracting interesting information, four cross-sections are also included in figure 4.2 (b-e). On one hand, we observe a decreasing behavior of $1 / T_{1}$ with $\omega_{z}$ for $\mathbf{F} \perp \mathbf{B}$, figure 4.2 (c), while it remains constant for $\mathbf{F} \| \mathbf{B}$, figure $4.2(\mathrm{~b})$. On the other hand, the dependence of $1 / T_{1}$ on the polar angle $\theta$ shows opposite behavior for the two vertical confinements considered. It increases for $\omega_{z}=10 \mathrm{meV}$, figure $4.2(\mathrm{~d})$, and decreases for $\omega_{z}=100 \mathrm{meV}$, figure $4.2(\mathrm{e})$.

These results can be justified following the same strategy as for DSOI Then, the spin relaxation rate is mainly determined by the strength of the RSOI, which can be understood by analyzing the terms in $H_{S I A}^{C B}$, equation (2.24), contributing to spin flips. The spin mixing part is as follows

$$
H_{S I A}^{\operatorname{mix}}=r_{41}\left[F_{z}\left(p_{y} \sigma_{x}-p_{x} \sigma_{y}\right)+F_{x} p_{z} \sigma_{y}-F_{y} p_{z} \sigma_{x}\right] .
$$

The first term in (4.6) corresponds to figure 4.2(b) ( F \| B ). It is worth mentioning that this is the only term included in most studies on quasi2D QDs, [54] This term does not depend on the vertical carrier motion, i.e. does not contain $p_{z}$, explaining the flat dependence with $\omega_{z}$. Contrarily, the 


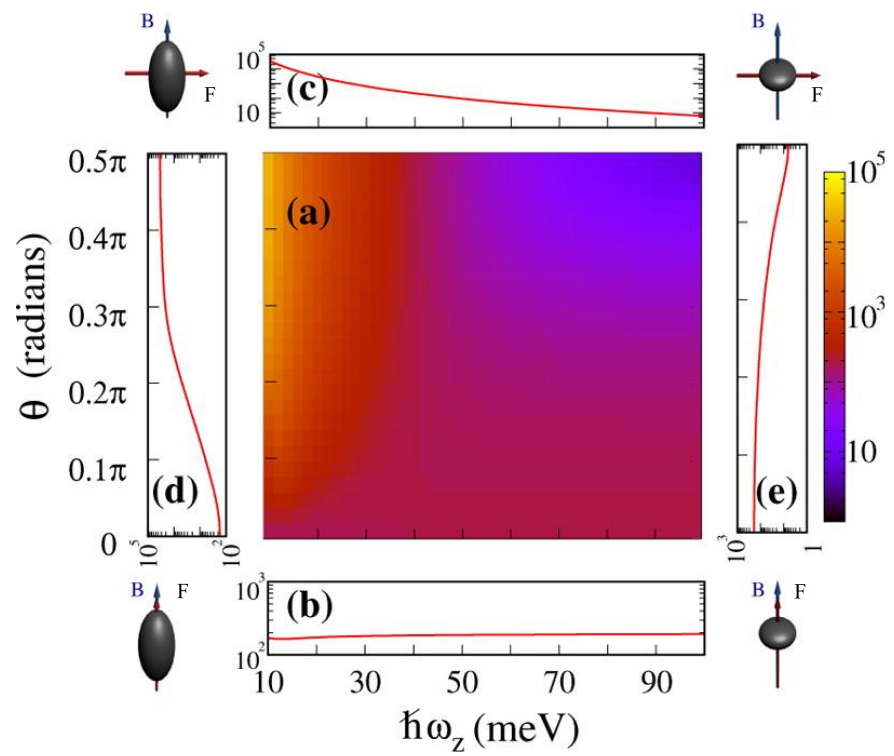

Figure 4.2: Spin relaxation rate in a $\mathrm{QD}$ with $\hbar \omega_{\perp}=50 \mathrm{meV}$ in the presence of RSOI All calculations are carried out for $\mathbf{F}=30 \mathrm{kV} / \mathrm{cm}$ and $B=5 \mathrm{~T}$. (a) Contour plot of $1 / T_{1}$ as a function of vertical confinement and the orientation of the electric field. Panels (b) and (c) are cross sections for $\theta=0$ and $\theta=\pi / 2$, respectively. Panels (d) and (e) are cross sections for $\hbar \omega_{z}=10 \mathrm{meV}$ and $\hbar \omega_{z}=100 \mathrm{meV}$, respectively. The schematics at the corners illustrate the QD shape and the orientation of $\mathbf{B}$ and $\mathbf{F}$.

other two terms $(\mathbf{F} \perp \mathbf{B})$ do depend on the vertical confinement, justifying the high influence of $\omega_{z}$ on $1 / T_{1}$ in figure 4.2 (c). As for the opposite behavior observed in panels (d) and (e), we have to take into account the states that Hamiltonian (4.6) couples. One can see that all three terms couple the ground state $|0,0,0\rangle$ to excited states with a node in a direction perpendicular to the electric field. As $\mathbf{F}$ is tilted the dominant term changes and so does the excited states involved in the coupling. Thus, when $\mathbf{F}$ is perpendicular (parallel) to the direction of strongest confinement, $H_{S I A}^{\operatorname{mix}}$ couples the ground state with excited states of higher (lower) energy, hence the coupling is inhibited (enhanced) and so is the relaxation rate.

\subsubsection{Hole spin relaxation}

Here we extend the study on electron spin relaxation discussed in the previous section to the spin of holes. Several theoretical works have investi- 
gated the spin dynamics of single holes in quasi-2D QDs considering different sources of spin mixing, namely lh-hh coupling, [113, 118] cubic-in- $k$ DSOI, 119. linear-in- $k$ DSOI, 120, and the e-h exchange interaction together with strain in holes forming excitons. [121] In all these works, one spin mixing mechanism is assumed as dominant, while the others are neglected without any comparison between them. In order to shed light on this matter, we investigate the dependence of the hole spin lifetime on the QD geometry by considering simultaneously all relevant sources of spin admixing. In this way, it is possible to identify the dominant mechanisms in hole spin scattering processes and establish their regime of application. In addition, some of the aforementioned works report opposite results, [113, 118, or predict results not observed in experiments. [119] In this respect, we explicitly compare our results with those apparently controversial.

The Hamiltonian describing the hole states in 3D spheroidal QDs reads

$$
H=H_{Z B}^{L K}+H_{B I A}^{V B}+V_{Q D} \mathcal{I}+H_{Z},
$$

where $\mathcal{I}$ is the $4 \times 4$ identity matrix. $H_{Z B}^{L K}$ is the four-band Luttinger-Kohn Hamiltonian, [122] whose matrix form is given in appendix A, section A.1.1. The second term in (4.7) corresponds to the DSOI Hamiltonian, equation 2.23). 33. It includes linear- and cubic-in- $k$ terms, and its matrix representation can be found in section B.1.2. The RSOI is disregarded in this study because it is an extrinsic effect, and for holes it is less efficient than DSOI under moderate magnetic fields. [119] The third term denotes the confining potential, $V_{Q D}$, modeling QDs with parabolic confinement:

$$
V_{Q D}=-\frac{1}{2} m_{\perp}^{*} \omega_{\perp}^{2}\left(x^{2}+y^{2}\right)-\frac{1}{2} m_{z}^{*} \omega_{z}^{2} z^{2},
$$

with $\omega_{j}$ standing for the frequency of the confining parabola. Equation 4.8 simulate 3D spheroidal QDs with different aspect ratios. Finally, last term in 4.7 is the Hamiltonian describing the splitting of the hole states by an effective axial magnetic field:

$$
H_{Z}=\frac{1}{2}\left(\begin{array}{cccc}
\Delta & 0 & 0 & 0 \\
0 & \frac{1}{3} \Delta & 0 & 0 \\
0 & 0 & -\frac{1}{3} \Delta & 0 \\
0 & 0 & 0 & -\Delta
\end{array}\right)
$$

Here, $\Delta$ is the value of the energy splitting, which is three times larger for $\mathrm{hh}$ than for $\mathrm{lh}$. The origin of this splitting could be the e- $\mathrm{h}$ exchange interaction or a Zeeman effect.

Equation (4.7) is solved following the same procedure as for the $\mathrm{CB}$. Nevertheless, we now deal with a four-band model where each subband 
has different mass and, hence, also different oscillator frequency. Then, we rewrite all coordinates and derivatives of equation (4.7) in terms of the hh harmonic oscillator Hamiltonians. The resulting Hamiltonian is then projected onto the $1 \mathrm{D}$ hh eigenfunctions.

Hole spin lifetimes are calculated within the theoretical formalism exposed in section 4.1, i.e. the Fermi's golden rule. The specific expressions for a four-band $\mathrm{VB}$ model are given in appendix $\mathrm{D}$. Simulations are carried out for InAs QDs embedded in a GaAs matrix. The material parameters compiled in table 1 of the published manuscript (see page 205).

\section{Geometry and spin splitting dependence}

We start investigating the role of the QD aspect ratio and the spin splitting magnitude on the hole spin lifetime, $T_{1}^{h}$. The results obtained are summarized in figure 4.3 . For the sake of comparison, both hole (red solid line) and electron (blue dotted line) spin relaxation times are depicted. Calculations for electrons and holes are performed considering a QD with the same size, i.e. defining the confining parabola with the same force constants $m_{j}^{h h}\left(\omega_{j}^{h h}\right)^{2}=m_{j}^{e}\left(\omega_{j}^{e}\right)^{2}$ with $j=\perp, z$.

Figure 4.3(a) shows $T_{1}$ as a function of $\omega_{\perp}$ in InAs QDs with strong vertical confinement. One can see that $T_{1}^{e}$ increases with the vertical confinement because DSOI is gradually suppressed as we get closer to a spherical QD $\left(\omega_{\perp}^{e}=\omega_{z}^{e}\right)$, in agreement with the results of the preceding section. On the other hand, $T_{1}^{h}$ has a non-monotonic behavior, presenting a minimum at $\hbar \omega_{\perp}^{h h}=28 \mathrm{meV}$. Previous works have reported an opposite dependence of $T_{1}^{h}$ on $\omega_{\perp}^{h h}$ : Woods et al. [118] predicted the hole spin lifetime to increase with the lateral confinement, while Lü et al. [113] predicted the opposite trend in a similar study. These apparently contradictory results are both compatible with figure 4.3(a), corresponding to the right and left side of the minimum.

We next study the dependence of the spin relaxation on the vertical confinement. In QDs with moderately strong lateral confinement, figure 4.3(b), which can be roughly seen as self-assembled QDs, electrons and holes present opposite behavior. $T_{1}^{e}$ decreases with $\omega_{z}$ since the system becomes flatter, i.e. less spheric. The hole spin lifetime, instead, increases in general but a shallow minimum can be observed at $\hbar \omega_{z}=14 \mathrm{meV}$, showing that different trends for $T_{1}^{h}$ are also possible when varying $\omega_{z}$. In figure 4.3.(c) we consider structures with weak lateral confinement, which are comparable to electrostatic QDs. Electrons present the same qualitative behavior 
64 Chapter 4. Spin-orbit-induced spin relaxation in semiconductor QDs
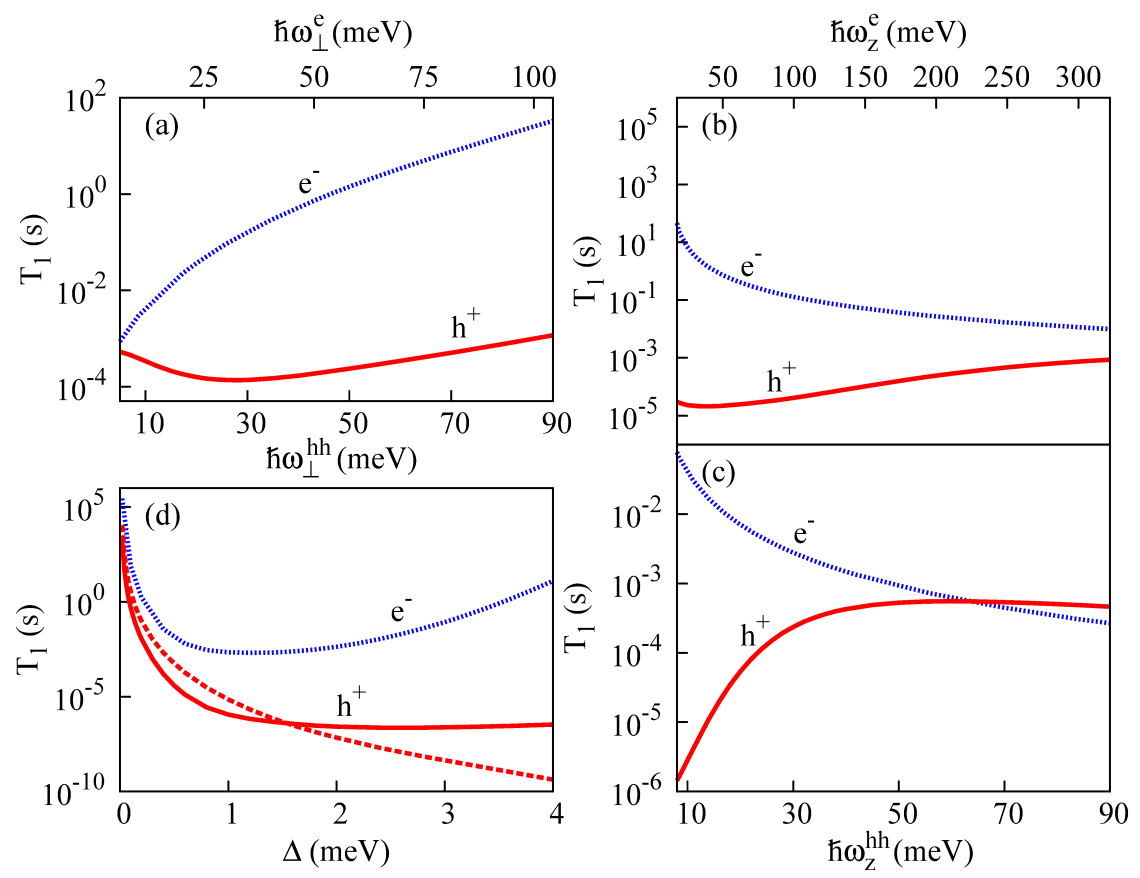

Figure 4.3: Hole (red solid line) and electron (blue dotted line) spin relaxation time as a function of (a) lateral confinement, (b-c) vertical confinement, and (d) energy splitting $\Delta$. (a) $\hbar \omega_{z}^{h h}=50 \mathrm{meV}$, $\hbar \omega_{z}^{e}=179 \mathrm{meV}, \Delta=0.4 \mathrm{meV}$. (b) $\hbar \omega_{\perp}^{h h}=20 \mathrm{meV}, \hbar \omega_{\perp}^{e}=23.2 \mathrm{meV}$, $\Delta=0.4 \mathrm{meV}$. (c) $\hbar \omega_{\perp}^{h h}=5 \mathrm{meV}, \hbar \omega_{\perp}^{e}=5.8 \mathrm{meV}, \Delta=0.4 \mathrm{meV}$. (d) $\hbar \omega_{\perp}^{h h}=20 \mathrm{meV}$ and $\hbar \omega_{z}^{h h}=50 \mathrm{meV} ; \hbar \omega_{\perp}^{e}=23.2 \mathrm{meV}$ and $\hbar \omega_{z}^{e}=$ $179 \mathrm{meV} ; \hbar \omega_{\perp}^{h h}=40 \mathrm{meV}$ and $\hbar \omega_{z}^{h h}=5 \mathrm{meV}$ (red dashed line).

as before, while the minimum of $T_{1}^{h}$ is now absent since it is shifted towards smaller $\omega_{z}$. Remarkably, we find that the spin lifetime of holes may exceed that of electrons in flat enough QDs. This result corroborates the prediction of Bulaev and Loss [119], who reported the possibility of having $T_{1}^{h}>T_{1}^{e}$ in gated structures. For self-assembled QDs, instead, $T_{1}^{h}$ is one order of magnitude shorter than $T_{1}^{e}$, what also goes along with experimental observations. 123 .

The electron spin relaxation is known to be maximum when the phonon wavelength is similar to the carrier wave function extension, but it decreases for smaller or larger spin splitting energy $\Delta$. Figure $4.3(\mathrm{~d})$ displays $T_{1}$ as a function of $\Delta$ for a self-assembled-like QD. For electrons a minimum is found $(\Delta \approx 1 \mathrm{meV})$, as expected. However, $T_{1}^{h}$ becomes constant for $\Delta>1.5 \mathrm{meV}$. This different behavior can be understood from their distinct effective masses which, despite considering QDs of the same size, result in 
unlike characteristic oscillator lengths. As a result, a larger $\Delta$ is needed for $T_{1}^{h}$ to increase again. We also run simulations for a nanorod-like structure (red dashed line), for comparison. In this case, the plateau in $T_{1}^{h}$ disappears and the relaxation is sensitive to $\Delta$ for all the range under study.

\section{Mechanisms of spin admixture}

As we have seen for electrons in the preceding section, in most cases the spin relaxation is mainly determined by the degree of spin admixing. Therefore, it is crucial to establish which mechanisms are dominant in order to understand the dependence of spin lifetimes on system geometry. To this aim, we repeat all calculations depicted in figure 4.3 but now taking into account each source of mixing individually. Notice that henceforth only the spin of holes is considered, so the hh superscript is dropped. The results for the spin relaxation rate, $1 / T_{1}$, are summarized in figure 4.4 .

The two relevant spin-mixing mechanisms for the $\mathrm{VB}$ are the lh-hh coupling originating from the Luttinger-Kohn Hamiltonian $H_{Z B}^{L K}$, and the DSOI, which can be further divided into its various terms. Then, the simulations are performed including the diagonal terms of $H_{Z B}^{L K}$ plus different combinations of the off-diagonal $H_{Z B}^{L K}$ Hamiltonian and some DSOI terms, as shown in figure 4.4 .

We analyze first the influence of the lateral confinement on the spin relaxation main mechanisms, figure 4.4(a). It is found that lh-hh coupling is more important than DSOI alone for large $\omega_{\perp}$. However, both contributions are comparable for moderate lateral confinement (self-assembled-like QDs), and $H_{B I A}$ becomes dominant for weak laterally confined structures (e.g. gated dots). As for the contribution of the different $H_{B I A}^{V B}$ terms, figure 4.4 (a) reveals that $H_{b_{41}}$ is the most relevant one. In fact, by only considering the lh-hh coupling and the $H_{b_{41}}$ term (green dotted line) we almost recover the spin relaxation rate obtained employing the full Hamiltonian (black line). In contrast, the linear-in-k term $H_{C_{k}}$ has a negligible influence, oppositely to reference [120] where it has been proposed as the dominant SOI term.

The behavior when changing the vertical confinement is in agreement with that seen in figure 4.4(a). In QDs with moderate lateral confinement, figure 4.4(b), lh-hh coupling dominates for all $\omega_{z}$ under study, but for weak lateral confinement, figure 4.4 (c), the strength of both mechanisms is comparable. Indeed, a transition from a dominant $H_{L}$ to a dominant $H_{B I A}$ situation takes place as $\omega_{z}$ decreases. Interestingly, $H_{B I A}$ provides a lower bound to $1 / T_{1}$ that is responsible for the origin of a plateau for $\omega_{z}>40 \mathrm{meV}$. 
66 Chapter 4. Spin-orbit-induced spin relaxation in semiconductor QDs
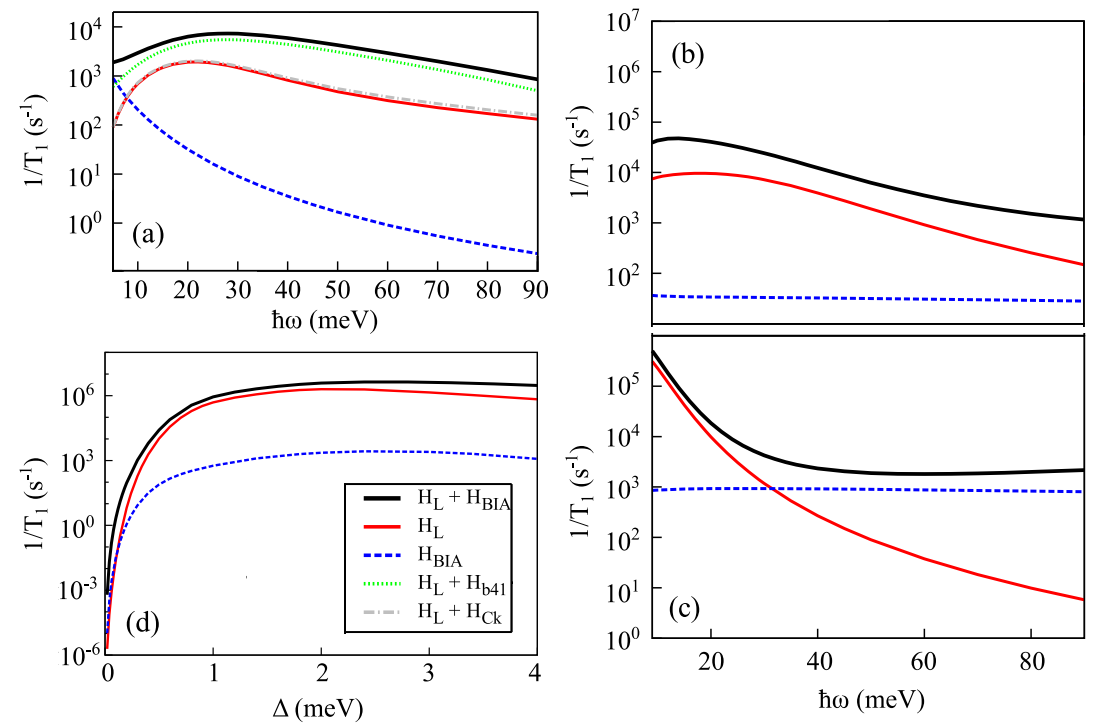

Figure 4.4: Same as figure 4.3, but considering the most relevant mechanisms of spin admixture individually. That is, the diagonal terms of $H_{Z B}^{L K}$ plus: off-diagonal $H_{Z B}^{L K}$ terms only, $H_{L}$ (red solid line); all DSOI terms, $H_{B I A}$ (blue dashed line); off-diagonal $H_{Z B}^{L K}$ terms and $b_{41}^{V B} \overline{\mathrm{DSOI}}$ term, $H_{b_{41}}$ (green dotted line); off-diagonal $H_{Z B}^{L K}$ terms and $C_{k}$ DSOI term, $H_{C_{k}}$ (gray dash-dotted line); and full Hamiltonian $H_{L}+H_{B I A}$ (thick black line). Only the spin relaxation of holes is included. Note that here we represent the spin relaxation rate $1 / T_{1}$, while in figure 4.3 we represent the spin lifetime $T_{1}$.

The influence of the spin splitting $\Delta$ on the mechanisms of spin admixture is explored in figure 4.4(d). The lh-hh coupling has a dominant contribution for most $\Delta$, but $H_{B I A}$ becomes equally important when $\Delta \rightarrow 0$. For such small energy splitting, the hole-phonon coupling becomes very inefficient and the relaxation rate decreases rapidly. The presence of DSOI. however, originates a small zero-field spin splitting that becomes significant for $\Delta \approx 0$, thus causing $H_{B I A}$ to prevail.

In summary, in this section we have shown the substantial effect of the geometry anisotropy on the spin relaxation of both electrons and holes by changing the aspect ratio of spheroidal QDs, In addition, we have found that accounting for the $3 \mathrm{D}$ nature of the structures is crucial for a proper description of the SOI. In the subsequent sections, we also study SOI-induced effects in more complex systems in which three-dimensionality is expected to play and important role. 


\subsection{Electron spin-relaxation anisotropy in [001] and [111] grown QDs}

In the preceding section we have shown the anisotropy of the spin-orbitinduced spin relaxation by changing the aspect ratio of 3D spheroidal QDs with circular lateral confinement. Other theoretical works investigating also QDs with circular symmetry have reported an in-plane spin relaxation anisotropy with the magnetic field orientation due to the interplay between RSOI and DSOI. 124, 125. In those studies, the spin lifetime becomes maximum (minimum) when the magnetic field is along the [110] ([110]) crystallographic direction. Such anisotropic angular dependence has been confirmed experimentally by Scarlino et al. [126], but the singular points of the $T_{1}$ curve obtained are deviated from the theoretical angles. This fact is ascribed to the elongated geometry of the QDs in the experiments, what goes along with some theoretical works that have pointed out that deviations from the in-plane circular symmetry affect the spin relaxation anisotropy. 127 129] It is also worth noting that all the aforementioned theoretical works have considered 2D models ignoring the contribution of cubic DSOI terms. Nonetheless, we have already seen above the important role of these terms on the $\mathrm{SOI}$ anisotropy in $3 \mathrm{D}$ QDs, so that one can also expect them to have a relevant influence on the in-plane spin relaxation anisotropy.

Here we study the in-plane electron spin relaxation anisotropy including all terms of RSOI and DSOI in a fully 3D model. The spin relaxation rate is monitored by modifying the orientation of the externally applied electric $\mathbf{F}$ and magnetic $\mathbf{B}$ fields, see figure 4.5. In particular, we consider cuboidal GaAs QDs with different heights and base shapes in order to gain insight into the role of three-dimensionality and QD elongation. Additionally, we investigate QDs with various crystallographic orientations, particularly QDs rotated around the $z$ axis and QDs grown along the [111] crystal direction. The latter are particularly interesting for optical spin preparation. [130] In fact, the spin relaxation has already been extensively discussed in [111] quantum wells, [131-133] but it is still poorly understood in zero-dimensional structures.

The Hamiltonian describing the electronic states of such systems is as follows:

$$
H=\frac{\mathbf{p}^{2}}{2 m^{*}}+V_{Q D}-e \mathbf{F r}+H_{Z}+H_{B I A}^{C B}+H_{S I A}^{C B},
$$

with $\mathbf{p}=-i \hbar \boldsymbol{\nabla}-e \mathbf{A}$, and $V_{Q D}$ standing for the confining potential. The vector potential employed to define the in-plane magnetic field is $\mathbf{A}=$ 


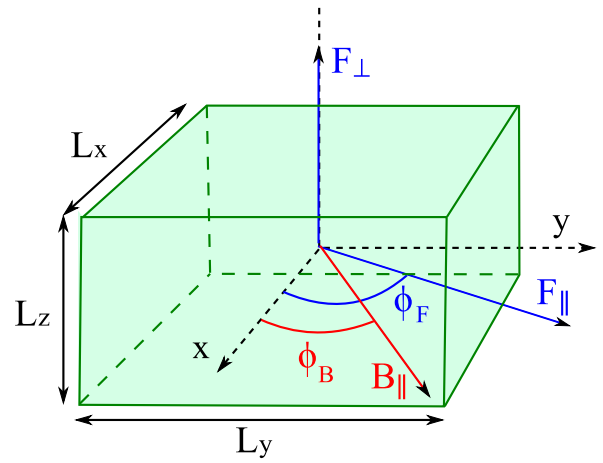

Figure 4.5: Schematic representation of the cuboidal GaAs QD The parameters defining the system dimensions and the orientation of the external electric and magnetic fields are indicated.

$z B\left(\sin \phi_{B},-\cos \phi_{B}, 0\right)$. The third term in 4.10 is the electric field potential, equation (2.19), with $e=-1$ for electrons. The fourth term $H_{Z}$ is the Zeeman splitting, equation (2.20). Finally, last two terms in 4.10 $H_{B I A}^{C B}$ and $H_{S I A}^{C B}$ correspond to the DSOI, equation 2.22), and the RSOI. equation (2.24), respectively.

Hamiltonian 4.10) is only valid for ZB|QDs grown along the [001] crystal direction. In order to study systems grown along other directions, new expressions need to be derived. To this purpose, we consider the confinement potential to be fixed in space and perform a rotation of the crystalline structure. In this way, we guarantee the accuracy of the calculations since the mesh always fits the cuboidal geometry in the same way independently of the QD orientation. A rotation of the crystalline structure causes changes in the internal coordinates and, thus, changes in the Hamiltonian. Next, we analyze how the different terms in equation (4.10) are affected by rotations. Since the mass of the $\mathrm{CB}$ is isotropic, the kinetic energy term has spheric symmetry and is invariant under rotations. The confinement potential and the external fields are not rotated, so their corresponding terms remain also unaltered. As for the Zeeman term, we need to take into account that the dot product of two vectors defined with respect to the same coordinate system is invariant as long as they rotate simultaneously. This is indeed the case of the magnetic field and the spin in $H_{Z}$. However, $H_{B I A}^{C B}$ and $H_{S I A}^{C B}$ do change when the crystalline structure is rotated and the expressions introduced in section 2.3 must be recalculated.

First, we obtain the SOI Hamiltonians corresponding to an in-plane 
rotation $\theta_{z}$ around the $z$ axis. The resulting Hamiltonians read:

$$
H_{S I A}^{[001]}\left(\theta_{z}\right)=r_{41} F_{z}\left(\sigma_{x} p_{y}-\sigma_{y} p_{x}\right),
$$

and

$$
\begin{gathered}
H_{B I A}^{[001]}\left(\theta_{z}\right)=b_{41}^{C B} \cos 2 \theta_{z}\left[\sigma_{x} p_{x}\left(p_{y}^{2}-p_{z}^{2}\right)+\sigma_{y} p_{y}\left(p_{z}^{2}-p_{x}^{2}\right)+\sigma_{z} p_{z}\left(p_{x}^{2}-p_{y}^{2}\right)\right]+ \\
b_{41}^{C B} \sin 2 \theta_{z}\left[p_{z}^{2}\left(\sigma_{y} p_{x}+\sigma_{x} p_{y}\right)-2 \sigma_{z} p_{x} p_{y} p_{z}+\frac{1}{2}\left(p_{x}^{2}-p_{y}^{2}\right)\left(\sigma_{x} p_{y}-\sigma_{y} p_{x}\right)\right]
\end{gathered}
$$

Note that we have restricted ourselves to an axially applied electric field $F_{z}$ and, in such a case, the Rashba Hamiltonian (4.11) is independent of $\theta_{z}$.

We consider next QDs grown along the [111] direction. This orientation is reached by rotating the crystalline structure by the following Euler angles: $\theta=\arccos (1 / \sqrt{3}), \phi=45$ and $\alpha=-45$. The rotated SOI Hamiltonians have the following form:

$$
H_{S I A}^{[111]}=\frac{r_{41} F_{z}}{\sqrt{3}}\left[\sigma_{z}\left(p_{y}-p_{x}\right)-\sigma_{y}\left(p_{x}+p_{z}\right)+\sigma_{x}\left(p_{y}+p_{z}\right)\right],
$$

and

$$
\begin{aligned}
H_{B I A}^{[111]}= & \frac{b_{41}^{C B}}{2 \sqrt{3}}\left[\left(p_{x}^{2}+p_{y}^{2}-4 p_{z}^{2}\right)\left(p_{x} \sigma_{y}-p_{y} \sigma_{x}\right)+p_{z}\left(p_{x}^{2}-p_{y}^{2}\right)\left(\sigma_{x}+\sigma_{y}\right)+\right. \\
& \left.2 p_{x} p_{y} p_{z}\left(\sigma_{x}-\sigma_{y}\right)-\sigma_{z} p_{x}^{2}\left(p_{x}+3 p_{y}\right)+\sigma_{z} p_{y}^{2}\left(p_{y}+3 p_{x}\right)\right] .
\end{aligned}
$$

Once the electron states have been calculated, the spin relaxation rate between Zeeman-split sublevels is estimated using the Fermi's golden rule as explained in section 4.1 .

The eigenvalue problem is solved numerically using a finite-difference scheme. Accounting for the SOI terms, which present third-order derivatives and are small in magnitude, requires high precision in the simulations. In general, higher precision can be achieved by increasing either the number of mesh points or the number of points in the discretization of the derivatives. In our particular case, after a series of convergence tests, we have found that a seven-point stencil central difference scheme and a number of 42875 mesh points provides an appropriate description of the system at a reasonable computational cost.

All calculations are carried out, unless otherwise stated, considering an in-plane magnetic field $B_{\|}=1 \mathrm{~T}$ and an axial electric field $F_{z}=10 \mathrm{kV} / \mathrm{cm}$. The material parameters used are those of GaAs. For specific values see the published paper in page 193 . 


\subsubsection{Effect of the QD geometry}

First, we explore the influence of the QD shape on the electron spin relaxation. To this aim, we consider QDs with square $\left(L_{x}=L_{y}=80 \mathrm{~nm}\right)$ or rectangular $\left(L_{x}=70 \mathrm{~nm}\right.$ and $\left.L_{y}=90 \mathrm{~nm}\right)$ base and with several heights ranging from $L_{z}=10 \mathrm{~nm}$ to $L_{z}=40 \mathrm{~nm}$. The spin relaxation rate is calculated for varying in-plane magnetic field orientation, as represented in figure 4.6(a) for three values of the magnetic field angle $\phi_{B}$.

We note that, after a series of preliminary calculations, it is seen that the spin relaxation is much slower when mediated by RSOI than by DSOI. This is because our structure does not present any potential gradient, so the relatively weak external electric field is the only factor breaking the inversion symmetry of the system. Because of this, in figure 4.6 and hereafter the two spin-orbit couplings are only taken into account individually.

When only RSOI is included, figure 4.6(b), the angular dependence is completely flat for QDs with square base (solid line), but shows a clear anisotropic behavior in rectangular dots (dashed line). In this case, $1 / T_{1}$ is maximum (minimum) when the magnetic field is oriented along the direction of weaker (stronger) confinement. In both cases, the spin relaxation rate is independent of the $\mathrm{QD}$ height within the calculated range, $L_{z}=10-40 \mathrm{~nm}$.

On the other hand, for DSOI, figures 4.6(c) and 4.6(d), the spin relaxation is remarkably different for short and tall QDs, evidencing that accounting for the three-dimensionality of the dots is critical to properly investigate the spin relaxation. In square dots with $L_{z}=10 \mathrm{~nm}$, figure 4.6(c), the relaxation rate does not significantly change with the magnetic field orientation. Contrarily, the $1 / T_{1}$ curves for larger QDs, $L_{z}=20,30,40 \mathrm{~nm}$, exhibit striking minima at $\phi_{B}=45$ and $\phi_{B}=135$. The same calculations but for a rectangular QD are shown in figure 4.6(d). Now the relaxation rate varies smoothly for all heights investigated and the sharp minima are removed. The results for different $L_{z}$ show an opposite trend, when $B_{\|}$is along the direction of weaker confinement $\phi_{B}=90$ the relaxation is maximum (minimum) for tall (short) QDs. This behavior is inverted for $\phi_{B}=0$.

In order to understand the results found in figure 4.6(b) we need to analyze the degree of spin admixing of the states involved in the transition. A perturbative analysis ${ }^{1}$ shows that the spin mixing originates from the coupling between the ground state $\psi_{000}$ and the excited states $\psi_{100}$ and $\psi_{010}$, where the three numbers in the subscript represent in this order the number

\footnotetext{
${ }^{1}$ The details are omitted here for brevity but the reader can find them in the publication (see page 193).
} 

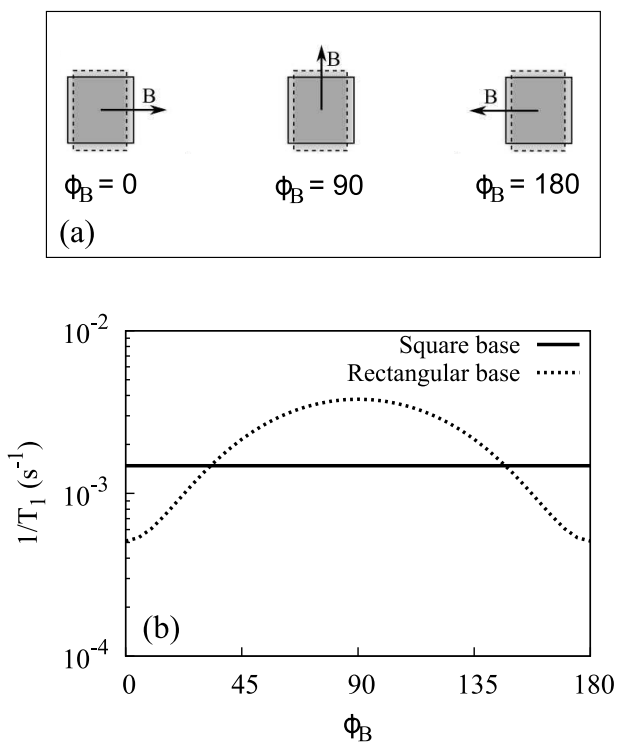

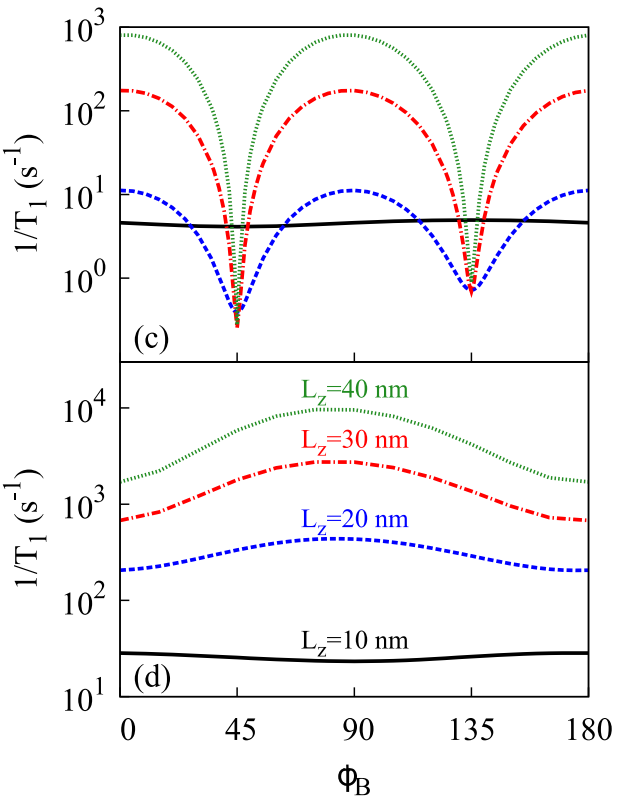

Figure 4.6: (a) Schematic drawing of the QD base (squared or rectangular) showing the relative orientation of the magnetic field for three specific angles $\phi_{B}=0,90,180$. (b) Electron spin relaxation rate as a function of the magnetic field orientation considering only RSOI QDs of $10 \mathrm{~nm}$ height with square (solid line) and rectangular (dashed line) base are considered. (c) $1 / T_{1}$ versus $\phi_{B}$ in QDs with square base when only DSOI is taken into account. Various dot heights are studied: $L_{z}=10 \mathrm{~nm}$ (black solid line), $L_{z}=20 \mathrm{~nm}$ (blue dashed line), $L_{z}=30 \mathrm{~nm}$ (red dash-dotted line), and $L_{z}=40 \mathrm{~nm}$ (green dotted line). (d) Same as in panel (c), but for a rectangular base QD

of nodes in the $x, y$ and $z$ direction. The specific excited state that couples with the ground state is determined by the magnetic field orientation. Then, square QDs, where both in-plane directions are equivalent, give rise to an isotropic behavior. For dots with rectangular base, instead, the excited state with a node in the direction of weaker confinement becomes closer in energy, justifying the spin relaxation anisotropy seen for varying $\phi_{B}$.

To analyze the case of DSOI we split Hamiltonian (2.22) as $H_{B I A}^{C B}=H_{z}+$ $H_{x y}$, with $H_{z}=b_{41}^{C B} p_{z}^{2}\left(p_{y} \sigma_{y}-p_{x} \sigma_{x}\right)$ and $H_{x y}=H_{x}+H_{y}=b_{41}^{C B}\left[p_{x}^{2}\left(p_{z} \sigma_{z}-\right.\right.$ $\left.\left.p_{y} \sigma_{y}\right)+p_{y}^{2}\left(p_{x} \sigma_{x}-p_{z} \sigma_{z}\right)\right]$, and perform calculations considering them individually (not shown). For QDs with $L_{z}=10 \mathrm{~nm} H_{z}$ dominates, as expected for quasi-2D systems. Then, the flat $1 / T_{1}$ dependence for square QDs and the minimum at $\phi_{B}=90$ for rectangular ones can be justified following the 
same perturbative study for $H_{z}$ as for RSOI As the dot height is increased, $H_{x y}$ soon takes over $H_{z}$ as the dominant contribution. The individual $H_{x}$ and $H_{y}$ terms show opposite behavior. $H_{x}$ presents maximum (minimum) $1 / T_{1}$ for $\phi_{B}=90\left(\phi_{B}=0\right)$ and $H_{y}$ for $\phi_{B}=0\left(\phi_{B}=90\right)$, independently of the base shape. In rectangular $\mathrm{QD}$, the different confinement strength in each in-plane direction determines which term, $H_{x}$ or $H_{y}$, predominates, so the spin relaxation exhibits its dependence with $\phi_{B}$. For square QDs, $H_{x}$ and $H_{y}$ cancel each other out at $\phi_{B}=45$ and $\phi_{B}=135$, originating the minima found in figure 4.6 (c) at these angles.

\subsubsection{In-plane rotation of the QD}

In this section, we investigate the effect of rotating the QD confinement potential with respect to the crystalline structure on the spin dynamics. The rotation angle $\theta_{z}$ is defined between the $x$ axis of the dot and the [100] crystal direction, as represented in the inset of figure 4.7(a). The QDs considered are the same as the ones with square base in section 4.3.1 with heights $L_{z}=10 \mathrm{~nm}$ and $L_{z}=20 \mathrm{~nm} 2^{2}$ The in-plane magnetic field is kept still at $\phi_{B}=0$ in all cases.

The spin relaxation versus $\theta_{z}$ when only RSOI is taken into account is shown in figure 4.7(a). The results are exactly the same for both dot heights. As expected from equation 4.12, which does not depend on $\theta_{z}$, the magnitude of $1 / T_{1}$ remains unaltered with the system rotation.

Conversely, for pure DSOI the spin relaxation rate as a function of the rotation angle presents an anisotropy with a $45^{\circ}$ periodicity, showing profound minima at $\theta_{z}=0,45,90$, see figure 4.7 (b). At these particular angles, the value of the relaxation rate drops approximately five orders of magnitude. This behavior can be explained from the form of the Hamiltonian (4.14) if one notices that half of the terms depend on $\sin 2 \theta_{z}$ and the other half on $\cos 2 \theta_{z}$. Consequently, for $\theta_{z}=0,90$ the first part of (4.14) vanishes and for $\theta_{z}=45,135$ the second one, thus originating the quenching of the spin mixing and, by extension, of the relaxation rate.

It is interesting to note that the suppression of the spin relaxation at specific rotation angles is caused by $H_{x y}$, while $H_{z}$ is completely flat, see the inset of figure 4.7(b). This fact stresses the important role of cubic DSOI terms in the SOI anisotropy even in flat structures, $L_{z}=10 \mathrm{~nm}$.

\footnotetext{
${ }^{2}$ Rectangular QDs show the same qualitative trends and are omitted here for the sake of brevity.
} 

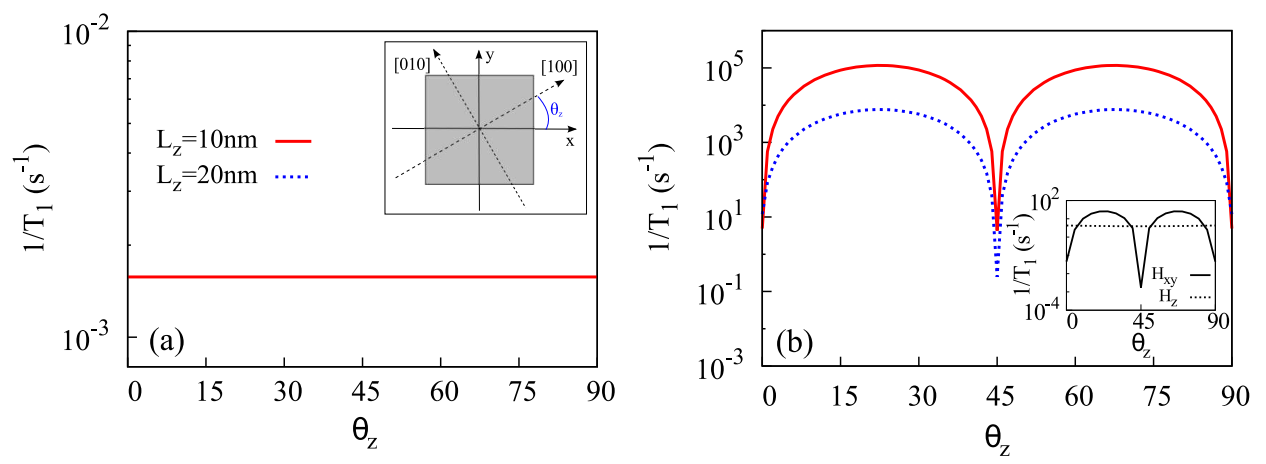

Figure 4.7: Spin dynamics as a function of the QD orientation for (a) pure RSOI and (b) pure DSOI in QDs with square base. Two dot heights are studied: $L_{z}=10 \mathrm{~nm}$ (red solid line) and $L_{z}=20 \mathrm{~nm}$ (blue dotted line). The magnetic field is directed along the $x$ axis $\left(\phi_{B}=0\right)$ in all calculations. The inset in panel (a) depicts an illustration of the system in which the rotation angle $\theta_{z}$ is defined. The inset in panel (b) shows $1 / T_{1}$ when the DSOI Hamiltonian is split into $H_{x y}$ (solid line) and $H_{z}$ (dashed line) for the dot with $L_{z}=10 \mathrm{~nm}$.

Once we have studied the influence of geometry and in-plane QD orientation on the spin relaxation anisotropy, we are in a position to make sense of the results reported in the experiments of Scarlino et al. [126]. They measured the spin lifetime for rotating in-plane magnetic field and found a $180^{\circ}$ periodicity with a small deviation of the extrema from the theoretical [110] direction. The periodicity was ascribed to the QD elongation assuming RSOI and DSOI to have the same weight, and the deviation from [110] to the misalignment of the dot principal axes with respect to the crystallographic main directions as well as to the RSOI to DSOI strength ratio. These two last factors are unknown in the experimental setup.

We investigate the effect of $\mathrm{QD}$ base elongation and in-plane rotation in the angle at which the relaxation rate becomes minimum, $\phi_{B}^{\min }$. The cubic DSOI terms that were ignored in their analysis, are taken into account here. In addition, the coefficient $r_{41}$ has been changed to make RSOI as strong as the linear DSOI term $H_{z}$, i.e. $r_{41}=b_{41}^{C B}\left\langle p_{z}^{2}\right\rangle / F_{z}$.

Figure 4.8 illustrates $\phi_{B}^{m i n}$ versus the QD orientation $\theta_{z}$. Calculations are carried out for dots with $L_{x}=80 \mathrm{~nm}$ and $L_{y}=80,90,110,150 \mathrm{~nm}$. The results for a strongly elongated $\mathrm{QD}$ (green dotted line) shows that $\phi_{B}^{\min }$ takes place when $B_{\|}$points approximately along [110], which is consistent with the results of Scarlino et al. [126]. We attribute the small deviations observed to the cubic DSOI terms. As the elongation is reduced, these 
74 Chapter 4. Spin-orbit-induced spin relaxation in semiconductor QDs

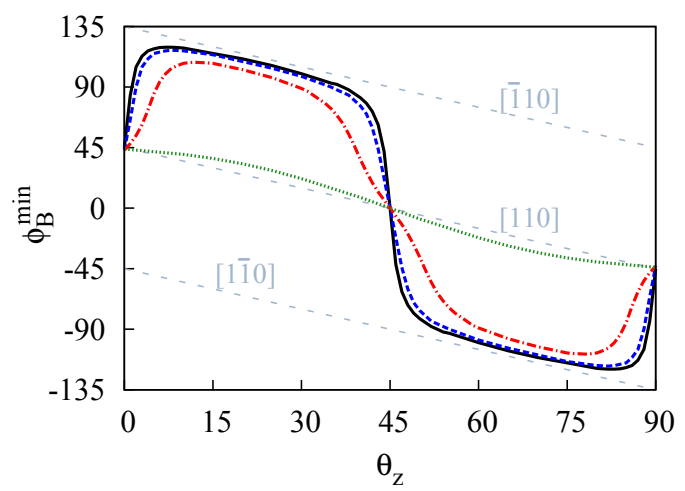

Figure 4.8: Magnetic field angle at which the $1 / T_{1}$ curve presents a minimum as a function of the in-plane dot orientation $\theta_{z}$. QDS with $L_{x}=80 \mathrm{~nm}$, variable $L_{y}$ and $L_{z}=10 \mathrm{~nm}$ are considered. The $L_{y}$ studied are: $L_{y}=80 \mathrm{~nm}$ (black solid line), $L_{y}=90 \mathrm{~nm}$ (blue dashed line), $L_{y}=110 \mathrm{~nm}$ (red dash-dotted line), and $L_{y}=150 \mathrm{~nm}$ (green dotted line). The values of $\phi_{B}$ corresponding to characteristic crystal directions [110], [110] and [110] are indicated as gray dashed lines for reference.

deviations become much larger and the behavior with $\theta_{z}$ richer. In this case, the minimum relaxation is found when the magnetic field is along [110] only for $\theta_{z}=0,45,90$, and rapidly deviates for other orientations. As can be seen in figure 4.8, for $0<\theta_{z}<45$ it is found when $B_{\|}$is along [110] and for $45<\theta_{z}<90$ when is along [110]. This behavior can be understood considering the inset of figure 4.7(b). When $\theta_{z}=0,45,90$ the linear DSOI term $H_{z}$ dominates, while $H_{x y}$ takes over for any other $\theta_{z}$. Then, the assumption of RSOI and $H_{z}$ of similar strength to interpret the deviations of the electron spin relaxation is no longer valid. This stresses the important role of DSOI cubic terms in SOI anisotropy if the QDs are not strongly elongated.

\subsection{3 [111] grown QDs}

Lastly, we explore the spin dynamics as a function of the in-plane magnetic field orientation in the same square $\mathrm{QD}$ system as in section $4.3 .1^{3}$ but now considering the dot is grown in the [111] crystal direction. The results for three different $\mathrm{QD}$ heights, $L_{z}=10,20,30 \mathrm{~nm}$, are depicted in figure 4.9 .

\footnotetext{
${ }^{3}$ Rectangular dots yield the same qualitative behavior, the only difference is that the minima is slightly shifted for pure DSOI
} 

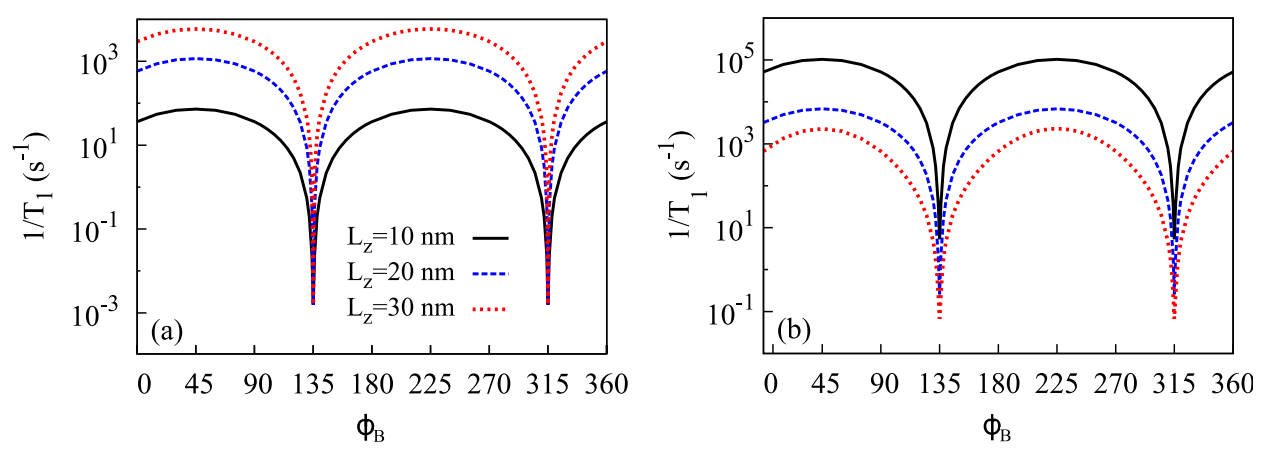

Figure 4.9: $1 / T_{1}$ versus $\phi_{B}$ in square QDs grown along the [111] crystallographic direction. Simulations considering (a) pure RSOI and (b) pure DSOI are presented for three dot heights: $L_{z}=10 \mathrm{~nm}$ (black solid line), $L_{z}=20 \mathrm{~nm}$ (blue dashed line), and $L_{z}=30 \mathrm{~nm}$ (red dotted line).

Overall, a moderate increase in the spin relaxation rate is found in [111] grown QDs, figure 4.9, in comparison to [001] QDs, figure 4.6. Interestingly, both spin mixing mechanisms show the same angular dependence. That is, a periodicity of $180^{\circ}$ with sharp minima at $\phi_{B}=135$ and $\phi_{B}=315$. The qualitative trend is the same for the three $\mathrm{QD}$ heights studied, with the only difference that $1 / T_{1}$ increases for RSOI while it decreases for DSOI. Therefore, since the difference in magnitude is relatively small, the dominant coupling depends on the height of the QD.

The identical dependence observed for both SOIs is due to the formal equivalences between $H_{S I A}^{[111]}$ and $H_{B I A}^{[111]}$, as already discussed in literature for quantum wells. [131, 134] As for the strong suppression of the spin relaxation at $\phi_{B}=135$ and $\phi_{B}=315$, it can be justified if one notices that at these specific angles the canonical momenta $p_{x}=-i \hbar d / d x+z B \sin \phi_{B}$ and $p_{y}=$ $-i \hbar d / d y-z B \cos \phi_{B}$ are exactly equal in magnitude since $L_{x}=L_{y}$. As a result, the first term in equation 4.13 and several terms in equation 4.14) cancel out, giving rise to a sharp decrease in $1 / T_{1}$.

\subsection{Hole spin relaxation in InAs/GaAs quantum dot molecules}

Quantum dot molecules are ensembles of two QDs which are close enough to couple via tunneling. The coherent tunneling leads to the formation of 


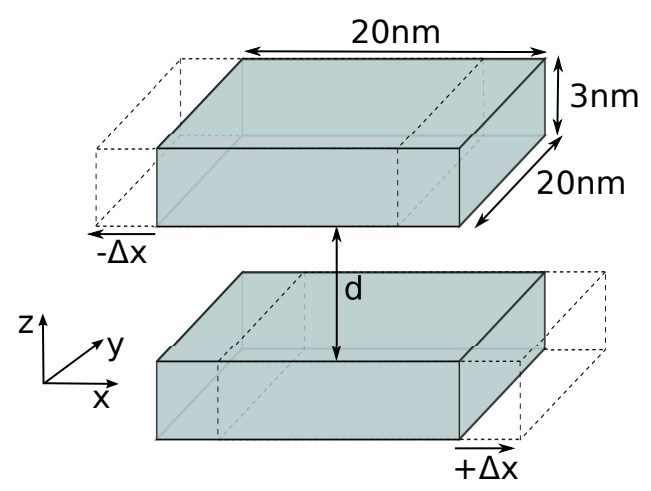

Figure 4.10: Drawing of the DQD cuboidal system. The dimensions of the QDs and the variables corresponding to interdot barrier thickness, $d$, and the shift in opposite directions along the $x$ direction, $\Delta_{x}$, are indicated. Dashed lines represent the $\mathrm{DQD}$ with misalignment.

states delocalized over the entire system that are truly molecular in nature, analogously to the hydrogen molecule. In general, they present the same properties as the constituent single QDs, but with the important advantage of being more versatile. That is, the localization of the wave function can be tuned by modifying the intensity of an externally applied electric field. [135138. Therefore, one can change from an atomic-like state that is confined in one QD for large electric fields, to a fully molecular-like state when the energy of the QDs is modulated to be the same in both of them. [138] This clearly offers an additional control mechanism that might be very useful in the development of applications. For instance, quantum dot molecules have been suggested as a way to use independent optical transitions for spin preparation, manipulation and readout,[139] as well as in multiple qubit architectures. [140]

In particular, in this section we discuss the hole spin relaxation in a vertically coupled double quantum dot (DQD) formed by two identical InAs cuboidal dots embedded in a GaAs matrix, see drawing in figure 4.10. We consider systems with various relative positions of the individual QDS in order to assess the effect of different tunneling regimes and dots misalignments. The parameters that control the geometry of the structure are the interdot separation, $d$, and the offset along the $x$ direction, $\Delta_{x}$. It is obvious that to properly describe such a system, a 3D model is indispensable.

The four-band Hamiltonian describing the hole states in a DQD under an external electric field $\mathbf{F}=\left(0,0, F_{z}\right)$ and a magnetic field $\mathbf{B}=\left(0,0, B_{0}\right)$, 
both applied along the growth direction, reads:

$$
H=H_{Z B}^{L K}+H_{B}+\left(V_{Q D}+e F_{z} z\right) \mathcal{I}+H_{B I A}^{V B},
$$

where $H_{Z B}^{L K}$ is the Luttinger-Kohn Hamiltonian, equation (A.1). $H_{B}$ stands for the terms coming from the implementation of the magnetic field obeying the procedure introduced in section 2.2 .2 for the $\mathrm{VB}$. The third and fourth terms in 4.15) are the confining potential $V_{Q D}$ and the electric field potential (see section 2.2.1), respectively. Finally, the last term in the Hamiltonian stands for the DSOI, equation (2.23). Note that RSOI is disregarded here because preliminary calculations (not included) showed its negligible influence. This is due to the minimal asymmetry of the system in the growth direction under resonant electric fields.

The hole spin relaxation rate between Zeeman-split sublevels is computed by means of the Fermi's golden rule following the procedure described in section 4.1. Both lh-hh coupling and DSOI sources of spin admixing are taken into account in the scattering processes mediated by acoustic phonons.

The multi-band Hamiltonian 4.15 is integrated numerically by using the finite differences method. A 5-point stencil central difference scheme is employed since a series of convergence tests yielded the desired accuracy while maintaining a reasonable computational cost. On account of using a constant-mass model, all parameters used in the simulations correspond to InAs, except for the ones defining the phonons where parameters of the GaAs matrix are considered, as we assume bulk phonons.

We investigate the dependence of the spin relaxation on the electric field strength $F_{z}$ in a $\mathrm{DQD}$ with strong tunneling, $d=3 \mathrm{~nm}$. Figure 4.11 illustrates the energy spectrum and hole spin lifetime in DQDs with two different relative positions of the constituent dots: aligned (left panels) and misaligned (right panels). The displacement of the QDs takes place along the $x$ direction by an offset $\Delta_{x}=3.3 \mathrm{~nm}$ as represented in figure 4.10 , which is relatively big but still realistic.[141] The investigated transition between the spin-split sublevels of the ground state is indicated by orange arrows in the energy spectra 4 The wave function of these sublevels present hh character, as expected of flat systems. Therefore, the transition takes place from states with $J_{z}=+3 / 2$ (介 in figure 4.11 insets) to states with $J_{z}=-3 / 2$ $(\Downarrow)$. All calculations are performed considering an uniform axial magnetic field $B_{0}=2 \mathrm{~T}$.

To begin with, we focus on the energy spectra (top panels in figure 4.11). For both alignments, we observe a clear anticrossing at $F_{z}=0$ between the

\footnotetext{
${ }^{4}$ Notice that the $\mathrm{VB}$ has negative energies, so that the ground state corresponds to the state with lowest absolute value of energy.
} 
two lowest-lying states. The origin of this anticrossing lies in the change of localization of the wave function when varying $F_{z}$. As represented in the insets of figures 4.11(a) and 4.11(b), for large negative $F_{z}$ the wave function is mainly localized at the bottom (top) QD for the ground (firstexcited) state, while the situation is inverted for positive $F_{z}$. We see that the magnitude of this charge transfer anticrossing is smaller in the misaligned case as a consequence of the weaker tunneling between the dots. [141] It is also worth mentioning that, since the two QDs are identical in size, in the absence of an external electric field the dots are in resonance and the wave function is equally delocalized over the entire structure, i.e. we have molecular-like states.

As for the hole spin relaxation (bottom panels), two series of calculations are carried out: one taking into account only the Luttinger-Kohn Hamiltonian $H_{Z B}^{L K}$ (black solid line), and the other including also the contribution of DSOI (blue dashed line). For both alignments the dependence of the relaxation time with the electric field is similar. When only the lh-hh spin mixing is considered, the spin relaxation time is maximum for molecular states, $F_{z}=0$, and decreases as the states become more atomic-like at finite $F_{z}$. When the DSOI mechanism of spin mixing is added to the model, $T_{1}$ is obviously diminished. The lifetime reduction is about one order of magnitude for molecular states and somewhat less for finite $F_{z}$. In addition, now the $T_{1}$ curve at $F_{z}=0$ is smoothed, becoming almost flat. With regard to the misalignment, we observe two main effects: an overall reduction of about one order of magnitude for both situations studied, $H_{L}$ and $H_{B I A}$, and a faster decrease of $T_{1}$ with $F_{z}$ for $H_{L}$. Both differences are also attributed to the fact that the molecular states disappear at smaller electric fields due to the weaker tunneling.

To justify the above results we need to analyze the degree of spin mixing in each situation. Other factors influencing $T_{1}$, such as the density of phonons, are not relevant since all calculations are performed at the same magnetic field. The strength of the $\mathrm{SOI}$ is related to the symmetry of the system. In general, a lowering in symmetry implies the activation of new mixing channels and, thus, an enhancement of the scattering mechanisms. 33. The symmetry of the system for the most relevant situations is indicated in figures 4.11(c) and 4.11(d) taking into account the $\mathrm{ZB}$ crystal structure, the confining potential and the presence of an axial magnetic field. An aligned homonuclear DQD considering only lh-hh mixing $\left(H_{L}\right)$ has $C_{4 h}$ symmetry. The inclusion of an electric field breaks the parity symmetry in $z$, reducing the system symmetry to $C_{4}$, which justifies the decrease in $T_{1}$ with $F_{z}$. If we include the DSOI instead, the descent in symmetry is more important $\left(C_{2}\right)$ and, consequently, the hole spin relaxation quenching is larger. Finally, 

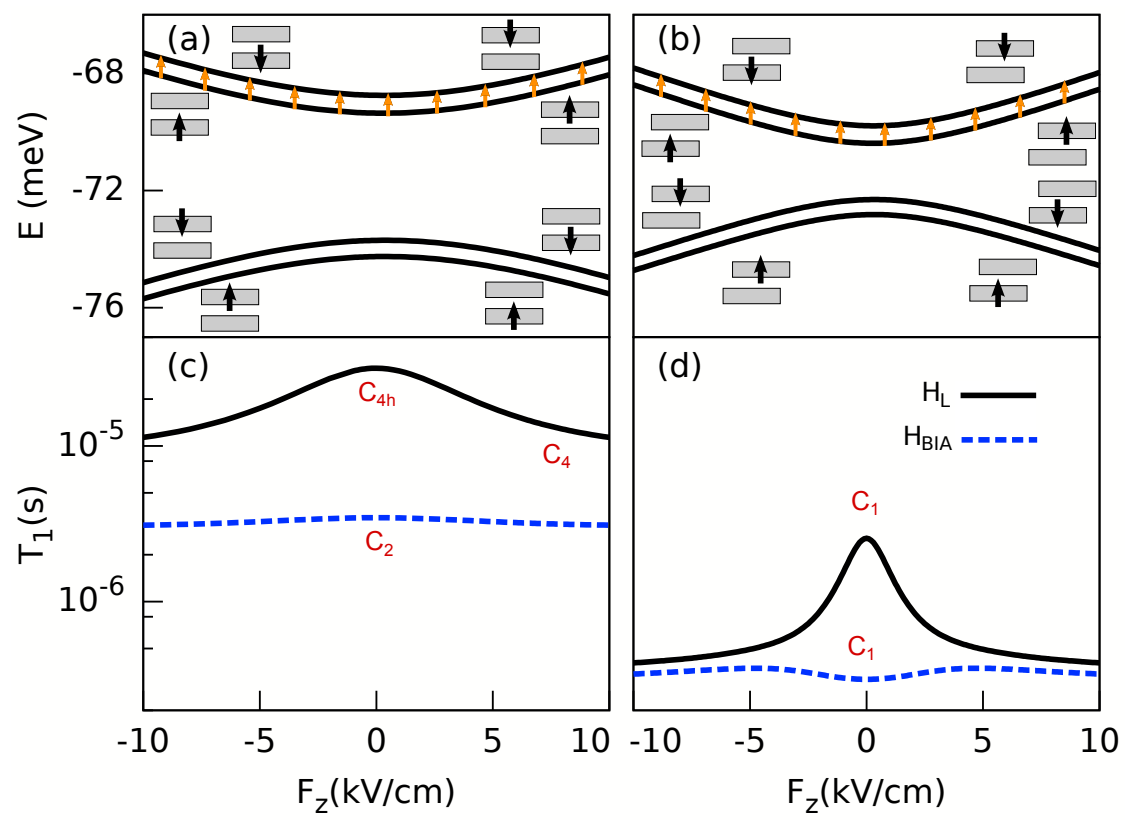

Figure 4.11: (a-b) Hole energy spectra and (c-d) hole spin lifetimes as a function of the electric field intensity $F_{z}$ for a InAs/GaAs DQD in the strong tunneling regime, $d=3 \mathrm{~nm}$. Top panels include orange arrows indicating the investigated transition, and insets showing the localization of the wave function and its dominant component: $J_{z}=$ $+3 / 2(\Uparrow)$ or $J_{z}=-3 / 2(\Downarrow)$. Bottom panels illustrate $T_{1}$ calculated by considering: only $H_{Z B}^{L K}$ (black solid line $H_{L}$ ), i.e. lh-hh coupling, or both $H_{Z B}^{L K}$ and DSOI Hamiltonian $H_{B I A}^{V B}$ (blue dashed line $H_{B I A}$ ). Labels denoting the symmetry point group have also been added. Lastly, left (right) panels correspond to aligned (misaligned) QDs

adding an external electric field to the $H_{B I A}$ case does not further reduce the system symmetry and a flat dependence is obtained. On the other hand, in the $\mathrm{DQD}$ with misalignment the combination of the confining potential and the magnetic field lifts all symmetries, group $C_{1}$. This explains the reduction in the hole spin lifetime by one order of magnitude or more compared to the aligned case. Introducing an electric field or the DSOI to the model cannot reduce the symmetry further, but it improves the efficiency of the scattering by opening new channels of mixing, thus making $T_{1}$ even shorter.

Calculations for a $\mathrm{DQD}$ with weaker tunneling, $d=9 \mathrm{~nm}$, have been also carried out. The complete results are not included here for brevity, since they are qualitatively the same as the ones for strong tunneling, but can 
be found in the article (page 227). The hole spin lifetimes are of the same order of magnitude as those in figure 4.11 and also exhibit maximum $T_{1}$ for molecular states, $F_{z}=0$. Nevertheless, the range of electric fields presenting enhanced lifetimes is now much narrower due to the weaker tunneling.

\subsection{Control of electron spin-orbit anisotropy in pyramidal QDs}

To close this chapter we investigate another experimental signature of SOI in semiconductor QDs, particularly the emergence of spin anticrossings in the energy spectrum. The magnitude of the gap opened at the anticrossing is known to be proportional to the SOI intensity, 142 144] offering the possibility to study the intrinsic anisotropy of RSOI and DSOI.

In relation to this, a experimental work by Takahashi et al. [116] reported a strong in-plane $\mathrm{SOI}$ anisotropy in InAs self-assembled QDs by measuring the size of the anticrossings as an external magnetic field is rotated. They found a dependence on the magnetic field azimuthal angle $\phi$ that fits the form of an absolute cosine function with an offset $\phi_{0}$, i.e. $f(\phi) \propto \mid \cos (\phi-$ $\left.\phi_{0}\right) \mid$. In the same publication it was suggested that the origin of this offset might be a consequence of the QD elongated pyramidal geometry along with the contribution of only RSOI. Subsequently, a theoretical study by Nowak et al. 145, proposed an alternative origin. They attributed the offset to the combined action of both RSOI and DSOI in elongated QDs, and not solely to RSOI. The model they used consists in a cuboidal QD in which the confinement potential is taken as separable, $V(\mathbf{r})=V_{x}(x)+V_{y}(y)+V_{z}(z)$. All results discussed throughout the preceding sections of this chapter have pointed out the crucial role of the geometry on the SOI strength, hence one wonders if using a simplified cuboidal system is enough to describe the behavior of realistic pyramidal QDs.

In view of the above, we investigate here the dependence of the anticrossing energy, $E_{A C}$, on the in-plane magnetic field orientation, $\phi$, in a $\mathrm{QD}$ with similar geometry to the one used in the experiments of reference [116]. It consists in an uncapped InAs pyramidal QD grown on top of a GaAs wetting layer. Uncapped dots are usually oxidized on the surface, so the tip may acquire an insulating character and, thus, a truncated structure offers a more realistic description than a pyramidal one.[146] In addition, the base of the QD is rectangular with the longer side along the [100] crystallographic direction. A schematic drawing of the QD modeled is depicted in figure 4.12 (a) together with all system dimensions. 
(a)

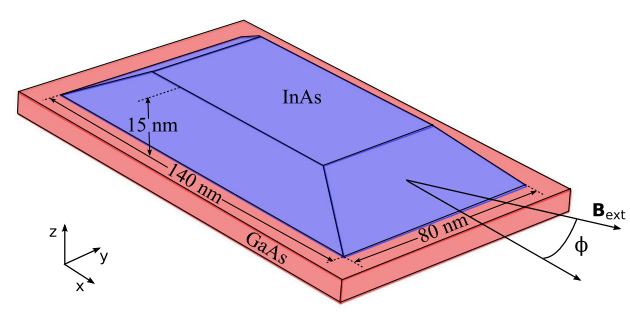

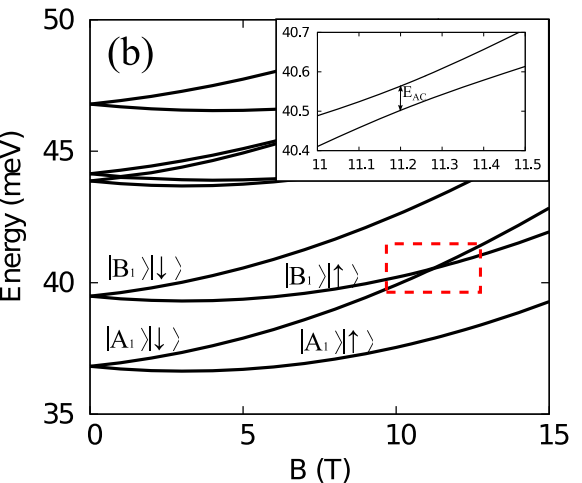

Figure 4.12: (a) Schematic representation of the uncapped pyramidal InAs $\mathrm{QD}$ grown on top of a GaAs wetting layer. The specific dimensions of the modeled geometry are indicated as well as the orientation of the magnetic field. The truncated pyramid is defined as having the upper base 0.6 times the size of the lower one. (b) Energy spectrum as a function of the magnetic field strength $(\phi=0)$ in the absence of SOI The orbitals are labeled with its symmetry and spin orientation for $B=0$ and no $\mathrm{SOI}$. The inset is a zoom-in of the region marked by the red dashed box when both SOIs are activated.

The electronic states of such a system in the presence of an axial electric field and an in-plane magnetic field are described by employing the following constant-mass 3D Hamiltonian:

$$
H=\frac{\mathbf{p}^{2}}{2 m^{*}}+V(\mathbf{r})-e \mathbf{F r}+H_{Z}+H_{S I A}^{C B}+H_{B I A}^{C B},
$$

where $\mathbf{p}=-i \hbar \boldsymbol{\nabla}-e \mathbf{A}$ and $e=-1$. The in-plane magnetic field $\mathbf{B}=$ $B(\cos \phi, \sin \phi, 0)$ is defined by the vector potential $\mathbf{A}=z B(\sin \phi, \cos \phi, 0)$, with $\phi$ being the azimuthal angle with respect to the $x$ axis as represented in figure 4.12(a). The terms in equation 4.16) are, in this order, the kinetic energy (equation (2.20), the confining potential, the external electric field (equation (2.19), the Zeeman splitting (equation (2.20), the RSOI Hamiltonian (equation (2.24), and the DSOI Hamiltonian (equation (2.22)).

Hamiltonian 4.16) is solved using a finite-difference method on a regular 3D grid. Simulations are carried out taking the experimental effective g-factor instead of the bulk one, which is consistent with the observed gfactor reduction due to confinement. The axial electric field is estimated to be $F_{z} \approx-15 \mathrm{kV} / \mathrm{cm}$ from the supplemental material of [116]. In addition, unless otherwise stated we assume the QD to have a composition of $66 \%$ In, accounting for the diffusion of Ga atoms from the wetting layer into 


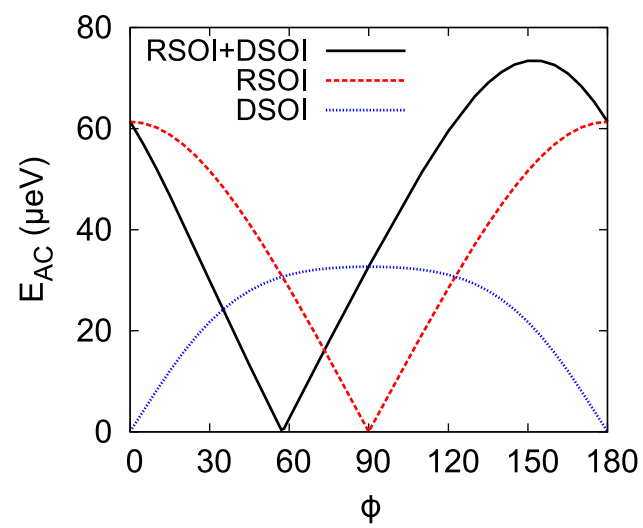

Figure 4.13: Anticrossing energy $E_{A C} v s$. magnetic field azimuthal angle $\phi$. Calculations are performed including only RSOI (red dashed line), only DSOI (blue dotted line), and both RSOI and DSOI (black solid line).

the QD. Similar alloy compositions have been found experimentally in InAs self-assembled QDs, 147, 148, Consequently, the system parameters are calculated as the linear interpolation from the pure InAs and GaAs material parameters.

\subsubsection{Angular dependence of the SOI}

As mentioned above, we analyze the anticrossing energy $E_{A C}$ for varying magnetic field orientation $\phi$. We consider the intersection of states marked by a red dashed box in figure 4.12(b), where the energy spectrum in the absence of $\mathrm{SOI}$ is shown. We see that no avoided crossing between the states emerges. This is due to the different symmetry of the states involved, which are labeled in the plot according to their orbital symmetry $\left(C_{2 v}\right.$ point group) and spin orientation at $B=0$. Nevertheless, when the $\mathrm{SOI}$ is included the symmetry is reduced and an anticrossing appears, see inset in figure 4.12(b). The size of the opened gap is defined as the anticrossing energy $E_{A C}$.

In figure 4.13, we summarize the dependence of the spin anticrossing energy on $\phi$ when DSOI and RSOI are considered individually and also simultaneously. The results clearly evidence the strong anisotropy of the two SOI mechanisms. When only RSOI is taken into account, $E_{A C}$ is maximum (minimum) for a magnetic field oriented along the $x$ axis, $\phi=0$ ( $y$ axis, $\phi=90$ ). If only DSOI is considered, instead, the behavior is the opposite. Including both couplings at the same time gives rise to a curve with similar 
form to the ones obtained for the individual cases, but the singular points are no longer found for $\mathbf{B}$ pointing toward the main axes of the QD. The anticrossing energy vanishes exactly at the point where the two individual curves cross, indicating that the two terms cancel each other out. Based on this fact and after analyzing figure 4.13 carefully, it can be inferred that the curve including both terms can be derived from the absolute value of the addition or subtraction of the curves for the individual SOI mechanisms. The angular dependence of $E_{A C}$ can be fitted well using the absolute value of a cosine function with an offset $\phi_{0}$, i.e. $E_{A C} \propto \cos \left(\phi-\phi_{0}\right)$. Since the minimum of the function is determined by the crossing of the individual curves, the magnitude of $\phi_{0}$ depends on the relative strength of the RSOI and DSOI contributions.

These results demonstrate the need of considering both SOIs for the offset $\phi_{0}$ to exist. This is qualitatively the same behavior found by Nowak et al. [145], thus confirming the validity of their simplified cuboidal model in explaining the origin of the offset.

\subsubsection{Effect of the QD composition and height}

From the point of view of taking advantage of the SOI anisotropy in the development of spin-based applications, knowing under which circumstances the SOI vanish is of vital importance. For instance, it may be useful in spin control protocols and also in hindering spin decoherence mechanisms. In this regard, we investigate how the $E_{A C}$ dependence is affected by two factors, namely the diffusion of the matrix material into the $\mathrm{QD}$ and the dot height.

Diffusion effects are generally relevant in InAs/GaAs self-assembled QD islands, leading to important variations in their composition. [147, 148] In figure 4.14(a), we consider four $\mathrm{In}_{\mathrm{x}} \mathrm{Ga}_{1-\mathrm{x}}$ As alloys with a uniform concentration ranging from $50 \%$ In to $100 \%$ In. We observe that when the diffusion of $\mathrm{Ga}$ atoms becomes more important, the maximum in $E_{A C}$ decreases and the angle at which $[\mathrm{SOI}$ vanish is moved towards lower values. This behavior can be justified taking into account the values of the SOI coefficients. On one hand, the DSOI coefficients are similar for both materials, so its strength does not vary noticeably when changing the alloy composition. On the other hand, the InAs RSOI parameter is approximately 23 times higher than the GaAs one. As a consequence, increasing the Ga content results in weaker RSOI, thus shifting the angle where the anticrossing disappears towards the DSOI limit, $\phi=0$.

Next, various QD heights are investigated in figure 4.14(b): $L_{z}=10,15$, $20 \mathrm{~nm}$. A clear modulation of the overall[SOI] strength and the $\phi_{0}$ value with 
84 Chapter 4. Spin-orbit-induced spin relaxation in semiconductor QDs
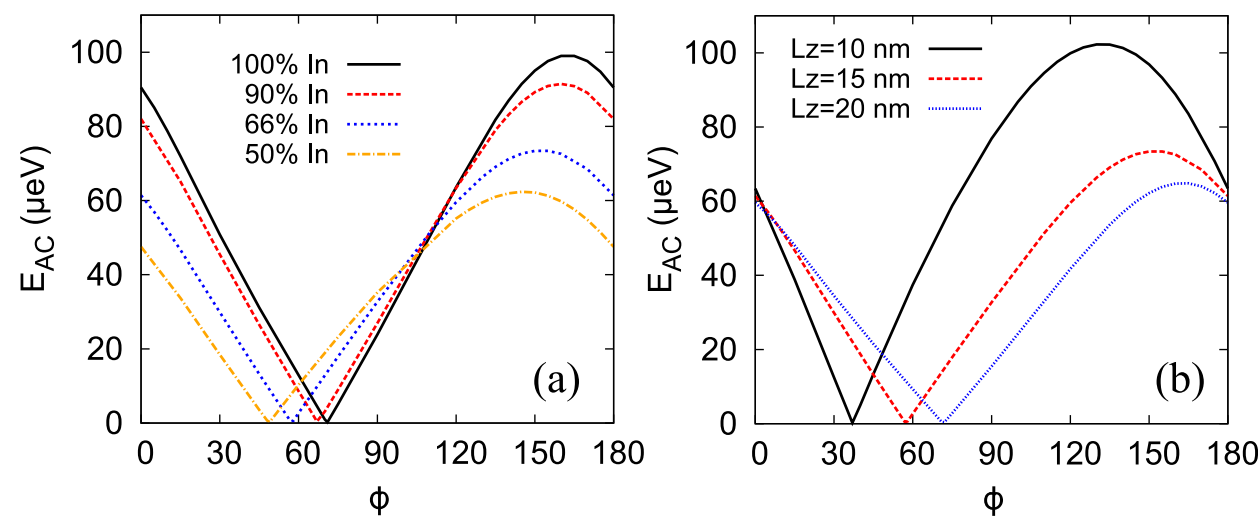

Figure 4.14: Anticrossing energy $E_{A C}$ as a function of the magnetic field in-plane orientation for various (a) QD compositions and (b) QD heights. In panel (a) results for four QDs with different In content are shown: $100 \%$ In (black solid line), $90 \%$ In (red dashed line), $66 \%$ In (blue dotted line), and $50 \%$ In (orange dash-dotted line). Panel (b) presents the $E_{A C}$ dependence for QDs with a height of $10 \mathrm{~nm}$ (black solid line), $15 \mathrm{~nm}$ (red dashed line), and $20 \mathrm{~nm}$ (blued dotted line).

$L_{z}$ is found. Taller (shorter) dots give rise to smaller (larger) gaps and the cancellation angle is shifted towards larger (smaller) values. The effect of $L_{z}$ can be explained if one analyzes the form of the SOI Hamiltonians. In the pyramidal QDs considered, the confinement along the $z$ direction is stronger than in the $x y$ plane, so that the dominant term in the DSOI Hamiltonian, equation (2.22), is $b_{41}^{C B} k_{z}^{2}\left(\sigma_{y} k_{y}-\sigma_{x} k_{x}\right)$. Consequently, changes in the QD height modify the strength of DSOI approximately as $H_{B I A} \propto\left\langle k_{z}^{2}\right\rangle \propto 1 / L_{z}^{2}$, while the RSOI Hamiltonian, equation (2.24), remains unaltered. Therefore, an increase in $L_{z}$ weakens the DSOI while RSOI remains roughly the same, thus leading to smaller anticrossing gaps and the zero SOI angle is shifted towards the RSOI limit, $\phi=90$.

\subsection{Summary}

In this chapter we have studied the spin relaxation of electrons and holes, paying special attention to the $\mathrm{SOI}$ as a source of spin admixture, which allows the energy relaxation in the phonon bath. To this end, we have developed truly 3D models, going beyond the commonly employed quasi2D simplified descriptions. Both semianalytic integration of Hamiltonians with harmonic confining potential, appropriate to describe ellipsoids of ar- 
bitrary shapes, and highly accurated numerical integration of Hamiltonians for pyramidal or cuboidal QDs have been carried out. Our numerical results reveal the need of including all three spatial dimensions for a proper description of the studied phenomena, since the physics of $3 \mathrm{D}$ systems, e.g. typical self-assembled dots or core-shell nanocrystals, cannot be inferred from that of flat systems modeled with quasi-2D simplified Hamiltonians. With our model we have been able to describe the highly anisotropic character of the spin relaxation with respect to the QD aspect ratio and the orientation of external magnetic fields for various crystal growth directions, the high sensitivity to alignment in $\mathrm{QD}$ molecules, etc., so that some apparent, previously reported, contradictory results have been harmonized and experimental results theoretically rationalized. 



\section{Strain and piezoelectricity in wurtzite and polytype QDs}

In the previous chapter, we have seen the crucial role of strain and piezoelectricity as the main carrier-phonon coupling mechanism in spin scattering processes induced by SOI in QDs, Nevertheless, the influence of these phenomena is not restricted to the field of spin dynamics. Indeed, both strain and piezoelectricity can lead to important changes in the electronic and optical properties of confined systems. Thus, understanding and controlling their effects is critical for the fabrication of novel QD based devices.

Strain in semiconductor nanostructures is caused by the mismatch of the lattice constants of the constituent materials. In most applications strain is undesirable since it may originate interface defects that reduce PL efficiency. [149, 150] However, it also offers the opportunity to tailor the system properties by means of strain engineering. For instance, strain can be used to modify the $\mathrm{CB}$ and $\mathrm{VB}$ energies in order to induce changes in the band alignment of the materials, such as an indirect-to-direct band gap transition in germanium, [151] or from a type-I to a type-II heterostructure in $\mathrm{CdTe} / \mathrm{ZnSe} \mathrm{QDs}$, 152 In addition, strain has also been used to change the $\mathrm{VB}$ ground state character between $\mathrm{lh}$ and $\mathrm{hh}$ in excitons. 153] As for the strain-induced piezoelectric fields, they are known to impact the spatial separation between electrons and holes in colloidal QDs which, in turn, may affect the exciton lifetimes, [154, 155] e-h] exchange interaction, [156] and other properties relevant for opto-electronic devices. [1]

Piezoelectricity emerges in non-centrosymmetric crystals, e.g. ZB and WZ, when the structure is under strain or stress. Due to symmetry considerations, $\mathrm{ZB}$ presents quadrupole piezoelectric polarization, while $\mathrm{WZ}$ 
presents a stronger dipolar polarization. [157] Moreover, WZ crystals have an additional contribution to the total polarization, the so-called spontaneous polarization, $P_{s p}$. As a consequence, piezoelectric effects are, in general, far more important in WZ QDs than in $\mathrm{ZB}$ ones. In fact, typical piezoelectric fields in WZ materials are larger than $10^{6} \mathrm{~V} / \mathrm{cm}$, an order of magnitude stronger than in $\mathrm{ZB}, 158$,

The spontaneous polarization in heterostructures is stronger when the constituent materials have very different $P_{s p}$ parameters. Hence, this phenomenon is expected to be especially relevant in polytype QDs. Such novel semiconductor structures are synthesized by growing alternate segments of $\mathrm{WZ}$ and $\mathrm{ZB}$ phases, i.e. alternating regions where spontaneous polarization is present with others where it is absent. As a result of the abrupt changes in $P_{s p}$ at the WZ/ZB and $\mathrm{ZB} / \mathrm{WZ}$ interfaces, one expects polytype systems to exhibit strong polarizations that give rise to important built-in electric fields. In spite of this, most theoretical works dealing with polytype QDs have neglected this effect, [20, 159, 160] so that further studies are required in order to gain a deeper understanding.

In light of the above, we investigate the role of strain and piezoelectricity, including $P_{s p}$, in $\mathrm{WZ}$ and polytype QDs. In particular, we focus on the behavior of excitons in colloidal CdSe/CdS nanocrystals and GaAs polytype QDs, We find out that piezoelectricity in core-shell colloidal structures and spontaneous polarization in polytype QDs are efficient mechanisms of charge separation for large enough systems. The spatial separation of electrons and holes governs the e-h overlap and, in turn, the exciton lifetime, thus representing a new degree of freedom for engineering nanodevices with the desired properties. The contents of the present chapter are based on the results of two articles, which can be consulted in pages 235 and 245.

\subsection{Piezoelectric control of exciton wave function in wurtzite QDs}

Excitons are bound states composed of an electron and a hole that are attracted to each other by the electrostatic Coulomb interaction. Consequently, the spatial separation between electrons and holes strongly affects many exciton properties, e.g. exciton emission lifetime, [154] Auger recombination rate, 161, 162] and e-h exchange interaction, [156] to name a few.

In colloidal core-shell nanocrystals the localization of the carriers has been typically tailored by means of band gap engineering. That is, electrons 
and holes can be confined into the core or the shell by properly combining materials with different band gap. Depending on the material combination we can have both carriers in the core (type-I band alignment), both in the shell (inverted type-I), one in the core and the other in the shell (type-II), and also one in the core and the other delocalized over both core and shell (quasi-type-II).

Additionally, in systems with large lattice mismatch strain has also been proposed as a mechanism to control carriers separation. In such a case, strain produces shifts of different magnitude in the band edges of core and shell materials, leading to transitions from one band alignment to another. This control mechanism has been investigated in CdTe/ZnSe, 152 CdSe/CdTe, 163 and ZnSe/ZnTe nanocrystals, [164 among others. However, it cannot be efficiently employed in CdSe/CdS QDs owing to their weak strain, hence the e-h separation in such structures has been traditionally controlled by quantum confinement. This is unfortunate because this system is particularly of interest due to their monodispersity, narrow emission linewidth, reduced blinking and high PL quantum yield. [165, 166] Nevertheless, a recent experiment in a "giant" CdSe/CdS rod-in-rod system has reported extremely long exciton lifetimes as a consequence of a relatively strong strain-induced piezoelectric field. [155] One then wonders if piezoelectricity may be also important in other CdSe/CdS systems and, if so, under which circumstances.

In this section, we explore how strain and piezoelectricity $\left.\right|^{1}$ affect the exciton wave function in $3 \mathrm{D}$ dot-in-dot WZ CdSe/CdS nanocrystals. We describe electrons and holes using a 2- and 6-band k.p Hamiltonian, respectively, taking into account strain and piezoelectricity within the continuous elastic theory, as discussed in section 2.4. The position-dependent Hamiltonian for electrons reads:

$$
H_{e}=\mathbf{p} \frac{1}{2 m^{*}} \mathbf{p}+V_{Q D}+H_{\epsilon, C B}^{W Z}+H_{p z},
$$

where $m^{*}$ is the electron effective mass, $\mathbf{p}=-i \hbar \nabla$ and $V_{Q D}$ stands for the confining potential. $H_{\epsilon, C B}^{W Z}$ represents the strain Hamiltonian for the WZ $\mathrm{CB}$, equation (C.4), and $H_{p z}$ represents the diagonal piezoelectric potential, equation (2.41). The Hamiltonian for holes is as follows:

$$
H_{h}=H_{W Z}+V_{Q D}+H_{\epsilon, V B}^{W Z}+H_{p z} .
$$

Here, $H_{W Z}$ is the six-band Hamiltonian describing the WZ VB, equation A.4. $H_{\epsilon, V B}^{W Z}$ denotes the WZ strain Hamiltonian for holes, equation (C.5), while $H_{p z}$ is the same as for electrons, but using hole's charge.

\footnotetext{
${ }^{1}$ For simplicity, the contribution of the spontaneous polarization is disregarded since preliminary results show that it does not change the qualitative trends.
} 
90 Chapter 5. Strain and piezoelectricity in wurtzite and polytype QDs

The Coulomb interaction between electrons and holes is taken into account by iteratively solving the Schrödinger-Poisson equation. First, we solve the independent single-particle problem for the CB. Next, the calculation is carried out for the $\mathrm{VB}$ including the electron charge density from the previous step via the Poisson equation, equation (2.36). Then, equation (5.1) for electrons is solved again, but now incorporating the calculated hole charge density. Lastly, this procedure of alternately solving electrons and holes considering the charge density of the other carrier from the previous step is repeated until convergence is reached.

Hamiltonians (5.1) and (5.2), and the Poisson equation are solved using the commercial software Comsol 4.2, which employs the finite elements method in a $3 \mathrm{D}$ mesh. Material parameters are given in the Supplementary Material of the article, page 245.

\subsubsection{Spheroidal dot-in-dot systems}

We start investigating systems of spheroidal shape in which CdSe core and CdS shell are equally elongated. Three different structures are considered: spherical, figure 5.1(a), prolate, figure 5.1(b), and oblate, figure 5.1(c).

Analyzing the form of the polarization vector for WZ materials, equation 2.40), it can be seen that the usually larger diagonal strain components appear only in the $P_{z}$ component. Thus, one expects polarization effects to be more important along the $c$-axis. Because of this, we study the potential profiles along the growth direction $z$, figures 5.1(d-f). Black dashed lines indicate $\mathrm{CB}$ and $\mathrm{VB}$ confining potentials for reference. The total potential $V^{t o t}=V_{Q D}+V_{s t r}+V_{p z}$ for each subband is also depicted as solid lines of different colors.

In general, we find a similar behavior for the three geometries. For the $\mathrm{CB}$, orange curve, we observe that the core potential well becomes shallower and the core bottom develops a built-in electric field of approximately $15 \mathrm{mV} / \mathrm{nm}$. The potential of the shell is increased by $V_{s t r}$ and $V_{p z}$ at one side of the well, while it is decreased at the other one. Remarkably, the potential minima, indicated by red arrows in the plots, are lower in energy than the core bottom, thus favoring the leakage of electrons into the shell. As for the $\mathrm{VB}$, subbands $\mathrm{A}, \mathrm{B}$ and $\mathrm{C}$ are represented as red, green and blue curves, respectively. In this case, the core bottom is moved upwards, originating a deeper well. A built-in electric field similar to that in the $\mathrm{CB}$ is also present. Holes are pushed towards the contrary direction compared to electrons provided their opposite charge sign, thus favoring $\mathrm{e}-\mathrm{h}$ separation. 

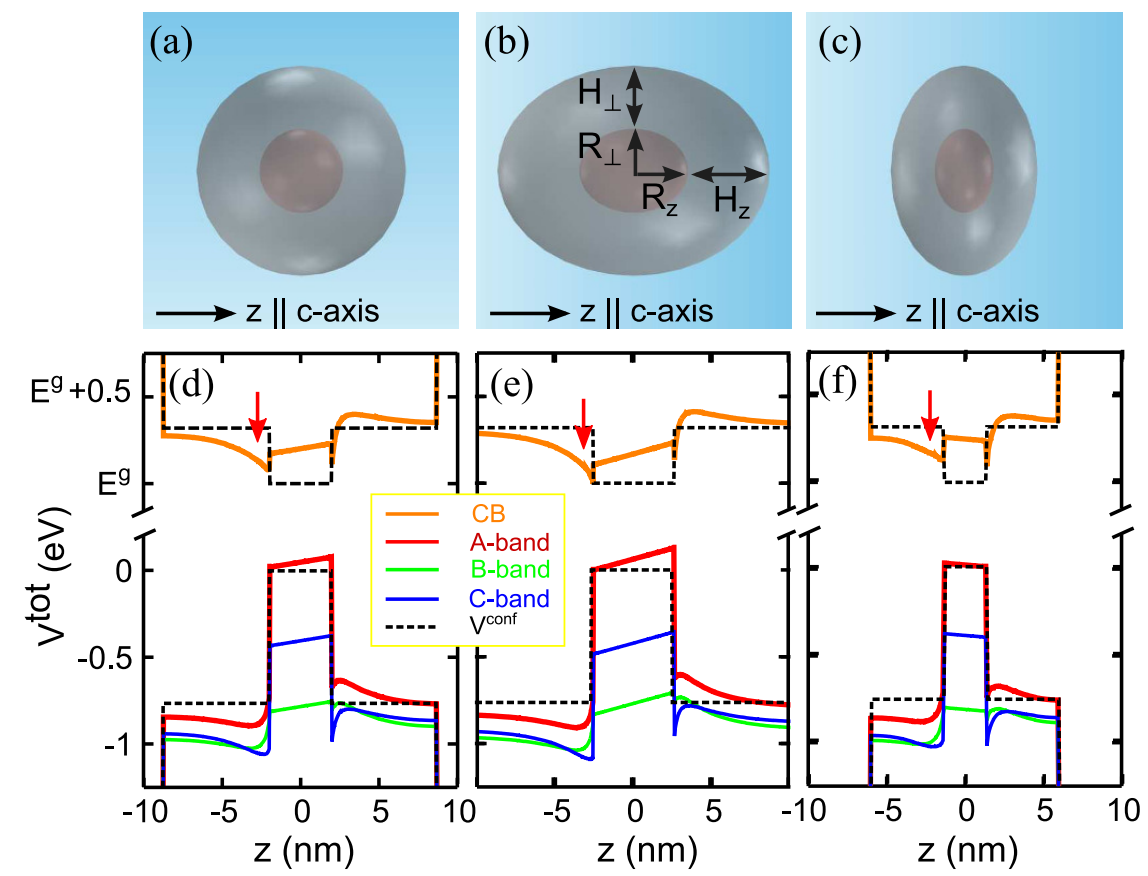

Figure 5.1: Schematic drawing of (a) spherical, (b) prolate, and (c) oblate $\mathrm{CdSe} / \mathrm{CdS}$ core-shell nanocrystals. The $c$-axis is indicated together with the core radii $R_{(\perp, z)}$ and shell thicknesses $H_{(\perp, z)}$. (a) $R_{\perp}=R_{z}=2 \mathrm{~nm}$ and $H_{\perp}=H_{z}=7 \mathrm{~nm}$. (b) Same as (a) in the inplane direction and $R_{z}=1.3 R_{\perp}$ and $H_{z}=1.3 H_{\perp}$. (c) Same but with $R_{z}=0.7 R_{\perp}$ and $H_{z}=0.7 H_{\perp}$. Panels (d) to (f) show the $\mathrm{CB}$ and $\mathrm{VB}$ potential profiles along the $z$ direction for the (d) spherical, (e) prolate, and (f) oblate systems. The plots include the confining potential (black dashed line), and the total potential for the $\mathrm{CB}$ (orange line), A-band (red line), B-band (green line), and C-band (blue line).

The potentials in the shell are also similar to those in the $\mathrm{CB}$, but now the potential well remains deep enough to guarantee the localization of the holes inside the core.

In order to understand the origin of this behavior we show in figure 5.2 the individual effect of strain and piezoelectricity. The potentials shown correspond to the $\mathrm{CB}$, but are also extensive to the $\mathrm{VB}$. We see that strain $V_{s t r}$, figure $5.2(\mathrm{a}-\mathrm{c})$, increases the energy inside the core, while in the shell it decreases the energy in a small region next to the interface. This explains the shallower well found for the $\mathrm{CB}$. The piezoelectric potential $V_{p z}$, figure 5.2 (d-f), forms a dipole due to the accumulation of charges of opposite sign on each CdSe/CdS interface, and a linear built-in electric field in the 

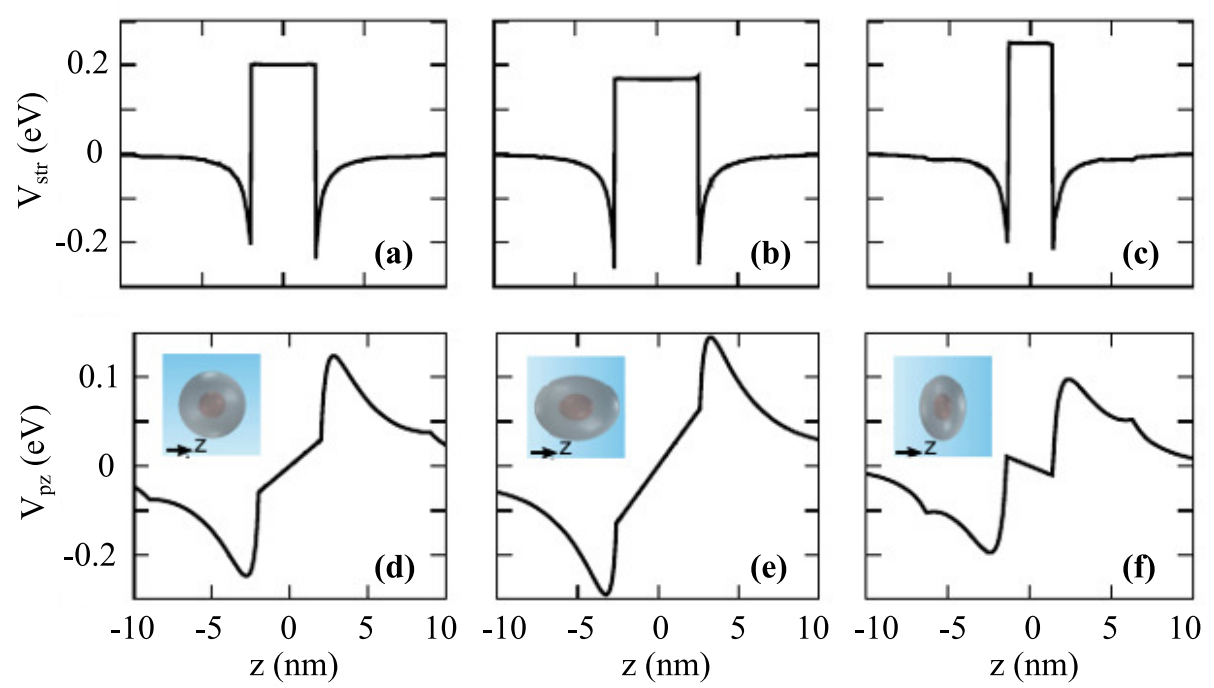

Figure 5.2: (a-c) $V_{s t r}$ and (d-f) $V_{p z}$ along the $c$-axis of the WZ structure. Results for spheric (left column), prolate (central column), and oblate (right column) are presented.

core. Obviously, the potential created in the shell by these charges has opposite sign at each side of the core, and its magnitude is large enough to compensate the $\mathrm{CB}$ core well depth, allowing electron delocalization. It is also interesting to notice that the core electric field is larger (smaller) in prolate (oblate) systems. Indeed, in oblate QDs the electric field can even change sign, as can be observed in figure 5.2 (f).

The dependence of the polarization field on the QD geometry can be justified by analyzing the axial and in-plane strain components $\epsilon_{z}$ and $\epsilon_{\perp}$, respectively. Figure 5.3 illustrates the values of $\epsilon_{z}$ and $\epsilon_{\perp}$ in the cut plane $x z$ for the three geometries. The polarization along the $c$-axis is given by $P_{z}=e_{31}\left(\epsilon_{x x}+\epsilon_{y y}\right)+e_{33} \epsilon_{z z}$. Since $e_{33} \approx-2 e_{31}$, the sign of the polarization is determined by the relative magnitude of $\epsilon_{\perp}$ and $\epsilon_{z z}$. In figures 5.3(a) and $5.3(\mathrm{~d})$, spheric system, the compressive strain in the core is slightly anisotropic with $\left|\epsilon_{z z}\right|>\left|\epsilon_{\perp}\right|$, which yields a small negative $P_{z}$. In prolate nanocrystals, figures $5.3(\mathrm{~b})$ and $5.3(\mathrm{e})$, the strain $\epsilon_{z z}$ increases further, explaining the strongest $P_{z}$ and $V_{p z}$. Finally, in oblate systems, figures 5.3.(c) and $5.3(\mathrm{f}), \epsilon_{z z}$ in the core decreases while $\epsilon_{\perp}$ increases. In fact we have $\left|\epsilon_{z z}\right|<\left|\epsilon_{\perp}\right|$, yielding a positive polarization $P_{z}$ that justifies the sign reversal in figure $5.2(\mathrm{f})$.

If we focus now on the strain components in the shell, it can be seen that 


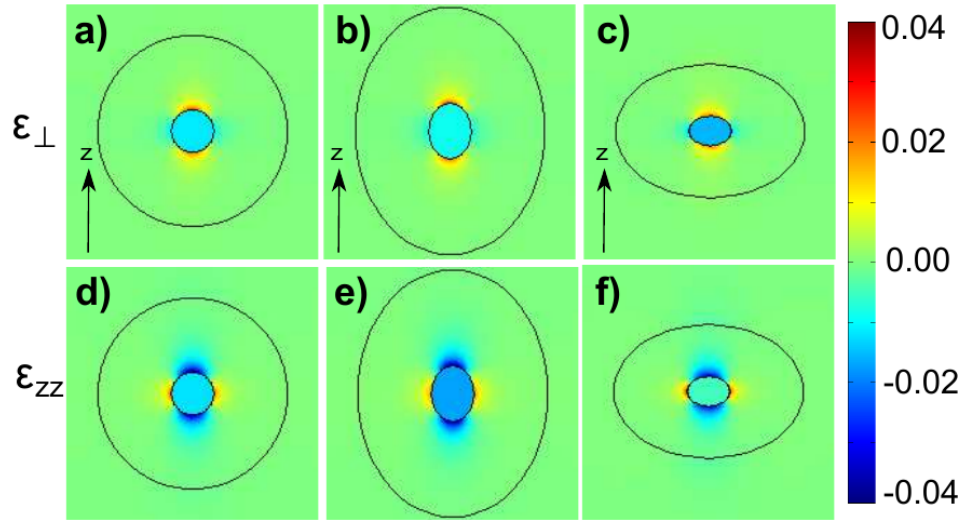

Figure 5.3: Diagonal strain along the $c$-axis $\epsilon_{z z}$ (top row) and in the $x y$ plane $\epsilon_{\perp}=1 / 2\left(\epsilon_{x x}+\epsilon_{y y}\right)$ (bottom row). Left column corresponds to spherical QDs, central column to prolate QDs, and right column to oblate QDs

the anisotropy is much more important. In the $z$ direction, $\epsilon_{\perp}$ is tensile, $\left|\epsilon_{\perp}\right|>0$, while $\epsilon_{z z}$ is compressive, $\left|\epsilon_{z z}\right|<0$. This originates a strong $V_{p z}$ in the shell with an abrupt change at the interface, as seen in figure 5.2 (d-f).

Now that we know the form of strain and piezoelectric potentials, we investigate the carriers localization in CdSe/CdS dot-in-dots for various core radii $R$ and shell thicknesses $H$. We have seen in figure 5.1 that the piezoelectric field pushes electrons towards the shell, but we need to explore if this is strong enough to surpass the Coulomb interaction. Figure 5.4 depicts the charge density of electrons, panels (a-d), and holes, panels (e-h). Overall, we find that the electron charge density can be localized in the core or the shell depending on the system dimensions, while holes are localized into the core in all cases.

For small radius and thin shell, figures 5.4(a) and 5.4(e), both electron and hole are localized in the CdSe core, although the electron density penetrates a little into the shell due to the shallower potential well. When the shell is enlarged, figures 5.4(b) and 5.4(f), the core is more compressed and, thus, the strongest polarization makes the electron to leak partially into the shell. Contrarily, the deeper potential well of the $\mathrm{VB}$ prevents the hole delocalization. If we increase the core radius and maintain a thin shell instead, figures 5.4 (c) and 5.4 (g), both carriers remain inside the core but electron and hole are pushed downwards and upwards, respectively, in spite of the Coulomb interaction. Interestingly, in a large core with a giant shell, fig- 
94 Chapter 5. Strain and piezoelectricity in wurtzite and polytype QDs

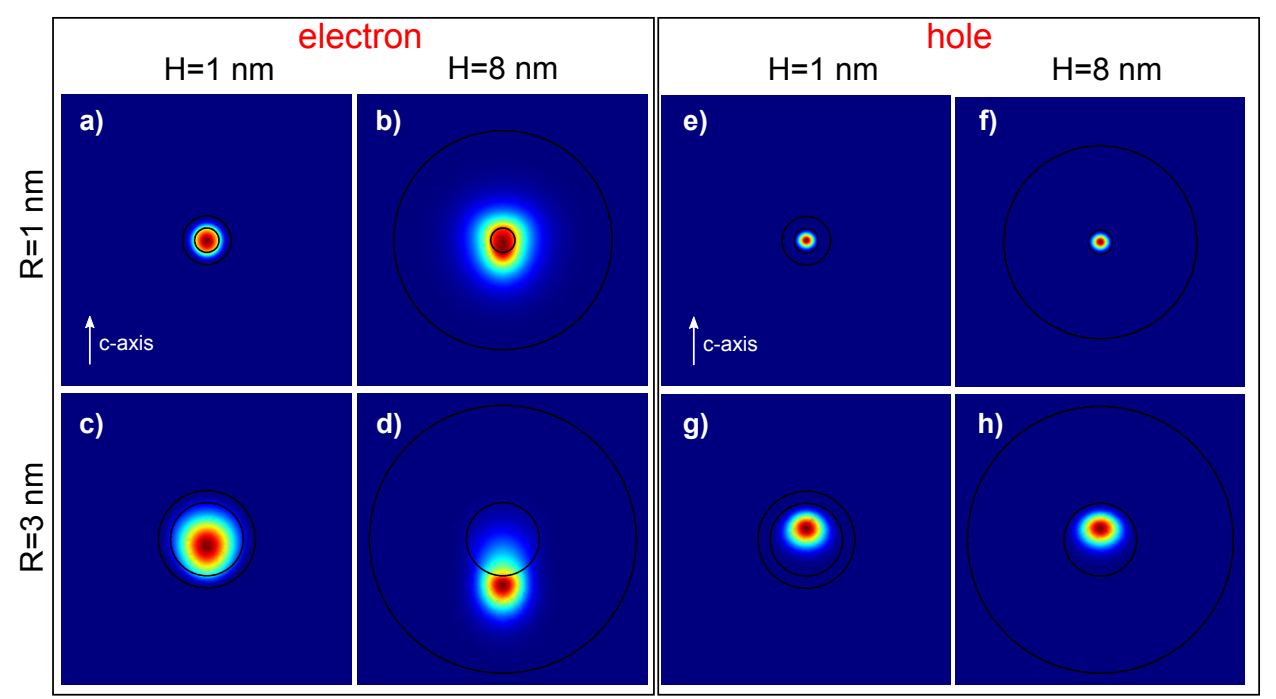

Figure 5.4: (a-d) Electron charge density in spheric core-shell QDs of various radii and shell thicknesses, as indicated in the plot. (e-h) Same for the hole charge density.

ures 5.4(d) and 5.4 (h), the electron density is mainly localized in the shell near the interface while the hole remains in the core. This demonstrates that piezoelectricity is capable of originating an evident charge separation in WZ dot-in-dot systems as a consequence of the transition from type-I to type-II band alignment when growing larger shells.

In order to systematically study the dependence of carriers separation on core-shell QD dimensions we compute the e-h overlap integral squared $S_{e h}^{2}=\left\langle\Psi_{e} \mid \Psi_{h}\right\rangle^{2}$ as a function of $R$ and $H$. The results are summarized in figure 5.5 for $V_{s t r}=0$ and $V_{p z}=0$, panel (a), $V_{s t r} \neq 0$ and $V_{p z}=0$, panel (b), and $V_{s t r} \neq 0$ and $V_{p z} \neq 0$, panel (c). In general, by comparing the three series of calculations we observe an important reduction in $S_{e h}^{2}$ as strain and piezoelectricity are included in the simulations.

When only quantum confinement effects and Coulomb interaction are considered, figure 5.5(a), we obtain high e-h overlaps for moderate and large cores independently of the shell thickness. For small CdSe core radius, $R \approx$ $1-1.5 \mathrm{~nm}$, the overlap is strong for thin shells but decreases substantially as the shell becomes thicker. In systems of these dimensions, i.e. small $R$ and large $H$, the band alignment is quasi-type-II explaining the small value of $S_{e h}^{2}$, see the marked regions in figure 5.5(a). Here, electron density penetrates into the shell as a result of the high kinetic energy in small QDs 


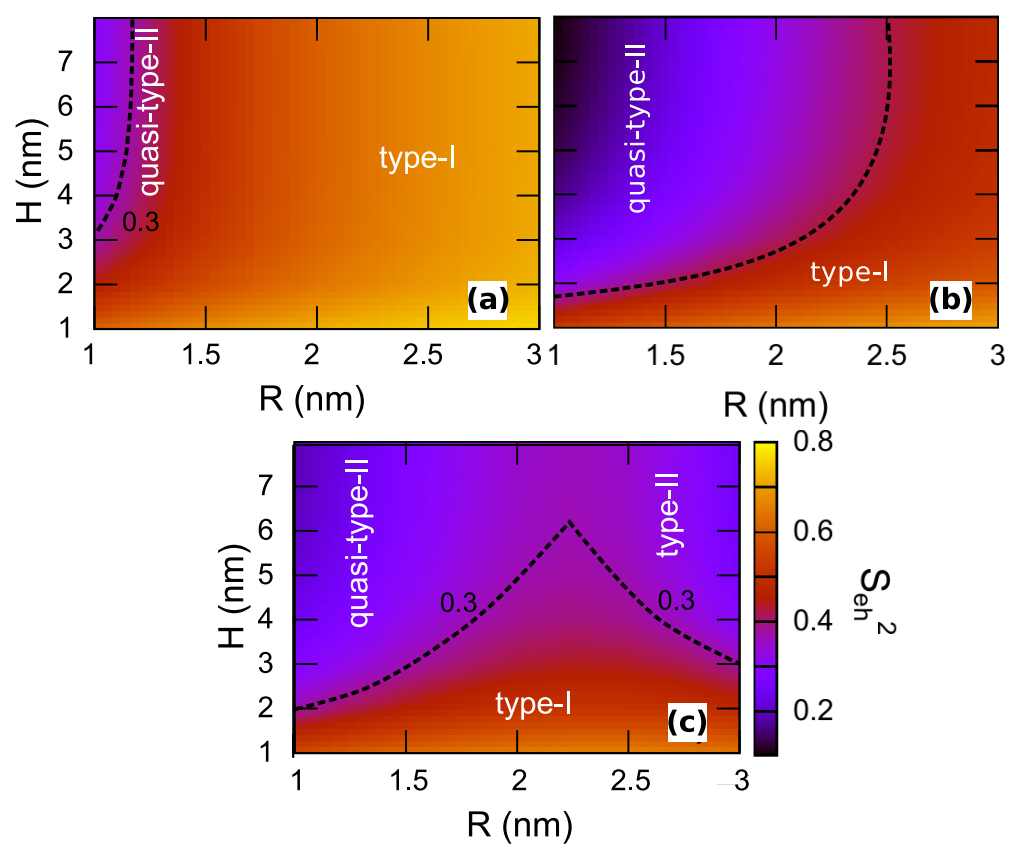

Figure 5.5: Map of the squared e-h overlap $S_{e h}^{2}$ as a function of $R$ and $H$ for excitons in spherical CdSe/CdS dot-in-dot systems. Calculations are carried out without considering strain and piezoelectricity, panel (a), including only strain, panel (b), and also considering both of them, panel (c).

and the shallow potential well. Figure 5.5(b) shows the exciton overlap squared when strain is included. One can see that the region with quasitype-II regime is extended to larger cores. This is because strain lessens the potential well depth, so that weaker kinetic energies suffice to produce electron delocalization. Lastly, the results when both effects are taken into account are depicted in figure 5.5(c). For moderately small core radius, $R<2.5 \mathrm{~nm}$, the results are similar to those with only strain. However, for larger dots piezoelectricity starts playing a role and we see the emergence of a type-II region for large enough shells. This behavior corresponds to figures 5.4(d) and 5.4(h) where electrons are localized in the shell while holes stay inside the core.

It is worth noting that the exciton overlap behavior is robust against small elongations of the system as seen in calculations for the same prolate and oblate structures of figures 5.1(b) and 5.1(c), respectively. The corresponding results are not included here for brevity, but can be found in figure S5 of the publication's Supplementary Material, page 245. 
All the above results have been confirmed experimentally in a series of giant-shell $\mathrm{CdSe} / \mathrm{CdS}$ dot-in-dot structures. In those experiments, timeresolved $\mathrm{PL}$ measurements reveal longer exciton lifetimes with increasing core radius, in agreement with the smaller exciton overlap predicted theoretically. The experiments were carried out by A. Polovitsyn and I. Moreels from the Istituto Italiano di Tecnologia in Genova (Italy) in a collaboration with our group. The experimental results and the details of the synthesis and measurement methods can be found in the published article, page 245 .

\subsubsection{Other CdSe/CdS core-shell structures}

For the sake of completeness, we also explore the influence of strain and piezoelectricity on the e-h overlap in nanostructures with other geometries, namely dot-in-rods, dot-in-plates and rod-in-rods. Such systems are modeled as highly elongated ellipsoids in either the $z$ or the in-plane direction.

Figure 5.6 shows the electron and hole charge densities for such structures with various core and shell dimensions. In general, we find that piezoelectricity is an efficient mechanism of charge separation in all the studied wurtzite CdSe/CdS core-shell structures as long as both core and shell are large. This is clearly seen in rod-in-rods with large cores and giant shells, figure 5.6(d), which goes along with the extremely long exciton lifetimes reported for this system. [155] It is worth stressing that a thick shell is necessary not only in the growth direction, where the polarization field emerges, but also in the lateral one. For example, in systems with thin shells in one direction such as dot-in-rods, figure 5.6(a), and dot-in-plates, figure 5.6(c), of typical dimensions, carriers are localized inside the core since the shell cannot compress much the core, thus yielding a weak core strain and in turn a weak polarization field. This becomes evident in figure 5.6(b) (5.6(e)) where carriers separation is enhanced (suppressed) when the lateral confinement is weakeaned (strengthened). Therefore, it can be concluded that the e-h overlap induced by piezoelectricity can be modulated in all the structures investigated by properly controlling their shell lateral confinement.

\subsection{Spontaneous polarization in GaAs polytype QDs}

Polytype QDs are a new type of semiconductor nanostructures that have been successfully synthesized in the past few years. [20, 21] They consist of 

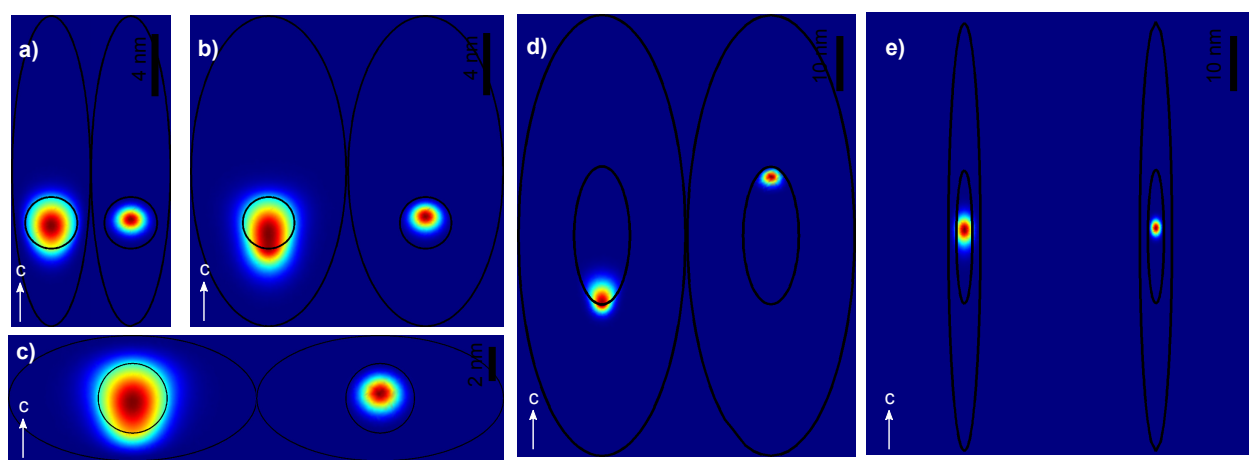

Figure 5.6: Excitonic charge density in various wurtzite $\mathrm{CdSe} / \mathrm{CdS}$ nanocrystals: (a) dot-in-rod with standard dimensions; (b) dot-in-rod with enlarged lateral shell; (c) dot-in-plate; (d) rod-in-rod with large core and giant shell; (e) rod-in-rod with core and shell of small lateral size. In each panel, electrons are represented on the left and holes on the right side.

a nanowire system in which $\overline{\mathrm{ZB}}$ and $\mathrm{WZ}$ crystal structures of the same material coexist, i.e. the system presents alternate segments of both crystal phases. These two phases have somewhat different band gaps, hence band offsets are formed and carriers can be confined originating QDs. [167] Since all regions are composed of the same atoms with the only difference being their spatial arrangement, these dots present negligible strain and atomically sharp interfaces. [168, 169] This offers the opportunity to control the geometry with a single atomic layer precision, which is a great advantage compared to self-assembled QDs where strain and alloying effects limit the optoelectronic performance. Consequently, polytype QDs are expected to have excellent optical properties, which makes them promising for future applications.

Recent advances in the synthesis techniques have allowed the fabrication of single crystal phase QDs with good control on their dimensions. In particular, Vainorius et al. [159] have reported the synthesis of GaAs polytype QDs with exact control on the dot thickness, while Loitsch et al. [170] have grown samples of the same system with various nanowire diameters down to $7 \mathrm{~nm}$. Together, these experimental works show that precise tailoring of the QD geometry and, thus, of the energy structure is possible, which represents the first step towards the development of real devices.

From a theoretical point of view, however, crystal phase QDs have not been extensively investigated yet and the influence of several factors is still unclear. Among them, here we pay special attention to the role of quantum 

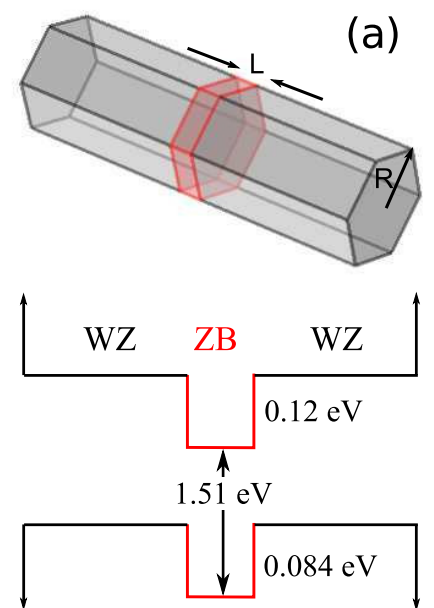
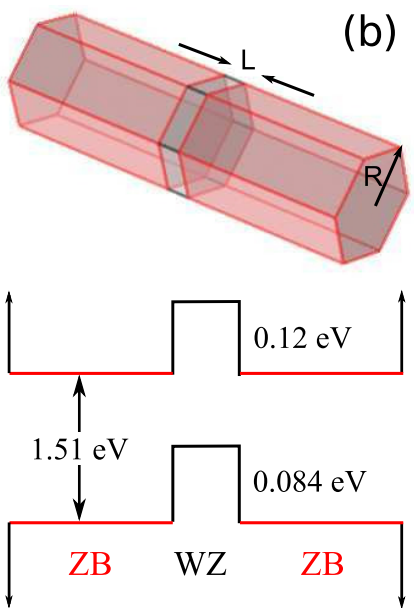

Figure 5.7: Schematic drawings of the hexagonal polytype structures and the potential profiles along the growth direction for (a) ZB QD in a WZ wire and (b) WZ QD in a ZB wire. Band offsets, band gaps and parameters defining the structure size are also indicated.

confinement, spontaneous polarization, $\mathrm{VB}$ mixing and exciton Coulomb interaction. Polytype QDs often present a type-II band alignment, e.g. in GaAs [159] and InP [169, giving rise to effective e-h separation. Nevertheless, the band offsets between $\overline{\mathrm{WZ}}$ and $\mathrm{ZB}$ are commonly small and the high kinetic energy in small systems may lead to significant electron and hole wave function leakage into each other's phase. Additionally, as seen in the preceding section, polarization fields and Coulomb interaction strongly affect carrier localization and, hence, they may drastically change the exciton properties. Lastly, to date the role of $\mathrm{VB}$ mixing is poorly understood and further assessment to determine the dominant subband for different size regimes is needed.

Particularly, in the present section we focus on GaAs polytype QDs within the confinement ranges reported by Vainorius et al. [159] and Loitsch et al. [170. We study hexagonal nanowires with variable dot thickness $L$ and radius $R$, as illustrated in figure 5.7. Since GaAs ZB/WZ structures have type-II band alignment, electrons and holes are localized in different regions of the system. Thus, we investigate excitons considering the two possibilities of defining a single polytype QD namely a $\mathrm{ZB} \mathrm{QD}$ embedded in a WZ nanowire, figure 5.7(a), and a WZ QD embedded in a ZB wire, figure 5.7(b). Such polytypical structures are modeled using the $\mathrm{k} \cdot \mathrm{p}$ method as explained in section 2.1.5. 
Electrons are described using a single-band Hamiltonian. This is justified in $\mathrm{ZB}$ structures where the lowest subband is well separated from other remote bands. However, the situation is different in WZ GaAs where $\Gamma_{8 c}$ and $\Gamma_{7 c}$ bands are close to each other. In spite of this, the lack of mass parameters does not allow to model both bands simultaneously. Instead, we use a single-band Hamiltonian of hybrid character: $\Gamma_{8 c}$ masses but optically bright as $\Gamma_{7 c}$ band.[171, 172] The polytype $\mathrm{CB}$ Hamiltonian reads

$$
H_{e}=\mathbf{p} \frac{1}{2 m^{*}} \mathbf{p}+V_{Q D}+H_{p z}
$$

where $\mathbf{p}=-i \hbar \boldsymbol{\nabla}, V_{Q D}$ is the confining potential defined by the ZB/WZ band offset, and $H_{p z}$ stands for the total polarization field potential, equation (2.41).

Holes are described employing a six-band position-dependent Hamiltonian spanned on the same basis of Bloch functions in both crystal structures. The specific basis set used here is that of lower symmetry, i.e. the one for $\mathrm{WZ}$ materials given in equation 2.16. The $\mathrm{VB}$ Hamiltonian is as follows:

$$
H_{h}=H_{Z B / W Z}^{B F}+\left(V_{Q D}+H_{p z}-\frac{\Delta_{s o}}{3} Y_{Z B}\right) \mathcal{I}_{6 x 6},
$$

with $H_{Z B / W Z}^{B F}$ denoting the $\mathrm{VB}$ polytype Hamiltonian given in equation (A.6). As in the CB, equation (5.3), $V_{Q D}$ and $H_{p z}$ are the confining and polarization field potentials, respectively. Last term in (5.4) corrects the Hamiltonian when using $\mathrm{ZB}$ parameters by subtracting $\Delta_{s o} / 3$ in all diagonal elements. Then, $Y_{Z B}$ is a heaviside function which takes $Y_{Z B}=0$ in WZ and $Y_{Z B}=1$ in $\mathrm{ZB}$ crystal phase.

As already discussed in section 2.1.5, polytype QDs are formed by segments of ZB phase grown along the [111] crystal direction and segments of WZ phase grown along [0001]. These two crystal phases are constituted by the same atoms and only differ in the stacking order of the layers. Furthermore, the atoms have the same tetrahedral coordination in both crystal structures. As a result, the lattice mismatch at the $\mathrm{ZB} / \mathrm{WZ}$ interface is insignificant and strain effects can be safely disregarded in equations (5.3) and 5.4. This is in agreement with experimental works that have synthesized defect-free dots. [159, 170]

If strain is negligible, so is the strain-induced piezoelectric polarization. Therefore, spontaneous polarization is the only significant source of polarization in these systems. Relevantly, such phenomenon is expected to be particularly strong in polytype QDs owing to the alternation of phases 
100 Chapter 5. Strain and piezoelectricity in wurtzite and polytype QDs

where the spontaneous polarization is absent $(\overline{\mathrm{ZB}})$ with others where symmetry allows its presence (WZ). The abrupt change in $P_{s p}$ at the interfaces should result in a substantial overall polarization field. Nonetheless, WZ GaAs presents one order of magnitude weaker $P_{s p}$ in comparison to other materials, so its effect might be limited.

Finally, neutral excitons are described by the following Hamiltonian

$$
H_{X}=H_{e}+H_{h}^{1 B}+V_{e h},
$$

where $V_{e h}$ is the e-h Coulomb interaction. For the sake of simplicity, we use here a single-band model for the $\mathrm{VB}, H_{h}^{1 B}$, obtained after decoupling the $F$ band from the rest of the matrix in Hamiltonian (5.4), which is justified under certain conditions ${ }^{2}$ as will be shown in section 5.2.2. Please note that in this section we refer to the WZ $A, B$ and $C$ hole subbands as $F$, $G$ and $\lambda$, respectively, which is an alternative notation commonly used in literature. The resulting one-band Hamiltonian is

$$
H_{h}^{1 B}=\Delta_{1}+\Delta_{2}+\mathbf{p}_{\perp} \frac{1}{2 m_{\perp}^{*}} \mathbf{p}_{\perp}+\mathbf{p}_{z} \frac{1}{2 m_{z}^{*}} \mathbf{p}_{z}+V_{Q D}+H_{p z}-\frac{\Delta_{s o}}{3} Y_{Z B}
$$

Equation (5.5) is solved by taking into account $V_{e h}$ using exactly the same procedure introduced in the previous section, in which Hamiltonians (5.3) and (5.4), and the Poisson equation are iteratively solved until convergence.

Simulations are carried out considering the nanowire to be surrounded by an insulating material by setting in this region $\left|V_{Q D}\right|=5 \mathrm{eV}$ and the relative dielectric constant $\varepsilon_{r}=4$. We use Comsol 4.2 software in the calculations, which employs a finite elements scheme on a $3 \mathrm{D}$ adaptive mesh. The GaAs parameters used to model the $\mathrm{ZB}$ and $\mathrm{WZ}$ materials can be found in the corresponding publication, page 235 .

\subsubsection{Electrons in GaAs $\mathrm{WZ} / \mathrm{ZB} / \mathrm{WZ}$ polytype QDs}

We first investigate a single electron confined in a $\mathrm{ZB} \mid \mathrm{QD}$ as the one represented in figure 5.7(a). We consider a nanowire with radius $R=50 \mathrm{~nm}$, and calculate the electron energy as a function of the dot thickness $L$. Figure 5.8(a) compares the results for two values of the GaAs spontaneous polarization: the one reported in literature $P_{s p}=2.3 \times 10^{-3} \mathrm{C} \mathrm{m}^{-2}$ (solid line) and a value weakened artificially one order of magnitude $P_{s p}=2.3 \times 10^{-4} \mathrm{C} \mathrm{m}^{-2}$

\footnotetext{
${ }^{2} \mathrm{In}$ WZ holes, the uppermost $F$ band is separated by the spin-orbit $\left(\Delta_{\text {so }}=3 \Delta_{2}\right)$ and crystal field splittings $\left(\Delta_{c r}=\Delta_{1}\right)$. In $\mathrm{ZB}$ instead, hh and $\mathrm{hh}$ subbands are degenerate but they are split by the action of quantum confinement.
} 

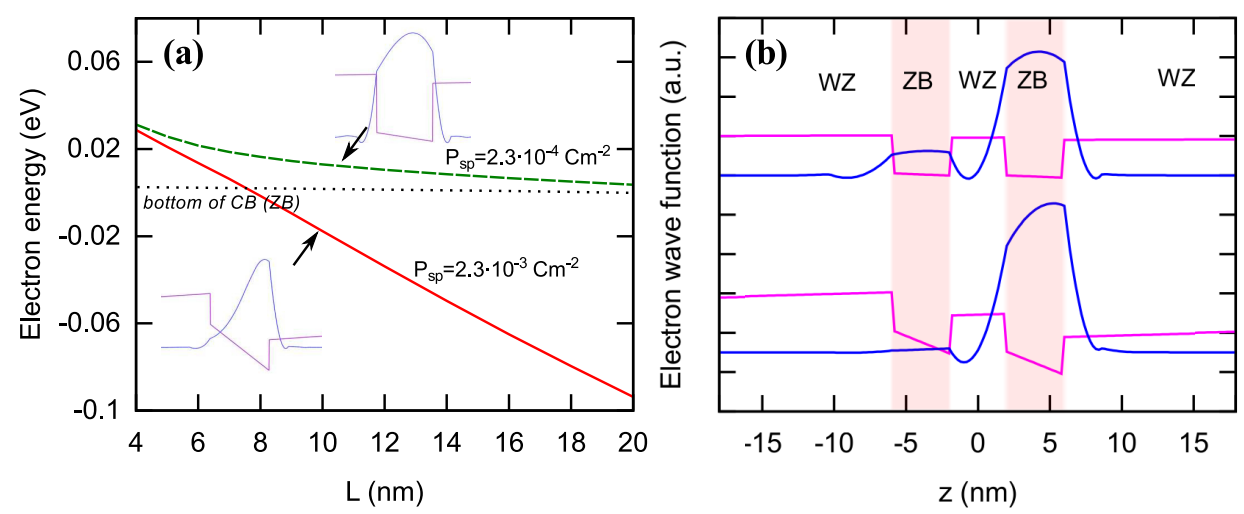

Figure 5.8: (a) Electron ground state energy for varying $Q D$ thickness in a $\mathrm{ZB} Q \mathrm{QD}$ with fixed radius $R=50 \mathrm{~nm}$. Calculations for realistic (solid line) and weakened (dashed line) GaAs spontaneous polarization are presented. The bottom of the $\mathrm{QD} \mathrm{CB}$ is indicated as a dotted line for reference. The insets show the localization of the wave function in both cases studied. (b) Wave function and confining potential cut line along the growth direction for a vertically symmetric GaAs $\mathrm{ZB} \mathrm{DQD}$. Results for weakened (top) and full $P_{s p}$ (bottom) are shown. Both QDs have $L=4 \mathrm{~nm}$.

(dashed line). By comparing the two curves, one can see the big influence of spontaneous polarization on the ground state energy, specially for large dot thickness. This clearly points out that $P_{s p}$ cannot be disregarded in moderately large systems. In the case of a realistic $P_{s p}$, the curve presents a linear dependence with $L$ that stabilizes the ground state well below the bottom of the $\mathrm{CB}$ as a consequence of the built-in electric field. The wave function is then pushed towards the upper ZB/WZ interface partially leaking into the WZ phase, see the corresponding inset in figure 5.8 (a). Contrarily, when the spontaneous polarization is reduced we find a quadratic regime for $L<10 \mathrm{~nm}$, governed by quantum confinement, that becomes linear for larger $L$. In such a case, the energy stabilization is much less pronounced and the wave function is mainly localized inside the $\mathrm{ZB}$ dot, see inset.

Similarly to traditional heterostructures where quantum confinement is originated by growing regions of different materials, polytypes can also be used to build systems of coupled QDs. In fact, the exact control in the synthesis of atomically sharp interfaces opens the possibility to produce homonuclear molecular states by fabricating perfectly symmetric pairs of QDs. Oppositely to self-assembled DQDs, polytype DQDs would not require the help of external electric fields to get states with homonuclear 
character. However, as seen in the insets of figure 5.8 (a), the polarization fields originate a significant distortion of the wave function that implies the breaking of its symmetry. Indeed, this fact is confirmed in figure 5.8 (b), where the wave function along the $z$ direction is depicted for the same $P_{s p}$ values of figure 5.8 (a). It can be observed that, even when the spontaneous polarization is reduced, the wave function is mainly localized in the upper dot, thus confirming $P_{s p}$ as a factor to be accounted for in the fabrication of polytype DQDs with homonuclear molecular states.

\subsubsection{Holes in GaAs ZB/WZ/ZB polytype QDs}

Holes are confined in the WZ phase in GaAs polytype structures. Thus, we study the $\mathrm{VB}$ of WZ QDs embedded in a $\mathrm{ZB}$ nanowire, see figure 5.7(b) for a schematic representation. The behavior of the hole energy under the effect of spontaneous polarization is similar to that of electrons, so we do not include the corresponding results here. Instead, we focus on the $\mathrm{VB}$ mixing and, more specifically, on determining the subband that contributes the most to the ground state.

The $\mathrm{VB}$ wave function is a six-component spinor of the form: $\Psi_{h}=$ $\sum_{i=1}^{6} f_{i}(\mathbf{r})\left|u_{i}\right\rangle$, with $f_{i}(\mathbf{r})$ standing for the envelope function and $\left|u_{i}\right\rangle$ for the associated Bloch function. Previous works have assumed a ground state with dominating well-defined hh character. [159, 160, 170] In Hamiltonian (5.4) the hh corresponds to the $F$ subbands, i.e. the first $\left(\left|u_{1}\right\rangle\right)$ and the fourth $\left(\left|u_{4}\right\rangle\right)$ components which denote spin-up $\left(F_{z}=+3 / 2\right)$ and spin-down $\left(F_{z}=-3 / 2\right)$, respectively. In order to disentangle these two components, a small Zeeman splitting is added, $\Delta_{z}=B_{z} \mu_{B} g \mathbb{J}_{z}$, with $B_{z}=1 \mathrm{~T}$ and $g=4 / 3$.

Figure 5.9 (a) presents the weight of the subbands with hh character, $\left(\left|f_{1}\right|^{2}+\left|f_{4}\right|^{2}\right) / \sum\left|f_{i}\right|^{2}$, for varying $R$ and $L$ in a system with spontaneous polarization. The $F$ subbands are clearly dominant for moderate and large QD radii, $R>5 \mathrm{~nm}$, while the thickness does not substantially affect the $\mathrm{VB}$ mixing in the range of $L$ under study. The inset of figure 5.9(a) shows that this abrupt change in the hole composition for radii under $5 \mathrm{~nm}$ is due to a transition from a $F$ band (hh) to a $\lambda$ band (so). In large systems, the $F$ band dominates because the bulk spin-orbit $\Delta_{s o}$ and crystal-field $\Delta_{c r}$ splittings stabilize this subband. Nevertheless, in small enough dots the high kinetic energy may take over and the character of the ground state is then determined by the effective masses of the subbands. In this case, we have $m_{\perp}^{F}=m_{\perp}^{G}=1 /\left(A_{2}+A_{4}\right)=-0.13$ and $m_{\perp}^{\lambda}=1 / A_{2}=-0.617$. Therefore, the lighter mass of the $\lambda$ band in the in-plane direction, $\left|m_{\perp}^{F}\right|<\left|m_{\perp}^{\lambda}\right|$, 

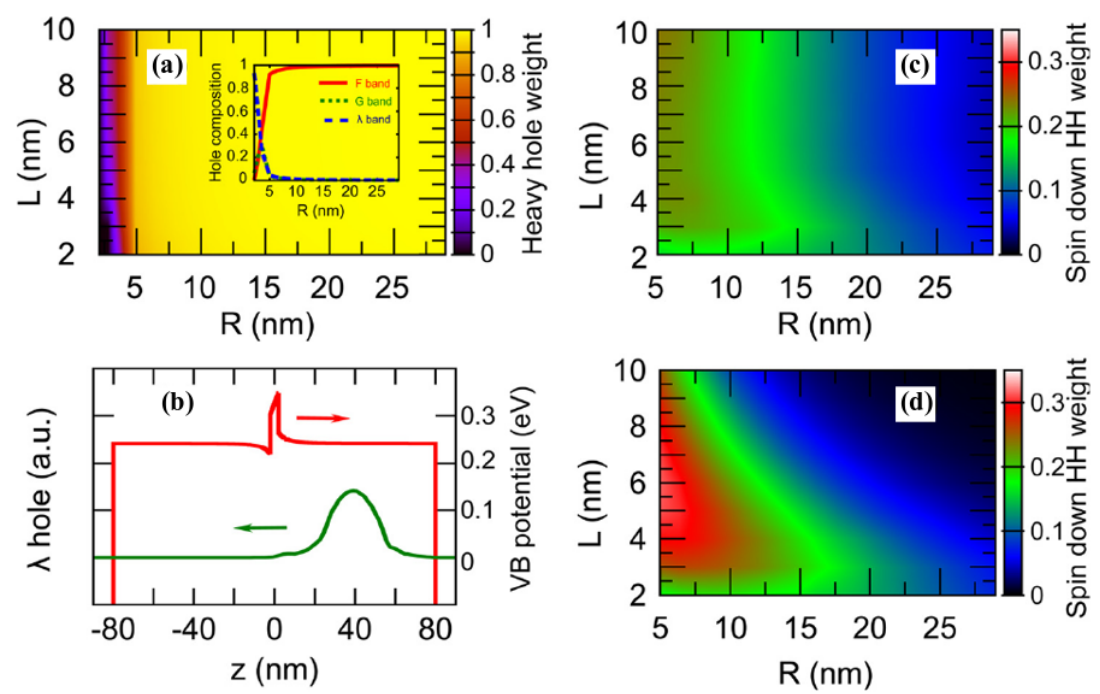

Figure 5.9: (a) Contour map showing the weight of the $F$ band in the ground state as a function of the QD thickness $L$ and radius $R$. Inset: same but for only varying $R(L=4 \mathrm{~nm})$ and including also the $G$ and $\lambda$ bands in the plot. (b) Confining potential (red) and $\lambda$ hole wave function (green) along the nanowire growth direction in a QD with $R=2.5 \mathrm{~nm}$ and $L=4 \mathrm{~nm}$. (c-d) Weight of the envelope function $f_{4}$, spin-down hh in the (c) presence and (d) absence of $P_{s p}$.

makes such band more stable than the hh one in QDs with strong lateral confinement. The effective mass of the $\lambda$ band is even lighter in the $z$ direction, $m_{z}^{\lambda}=1 / A_{1}=-0.05$, thus causing the wave function to mainly localize outside the WZ QD, see figure $5.9(\mathrm{~b})$.

We next explore the spin purity of the ground state by calculating the weight of the spin-down $F$ subband, $\left|f_{4}\right|^{2} / \sum\left|f_{i}\right|^{2}$. Calculations with $P_{s p}$, figure 5.9 (c), and without $P_{s p}$, figure 5.9(d), are compared. In general, we observe an insignificant contribution of the spin-down hh in large systems that increases as the size of the QD is reduced. The inclusion of spontaneous polarization in the model does not remarkably modify this trend and only smoothens the regions of maximum and minimum weight in figure 5.9(d). It is worth mentioning that the coupling between Zeeman-split hh bands is not direct and takes place solely at second order through the coupling with intermediate $G$ and $\lambda$ subbands, see equation (A.6). Interestingly, in spite of this fact, the contribution of these intermediate subbands to the hole ground state is nearly zero, as seen in figure 5.9(a). 
104 Chapter 5. Strain and piezoelectricity in wurtzite and polytype QDs

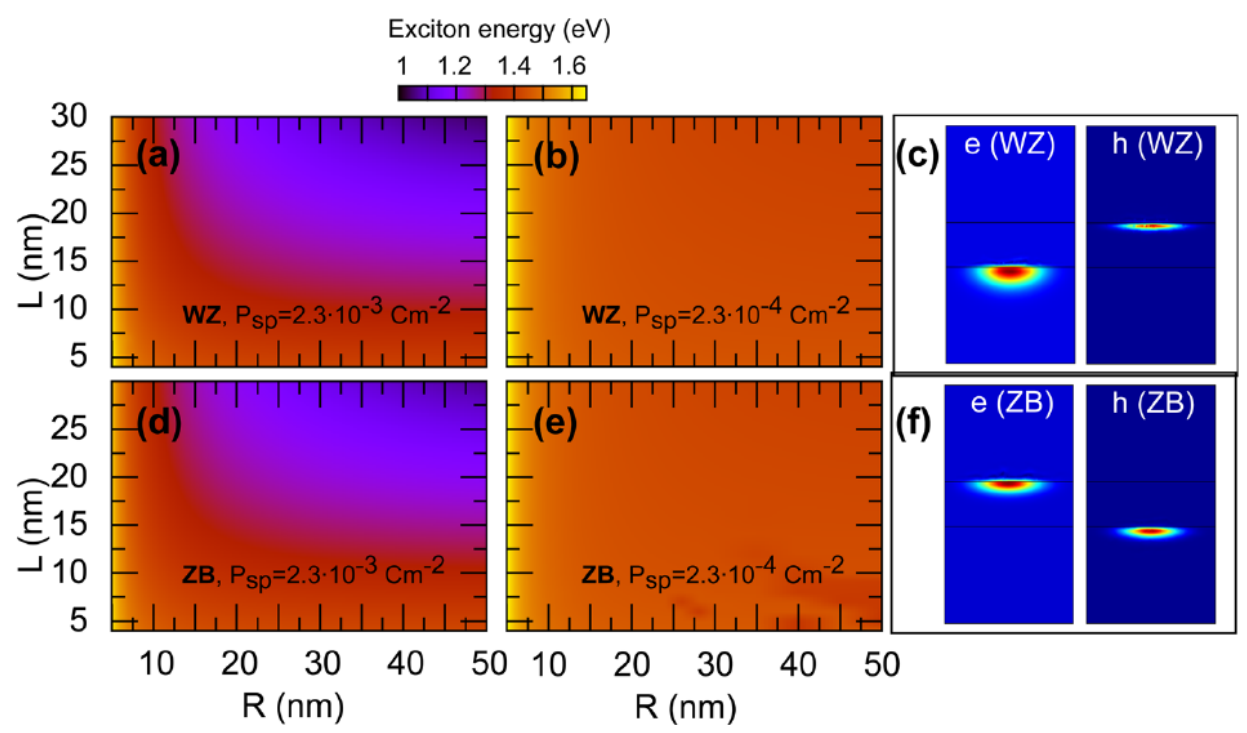

Figure 5.10: (a-b) Exciton energy versus WZ QD radius $R$ and thickness $L$ in a ZB/WZ/ZB system. The energy is computed considering (a) full and (b) weakened $P_{s p}$. (c) Electron (left) and hole (right) wave functions in a WZ QD with $R=50 \mathrm{~nm}$ and $L=30 \mathrm{~nm}$. Full $P_{s p}$ used in the simulations. (d-f) Same but for a ZB QD.

Finally, we stress that using a single-band hh model is justified except for polytype QDs with small radius, where the ground state switches from being mainly a $F$ band to a $\lambda$ band. This change in the hole ground state character should be found in the thin polytype nanowires synthesized by Loitsch et al. [170], e.g. by analyzing the polarization of interband optical transitions.

\subsubsection{Excitons in GaAs $\mathrm{WZ} / \mathrm{ZB} / \mathrm{WZ}$ and $\mathrm{ZB} / \mathrm{WZ} / \mathrm{ZB}$ poly- type structures}

Lastly, the behavior of excitons in polytype QDs is investigated. We restrict to dots with $R>5 \mathrm{~nm}$, so that a single-band model including only one hh subband, equation (5.5), is enough to describe the $\mathrm{VB}$ states satisfactorily. The two possibilities of defining a $\mathrm{QD}$ in a system with type-II band alignment are taken into account, namely $\mathrm{ZB}$ QDs embedded in a WZ nanowire and vice versa.

First, we analize the exciton energy as a function of the system dimensions. For a WZ QD in the presence of $P_{s p}$, figure 5.10(a), a striking 

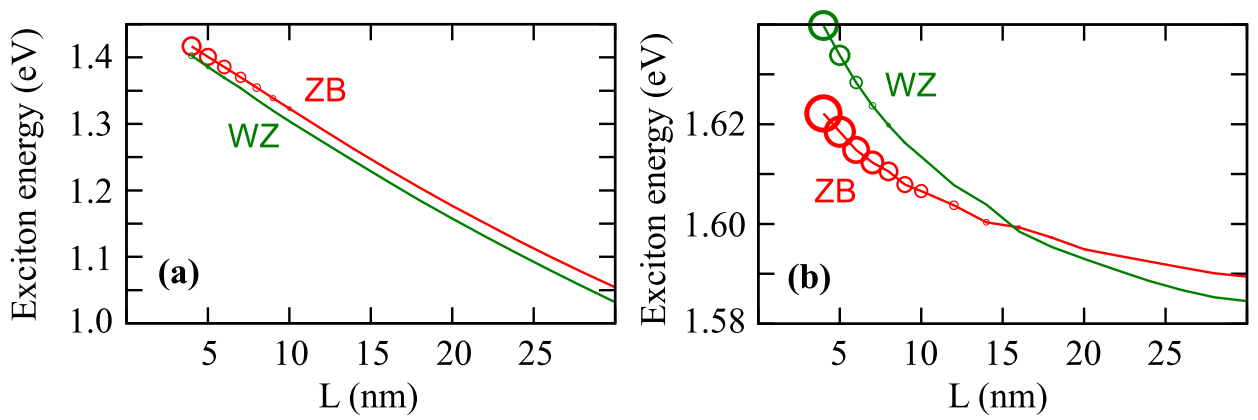

Figure 5.11: Calculated exciton energy in WZ (green line) and ZB (red line) QDs for varying dot thickness $L$. Two different nanowire radii are considered: (a) $R=50 \mathrm{~nm}$ and (b) $R=5 \mathrm{~nm}$. The size of the circles indicates the relative magnitude of the e-h overlap.

tunability of the exciton energy is found from $E_{X} \approx 1 \mathrm{eV}$ (near infrared) in large dots to $E_{X} \approx 1.6 \mathrm{eV}$ (visible) in small ones. When the spontaneous polarization is weakened, figure 5.10 (b), such tunability is strongly reduced, evidencing again the importance of this phenomenon in polytype structures. For excitons in ZB|QDs, bottom row in figure 5.10 , we find exactly the same results as for WZ QDS.

We next investigate the e-h overlap behavior in the presence of full $P_{s p}$. Figures 5.10 (c) and 5.10 (f) illustrate the electron and hole wave functions for large QDs, i.e. $R=50 \mathrm{~nm}$ and $L=30 \mathrm{~nm}$. In both WZ and $\mathrm{ZB}$ dots, carriers are separated despite the attractive Coulomb interaction, yielding weak $\mathrm{e}-\mathrm{h}$ overlaps and, hence, dark character to the excitons. This demonstrates that spontaneous polarization is capable of inducing $\mathrm{e}-\mathrm{h}$ separation in large enough polytypical structures.

In order to assess the differences between $\mathrm{ZB}$ and WZ|QDs, in figure 5.11 we directly compare the exciton energy of both systems by plotting $E_{X}$ versus the dot thickness. Simulations for two representative size regimes are carried out: typical QDs with large radius $R=50 \mathrm{~nm}$ in figure 5.11(a), and QDs with strong lateral confinement $R=5 \mathrm{~nm}$ in figure 5.11(b). A comparison between both plots shows that in QDs with large radius the excitonic energy scales linearly with $L$ while the dependence is quadratic for small radius. In dots with weak lateral confinement the thickness dependence is linear due to the built-in electric field induced by $P_{s p}$, which prevails over Coulomb interactions. In strongly confined dots, instead, the high kinetic energy makes the quantum confinement to take over and the dependence becomes quadratic. If we compare now the curves of both crystal phases, 
106 Chapter 5. Strain and piezoelectricity in wurtzite and polytype QDs

we also observe a remarkable distinct behavior: for large $R$ excitons in WZ QDs haver lower energy than those in ZB QDs regardless of $L$, while the curves cross at $L \approx 15 \mathrm{~nm}$ for small $R$. These results can be understood taking into account the effective masses $m_{z}$ of the confined carriers. Electrons in $\mathrm{ZB}$ have lighter mass than holes in WZ, causing excitons in ZB to have higher energy as can be seen in figure 5.11(a). This reasoning is also valid in figure 5.11(b) for large dot thickness, but for $L<15 \mathrm{~nm}$ the high kinetic energy of electrons allows them to escape into the WZ phase, becoming more delocalized, i.e. more stable. In contrast, the heavier mass of holes limits their penetration outside the WZs QD and the exciton energy rapidly increases with decreasing dot thickness.

We turn now our attention to the e-h overlap, represented in figure 5.11 by circles of different size. It can be seen that the overlap is substantially enhanced in QDs with small $R$, which is also connected with the delocalization of the confined carriers due to their higher kinetic energy. This points out that a transition from the usual type-II band alignment in GaAs polytype QDs to a type-I one is produced by the interplay of the spatial confinement and the $P_{s p}$-induced polarization. Therefore, the $\mathrm{QD}$ thickness can be used as a mechanism to control the $\mathrm{e}-\mathrm{h}$ overlap and also related properties such as the intensity of absorption processes and exciton lifetimes.

To close this section, we briefly compare the results of figure 5.11 with the experimental observations reported by recent works that investigate GaAs polytype QDs of similar size to ours. On one hand, Vainorius et al. [159] investigated the $\mathrm{PL}$ of both $\mathrm{ZB}$ and $\mathrm{WZ}$ QDs with large radii, $R=$ $45-60 \mathrm{~nm}$, and variable dot thickness. They obtained a WZ emission redshifted with respect to $\mathrm{ZB}$ one by a few tens of $\mathrm{meV}$. This is consistent with the higher energy found for ZB dots in figure 5.11(a), which corresponds to the same size regime of the samples. However, in the experiments the change in the emission energy with $L$ is much less pronounced, about one order of magnitude, than the calculated one in figure 5.11(a). In fact, such variation is found when disregarding the effect of spontaneous polarization and only quantum confinement is included in the model (results not shown). This fact suggests that the spontaneous polarization is somehow suppressed in the experimental setup. On the other hand, Loitsch et al. [170. studied the $\mathrm{PL}$ energy of GaAs polytype structures as a function of the dot radii. They found an exciton energy up to $1.610 \mathrm{eV}$ for WZ QDs, presenting a blueshift as large as $100 \mathrm{meV}$ with respect to bulk GaAs for the thinnest nanowires investigated, $R \approx 5 \mathrm{~nm}$. In figure 5.11(b) we have seen that this blueshift could be even larger for WZ QDs with small $L$ (the GaAs bulk band gap is $1.51 \mathrm{eV}$ ). Another work of the same authors studying the same system has also reported fast radiative lifetimes (below $1 \mathrm{~ns}$ ) in very 
thin nanowires, [160] in contrast to the commonly long lifetimes ( $>3 \mathrm{ns)}$ exhibited by typical large-diameter structures. In their work, the fastest exciton decay for small $R$ was ascribed to a transition from type-II to typeI band alignment, which goes along with our prediction in figure 5.11(b). 



\section{CHAPTER 6}

\section{Edge states in monolayer $\mathrm{MoS}_{2}$ nanostructures}

Over the last few years the emergence of atomically thin materials has revolutionized the fields of solid-state physics and material science, chiefly because they exhibit dramatically different, and often superior, properties compared to their bulk counterparts. The main reason for this dissimilarity lies in their distinct dimensionality. The first truly $2 \mathrm{D}$ system was graphene. It was discovered by Novoselov et al. 22 in 2004 and, since then, many groups have dedicated big efforts to investigate these novel systems. As a result, nowadays several new 2D materials have been prepared.[173, 174]

Particularly, in this work we focus on monolayer TMDC. rich variety of TMDCs, we investigate those with semiconducting behavior, specifically $\mathrm{MoS}_{2}$. Unlike graphene, $\mathrm{MoS}_{2}$ and other TMDCs present a finite band gap which is indirect in bulk form but becomes direct in the monolayer limit.[175] The direct band gap makes single-layer TMDCs especially attractive for electronic and optoelectronic applications. 176 178

Similarly to traditional 3D bulk semiconductors, single-layer materials also offer the possibility of fabricating nanostructures with lower dimensionality, e.g. nanoribbons (1D) and QDs (0D). To date, these finite structures have not been extensively investigated yet and a more detailed understanding of their electronic structure is needed for the development of possible

${ }^{1}$ The 2D form of TMDC materials $\left(\mathrm{MX}_{2}\right)$ is commonly called monolayer, but it is actually composed by three layers of atoms (X-M-X): i.e. one layer of metal atoms sandwiched between two layers of chalcogenide atoms. The atoms in each layer are arranged as a triangular lattice. 
devices. Several works have reported the existence of edge states in finite $\mathrm{MoS}_{2}$ systems under different conditions. [179 182] The presence of these edge states is very relevant since they form 1D metallic channels along the edges, thus affecting transport and optical properties.

In this chapter, we deal with the electronic structure of monolayer $\mathrm{MoS}_{2}$ nanostructures, namely nanoribbons and QDs, Special attention is paid to the origin of edge states in these finite systems and its connection with topological insulators. Topological insulators have been intensely discussed in recent literature due to their unique properties. [183, 184. They are materials with insulating behavior in the bulk, but present gapless conducting states at the edges/surfaces of the system.[185, 186] Surface states may originate from different sources, e.g. dangling bonds or polar discontinuities [187, 188, but what makes topological insulators special is that metallic states are protected by time-reversal symmetry. Therefore, they are robust against backscattering and in the presence of non-magnetic perturbations.

The contents of the present chapter are based on an article published in collaboration with professor Sergio E. Ulloa from Ohio University (USA) and are the result of a research short stay in his group. The full version of the publication can be found in page 267 .

\subsection{Effective Hamiltonian}

Apart from their dimensionality, monolayer TMDCs and the traditional semiconductors studied in the previous chapters present more structural differences. In monolayer $\mathrm{MoS}_{2}$ the metal atoms have trigonal prismatic coordination with the chalcogenide ones, and the direct gap of the band structure is situated at the two nonequivalent points $K$ and $K^{\prime}$ of the Brillouin zone. Contrarily, $\mathrm{WZ}$ and $\mathrm{ZB}$ semiconductors present tetrahedral coordination and the direct gap is at the center of the Brillouin zone $(\Gamma$ point). As a consequence, the Hamiltonians introduced in chapter 2 are not valid and several authors have derived effective $\mathrm{k} \cdot \mathrm{p}$ models to study the low-energy physics of TMDC monolayers. [189 192] Since we deal with edge states in the gap, we should employ a Hamiltonian including both $\mathrm{CB}$ and $\mathrm{VB}$. A simple two-band model describing such bands up to second order in $k$ suffices for our exploratory purposes. It can be written as:

$$
H=\left(\begin{array}{cc}
\varepsilon_{v}+\alpha k^{2} & \tau \gamma k_{-} \\
\tau \gamma k_{+} & \varepsilon_{c}+\beta k^{2}
\end{array}\right),
$$

where $k_{ \pm}=k_{x} \pm i \tau k_{y}$, and $\varepsilon_{c}=\Delta / 2$ and $\varepsilon_{v}=-\Delta / 2$ are the band-edge energies with $\Delta=1.9 \mathrm{eV}$ standing for the material band gap; $\mathbf{k}$ is the 
momentum relative to the $\mathrm{K} / \mathrm{K}^{\prime}$ points. The constants $\alpha, \beta$ and $\gamma$ are material parameters, while $\tau$ identifies the valley $\mathrm{K}(\tau=1)$ or $\mathrm{K}^{\prime}(\tau=-1)$. In literature, different authors report different values for these parameters. We use in all calculations presented here the ones suggested in reference [191] unless otherwise specified. These parameters are $\alpha=1.72 \mathrm{eV} \AA^{2}, \beta=$ $-0.13 \mathrm{eV} \AA^{2}$ and $\gamma=3.82 \mathrm{eV} \AA$, as fitted from density functional theory calculations.

Hamiltonian (6.1) takes into account the electron-hole symmetry breaking observed in first-principles simulations by using unlike values for $\alpha$ and $\beta$. However, for the sake of simplicity, trigonal warping and other minor contributions have been disregarded as they do not change the qualitative results. Additionally, hard-wall boundary conditions are employed to describe the edges of the nanostructures. These boundary conditions do not produce coupling between valleys or spins, so each valley/spin can be discussed independently and a two-band model can be used. Such situation is also expected of zigzag edges, although further investigations are required to confirm the equivalence of both conditions.

\subsection{Results and discussion}

In this section we apply the above presented model to study the electronic behavior of $\mathrm{MoS}_{2}$ nanoribbons and QDs. All simulations are carried out using COMSOL utilities, a commercial software that uses the numerical finite element method.

\subsection{1 $\quad \mathrm{MoS}_{2}$ nanoribbons}

Nanoribbons are structures of finite width where particles are confined in one direction of space and move freely in the perpendicular one. We define the nanoribbons in our calculations to be translational invariant along the $y$ direction, so that the momentum $k_{y}$ is a good quantum number and the two-component wave function can be written as $\psi(x, y)=e^{i k_{y} y} \phi(x)$, where $\psi$ and $\phi$ have components over the conduction $c$ and valence $v$ basis. Substituting $\psi(x, y)$ into Hamiltonian (6.1) results in a two coupled secondorder differential equation system in one dimension that can be numerically solved for a given $k_{y}$.

The band dispersion obtained for a $10 \mathrm{~nm}$-width $\mathrm{MoS}_{2}$ nanoribbon is shown in figure 6.1(b). Interestingly, we find two states inside the band 


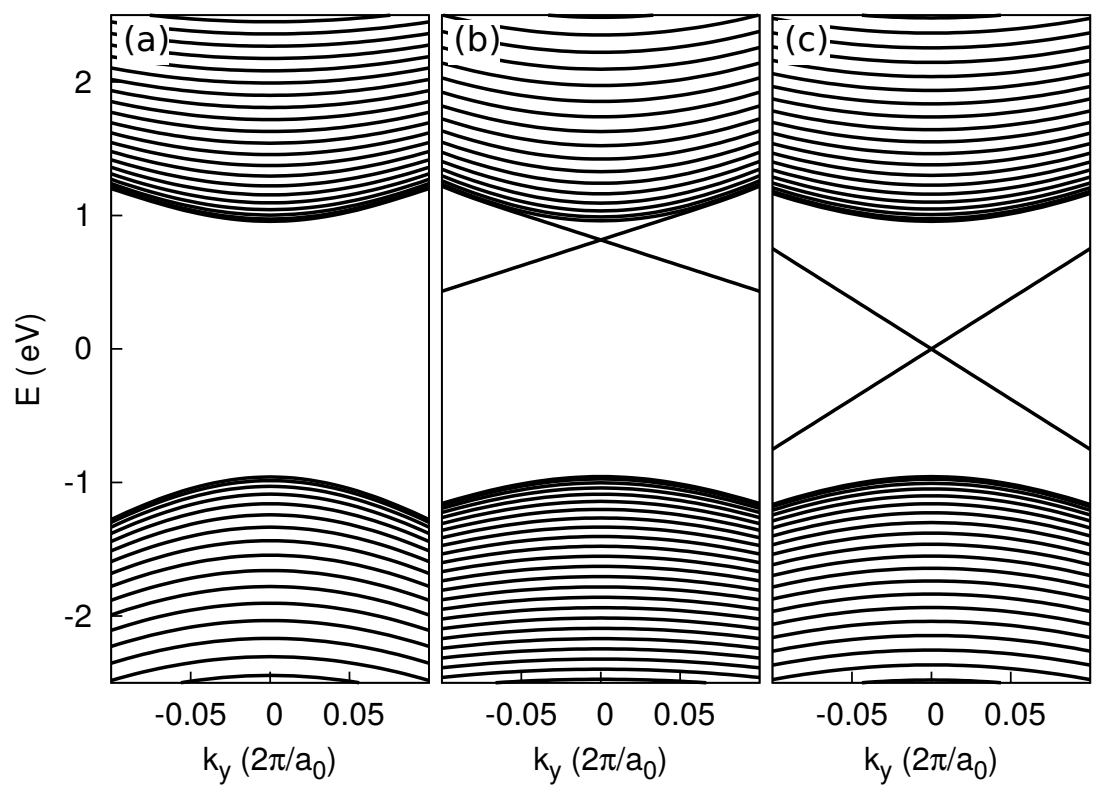

Figure 6.1: Energy-band dispersion for $\mathrm{MoS}_{2}$ nanoribbons considering different values of $\alpha$ and $\beta$ : (a) $\alpha=-1.72 \mathrm{eV}^{2}$ and $\beta=0.13 \mathrm{eVA}^{2}$, (b) $\alpha=1.72 \mathrm{eV}^{2}$ and $\beta=-0.13 \mathrm{eV} \AA^{2}$, and (c) $\alpha=1.72 \mathrm{eVA}^{2}$ and $\beta=-1.72 \mathrm{eV}^{2}$. The edges are parallel to the $y$ direction, and the wave vector $k_{y}$ is measured with respect to the $\mathrm{K}$ valley, where $a_{0}=3.193 \AA$ is the lattice constant.

gap with a nearly linear dispersion. These levels cross at $k_{y}=0$ and $E=0.816 \mathrm{eV}$, and have energies very close to the $\mathrm{CB}$ edge. In fact, they disperse upwards in energy coming very close to the $\mathrm{CB}$ for not large $k_{y}$, and soon admix with the band states, becoming indistinguishable from them. The states of lower energy, instead, remain far from the $\mathrm{VB}$ and are not hybridized in the range of $k_{y}$ considered.

In order to study the origin of these states, we repeat the same calculations but for other sets of parameters. We only tune $\alpha$ and $\beta$ since $\gamma$ does not affect the presence of midgap states. First, the sign of both $\alpha$ and $\beta$ is changed: $\alpha=-1.72 \mathrm{eV} \AA^{2}$ and $\beta=0.13 \mathrm{eV} \AA^{2}$. Figure. 6.1 (a) shows that the states lying inside the gap are now absent. Subsequently, in figure. 6.1 (c) we keep the signs unaltered to those in panel (b) but modify $\beta$ to have the same absolute value of $\alpha: \alpha=1.72 \mathrm{eV}^{2}$ and $\beta=-1.72 \mathrm{eV} \AA^{2}$. In this case, the two states inside the gap are still present but they have lower energies compared to figure 6.1(b). The dispersion bands now cross exactly at $k_{y}=0$ and $E=0$. This is as expected from symmetry considerations 

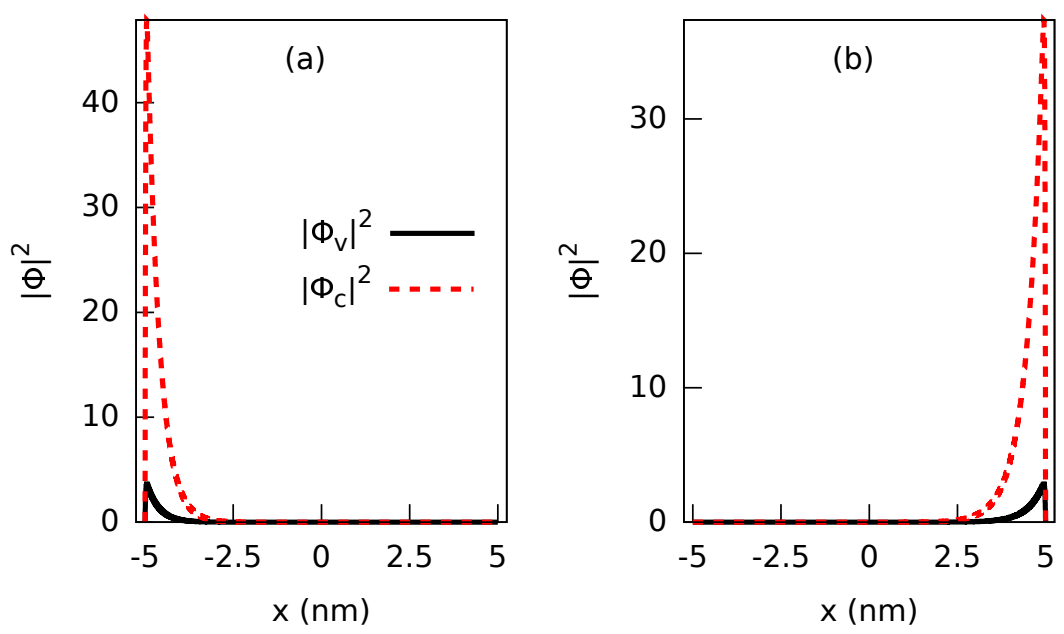

Figure 6.2: Squared modulus $|\phi|^{2}$ of the two components of the wave function for the two states with energies lying in the band gap in figure 6.1(b). The states correspond to $k_{y}=0.01 \times 2 \pi / a_{0}$ and have the following energies: (a) $E=0.778 \mathrm{eV}$, and (b) $E=0.855 \mathrm{eV}$. Black solid lines represent the $\mathrm{VB}$ component $\phi_{v}$ and red dashed lines the $\mathrm{CB}$ component $\phi_{c}$.

because $\alpha=\beta$ confers electron-hole symmetry to the Hamiltonian. We also stress that a situation with $\alpha$ and $\beta$ having the same sign is not taken into account since in such a case there is not a real gap separating the bands.

From the analysis of the results for the three sets of parameters in figure 6.1, it is clear that the presence/absence of midgap states is determined by the sign of $\alpha$ and $\beta$. It can be inferred that they exist if $\alpha>0$ and $\beta<0$, and are absent if $\alpha<0$ and $\beta>0$. The energy and, therefore, the position of the states inside the gap is determined by the relative value of the two parameters. When $|\alpha|>|\beta|$ the states are closer to the $\mathrm{CB}$ as in figure 6.1(b), and when $|\alpha|<|\beta|$ they become closer to the $\mathrm{VB}$.

Midgap states are typically associated to states localized at the edge of finite structures, the so-called edge states. Thus, to further explore their nature we examine the form of the wave functions. As an example, we represent in figure 6.2 the midgap states corresponding to $k_{y}=0.01 \times 2 \pi / a_{0}$ in figure 6.1(b). We choose this value of $k_{y}$ to avoid problems derived from degeneracies and mixing with the $\mathrm{CB}$. Figure 6.2(a) illustrates the wave function squared modulus of the lower state at $E=0.778 \mathrm{eV}$ and figure 6.2(b) of the higher one at $E=0.855 \mathrm{eV}$. We clearly observe that both states are localized at opposite edges of the $\mathrm{MoS}_{2}$ nanoribbon, thus 
confirming that they are in effect edge states. Figure 6.2 also reveals that the width of the nanoribbon is large enough to ensure decoupled states on both edges.

By comparing the height of the components in figure 6.2, it is also evident that the $\mathrm{CB}$ component (red dashed line) is the main contribution to the wave function in both states. The calculation of the relative weight of the two components yields $w\left(\phi_{c}\right)=93 \%$ and $w\left(\phi_{v}\right)=7 \%$ for the conductionand valence-components, respectively. These values can be directly obtained from the parameters $\alpha$ and $\beta$ using the expressions $w\left(\phi_{c}\right)=|\beta| /(|\alpha|+|\beta|)$ and $w\left(\phi_{v}\right)=|\alpha| /(|\alpha|+|\beta|)$. These expressions hold as long as the edge states are relatively far from the bulk bands. Moreover, it can be seen that the wave functions of the two states are slightly different, e.g. by comparing the maximum value of $\left|\phi_{c}\right|^{2}$ or the $x$ extension. This asymmetry is due to the different proximity of the $\mathrm{CB}$. The edge state in panel (b) is closer to the $\mathrm{CB}$ and, thus, it is slightly more admixed with the bulk states and its wave function is somewhat more delocalized.

The results summarized in figure 6.1 and figure 6.2 can be related to those coming from the model proposed by Bernevig, Hughes, and Zhang (BHZ).[193] In this model, the observation of the quantum spin Hall effect (QSHE) was predicted in HgTe quantum wells larger than a critical thickness, due to a band inversion, i.e. a change in $\Delta$ 's sign. In that work, for $\Delta<0$ bands are inverted and the system shows topological behavior. This means that edge states will form when a transition between two distinct topological phases takes place, as predicted by the principle of bulk-edge correspondence. [185] In our system we have $\Delta>0$, which is apparently trivial, but the sign of the band curvatures $(\alpha>0$ and $\beta<0)$ yields also a situation with inverted band $2^{2}$ similar to the $\mathrm{BHZ}$ model, so that the origin of the edge states can be analyzed in terms of the topological character of Hamiltonian 6.1).

In order to make the above reasoning clear, we perform calculations of the energy spectrum as a function of $\Delta$. Results are shown in figure 6.3. We fix $k_{y}=0$ and consider two sets of band curvatures: $\alpha=1.72 \mathrm{eV} \AA^{2}$ and $\beta=-0.13 \mathrm{eV} \AA^{2}$ in figure 6.3 (a), and $\alpha=1.72 \mathrm{eV} \AA^{2}$ and $\beta=-1.72 \mathrm{eV} \AA^{2}$ in figure 6.3(b). Two red dashed lines showing the limits of the band gap have been added in each plot to improve the readability. In both cases, a trivial situation with no states in the gap is observed at large negative $\Delta$. As $\Delta$ increases and changes sign two degenerate edge states appear with energies

\footnotetext{
${ }^{2}$ The "bare" effective masses for the $\mathrm{VB}$ and $\mathrm{CB}$ are determined by the $\alpha$ and $\beta$ coefficients, respectively. A negative $\beta$, corresponding to a negative mass $\approx 1 / \beta$, is "inverted", and that symmetry is present in the states even after the mixing due to $\gamma$.
} 


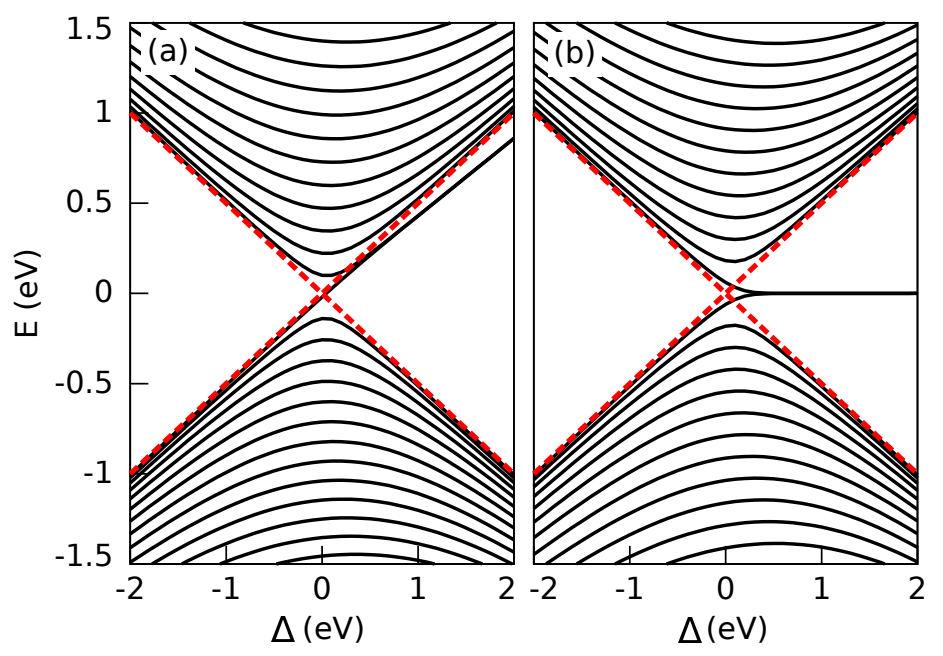

Figure 6.3: Energy spectrum of a $\mathrm{MoS}_{2}$ nanoribbon as a function of the band gap $\Delta$, for $k_{y}=0$. Two sets of parameters are considered: (a) $\alpha=1.72 \mathrm{eV} \AA^{2}$ and $\beta=-0.13 \mathrm{eV} \AA^{2}$, and (b) $\alpha=1.72 \mathrm{eV} \AA^{2}=$ $-\beta$. Red dashed lines indicate the edges of the band gap.

clearly lying in the gap. This behavior confirms the connection between edge states and band inversion as in the BHZ model. As already discussed in figure 6.1. midgap states are closer to the $\mathrm{CB}$ when $|\alpha|>|\beta|$, panel (a), and become perfectly equidistant from the $\mathrm{CB}$ and $\mathrm{VB}$ for $|\alpha|=|\beta|$ due to electron-hole symmetry, panel (b).

To explore the topological behavior further, we analyze the results with the help of the Chern number associated with the occupied band. The Chern number is a topological invariant (its value cannot change under smooth deformations of the Hamiltonian parameters) that characterizes a state as trivial $(c=0)$ or nontrivial $(c \neq 0)$. For a two-level Hamiltonian like that in equation (6.1), once we rewrite it in the form $H(\mathbf{k})=\mathbf{g}(\mathbf{k}) \cdot \boldsymbol{\sigma}$, where $\boldsymbol{\sigma}$ is a vector with the Pauli matrices as components, the Chern number is given by 185 .

$$
c=\frac{1}{4 \pi} \int d^{2} k\left(\partial_{k_{x}} \hat{\mathbf{g}} \times \partial_{k_{y}} \hat{\mathbf{g}}\right) \cdot \hat{\mathbf{g}},
$$

where $\hat{\mathbf{g}}=\mathbf{g} /|\mathbf{g}|$ and the integral is computed over the entire Brillouin zone. For Hamiltonian (6.1), equation (6.2) yields $c=\tau / 2[\operatorname{sgn}(\Delta)+\operatorname{sgn}(\alpha-\beta)]$. Then, for $\Delta>0$ one obtains $c=0$ if $\alpha<\beta$ and $c=\tau$ if $\alpha>\beta$. When $\alpha=1.72 \mathrm{eV} \AA^{2}$ and $\beta=-0.13 \mathrm{eV} \AA^{2}$ we have $c \neq 0$, denoting the non-trivial character of the $\mathrm{MoS}_{2}$ Hamiltonian. As a consequence, gapless states must be present at the domain wall separating the nanoribbon (non-trivial) and 
the vacuum (trivial), according to the bulk-edge correspondence. This goes along with the previous discussion based on band inversion arguments.

It is important to note, however, that the contribution of the valleys $\mathrm{K}$ and $\mathrm{K}$ ' to the topological invariant has opposite signs, originating an overall $c=0$. Therefore, strictly speaking multivalley materials such as graphene or $\mathrm{MoS}_{2}$ are topologically trivial. In spite of this, the origin of edge states in gapped and bilayer graphene has been discussed in terms of the marginal topological properties of the single-valley Hamiltonians. [194, 195] This analysis is possible owing to the close analogy between graphene systems and $2 \mathrm{D}$ topological insulators, and can also be applied to monolayer $\mathrm{MoS}_{2}$. Nevertheless, this analogy has important limitations. Since $c$ per valley is not a well-defined topological invariant, $c \neq 0$ does not guarantee the existence of edge states at the boundaries with the vacuum. Furthermore, they are not topologically protected against backscattering and can then be affected by any mechanism of disorder or valley coupling. All the same, edge states in bilayer graphene have been shown to be robust under moderate disorder, [196] and to exhibit pure valley currents, as indicated by the local valley Berry curvature.[197]

\subsection{2 $\quad \mathrm{MoS}_{2}$ triangular QDs}

We next investigate the electronic structure of monolayer $\mathrm{MoS}_{2}$ QDs formed by finite-size flakes. The flakes are modeled as equilateral triangles since it is the most common shape obtained in the laboratory.[198 200] The side length of the QDs is $10 \mathrm{~nm}$ in all calculations. As for the material parameters, we employ the same ones as in figure 6.2(b) of previous section: $\alpha=1.72 \mathrm{eV} \AA^{2}$ and $\beta=-0.13 \mathrm{eV} \AA^{2}$.

Similarly to $\mathrm{MoS}_{2}$ nanoribbons, midgap states also emerge for this set of parameters. However, several states with energies lying in the band gap are found for $\mathrm{MoS}_{2}$ QDs, in contrast to only one state per edge found for nanoribbons. This difference can be understood considering the finite size of the edges in QDs. The edge states are then confined along each border, thus originating the discretization of this band and the corresponding energy quantization.

To illustrate this, we show in figure 6.4 the squared modulus of the wave function for a selection of states with energy close to the $\mathrm{CB}(E \approx 0.95 \mathrm{eV})$. We choose this range of energies because we know that for these parameters the edge states are closer to the $\mathrm{CB}$. Besides, it offers the possibility to compare the "bulk" and edge states in the flake. In figure. 6.4 the states 

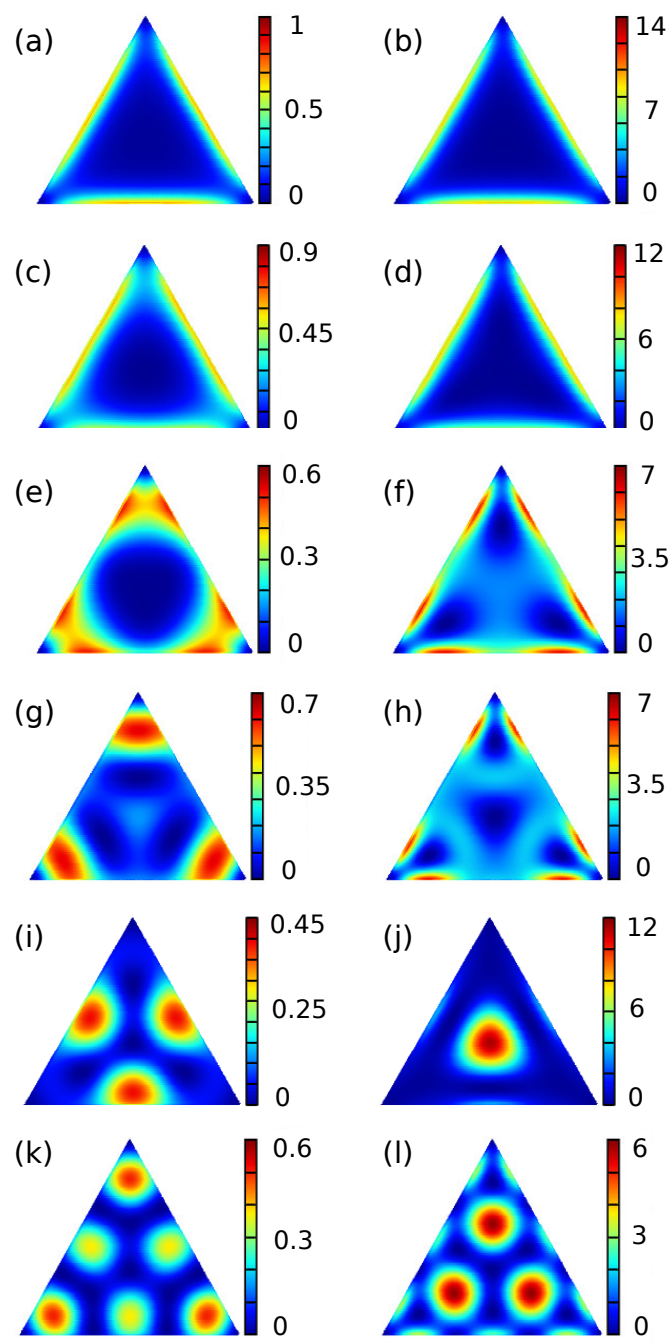

Figure 6.4: Wave-function squared modulus of a selection of states close to the $\mathrm{CB}$ edge energy. Left and right columns illustrate the VB $\left|\phi_{v}\right|^{2}$, and $\mathrm{CB}\left|\phi_{c}\right|^{2}$, components, respectively. Different states are arranged in rows with increasing energy: (a)-(b) $E=0.907 \mathrm{eV}$, (c)-(d) $E=0.962 \mathrm{eV},(\mathrm{e})-(\mathrm{f}) E=1.015 \mathrm{eV}$, (g)-(h) $E=1.022 \mathrm{eV}$, (i)-(j) $E=1.057 \mathrm{eV}$ and (k)-(l) $E=1.100 \mathrm{eV}$. 
are presented in order of increasing energy. Each row corresponds to a different state, with $\left|\phi_{v}\right|^{2}$ and $\left|\phi_{c}\right|^{2}$ components depicted on the left and right column, respectively. The first two states shown, panels (a)-(d), are clearly edge states with their wave functions localized near the triangle border. Next two states in energy, figure 6.4(e)-(h), also show their wave functions mainly near the edges, but noticeably more delocalized than the previous two. This suggests that they are partially admixed with the $\mathrm{CB}$ states due to their energy proximity. Finally, figure 6.4(i)-(l) show two conduction states with the wave function completely delocalized over the entire QD. A representation of the real and imaginary parts of the wave functions (not shown) allows one to see the wave function nodes more clearly. It is seen that the number of nodes increases with the energy of the states, a typical signature of quantization.

It is worth mentioning that calculations using other sets of parameters have also been carried out for $\mathrm{MoS}_{2}$ QDs. The corresponding results show exactly the same dependence between the presence/absence of edge states and the curvature parameters as in the case of nanoribbons. Therefore, one can also invoke the marginal topological character of the Hamiltonian as the origin of edge states in these zero-dimensional nanostructures.

In summary, $\mathrm{MoS}_{2}$ nanoribbons and QDs exhibit edge states spatially localized on the edges with energies lying in the band gap. Such states originate from the $\mathrm{CB}$ and $\mathrm{VB}$ curvatures and are related to the marginal topological properties of the $\mathrm{MoS}_{2}$ single-valley Hamiltonian. 


\section{CHAPTER 7}

\section{Conclusions}

The aim of this Thesis is to theoretically determine the effect of externally applied fields and the interaction with the environment on the optical and electronic properties of QDs with possible technological interest. To this end, we have employed effective $\mathrm{k} \cdot \mathrm{p}$ models within the EMA and the EFA, which offer satisfactory results for the electronic structure at a reasonable computational cost. In order to account for the various phenomena investigated, namely external electric and magnetic fields, SOI, strain and piezoelectricity, the $\mathrm{k} \cdot \mathrm{p}$ Hamiltonians have been supplemented with the corresponding additional terms. We outline next the main obtained results.

First, we have derived a six-band position-dependent Hamiltonian in cylindrical coordinates to study the $\mathrm{VB}$ of $\mathrm{ZB}$ GaN/AlN QDs. Using this model we have found a high spin purity in QDs of typical dimensions, i.e. with small aspect ratio, which is reduced in taller dots. In fact, the symmetry of the hole ground state can be switched in dots with aspect ratio approximately unity by applying external magnetic fields of different intensity, thus providing an easy way to modulate the optical answer of this aspect ratio systems.

The effect of axially applied external magnetic fields has also been investigated in hexagonal QRs. Similarly to circular ones, the $\mathrm{AB}$ effect emerge in such doubly-connected structures as a consequence of the topology, but some distinct symmetry-related features are found in this particular case. On one hand, in large hexagonal rings populated with six electrons in a regime of low electronic density, the typical $\mathrm{AB}$ oscillations are completely suppressed and the magnetization profile becomes flat. This behavior is due to an anticrossing between the multi-particle ground state and a highly excited state of the same symmetry. In other words, symmetry overpasses 
topology in this case. We have also demonstrated in nanowires presenting a hexagonal ring in their cross section and pierced by weak magnetic fields, that the AB-like magnetoconductance oscillations can disappear or resurface by varying the voltage strength in both gate-all-around and backgate device configurations. In addition, in the high-magnetic-field regime we have shown that several field-induced transitions take place due to the electron distribution relocalization and to the complete electron depletion of excited bands. Signatures of such transitions should be observed in magnetoconductance experiments as a steplike behavior.

We have also addressed another topology-related problem: the emergence of edge states in monolayer $\mathrm{MoS}_{2}$ nanoribbons and QDs. We have shown that these atomically thin nanostructures exhibit states spatially localized on the borders and with energies lying in the band gap. The origin of such edge states has been related to the marginal topological character of the single-valley $\mathrm{MoS}_{2}$ Hamiltonian, which is governed in turn by the curvature of conduction and valence bands.

We have next turned our attention to the relaxation processes of the spin degree of freedom confined in QDs. The spin-orbit-induced spin relaxation is calculated for electrons and holes employing fully 3D models, going beyond the common quasi-2D ones. By using this more realistic description the important role of cubic DSOI becomes evident even in moderately short dots. It has also been found that the spin relaxation presents a remarkable anisotropy with the orientation of external fields and with anisotropies in the QD shape. Such behavior has been observed for both $\mathrm{CB}$ and $\mathrm{VB}$ in several structures of different materials, shapes, and grown along various crystallographic orientations, thus opening the possibility to enhance or suppress the spin lifetimes in these systems by properly designing them. Furthermore, for the VB we have provided insight into the dominant contribution of the various spin-mixing mechanisms. In particular, SOI or lh-hh couplings prevail depending on the dot aspect ratio. Additionally, we have shown that the spin lifetime of holes exceeds that of electrons in flat enough QDs, Moreover, spin relaxation has also been explored in vertical DQDs under axial electric fields. Maximum hole spin lifetimes are obtained for molecular states, but they rapidly decrease when the symmetry of the system is lowered by misalignment of the dots, non-resonant fields or inclusion of DSOI

Finally, we have dealt with the influence of the environment on the QD properties, specifically how strain and piezoelectricity affect carriers spatial separation and, thus, exciton performance. Particularly, in WZ core-shell nanocrystals, strong built-in piezoelectric fields along the growth direction 
are found in dot-in-dot structures when cores and shells are large, leading to effective e- $\mathrm{h}$ separation in spite of the attractive Coulomb interaction. The same is true for other systems such as dot-in-rods, dot-in-plates and rod-inrods as long as the core is large and the shell thick. Surprisingly enough, a thick shell is needed not only in the axial direction but also in the lateral one. This is because thin shells reduce the overall system strain independently of the direction, thus yielding weak polarization fields. This explains why piezoelectricity has not been reported yet as a feasible control mechanism in experiments studying dot-in-dot and dot-in-rod systems. The effect of polarization fields on excitons is also explored in polytype QDs, where strain and piezoelectricity are negligible, and spontaneous polarization is dominant. In such a system, the exciton energy has been proved to be very sensitive to the QD dimensions in the presence of spontaneous polarization, confirming its important action. Remarkably, radial confinement induces a gradual transition from type-II to type-I band alignment that results in a substantial modulation of the e-h overlap. For both crystal structures it can be concluded that a wide exciton wavelength and lifetime tunability is possible thanks to the emergence of strong polarizations when growing QDs with the appropriate dimensions. 



\section{Valence band Hamiltonians}

\section{A.1 Zinc-blende crystal structure}

\section{A.1.1 Four-band Luttinger-Kohn Hamiltonian}

The Hamiltonian proposed by Luttinger and Kohn [122] in 1955 is one of the most commonly used to describe systems with constant mass. The matrix form reads:

$$
H_{Z B}^{L K}=-\left(\begin{array}{cccc}
\left|\frac{3}{2} \frac{+3}{2}\right\rangle & \left|\frac{3}{2} \frac{+1}{2}\right\rangle & \left|\frac{3}{2} \frac{-1}{2}\right\rangle & \left|\frac{3}{2} \frac{-3}{2}\right\rangle \\
-S^{\dagger} & P-Q & R & 0 \\
R^{\dagger} & 0 & P & R \\
0 & R^{\dagger} & S^{\dagger} & P+Q
\end{array}\right),
$$

with

$$
\begin{aligned}
P & =\frac{\hbar^{2}}{2 m_{0}} \gamma_{1}\left(k_{x}^{2}+k_{y}^{2}+k_{z}^{2}\right), \\
Q & =\frac{\hbar^{2}}{2 m_{0}} \gamma_{2}\left(k_{x}^{2}+k_{y}^{2}-2 k_{z}^{2}\right), \\
R & =\frac{\hbar^{2}}{2 m_{0}}\left[-\sqrt{3} \gamma_{2}\left(k_{x}^{2}-k_{y}^{2}\right)+2 i \sqrt{3} \gamma_{3} k_{x} k_{y}\right], \\
S & =\frac{\hbar^{2}}{m_{0}} \sqrt{3} \gamma_{3}\left(k_{x}-i k_{y}\right) k_{z} .
\end{aligned}
$$

Here $m_{0}$ is the free electron mass and $\gamma_{i}$ are the Luttinger parameters. 


\section{A.1.2 Six-band Burt-Foreman Hamiltonian}

The position-dependent Hamiltonian derived by Foreman 40, 41] using Burt's formalism [37-39] to investigate the $\mathrm{VB}$ of $\mathrm{ZB}$ materials reads

$$
H_{Z B}^{B F}=-\left(\begin{array}{cccccc}
\left|\frac{3}{2}, \frac{3}{2}\right\rangle & \left|\frac{3}{2}, \frac{1}{2}\right\rangle & \left|\frac{3}{2}, \frac{-1}{2}\right\rangle & \left|\frac{3}{2}, \frac{-3}{2}\right\rangle & \left|\frac{1}{2}, \frac{1}{2}\right\rangle & \left|\frac{1}{2}, \frac{-1}{2}\right\rangle \\
P^{\prime} & S_{-} & -R & 0 & -\frac{1}{\sqrt{2}} S_{-} & -\sqrt{2} R \\
S_{-}^{\dagger} & P^{\prime \prime} & -C & R & -\sqrt{2} Q & -\sqrt{\frac{3}{2}} \Sigma_{-} \\
-R^{\dagger} & -C^{\dagger} & P^{\prime \prime *} & S_{+}^{\dagger} & \sqrt{\frac{3}{2}} \Sigma_{+} & -\sqrt{2} Q^{*} \\
0 & R^{\dagger} & S_{+} & P^{\prime *} & \sqrt{2} R^{\dagger} & -\frac{1}{\sqrt{2}} S_{+} \\
-\frac{1}{\sqrt{2}} S_{-}^{\dagger} & -\sqrt{2} Q^{\dagger} & \sqrt{\frac{3}{2}} \Sigma_{+}^{\dagger} & \sqrt{2} R & P^{\prime \prime \prime}-\Delta_{s o} & C \\
-\sqrt{2} R^{\dagger} & -\sqrt{\frac{3}{2}} \Sigma_{-}^{\dagger} & -\sqrt{2} Q^{*} & -\frac{1}{\sqrt{2}} S_{+}^{\dagger} & C^{\dagger} & P^{\prime \prime \prime *}-\Delta_{s o}
\end{array}\right),
$$

with

$$
\begin{aligned}
P^{\prime}= & \frac{1}{2}\left\{k_{x}(L+M) k_{x}+k_{y}(L+M) k_{y}+k_{z} 2 M k_{z}\right\} \\
& +\frac{i}{2}\left\{k_{x}\left(F-G-H_{1}+H_{2}\right) k_{y}-k_{y}\left(F-G-H_{1}+H_{2}\right) k_{x}\right\}, \\
P^{\prime \prime}= & \frac{1}{6}\left\{k_{x}(L+5 M) k_{x}+k_{y}(L+5 M) k_{y}+2 k_{z}(2 L+M) k_{z}\right\} \\
& +\frac{i}{6}\left\{k_{x}\left(F-G-H_{1}+H_{2}\right) k_{y}-k_{y}\left(F-G-H_{1}+H_{2}\right) k_{x}\right\}, \\
P^{\prime \prime \prime}= & \frac{1}{3}\left\{k_{x}(L+2 M) k_{x}+k_{y}(L+2 M) k_{y}+k_{z}(L+2 M) k_{z}\right\} \\
& +\frac{i}{3}\left\{k_{x}\left(F-G-H_{1}+H_{2}\right) k_{y}-k_{y}\left(F-G-H_{1}+H_{2}\right) k_{x}\right\}, \\
Q= & -\frac{1}{6}\left\{k_{x}(L-M) k_{x}+k_{y}(L-M) k_{y}-2 k_{z}(L-M) k_{z}\right. \\
& \left.+i\left[k_{x}\left(F-G-H_{1}+H_{2}\right) k_{y}-k_{y}\left(F-G-H_{1}+H_{2}\right) k_{x}\right]\right\}, \\
R= & \frac{1}{2 \sqrt{3}}\left\{k_{x}(L-M) k_{x}-k_{y}(L-M) k_{y}-i\left[k_{x} N k_{y}+k_{y} N k_{x}\right]\right\}, \\
S_{ \pm}= & -\frac{1}{\sqrt{3}}\left\{k_{ \pm}(F-G) k_{z}+k_{z}\left(H_{1}-H_{2}\right) k_{ \pm}\right\} \\
C= & -\frac{1}{3}\left\{k_{z}\left(F-G-H_{1}+H_{2}\right) k_{-}-k_{-}\left(F-G-H_{1}+H_{2}\right) k_{z}\right\}, \\
\Sigma_{ \pm}= & -\frac{1}{3 \sqrt{3}}\left\{k_{ \pm}\left(F-G+2 H_{1}-2 H_{2}\right) k_{z}+k_{z}\left(2 F-2 G+H_{1}-H_{2}\right) k_{ \pm}\right\} .
\end{aligned}
$$

where $L, M, F, G, H_{1}$ and $H_{2}$ are mass parameters and $\Delta_{s o}$ is the spinorbit energy. This set of parameters can be re-expressed in terms of the well-known Luttinger parameters if necessary. 


\section{A.1.3 Six-band Burt-Foreman Hamiltonian in cylindrical co- ordinates}

The position-dependent Hamiltonian in cylindrical coordinates has been derived from A.2 in the present work, since the six-band version was not available in literature. In order to do this, all Cartesian differential operators are replaced by their cylindrical counterparts. Then, the axial approximation $\tilde{\gamma}=\frac{1}{2}\left(\gamma_{2}+\gamma_{3}\right)[77$, 78] is taken into account (only the $\mathrm{R}$ terms of A.2 are changed) and the resulting Hamiltonian reads

$$
H_{B F}^{Z B}\left(F_{z}\right)=\frac{\hbar^{2}}{2 m_{0}} M_{6 x 6},
$$

with

$$
\begin{aligned}
M_{11} & =\frac{\partial}{\partial \rho}\left(\gamma_{1}+\gamma_{2}\right) \frac{\partial}{\partial \rho}+\frac{\left(\gamma_{1}+\gamma_{2}\right)}{\rho} \frac{\partial}{\partial \rho}+\frac{\partial}{\partial z}\left(\gamma_{1}-2 \gamma_{2}\right) \frac{\partial}{\partial z}-\frac{\left(F_{z}-\frac{3}{2}\right)^{2}}{\rho^{2}}\left(\gamma_{1}+\gamma_{2}\right) \\
& +\frac{\left(F_{z}-\frac{3}{2}\right)}{2 \rho}\left[\frac{\partial}{\partial \rho}\left(c_{1}+c_{2}\right)-\left(c_{1}+c_{2}\right) \frac{\partial}{\partial \rho}\right], \\
M_{12} & =\frac{1}{\sqrt{3}}\left\{\frac{\partial}{\partial \rho} c_{1} \frac{\partial}{\partial z}-\frac{\partial}{\partial z} c_{2} \frac{\partial}{\partial \rho}+\frac{\left(F_{z}-\frac{1}{2}\right)}{\rho}\left[c_{1} \frac{\partial}{\partial z}-\frac{\partial}{\partial z} c_{2}\right]\right\}, \\
M_{13} & =-\sqrt{3}\left\{\frac{\partial}{\partial \rho} \tilde{\gamma} \frac{\partial}{\partial \rho}+\frac{\left(F_{z}+\frac{1}{2}\right)}{\rho} \frac{\partial}{\partial \rho} \tilde{\gamma}+\frac{\left(F_{z}-\frac{1}{2}\right)}{\rho} \tilde{\gamma} \frac{\partial}{\partial \rho}+\frac{\left(F_{z}-\frac{3}{2}\right)\left(F_{z}+\frac{1}{2}\right)}{\rho^{2}} \tilde{\gamma}\right\}, \\
M_{14} & =0, \\
M_{15} & =-\frac{1}{\sqrt{6}}\left\{\frac{\partial}{\partial \rho} c_{1} \frac{\partial}{\partial z}-\frac{\partial}{\partial z} c_{2} \frac{\partial}{\partial \rho}+\frac{\left(F_{z}-\frac{1}{2}\right)}{\rho}\left[c_{1} \frac{\partial}{\partial z}-\frac{\partial}{\partial z} c_{2}\right]\right\}, \\
M_{16} & =-\sqrt{6}\left\{\frac{\partial}{\partial \rho} \tilde{\gamma} \frac{\partial}{\partial \rho}+\frac{\left(F_{z}+\frac{1}{2}\right)}{\rho} \frac{\partial}{\partial \rho} \tilde{\gamma}+\frac{\left(F_{z}-\frac{1}{2}\right)}{\rho} \tilde{\gamma} \frac{\partial}{\partial \rho}+\frac{\left(F_{z}-\frac{3}{2}\right)\left(F_{z}+\frac{1}{2}\right)}{\rho^{2}} \tilde{\gamma}\right\}, \\
M_{21} & =\frac{1}{\sqrt{3}}\left\{\frac{\partial}{\partial z} c_{1} \frac{\partial}{\partial \rho}-\frac{\partial}{\partial \rho} c_{2} \frac{\partial}{\partial z}+\frac{\left(F_{z}-\frac{3}{2}\right)}{\rho}\left[c_{2} \frac{\partial}{\partial z}-\frac{\partial}{\partial z} c_{1}\right]\right\}, \\
M_{22} & =\frac{\partial}{\partial \rho}\left(\gamma_{1}-\gamma_{2}\right) \frac{\partial}{\partial \rho}+\frac{\left(\gamma_{1}-\gamma_{2}\right)}{\rho} \frac{\partial}{\partial \rho}+\frac{\partial}{\partial z}\left(\gamma_{1}+2 \gamma_{2}\right) \frac{\partial}{\partial z}-\frac{\left(F_{z}-\frac{1}{2}\right)^{2}}{\rho^{2}}\left(\gamma_{1}-\gamma_{2}\right) \\
& +\frac{\left(F_{z}-\frac{1}{2}\right)}{6 \rho}\left[\frac{\partial}{\partial \rho}\left(c_{1}+c_{2}\right)-\left(c_{1}+c_{2}\right) \frac{\partial}{\partial \rho}\right], \\
M_{23}= & \frac{1}{3}\left\{\frac{\partial}{\partial \rho}\left(c_{1}+c_{2}\right) \frac{\partial}{\partial z}-\frac{\partial}{\partial z}\left(c_{1}+c_{2}\right) \frac{\partial}{\partial \rho}\right. \\
& \left.\frac{\left(F_{z}+\frac{1}{2}\right)}{\rho}\left[\left(c_{1}+c_{2}\right) \frac{\partial}{\partial z}-\frac{\partial}{\partial z}\left(c_{1}+c_{2}\right)\right]\right\},
\end{aligned}
$$




$$
\begin{aligned}
& M_{24}=\sqrt{3}\left\{\frac{\partial}{\partial \rho} \tilde{\gamma} \frac{\partial}{\partial \rho}+\frac{\left(F_{z}+\frac{3}{2}\right)}{\rho} \frac{\partial}{\partial \rho} \tilde{\gamma}+\frac{\left(F_{z}+\frac{1}{2}\right)}{\rho} \tilde{\gamma} \frac{\partial}{\partial \rho}+\frac{\left(F_{z}+\frac{3}{2}\right)\left(F_{z}-\frac{1}{2}\right)}{\rho^{2}} \tilde{\gamma}\right\}, \\
& M_{25}=\sqrt{2}\left\{\frac{\partial}{\partial \rho} \gamma_{2} \frac{\partial}{\partial \rho}-2 \frac{\partial}{\partial z} \gamma_{2} \frac{\partial}{\partial z}+\frac{\gamma_{2}}{\rho} \frac{\partial}{\partial \rho}-\frac{\left(F_{z}-\frac{1}{2}\right)^{2}}{\rho^{2}} \gamma_{2}\right. \\
& \left.+\frac{\left(F_{z}-\frac{1}{2}\right)}{6 \rho}\left[\frac{\partial}{\partial \rho}\left(c_{1}+c_{2}\right)-\left(c_{1}+c_{2}\right) \frac{\partial}{\partial \rho}\right]\right\} \\
& M_{26}=-\frac{1}{3 \sqrt{2}}\left\{\frac{\partial}{\partial \rho}\left(c_{1}-2 c_{2}\right) \frac{\partial}{\partial z}+\frac{\partial}{\partial z}\left(2 c_{1}-c_{2}\right) \frac{\partial}{\partial \rho}\right. \\
& \left.+\frac{\left(F_{z}+\frac{1}{2}\right)}{\rho}\left[\left(c_{1}-2 c_{2}\right) \frac{\partial}{\partial z}+\frac{\partial}{\partial z}\left(2 c_{1}-c_{2}\right)\right]\right\}, \\
& M_{31}=-\sqrt{3}\left\{\frac{\partial}{\partial \rho} \tilde{\gamma} \frac{\partial}{\partial \rho}-\frac{\left(F_{z}-\frac{3}{2}\right)}{\rho} \frac{\partial}{\partial \rho} \tilde{\gamma}-\frac{\left(F_{z}-\frac{1}{2}\right)}{\rho} \tilde{\gamma} \frac{\partial}{\partial \rho}+\frac{\left(F_{z}-\frac{3}{2}\right)\left(F_{z}+\frac{1}{2}\right)}{\rho^{2}} \tilde{\gamma}\right\} \text {, } \\
& M_{32}=\frac{1}{3}\left\{\frac{\partial}{\partial z}\left(c_{1}+c_{2}\right) \frac{\partial}{\partial \rho}-\frac{\partial}{\partial \rho}\left(c_{1}+c_{2}\right) \frac{\partial}{\partial z}\right. \\
& \left.+\frac{\left(F_{z}-\frac{1}{2}\right)}{\rho}\left[\left(c_{1}+c_{2}\right) \frac{\partial}{\partial z}-\frac{\partial}{\partial z}\left(c_{1}+c_{2}\right)\right]\right\} \\
& M_{33}=\frac{\partial}{\partial \rho}\left(\gamma_{1}-\gamma_{2}\right) \frac{\partial}{\partial \rho}+\frac{\left(\gamma_{1}-\gamma_{2}\right)}{\rho} \frac{\partial}{\partial \rho}+\frac{\partial}{\partial z}\left(\gamma_{1}+2 \gamma_{2}\right) \frac{\partial}{\partial z} \\
& -\frac{\left(F_{z}+\frac{1}{2}\right)^{2}}{\rho^{2}}\left(\gamma_{1}-\gamma_{2}\right)-\frac{\left(F_{z}+\frac{1}{2}\right)}{6 \rho}\left[\frac{\partial}{\partial \rho}\left(c_{1}+c_{2}\right)-\left(c_{1}+c_{2}\right) \frac{\partial}{\partial \rho}\right], \\
& M_{34}=\frac{1}{\sqrt{3}}\left\{\frac{\partial}{\partial z} c_{1} \frac{\partial}{\partial \rho}-\frac{\partial}{\partial \rho} c_{2} \frac{\partial}{\partial z}-\frac{\left(F_{z}+\frac{3}{2}\right)}{\rho}\left[c_{2} \frac{\partial}{\partial z}-\frac{\partial}{\partial z} c_{1}\right]\right\}, \\
& M_{35}=\frac{1}{3 \sqrt{2}}\left\{\frac{\partial}{\partial \rho}\left(c_{1}-2 c_{2}\right) \frac{\partial}{\partial z}+\frac{\partial}{\partial z}\left(2 c_{1}-c_{2}\right) \frac{\partial}{\partial \rho}\right. \\
& \left.-\frac{\left(F_{z}-\frac{1}{2}\right)}{\rho}\left[\left(c_{1}-2 c_{2}\right) \frac{\partial}{\partial z}+\frac{\partial}{\partial z}\left(2 c_{1}-c_{2}\right)\right]\right\}, \\
& M_{36}=\sqrt{2}\left\{\frac{\partial}{\partial \rho} \gamma_{2} \frac{\partial}{\partial \rho}-2 \frac{\partial}{\partial z} \gamma_{2} \frac{\partial}{\partial z}+\frac{\gamma_{2}}{\rho} \frac{\partial}{\partial \rho}-\frac{\left(F_{z}+\frac{1}{2}\right)^{2}}{\rho^{2}} \gamma_{2}\right. \\
& \left.-\frac{\left(F_{z}+\frac{1}{2}\right)}{6 \rho}\left[\frac{\partial}{\partial \rho}\left(c_{1}+c_{2}\right)-\left(c_{1}+c_{2}\right) \frac{\partial}{\partial \rho}\right]\right\} \\
& M_{41}=0 \text {, } \\
& M_{42}=\sqrt{3}\left\{\frac{\partial}{\partial \rho} \tilde{\gamma} \frac{\partial}{\partial \rho}-\frac{\left(F_{z}-\frac{1}{2}\right)}{\rho} \frac{\partial}{\partial \rho} \tilde{\gamma}-\frac{\left(F_{z}+\frac{1}{2}\right)}{\rho} \tilde{\gamma} \frac{\partial}{\partial \rho}+\frac{\left(F_{z}+\frac{3}{2}\right)\left(F_{z}-\frac{1}{2}\right)}{\rho^{2}} \tilde{\gamma}\right\},
\end{aligned}
$$




$$
\begin{aligned}
& M_{43}=\frac{1}{\sqrt{3}}\left\{\frac{\partial}{\partial \rho} c_{1} \frac{\partial}{\partial z}-\frac{\partial}{\partial z} c_{2} \frac{\partial}{\partial \rho}-\frac{\left(F_{z}+\frac{1}{2}\right)}{\rho}\left[c_{1} \frac{\partial}{\partial z}-\frac{\partial}{\partial z} c_{2}\right]\right\}, \\
& M_{44}=\frac{\partial}{\partial \rho}\left(\gamma_{1}+\gamma_{2}\right) \frac{\partial}{\partial \rho}+\frac{\left(\gamma_{1}+\gamma_{2}\right)}{\rho} \frac{\partial}{\partial \rho}+\frac{\partial}{\partial z}\left(\gamma_{1}-2 \gamma_{2}\right) \frac{\partial}{\partial z}-\frac{\left(F_{z}+\frac{3}{2}\right)^{2}}{\rho^{2}}\left(\gamma_{1}+\gamma_{2}\right) \\
& -\frac{\left(F_{z}+\frac{3}{2}\right)}{2 \rho}\left[\frac{\partial}{\partial \rho}\left(c_{1}+c_{2}\right)-\left(c_{1}+c_{2}\right) \frac{\partial}{\partial \rho}\right] \\
& M_{45}=\sqrt{6}\left\{\frac{\partial}{\partial \rho} \tilde{\gamma} \frac{\partial}{\partial \rho}-\frac{\left(F_{z}-\frac{1}{2}\right)}{\rho} \frac{\partial}{\partial \rho} \tilde{\gamma}-\frac{\left(F_{z}+\frac{1}{2}\right)}{\rho} \tilde{\gamma} \frac{\partial}{\partial \rho}+\frac{\left(F_{z}+\frac{3}{2}\right)\left(F_{z}-\frac{1}{2}\right)}{\rho^{2}} \tilde{\gamma}\right\} \text {, } \\
& M_{46}=-\frac{1}{\sqrt{6}}\left\{\frac{\partial}{\partial \rho} c_{1} \frac{\partial}{\partial z}-\frac{\partial}{\partial z} c_{2} \frac{\partial}{\partial \rho}-\frac{\left(F_{z}+\frac{1}{2}\right)}{\rho}\left[c_{1} \frac{\partial}{\partial z}-\frac{\partial}{\partial z} c_{2}\right]\right\} \text {, } \\
& M_{51}=-\frac{1}{\sqrt{6}}\left\{\frac{\partial}{\partial z} c_{1} \frac{\partial}{\partial \rho}-\frac{\partial}{\partial \rho} c_{2} \frac{\partial}{\partial z}+\frac{\left(F_{z}-\frac{3}{2}\right)}{\rho}\left[c_{2} \frac{\partial}{\partial z}-\frac{\partial}{\partial z} c_{1}\right]\right\} \text {, } \\
& M_{52}=\sqrt{2}\left\{\frac{\partial}{\partial \rho} \gamma_{2} \frac{\partial}{\partial \rho}-2 \frac{\partial}{\partial z} \gamma_{2} \frac{\partial}{\partial z}+\frac{\gamma_{2}}{\rho} \frac{\partial}{\partial \rho}-\frac{\left(F_{z}-\frac{1}{2}\right)^{2}}{\rho^{2}} \gamma_{2}\right. \\
& \left.+\frac{\left(F_{z}-\frac{1}{2}\right)}{6 \rho}\left[\frac{\partial}{\partial \rho}\left(c_{1}+c_{2}\right)-\left(c_{1}+c_{2}\right) \frac{\partial}{\partial \rho}\right]\right\}, \\
& M_{53}=\frac{1}{3 \sqrt{2}}\left\{\frac{\partial}{\partial \rho}\left(2 c_{1}-c_{2}\right) \frac{\partial}{\partial z}+\frac{\partial}{\partial z}\left(c_{1}-2 c_{2}\right) \frac{\partial}{\partial \rho}\right. \\
& \left.+\frac{\left(F_{z}+\frac{1}{2}\right)}{\rho}\left[\left(2 c_{1}-c_{2}\right) \frac{\partial}{\partial z}+\frac{\partial}{\partial z}\left(c_{1}-2 c_{2}\right)\right]\right\} \\
& M_{54}=\sqrt{6}\left\{\frac{\partial}{\partial \rho} \tilde{\gamma} \frac{\partial}{\partial \rho}+\frac{\left(F_{z}+\frac{3}{2}\right)}{\rho} \frac{\partial}{\partial \rho} \tilde{\gamma}+\frac{\left(F_{z}+\frac{1}{2}\right)}{\rho} \tilde{\gamma} \frac{\partial}{\partial \rho}+\frac{\left(F_{z}+\frac{3}{2}\right)\left(F_{z}-\frac{1}{2}\right)}{\rho^{2}} \tilde{\gamma}\right\} \text {, } \\
& M_{55}=\frac{\partial}{\partial \rho} \gamma_{1} \frac{\partial}{\partial \rho}+\frac{\partial}{\partial z} \gamma_{1} \frac{\partial}{\partial z}+\frac{\gamma_{1}}{\rho} \frac{\partial}{\partial \rho}-\frac{\left(F_{z}-\frac{1}{2}\right)^{2}}{\rho^{2}} \gamma_{1} \\
& +\frac{\left(F_{z}-\frac{1}{2}\right)}{3 \rho}\left[\frac{\partial}{\partial \rho}\left(c_{1}+c_{2}\right)-\left(c_{1}+c_{2}\right) \frac{\partial}{\partial \rho}\right]-2 \Delta_{s o}, \\
& M_{56}=-\frac{1}{3}\left\{\frac{\partial}{\partial \rho}\left(c_{1}+c_{2}\right) \frac{\partial}{\partial z}-\frac{\partial}{\partial z}\left(c_{1}+c_{2}\right) \frac{\partial}{\partial \rho}\right. \\
& \left.+\frac{\left(F_{z}+\frac{1}{2}\right)}{\rho}\left[\left(c_{1}+c_{2}\right) \frac{\partial}{\partial z}-\frac{\partial}{\partial z}\left(c_{1}+c_{2}\right)\right]\right\} \\
& M_{61}=-\sqrt{6}\left\{\frac{\partial}{\partial \rho} \tilde{\gamma} \frac{\partial}{\partial \rho}-\frac{\left(F_{z}-\frac{3}{2}\right)}{\rho} \frac{\partial}{\partial \rho} \tilde{\gamma}-\frac{\left(F_{z}-\frac{1}{2}\right)}{\rho} \tilde{\gamma} \frac{\partial}{\partial \rho}\right. \\
& \left.+\frac{\left(F_{z}-\frac{3}{2}\right)\left(F_{z}+\frac{1}{2}\right)}{\rho^{2}} \tilde{\gamma}\right\},
\end{aligned}
$$




$$
\begin{aligned}
M_{62} & =-\frac{1}{3 \sqrt{2}}\left\{\frac{\partial}{\partial \rho}\left(2 c_{1}-c_{2}\right) \frac{\partial}{\partial z}+\frac{\partial}{\partial z}\left(c_{1}-2 c_{2}\right) \frac{\partial}{\partial \rho}\right. \\
& \left.-\frac{\left(F_{z}-\frac{1}{2}\right)}{\rho}\left[\left(2 c_{1}-c_{2}\right) \frac{\partial}{\partial z}+\frac{\partial}{\partial z}\left(c_{1}-2 c_{2}\right)\right]\right\}, \\
M_{63} & =\sqrt{2}\left\{\frac{\partial}{\partial \rho} \gamma_{2} \frac{\partial}{\partial \rho}-2 \frac{\partial}{\partial z} \gamma_{2} \frac{\partial}{\partial z}+\frac{\gamma_{2}}{\rho} \frac{\partial}{\partial \rho}-\frac{\left(F_{z}+\frac{1}{2}\right)^{2}}{\rho^{2}} \gamma_{2}\right. \\
& \left.-\frac{\left(F_{z}+\frac{1}{2}\right)}{6 \rho}\left[\frac{\partial}{\partial \rho}\left(c_{1}+c_{2}\right)-\left(c_{1}+c_{2}\right) \frac{\partial}{\partial \rho}\right]\right\}, \\
M_{64} & =-\frac{1}{\sqrt{6}}\left\{\frac{\partial}{\partial z} c_{1} \frac{\partial}{\partial \rho}-\frac{\partial}{\partial \rho} c_{2} \frac{\partial}{\partial z}-\frac{\left(F_{z}+\frac{3}{2}\right)}{\rho}\left[c_{2} \frac{\partial}{\partial z}-\frac{\partial}{\partial z} c_{1}\right]\right\}, \\
M_{65} & =-\frac{1}{3}\left\{\frac{\partial}{\partial z}\left(c_{1}+c_{2}\right) \frac{\partial}{\partial \rho}-\frac{\partial}{\partial \rho}\left(c_{1}+c_{2}\right) \frac{\partial}{\partial z}\right. \\
& \left.+\frac{\left(F_{z}-\frac{1}{2}\right)}{\rho}\left[\left(c_{1}+c_{2}\right) \frac{\partial}{\partial z}-\frac{\partial}{\partial z}\left(c_{1}+c_{2}\right)\right]\right\}, \\
M_{66} & =\frac{\partial}{\partial \rho} \gamma_{1} \frac{\partial}{\partial \rho}+\frac{\partial}{\partial z} \gamma_{1} \frac{\partial}{\partial z}+\frac{\gamma_{1}}{\rho} \frac{\partial}{\partial \rho}-\frac{\left(F_{z}+\frac{1}{2}\right)^{2}}{\rho^{2}} \gamma_{1} \\
& -\frac{\left(F_{z}+\frac{1}{2}\right)}{3 \rho}\left[\frac{\partial}{\partial \rho}\left(c_{1}+c_{2}\right)-\left(c_{1}+c_{2}\right) \frac{\partial}{\partial \rho}\right]-2 \Delta_{s o} .
\end{aligned}
$$

where $\gamma_{1}, \gamma_{2}$ and $\tilde{\gamma}$ are the Luttinger mass parameters, $\Delta_{s o}$ is the spin-orbit splitting and $F_{z}$ stands for the total angular momentum. 


\section{A.2 Wurtzite crystal structure}

The position-dependent Hamiltonian for the $\mathrm{VB}$ of WZ materials derived with Burt-Foreman operator ordering is: 45 .

$$
H_{W Z}=-\left[\begin{array}{cccccc}
\left|u_{1}\right\rangle & \left|u_{2}\right\rangle & \left|u_{3}\right\rangle & \left|u_{4}\right\rangle & \left|u_{5}\right\rangle & \left|u_{6}\right\rangle \\
F-\rho & \kappa & \xi^{*} & 0 & 0 & 0 \\
\kappa^{*} & G+\rho & -\xi & 0 & 0 & \sqrt{2} \Delta_{3} \\
\eta & -\eta^{*} & \lambda & 0 & \sqrt{2} \Delta_{3} & 0 \\
0 & 0 & 0 & F+\rho & \kappa^{*} & -\xi \\
0 & 0 & \sqrt{2} \Delta_{3} & \kappa & G-\rho & \xi^{*} \\
0 & \sqrt{2} \Delta_{3} & 0 & -\eta^{*} & \eta & \lambda
\end{array}\right] \text {, }
$$

where

$$
\begin{aligned}
F & =\Delta_{1}+\Delta_{2}+\lambda+\theta \\
G & =\Delta_{1}-\Delta_{2}+\lambda+\theta, \\
\lambda & =\frac{\hbar^{2}}{2 m_{0}}\left[k_{z} A_{1} k_{z}+k_{x} A_{2} k_{x}+k_{y} A_{2} k_{y}\right], \\
\theta & =\frac{\hbar^{2}}{2 m_{0}}\left[k_{z} A_{3} k_{z}+k_{x} A_{4} k_{x}+k_{y} A_{4} k_{y}\right], \\
\kappa & =\frac{\hbar^{2}}{2 m_{0}}\left[-k_{x} A_{5} k_{x}+k_{y} A_{5} k_{y}+i\left(k_{x} A_{5} k_{y}+k_{y} A_{5} k_{x}\right)\right], \\
\eta & =\frac{\hbar^{2}}{2 m_{0}}\left[-k_{z} A_{6}^{(+)} k_{+}-k_{+} A_{6}^{(-)} k_{z}\right], \\
\xi & =\frac{\hbar^{2}}{2 m_{0}}\left[-k_{z} A_{6}^{(-)} k_{+}-k_{+} A_{6}^{(+)} k_{z}\right], \\
\rho & =\frac{\hbar^{2}}{2 m_{0}}\left[i k_{y}\left(A_{5}^{(+)}-A_{5}^{(-)}\right) k_{x}-i k_{x}\left(A_{5}^{(+)}-A_{5}^{(-)}\right) k_{y}\right] .
\end{aligned}
$$

Here, $m_{0}$ is the free electron mass and $A_{i}$ are material mass parameters, with $A_{5}=A_{5}^{(+)}+A_{5}^{(-)}$and $A_{6}=A_{6}^{(+)}+A_{6}^{(-)}$. In addition, the crystal-field splitting is denoted by $\Delta_{1}=\Delta_{c r}$, and $\Delta_{2}$ and $\Delta_{3}$ are the spin-orbit terms. In the so-called cubic approximation we have $\Delta_{2}=\Delta_{3}=\Delta_{s o} / 3$.

It should be stressed that coefficients $A_{5}^{( \pm)}$and $A_{6}^{( \pm)}$are not generally available in literature. Here we take the complete asymmetric operator order, i.e. $A_{i}^{(+)}=A_{i}$ and $A_{i}^{(-)}=0$, following the criteria suggested by Veprek et al. [201, 202]. In their works it is shown that the emergence of spurious solutions within the $\mathrm{k} \cdot \mathrm{p}$ method comes from the non-ellipticity of the 
coupled differential equation system. After analyzing various sets of mass parameters for several materials, they found that taking $A_{i}^{(-)}=0$ grants the ellipticity of the Hamiltonian, thus avoiding non-physical solutions. We have reached the same result for the materials studied in this work.

\section{A.3 Polytypes}

\section{A.3.1 Constant-mass Hamiltonian}

The six-band Hamiltonian for studying the $\mathrm{VB}$ of $\mathrm{ZB} \mathrm{WZ}$ polytypes is as follows: 46

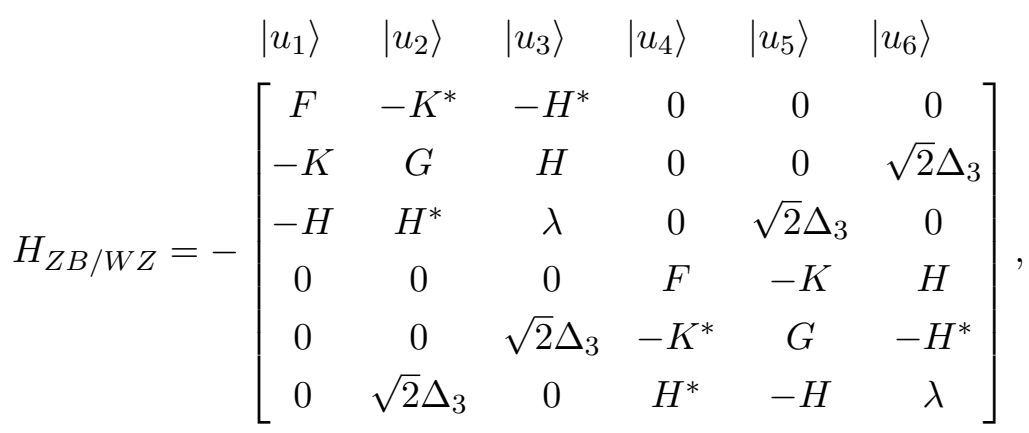

where

$$
\begin{aligned}
F & =\Delta_{1}+\Delta_{2}+\lambda+\theta, \\
G & =\Delta_{1}-\Delta_{2}+\lambda+\theta, \\
\lambda & =\frac{\hbar^{2}}{2 m_{0}}\left[A_{1} k_{z}^{2}+A_{2} k_{\perp}^{2}\right], \\
\theta & =\frac{\hbar^{2}}{2 m_{0}}\left[A_{3} k_{z}^{2}+A_{4} k_{\perp}^{2}\right], \\
K & =\frac{\hbar^{2}}{2 m_{0}} A_{5} k_{+}^{2}+\Delta K, \\
H & =\frac{\hbar^{2}}{2 m_{0}} A_{6} k_{+} k_{z}+\Delta H, \\
\Delta K & =2 \sqrt{2} \frac{\hbar^{2}}{2 m_{0}} A_{z} k_{-} k_{z}, \\
\Delta H & =\frac{\hbar^{2}}{2 m_{0}} A_{z} k_{-}^{2} .
\end{aligned}
$$

Here, $m_{0}$ is the free electron mass, $A_{i}$ are effective mass parameters, $k_{\perp}=$ $k_{x}^{2}+k_{y}^{2}, k_{ \pm}=k_{x} \pm i k_{y}, \Delta_{1}$ is the crystal field splitting, and $\Delta_{2}$ and $\Delta_{3}$ are spin-orbit matrix elements. 


\section{A.3.2 Variable mass Hamiltonian}

The position-dependent Burt-Foreman version of Hamiltonian A.5 reads:

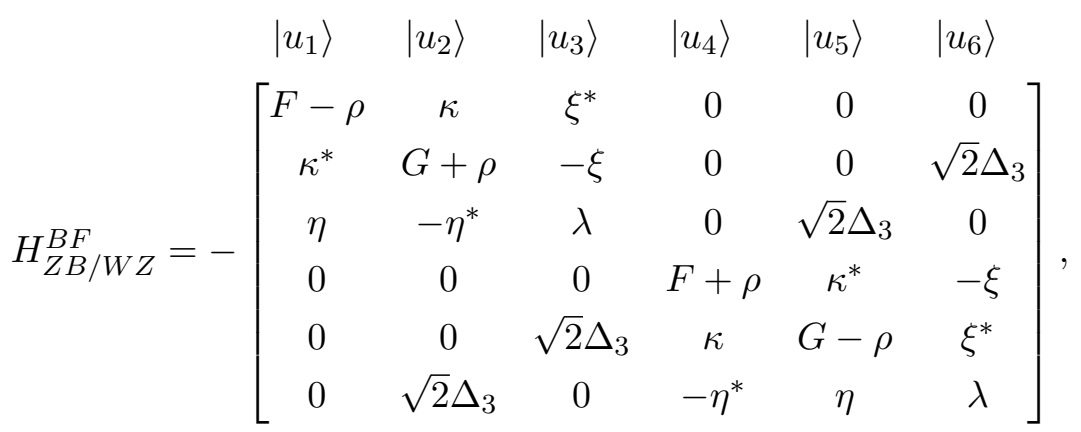

where

$$
\begin{aligned}
F & =\Delta_{1}+\Delta_{2}+\lambda+\theta, \\
G & =\Delta_{1}-\Delta_{2}+\lambda+\theta, \\
\lambda & =\frac{\hbar^{2}}{2 m_{0}}\left[k_{z} A_{1} k_{z}+k_{x} A_{2} k_{x}+k_{y} A_{2} k_{y}\right], \\
\theta & =\frac{\hbar^{2}}{2 m_{0}}\left[k_{z} A_{3} k_{z}+k_{x} A_{4} k_{x}+k_{y} A_{4} k_{y}\right], \\
\kappa & =\frac{\hbar^{2}}{2 m_{0}}\left[-k_{x} A_{5} k_{x}+k_{y} A_{5} k_{y}+i\left(k_{x} A_{5} k_{y}+k_{y} A_{5} k_{x}\right)\right]+\Delta \kappa, \\
\eta & =\frac{\hbar^{2}}{2 m_{0}}\left[-k_{z} A_{6}^{(+)} k_{+}-k_{+} A_{6}^{(-)} k_{z}\right]+\Delta \eta, \\
\xi & =\frac{\hbar^{2}}{2 m_{0}}\left[-k_{z} A_{6}^{(-)} k_{+}-k_{+} A_{6}^{(+)} k_{z}\right]+\Delta \xi, \\
\rho & =\frac{\hbar^{2}}{2 m_{0}}\left[i k_{y}\left(A_{5}^{(+)}-A_{5}^{(-)}\right) k_{x}-i k_{x}\left(A_{5}^{(+)}-A_{5}^{(-)}\right) k_{y}\right], \\
\Delta \xi & =\frac{\hbar^{2}}{2 m_{0}}\left[-\left(k_{x}-i k_{y}\right) A_{z}\left(k_{x}-i k_{y}\right)\right], \\
\Delta \eta & =\Delta \xi \\
\Delta \kappa & =-2 \sqrt{2} \frac{\hbar^{2}}{2 m_{0}}\left[\left(k_{x}+i k_{y}\right) A_{z}^{(+)} k_{z}+k_{z} A_{z}^{(-)}\left(k_{x}+i k_{y}\right)\right] .
\end{aligned}
$$

Here, $m_{0}$ is the free electron mass, $A_{i}$ are material mass parameters, $A_{5}=$ $A_{5}^{(+)}+A_{5}^{(-)}, A_{6}=A_{6}^{(+)}+A_{6}^{(-)}, \Delta_{1}=\Delta_{c r}$ the crystal-field splitting and $\Delta_{2}=\Delta_{3}=\Delta_{\text {so }} / 3$ the spin-orbit energy terms within the quasi-cubic approximation. 
Following the same reasoning as for the $\mathrm{WZ} / \mathrm{VB}$ Hamiltonian in section A.2. we also take complete asymmetric operator ordering $\left(A_{i}^{(+)}=A_{i}\right.$ and $\left.A_{i}^{(-)}=0\right)$ in order to ensure equation system ellipticity. [201, 202], 
APPENDIX $\mathbf{B}$

\section{Spin-orbit Hamiltonians in matrix form}

This appendix collects the explicit matrix form of the SOI Hamiltonians introduced in section 2.3 .

\section{B.1 Dresselhaus SOI}

\section{B.1.1 Conduction band DSOI Hamiltonian}

The DSOI Hamiltonian accounting for the spin-up and spin-down bands of the $\mathrm{CB}$ is:

$H_{B I A}^{C B}=b_{41}^{C B}\left(\begin{array}{cc}\frac{1}{2}\left\{k_{+}^{2}+k_{-}^{2}, k_{z}\right\} & \frac{1}{4}\left\{k_{+}^{2}-k_{-}^{2}, k_{-}\right\}-\left\{k_{z}^{2}, k_{+}\right\} \\ \frac{1}{4}\left\{k_{-}^{2}-k_{+}^{2}, k_{+}\right\}-\left\{k_{z}^{2}, k_{-}\right\} & -\frac{1}{2}\left\{k_{+}^{2}+k_{-}^{2}, k_{z}\right\}\end{array}\right)$,

where $k_{ \pm}=k_{x} \pm i k_{y}$. 


\section{B.1.2 Valence band DSOI Hamiltonian}

Matrix form of the four-band DSOI Hamiltonian in Cartesian coordinates:

$$
H_{B I A}^{V B}=H_{C_{k}}+H_{b_{41}}+H_{b_{42}}+H_{b_{51}}+H_{b_{52}}
$$

where:

$$
H_{C_{k}}=C_{k}\left(\begin{array}{cccc}
0 & -\frac{k_{-}}{2} & k_{z} & -\frac{\sqrt{3} k_{-}}{2} \\
-\frac{k_{+}}{2} & 0 & \frac{\sqrt{3} k_{+}}{2} & -k_{z} \\
k_{z} & \frac{\sqrt{3} k_{-}}{2} & 0 & -\frac{k_{-}}{2} \\
-\frac{\sqrt{3} k_{+}}{2} & -k_{z} & -\frac{k_{+}}{2} & 0
\end{array}\right)
$$

with $k_{ \pm}=k_{x} \pm i k_{y}$

$$
H_{b_{41}}=b_{41}^{V B}\left(\begin{array}{cccc}
\frac{3}{2} P_{41} & \frac{\sqrt{3}}{2} L_{41} & 0 & 0 \\
\frac{\sqrt{3}}{2} L_{41}^{\dagger} & \frac{1}{2} P_{41} & L_{41} & 0 \\
0 & L_{41}^{\dagger} & -\frac{1}{2} P_{41} & \frac{\sqrt{3}}{2} L_{41} \\
0 & 0 & \frac{\sqrt{3}}{2} L_{41}^{\dagger} & -\frac{3}{2} P_{41}
\end{array}\right)
$$

where $P_{41}=\left(k_{x}^{2}-k_{y}^{2}\right) k_{z}$ and $L_{41}=i k_{-} k_{x} k_{y}-k_{+} k_{z}^{2}$.

$$
H_{b_{42}}=b_{42}\left(\begin{array}{cccc}
\frac{27}{8} P_{41} & \frac{7 \sqrt{3}}{8} L_{41} & 0 & -\frac{3}{4} L_{42} \\
\frac{7 \sqrt{3}}{8} L_{41}^{\dagger} & \frac{1}{8} P_{41} & \frac{5}{2} L_{41} & 0 \\
0 & \frac{5}{2} L_{41}^{\dagger} & -\frac{1}{8} P_{41} & \frac{7 \sqrt{3}}{8} L_{41} \\
\frac{3}{4} L_{42}^{\dagger} & 0 & \frac{7 \sqrt{3}}{8} L_{41}^{\dagger} & -\frac{27}{8} P_{41}
\end{array}\right),
$$

where $L_{42}=i k_{+} k_{x} k_{y}+k_{-} k_{z}^{2}$.

$$
H_{b_{51}}=b_{51}\left(\begin{array}{cccc}
0 & -\frac{\sqrt{3}}{4} K_{+} & \frac{\sqrt{3}}{2} K_{z} & -\frac{3}{4} K_{-} \\
-\frac{\sqrt{3}}{4} K_{-} & 0 & \frac{3}{4} K_{+} & -\frac{\sqrt{3}}{2} K_{z} \\
\frac{\sqrt{3}}{2} K_{z} & \frac{3}{4} K_{-} & 0 & -\frac{\sqrt{3}}{4} K_{+} \\
-\frac{3}{4} K_{+} & -\frac{\sqrt{3}}{2} K_{z} & -\frac{\sqrt{3}}{4} K_{-} & 0
\end{array}\right),
$$

where $K_{+}=K_{x}+i K_{y}, K_{-}=K_{x}-i K_{y}, K_{x}=k_{x}\left(k_{y}^{2}+k_{z}^{2}\right), K_{y}=k_{y}\left(k_{x}^{2}+\right.$ $\left.k_{z}^{2}\right)$, and $K_{z}=k_{z}\left(k_{x}^{2}+k_{y}^{2}\right)$.

$$
H_{b_{52}}=b_{52}\left(\begin{array}{cccc}
0 & -\frac{\sqrt{3}}{4} M_{+} & \frac{\sqrt{3}}{2} k_{z}^{3} & -\frac{3}{4} M_{-} \\
-\frac{\sqrt{3}}{4} M_{-} & 0 & \frac{3}{4} M_{+} & -\frac{\sqrt{3}}{2} k_{z}^{3} \\
\frac{\sqrt{3}}{2} k_{z}^{3} & \frac{3}{4} M_{-} & 0 & -\frac{\sqrt{3}}{4} M_{+} \\
-\frac{3}{4} M_{+} & -\frac{\sqrt{3}}{2} k_{z}^{3} & -\frac{\sqrt{3}}{4} M_{-} & 0
\end{array}\right),
$$

where $M_{+}=k_{x}^{3}+i k_{y}^{3}$ and $M_{-}=k_{x}^{3}-i k_{y}^{3}$. 


\section{B.2 Rashba SOI}

\section{B.2.1 Conduction band RSOI Hamiltonian}

The electron RSOI Hamiltonian reads:

$$
H_{S I A}^{C B}=r_{41}\left(\begin{array}{cc}
-F_{x} k_{y}+F_{y} k_{x} & -\left(i F_{x}+F_{y}\right) k_{z}+i F_{z} k_{-} \\
\left(i F_{x}+F_{y}\right) k_{z}-i F_{z} k_{+} & F_{x} k_{y}-F_{y} k_{x}
\end{array}\right),
$$

where $F_{i}$ are the components of the external electric field $\mathbf{F}$ and $k_{ \pm}=$ $k_{x} \pm i k_{y}$. 



\section{APPENDIX \\ Strain Hamiltonians}

The implementation of strain into the $\mathrm{k} \cdot \mathrm{p}$ models is carried out following Bir and Pikus [32. In this appendix we present the strain Hamiltonians for the crystal structures studied throughout the Thesis.

\section{C.1 Zinc-blende QDs}

\section{C.1.1 Conduction band}

The one-band electron Hamiltonian of equation (2.14 has to be supplemented with

$$
H_{\epsilon}^{Z B}=a_{c}\left(\epsilon_{x x}+\epsilon_{y y}+\epsilon_{z z}\right),
$$

with $a_{c}$ standing for the $\mathrm{CB}$ deformation potential.

\section{C.1.2 Valence band}

The four-band VB strain Hamiltonian is derived from Hamiltonian A.1 after performing the following substitutions:

$$
\begin{aligned}
\frac{\hbar^{2}}{2 m_{0}} \gamma_{1} & \rightarrow-a_{v}, \\
\frac{\hbar^{2}}{2 m_{0}} \gamma_{2} & \rightarrow-\frac{b}{2}, \\
\frac{\hbar^{2}}{2 m_{0}} \gamma_{3} & \rightarrow-\frac{d}{2 \sqrt{3}} .
\end{aligned}
$$


Here, $a_{v}$ is the hydrostatic $\mathrm{VB}$ deformation potential, and $b$ and $d$ are shear $\mathrm{VB}$ deformation potentials. The strain Hamiltonian is as follows:

$$
H_{\epsilon}^{Z B}=-\left(\begin{array}{cccc}
\left|\frac{3}{2} \frac{+3}{2}\right\rangle & \left|\frac{3}{2} \frac{+1}{2}\right\rangle & \left|\frac{3}{2} \frac{-1}{2}\right\rangle & \left|\frac{3}{2} \frac{-3}{2}\right\rangle \\
P_{\epsilon}+Q_{\epsilon} & -S_{\epsilon} & R_{\epsilon} & 0 \\
-S_{\epsilon}^{\dagger} & P_{\epsilon}-Q_{\epsilon} & 0 & R_{\epsilon} \\
R_{\epsilon}^{\dagger} & 0 & P_{\epsilon}-Q_{\epsilon} & S_{\epsilon} \\
0 & R_{\epsilon}^{\dagger} & S_{\epsilon}^{\dagger} & P_{\epsilon}+Q_{\epsilon}
\end{array}\right),
$$

with

$$
\begin{aligned}
P_{\epsilon} & =-a_{v}\left(\epsilon_{x x}+\epsilon_{y y}+\epsilon_{z z}\right), \\
Q_{\epsilon} & =-\frac{b}{2}\left(\epsilon_{x x}+\epsilon_{y y}-2 \epsilon_{z z}\right), \\
R_{\epsilon} & =\frac{\sqrt{3}}{2} b\left(\epsilon_{x x}-\epsilon_{y y}\right)-i d \epsilon_{x y} \\
S_{\epsilon} & =-d\left(\epsilon_{z x}-i \epsilon_{y z}\right) .
\end{aligned}
$$

\section{C.2 Wurtzite QDs}

\section{C.2.1 Conduction band}

WZ crystals are hexagonal, so they have different lattice parameters in the axial and in-plane directions. Therefore, the strain is clearly anisotropic and this is also reflected in the strain Hamiltonian

$$
H_{\epsilon}^{W Z}=a_{c}^{\perp}\left(\epsilon_{x x}+\epsilon_{y y}\right)+a_{c}^{z} \epsilon_{z z},
$$

where $a_{c}^{\perp}$ and $a_{c}^{z}$ are the $\mathrm{CB}$ deformation potentials for the in-plane and growth directions, respectively. 


\section{C.2.2 Valence band}

The hole Hamiltonian for strained WZ systems is obtained by substituting the effective mass parameters $\frac{\hbar^{2}}{2 m_{0}} A_{i}$ by the deformation potentials $D_{i}$ in equation (A.4). The resulting strain Hamiltonian reads:

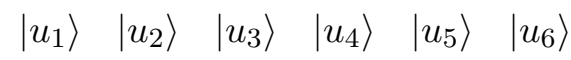

$$
\begin{aligned}
& H_{\epsilon}^{W Z}=\left[\begin{array}{cccccc}
F_{\epsilon} & \kappa_{\epsilon} & \xi_{\epsilon}^{*} & 0 & 0 & 0 \\
\kappa_{\epsilon}^{*} & F_{\epsilon} & -\xi_{\epsilon} & 0 & 0 & 0 \\
\xi_{\epsilon} & -\xi_{\epsilon}^{*} & \lambda_{\epsilon} & 0 & 0 & 0 \\
0 & 0 & 0 & F_{\epsilon} & \kappa_{\epsilon}^{*} & -\xi_{\epsilon} \\
0 & 0 & 0 & \kappa_{\epsilon} & F_{\epsilon} & \xi_{\epsilon}^{*} \\
0 & 0 & 0 & -\xi_{\epsilon}^{*} & \xi_{\epsilon} & \lambda_{\epsilon}
\end{array}\right] \text {, }
\end{aligned}
$$

where

$$
\begin{aligned}
F_{\epsilon} & =\left(D_{1}+D_{3}\right) \epsilon_{z z}+\left(D_{2}+D_{4}\right)\left(\epsilon_{x x}+\epsilon_{y y}\right), \\
\lambda_{\epsilon} & =D_{1} \epsilon_{z z}+D_{2}\left(\epsilon_{x x}+\epsilon_{y y}\right), \\
\kappa_{\epsilon} & =D_{5}\left(-\epsilon_{x x}+\epsilon_{y y}+2 i \epsilon_{x y}\right), \\
\xi_{\epsilon} & =-D_{6}\left(\epsilon_{x z}+i \epsilon_{y z}\right) .
\end{aligned}
$$





\section{Carrier-phonon interaction Hamiltonians}

This appendix outlines the derivation and contains complete expressions for the deformation-potential and piezoelectric Hamiltonians, equation (4.1), in terms of the phonon normal modes of vibration. Only $\mathrm{ZB}$ semiconductors are considered since spin scattering phenomena in $\mathrm{WZ}$ are not investigated in the present Thesis.

The origin of these interaction potentials lies in the displacement of lattice atoms from their equilibrium positions, producing strain and this strain yielding piezoelectricity. Therefore, we can use the expressions introduced for the strain and piezoelectricity in section 2.4.1, which depend on the strain tensor components $\epsilon_{i j}$, and then relate them to the normal modes of vibration.

First, we write the displacement of crystal atoms $\mathbf{u}_{\lambda}(\mathbf{r})$ in terms of the phonon creation and annihilation operators, $a_{\mathbf{q}}^{\dagger}$ and $a_{\mathbf{q}}$, respectively: 203.

$$
\mathbf{u}_{\lambda}(\mathbf{r})=\sum_{\mathbf{q}} \eta_{\lambda}(\mathbf{q}) \sqrt{\frac{\hbar}{2 M N \omega_{q \lambda}}}\left(a_{\mathbf{q}} e^{i \mathbf{q r}}+a_{\mathbf{q}}^{\dagger} e^{-i \mathbf{q r}}\right),
$$

where $M$ and $N$ are the mass and number of atoms in the crystal, $\mathbf{q}$ is the wave vector, and $\lambda$ indicates the phonon branch: longitudinal $(\lambda=l)$ or transversal $\left(\lambda=t_{1}, t_{2}\right) \cdot \eta_{\lambda}(\mathbf{q})$ stands for the polarization vector. Here we use: 118

$$
\eta_{l}(\mathbf{q})=\frac{1}{q}\left(\begin{array}{c}
q_{x} \\
q_{y} \\
q_{z}
\end{array}\right), \quad \eta_{t_{1}}(\mathbf{q})=\frac{1}{q q_{\perp}}\left(\begin{array}{c}
q_{x} q_{z} \\
q_{y} q_{z} \\
-q_{\perp}^{2}
\end{array}\right), \quad \eta_{t_{2}}(\mathbf{q})=\frac{1}{q_{\perp}}\left(\begin{array}{c}
q_{y} \\
-q_{x} \\
0
\end{array}\right),
$$


with $q_{\perp}=\sqrt{q_{x}^{2}+q_{y}^{2}}$.

Taking into account equation (D.2) and the definition of the strain tensor in terms of displacements, equation (2.25), after some algebra one obtains:

$$
\epsilon_{i j}^{\lambda}=-\frac{i}{2} \sum_{\mathbf{q}} U^{\lambda}(q)\left(\eta_{\lambda}^{i}(\mathbf{q}) q_{j}+\eta_{\lambda}^{j}(\mathbf{q}) q_{i}\right) F(\mathbf{q}, \mathbf{r}),
$$

where $F(\mathbf{q}, \mathbf{r})=a_{\mathbf{q}}^{\dagger} e^{-i \mathbf{q r}}$ and $U^{\lambda}(q)=\sqrt{\hbar /\left(2 \rho V \omega_{q \lambda}\right)}$. Here, the annihilation operator term has been dropped since we have assumed zero temperature, i.e. no emission processes, and $M N=\rho V$, with $\rho$ and $V$ standing for the crystal density and volume.

Equation (D.3) is the general form of the strain components. The specific expressions for the six independent strain components and three phonon branches can be easily obtained from the general one. Thus, we show here the case of $\epsilon_{x x}^{l}$ as an example and omit the others for brevity. After substituting the polarization vector $\eta_{l}(\mathbf{q})$ given in equation (D.2) into (D.3), one obtains:

$$
\epsilon_{x x}^{l}=-i \sum_{\mathbf{q}} U^{\lambda}(q)\left(\frac{q_{x}^{2}}{q}\right) F(\mathbf{q}, \mathbf{r}) .
$$

Following a similar procedure one can calculate the remaining strain components, which will be further substituted in the strain and piezoelectric Hamiltonians as shown below.

\section{D.1 Piezoelectric potential}

The piezoelectric potential is given by:[204]

$$
\phi_{p z}^{\lambda}=-\sum_{\mathbf{q}} \frac{4 \pi i}{\varepsilon_{r} q^{2}} h_{14}\left(q_{x} \epsilon_{y z}^{\lambda}+q_{y} \epsilon_{x z}^{\lambda}+q_{z} \epsilon_{x y}^{\lambda}\right) .
$$

where $\varepsilon_{r}$ is the relative dielectric constant and $h_{14}$ is the piezoelectric constant. The corresponding expressions for the different phonon branches are

$$
\begin{aligned}
\phi_{p z}^{l} & =-\frac{12 \pi h_{14}}{\epsilon_{r}} U^{l}(q) \sum_{\mathbf{q}} \frac{q_{x} q_{y} q_{z}}{q^{3}} F(\mathbf{q}, \mathbf{r}), \\
\phi_{p z}^{t_{1}} & =-\frac{4 \pi h_{14}}{\epsilon_{r}} U^{t}(q) \sum_{\mathbf{q}} \frac{q_{x} q_{y}\left(2 q_{z}^{2}-q_{\perp}^{2}\right)}{q^{3} q_{\perp}} F(\mathbf{q}, \mathbf{r}),
\end{aligned}
$$




$$
\phi_{p z}^{t_{2}}=-\frac{4 \pi h_{14}}{\epsilon_{r}} U^{t}(q) \sum_{\mathbf{q}} \frac{q_{z}\left(q_{y}^{2}-q_{x}^{2}\right)}{q^{2} q_{\perp}} F(\mathbf{q}, \mathbf{r}),
$$

It is worth noting that $\phi_{p z}$ is a potential diagonal term and the expressions in (D.6) are valid for both electrons and holes.

\section{D.2 Deformation potential}

The carrier-phonon interaction Hamiltonian for the deformation-potential relaxation mechanism, $H_{d p}^{\lambda}$, is derived by simply substituting the strain components calculated above into the corresponding strain Hamiltonians presented in appendix C.

\section{D.2.1 Conduction band}

For electrons, only longitudinal phonon modes contribute to the deformationpotential scattering. After carrying out the aforementioned substitution into equation C.1 one gets

$$
H_{d p}^{l}=-i a_{c} U^{l}(q) \sum_{\mathbf{q}} q F(\mathbf{q}, \mathbf{r})
$$

with $a_{c}$ denoting the $\mathrm{CB}$ deformation potential.

\section{D.2.2 Valence band}

The deformation potential term is given by the four-band Bir-Pikus strain Hamiltonian, equation (C.2). The strain operators for the three branches become

$$
\begin{aligned}
& p^{l}=i a_{v} U^{l}(q) \sum_{\mathbf{q}} q F(\mathbf{q}, \mathbf{r}), \\
& q^{l}=i \frac{b}{2} U^{l}(q) \sum_{\mathbf{q}}\left(q-3 \frac{q_{z}^{2}}{q}\right) F(\mathbf{q}, \mathbf{r}), \\
& r^{l}=-i U^{l}(q) \sum_{\mathbf{q}}\left(\frac{\sqrt{3}}{2} b \frac{q_{x}^{2}-q_{y}^{2}}{q}-i d \frac{q_{x} q_{y}}{q}\right) F(\mathbf{q}, \mathbf{r}), \\
& s^{l}=i d U^{l}(q) \sum_{\mathbf{q}} \frac{q_{z}\left(q_{x}-i q_{y}\right)}{q} F(\mathbf{q}, \mathbf{r}),
\end{aligned}
$$


for longitudinal phonons,

$$
\begin{aligned}
p^{t_{1}} & =0 \\
q^{t_{1}} & =i \frac{b}{2} U^{t}(q) \sum_{\mathbf{q}}\left(\frac{3 q_{z} q_{\perp}}{q}\right) F(\mathbf{q}, \mathbf{r}), \\
r^{t_{1}} & =-i U^{t}(q) \sum_{\mathbf{q}}\left(\frac{\sqrt{3}}{2} b \frac{q_{z}\left(q_{x}^{2}-q_{y}^{2}\right)}{q q_{\perp}}-i d \frac{q_{x} q_{y} q_{z}}{q q_{\perp}}\right) F(\mathbf{q}, \mathbf{r}), \\
s^{t_{1}} & =i \frac{d}{2} U^{t}(q) \sum_{\mathbf{q}} \frac{\left(q_{z}^{2}-q_{\perp}^{2}\right)\left(q_{x}-i q_{y}\right)}{q_{\perp} q} F(\mathbf{q}, \mathbf{r}),
\end{aligned}
$$

for transversal $t_{1}$ phonons, and

$$
\begin{aligned}
p^{t_{2}} & =0 \\
q^{t_{2}} & =0 \\
r^{t_{2}} & =-i U^{t}(q) \sum_{\mathbf{q}}\left(\sqrt{3} b \frac{q_{x} q_{y}}{q_{\perp}}-i \frac{d}{2} \frac{q_{y}^{2}-q_{x}^{2}}{q_{\perp}}\right) F(\mathbf{q}, \mathbf{r}), \\
s^{t_{2}} & =-\frac{d}{2} U^{t}(q) \sum_{\mathbf{q}} \frac{q_{z}\left(q_{x}-i q_{y}\right)}{q_{\perp}} F(\mathbf{q}, \mathbf{r}),
\end{aligned}
$$

for transversal $t_{2}$ phonons. Parameters $a_{v}, b$ and $d$ stand for the VB deformation potential. 


\section{Resum}

El treball presentat en aquesta Tesi doctoral s'emmarca dintre del camp de la nanotecnologia, és a dir, estudia les propietats de sistemes amb grandàries en escala nanomètrica. Aquesta escala propicia l'aparició de fenòmens quàntics perquè quan els portadors de càrrega, tant electrons com forats, es troben confinats en un espai de dimensions del mateix ordre o inferior a la seua longitud d'ona de de Broglie, es comporten seguint les lleis de la mecànica quàntica, de manera que una descripció clàssica deixa de ser vàlida. Aquest fet dóna lloc a una sèrie de propietats no habituals en sistems tradicionals que fan que aquestes nanoestructures siguen especialment prometedores per al desenvolupament de nombroses aplicacions tecnològiques en camps tan diversos com medicina, electrònica, cèllules solars, computació, etc. [1, 4, 8]

Entre la gran diversitat de sistemes nanoscòpics, nosaltres ens centrem principalment en nanoestructures semiconductores de baixa dimensionalitat, concretament en aquelles que confinen els portadors de càrrega en les tres direccions de l'espai, anomenades normalment punts quàntics o quantum dots (QDs). Aquestes estructures es caracteritzen per presentar un espectre d'energia discret paregut al dels àtoms, pel que de vegades se les coneix també com àtoms artificials. 2] A més, els punts quàntics presenten l'avantatge de poder ser sintetitzats en una gran varietat de formes, grandàries, materials i de ser poblats de forma controlada amb un nombre de portadors determinat. [10, 11] Aquesta gran flexibilitat permet el disseny de punts quàntics molt diversos, podent decidir en cada cas quin sistema és el més adient per a la finalitat que ha de complir.

Els mètodes de fabricació més importants són bàsicament tres: tècniques litogràfiques, [13, 14] creixement auto-ordenat [16, 17] i tècniques de química humida. 18] Cadascun d'ells proporciona punts quàntics amb unes característiques diferents. Els més estudiats en aquesta memòria són els obtinguts a partir de les dues darreres tècniques per motiu de la seua tridimensionalitat. Els punts quàntics auto-ordenats solen presentar forma piramidal, de 
piràmide truncada, lents planes o anells, amb alçades de l'ordre de $25 \mathrm{~nm}$ i bases de $20 \mathrm{~nm}$ d'amplada. Pel que fa als nanocristalls colloïdals fabricats per via humida, solen ser pràcticament esfèrics amb radis menuts (d'aproximadament 1.2-10 nm). Quant als materials emprats, els punts quàntics típics estan compostos per semiconductors binaris amb estructura cristallina zinc-blenda (ZB) o wurtzita (WZ), però recentment també s'ha aconseguit sintetitzar punts quàntics politípics, en els que coexisteixen ambdues fases cristallines d'un mateix material. A més a més, en els darrers anys també ha sigut possible obtenir punts quàntics a partir de materials purament bidimensionals, com per exemple grafé o d'altres més recents com ara el $\mathrm{MoS}_{2}$ monocapa.

Per tal de poder implementar satisfactòriament els punts quàntics en dispositius tecnològics que algun dia arriben a comercialitzar-se, és fonamental entendre en profunditat les seues propietats, tant des d'un punt de vista teòric com experimental. A aquest respecte, cal tindre present que no es tracta de sistemes aïllats del seu voltant i que la interacció amb el medi exterior pot provocar canvis substàncials en el seu comportament. Per aquest motiu, un dels principals objectius d'aquesta Tesi és investigar l'efecte que el medi que envolta als punts quàntics té sobre la seua estructura electrònica. En particular, estudiem la influència de les tensions que sorgeixen en la interfase entre dos materials com a conseqüència de tenir constants de xarxa diferents, així com dels camps piezoelèctrics derivats d'aquestes forces de tensió. Aquests dos factors poden donar lloc a canvis en l'estructura electrònica, separació d'electrons i forats en excitons, i són també els principals mecanismes de relaxació d'espín. D'altra banda, tant important com entendre les propietats electròniques i òptiques dels punts quàntics, ho és també disposar dels mitjans per a manipular-les externament de forma reversible i així poder controlar fàcilment la seua resposta. Açò sol fer-se mitjançant camps elèctrics i magnètics externament aplicats, de forma que esdevé de vital importància conèixer com afecten aquests a l'estructura electrònica i a la resposta dels punts quàntics.

L'objectiu principal d'aquesta Tesi és estudiar teòricament l'estructura electrònica de punts quàntics semiconductors sota la influència de camps externament aplicats i del medi que els envolta. La metodologia emprada és bàsicament el mètode $\mathrm{k} \cdot \mathrm{p}$ en el marc de les aproximacions de massa efectiva (EMA) i funció envolupant (EFA) 1 El mètode $\mathrm{k} \cdot \mathrm{p}$ és un model continu basat en la teoria de pertorbacions que té en compte les simetries dels cristalls per descriure l'estructura electrònica en funció d'un reduït

\footnotetext{
${ }^{1}$ Altres formalismes s'han utilitzat en l'estudi de sistemes correlacionats però només de forma puntual.
} 
nombre de paràmetres empírics, que es determinen experimentalment o a partir de càlculs ab initio. Malgrat la seua senzillesa, aquest model permet estimar satisfactòriament les propietats d'electrons i forats amb una exigència computacional raonable. Adicionalment, el mètode $\mathrm{k} \cdot \mathrm{p}$ permet estudiar amb relativa facilitat camps magnètics i elèctrics aplicats externament, la interacció espín-òrbita, les forces de tensió i deformació, i també la piezoelectricitat, que són els fenòmens analitzats en aquesta Tesi. Pel que fa als dos factors enumerats en darrer lloc, els camps que aquests originen i que després entren en els Hamiltonians, es calculen utilitzant la teoria contínua de l'elasticitat.

La metodologia emprada consisteix en la modelització teòrica de les propietats de les nanoestructures mitjançant el desenvolupament de codis computacionals fent ús dels programes Fortran i Matlab. Per a la integració numèrica dels Hamiltonians utilitzem els mètodes de diferències finites o elements finits. Adicionalment, també s'ha usat el programari comercial Comsol Multiphysics en alguns treballs, específicament en aquells que investiguen les forces de tensió i la piezoelectricitat.

En primer lloc, estudiem els efectes resultants de l'aplicació de camps magnètics externs en dos sistemes diferents. D'una banda, explorem l'estructura electrònica de la banda de valència de punts quàntics de GaN/AlN amb estructura cristallina zinc-blenda. Aquests materials tenen la banda de split-off (so) molt pròxima a les de forat pesat (hh) i lleuger (lh), de forma que és d'esperar que hi haja una interacció no rebutjable de les bandes esmentades, fet pel qual esdevé necessari l'ús d'un model de sis bandes. Com els punts considerats presenten simetria axial i els paràmetres màssics dels dos materials són prou diferents, construïm un Hamiltonià de massa variable en coordenades cilíndriques, de manera que el problema es redueix de tres a dues dimensions. Els resultats obtinguts ens indiquen que la puresa de espín de punts quàntics de GaN/AlN de dimensions típiques és extraordinàriament gran. Fins i tot major que la de punts quàntics de InGaAs/GaAs. Resultat aquest sorprenent atesa la massa efectiva més gran del GaN que origina una major densitats d'estats i la menor interacció espín-òrbita que implica una major proximitat de la banda de split-off. La justificació la trobem en el valor petit del paràmetre de mescla $\tilde{\gamma}$ que apareix en els termes extradiagonals de l'Hamiltonià. Un segon resultat remarcable és la possibilitat de creuament entre un estat fonamental hh $\mathrm{i}$ un excitat ih quan la proporció alçada/diàmetre és aproximadament la unitat, creuament originat per l'acció d'un camp magnètic extern axialment aplicat. Aquest fet significa que és possible controlar magnèticament les propietats òptiques, com ara la polarització d'emissió excitònica. 
Tot seguit, analitzem el paper d'un camp magnètic axialment aplicat en anells quàntics de forma hexagonal poblats amb un nombre reduït d'electrons. És ben conegut que l'acció d'un camp magnètic aplicat axialment sobre un sistema anular origina l'anomenat efecte Aharonov-Bohm. Aquest efecte, que és conseqüència de la topologia doblement connexa del sistema, dóna lloc a un espectre energètic que es repeteix periòdicament amb el flux magnètic. Aquest fenòmen ha sigut àmpliament estudiat en anells amb simetria circular però les conseqüències d'una reducció de simetria han estat poc o gens explorades. Realitzem una sèrie de càlculs canviant el nombre d'electrons que poblen l'anell des d'un fins a set i analitzem la forma dels espectres d'energia. El resultat més llamatiu és la completa supressió de les oscillacions típiques associades a l'efecte Aharonov-Bohm en el cas del sistema poblat amb sis electrons. Aquest fet és conseqüència directa de la simetria hexagonal dels anells i s'ha comprovat que només pot ocórrer en el cas de sis electrons, i sempre que el sistema estiga en un règim de baixa densitat electrònica. Estudiem també la influència d'un camp magnètic axial en nanofils hexagonals multi-capa, la secció dels quals origina un pou de potencial en forma d'anell hexagonal. Els resultats mostren senyals característiques de la simetria hexagonal, així com un patró típic d'oscillacions d'Aharonov-Bohm. A camps febles aquestes oscillacions poden desaparèixer o ressorgir en funció de la intensitat dels camps aplicats, independentment de la configuració dels electròdes en el dispositiu. Aquests resultats permeten entendre les observacions experimentals de treballs recents on es realitzen experiments de magneto-conductància en nanofils similars. Quan els camps són més forts sorgeixen diverses transicions en l'espectre d'energia que són induïdes pel camp magnètic. El seu origen són tant els canvis en la distribució electrònica com el buidatge o despoblament electrònic de bandes excitades. Des d'un punt de vista experimental, aquestes transicions haurien d'observar-se en mesures de magneto-conductància com a corbes amb forma d'escaló.

Realitzem un segon estudi on la topologia juga un paper clau: l'existència o no d'estats localitzats en la frontera física del sistema, amb energies situades en la zona prohibida (band gap), en el cas de nanocintes i punts quàntics fabricats a partir de monocapes de $\mathrm{MoS}_{2}$. Aquest material pertany a la familia dels dicalcogenurs de metalls de transició i en la seua forma purament bidimensional es comporta com un semiconductor de gap directe. En el cas de les nanocintes mostrem que aquestes estructures finites efectivament presenten estats espacialment localitzats a les vores o frontera física del sistema amb energies situades dintre de la banda prohibida. L'origen d'aquests estats localitzats s'ha pogut relacionar amb el caràcter topològic marginal de l'Hamiltonià del $\mathrm{MoS}_{2}$. Aquesta topologia està direc- 
tament determinada per la curvatura de les bandes de conducció i valència. Pel que fa a punts quàntics, el comportament observat és qualitativament el mateix però ara les bandes dels estats de vora es troben quantitzades, de forma que hi ha un nombre discret d'estats a la banda prohibida.

Investiguem també els processos de relaxació d'espín en punts quàntics. En particular, tenim en compte transicions entre els subnivells d'espín contrari en què es desdobla l'estat fonamental per l'acció de l'efecte Zeeman en presència d'un camp magnètic. Com la separació energètica entre els estats implicats és d'uns pocs meV, el principal mecanisme de relaxació és la interacció amb fonons acústics. Adicionalment, per a que la relaxació tinga lloc fa falta una font de mescla d'espín que permeta la transició. En els sistemes abordats ací, aquesta mescla ve originada per la interacció espín-òrbita. Tradicionalment, els treballs que han estudiat els efectes de la interacció espín-òrbita en la literatura han considerat models bidimensionals, els quals descriuen correctament punts quàntics electrostàtics. Tanmateix, en punts auto-ordenats i nanocristalls l'alçada de les estructures pot arribar a ser important i, per tant, s'espera que aquests models simplificats comencen a fallar. Per aquest motiu, nosaltres estudiem l'efecte de la tridimensionalitat en la relaxació d'espín, prestant especial atenció a la seua anisotropia. Primer, examinem com afecta la forma del confinament espacial a la relaxació d'espín fent ús d'un model de punts quàntics de forma esferoïdal. Trobem, tant en la banda de conducció com en la de valència, que la tridimensionalitat dels punts és rellevant, evidenciant que els models bidimensionals utilitzats fins ara no són suficient per estudiar aquests sistemes de forma rigorosa. A més, observem una gran anisotropia en la relaxació quan es canvia la forma dels punts com a conseqüència de la gran influència de la simetria del sistema en els Hamiltonians d'espín-òrbita, fet que determina el grau de mescla d'espín i, per tant, l'eficiència dels mecanismes de relaxació. Tot seguit, passem a explorar punts quàntics més realistes on la tridimensionalitat és a priori important, com per exemple punts quàntics piramidals o molècules de dos punts quàntics acoblats verticalment. En aquests casos observem també una gran anisotropia de la relaxació d'espín quan es rota l'orientació de camps aplicats externament, evidenciant que és possible maximitzar o minimitzar els processos de relaxació orientant els camps en la direcció adequada. Aquest comportament anisotropic és, en general, robust davant de canvis en la geometria i en la orientació cristallogràfica dels sistemes considerats. En particular, observem que, en molècules de punts quàntics, el temps de vida mitjana és màxim quan la funció d'ona forma estats moleculars homonuclears, però es redueix ràpidament en presència de qualsevol factor que minve la seua simetria. 
Seguidament analitzem els efectes que té el medi que envolta als punts quàntics sobre les seues propietats. En concret, ens centrem en les forces de tensió i la piezoelectricitat que s'originen en la diferència entre les constants de xarxa del material del punt i el de la matriu que l'envolta. Incloem també la polarització espontània quan és rellevant. Simulem el comportament d'electrons i forats formant excitons en dos sistemes amb estructura critallina diferent. Per un costat, en nanocristalls esfèrics de CdSe/CdS amb estructura wurtzita amb cor i capa exterior grans ${ }^{2}$ (dot-in-dot) sorgeixen forts camps piezoelèctrics dipolars en la direcció de creixement, mentre que la polarització espontània és menyspreable. Aquests forts camps de polarització produeixen una clara separació d'electrons i forats malgrat el potencial atractiu de Coulomb que actua en sentit contrari. La separació de les dues partícules origina que el solapament de les funcions d'ona siga feble i, per tant, els temps de vida mitjana de l'excitó llargs. Es troben resultats semblants quan estudiem altres geometries sempre que tant cor com capa externa siguen el suficientment grans. Les diverses estructures considerades s'obtenen elongant el cor, la capa exterior o tots dos al mateix temps, de manera que representen de forma aproximada sistemes anomenats en anglès dot-in-rod, dot-in-plate i rod-in-rod. Cal destacar que per obtenir excitons amb temps de vida mitjana llargs, cal que la capa exterior siga gran en la direcció axial, tal com podem esperar, però sorprenentment cal que siga també gran en la direcció lateral, ja que aquesta capa afecta a la magnitud global de les forces de tensió. Explorem també el paper d'aquests efectes en punts quàntics politípics de GaAs. Aquestes estructures estan formades per segments amb estructura critallina zinc-blenda en la direcció [111] que s'alternen amb altres que presenten estructura wurtzita [0001]. Degut a la gran similitud d'ambdues estructures cristallines i al fet d'estar formades pel mateix material, les forces de tensió i la piezoelectricitat són rebutjables. En canvi, la polarització espontània s'espera que siga molt important ja que en les interfases es passa d'una regió on la polarització espontània és zero a una altra on no ho és, cosa que pot originar forts camps de polarització en la direcció de creixement. En estudiar aquest fenòmen comprovem que efectivament la polarització espontània no és menyspreable i afecta notablement al comportament dels excitons. D'una banda, l'energia de l'excitó és molt sensible a canvis en les dimensions dels punts quàntics. D'altra banda, controlant el confinament lateral es pot induir una transició gradual entre excitons directes i indirectes, fet que influeix en gran mesura a la separació d'electrons i forats i, per extensió, a les propietats òptiques dels punts politípics.

\footnotetext{
${ }^{2}$ Utilitzem "cor" i "capa exterior" per referir-nos a core i external shell.
} 
Tots els continguts presentats en la present Tesi doctoral es basen en els onze articles d'investigació en què l'autor ha particitat durant els darrers quatre anys. Tots ells estan publicats en revistes especialitzades de reconegut prestigi internacional. Una còpia d'aquests es recull al final d'aquesta memòria.

1. C. Segarra, J. I. Climente, and J. Planelles, Valence band mixing of cubic GaN/AlN quantum dots, J. Phys.: Condens. Matter 24, 115801 (2012)

2. J. Planelles, J. I. Climente, and C. Segarra, Electron spin relaxation in 3D quantum dots: geometrical suppression of Dresselhaus and Rashba spin-orbit interaction, J. Phys. Chem. C 116, 25143 (2012)

3. J. I. Climente, C. Segarra, and J. Planelles, Spin-orbit-induced hole spin relaxation in InAs and GaAs quantum dots, New J. Phys. 15, 093009 (2013)

4. A. Ballester, C. Segarra, A. Bertoni, and J. Planelles, Suppression of the Aharonov-Bohm effect in hexagonal quantum rings, EPL 104, 67004 (2013)

5. M. Royo, C. Segarra, A. Bertoni, G. Goldoni, and J. Planelles, AharonovBohm oscillations and electron gas transitions in hexagonal core-shell nanowires with an axial magnetic field, Phys. Rev. B 91, 115440 (2015)

6. C. Segarra, J. Planelles, and J. I. Climente, Control of electron spinorbit anisotropy in pyramidal InAs quantum dots, Physica E 66, 234 (2015)

7. C. Segarra, J. Planelles, J. I. Climente, and F. Rajadell, Anisotropy of spin-orbit induced electron spin relaxation in [001] and [111] grown GaAs quantum dots, New J. Phys. 17, 033014 (2015)

8. C. Segarra, J. I. Climente, F. Rajadell, and J. Planelles, Hole spin relaxation in InAs/GaAs quantum dot molecules, J. Phys.: Condens. Matter 27, 415301 (2015)

9. C. Segarra, J. Planelles, and S. E. Ulloa, Edge states in dichalcogenide nanoribbons and triangular quantum dots, Phys. Rev. B 93, 085312 (2016)

10. J. I. Climente, C. Segarra, F. Rajadell, and J. Planelles, Electrons, holes, and excitons in GaAs polytype quantum dots, J. Appl. Phys. 119, 125705 (2016)

11. C. Segarra, J. I. Climente, A. Polovitsyn, F. Rajadell, I. Moreels, and J. Planelles, Piezoelectric control of the exciton wave function in colloidal CdSe/CdS nanocrystals, J. Phys. Chem. Lett. 7, 2182 (2016) 



\section{Publications}

1 Valence band mixing of cubic GaN/AlN QDs . . . . . . . . 155

2 Suppression of the Aharonov-Bohm effect in hexagonal QRs . . . . 163

3 Aharonov-Bohm oscillations and electron gas transitions in hexagonal core-shell nanowires with an axial magnetic field . . . . . 169

4 Control of electron spin-orbit anisotropy in pyramidal InAs QDs . 179

5 Electron spin relaxation in 3D QDs: geometrical suppression of Dresselhaus and Rashba spin-orbit interaction . . . . . . . . . . 183

6 Anisotropy of spin-orbit induced electron spin relaxation in [001] and $[111]$ grown GaAs QDs . . . . . . . . . . . . . 193

7 Spin-orbit-induced hole spin relaxation in InAs and GaAs QDs . . 205

8 Hole spin relaxation in InAs/GaAs quantum dot molecules . . . . 227

9 Electrons, holes and excitons in GaAs polytype quantum dots . . . 235

10 Piezoelectric control of the exciton wave function in colloidal CdSe/CdS nanocrystals . . . . . . . . . . . . . . . . 245

11 Edge states in dichalcogenide nanoribbons and triangular QDs . . 267 
Valence band mixing of cubic GaN/AIN quantum dots / C. Segarra; J.I. Climente; J. Planelles

Journal of Physics: Condensed Matter, 24, 11, 2012, p. 155- 162

DOI: http://dx.doi.org/10.1088/0953-8984/24/11/115801

\begin{abstract}
:
We study the spin purity of the hole ground state in nearly axially symmetric GaN/AIN quantum dots (QDs). To this end, we develop a six-band Burt-Foreman Hamiltonian describing the valence band structure of zinc-blende nanostructures with cylindrical symmetry, and calculate the effects of eccentricity variationally. We show that that the aspect ratio is a key factor for spin purity. In typical QDs with small aspect ratio the ground state is essentially a heavy hole $(\mathrm{HH})$ whose spin purity is even higher than that of InGaAs QDs of similar size. When the aspect ratio increases, mixing with lighthole ( $\mathrm{LH}$ ) and split-off (SO) subbands becomes important and, additionally, the ground state becomes sensitive to QD anisotropy, which further enhances the mixing. We finally show that despite the large GaN hole effective mass, an efficient magnetic modulation is feasible in QDs with aspect ratio 1, which can be used to modify the ground state symmetry and hence the optical spectrum properties
\end{abstract}

Enlace Repositori UJI (versión postprint): http://hdl.handle.net/10234/67117 


\section{Suppression of the Aharonov-Bohm effect in hexagonal quantum}

Rings / A. Ballester, C. Segarra, A. Bertoni and J. Planelles

EPL (Europhysics Letters), Volume 104, Number 6, 2013 p. 163 - 168

DOI: http://dx.doi.org/10.1209/0295-5075/104/67004

Abstract:

Few-electron states of AlAs-GaAs-AlAs hexagonal quantum rings pierced by an axial magnetic field are computed through full configuration interaction calculations. The quantum ring is in the low-density regime, populated with $N=1$ up to $N=7$ electrons. Similar to circular rings, the energy spectra of the hexagonal ones reflect an integer and fractional Aharonov-Bohm regular oscillation pattern for $N=1$ and $N=2,3$, respectively. Deviations from the regular fractional period with increasing electron density become apparent for larger $N$. Remarkably, for $N=6$ the Aharonov-Bohm effect is completely suppressed. This is a unique symmetry-related feature of hexagonal rings that only can emerge in the low-density regime. 


\title{
Aharonov-Bohm oscillations and electron gas transitions in hexagonal core-shell nanowires with an axial magnetic field
}

\author{
Miquel Royo,,${ }^{1,2,}$ Carlos Segarra, ${ }^{1}$ Andrea Bertoni, ${ }^{2}$ Guido Goldoni, ${ }^{2,3}$ and Josep Planelles ${ }^{1}$ \\ ${ }^{1}$ Departament de Química Física i Analítica, Universitat Jaume I, E-12080 Castelló, Spain \\ ${ }^{2}$ CNR-NANO S3, Institute for Nanoscience, Via Campi 213/a, 41125 Modena, Italy \\ ${ }^{3}$ Department of Physics, Informatics and Mathematics, University of Modena and Reggio Emilia, Italy \\ (Received 27 January 2015; revised manuscript received 17 March 2015; published 30 March 2015)
}

\begin{abstract}
We use spin-density-functional theory within an envelope function approach to calculate electronic states in a GaAs/InAs core-shell nanowire pierced by an axial magnetic field. Our fully three-dimensional quantum modeling includes explicitly a description of the realistic cross section and composition of the sample, and the electrostatic field induced by external gates in two different device geometries: gate-all-around and back-gate. At low magnetic fields, we investigate Aharonov-Bohm oscillations and signatures therein of the discrete symmetry of the electronic system, and we critically analyze recent magnetoconductance observations. At high magnetic fields, we find that several charge and spin transitions occur. We discuss the origin of these transitions in terms of different localization and Coulomb regimes, and we predict their signatures in magnetoconductance experiments.
\end{abstract}

\section{INTRODUCTION}

Gated semiconductor nanowire (NW) devices represent flexible test beds to study transport phenomena in the quasione-dimensional quantum regime. In this context, InAs-based NWs offer privileged properties derived, for instance, from the light InAs electron effective mass, which enables the experimental observation of the subband spectrum quantization even in NWs of a relatively large section, [1-3] or from its large spin-orbit interaction and Landé factor [4,5]. This boosts their prospective applications in spintronics [6], even at relatively high temperature [7]. Furthermore, in this narrow-gap material, the Fermi energy, $E_{F}$, is pinned by surface states above the conduction-band edge [8], leading to an accumulation of electrons at the NW surface and facilitating the fabrication of Ohmic contacts $[1,9]$.

The resulting tubular shape of the conducting channel points toward interesting quantum phenomena under external magnetic fields [10]. In particular, an axial field may lead to Aharonov-Bohm (AB) field-periodic modulation of the electron energy spectrum [11] and, if the phase-coherent length exceeds the perimeter of the NW, the observation of magnetoconductance oscillations [12,13]. Indeed, several observations of AB-like oscillations in magnetotransport experiments performed on radial heterostructures have been reported [14-18]. Recently, Gül et al. [16] observed fluxperiodic magnetoconductance oscillations in GaAs/InAs coreshell NWs. The oscillations persisted at different density regimes, modulated by a back-gate, exhibiting phase shifts as the back-gate voltage was gradually increased. A field-periodic magnetoconductance has also been observed in the same system with superconductor contacts [17] and, after removal of the GaAs core, in a hollow InAs shell [18].

The single-crystal NW-based heterostructures investigated in these experiments have a prismatic hexagonal cross section. However, the experimental observations were analyzed in terms of simplified cylindrical electronic systems, and

\footnotetext{
*mroyo@qfa.uji.es
}

the potential induced by the back-gate voltage, which also removes the cylindrical symmetry, was neglected. Likewise, theoretical calculations dealing with radial electronic systems with an axial magnetic field usually assume a cylindrical symmetry $[5,12,13,19,20]$. Ferrari et al. [10] investigated the effect of an axial magnetic field in prismatic systems, but the single-particle model adopted did not allow for a direct comparison with experiments.

Such approximations are particularly severe in radial heterostructures, where coupling between the discrete (hexagonal in InAs or GaAs) symmetry and many-electron interactions leads to strongly inhomogeneously distributed electron gas and, in turn, to the coexistence of one-dimensional (1D) and $2 \mathrm{D}$ channels at the corners and facets of the hexagonal heterointerfaces [21-23]. Strong anisotropy-induced effects are predicted in this case, such as negative magnetoresistance in a transverse magnetic field [24] and symmetry-induced cancellation of the AB effect in hexagonal quantum rings [25]. The inhomogeneous electron gas localization was crucially exposed in the recent observation of intra- and interband excitations $[23,26]$.

In this paper, we study the electronic states and magnetoconductance in GaAs/InAs core-shell NWs with an axial magnetic field within a spin-density-functional theory (SDFT) approach. Our fully 3D modeling explicitly includes the description of the quantum states within an envelope function approach with a realistic cross section and composition of the sample, and it includes the electrostatic field induced by external gates in two different device geometries, namely gate-all-around and back-gate. At low magnetic fields, we investigate the nature of the magnetoconductance oscillations, as measured in Ref. [16], predicting specific signatures of the discrete symmetry of the electronic system in the $A B$ magnetoconductance oscillations, and justifying the observation of $\mathrm{AB}$ oscillations despite the broken symmetry induced by the back-gate voltage. At high magnetic fields, we found that several charge and spin transitions occur. We discuss the origin of these transitions in terms of different magnetic-fieldinduced localization and Coulomb regimes, and we predict their signatures in magnetoconductance experiments. 


\section{THEORETICAL MODEL}

Within a parabolic single-band envelope-function description, the effective Kohn-Sham Hamiltonian under an external magnetic field reads

$$
\begin{aligned}
\hat{H}= & \frac{1}{2}(\hat{\mathbf{P}}-e \mathbf{A}(\mathbf{R})) \frac{1}{m^{*}(\mathbf{R})}(\hat{\mathbf{P}}-e \mathbf{A}(\mathbf{R}))+V_{\text {conf }}(\mathbf{R}) \\
& +V_{Z}^{\sigma}(\mathbf{R})+V_{H}(\mathbf{R})+V_{\mathrm{XC}}^{\sigma}(\mathbf{R}) .
\end{aligned}
$$

Here, $\mathbf{R}=(x, y, z), \hat{\mathbf{P}}$ is the momentum operator, $\mathbf{A}(\mathbf{R})$ is the vector potential, $e$ is the elementary charge, and $m^{*}(\mathbf{R})$ is the material-dependent electron effective mass. $V_{\text {conf }}(\mathbf{R})$ is the spatial confinement potential induced by the heterostructure, and $V_{H}(\mathbf{R})$ is the Hartree potential energy. The Zeeman energy $V_{Z}^{\sigma}(\mathbf{R})$ and the exchange-correlation potential $V_{\mathrm{XC}}^{\sigma}(\mathbf{R})$ depend on the the spin index $\sigma=\uparrow, \downarrow$ of the electrons.

We consider an infinitely long NW extending along the $z$ direction. To describe an axial magnetic field, we adopt the symmetric gauge $\mathbf{A}(\mathbf{R})=B / 2(-y, x, 0)$ (see Fig. 1 for axis definition). The axial field does not break the spatial invariance along the $z$ axis. Therefore, the single-particle eigenfunctions of (1) can be written as $\Psi_{n, k, \sigma}(\mathbf{R})=e^{i k z} \phi_{n, \sigma}(\mathbf{r})$, with $\mathbf{r} \equiv$ $(x, y), n$ the principal quantum number, and $k$ the wave number along direction $z$. Substituting $\Psi_{n, k, \sigma}(\mathbf{R})$ and $\mathbf{A}(\mathbf{R})$ in (1), we obtain the spin-dependent Kohn-Sham equation

$$
\begin{aligned}
& {\left[-\frac{\hbar^{2}}{2} \nabla_{\mathbf{r}} \frac{1}{m^{*}(\mathbf{r})} \nabla_{\mathbf{r}}+\frac{e B}{2 m^{*}(\mathbf{r})} \hat{L}_{z}+\frac{e^{2} B^{2}}{8 m^{*}(\mathbf{r})}\left(x^{2}+y^{2}\right)\right.} \\
& \left.\quad+v_{\mathrm{conf}}(\mathbf{r})+v_{Z}^{\sigma}(\mathbf{r})+v_{H}(\mathbf{r})+v_{\mathrm{xc}}^{\sigma}(\mathbf{r})\right] \phi_{n, \sigma}(\mathbf{r}) \\
& =\epsilon_{n, k, \sigma} \phi_{n, \sigma}(\mathbf{r}) .
\end{aligned}
$$

Here, $\epsilon_{n, k, \sigma}=\varepsilon_{n, \sigma}+\frac{\hbar^{2} k^{2}}{2 m_{z}^{*}}$ includes the 1D parabolic dispersion along the $z$ axis, and $\hat{L}_{z}=-i \hbar\left(x \frac{\partial}{\partial y}-y \frac{\partial}{\partial x}\right)$ is the
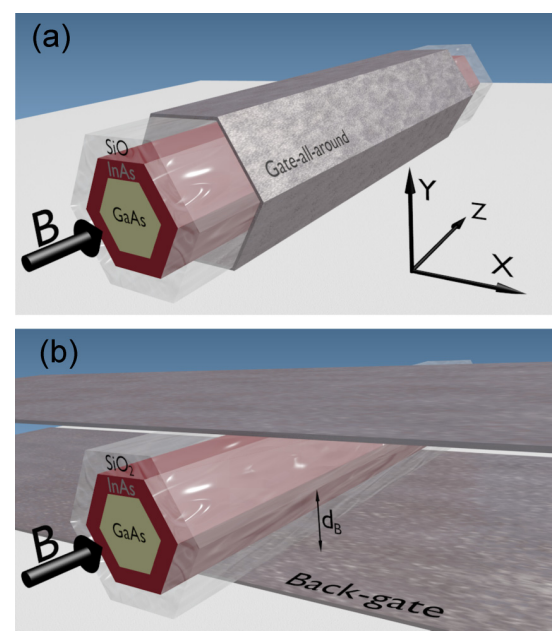

FIG. 1. (Color online) Schematics of a core-shell NW in the (a) gate-all-around and (b) back-gate device configurations. azimuthal angular momentum operator. To obtain Eq. (2), it is necessary to assume that the $z$ component of the effective mass, $m_{z}^{*}$, does not depend on $\mathbf{r}$, i.e., on the material. This approximation is expected to have a small effect [27] and enables us to decouple the electron motion in the longitudinal and transverse directions.

The confinement potential $v_{\text {conf }}(\mathbf{r})$ is set by the conductionband offsets among the different materials that are radially modulated in the NW cross section. The Zeeman term is

$$
v_{Z}^{\sigma}(\mathbf{r})=g^{*}(\mathbf{r}) \mu_{B} B \eta_{\sigma},
$$

where $g^{*}(\mathbf{r})$ is the material-dependent Lande factor, $\mu_{B}$ is the Bohr magneton, and $\eta_{\sigma}=+1 / 2(-1 / 2)$ for $\sigma=\uparrow(\downarrow)$.

The Hartree potential energy, $v_{H}(\mathbf{r})$, is calculated from the electrostatic potential, $v_{H}(\mathbf{r})=-e \Phi(\mathbf{r})$, via the Poisson equation

$$
\nabla \varepsilon(\mathbf{r}) \nabla \Phi(\mathbf{r})=\frac{1}{\varepsilon_{0}} e\left[n(\mathbf{r})-n_{D}(\mathbf{r})\right]
$$

Here, $n(\mathbf{r})=n_{\uparrow}(\mathbf{r})+n_{\downarrow}(\mathbf{r})$ is the total free-electron charge density calculated, using the Kohn-Sham eigenstates obtained from Eq. (2), as

$$
n_{\sigma}(\mathbf{r})=\frac{1}{2 \pi} \sum_{n}\left|\phi_{n, \sigma}(\mathbf{r})\right|^{2} \int_{-\infty}^{\infty} d k f\left(\epsilon_{n, k, \sigma}-E_{F}, T\right),
$$

where

$$
f\left(\epsilon_{n, k, \sigma}-E_{F}, T\right)=\frac{1}{1+e^{\left(\epsilon_{n, k, \sigma}-E_{F}\right) / k_{B} T}}
$$

is the Fermi occupation, with $E_{F}, T$, and $k_{B}$ being, respectively, the Fermi energy, temperature, and Boltzmann constant. In Eq. (4), $n_{D}(\mathbf{r})$ is the density of static donors and $\varepsilon(\mathbf{r})$ is the material-dependent static dielectric constant.

The exchange and correlation potential, $v_{\mathrm{xc}}^{\sigma}(\mathbf{r})$, in the local-spin-density approximation (LSDA) [28] is given by the functional derivative

$$
v_{\mathrm{xc}}^{\sigma}(\mathbf{r})=\frac{\delta \varepsilon_{\mathrm{xc}}(n(\mathbf{r}), \zeta(\mathbf{r}))}{\delta n_{\sigma}(\mathbf{r})},
$$

where $\varepsilon_{\mathrm{xc}}(n(\mathbf{r}), \zeta(\mathbf{r}))$ is the exchange and correlation energy density, and

$$
\zeta(\mathbf{r})=\frac{n_{\uparrow}(\mathbf{r})-n_{\downarrow}(\mathbf{r})}{n(\mathbf{r})}
$$

is the local spin polarization. In the present paper, we use the correlation functional proposed by Perdew and Wang [29].

From the solutions of the Kohn-Sham equations, we also obtain the total free energy per unit length from [30]

$$
\begin{aligned}
E= & \frac{1}{2 \pi} \sum_{n, \sigma} \int_{-\infty}^{\infty} d k \epsilon_{n, k, \sigma} f_{n, k, \sigma} \\
& -\frac{1}{2} \int d \mathbf{r} v_{H}(\mathbf{r}) n(\mathbf{r})-\sum_{\sigma} \int d \mathbf{r} v_{\mathrm{xc}}^{\sigma}(\mathbf{r}) n_{\sigma}(\mathbf{r}) \\
& +\int d \mathbf{r} \varepsilon_{\mathrm{xc}}(n(\mathbf{r}), \zeta(\mathbf{r}))+\frac{k_{B} T}{2 \pi} \sum_{n, \sigma} \int_{-\infty}^{\infty} d k \\
& \times\left[f_{n, k, \sigma} \ln f_{n, k, \sigma}+\left(1-f_{n, k, \sigma}\right) \ln \left(1-f_{n, k, \sigma}\right)\right] .
\end{aligned}
$$

Here, the second term on the right-hand side is the Hartree energy per unit length with the sign inverted, the fourth term 
Aharonov-Bohm oscillations and electron gas transitions in hexagonal core-shell nanowires with an axial magnetic field

is the exchange and correlation energy per unit length, and the last term is an entropy functional, where $f_{n, k, \sigma}=f\left(\epsilon_{n, k, \sigma}\right.$ $\left.E_{F}, T\right)$.

Equations (2)-(9) are solved iteratively until selfconsistency is reached, which we consider to occur when two convergence criteria are simultaneously fulfilled in two consecutive iterations: first, the relative variation of the charge density is lower than $10^{-4}$ at any point of the discretization domain, and second, the relative variation in total free energy per unit length [Eq. (9)] is lower than $10^{-8}$.

Equations (2) and (4) are numerically integrated in a real-space hexagonal domain. We use the same symmetrypreserving triangular grid with $\sim 1.14$ points $/ \mathrm{nm}^{2}$ for both formulas, and we integrate Eqs. (2) and (4) with the methods of finite elements and finite volumes, respectively. Dirichlet boundary conditions are assumed in both cases, generally forcing the solutions to vanish at the boundaries. To simulate the effect of a gate-all-around [see Fig. 1(a)], the electrostatic potential in the Poisson equation is forced to take the gate voltage $V_{g}$ at the domain boundaries. For a back-gate, we assume that the hexagonal domain is sandwiched by two flat infinite electrodes [see Fig. 1(b)] and the electrostatic potential is set at the gate voltage $V_{g}$ at the bottom facet and zero at the top one. Accordingly, at the lateral boundaries the electrostatic potential is set to $F d_{B}(\mathbf{r})$, with $F$ and $d_{B}(\mathbf{r})$ being, respectively, the electric field in the capacitor and the vertical distance from the boundary point to the bottom electrode [see Fig. 1(b)]

Finally, we also calculate the spin-projected free charge density per unit length,

$$
\bar{n}_{\sigma}=\int n_{\sigma}(\mathbf{r}) d \mathbf{r}
$$

and the spin-projected ballistic conductance of the NW by means of the linear-response Landauer formula [31],

$$
G^{\sigma}=\frac{e^{2}}{h} \sum_{n} \int_{\mathcal{B}_{n, \sigma}}-\frac{\partial f\left(E-E_{F}, T\right)}{\partial E} d E,
$$

where the integral is performed along each energy spinsubband $\mathcal{B}_{n, \sigma}$. Note that the integrand makes a significant contribution only in the energy region close to the crossings of the subband with the Fermi energy $E_{F}$.

\section{NUMERICAL RESULTS}

We consider a GaAs/InAs core-shell hexagonal NW such as the one measured in Ref. [16] and outlined in Fig. 1. It is composed of a GaAs NW core with a minimal diameter of 100 $\mathrm{nm}$ and an InAs shell with a thickness of $25 \mathrm{~nm}$. In addition, we include in the device an external 30-nm-thick layer of $\mathrm{SiO}_{2}$ intended to simulate the insulating layer that separates the conducting channel from a back-gate in the experiment [16]. The GaAs core is doped with a homogeneous density of donors $n_{D}=5 \times 10^{15} \mathrm{~cm}^{-3}$ (as in Ref. [16]). The material parameters used in the calculations are listed in Table I, where the conduction-band edge, $E_{\mathrm{CB}}$, is obtained with the so-called 40:60 rule [32,33] from the band gap. Calculations have been conducted assuming a Fermi energy placed $75 \mathrm{meV}$ above the InAs conduction-band edge (as in Ref. [16]), a temperature of $1.8 \mathrm{~K}$, and the InAs effective mass as the constant mass factor
TABLE I. Material parameters used in the simulations; electron effective mass $\left(m^{*}\right)$, dielectric constant $(\varepsilon)$, effective Landé factor $\left(g^{*}\right)$, and conduction-band edge $\left(E_{\mathrm{CB}}\right)$.

\begin{tabular}{lccl}
\hline \hline & GaAs & InAs & $\mathrm{SiO}_{2}$ \\
\hline$m^{*}$ & 0.067 & 0.028 & 0.41 \\
$\varepsilon$ & 13.18 & 15.5 & 3.9 \\
$g^{*}$ & -0.484 & -14.3 & 2.0 \\
$E_{\mathrm{CB}}(\mathrm{eV})$ & 0.858 & 0.252 & 5.4 \\
\hline \hline
\end{tabular}

$\left(m_{z}^{*}\right)$ arising in the parabolic dispersion of the 1D subbands [see Eq. (2)].

\section{A. Low-magnetic-field regime: Magnetoconductance} oscillations

In Fig. 2, we show the ground-state properties and magnetoconductance of the investigated core-shell NW at $V_{g}=0$. The density distribution of conduction-band electrons [Fig. 2(b)] shows that charge is exclusively accumulated in the InAs shell and preferentially localized at the corners of the hexagonal section. As reported for several core-(multi)shell hexagonal NWs [21-23,34], such distribution is favored by Coulomb interactions, which tend to increase the interelectron distance. In Fig. 2(a), we show the energies of the spin-subband edges at different magnetic fields, hereafter referred to as magnetic spin-subbands (MSS), with spin up ( $\uparrow$-MSSs) and spin down ( $\downarrow$-MSSs). Due to the hexagonal symmetry of the self-consistent potential, the low-energy spectrum is at low fields formed out of groups of 12 MSSs arising from the six irreducible representations of the $\mathrm{C}_{6}$ symmetry group. Each of these groups is further spin-split by the strong Zeeman effect
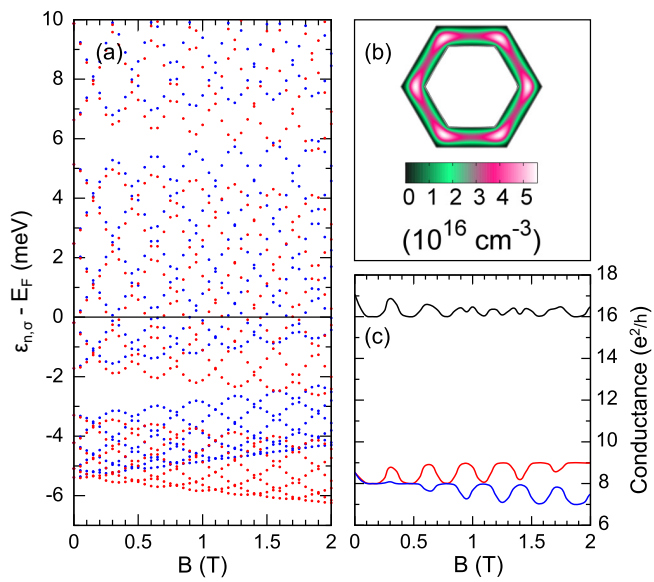

FIG. 2. (Color online) (a) Magnetic spin-subbands (MSS) in the low-field regime. Red and blue dots indicate $\downarrow$ - MSSs and $\uparrow$-MSSs, respectively. The horizontal black line is set at $E_{F}$. (b) Self-consistent electron density distribution, $n(\mathbf{r})$, for the InAs/GaAs NW at $B=0$. (c) Spin-projected magnetoconductances $G_{\downarrow}$ (red) and $G_{\uparrow}$ (blue), and total magnetoconductance (black). 
forming two bunches, one of $\uparrow$-MSS and the other of $\downarrow$-MSSs, of six braided MSSs.

Within each group, the MSSs oscillate due to the $\mathrm{AB}$ effect, developing crossings with MSSs of their same group, which have different symmetry and/or different spin, and anticrossings with MSSs of neighboring groups with the same symmetry and spin. The oscillation period is $\sim 0.32 \mathrm{~T}$. Since the calculated expectation value of the radial position, $\rho=$ $\sqrt{x^{2}+y^{2}}$, of the electron system is $66.36 \mathrm{~nm}$, this periodicity corresponds fairly well to the periodicity of $\sim 0.30 \mathrm{~T}$ of the corresponding circular system.

In Fig. 2(c), we show the spin-projected magnetoconductances $G_{\sigma}(B)$ and the total magnetoconductance $G(B)=$ $G_{\uparrow}(B)+G_{\downarrow}(B)$. Even though both $G_{\sigma}(B)$ exhibit regular flux-periodic oscillations, $G(B)$ only does so at very low fields. After the second oscillation cycle, the $G(B)$ periodicity is suppressed by the Zeeman effect, which breaks the periodicity of the MSS spectrum [12,13]. Apart from this, $G(B)$ does not differ qualitatively from that of an electron system in a cylindrical tube $[12,13,16]$. Indeed, in the present case, $E_{F}$ lies within one group of braided MSSs, and the spectrum around $E_{F}$ is similar to that of a cylindrical system. However, in an experiment $E_{F}$ can be tuned by means of external gates. Therefore, we next study the system at different Fermi levels $E_{F}$ or applied gate voltages $V_{g}$.

In Fig. 3, we show the effect of a gate-all-around voltage. This geometry tunes the position of the MSSs with respect to $E_{F}$, modulating the total density in the system while preserving the hexagonal symmetry. As shown in Fig. 3(a), the oscillatory behavior of the magnetoconductance due to the $\mathrm{AB}$ effect is absent at certain voltages. For instance, at $V_{g}=80 \mathrm{mV}$ the magnetoconductance is flat. This is due to the positioning of $E_{F}$ in the energy gap between the second and third group of MSSs, as shown in Fig. 3(c). Since $E_{F}$ does not cross any MSS, the number of conducting channels is constant. Comparing Figs. 3(b) and 3(c), which correspond to $V_{g}=-60$ and $80 \mathrm{mV}$, respectively, we also observe that the gate voltage affects both the width of the MSSs groups and the gaps between them. In fact, $V_{g}$ affects the total electron density and, hence, electron localization. As shown in the insets of Figs. 3(b) and 3(c), a $V_{g}>0$ favors localization in the corners of the InAs shell, due to the larger electron-electron interaction. This, in turn, reduces the tunneling among states at the corners and, hence, the splittings within bunches of MSSs, while it increases the gaps between consecutive bunches [10]. Note that, since the latter gaps are a direct consequence of the discrete symmetry of the system, flat magnetoconductance is a direct signature of the hexagonal symmetry, which is more likely to be observed at positive gate voltages.

Observation of flat magnetoconductance when sweeping $V_{g}$ has not been reported in the transport measurements performed hitherto on hexagonal NWs under axial magnetic fields $[9,16-18]$. However, in these works the electron density was normally modulated by a back-gate instead of a gateall-around. The electrostatic field generated by a back-gate removes the hexagonal symmetry of the electronic system, and it could even destroy the doubly connected topology that originates the $\mathrm{AB}$ effect. Therefore, one may wonder why flux-periodic oscillations in the magnetoconductance are observed at all.
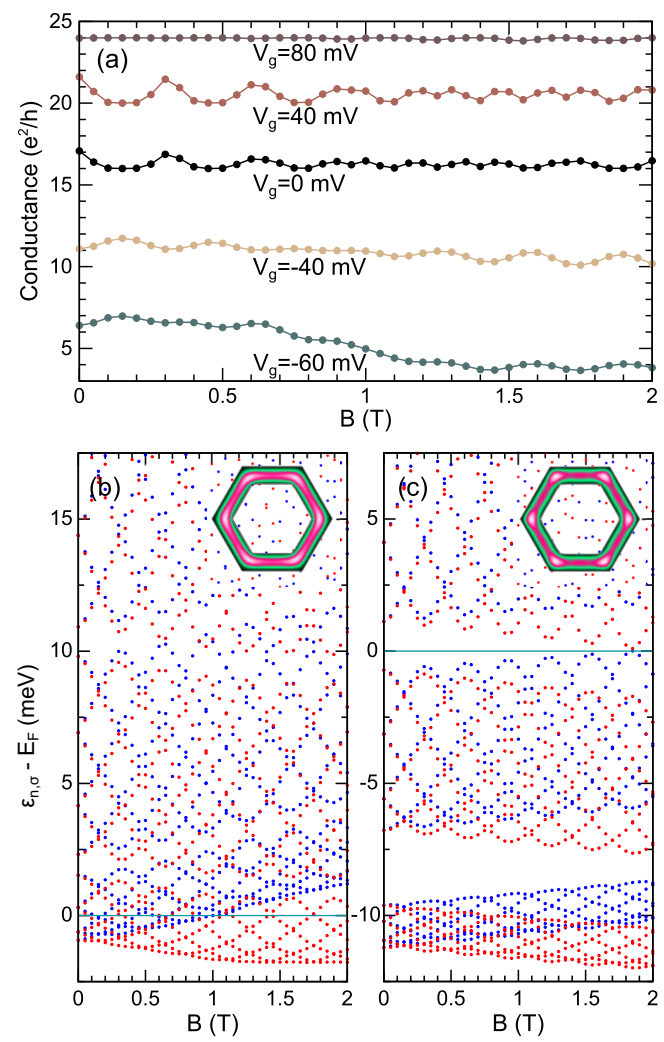

FIG. 3. (Color online) (a) Total magnetoconductance at selected gate-all-around voltages, $V_{g}$, as indicated by labels [the $V_{g}=0$ curve is the same as the black line in Fig. 2(c)]. (b) MSSs at $V_{g}=-60 \mathrm{mV}$. (c) MSSs at $V_{g}=80 \mathrm{mV}$. Insets in (b) and (c) show the corresponding $n(\mathbf{r})$.

To assess this point, in Fig. 4 we show the results of simulations performed at different back-gate voltages. As shown in the insets of Figs. 4(b) and 4(c), the applied voltage strongly reshapes the electron density distribution in the NW. At negative (positive) $V_{g}$ the total density in the system is reduced (increased) and concentrated in the top (bottom) half of the InAs shell. However, whereas the doubly connected topology that results in $\mathrm{AB}$ oscillations is removed at sufficiently negative voltages (e.g., $V_{g}=-80 \mathrm{mV}$ ), it is robust for $V_{g}>0$. The origin of this difference can be appreciated from the corresponding MSSs [Figs. 4(b) and 4(c)]. The lowest-lying MSSs are strongly affected by the gate, losing the doubly connected topology and showing an almost linear dispersion with the magnetic field. Higher-energy MSSs, on the contrary, being more delocalized over the NW section, still show doubly connected topology. Since at $V_{g}=-80$ and $-100 \mathrm{mV}$ only low-lying MSSs are occupied [see Fig. 4(b)], the total electron density loses the doubly connected 
Aharonov-Bohm oscillations and electron gas transitions in hexagonal core-shell nanowires with an axial magnetic field
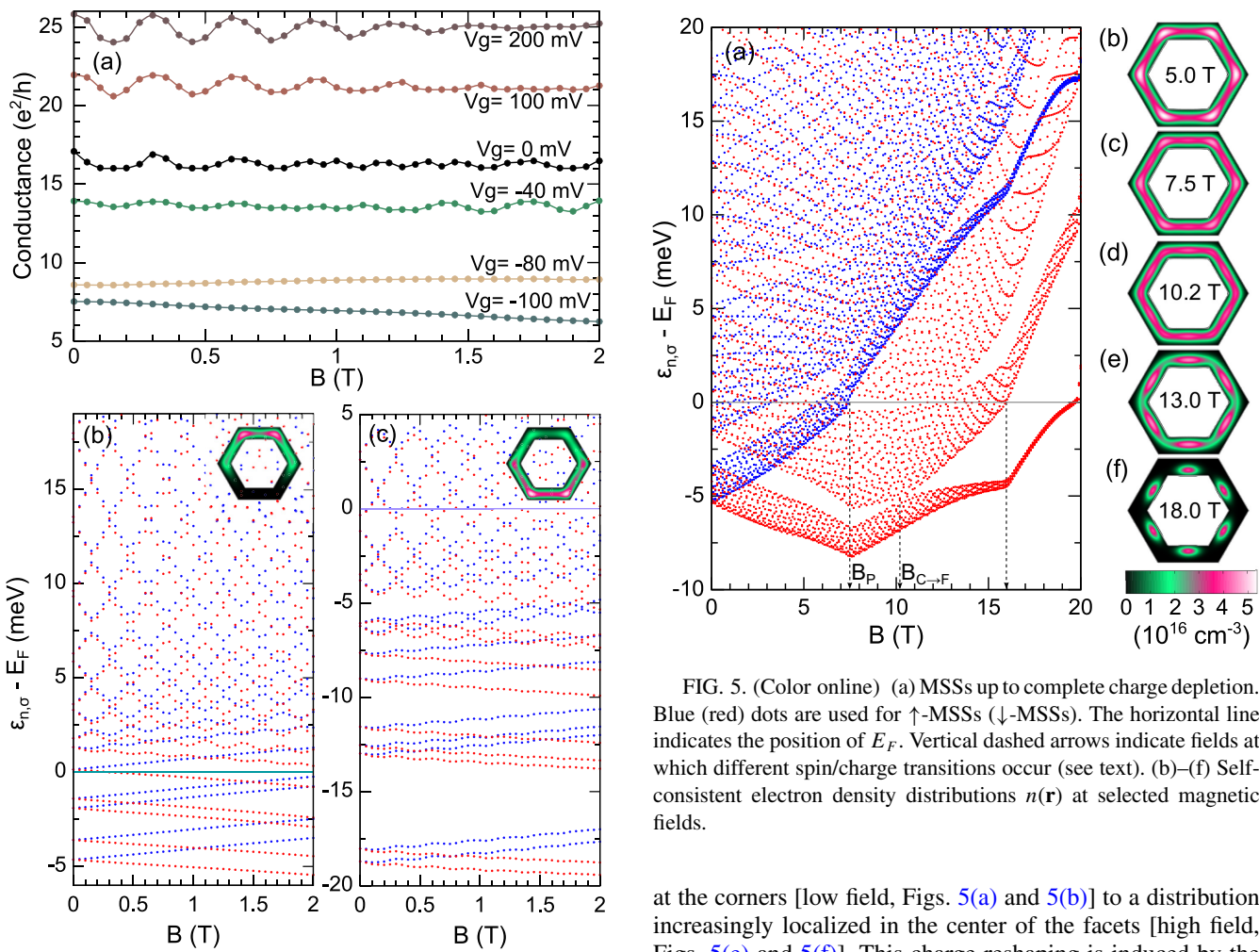

FIG. 4. (Color online) (a) Same as in Fig. 3 but for a back-gate device. (b) MSSs at $V_{g}=-80 \mathrm{mV}$, (c) MSSs at $V_{g}=200 \mathrm{mV}$.

topology, and the corresponding magnetoconductance does not show $\mathrm{AB}$ oscillations. In contrast, at $V_{g}>0$ several states with doubly connected topology are occupied, and the $\mathrm{AB}$ oscillations of the magnetoconductance persist [see Fig. 4(a)] The latter is indeed the usual regime in magnetotransport experiments $[16,17]$ where, therefore, periodic oscillations in the magnetoconductance are observed despite the symmetry reduction.

B. High-magnetic-field regime: Spin and charge transitions

We next study the high-magnetic-field regime, up to the limit of complete electron depletion, which occurs at $B \sim 20 \mathrm{~T}$ in this sample. Figure 5 shows the MSSs and the self-consistent total electron density distributions at selected fields (spin-projected electron densities show only minor differences and are not shown here). All simulations in this section are performed at $V_{g}=0$. The overall behavior of MSSs shows that, in addition to the diamagnetic shift, several transitions occur at discrete fields, as we discuss below.

The evolution of $n(\mathbf{r})$ in Figs. 5(b)-5(f) shows that the axial field induces a transition from an electron distribution localized

FIG. 5. (Color online) (a) MSSs up to complete charge depletion. Blue (red) dots are used for $\uparrow$-MSSs ( $\downarrow$-MSSs). The horizontal line indicates the position of $E_{F}$. Vertical dashed arrows indicate fields at which different spin/charge transitions occur (see text). (b)-(f) Selfconsistent electron density distributions $n(\mathbf{r})$ at selected magnetic fields.

at the corners [low field, Figs. 5(a) and 5(b)] to a distribution increasingly localized in the center of the facets [high field, Figs. 5(e) and 5(f)]. This charge reshaping is induced by the diamagnetic term [third term on the left-hand side of Eq. (2)], which constrains the electron density to adopt distributions with lower radius as the field is increased, counteracted by Coulomb interactions.

Such a corner-to-facet transition can be correlated with the evolution of the MSSs. In Fig. 5(a), the lowest-lying bunch of $12 \mathrm{MSS}$ at $B=0$ corresponds to states localized at the corners, whereas the second set of states are localized at the facets for orthogonality. As the field is increased, Zeeman spinsplitting takes place and the two sets of six $\downarrow$-MSSs approach in energy, eventually overlapping at $B_{C \rightarrow F} \sim 10.2 \mathrm{~T}$. At this point, the $2 \mathrm{D}$ electron density integrated along the minimal (facet-to-facet) and maximal (corner-to-corner) diameter [22] is nearly the same [see Fig. 5(d)]. At $B>B_{C \rightarrow F}$, the six lowest $\downarrow$-MSSs are localized at the facets of the inner interface, while corner states are much higher in energy, corresponding to the third group of six $\downarrow$-MSS. The same transition occurs for $\uparrow$-MSS, however these states are already depopulated at the transition field.

Apart from this smooth spatial localization transition, two abrupt changes of slope appear in the calculated MSSs. The first one occurs at $B_{P}=7.5 \mathrm{~T}$ and corresponds to complete spin polarization, as demonstrated by the spin-projected electron densities $\bar{n}_{\uparrow}, \bar{n}_{\downarrow}$ shown in Fig. 6(a) and the corresponding spin polarization in Fig. 6(b), which marks a clear transition to a ferromagnetic state at $B_{P}$. Note that the total density 


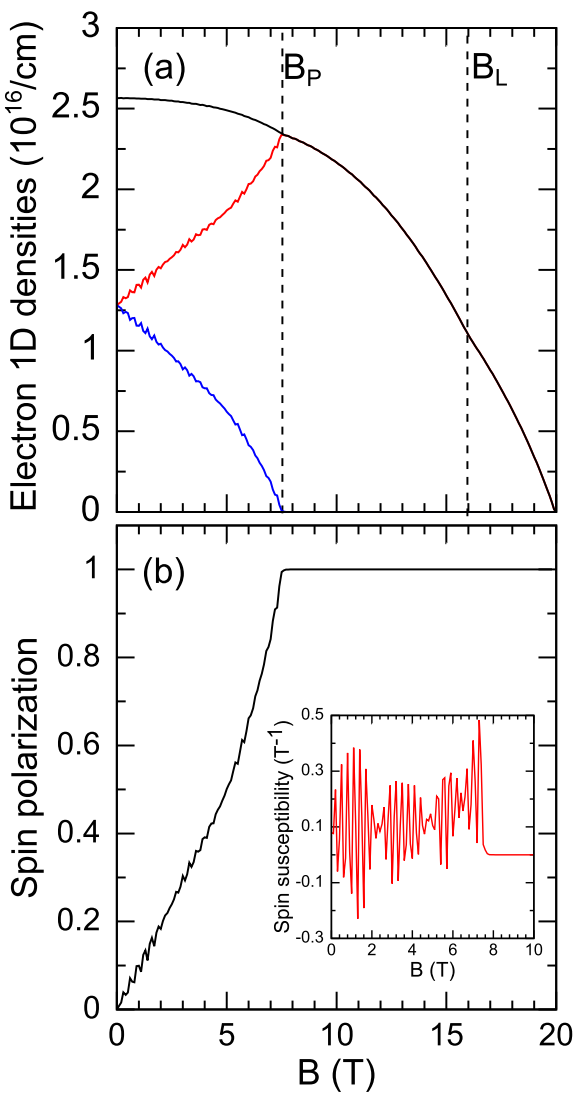

FIG. 6. (Color online) (a) Total density $\hat{n}$ (black lines) and spinprojected densities $\bar{n}_{\uparrow}$ (blue lines) and $\bar{n}_{\downarrow}$ (red lines) as a function of the field intensity $B$. Vertical dashed lines illustrate the transition fields in Fig. 5(a). (b) Spin polarization as a function of the magnetic field. Inset: spin susceptibility.

[black line in Fig. 6(a)] is reduced by the magnetic field with a roughly parabolic trend due to the depletion of successive, high-energy MSSs. However, the curve shows a change of slope at $\left(B_{P}\right)$. At fields right after $B_{P}$, the rate at which the NW is depleted decreases momentarily. $\bar{n}_{\downarrow}$ passes abruptly from being increased to decreased at $B_{P}$, in agreement with the inversion of the $\downarrow$-MMSs slope exposed in Fig. 5(a)

The singular behavior of the spin polarization [Fig. 6(b)] is reminiscent of the first-order phase transition of a $2 \mathrm{D}$ electron gas with an in-plane magnetic field $[35,36]$ (note that in our system, the Seitz radius $r_{s} \sim 0.07$ at zero field, which is a very weakly correlated regime), although it is difficult in our numerical treatment to establish whether it is a weakly firstorder or continuous transition. The inset in Fig. 6(b) shows the spin susceptibility, i.e., the magnetic-field derivative of the spin polarization. This magnitude oscillates with the field as

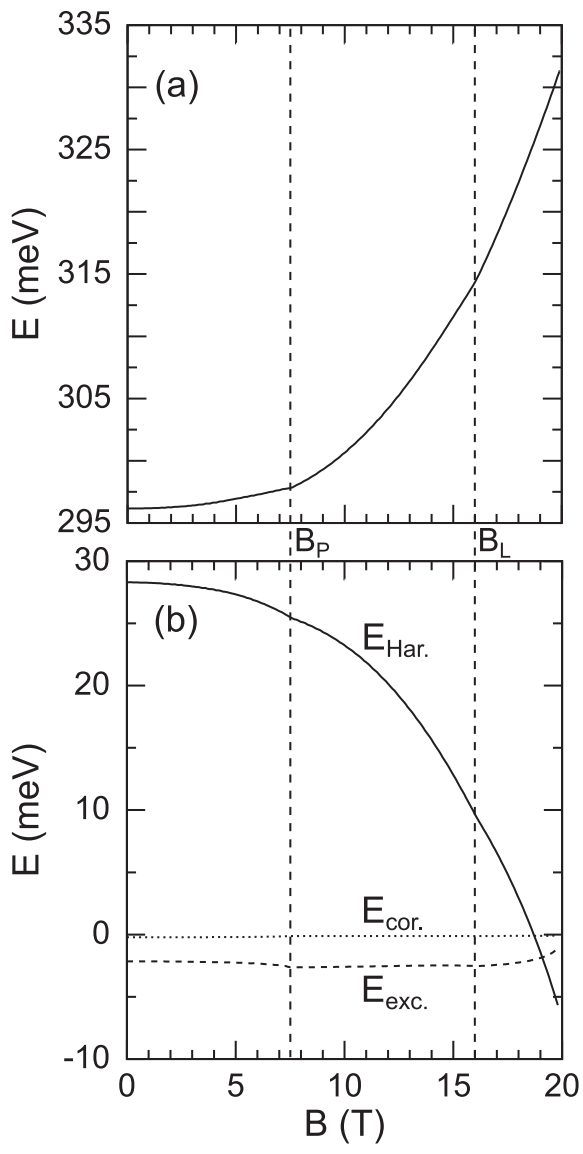

FIG. 7. (a) Total free energy per electron and (b) Hartree (solid), exchange (dashed), and correlation (dotted) energies per electron as a function of the field $B$. Vertical dashed lines indicate the transition fields in Fig. 5.

a consequence of the interplay between the $\mathrm{AB}$ effect and the Zeeman splitting, which produce short-period modulations of the spin densities.

At fields higher than $B_{P}$ and $B_{C \rightarrow F}$, the MSSs shown in Fig. 5(a) rearrange in groups of six, which tend to form Landau-like bands. Finally, at a larger field $B_{L}=16 \mathrm{~T}$ the spectrum shows an additional transition. This corresponds to complete depletion of the incipient second Landau-like band. The transition is also marked by a weak but visible kink in $\bar{n}(B)$, as shown in Fig. 6(a), which, as for the ferromagnetic transition, indicates a sudden decrease in the depletion rate.

The free energy per electron and the many-electron energy contributions per electron are calculated dividing the corresponding magnitudes per unit length by the total electron density and plotted in Figs. 7(a) and 7(b), respectively. All energy contributions show weak kinks at $B_{P}$ and $B_{L}$. The 
Aharonov-Bohm oscillations and electron gas transitions in hexagonal core-shell nanowires with an axial magnetic field

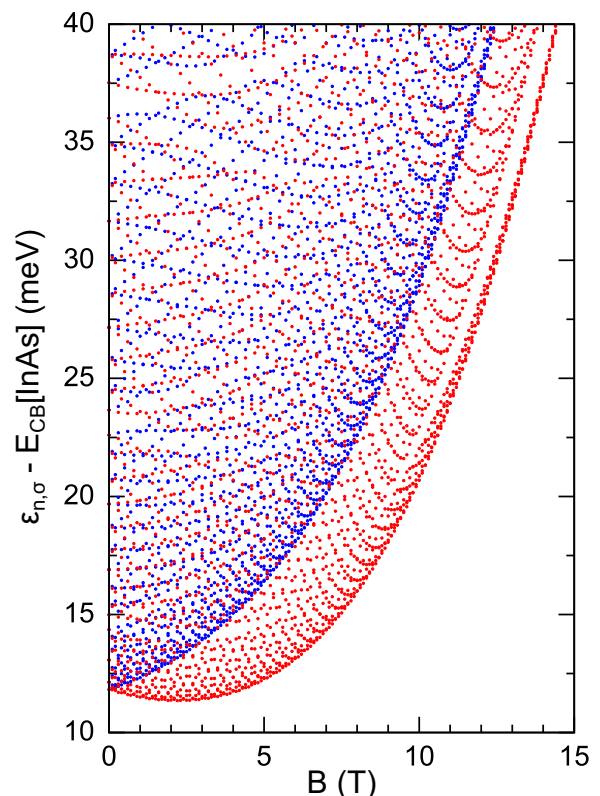

FIG. 8. (Color online) Noninteracting MSS edges with respect to the InAs conduction-band edge. Blue (red) dots are used for $\uparrow$-MSS $(\downarrow$-MSSs)

free energy per electron increases with $B$ due to the increase in magnetic confinement. However, the Hartree energy per electron [Fig. 7(b)] is reduced with $B$ due to field-induced charge depopulation. At high magnetic fields, $B>18.5 \mathrm{~T}$, the Hartree energy changes sign because the free-electron density is lower than the total density of static donors included in the simulation in the NW GaAs core. Note from Fig. 7(b) that the direct Hartree energy is one and two orders of magnitude larger than the exchange and correlation contributions, respectively, and therefore it will rule many-electron effects in the system.

To assess the role of many-electron contributions, in Fig. 8 we show the MSSs calculated in a noninteracting model, i.e. $v_{H}=0$ and $v_{\mathrm{XC}}=0$. The MSSs follow in this case a smooth evolution with $B$, which evidences the many-electron origin of the two transitions at $B_{P}$ and $B_{L}$ in the SDFT calculation. We have also checked that such transitions persist when only $v_{\mathrm{XC}}=0$ (data not shown here), as was expected from the weak effect of the exchange and correlation contributions in the present system [see Fig. 7(b)]

Indeed, the transitions at $B_{P}$ and $B_{L}$ result from the balance between the two main energy contributions: the magnetic confinement, which increases the system energy with $B$, and the direct Coulomb or Hartree energy, which is reduced with $B$ due to the charge depletion decreasing the system energy in this way. Thus, the first transition at $B_{P}$, which produces an inversion in the slope of the $\downarrow$-MSSs, can be understood as a transition between a regime, $B<B_{P}$, in which the reduction in Hartree energy dominates over the magnetic confinement, to

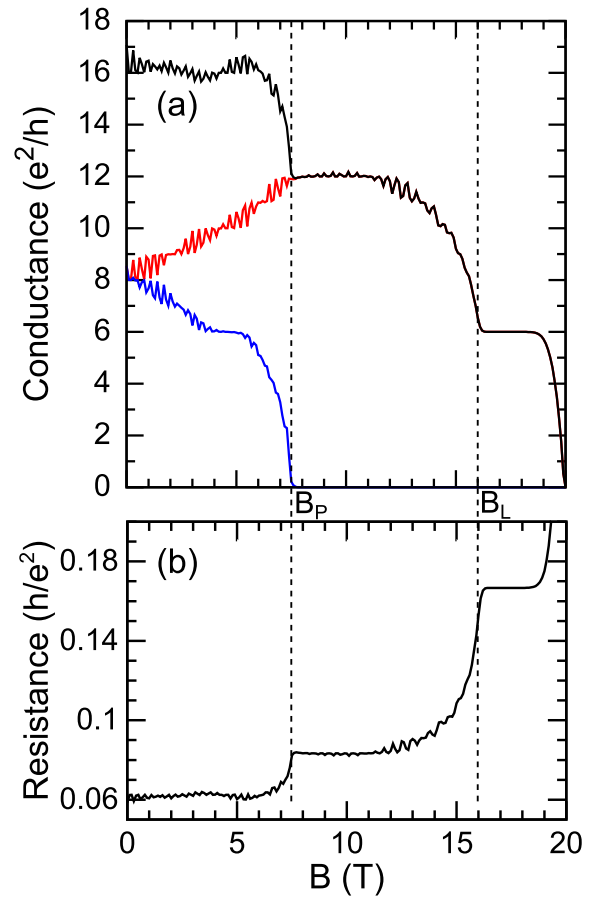

FIG. 9. (Color online) (a) Total magnetoconductance $G=G_{\uparrow}+$ $G_{\downarrow}$ (black) and spin-resolved magnetoconductances, $G_{\uparrow}$ (blue) an $G_{\downarrow}$ (red). (b) Total magnetoresistance. Vertical dashed lines indicate the transition fields in Fig. 5.

another regime, $B>B_{P}$, in which the magnetic confinement dominates. The key difference before and after $B_{P}$ is the magnitude of the Hartree energy that is lost per depleted state, which is larger at $B<B_{P}$. This is because when the system is not spin-polarized, the Hartree energy also arises from the interactions between electrons with antiparallel spin. The latter, which are absent in the ferromagnetic phase, are stronger than interactions between parallel spin electrons due to the lack of a Fermi hole.

The transition at $B_{L}$, which produces an abrupt increase of the MSSs, is also interpreted with similar arguments, i.e., the Hartree energy lost per depleted state is lower at $B>B_{L}$. This is due to the larger localization of the electron density at $B>B_{L}$ [cf. Figs. 5(e) and 5(f)], which entails a larger Fermi hole in the direct Coulomb interaction in this regime. Indeed, it has been proven that the conditional probability of finding an electron with a given spin when there is already another electron with the same spin nearby is lower when the former is localized [37].

The spin-projected magnetoconductances $G_{\uparrow}, G_{\downarrow}$ and the total magnetoconductance $G=G_{\uparrow}+G_{\downarrow}$ calculated from the SDFT modeling are shown in Fig. 9(a). Starting from low fields, the total magnetoconductance oscillates, due to oscillating MSSs crossing $E_{F}$, around an average value of 16 
$e^{2} / h$ up to a magnetic field $B \sim 6 \mathrm{~T}$. As the field approaches $B_{P}$, a sudden steplike reduction of four magnetoconductance units is caused by the sudden depletion of the lowest set of $\uparrow$ MSSs [see Fig. 5(a)] induced by the ferromagnetic transition.

At $B>B_{P}$, the magnetoconductance shows an almost flat plateau that lasts up to $B \sim 12 \mathrm{~T}$. This originates in the location of $E_{F}$ in the symmetry-induced energy gap between the second and third group of six $\downarrow$-MSSs [see Fig. 5(a)]. As $E_{F}$ merges in the second group of $\downarrow$-MSSs, the magnetoconductance starts to oscillate again, while reducing in average at an increasing rate approaching $B_{L}$. At $B>B_{L}$,

$E_{F}$ lies in the wide energy gap between the first and second Landau bands, hence $G$ is constant.

Finally, $G(B)$ drops to zero when the first incipient Landau band crosses $E_{F}$ and the conduction band gets completely depleted. In Fig. 9(b), we also plot the magnetoresistance $1 / G(B)$ to illustrate the kink observed at $B_{P}$, which corresponds to that observed in experimental measures [38,39] of ferromagnetic transitions in flat quasi-2D electron systems under in-plane magnetic fields.

\section{SUMMARY AND CONCLUSIONS}

We performed a SDFT study of the electronic structure and magnetoconductance of hexagonal core-shell NWs pierced by an axial magnetic field. Critically, our modeling goes beyond often employed cylindrical and/or single-particle approximations to simulate radial heterostructures, which neglect the strongly inhomogeneous, field-dependent distribution of the electron gas.

In the low-field regime $(B \lesssim 2 \mathrm{~T})$, we predict that $\mathrm{AB}$ magnetoconductance oscillations may disappear/resurface as a function of the gate-all-around voltage as a direct consequence of the presence of discrete symmetry-induced energy gaps. Our calculations also allowed us to critically analyze recent experiments $[16,17]$ and justify the observation of $\mathrm{AB}$ oscillations in spite of the broken symmetry induced by the back-gate voltage.

In the high-magnetic-field regime, we found several fieldinduced transitions. First, the diamagnetic confinement induces a strong reshaping of the electron gas, which goes through a smooth transition from a low-field electron density distribution concentrated in the corners to a high-field distribution strongly localized in the facets of the radial heterojunction. Several experimental consequences of such reshaping are expected, for example in optical recombination experiments, due to the different localization of electrons and holes [26].

In addition, two abrupt transitions occur at discrete fields that are related to the depletion of higher MSSs. These depletions are either of the lowest antiparallel spin MSSs, leading to spin polarization, or of the second incipient Landau-like band with parallel spin. The origin of these transitions lies in the increase of the effective Fermi hole occurring at each transition, which affects the amount of Hartree energy that is lost per depleted state. As a consequence, such abrupt transitions are clearly marked in the calculated magnetoconductance by steplike behaviors.

\section{ACKNOWLEDGMENTS}

We acknowledge partial financial support from Universitat Jaume I, Projects No. P1-1B2011-01 and P1.1B2014-24, MINECO Project No. CTQ2011-27324, APOSTD/2013/052 Generalitat Valenciana Vali+d Grant (M.R.), and a MINECO FPU Grant (C.S.), from EU-FP7 Initial Training Network INDEX, and from Universitá di Modena e Reggio Emilia, through the Grant "Nano- and emerging materials and systems for sustainable technologies."
[1] S. Chuang, Q. Gao, R. Kapadia, A. C. Ford, J. Guo, and A. Javey, Nano Lett. 13, 555 (2013).

[2] F. Vigneau, V. Prudkovkiy, I. Duchemin, W. Escoffier, P. Caroff, Y.-M. Niquet, R. Leturcq, M. Goiran, and B. Raquet, Phys. Rev. Lett. 112, 076801 (2014)

[3] E. Halpern, A. Henning, H. Shtrikman, R. Rurali, X. Cartoixà, and Y. Rosenwaks, Nano Lett. 15, 481 (2015).

[4] D. Liang and X. P. Gao, Nano Lett. 12, 3263 (2012).

[5] A. Bringer and T. Schäpers, Phys. Rev. B 83, 115305 (2011).

[6] S. Nadj-Perge, S. Frolov, E. Bakkers, and L. P. Kouwenhoven, Nature (London) 468, 1084 (2010)

[7] F. Rossella, A. Bertoni, D. Ercolani, M. Rontani, L. Sorba, F. Beltram, and S. Roddaro, Nat. Nanotech. 9, 997 (2014).

[8] H. Lüth, Solid Surfaces, Interfaces and Thin Films, 6 ed. (Springer-Verlag, Berlin, Heidelberg, 2015).

[9] C. Blomers, M. I. Lepsa, M. Luysberg, D. Grutzmacher, H. Luth, and T. Schapers, Nano Lett. 11, 3550 (2011).

[10] G. Ferrari, G. Goldoni, A. Bertoni, G. Cuoghi, and E. Molinari, Nano Lett. 9, 1631 (2009).

[11] J. Planelles, W. Jaskólski, and J. I. Aliaga, Phys. Rev. B 65, 033306 (2001).
[12] Y. Tserkovnyak and B. I. Halperin, Phys. Rev. B 74, 245327 (2006).

[13] T. O. Rosdahl, A. Manolescu, and V. Gudmundsson, Phys. Rev. B 90, 035421 (2014).

[14] M. Jung, J. S. Lee, W. Song, Y. H. Kim, S. D. Lee, N. Kim, J. Park, M.-S. Choi, S. Katsumoto, H. Lee, and J. Kim, Nano Lett. 8, 3189 (2008).

[15] T. Richter, C. Blömers, H. Lüth, R. Calarco, M. Indlekofer, M. Marso, and T. Schäpers, Nano Lett. 8, 2834 (2008).

[16] Ö. Gül, N. Demarina, C. Blömers, T. Rieger, H. Lüth, M. I. Lepsa, D. Grützmacher, and T. Schäpers, Phys. Rev. B 89, 045417 (2014).

[17] Ö. Gül, H. Y. Günel, H. Lüth, T. Rieger, T. Wenz, F. Haas, M Lepsa, G. Panaitov, D. Grützmacher, and T. Schäpers, Nano Lett. 14, 6269 (2014).

[18] T. Wenz, M. Rosien, F. Haas, T. Rieger, N. Demarina, M. I. Lepsa, H. Lüth, D. Grützmacher, and T. Schäpers, Appl. Phys. Lett. 105, 113111 (2014).

[19] V. N. Gladilin, J. Tempere, J. T. Devreese, and P. M. Koenraad, Phys. Rev. B 87, 165424 (2013).

[20] T. O. Rosdahl, A. Manolescu, and V. Gudmundsson, Nano Lett. 15, 254 (2015) 
Aharonov-Bohm oscillations and electron gas transitions in hexagonal core-shell nanowires with an axial magnetic field

[21] B. M. Wong, F. Léonard, Q. Li, and G. T. Wang, Nano Lett. 11, 3074 (2011)

[22] A. Bertoni, M. Royo, F. Mahawish, and G. Goldoni, Phys. Rev. B 84, 205323 (2011).

[23] S. Funk, M. Royo, I. Zardo, D. Rudolph, S. Morkötter, B. Mayer, J. Becker, A. Bechtold, S. Matich, M. Döblinger, M. Bichler, G. Koblmüller, J. J. Finley, A. Bertoni, G. Goldoni, and G. Abstreiter, Nano Lett. 13, 6189 (2013).

[24] M. Royo, A. Bertoni, and G. Goldoni, Phys. Rev. B 87, 115316 (2013).

[25] A. Ballester, C. Segarra, A. Bertoni, and J. Planelles, Europhys. Lett. 104, 67004 (2013).

[26] J. Jadczak, P. Plochocka, A. Mitioglu, I. Breslavetz, M. Royo, A. Bertoni, G. Goldoni, T. Smolenski, P. Kossacki, A. Kretinin, H. Shtrikman, and D. K. Maude, Nano Lett. 14, 2807 (2014).

[27] T. Ando, J. Phys. Soc. Jpn. 51, 3893 (1982)

[28] The validity of the LSDA for the $B$ values of interest was assessed in Ref. [40] by comparing the obtained results for a single QD with those given by current-spin-density-functional theory [41], which is better suited to study many-electron systems in magnetic fields.

[29] J. P. Perdew and Y. Wang, Phys. Rev. B 45, 13244 (1992).

[30] K. Hirose, S.-S. Li, and N. S. Wingreen, Phys. Rev. B 63, 033315 (2001)
[31] This formula assumes a fully ballistic regime, which is not exactly the experimentally attainable regime (although quasiballistic transmissions as large as 0.8 have been experimentally observed, even at room temperature, in InAs NW FETs [1]). We employ this simplified formula since it can surely yield a correct qualitative description of the magnetoconductance.

[32] S. Adachi (ed.), Properties of Aluminum Gallium Arsenide, EMIS Datareviews Series No. 7 (INSPEC; The Institution of Electrical Engineers, London, 1993).

[33] N. Debbar, D. Biswas, and P. Bhattacharya, Phys. Rev. B 40, 1058 (1989).

[34] G. Martínez-Criado, A. Homs, B. Alén, J. A. Sans, J. SeguraRuiz, A. Molina-Sánchez, J. Susini, J. Yoo, and G.-C. Yi, Nano Lett. 12, 5829 (2012).

[35] A. L. Subaşi and B. Tanatar, Phys. Rev. B 78, 155304 (2008).

[36] Y. Zhang and S. Das Sarma, Phys. Rev. Lett. 96, 196602 (2006).

[37] E. Matito, B. Silvi, M. Duran, and M. Solà, J. Chem. Phys. 125, 024301 (2006).

[38] B. A. Piot, D. K. Maude, U. Gennser, A. Cavanna, and D. Mailly, Phys. Rev. B 80, 115337 (2009).

[39] E. Tutuc, S. Melinte, E. P. De Poortere, M. Shayegan, and R. Winkler, Phys. Rev. B 67, 241309 (2003).

[40] F. Ancilotto, D. G. Austing, M. Barranco, R. Mayol, K. Muraki, M. Pi, S. Sasaki, and S. Tarucha, Phys. Rev. B 67, 205311 (2003).

[41] M. Ferconi and G. Vignale, Phys. Rev. B 50, 14722 (1994). 
Control of electron spin-orbit anisotropy in pyramidal InAs quantum dots / C. Segarra, J. Planelles, J.I. Climente

Physica E: Low-dimensional Systems and Nanostructures, Volume 66, February 2015, Pages 234-237

DOI: http://dx.doi.org/10.1016/j.physe.2014.10.030

\section{Abstract:}

We investigate the electron spin-orbit interaction anisotropy of pyramidal InAs quantum dots using a fully three-dimensional Hamiltonian. The dependence of the spin-orbit interaction strength on the orientation of externally applied in-plane magnetic fields is consistent with recent experiments, and it can be explained from the interplay between Rashba and Dresselhaus spin-orbit terms in dots with asymmetric confinement. Based on this, we propose manipulating the dot composition and height as efficient means for controlling the spin-orbit anisotropy. 
Electron Spin Relaxation in 3D Quantum Dots: Geometrical Suppression of Dresselhaus and Rashba Spin-Orbit Interaction/ Josep Planelles, Juan I. Climente, Carlos Segarra

The Journal of Physical Chemistry C , 116 (47), p. 25143-25146

DOI: http://dx.doi.org/10.1021/jp309389p

\begin{abstract}
:
We investigate the electron spin relaxation between Zeeman sublevels of fully threedimensional quantum dots. By going beyond the usual two-dimensional description of Rashba and Dresselhaus spin-orbit interactions (SOI), we provide a general overview of the effect of the quantum dot shape. It is shown that, in spherical quantum dots, the Dresselhaus $\mathrm{SO}$ is severely suppressed, leading to slow relaxation rates and a strong $\left(B^{9}\right)$ dependence on the magnetic field.
\end{abstract}




\title{
Anisotropy of spin-orbit induced electron spin relaxation in [001] and [111] grown GaAs quantum dots
}

C Segarra, J Planelles, J I Climente and F Rajadell

Departament de Química Física i Analítica, Universitat Jaume I, Castelló de la Plana, Spain

E-mail: josep.planelles@uji.es

Keywords: spin-orbit interaction, spin relaxation, quantum dot, magnetic field

\begin{abstract}
We report a systematic study of the spin relaxation anisotropy between single electron Zeeman sublevels in three-dimensional cuboidal GaAs quantum dots (QDs). The QDs are subject to an inplane magnetic field. As the field orientation varies, the relaxation rate oscillates periodically, showing 'magic' angles where the relaxation rate is suppressed by several orders of magnitude. This behavior is found in QDs with different shapes, heights, crystallographic orientations and external fields. The origin of these angles can be traced back to the symmetries of the spin admixing terms of the Hamiltonian. Our results evidence that cubic Dresselhaus terms play an important role in determining the spin relaxation anisotropy, which can induce deviations of the 'magic' angles from the crystallographic directions reported in recent experiments (P Scarlino et al 2014 Phys. Rev. Lett. 113 256802).
\end{abstract}

\section{Introduction}

The electron spin confined in semiconductor quatum dots (QDs) is a promising candidate for the realization of quantum computing and the development of spin-based devices in spintronics [1,2]. Using the spin of electrons as qubits was first proposed by Loss and DiVincenzo [3] and, since then, a lot of effort has been devoted to its accomplishment [4]. QDs offer the possibility of isolating single electron spins which exhibit longer lifetimes than in delocalized systems since quantum confinement suppresses the main bulk decoherence mechanisms [5]. Nevertheless, coupling between the electron spin and the surrounding environment cannot be avoided, resulting in spin relaxation and decoherence. Therefore, a good understanding of the relaxation mechanisms in QDs is needed for the development of spin-based applications.

The two main mechanisms of spin relaxation in III-V zinc-blende semiconductor QDs are the hyperfine coupling with the nuclear spins of the lattice and the spin-orbit interaction (SOI) [4]. The hyperfine interaction is generally important at relatively weak magnetic fields while for moderate and strong fields the phononmediated relaxation due to SOI predominates. In semiconductors without inversion symmetry, e.g. GaAs, SOI can be originated by the bulk inversion asymmetry of the material (Dresselhaus SOI) [6] and the structure inversion asymmetry of the confining potential (Rashba SOI) [7]. The Hamiltonians describing both SOI have different symmetries and exhibit an anisotropic behavior [8]. This anisotropy can be exploited to externally control and manipulate the electron spin by changing the orientation of applied magnetic or electric fields [911]. As a consequence, the anisotropy of the spin relaxation and its control via external means has been intensively studied [12-20].

Most previous theoretical works have dealt with two-dimensional (2D) circular QDs grown along the [001] crystal direction [4, 12-14,21], where in-plane anisotropy arises from the interference between Rashba and Dresselhaus SOI. However, QDs are prone to deviate from the circular symmetry and there is gathering evidence that this has a primary influence on the spin relaxation anisotropy [15-18]. This fact has been confirmed in very recent experiments by Scarlino and co-workers [22]. Relevantly, all the studies analyzing the influence of noncircular confinement on the spin relaxation anisotropy of single QDs have so far missed the effect of cubic Dresselhaus SOI terms and that of three-dimensionality (3D). Cubic terms are expected to become particularly 


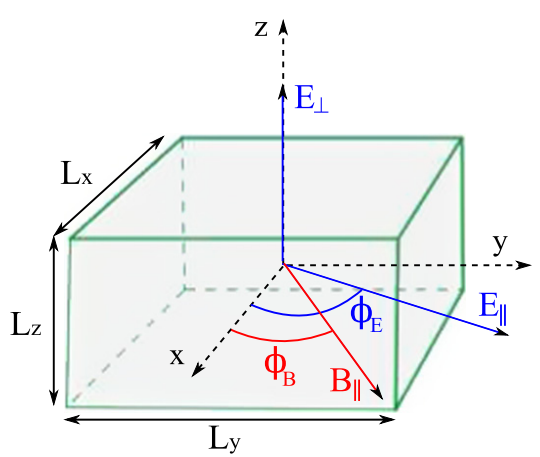

Figure 1. Schematic representation of the cuboidal QD system. The orientation of the external electric and magnetic fields is indicated.

important in tall QDs [23], which are increasingly available owing to recent progress in synthetic control $[24,25]$. On the other hand, going beyond [001] grown QDs is also of interest, especially in view of the convenience of [111] grown QDs for optical spin preparation [26]. The effect of the crystallographic orientation on the spin dynamics has been well studied in quantum wells [27-29], but further work is needed in relation to fully localized spins, where studies are limited [18].

In this article, we study the anisotropy of the electron spin relaxation between Zeeman sublevels in cuboidal GaAs QDs. The anisotropy is monitored by varying the orientation of an externally applied in-plane magnetic field $\left(\phi_{B}\right)$. We consider QDs grown along both [001] and [111] crystal directions, including all linear and cubic terms of Rashba and Dresselhaus SOI in a fully 3D model. Different heights, base shapes, crystallographic orientations, magnetic field intensities and external electric fields are considered. The numerical results, together with perturbative interpretations, provide a wide overview on the effect of confinement asymmetry and $3 \mathrm{D}$ on the spin relaxation anisotropy.

We find that, in [001] grown QDs, the spin relaxation anisotropy is very different depending on the dominating spin-orbit mechanism, Rashba or Dresselhaus SOI. By contrast, in [111] grown QDs the anisotropy is the same for both terms. In all cases, the spin relaxation rate shows strong oscillations with $\phi_{B}$. Interestingly, cubic Dresselhaus terms are shown to be critical in determining such anisotropic behavior. This occurs not only in tall QDs, but — contrary to common belief-also in quasi-2D QDs, provided the high symmetry directions of the dot are not aligned with the main crystallographic axes. In both squared and rectangular QDs we observe order-of-magnitude suppressions of the spin relaxation rate at certain 'magic' magnetic field angles $\phi_{B}$, which can be understood from symmetry considerations. A 'magic' angle around [110] has actually been very recently reported in experiments with a single GaAs QD strongly elongated along one in-plane direction [22]. We generalize this study considering less elongated structures. We show that cubic Dresselhaus terms help explain the deviation from [110] observed in the experiment, and in less elongated structures they switch the 'magic' angle to $[\overline{1} 10]$ or $[1 \overline{1} 0]$.

The paper is organized as follows. Section 2 presents the model we use to compute the electron spin relaxation, including the SOI Hamiltonians for QDs rotated with respect to the main crystallographic axes. In section 3 we show and discuss the numerical results for the cases under study. Finally, conclusions are given in section 4.

\section{Theoretical model}

We study the electron spin relaxation driven by SOI between Zeeman split sublevels of cuboidal GaAs QDs subject to externally applied electric $\mathbf{E}$ and magnetic $\mathbf{B}$ fields (see figure 1). The isotropy of the conduction band of III-V semiconductors leads to an isotropic kinetic energy term in the 3D one-electron Hamiltonian which reads

$$
H=\frac{\mathbf{p}^{2}}{2 m^{*}}+V_{c}+\mathrm{Er}+H_{Z}+H_{\mathrm{SOI}}
$$

where $m^{*}$ stands for the electron effective mass, $V_{c}$ is the confinement potential, $\mathrm{E}$ is an external electric field and $\mathbf{p}=-i \hbar \nabla+\mathbf{A}$, where $\mathbf{A}$ is the vector potential. An in-plane magnetic field $\mathbf{B}=B\left(\cos \phi_{B}, \sin \phi_{B}, 0\right)$ rotated 
Anisotropy of spin-orbit induced electron spin relaxation in [001] and [111] grown GaAs QDs

an angle $\phi_{B}$ with respect to the $x$ axis of the dot is included. This field is described by the vector potential $\mathrm{A}=\left(z B \sin \phi_{B},-z B \cos \phi_{B}, 0\right)$. The Zeeman term is $H_{Z}=\frac{1}{2} g \mu_{B} \mathrm{~B} \boldsymbol{\sigma}$ with $g, \mu_{B}$ and $\boldsymbol{\sigma}$ standing for the electron g-factor, Bohr magneton and Pauli spin matrices, respectively.

The last term in (1) corresponds to the SOI, [8] $H_{\text {SOI }}=H_{R}+H_{D}$, with $H_{R}$ being the Rashba SOI

$$
H_{R}^{[001]}=\alpha_{r} \sigma(\mathbf{p} \times \mathrm{E}) \text {, }
$$

and $H_{D}$ the Dresselhaus SOI

$$
H_{D}^{[001]}=\beta_{d}\left[\sigma_{x} p_{x}\left(p_{y}^{2}-p_{z}^{2}\right)+\sigma_{y} p_{y}\left(p_{z}^{2}-p_{x}^{2}\right)+\sigma_{z} p_{z}\left(p_{x}^{2}-p_{y}^{2}\right)\right] .
$$

Here, $\alpha_{r}$ and $\beta_{d}$ are material-dependent coefficients determining the strength of the SOI and the superscript [001] indicates de growth direction of the QD.

Equations (2) and (3) correspond to QDs grown along the [001] crystal direction. In order to consider other orientations of the QD with respect to the crystal host we maintain the confinement potential fixed in space and perform a rotation of the crystalline structure. Since the confining potential as well as the externally applied fields are kept while the crystalline structure is rotated, only the $H_{\text {SOI }}$ part of the Hamiltonian is affected. In particular, the $H_{\text {SOI }}$ Hamiltonian corresponding to an axially applied electric field and a crystalline structure subject to an in-plane rotation $\theta_{z}$ around the $z$ axis reads:

$$
H_{R}^{[001]}\left(\theta_{z}\right)=\alpha_{r} E_{z}\left(\sigma_{x} p_{y}-\sigma_{y} p_{x}\right),
$$

and

$$
\begin{aligned}
H_{D}^{[001]}\left(\theta_{z}\right)= & \beta_{d} \cos 2 \theta_{z}\left[\sigma_{x} p_{x}\left(p_{y}^{2}-p_{z}^{2}\right)+\sigma_{y} p_{y}\left(p_{z}^{2}-p_{x}^{2}\right)+\sigma_{z} p_{z}\left(p_{x}^{2}-p_{y}^{2}\right)\right] \\
& +\beta_{d} \sin 2 \theta_{z}\left[p_{z}^{2}\left(\sigma_{y} p_{x}+\sigma_{x} p_{y}\right)-2 \sigma_{z} p_{x} p_{y} p_{z}+\frac{1}{2}\left(p_{x}^{2}-p_{y}^{2}\right)\left(\sigma_{x} p_{y}-\sigma_{y} p_{x}\right)\right] .
\end{aligned}
$$

Note that this particular case of an axially applied electric field yields a Rashba Hamiltonian (4) independent of $\theta_{z}$

We consider next QDs grown along the [111] direction. In particular, we consider the rotation $\chi=\arccos (1 / \sqrt{3})$ around the straight line $y=-x$, that corresponds to the Euler angles $\theta=\arccos (1 / \sqrt{3})$, $\phi=45$ and $\alpha=-45$. The rotated SOI Hamiltonians have the form

$$
H_{R}^{[111]}=\frac{\alpha_{r} E_{z}}{\sqrt{3}}\left[\sigma_{z}\left(p_{y}-p_{x}\right)-\sigma_{y}\left(p_{x}+p_{z}\right)+\sigma_{x}\left(p_{y}+p_{z}\right)\right]
$$

and

$$
\begin{aligned}
H_{D}^{[111]}= & \frac{\beta_{d}}{2 \sqrt{3}}\left[\left(p_{x}^{2}+p_{y}^{2}-4 p_{z}^{2}\right)\left(p_{x} \sigma_{y}-p_{y} \sigma_{x}\right)+p_{z}\left(p_{x}^{2}-p_{y}^{2}\right)\left(\sigma_{x}+\sigma_{y}\right)\right. \\
& \left.+2 p_{x} p_{y} p_{z}\left(\sigma_{x}-\sigma_{y}\right)-\sigma_{z} p_{x}^{2}\left(p_{x}+3 p_{y}\right)+\sigma_{z} p_{y}^{2}\left(p_{y}+3 p_{x}\right)\right],
\end{aligned}
$$

where the electric field is aligned with the dot $z$ axis.

The relaxation rate between the initial electron state $\left|\Psi_{i}\right\rangle$ and the final electron state $\left|\Psi_{f}\right\rangle$ is estimated by the Fermi golden rule

$$
\frac{1}{T_{1}}=\frac{2 \pi}{\hbar} \sum_{\lambda, \mathbf{q}}\left|M_{\lambda}(\mathbf{q})\right|^{2}\left|\left\langle\Psi_{f}\left|\mathrm{e}^{-\mathrm{iqr}}\right| \Psi_{i}\right\rangle\right|^{2} \delta\left(E_{f}-E_{i}-E_{q}\right) .
$$

Here, the sum is done over all possible decay channels and directions of the phonon wave vector $\mathbf{q} \cdot M_{\lambda}(\mathbf{q})$ denotes the scattering matrix element corresponding to the electron-phonon interaction including the piezoelectric and deformation potentials [30]. The expressions for $M_{\lambda}(\mathbf{q})$ are derived considering the three phonon modes $\lambda$ of the bulk zinc-blende crystals, one longitudinal and two transversals, as producing strain and this strain yielding piezoelectricity (see [31] for more details). We assume bulk phonons, which is an appropiate model for embedded QDs. As a consequence, the scattering matrix elements $M_{\lambda}(\mathbf{q})$ does not depend on the QD orientation. All calculations are carried out at zero temperature, thus only phonon emission processes are possible, i.e. those inducing transitions from the first excited to the ground electronic state. The splitting energy between Zeeman sublevels is small so that only acoustic phonons are important and the linear dispersion regime applies $E_{q}=\hbar c_{\lambda} q$, where $c_{\lambda}$ is the velocity of the longitudinal or transversal phonon branch [32]. Note that phonons cannot couple states with opposite spin and the spin admixture caused by SOI is essential for relaxation to take place. 


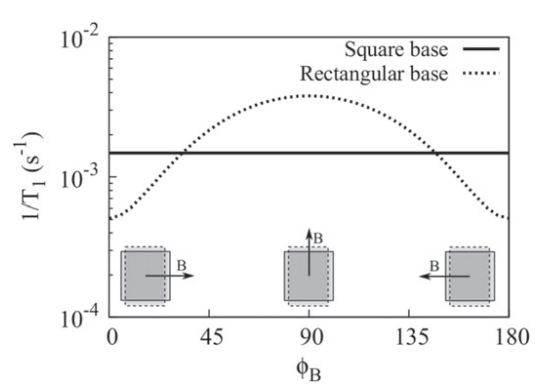

Figure 2. Electron spin relaxation rate as a function of the in-plane magnetic field orientation when only the Rashba SOI contribution is included. QDs of $10 \mathrm{~nm}$ height with rectangular (dotted line) and square base (solid line) are considered.

The eigenvalue problem is solved numerically using a finite difference method on a 3D grid. Accounting for SOI in the calculation of the energy spectra requires high numerical precision due to the small magnitude of this coupling and the presence of third-order derivatives. Accuracy in the derivatives in the finite difference method can be achieved by increasing the number of mesh nodes. However, the $3 \mathrm{D}$ character of the calculations is a serious hindrance, since the number of nodes increases as $n_{x} \cdot n_{y} \cdot n_{z}$, with $n_{i}$ the discretization along the axis $i$. We can also improve accuracy by increasing the points of the discretization of derivatives. We have explored the performance of 5, 7 and 15-point central difference schemes and, after a series of convergence tests, found that a seven-point stencil central difference scheme and a number of 42875 mesh nodes discretizing the 3D system guarantees an appropiate description at a reasonable computational cost. In order to preserve the accuracy we model QDs as hard-wall cuboids fitting exact numbers of nodes, so that the potential energy term does not introduce any additional inaccuracy. This idealized geometry has been shown to capture the basic features of the spin-orbit anisotropy of realistic InAs/GaAs QDs [11], while enabling a simple interpretation in terms of symmetries, which is the goal of this work.

We use GaAs material parameters, particularly electron effective mass $m^{\star}=0.067$, density $\rho=5310 \mathrm{~kg} \mathrm{~m}^{-3}$ , dielectric constant $\epsilon_{r}=12.9$, piezoelectric constant $h_{14}=1.45 \times 10^{9} \mathrm{~V} \mathrm{~m}^{-1}$, g-factor $g=-0.44$ and sound velocities $c_{l}=4720 \mathrm{~m} \mathrm{~s}^{-1}$ and $c_{t}=3340 \mathrm{~m} \mathrm{~s}^{-1}$. [33,34] For the SOI constants, we take $\beta_{d}=27.58 \mathrm{eV} \AA^{3}$ and $\alpha_{r}=5.026 e \AA^{2}$. [8] All simulations are carried out, unless otherwise stated, considering an axial electric field $E_{z}=10 \mathrm{kV} \mathrm{cm}^{-1}$ and an in-plane magnetic field $B_{\|}=1 \mathrm{~T}$.

\section{Results and discussion}

\subsection{Geometry dependence}

We investigate first the relaxation rate anisotropy for different dot geometries when applying an in-plane magnetic field at different orientations. The QDs considered have a base with square $\left(L_{x}=80 \mathrm{~nm}, L_{y}=80 \mathrm{~nm}\right)$ or rectangular $\left(L_{x}=70 \mathrm{~nm}, L_{y}=90 \mathrm{~nm}\right)$ shape and various heights ranging from $L_{z}=10 \mathrm{~nm}$ to $L_{z}=40 \mathrm{~nm}$.

Figure 2 shows the spin relaxation rate when only Rashba SOI is present. ${ }^{1}$ For QDs with square base the relaxation rate is constant for any $\phi_{B}$. In contrast, in rectangular QDs it presents an anisotropic behavior, where the maximum (minimum) corresponds to a magnetic field oriented along the direction of weaker (stronger) confinement. In both cases, $1 / T_{1}$ is independent of the QD height and, for the sake of clarity, only results for $L_{z}=10 \mathrm{~nm}$ are included in figure 2 .

In figure 3(a), we analyze the spin relaxation in the only presence of Dresselhaus SOI for QDs with square base. The relaxation rate for short $\mathrm{QDs}\left(L_{z}=10 \mathrm{~nm}\right)$ is almost isotropic with the orientation of the magnetic field. This is in sharp contrast with taller QDs, where strong quenchings are found at $\phi_{B}=45$ and $\phi_{B}=135$. On the other hand, when the QD base is rectangular, figure 3(b), only moderate modulations of $1 / T_{1}$ are observed. Again, the dependence on $\phi_{B}$ is different depending on the dot height. When $B_{\|}$is oriented along the direction of weaker confinement the relaxation is minimum for QDs with $L_{z}=10 \mathrm{~nm}$, but it changes into a maximum for $L_{z}=20,30,40 \mathrm{~nm}$.

${ }^{1}$ The relaxation is slower than in previous studies (e.g. [14,21]) because in our cuboidal QDs there is no potential gradient, so the only source of inversion asymmetry contributing to equation (2) is the (relatively weak) external field $\mathrm{E}$. The dependence on $\phi_{B}$ we describe below is however largely independent of the strength of the field 
Anisotropy of spin-orbit induced electron spin relaxation in [001] and [111] grown GaAs QDs

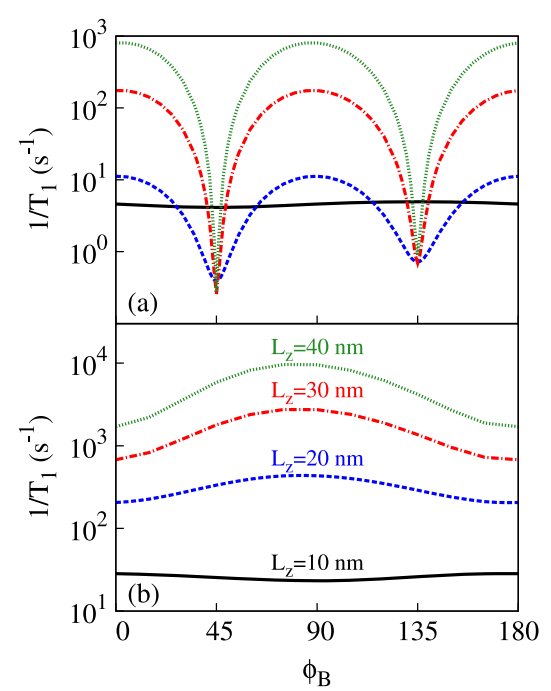

Figure 3. Calculated spin relaxation rate versus magnetic field orientation $\phi_{B}$ considering only Dresselhaus SOI in (a) square and (b) rectangular base QDs. Different dot heights are studied: $L_{z}=10 \mathrm{~nm}$ (solid black line), $L_{z}=20 \mathrm{~nm}$ (blue dashed line), $L_{z}=30 \mathrm{~nm}$ (red dash-dotted line) and $L_{z}=40 \mathrm{~nm}$ (green dotted line).

The preceding results reveal a strong sensitivity of the spin relaxation anisotropy to both the QD symmetry (squared or rectangular) and the QD height. Both factors can induce major, qualitative changes in the anisotropy. To understand such a behavior, we consider that the relaxation rate is proportional to the degree of spin admixture of the initial and final states of the transition, $\Psi_{i}$ and $\Psi_{f}$ in (8) [32]. These states can be approximated as:

$$
\begin{aligned}
& \Psi_{i} \approx \psi_{000}|\downarrow\rangle+c_{x}^{i} \psi_{100}|\uparrow\rangle+c_{y}^{i} \psi_{010}|\uparrow\rangle \\
& \Psi_{f} \approx \psi_{000}|\uparrow\rangle+c_{x}^{f} \psi_{100}|\downarrow\rangle+c_{y}^{f} \psi_{010}|\downarrow\rangle,
\end{aligned}
$$

where $\psi_{i j k}$ represents the electron orbital in the absence of SOI, with ijk the number of nodes in $x, y$ and $z$, respectively, while $|\uparrow\rangle(|\downarrow\rangle)$ represents parallel (antiparallel) spin alignment along the direction of the magnetic field. For the analysis we can focus on $\Psi_{i}$ (analogous reasoning is valid for $\Psi_{f}$ ). $\Psi_{i}$ is mostly a spin down state, with a little SOI induced spin admixture with excited levels. Notice that $\psi_{000}|\uparrow\rangle$ does not contribute to the spin admixture of $\Psi_{i}$ because the parity symmetry in $x$ and $y$ prevents direct SOI coupling with $\psi_{000}|\downarrow\rangle$. Thus, the degree of spin admixture is essentially captured by the coefficients $c_{x}^{i}$ and $c_{y}^{i}$, which can be estimated perturbatively as:

$$
c_{x}^{i}=-\frac{\left\langle\uparrow\left|\left\langle\psi_{100}\left|H_{\text {SOI }}\right| \psi_{000}\right\rangle\right| \downarrow\right\rangle}{\varepsilon_{100 \uparrow}-\varepsilon_{000 \downarrow}},
$$

and

$$
c_{y}^{i}=-\frac{\left\langle\uparrow\left|\left\langle\psi_{010}\left|H_{\text {SOI }}\right| \psi_{000}\right\rangle\right| \downarrow\right\rangle}{\varepsilon_{010 \uparrow}-\varepsilon_{000 \downarrow}} .
$$

The energy separations $\Delta \varepsilon_{x}=\varepsilon_{100 \uparrow}-\varepsilon_{000 \downarrow}$ and $\Delta \varepsilon_{y}=\varepsilon_{010 \uparrow}-\varepsilon_{000 \downarrow}$ do not vary with $\phi_{B}$. Thus, the origin of the anisotropy must be sought in the SOI matrix elements.

We consider first Rashba SOI, i.e. $H_{\mathrm{SOI}}=H_{R}^{[001]}(0)$. From (4) and parity considerations, it follows that, for $\phi_{B}=0$,

$$
c_{x}^{i}=\alpha_{r} E_{z} \frac{\left\langle\uparrow\left|\sigma_{y}\right| \downarrow\right\rangle\left\langle\psi_{100}\left|p_{x}\right| \psi_{000}\right\rangle}{\Delta \varepsilon_{x}}, \quad c_{y}^{i}=0
$$


while for $\phi_{B}=90$,

$$
c_{x}^{i}=0, \quad c_{y}^{i}=\alpha_{r} E_{z} \frac{\left\langle\uparrow\left|\sigma_{x}\right| \downarrow\right\rangle\left\langle\psi_{010}\left|p_{y}\right| \psi_{000}\right\rangle}{\Delta \varepsilon_{y}} .
$$

We see that depending on the orientation of the magnetic field the spin admixture is caused by the coupling to a different excited state. For QDs with square base $\Delta \varepsilon_{x}=\Delta \varepsilon_{y}$, and $\left\langle\psi_{100}\left|p_{x}\right| \psi_{000}\right\rangle=\left\langle\psi_{010}\left|p_{y}\right| \psi_{000}\right\rangle$. Consequently, the degree of spin mixing does not change at $\phi_{B}=0$ and $\phi_{B}=90$, in agreement with the isotropic $1 / T_{1}$ observed in figure 2. Conversely, in rectangular QDs with stronger confinement in $x, \Delta \varepsilon_{x}>\Delta \varepsilon_{y}$. Then, the admixture coefficients at $\phi_{B}=90$ are larger than at $\phi_{B}=0$, which justifies the anisotropy observed in figure 2 .

The anisotropy of Dresselhaus SOI induced spin relaxation, shown in figure 3, can be understood in similar terms. We split equation (3) as $H_{D}^{[001]}=H_{z}+H_{x y}$, where $H_{z}=\beta_{d} p_{z}^{2}\left(p_{y} \sigma_{y}-p_{x} \sigma_{x}\right)$ and

$H_{x y}=H_{x}+H_{y}=\beta_{d}\left[p_{x}^{2}\left(p_{z} \sigma_{z}-p_{y} \sigma_{y}\right)+p_{y}^{2}\left(p_{x} \sigma_{x}-p_{z} \sigma_{z}\right)\right]$. Calculations using these Hamiltonians independently show that $H_{z}$ dominates for $L_{z}=10 \mathrm{~nm}$, in agreement with the usual practice of approximating the Dresselhaus SOI by $H_{z}$ in quasi-2D systems. If we perform a similar analysis for $H_{z}$ as the one carried out for Rashba SOI, we find that coupling to $\psi_{010}$ and $\psi_{100}$ dominates at $\phi_{B}=0$ and $\phi_{B}=90$, respectively. This is exactly the opposite as for the Rashba SOI case, explaining the results obtained for $L_{z}=10 \mathrm{~nm} \mathrm{QDs}$ (see figure 3(b)). As the QD height is increased, however, $H_{x y}$ soon dominates over $H_{z}$. Indeed, for $L_{z}=20 \mathrm{~nm}$ it is already dominant. Considering individually $H_{x}$ and $H_{y}$ it can be shown that they present opposite behaviors with $\phi_{B} . H_{x}$ produces a maximum (minimum) relaxation for $\phi_{B}=90\left(\phi_{B}=0\right)$ and $H_{y}$ for $\phi_{B}=0\left(\phi_{B}=90\right)$. This dependence does not change with the base shape and a stronger confinement in one direction only determines which term, $H_{x}$ or $H_{y}$, prevails. In the rectangular dot of figure $3(\mathbf{b}), L_{x}<L_{y}$ so $H_{x}$ is more important and we observe its angular dependence. Instead, when the dot base is squared $H_{x}$ and $H_{y}$ cancel each other out at $\phi_{B}=45$ and $\phi_{B}=135$, thus giving rise to the pronounced minima of $1 / T_{1}$ observed in figure $3(\mathrm{a})$.

To summarize this section, the spin relaxation anisotropy of [001] grown GaAs QDs is determined by the spin admixture induced by SOI. This is qualitatively different in systems where Rashba or Dresselhaus SOI terms dominate. In the latter case, the anisotropy reflects whether $H_{z}$ or $H_{x y}$ prevails. It turns out that $H_{x y}$ is already dominant for $L_{z}=20 \mathrm{~nm}$ (height-to-base aspect ratio of 1:4), which points out at the early relevance of cubic Dresselhaus terms in structures where 3D starts becoming important. In this case, the use of QDs with symmetric $x-y$ confinement enables strong suppressions of the relaxation at certain magnetic field orientations.

3.1.1. The influence of strong magnetic fields

We study next the spin relaxation angular dependence in square QDs under strong magnetic fields. In such a case, the orbital effects of the magnetic field are expected to play an important role, especially in tall systems. We emphasize the need of a true 3D calculation to account for this effect, since it cannot be properly described using $2 \mathrm{D}$ models [14]. We calculate the relaxation rate for different values of the magnetic field up to $B_{\|}=10 T$ in QDs with $L_{x}=L_{y}=80 \mathrm{~nm}$ and $L_{z}=20 \mathrm{~nm}$.

The impact of the magnetic field strength on the angular dependence through the Rashba SOI is negligible and not shown. We enclose in figure 4 the spin relaxation yielded by the Dresselhaus SOI term only. Figure 4 shows that the minima of $1 / T_{1}$ at $\phi_{B}=45$ and $\phi_{B}=135$ is gradually removed for strong magnetic fields. This behavior can be understood in terms of the differential contribution of $H_{x y}$ and $H_{z}$, as pointed out previously. For QDs with $L_{z}=20 \mathrm{~nm}$ and $B_{\|}=1 T, H_{x y}$ dominates and we observe two pronounced minima (see inset in figure 4). When $B_{\|}$increases, $H_{z}$ rises up and it becomes dominant at $B_{\|}=10 T$, this being responsible for the suppression of the minima. It is noteworthy to mention that an increase in the height of the dot enhances the effects of the magnetic field but also diminishes the contribution of $H_{z}$ to the Hamiltonian. As a consequence, in taller QDs a balance of these two contributions will determine which term, $H_{x y}$ or $H_{z}$, dominates and, therefore, the angular dependence of the spin relaxation.

\subsection{In-plane confinement potential orientation}

In this section, we investigate the impact of the QD orientation with respect to the crystal host on the spin relaxation. The rotation angle $\theta_{z}$ is defined as the angle between the [001] crystal direction and the $x$ axis of the dot, see inset of figure 5 (a) for a schematic representation. All calculations are carried out with the magnetic field $B_{\|}=1 T$ oriented along the $x$ axis of the $\mathrm{QD}$ and an axial electric field $E_{z}=10 \mathrm{kV} \mathrm{cm}^{-1}$.

In figure 5(a), we plot the relaxation rate in the presence of Rashba SOI only for QDs with $L_{z}=10 \mathrm{~nm}$ (results for $L_{z}=20 \mathrm{~nm}$ are identical and are omitted for clarity). We find that $1 / T_{1}$ is not affected by changes in the dot orientation. This result is as expected since (4) does not depend on $\theta_{z}$. 
Anisotropy of spin-orbit induced electron spin relaxation in [001] and [111] grown GaAs QDs

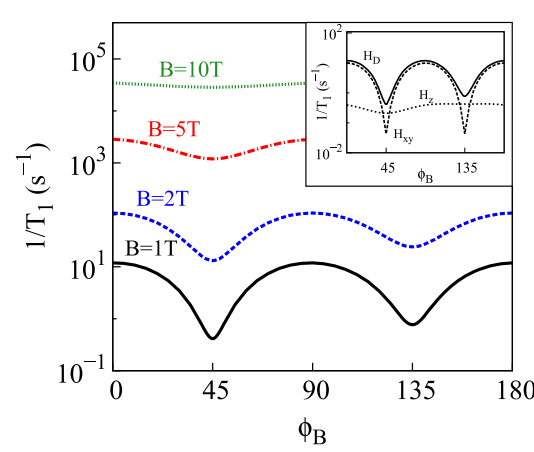

Figure 4. Spin dynamics of a square QD of $L_{z}=20 \mathrm{~nm}$ as a function of $\phi_{B}$ for different magnetic field intensities: $B_{\|}=1 T$ (black solid line), $B_{\|}=2 T$ (blue dashed line), $B_{\|}=5 T$ (red dash-dotted line) and $B_{\|}=10 T$ (green dotted line). Only the presence of Dresselhaus SOI is considered. The inset shows the spin relaxation for $B_{\|}=1 T$ when considering the full Dresselhaus Hamiltonian $H_{D}=H_{x y}+H_{z}$ (solid line), and also the partial contributions of $H_{x y}$ (dashed line) and $H_{z}$ (dotted line).

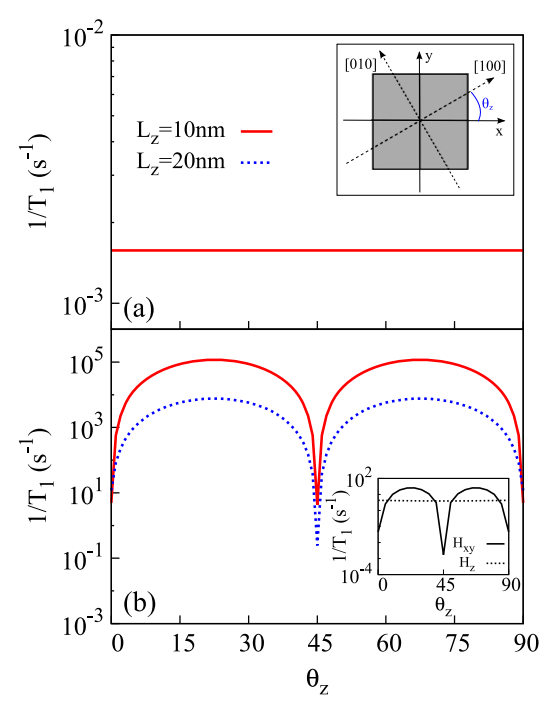

Figure 5. Spin relaxation rate as a function of the dot orientation $\theta_{z}$ for square base QDs with $L_{z}=10 \mathrm{~nm}$ (black solid curve) and $L_{z}=20 \mathrm{~nm}$ (blue dotted curve). Results are shown for (a) pure Rashba SOI and (b) pure Dresselhaus SOI. The in-plane magnetic field $B_{\|}=1 T$ is oriented along the dot $x$ axis $\left(\phi_{B}=0\right)$. The inset in (a) illustrates a representation of the system and the definition of the rotation angle. The inset in (b) shows the relaxation due to $H_{x y}$ and $H_{z}$ in the $L_{z}=10 \mathrm{~nm}$ dot.

For the Dresselhaus SOI case instead, figure 5(b) shows a strong dependence of $1 / T_{1}$ on the confinement potential rotation. In particular, one can see some specific rotation angles, $\theta_{z}=0,45,90$, where the spin relaxation is reduced by $4-5$ orders of magnitude as compared to others. This behavior can be understood from the form of the Hamiltonian in (5). The Dresselhaus SOI presents a $2 \theta_{z}$ dependence, with half of the terms multiplied by $\sin 2 \theta_{z}$ and the other half by $\cos 2 \theta_{z}$. Therefore, the first part of (5) cancels for $\theta_{z}=45$ and the second part for $\theta_{z}=0$ and $\theta_{z}=90$. This suppresses some of the SOI coupling channels, giving rise to slower relaxation rates than for intermediate angles.

It is noteworthy to mention that the dependence on $\theta_{z}$ originates in $H_{x y}$, with $H_{z}$ remaining isotropic, see figure 5(b) inset. This highlights the important role of the cubic terms of the Dresselhaus SOI Hamiltonian in GaAs QDs. As a matter of fact, the inset shows that even in the shortest QDs ( $L_{z}=10 \mathrm{~nm}$ ), save for the vicinity of 


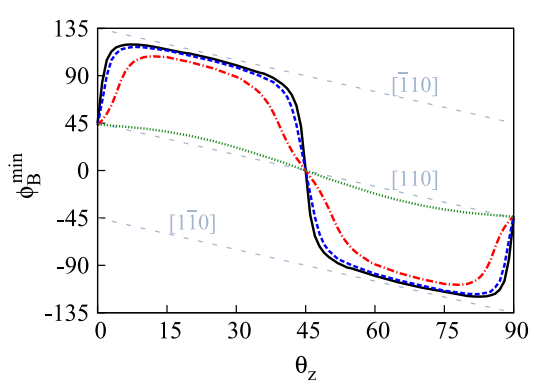

Figure 6. 'Magic' angle as a function of the dot orientation $\theta_{z}$ for a QD with $L_{z}=10 \mathrm{~nm}, L_{x}=80 \mathrm{~nm}$ and various $L_{y}: L_{y}=80 \mathrm{~nm}$ (black solid line), $L_{y}=90 \mathrm{~nm}$ (blue dashed line), $L_{y}=110 \mathrm{~nm}$ (red dash-dotted line) and $L_{y}=150 \mathrm{~nm}$ (green dotted line). The grey dashed lines correspond to $\phi_{B}$ pointing along[110], [i]10] and [11 0$]$ direction for each $\theta_{z}$.

the 'magic' rotation angles $\left(\theta_{z}=0,45,90\right)$ the main contribution to the relaxation rate does not come from $H_{z}$ but from $H_{x y}$.

These results are robust against changes in the QD geometry, such as height and base shape, which do not modify the qualitative trend. In particular, the minimum at $\theta_{z}=45$ remains unaltered while the minima at $\theta_{z}=0$ and $\theta_{z}=90$ are only slightly shifted in rectangular QDs.

Recent experiments by Scarlino and co-workers have also explored spin relaxation anisotropy of GaAs QDs. [22] For their specific QD, they observed a periodicity of 180 degrees in $\phi_{B}$, with a 'magic' angle near [110]. Both the periodicity and the relaxation suppression were explained assuming Rashba and Dresselhaus SOI terms had roughly the same weight and the QD was strongly elongated in one direction. It was shown that the deviation from the [110] direction could arise from the values of $\theta_{z}$ and the Rashba to Dresselhaus SOI strength ratio, which are unknown for their sample. Here we generalize this study by considering QDs with different in-plane shape, from square $\left(L_{x}=L_{y}\right)$ to strongly elongated $\left(L_{x} \ll L_{y}\right)$, and include the cubic Dresselhaus terms which are missing in their analysis. We set Rashba SOI to be as strong as the linear $\left(H_{z}\right)$ Dresselhaus term by setting $\alpha_{r}=\beta_{d}\left\langle p_{z}^{2}\right\rangle / E_{z}$. The results are shown in figure 6 .

One can see that for the strongly elongated QD, $L_{y}=150 \mathrm{~nm}$, the 'magic' angle $\phi_{B}^{\text {min }}$ takes place when the magnetic field points approximately along $[110]\left(\phi_{B}^{\min } \simeq 45-\theta_{z}\right)$. This is consistent with the estimates of Scarlino et al (figure 4(a) in [22]). The small deviations from [110] (dashed grey line in figure 6) are atributed to the influence of the cubic Dresselhaus terms. As the QD elongation is reduced, the anisotropy evolves towards a completely different limit, which is reached for the square QD, $L_{y}=80 \mathrm{~nm}$. In this case, the magic angle remains at [110] for $\theta_{z}=0,45,90$, but it rapidly deviates for any other $\theta_{z}$. For $0<\theta_{z}<45$ it switches to[110] $\left(\phi_{B}^{\min } \simeq 135-\theta_{z}\right)$, while for $45<\theta_{z}<90$ it switches to[110] $\left(\phi_{B}^{\min } \simeq-45-\theta_{z}\right)$. The origin of this distinct behavior is the same discussed in figure 5(b) inset. Namely, when the $x$ axis of the dot does not coincide with [100], [110] or [010], Dresselhaus $H_{x y}$ terms take over $H_{z}$ ones. This breaks the balance between Rashba and $H_{z}$ Dresselhaus SOI described in [22]. Because statistically QDs are likely to be tilted from $\theta_{z}=0,45,90$, it follows that cubic Dresselhaus terms can induce severe deviations from the spin-orbit anisotropy described in the experiment if the QDs are not strongly elongated.

\subsection{Effect of an additional in-plane electric field}

We next explore the influence of applying an in-plane electric field on the spin relaxation anisotropy. We consider the squared QD of section 3.1 with $B_{\|}=1 T$ and $E_{z}=10 \mathrm{kV} \mathrm{cm}^{-1}$, but now we add an additional electric field component $E_{\|}=10 \mathrm{kV} \mathrm{cm}^{-1}$. Calculations are performed rotating the in-plane electric field for some fixed magnetic field orientations.

In figures 7 (a) and (b), we present the relaxation rate obtained for pure Rashba and pure Dresselhaus SOI, respectively, at four different $\phi_{B}$ values. The most remarkable finding is that $1 / T_{1}$ is increased by several orders of magnitude in comparison with the case with only axial electric field (figures 2 and 3 ), although strong suppressions show up at some specific combinations of $\phi_{B}$ and $\phi_{E}$. For Rashba SOI the combination is $\phi_{B}-\phi_{E}=90,270$ and for Dresselhaus SOI $\phi_{B}+\phi_{E}=0,180$. Changes in the QD geometry do not modify significantly the qualitative results shown in figure 7 . Only small displacements of the cancellation angles and the moderation of some minima occur.

The influence of the in-plane electric field can be explained from the fact that $E_{\|}$breaks the parity symmetry in the direction $\phi_{E}$. This enables the otherwise forbidden SOI coupling between the Zeeman sublevels $\psi_{000}|\uparrow\rangle$ 
Anisotropy of spin-orbit induced electron spin relaxation in [001] and [111] grown GaAs QDs

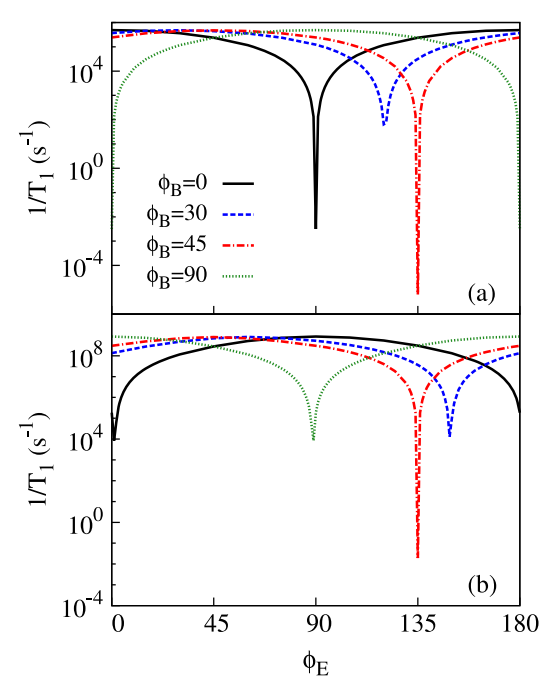

Figure 7. Electron spin relaxation as a function of the in-plane electric field orientation $\phi_{E}$ considering (a) only Rashba SOI and (b) only Dresselhaus SOI. The QDs studied have square base and $L_{z}=10 \mathrm{~nm}$. Calculations with the magnetic field oriented at some fixed angles are presented: $\phi_{B}=0$ (black solid line), $\phi_{B}=30$ (blue dashed line), $\phi_{B}=45$ (red dash-dotted line) and $\phi_{B}=90$ (green dotted line).

and $\psi_{000}|\downarrow\rangle \operatorname{in} \Psi_{i}$ and $\Psi_{f}$ (recall section 3.1). Since these states are very close in energy, the ensuing spin admixture is important, which justifies the large enhancement of $1 / T_{1}$. In order to understand the minima we carry out a similar perturbative analysis to that of section 3.1 but now focusing on the coupling between the two $\psi_{000}$ sublevels. Let us consider first the Dresselhaus SOI term. Assuming $H_{D}^{[001]} \approx H_{z}$ (as is the case for quasi-2D QDs and $\left.\theta_{z}=0\right)$, the $\phi_{B}=0$ matrix element is:

$$
\left\langle\psi_{000}\left\langle\uparrow\left|H_{z}\right| \psi_{000} \mid \downarrow\right\rangle=\beta_{d}\left\langle\downarrow\left|\sigma_{y}\right| \uparrow\right\rangle\left\langle\psi_{000}\left|p_{z}^{2} p_{y}\right| \psi_{000}\right\rangle .\right.
$$

The integral of the orbital part in (14) vanishes when $\phi_{E}=0$ because of the odd parity along $y$, but other orientations of the electric field break the parity symmetry in the $y$ direction and then $1 / T_{1}$ increases, as seen in figure 7(b) (black line). Similar reasoning shows that for $\phi_{B}=90$ the parity-induced minimum occurs at $\phi_{E}=90$. For intermediate magnetic field angles, however, the minimum no longer takes place when $E_{\|} \| \mathbf{B}$. Indeed, for $\phi_{B}=45$, the minimum is found at $\phi_{E}=135\left(E_{\|} \perp \mathrm{B}\right)$. To explain this, it is convenient to rotate the coordinate system 45 degrees from $(x, y)$ into $\left(x^{\prime}, y^{\prime}\right)$ so that the $\mathrm{x}^{\prime}$ axis is aligned with the direction of $\mathrm{B}$. As inferred from (5), the resulting SOI term is $H_{z}^{45}=\beta_{d} p_{z}^{2}\left(\sigma_{y^{\prime}} p_{x}^{\prime}+\sigma_{x^{\prime}} p_{y}^{\prime}\right)$ and the matrix element becomes:

$$
\left\langle\psi_{000}\left\langle\uparrow\left|H_{z}^{45}\right| \psi_{000} \mid \downarrow\right\rangle=\beta_{d}\left\langle\downarrow\left|\sigma_{y^{\prime}}\right| \uparrow\right\rangle\left\langle\psi_{000}\left|p_{z}^{2} p_{y}^{\prime}\right| \psi_{000}\right\rangle .\right.
$$

This integral vanishes due to the odd parity in x' when $E_{\|}$is parallel to the y' axis, i.e. when $\phi_{E}=135$ in the initial coordinate frame, in agreement with figure 5(b).

The minima in the presence of Rashba SOI can be explained in similar terms, but because $H_{R}^{[001]}$ has rotational symmetry, see equation (4), it does not change when rotating the coordinate system. Then, the minima always take place for $E_{\|} \perp \mathrm{B}$.

To summarize this section, the presence of in-plane electric fields greatly enhances spin relaxation due to the lowered orbital symmetry, but the anisotropy of both Rashba and Dresselhaus SOI makes it possible to find relative angles between $E_{\|}$and $\mathrm{B}$ such that the relaxation is severely reduced.

\section{4. [111] Grown QDs}

In figure 8 we plot the spin relaxation rate for the squared QD studied in section 3.1, but now considering the dot is grown along the [111] crystal direction. In general, faster relaxation rates are obtained for this orientation as compared to the [001] grown QDs. Interestingly, we observe the same angular dependence for both Rashba SOI (figure 8(a)) and Dresselhaus SOI (figure 8(b)). Both mechanisms show strong suppressions at $\phi_{B}=135$ and $\phi_{B}=315$. However, when increasing $L_{z}$ Rashba and Dresselhaus SOI mechanisms show opposite behaviors and 


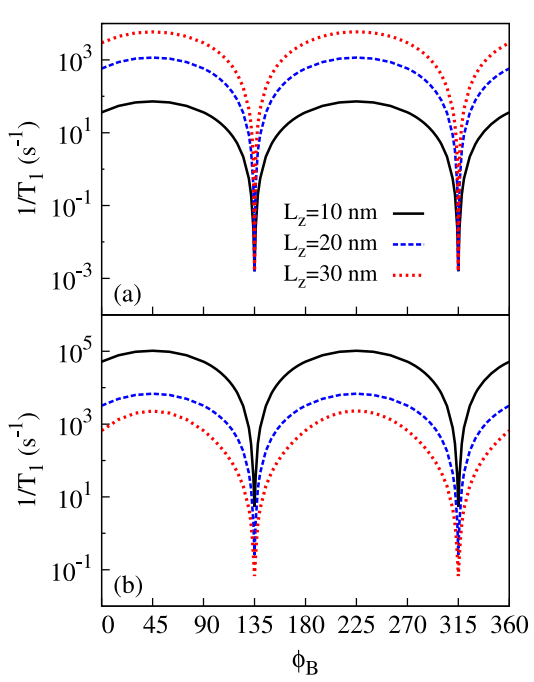

Figure 8. Electron spin dynamics of square QDs grown along the [111] crystallographic direction as a function of the magnetic field orientation. Simulations considering (a) the Rashba SOI and (b) the Dresselhaus SOI are included for three QD heights: $L_{z}=10 \mathrm{~nm}$ (black solid curve), $L_{z}=20 \mathrm{~nm}$ (blue dashed curve) and $L_{z}=30 \mathrm{~nm}$ (red dotted curve).

$1 / T_{1}$ increases and decreases, respectively. Therefore, the dot height determines which of the coupling mechanisms dominates.

The cancellation angles of the relaxation in figure 8 can be justified noting that the canonical momenta $p_{x}=-i \hbar \mathrm{d} / \mathrm{d}_{x}+z B \sin \phi_{B}$ and $p_{y}=-i \hbar \mathrm{d} / \mathrm{d}_{y}-z B \cos \phi_{B}$ have exactly the same form for $\phi_{B}=135$ and $\phi_{B}=315$ since $L_{x}=L_{y}$. As a result, the first term in (6) and several terms in (7) cancel out, yielding two sharp minima in the scattering rate curve.

The identical anisotropy of Rashba and Dresselhaus SOI induced spin relaxation in [111] QDs revealed by figure 8, which is a consequence of the formal equivalences between $H_{R}^{[111]}$ and $H_{D}^{[111]}$, [35], facilitates in practice the simultaneous quenching of both mechanisms. For magnetic fields where hyperfine interaction is negligible and square dots, this should lead to spin lifetimes in the range of seconds. We have further checked that changes in the QD base shape do not modify the qualitative behavior reported above, the minima being only slightly shifted for rectangular dots under Dresselhaus SOI.

\section{Conclusions}

We have investigated systematically the electron spin scattering anisotropy in 3D cuboidal GaAs QDs grown along the [001] and [111] directions. We have shown that the relaxation rate can be controlled by several orders of magnitude by varying the in-plane orientation of external magnetic and electric fields.

In [001] grown QDs under an axial electric field, the spin relaxation in-plane anisotropy is strongly dependent on the QD geometry and the nature of the dominating SOI term. For Rashba SOI, the relaxation is isotropic or anisotropic when the base is squared and rectangular, respectively, and it is not affected by changes in the QD height. On the other hand, for Dresselhaus SOI, the relaxation presents a different behavior depending not only on the base shape, but also on the QD height. In fact, short and tall dots can even show contrary angular dependence, evidencing the important role of QD 3D. In addition, we have demonstrated that the isotropic/ anisotropic behavior can be controlled by changing the magnetic field strength.

We have also shown that rotating the confinement potential in-plane with respect to the crystal structure causes an important modulation of the spin relaxation, that is severely suppressed when the high symmetry directions of the QD confinement match the main crystallographic axes. This modulation arises from the cubic Dresselhaus terms, which are important even for small heights. Such terms can explain the deviation of the slow spin relaxation direction of the magnetic field away from [110], as measured in very recent experiments [22], for strongly elongated QDs. For less elongated structures they can even switch it to [110] or [ 110$]$. 
Anisotropy of spin-orbit induced electron spin relaxation in [001] and [111] grown GaAs QDs

An additional in-plane electric field component causes a strong increase in the relaxation rate, but certain combinations of $\phi_{B}$ and $\phi_{E}$ lead to enhanced spin lifetimes. We find that these combinations are different for Rashba, $\phi_{B}-\phi_{E}=90,270$, and Dresselhaus SOI, $\phi_{B}+\phi_{E}=0,180$.

We have further studied QDs grown along the [111] direction. We have found that Rashba and Dresselhaus SOI present the same angular dependence with $\phi_{B}$, with pronounced minima at certain magnetic field orientations. This enables simultaneous suppression of Rashba and Dresselhaus SOI induced spin relaxation, which is an advantadge as compared to more conventional [001] grown QDs.

\section{Acknowledgments}

This work was supported by UJI-Bancaixa Project No. P1-1B2011-01, MINECO Project No. CTQ2011-27324, and FPU Grant (CS).

\section{References}

[1] Awschalom D D, Loss D and Samarth N 2002 Semiconductor Spintronics and Quantum Computation (Berlin: Springer)

[2] Fabian J, Matos-Abiague A, Ertler C, Stano P and Žutić I 2007 Acta Phys. Slovaca 57565

[3] Loss D and DiVincenzo D P 1998 Phys. Rev. A 57120

4] Hanson R, Kouwenhoven L P, Petta J R, Tarucha S and Vandersypen L M K 2007 Rev. Mod. Phys. 791217

[5] Khaetskii A V and Nazarov Y V 2000 Phys. Rev. B 6112639

[6] Dresselhaus G 1955 Phys. Rev. 100580

[7] Bychkov Y A and Rashba E I 1984 J. Phys. C 176039

[8] Winkler R 2003 Spin-Orbit Coupling Effects in Two-Dimensional Electron and Hole Systems (Berlin: Springer)

[9] Könemann J, Haug R J, Maude D K, Fal'ko V I and Altshuler B L 2005 Phys. Rev. Lett. 94226404

[10] Takahashi S, Deacon R S, Yoshida K, Oiwa A, Shibata K, Hirakawa K, Tokura Y and Tarucha S 2010 Phys. Rev. Lett. 104246801

[11] Nowak M P and Szafran B 2013 Phys. Rev. B 87205436

[12] Fal'ko V I, Altshuler B L and Tsyplyatyev O 2005 Phys. Rev. Lett. 95076603

[13] Destefani C F and Ulloa S E 2005 Phys. Rev. B 72115326

[14] Stano P and Fabian J 2006 Phys. Rev. Lett. 96186602

[15] Olendski O and Shahbazyan TV 2007 Phys. Rev. B 75041306

16] Amasha S, MacLean K, Radu I P, Zumbühl D M, Kastner M A, Hanson M P and Gossard A C 2008 Phys. Rev. Lett. 100046803

[17] Prabhakar S, Melnik R and Bonilla L L 2013 Phys. Rev. B 87235202

[18] Stano P and Fabian J 2006 Phys. Rev. B 74045320

[19] Nowak M P, Szafran B, Peeters F M, Partoens B and Pasek W J 2011 Phys. Rev. B 83245324

[20] Stepanenko D, Rudner M, Halperin B I and Loss D 2012 Phys. Rev. B 85075416

[21] Golovach V N, Khaetskii A and Loss D 2004 Phys. Rev. Lett. 93016601

[22] Scarlino P, Kawakami E, Stano P, Shafiei M, Reichl C, Wegscheider W and Vandersypen L M K 2014 Phys. Rev. Lett. 113256802

[23] Planelles J, Climente J I and Segarra C 2012 J. Phys. Chem. C 11625143

[24] Dalacu D, Mnaymneh K, Wu X, Lapointe J, Aers G C, Poole P J and Williams R L 2011 Appl. Phys. Lett. 98251101

[25] Pfund A, Shorubalko I, Leturcq R and Ensslin K 2006 Appl. Phys. Lett. 89252106

[26] Mano T, Abbarchi M, Kuroda T, McSkimming B, Ohtake A, Mitsuishi K and Sakoda K 2010 Appl. Phys. Express 3065203 and references therein

[27] Vurgaftman I and Meyer J R 2005 J. Appl. Phys. 97053707

[28] Biermann K, Hernández-Mínguez A, Hey R and Santos P V 2012 J. Appl. Phys. 112083913

[29] Balocchi A, Amand T, Wang G, Liu B L, Renucci P, Duong Q H and Marie X 2013 New J. Phys. 15095016

[30] Climente J I, Bertoni A, Goldoni G and Molinari E 2006 Phys. Rev. B 74035313

[31] Climente J I, Segarra C and Planelles J 2013 New J. Phys. 15093009

[32] Khaetskii A V and Nazarov Y V 2001 Phys. Rev. B 64125316

[33] Vurgaftman I, Meyer J R and Ram-Mohan L R 2001 J. Appl. Phys. 895815

[34] Madelung O (ed) 1982 Semiconductors. Physics of Group IV Elements and III-VCompounds (Landolt-Börnstein vol 17) (Berlin: Springer)

[35] Žutić I, Fabian J and Das Sarma S 2004 Rev. Mod. Phys. 76323 



\title{
Spin-orbit-induced hole spin relaxation in InAs and GaAs quantum dots
}

\author{
J I Climente, C Segarra and J Planelles ${ }^{1}$ \\ E-12080 Castelló, Spain \\ E-mail: josep.planelles@qfa.uji.es \\ New Journal of Physics 15 (2013) 093009 (21pp) \\ Received 4 April 2013 \\ Published 5 September 2013 \\ Online at http://www.njp.org/ \\ doi:10.1088/1367-2630/15/9/093009
}

Departament de Química Física i Analítica, Universitat Jaume I, Box 224,

\begin{abstract}
We study the effect of valence band spin-orbit interactions (SOI) on the acoustic phonon-assisted spin relaxation of holes confined in quantum dots (QDs). Heavy hole-light hole (hh-lh) mixing and all the spin-orbit terms arising from zinc-blende bulk inversion asymmetry (BIA) are considered on equal footing in a fully three-dimensional Hamiltonian. We show that hh-lh mixing and BIA have comparable contributions to the hole spin relaxation in self-assembled QDs, but BIA becomes dominant in gated QDs. Simultaneously accounting for both mechanisms is necessary for quantitatively correct results in quasi-two-dimensional QDs. The dependence of the hole spin relaxation on the QD geometry and spin splitting energy is drastically different from that of electrons, with a non-monotonic behavior which results from the interplay between SOI terms. Our results reconcile contradictory predictions of previous theoretical works and are consistent with experiments.
\end{abstract}

${ }^{1}$ Author to whom any correspondence should be addressed.

(i) Content from this work may be used under the terms of the Creative Commons Attribution 3.0 licence. Any further distribution of this work must maintain attribution to the author(s) and the title of the work, journal citation and DOI.

New Journal of Physics 15 (2013) 093009

$1367-2630 / 13 / 093009+21 \$ 33.00$

() IOP Publishing Ltd and Deutsche Physikalische Gesellschaft 


\section{Contents}

1. Introduction 2

2. Theoretical model 4

2.1. Hamiltonian . . . . . . . . . . . . . . . . . . . . . 4

2.2. Relaxation rate . . . . . . . . . . . . . . . . . . 8

3. Numerical results and discussion 9

3.1. Geometry and spin splitting dependence . . . . . . . . . . . . . . . . . . . 10

3.2. Mechanism of spin admixture . . . . . . . . . . . . . . . . 12

3.3. Light hole spin relaxation . . . . . . . . . . . . . . . . . . . . 15

3.4. Mechanism of hole-phonon coupling . . . . . . . . . . . . . 16

4. Summary 18

Acknowledgments $\quad 18$

Appendix. Dresselhaus Hamiltonian for holes 18

References $\quad 19$

\section{Introduction}

Over the last few years, the spin of holes confined in III-V semiconductor quantum dots (QDs) has emerged as a promising building block for spintronic and spin-based quantum information devices [1]. As compared to electrons, the p-like nature of the hole orbitals leads to weaker hyperfine interaction with the lattice nuclei, resulting in coherence times which hold promise for applications [2-7]. As a matter of fact, demonstrations of hole spin manipulation in QDs have been recently reported [8-10] and theoretical proposals of control mechanisms are being proposed [11-14]. In this context, the study of hole spin relaxation has become a subject of interest. Hole spin relaxation is also important for optical applications because it is believed to rule the exciton spin dynamics in both dark-to-bright exciton transitions [15-18], which affect exciton storage times [19-21], and transitions within the bright doublet [22], which affect light depolarization [17].

Experimental observations in self-assembled InAs QDs point at hole spin lifetimes ranging from $T_{1}^{\mathrm{h}} \sim 10 \mathrm{ps}$ to $1 \mathrm{~ms}$ [2, 23-26]. The large dispersion is partly attributed to the different relaxation mechanisms involved in different studies. When the energy splitting between orthogonal spin states is small, hyperfine interaction is the dominant relaxation channel [16, 27]. In this case, the lifetime is strongly dependent on the degree of hh-lh mixing. If the hole state is a pure hh, as in the ground state of flat (quasi-two-dimensional (2D)) QDs, the hyperfine interaction takes an Ising form and spin relaxation is slow, but it rapidly increases in non-flat QDs due to hh-lh mixing $[1,7]$. On the other hand, when the energy splitting exceeds the nuclear magnetic field, the valence band spin-orbit interaction (SOI) takes over as the main source of relaxation $[16,27]$. Long hole spin lifetimes have then been observed, reaching up to $T_{1}^{\mathrm{h}} \sim 0.25 \mathrm{~ms}$, which is only five to ten times shorter than electron spin lifetimes, $T_{1}^{\mathrm{e}}$ [26]. This result is encouraging for the use of holes in quantum information and optical applications, but it is surprising because the valence band $\mathrm{SOI}$ is known to be much stronger than that of the conduction band [28].

The above paradox has prompted a number of theoretical works trying to understand which factors determine the relaxation dynamics of single holes under magnetic fields [29-33] and 
that of holes forming excitons $[15,22,34,35]$ in quasi-2D InAs/GaAs QDs. For the relaxation to take place one needs a source of energy relaxation, which in these systems is provided by the acoustic phonon bath [23, 24], plus a source of spin admixture. Woods et al [29] and Lu et al [30] proposed that the latter is the coupling between hh and lh subbands. Other authors have suggested instead that the splitting between hh and lh subbands in flat QDs is large owing to confinement and strain, so that spin admixture must be due to other SOI mechanisms. It was then proposed that hole SOI should have an origin similar to that of conduction electrons, namely the bulk inversion asymmetry (BIA) of zinc-blende crystals, which gives rise to Dresselhaus SOI terms [28]. Bulaev and Loss assumed that the cubic-in- $k$ Dresselhaus term is dominant and showed that $T_{1}^{\mathrm{h}}$ could then become comparable to $T_{1}^{\mathrm{e}}$ in flat QDs [31]. Other studies followed this assumption and succeeded in explaining some experimental observations [15, 26, 32]. By contrast, Tsitsishvili et al [35] suggested that if the lateral confinement is weak, it is the linearin- $k$ term that dominates the mixing. Last, Roszak et al [34] suggested that for holes forming excitons, it is the electron-hole $(\mathrm{e}-\mathrm{h})$ exchange interaction together with the strain that gives rise to hole spin admixture.

It is worth noting that all the previous works assumed a dominating SOI mechanism without actually comparing it with others. In addition, simplified models disregarding hh-lh mixing become highly parametric, and different parameters were needed to explain different experimental observations even in the same system [15, 26, 32]. The lack of a comprehensive study translates into many open questions which show that hole spin relaxation in QDs is still not fully understood. To name a few: (i) while Woods et al [29] predict that $T_{1}^{\mathrm{h}}$ decreases with the QD diameter, Lu et al [30] predict exactly the opposite behavior; (ii) Bulaev and Loss [31] predicted $T_{1}^{\mathrm{h}}>T_{1}^{\mathrm{e}}$ in the limit of 2D QDs, but experiments on self-assembled QDs have only shown $T_{1}^{\mathrm{e}} / T_{1}^{\mathrm{h}}=5-10$ [26], so that one wonders if any realistic QD structure would actually permit holes relaxing slower than electrons; (iii) in excitons, the role of $\mathrm{e}-\mathrm{h}$ exchange energy is not clear: while experiments with self-assembled QDs have shown negligible dependence of $T_{1}^{\mathrm{h}}$ [24], a strong dependence has been found in colloidal quantum rods [36].

In this work, we aim at covering the existing gap in the understanding of hole spin relaxation in QDs. We study the hole spin dynamics considering simultaneously the most relevant intrinsic SOI terms of III-V QDs, namely hh-lh mixing and all the different Dresselhaus SOI terms arising from the BIA of zinc-blende crystals, along with the hole-acoustic phonon coupling. All the terms are described within a four-band $k \cdot p$ formalism and three-dimensional (3D) Hamiltonians, which allows us to provide a general overview on the effect of the QD size and geometry dependence while relying on well-known bulk parameters only. In this way, we are able to establish the regime of validity of previous studies which assumed a single dominating SOI mechanism. Furthermore, we explore spheroidal QDs beyond the usual quasi-2D limit, thus providing theoretical assessment for developing experimental research with spherical and prolate QDs [18, 36].

We find that hh-lh is the main SOI channel in prolated or spherical QDs, but Dresselhaus SOI has a comparable contribution in oblated QDs (such as self-assembled dots), and it becomes dominant in quasi-2D QDs with very weak confinement (as in electrostatically confined dots). The competition between SOI coupling terms and the energy splitting between hh and lh leads to a non-monotonic dependence of $T_{1}^{\mathrm{h}}$ with the QD geometry, in sharp contrast with the well-known case of electrons. This explains the opposite trends reported by different theoretical studies in the literature. The dependence of $T_{1}^{\mathrm{h}}$ on the $\mathrm{e}-\mathrm{h}$ exchange energy we predict is consistent with experiments on colloidal nanorods [36], but it suggests that 
two-phonon processes are relevant in self-assembled QDs. In prolate QDs, where the ground state is formed by $\mathrm{lh}$, the spin relaxation is shown to take place in similar timescales as for transitions between hh states. However, the coupling to acoustic phonons is different, with deformation potential interaction being the main mechanism even for vanishing spin splitting energy.

\section{Theoretical model}

We study the spin relaxation of holes confined in zinc-blende QDs grown along the [001] direction. The hole spin states are considered split energetically, for example by the $\mathrm{e}-\mathrm{h}$ exchange interaction in excitons or any other source that can be viewed as an effective axial magnetic field. Thus, similar results can be expected for transitions between Zeeman sublevels under moderate external magnetic fields.

\subsection{Hamiltonian}

The system Hamiltonian reads

$$
H=H_{\mathrm{h}}+H_{\mathrm{ph}}+H_{\mathrm{h}-\mathrm{ph}} \text {. }
$$

In equation (1), $H_{\mathrm{h}}$ is the hole Hamiltonian

$$
H_{\mathrm{h}}=H_{\mathrm{L}}+H_{\mathrm{BIA}}+V_{\mathrm{QD}} \mathcal{I}+H_{Z},
$$

where $H_{\mathrm{L}}$ is the four-band Luttinger Hamiltonian describing the coupled hh-lh bands [37]. It includes quadratic terms in $k$ only:

$$
\begin{aligned}
H_{\mathrm{L}}=\frac{1}{m_{0}}\left[\left(\gamma_{1}\right.\right. & \left.+\frac{5}{2} \gamma_{2}\right) \frac{k^{2}}{2}-\gamma_{2}\left(k_{x}^{2} J_{x}^{2}+k_{y}^{2} J_{y}^{2}+k_{z}^{2} J_{z}^{2}\right) \\
& \left.\quad-2 \gamma_{3}\left(\left\{k_{x}, k_{y}\right\}\left\{J_{x}, J_{y}\right\}+\left\{k_{y}, k_{z}\right\}\left\{J_{y}, J_{z}\right\}+\left\{k_{z}, k_{x}\right\}\left\{J_{z}, J_{x}\right\}\right)\right],
\end{aligned}
$$

where $m_{0}$ is the free electron mass, $\gamma_{i}$ are the Luttinger parameters, $k_{j}=-\mathrm{i} \hbar \partial_{j}$ the $j$ component of the linear momentum, $\{A, B\}=\frac{1}{2}(A B+B A)$ and $J_{i}$ is the $i$ th component of the angular momentum corresponding to the quantum number $J=3 / 2$. To obtain the matrix representation of this Hamiltonian we multiply the first term of equation (3) by the $4 \times 4$ unit matrix and employ the standard matrix representation of the $J=3 / 2$ components of the angular momentum [38]. We finally obtain

$$
H_{\mathrm{L}}=\left(\begin{array}{cccc}
P+Q & -S & R & 0 \\
-S^{\dagger} & P-Q & 0 & R \\
R^{\dagger} & 0 & P-Q & S \\
0 & R^{\dagger} & S^{\dagger} & P+Q
\end{array}\right)
$$

with

$$
\begin{aligned}
& P=\frac{1}{2 m_{0}} \gamma_{1}\left(k_{x}^{2}+k_{y}^{2}+k_{z}^{2}\right), \\
& Q=\frac{1}{2 m_{0}} \gamma_{2}\left(k_{x}^{2}+k_{y}^{2}-2 k_{z}^{2}\right),
\end{aligned}
$$




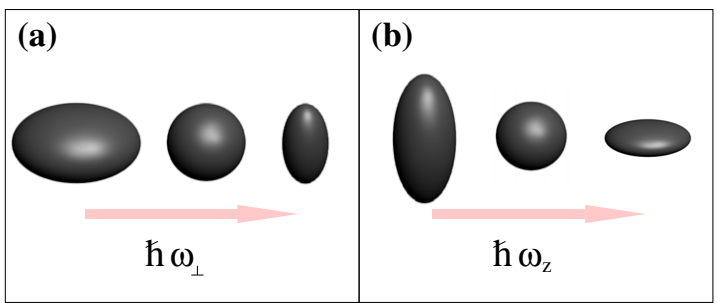

Figure 1. Geometry of QDs with varying lateral (a) and vertical (b) confinement frequency.

$$
\begin{aligned}
& R=\frac{1}{2 m_{0}}\left[-\sqrt{3} \gamma_{2}\left(k_{x}^{2}-k_{y}^{2}\right)+2 \mathrm{i} \sqrt{3} \gamma_{3} k_{x} k_{y}\right], \\
& S=\frac{1}{2 m_{0}} 2 \sqrt{3} \gamma_{3}\left(k_{x}-\mathrm{i} k_{y}\right) k_{z} .
\end{aligned}
$$

$H_{\mathrm{BIA}}$ includes the linear and the Dresselhaus SOI third order in $k$ terms [28]:

$$
\begin{gathered}
H_{\mathrm{BIA}}=\frac{2}{\sqrt{3}} C_{k}\left[k_{x}\left\{J_{x}, J_{y}^{2}-J_{z}^{2}\right\}+\mathrm{cp}\right]+b_{41}\left(\left\{k_{x}, k_{y}^{2}-k_{z}^{2}\right\} J_{x}+\mathrm{cp}\right)+b_{42}\left(\left\{k_{x}, k_{y}^{2}-k_{z}^{2}\right\} J_{x}^{3}+\mathrm{cp}\right) \\
+b_{51}\left(\left\{k_{x}, k_{y}^{2}+k_{z}^{2}\right\}\left\{J_{x}, J_{y}^{2}-J_{z}^{2}\right\}+\mathrm{cp}\right)+b_{52}\left(k_{x}^{3}\left\{J_{x}, J_{y}^{2}-J_{z}^{2}\right\}+\mathrm{cp}\right),
\end{gathered}
$$

where $C_{k}, b_{41}, b_{42}, b_{51}$ and $b_{52}$ are material-dependent coefficients and cp stands for cyclic permutations of the preceding terms. The matrix form of the Hamiltonian terms above is given in the appendix. One can note that all BIA terms provide direct mixing between hh spin-up and spin-down $\left(J_{z}=+3 / 2\right.$ and $\left.-3 / 2\right)$ components except for $b_{41}$, which requires the participation of the $\operatorname{lh}\left(J_{z}=+1 / 2\right.$ and $\left.-1 / 2\right)$ components. Rashba SOI is neglected in this study because it is an extrinsic effect, which depends on the details of the electric field felt by the system. Besides, for holes it couples energetically distant states so that, under moderate external fields, it is less efficient than Dresselhaus SOI [31]. $V_{\mathrm{QD}}$ describes the confining potential of the QD. We model QDs with parabolic confinement in the $x, y$ and $z$ directions:

$$
V_{\mathrm{QD}}=-\frac{1}{2} \chi_{\perp}\left(x^{2}+y^{2}\right)-\frac{1}{2} \chi_{z} z^{2}
$$

where $\chi_{\perp}$ and $\chi_{z}$ are the force constants perpendicular and parallel to the growth direction, respectively. Equation (10) allows us to simulate 3D spheroidal QDs with different aspect ratios, from flat (quasi-2D) to spherical or elongated (quasi-one-dimensional (1D)) structures, see figure $1 . H_{Z}$ is the Hamiltonian modeling the splitting of the hole states by an effective axial magnetic field, three times larger for heavy $\left(\left|J_{z}\right|=3 / 2\right)$ than for light holes $\left(\left|J_{z}\right|=1 / 2\right)$. This field could originate, e.g. from the e-h exchange interaction [34] or a spin Zeeman effect. Then, we assume that

$$
H_{\mathrm{Z}}=\frac{1}{2}\left(\begin{array}{cccc}
\Delta & 0 & 0 & 0 \\
0 & \frac{1}{3} \Delta & 0 & 0 \\
0 & 0 & -\frac{1}{3} \Delta & 0 \\
0 & 0 & 0 & -\Delta
\end{array}\right)
$$


To calculate the hole states from $H_{\mathrm{h}}$, we note that the diagonal terms correspond to harmonic oscillator Hamiltonians:

$$
P+Q+V_{\mathrm{QD}}=T_{\mathrm{hh}, \perp}-\frac{1}{2} \chi_{\perp}\left(x^{2}+y^{2}\right)+T_{\mathrm{hh}, z}-\frac{1}{2} \chi_{z} z^{2}
$$

and

$$
P-Q+V_{\mathrm{QD}}=T_{l h, \perp}-\frac{1}{2} \chi_{\perp}\left(x^{2}+y^{2}\right)+T_{\mathrm{lh}, z}-\frac{1}{2} \chi_{z} z^{2},
$$

where $T_{i, j}=\frac{\hbar^{2}}{2 m_{j}^{i}} k_{j}^{2}$, with $i=(\mathrm{hh}, \mathrm{hh}), j=(\perp, z), k_{\perp}=\left(k_{x}^{2}+k_{y}^{2}\right)^{1 / 2}, m_{\perp}^{\mathrm{hh}}=m_{0} /\left(\gamma_{1}+\gamma_{2}\right)$, $m_{z}^{\mathrm{hh}}=m_{0} /\left(\gamma_{1}-2 \gamma_{2}\right), m_{\perp}^{\mathrm{lh}}=m_{0} /\left(\gamma_{1}-\gamma_{2}\right)$ and $m_{z}^{\mathrm{lh}}=m_{0} /\left(\gamma_{1}+2 \gamma_{2}\right)$. This suggests rewriting all derivatives and coordinates of $H_{\mathrm{h}}$ in terms of harmonic oscillator ladder operators and then projecting it onto a basis formed by oscillator eigenstates. The problem is that equation (12a) has hh masses while equation $(12 b)$ has lh masses, and hence they have different oscillator frequency, $\omega_{j}^{i}=\left(\chi_{j} / m_{j}^{i}\right)^{1 / 2}$. Because the off-diagonal terms of $H_{\mathrm{L}}$ couple hh and lh components, it is convenient to use a single kind of oscillator state, e.g. hh state. This can be done by rewriting equation $(12 b)$ in terms of the hh harmonic oscillator Hamiltonians:

$P-Q+V_{\mathrm{QD}}=\frac{\gamma_{1}-\gamma_{2}}{\gamma_{1}+\gamma_{2}} H_{\mathrm{hh}, \perp}-\frac{\gamma_{2}}{\gamma_{1}+\gamma_{2}} \chi_{\perp}\left(x^{2}+y^{2}\right)+\frac{\gamma_{1}+2 \gamma_{2}}{\gamma_{1}-2 \gamma_{2}} H_{\mathrm{hh}, z}+\frac{2 \gamma_{2}}{\gamma_{1}-2 \gamma_{2}} \chi_{z} z^{2}$,

where $H_{\mathrm{hh}, \perp}=T_{\mathrm{hh}, \perp}-\chi_{\perp}\left(x^{2}+y^{2}\right) / 2$ and $H_{\mathrm{hh}, z}=T_{\mathrm{hh}, z}-\chi_{z}\left(z^{2}\right) / 2$. The resulting hole states are Luttinger spinors of the form

$$
\left|\Psi_{m}^{\mathrm{h}}\right\rangle=\sum_{r, J_{z}} c_{r, J_{z}}^{m}\left|v_{x}, v_{y}, v_{z}\right\rangle\left|3 / 2, J_{z}\right\rangle
$$

where $v_{j}=0,1,2 \ldots$ is the quantum number of the $1 \mathrm{D}$ hh harmonic oscillator along the $j$ direction, $r$ is the combined orbital quantum number, $r=\left(v_{x}, v_{y}, v_{z}\right)$ and $\left|3 / 2, J_{z}\right\rangle$ the Bloch function.

$H_{\mathrm{ph}}$ in equation (1) is the Hamiltonian of acoustic phonons, given by $H_{\mathrm{ph}}=$ $\sum_{\mathbf{q} \lambda} \hbar \omega_{q \lambda} a_{\mathbf{q} \lambda}^{\dagger} a_{\mathbf{q} \lambda}$, with $\omega_{q \lambda}$ standing for the phonon energy spectrum of branch $\lambda(\lambda=l, \mathrm{t} 1$, $\mathrm{t} 2$ for longitudinal and the two transversal phonon modes) and momentum q. We restrict to low phonon energies, where the linear dispersion regime applies, $\omega_{q \lambda}=c_{\lambda} q$, with $c_{\lambda}$ as the phonon velocity.

$H_{\mathrm{h}-\mathrm{ph}}$ is the hole-phonon interaction

$$
H_{\mathrm{h}-\mathrm{ph}}=e \phi_{\mathrm{pz}} \mathcal{I}+H_{\mathrm{dp}},
$$

where $e$ is the hole charge, $\phi_{\mathrm{pz}}$ the piezoelectric potential and $H_{\mathrm{dp}}$ the deformation potential term. These are the two relevant scattering mechanisms at low temperatures [30]. The piezoelectric potential is given by [39]

$$
\phi_{\mathrm{pz}}=\sum_{\lambda} \phi_{\mathrm{pz}}^{\lambda}=-\sum_{\lambda \mathbf{q}} \frac{4 \pi \mathrm{i}}{\epsilon_{\mathrm{r}} q^{2}} h_{14}\left(q_{x} \varepsilon_{y z}^{\lambda}+q_{y} \varepsilon_{z x}^{\lambda}+q_{z} \varepsilon_{x y}^{\lambda}\right),
$$

where $\epsilon_{\mathrm{r}}$ is the relative dielectric constant, $h_{14}$ is the piezoelectric constant and $\varepsilon_{i j}$ is the strain tensor component. On the other hand, the deformation potential term is given by the Bir-Pikus 
strain Hamiltonian:

$$
\mathcal{H}_{\mathrm{dp}}=\sum_{\lambda}\left(\begin{array}{cccc}
p^{\lambda}+q^{\lambda} & -s^{\lambda} & r^{\lambda} & 0 \\
-\left(s^{\lambda}\right)^{\dagger} & p^{\lambda}-q^{\lambda} & 0 & r^{\lambda} \\
\left(r^{\lambda}\right)^{\dagger} & 0 & p^{\lambda}-q^{\lambda} & s \\
0 & \left(r^{\lambda}\right)^{\dagger} & \left(s^{\lambda}\right)^{\dagger} & p^{\lambda}+q^{\lambda}
\end{array}\right),
$$

where

$$
\begin{aligned}
p^{\lambda} & =-a\left(\varepsilon_{x x}^{\lambda}+\varepsilon_{y y}^{\lambda}+\varepsilon_{z z}^{\lambda}\right), \\
q^{\lambda} & =-\frac{b}{2}\left(\varepsilon_{x x}^{\lambda}+\varepsilon_{y y}^{\lambda}-2 \varepsilon_{z z}^{\lambda}\right), \\
r^{\lambda} & =\frac{\sqrt{3}}{2} b\left(\varepsilon_{x x}^{\lambda}-\varepsilon_{y y}^{\lambda}\right)-\mathrm{i} d \varepsilon_{x y}^{\lambda}, \\
s^{\lambda} & =-d\left(\varepsilon_{z x}^{\lambda}-\mathrm{i} \varepsilon_{y z}^{\lambda}\right) .
\end{aligned}
$$

Here $a, b$ and $d$ are the valence band deformation potential constants.

The components of the strain tensor are rewritten using normal-modes coordinates [29]

$$
\varepsilon_{i j}^{\lambda}=-\frac{\mathrm{i}}{2} \sum_{\mathbf{q}} U^{\lambda}(q)\left(\eta_{\lambda}^{i}(\mathbf{q}) q_{j}+\eta_{\lambda}^{j}(\mathbf{q}) q_{i}\right) F(\mathbf{q}, \mathbf{r}),
$$

where $F(\mathbf{q}, \mathbf{r})=\left(\mathrm{e}^{-\mathrm{iqr}} a_{q}^{+}+\mathrm{e}^{\mathrm{i} \mathbf{q r}} a_{q}\right)$ and $U^{\lambda}(q)=\sqrt{\hbar / 2 \rho V \omega_{q \lambda}}$, with $\rho$ and $V$ standing for the crystal density and volume. $\eta_{\lambda}(\mathbf{q})$ are the phonon polarization vectors: $\boldsymbol{\eta}_{l}(\mathbf{q})=$ $\left(q_{x}, q_{y}, q_{z}\right) / q, \quad \boldsymbol{\eta}_{\mathrm{t} 1}(\mathbf{q})=\left(q_{x} q_{z}, q_{y} q_{z},-q_{\perp}^{2}\right) / q q_{\perp}$ and $\boldsymbol{\eta}_{12}(\mathbf{q})=\left(q_{y},-q_{x}, 0\right) / q_{\perp}$, with $q_{\perp}=$ $\sqrt{q_{x}^{2}+q_{y}^{2}}$. The piezoelectric potential now reads

$$
\begin{aligned}
& \phi_{\mathrm{pz}}^{1}=-\frac{12 \pi h_{14}}{\epsilon_{\mathrm{r}}} U^{\mathrm{1}}(q) \sum_{\mathbf{q}} \frac{q_{x} q_{y} q_{z}}{q^{3}} F(\mathbf{q}, \mathbf{r}), \\
& \phi_{\mathrm{pz}}^{\mathrm{t} 1}=-\frac{4 \pi h_{14}}{\epsilon_{\mathrm{r}}} U^{\mathrm{t}}(q) \sum_{\mathbf{q}} \frac{q_{x} q_{y}\left(2 q_{z}^{2}-q_{\perp}^{2}\right)}{q^{3} q_{\perp}} F(\mathbf{q}, \mathbf{r}), \\
& \phi_{\mathrm{pz}}^{\mathrm{t} 2}=-\frac{4 \pi h_{14}}{\epsilon_{\mathrm{r}}} U^{\mathrm{t}}(q) \sum_{\mathbf{q}} \frac{q_{z}\left(q_{y}^{2}-q_{x}^{2}\right)}{q^{2} q_{\perp}} F(\mathbf{q}, \mathbf{r}) .
\end{aligned}
$$

In turn, the deformation potential operators become

$$
\begin{aligned}
& p^{1}=\mathrm{i} a U^{1}(q) \sum_{\mathbf{q}} q F(\mathbf{q}, \mathbf{r}), \\
& q^{1}=\mathrm{i} \frac{b}{2} U^{1}(q) \sum_{\mathbf{q}}\left(q-3 \frac{q_{z}^{2}}{q}\right) F(\mathbf{q}, \mathbf{r}), \\
& r^{1}=-\mathrm{i} U^{1}(q) \sum_{\mathbf{q}}\left(\frac{\sqrt{3}}{2} b \frac{q_{x}^{2}-q_{y}^{2}}{q}-\mathrm{i} d \frac{q_{x} q_{y}}{q}\right) F(\mathbf{q}, \mathbf{r}), \\
& s^{1}=\mathrm{i} d U^{1}(q) \sum_{\mathbf{q}} \frac{q_{z}\left(q_{x}-\mathrm{i} q_{y}\right)}{q} F(\mathbf{q}, \mathbf{r})
\end{aligned}
$$


for longitudinal phonons,

$$
\begin{aligned}
& p^{\mathrm{t} 1}=0, \\
& q^{\mathrm{t} 1}=\mathrm{i} \frac{b}{2} U^{\mathrm{t}}(q) \sum_{\mathbf{q}}\left(\frac{3 q_{z} q_{\perp}}{q}\right) F(\mathbf{q}, \mathbf{r}), \\
& r^{\mathrm{t} 1}=-\mathrm{i} U^{\mathrm{t}}(q) \sum_{\mathbf{q}}\left(\frac{\sqrt{3}}{2} b \frac{q_{z}\left(q_{x}^{2}-q_{y}^{2}\right)}{q q_{\perp}}-\mathrm{i} d \frac{q_{x} q_{y} q_{z}}{q q_{\perp}}\right) F(\mathbf{q}, \mathbf{r}), \\
& s^{\mathrm{t} 1}=\mathrm{i} \frac{d}{2} U^{\mathrm{t}}(q) \sum_{\mathbf{q}} \frac{\left(q_{z}^{2}-q_{\perp}^{2}\right)\left(q_{x}-\mathrm{i} q_{y}\right)}{q_{\perp} q} F(\mathbf{q}, \mathbf{r})
\end{aligned}
$$

for transversal $\mathrm{t} 1$ phonons and

$$
\begin{aligned}
& p^{\mathrm{t} 2}=0, \\
& q^{\mathrm{t} 2}=0, \\
& r^{\mathrm{t} 2}=-\mathrm{i} U^{\mathrm{t}}(q) \sum_{\mathbf{q}}\left(\sqrt{3} b \frac{q_{x} q_{y}}{q_{\perp}}-\mathrm{i} \frac{d}{2} \frac{q_{y}^{2}-q_{x}^{2}}{q_{\perp}}\right) F(\mathbf{q}, \mathbf{r}), \\
& s^{\mathrm{t} 2}=-\frac{d}{2} U^{\mathrm{t}}(q) \sum_{\mathbf{q}} \frac{q_{z}\left(q_{x}-\mathrm{i} q_{y}\right)}{q_{\perp}} F(\mathbf{q}, \mathbf{r})
\end{aligned}
$$

for transversal $\mathrm{t} 2$ phonons.

\subsection{Relaxation rate}

We calculate the spin relaxation from an initial hole state $\left|\Psi_{\mathrm{i}}^{\mathrm{h}}\right\rangle$, with energy $E_{\mathrm{i}}^{\mathrm{h}}$, to a final hole state $\left|\Psi_{\mathrm{f}}^{\mathrm{h}}\right\rangle$, with energy $E_{\mathrm{f}}^{\mathrm{h}}$. The relaxation rate is estimated with a Fermi golden rule. We consider zero temperature, so that there is no phonon absorption. After integrating over phonon degrees of freedom, the rate is given by

$$
\tau_{\mathrm{i} \rightarrow \mathrm{f}}^{-1}=\frac{2 \pi}{\hbar} \sum_{\lambda, \mathbf{q}}\left|\left\langle\Psi_{\mathrm{f}}^{\mathrm{h}}\left|\mathcal{H}_{\mathrm{h}-\mathrm{ph}}^{\lambda q}\right| \Psi_{\mathrm{i}}^{\mathrm{h}}\right\rangle\right|^{2} \delta\left(\Delta E_{\mathrm{fi}}+\hbar c_{\lambda} q\right) .
$$

Here $\mathcal{H}_{\mathrm{h}-\mathrm{ph}}^{\lambda q}$ is the hole-phonon interaction Hamiltonian, equation (15), but for a fixed phonon branch $\lambda$ and momentum $\mathbf{q}$, and $\Delta E_{\mathrm{fi}}=E_{\mathrm{f}}^{\mathrm{h}}-E_{\mathrm{i}}^{\mathrm{h}}$. It can be seen from equations (23)-(26) that all the terms of $\mathcal{H}_{\mathrm{h}-\mathrm{ph}}^{\lambda q}$ contain a factor which depends on $\mathbf{q}$ only and $F(\mathbf{q}, \mathbf{r})$, which depends on spatial coordinates as well. Thus, when expanded, the matrix element $\left\langle\Psi_{\mathrm{f}}^{\mathrm{h}}\left|\mathcal{H}_{\mathrm{h}-\mathrm{ph}}^{\lambda q}\right| \Psi_{\mathrm{i}}^{\mathrm{h}}\right\rangle$ takes the form

$$
\left\langle\Psi_{\mathrm{f}}^{\mathrm{h}}\left|\mathcal{H}_{\mathrm{h}-\mathrm{ph}}^{\lambda q}\right| \Psi_{\mathrm{i}}^{\mathrm{h}}\right\rangle=\sum_{J_{z}^{\prime}, J_{z}, r^{\prime}, r}\left(c_{r^{\prime}, J_{z}^{\prime}}^{\mathrm{f}}\right)^{*} c_{r, J_{z}}^{\mathrm{i}} M_{J_{z}^{\prime}, J_{z}}^{\lambda}(\mathbf{q}) G_{r^{\prime}, r}(\mathbf{q})
$$

where $G_{r, r^{\prime}}(\mathbf{q})=\left\langle r^{\prime}\left|\mathrm{e}^{-\mathrm{i} \mathbf{q r}}\right| r\right\rangle$ and $M_{J_{2}^{\prime}, J_{z}}^{\lambda}(\mathbf{q})$ gathers the $\mathbf{q}$-dependent factor of the $\mathcal{H}_{\mathrm{h}-\mathrm{ph}}^{\lambda q}$ term coupling $J_{z}$ and $J_{z}^{\prime} . G_{r, r^{\prime}}(\mathbf{q})$ is calculated analytically using iterative procedures as described in [40]. The sum over $\mathbf{q}$ in equation (27) is then carried out using numerical integration. To this 
Table 1. Parameters used in the numerical calculations for InAs (left column) and GaAs (right column) QDs. GaAs parameters are used for the matrix in both cases. $\mathrm{e}^{-}, \mathrm{h}^{+}$and $\mathrm{ph}$ stand for electron, hole and phonon.

\begin{tabular}{lcccc}
\hline Parameter & Symbol & InAs & GaAs & \\
\hline$e^{-}$mass $\left(m_{0}\right)$ & $m_{\mathrm{e}}$ & 0.026 & 0.067 & {$[41]$} \\
$h^{+}$Luttinger param. & $\gamma_{1}$ & 20 & 6.98 & {$[41]$} \\
$h^{+}$Luttinger param. & $\gamma_{2}$ & 8.5 & 2.06 & {$[41]$} \\
$h^{+}$Luttinger param. & $\gamma_{3}$ & 9.2 & 2.93 & {$[41]$} \\
$e^{-}$deformation pot. $(\mathrm{eV})$ & $a_{c}$ & -5.08 & -7.17 & {$[41]$} \\
$h^{+}$deformation pot. $(\mathrm{eV})$ & $a$ & 1.0 & 1.16 & {$[41]$} \\
$h^{+}$deformation pot. $(\mathrm{eV})$ & $b$ & -1.8 & -2.0 & {$[41]$} \\
$h^{+}$deformation pot. $(\mathrm{eV})$ & $c$ & -3.6 & -4.8 & {$[41]$} \\
$e^{-}$BIA coeff. $\left(\mathrm{eV} \mathrm{\AA}{ }^{3}\right)$ & $b_{41}^{c}$ & 27.18 & 27.58 & {$[28]$} \\
$h^{+}$BIA coeff. $(\mathrm{eV} \AA$ ) & $C_{k}$ & -0.0112 & -0.0034 & {$[28]$} \\
$h^{+}$BIA coeff. $\left(\mathrm{eV}^{3}\right)$ & $b_{41}$ & -50.18 & -81.93 & {$[28]$} \\
$h^{+}$BIA coeff. $\left(\mathrm{eV} \mathrm{\AA}^{3}\right)$ & $b_{42}$ & 1.26 & 1.47 & {$[28]$} \\
$h^{+}$BIA coeff. $\left(\mathrm{eV} \mathrm{\AA}^{3}\right)$ & $b_{51}$ & 0.42 & 0.49 & {$[28]$} \\
$h^{+}$BIA coeff. $\left(\mathrm{eV} \mathrm{\AA}^{3}\right)$ & $b_{52}$ & -0.84 & -0.98 & {$[28]$} \\
Longitudinal ph speed $\left(\mathrm{m} \mathrm{s}^{-1}\right)$ & $c_{1}$ & 4720 & 4720 & {$[41]$} \\
Transversal ph speed $\left(\mathrm{m} \mathrm{s}^{-1}\right)$ & $c_{\mathrm{t}}$ & 3340 & 3340 & {$[41]$} \\
Crystal density $\left(\mathrm{kg} \mathrm{m}^{-3}\right)$ & $\rho$ & 5310 & 5310 & {$[41]$} \\
Piezoelectric coeff. $\left(\mathrm{V} \mathrm{cm}{ }^{-1}\right)$ & $h_{14}$ & $3.5 \times 10^{6}$ & $1.45 \times 10^{7}$ & {$[49]$} \\
\hline
\end{tabular}

end, it is convenient to use spherical coordinates, as the modulus $q$ is fixed by the resonance condition and we are left with a 2D integral.

Calculations are carried out for InAs QDs embedded in a GaAs matrix. When differences are expected, we also calculate GaAs QD embedded in an $\mathrm{Al}_{x} \mathrm{Ga}_{1-x}$ As matrix. Table 1 summarizes the parameters we use. The parameters correspond to the QD material, except for the crystal density and velocity of sound, which correspond to the matrix material because we assume bulk phonons (for simplicity, for $\mathrm{Al}_{x} \mathrm{Ga}_{1-x}$ As we assume $x \rightarrow 0$ and use GaAs phonon parameters). For the dielectric constant, an average value of $\epsilon_{\mathrm{r}}=12.9$ is taken all over the structure. The basis used to solve Hamiltonian (1) contains all the hh oscillator eigenstates with the quantum numbers $v_{x}, v_{y}<13$ and $v_{z}<9$.

\section{Numerical results and discussion}

We shall start this section by describing the dependence of the hole spin lifetime on the QD geometry and the spin splitting magnitude (section 3.1). The influence of each parameter can be understood by analyzing the spin admixture mechanisms, as we show in section 3.2. Next, in section 3.3, we study the effect of the ground state changing from mainly hh character, which is the case addressed in most previous studies, to mainly lh character. This transition is observed in QDs with large aspect ratio [42-45]. Last, in section 3.4, we compare the role of deformation potential and piezoelectric potential interactions in determining the efficiency of hole-phonon coupling. 

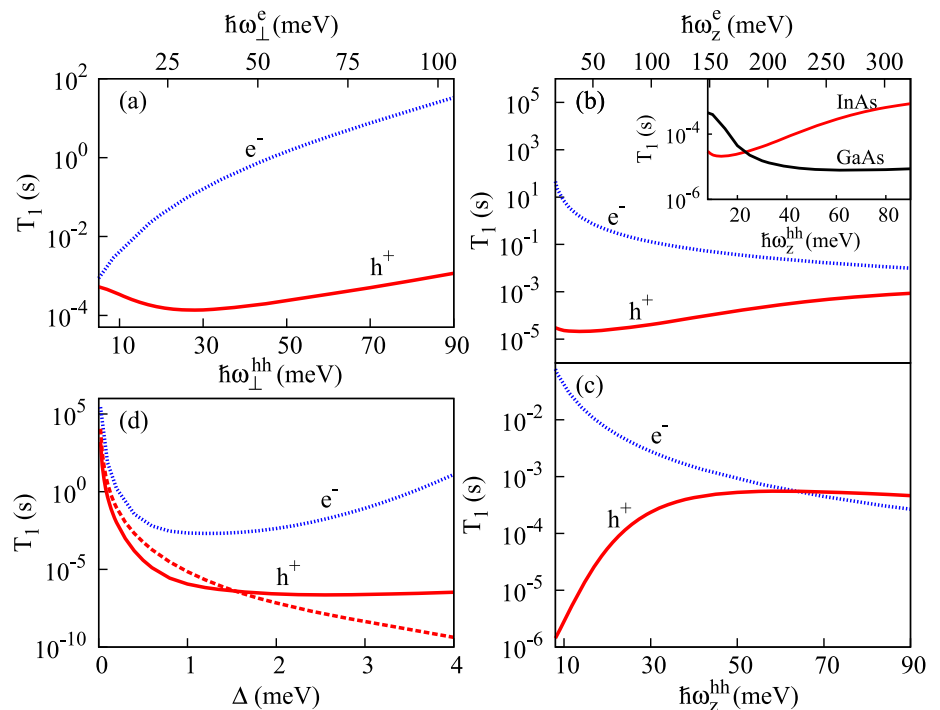

Figure 2. Hole (red solid line) and electron (blue dotted line) spin lifetime in InAs QDs, as a function of the lateral confinement (a), vertical confinement (b)-(c) and spin splitting energy (d). The inset in (b) compares $T_{1}^{\mathrm{h}}$ for InAs and GaAs. (a) $\hbar \omega_{z}^{\mathrm{hh}}=50 \mathrm{meV}, \hbar \omega_{z}^{\mathrm{e}}=179 \mathrm{meV}, \Delta=0.4 \mathrm{meV}$. (b) $\hbar \omega_{\perp}^{\mathrm{hh}}=$ $20 \mathrm{meV}, \hbar \omega_{\perp}^{\mathrm{e}}=23.2 \mathrm{meV}, \Delta=0.4 \mathrm{meV}$. (c) $\hbar \omega_{\perp}^{\text {hh }}=5 \mathrm{meV}, \hbar \omega_{\perp}^{\mathrm{e}}=5.8 \mathrm{meV}$, $\Delta=0.4 \mathrm{meV}$. (d) $\hbar \omega_{\perp}^{\mathrm{hh}}=20 \mathrm{meV}$ and $\hbar \omega_{z}^{\mathrm{hh}}=50 \mathrm{meV}$ (red solid line); $\hbar \omega_{\perp}^{\mathrm{e}}=$ $23.2 \mathrm{meV}$ and $\hbar \omega_{z}^{\mathrm{e}}=179 \mathrm{meV}$ (blue dotted line); $\hbar \omega_{\perp}^{\mathrm{hh}}=40 \mathrm{meV}$ and $\hbar \omega_{z}^{\mathrm{hh}}=$ $5 \mathrm{meV}$ (red dashed line).

\subsection{Geometry and spin splitting dependence}

Solid lines in figure 2 show the hole spin lifetime as a function of the QD geometry and the spin splitting energy of InAs QDs. For comparison, we also plot the electron spin lifetimes (dotted lines). The latter have been calculated using the same formalism as for holes but adapted for single-band conduction electrons [40]. Both electrons and holes are assumed to be confined in the same QD, hence they share the same force constants but have different confinement frequencies. One can see immediately in the figure that the behavior of holes differs drastically from the well-known case of electrons. Below we summarize the influence of each parameter.

Figure 2(a) shows the spin lifetime dependence on the lateral confinement in QDs with strong vertical confinement. $T_{1}^{\mathrm{e}}$ increases monotonically with $\omega_{\perp}$. This is because, as we approach the spherical confinement regime $\left(\omega_{z}^{\mathrm{e}}=\omega_{\perp}^{\mathrm{e}}\right)$, the Dresselhaus SOI of electrons is gradually suppressed [40]. No such trend is however observed for holes, as $H_{\mathrm{BIA}}$ does not cancel out even if the confinement is isotropic. As a matter of fact, $T_{1}^{\mathrm{h}}$ shows an evident non-monotonic behavior, with a minimum at $\omega_{\perp}^{\text {hh }}=28 \mathrm{meV}$ and increasing away from it. It is worth noting that a previous study by Woods et al [29] predicted $T_{1}^{\mathrm{h}}$ to decrease with the QD diameter, while a similar study by Lu et al [30] for somewhat larger QDs predicted the opposite trend. Figure 2(a) 
shows that both predictions are conciliable, corresponding to the right and left sides of the $T_{1}^{\mathrm{h}}$ minimum, respectively. The origin of the different trends will be discussed in section 3.2.

Figures 2(b) and (c) show the spin lifetime dependence on the vertical confinement in QDs with moderately strong and weak lateral confinement, respectively. These confinement strengths roughly correspond to self-assembled (panel (b)) [46, 47] and electrostatic (panel (c)) [48] QDs. As can be seen in figure 2(b), electrons and holes have opposite behaviors. $T_{1}^{\mathrm{e}}$ now decreases with $\omega_{z}^{\mathrm{e}}$ because the structure is becoming flatter (less isotropic). Instead, the behavior of $T_{1}^{\mathrm{h}}$ is similar to that observed for varying lateral confinement, with a shallow minimum at $\omega_{z}^{\mathrm{hh}}=14 \mathrm{meV}$. Previous studies have shown that $T_{1}^{\mathrm{h}}$ increases with the vertical confinement [30]. This is consistent with the right side of the $T_{1}^{\mathrm{h}}$ minimum in figure 2(b), but here we show that the opposite trend is also possible if the QD aspect ratio is large enough (left side of the minimum).

Figure 2(c) illustrates the case in which the lateral confinement is weak. The minimum of $T_{1}^{\mathrm{h}}$ is now shifted toward very small $\hbar \omega_{z}^{\text {hh }}$ values so that only the right side behavior is seen in the range under study. Interestingly, here $T_{1}^{\mathrm{h}}$ shows a clear saturation with increasing vertical confinement $\left(\hbar \omega_{z}^{\mathrm{hh}}>40 \mathrm{meV}\right)$, which has not been noticed before [30]. As we show in section 3.2, this saturation reflects the fact that $H_{\mathrm{BIA}}$ has replaced $\mathrm{hh}-\mathrm{lh}$ mixing as the main source of SOI.

The inset in figure 2(b) compares $T_{1}^{\mathrm{h}}$ in InAs and GaAs QDs with moderate lateral confinement. As can be observed, the spin lifetime in InAs QDs is longer than that in GaAs QDs when $\omega_{z}^{\mathrm{hh}}>\omega_{\perp}^{\mathrm{hh}}$, which is, e.g. the case of self-assembled QDs. This is an unexpected result because in bulk the valence band SOI of InAs is stronger than that of GaAs (compare the split-off band splittings [41] or the $\gamma_{2}$ and $\gamma_{3}$ coefficients appearing in $R$ and $S$ terms of equation (4), which couple hh to $\mathrm{lh}$ ). The underlying reason is that in confined structures, the cubic Dresselhaus SOI becomes important and it is stronger for GaAs (see $b_{41}$ in table 1).

Figure 2(d) shows the spin-flip lifetime of electrons and holes in a self-assembled-like QD as a function of the spin splitting energy. For electrons, $T_{1}^{\mathrm{e}}$ is largely determined by the efficiency of the carrier-phonon coupling [40]. It is short when the phonon wavelength is of the same order as the carrier wavefunction extension, but it increases for large (small) $\Delta$ values because the phonon wavelength becomes too short (long), as $q \approx \Delta / \hbar c$. The same happens for holes (notice that $G_{r, r^{\prime}}(\mathbf{q})$ in equation (28) vanishes in the limits of large and small phonon wavevector). However, figure 2(d) shows that $T_{1}^{\mathrm{h}}$ is only sensitive to $\Delta$ for small splittings, but then it reaches a plateau where $T_{1}^{\mathrm{h}} \sim \mu \mathrm{s}$. The different behavior of holes and electrons is due to the different effective mass along the growth direction, $m_{z}$. For $\hbar \omega_{z}=50 \mathrm{meV}$, the characteristic length of the oscillator states in the growth direction, $l_{z}=\sqrt{\hbar / m_{z} \omega_{z}}$, is $l_{z}^{\mathrm{e}}=4.5 \mathrm{~nm}$ for electrons and $l_{z}^{\mathrm{hh}}=2.4 \mathrm{~nm}$ for holes, i.e. the hole confinement is stronger. As a result, larger values of $\Delta$ than those used in figure $2(\mathrm{~d})$ are required for $T_{1}^{\mathrm{h}}$ to increase.

Experiments with excitons in self-assembled InGaAs QDs have revealed a negligible influence of $\mathrm{e}-\mathrm{h}$ interactions on $T_{1}^{\mathrm{h}}$ [24]. Our results would be consistent with such an observation in the regime of large $\Delta$. For self-assembled QDs, however, $\Delta \leqslant 0.5 \mathrm{meV}$. Thus, the insensitivity noted in the experiment is inconsistent with the single-phonon processes we consider in figure 2. This suggests that two-phonon processes dominate in these systems $[15,32]$. On the other hand, experiments with colloidal nanorods have shown a sizable increase of $T_{1}^{\mathrm{h}}$ when changing from type-I to type-II confinement, which modulates the e- $\mathrm{h}$ exchange energy from typical colloidal structure values (few $\mathrm{meV}$ ) to type-II system values (fractions of $\mathrm{meV}$ ) [36]. We have run simulations for a nanorod-like geometry (red dashed line in figure 2(d)) 


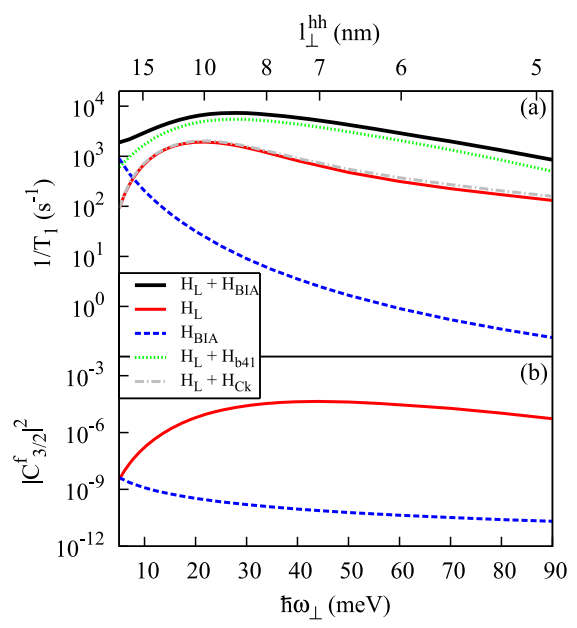

Figure 3. Hole spin relaxation (a) and weight of the minor hh component (b) as a function of the lateral confinement. Different SOI terms are considered. The system is the same as that of figure $2(a)$.

and find that the weak vertical confinement renders $T_{1}^{\mathrm{h}}$ sensitive to $\Delta$ for all the range under study, in agreement with the experiment. This indicates that the weak vertical confinement of rods renders single-phonon processes efficient.

To close this section, we notice that previous theoretical studies with simpler models had predicted that hole spin lifetimes can exceed those of electrons in very flat QDs [31]. Figure 2 confirms that this could actually be achieved in gated structures, where lateral confinement is very weak (see the crossing between $T_{1}^{\mathrm{e}}$ and $T_{1}^{\mathrm{h}}$ in panel (c)). However, for typical selfassembled InAs QDs, $T_{1}^{\mathrm{e}}$ is about one order of magnitude longer than $T_{1}^{\mathrm{h}}$ (see panel (b) for large $\left.\hbar \omega_{z}\right)^{2}{ }^{2}$ This is consistent with experimental measurements by Heiss et al [26].

\subsection{Mechanism of spin admixture}

Spin admixture is a requirement for spin-flip transitions to take place [50]. In this section, we compare the spin admixture resulting from all the SOI terms affecting the hole ground state. As we shall see, once the dominant SOI mechanism is determined, one can rationalize the geometry dependence of $T_{1}^{\mathrm{h}}$ described in the previous section. For convenience of the analysis, in what follows we plot and discuss relaxation rates, $1 / T_{1}^{\mathrm{h}}$. Furthermore, we shall often drop the $\mathrm{hh}$ superscript when referring to the confinement frequency, $\omega_{\perp}^{\text {hh }}$ or $\omega_{z}^{\text {hh }}$.

Figure 3(a) shows the relaxation rate for the InAs QDs of figure 2(a), but now obtained by calculating hole states with the diagonal terms of $H_{\mathrm{L}}$ plus different $\mathrm{SOI}$ terms: off-diagonal $H_{\mathrm{L}}$ terms (hereafter hh-lh coupling), full Dresselhaus Hamiltonian $\left(H_{\mathrm{BIA}}\right)$, hh-lh coupling plus linear-in- $k$ term $\left(H_{\mathrm{L}}+H_{C_{k}}\right)$ and hh-lh coupling plus the dominant cubic-in- $k$ Dresselhaus term

2 The same conclusions, albeit with somewhat lower $T_{1}^{\mathrm{e}}$, are obtained using larger electron masses to account, e.g. for Ga diffusion into the InAs QD. 
$\left(H_{\mathrm{L}}+H_{b_{41}}\right)$. The total rate, corresponding to $H_{\mathrm{L}}+H_{\mathrm{BIA}}$, is also shown (thick black line). One can see that $H_{\mathrm{L}}$ (red solid line) is more important than $H_{\mathrm{BIA}}$ (blue dashed line) for large $\omega_{\perp}$ values. However, as the lateral confinement is weakened and the system becomes flatter, $H_{\text {BIA }}$ gains weight. For self-assembled QDs $\left(\hbar \omega_{\perp} \approx 10-25 \mathrm{meV}\right), H_{\mathrm{BIA}}$ is already comparable to $H_{\mathrm{L}}$ and it becomes dominant for weakly confined (e.g. gated) QDs. Figure 3 also reveals that the linearin- $k$ BIA term (gray dot-dashed line) is but a secondary mechanism, which barely enhances the relaxation rate coming from $H_{\mathrm{L}}$. This is inspite of the fact that it is a source of direct admixture between hh states with opposite spin, with no participation of lh states. For this reason, it had been proposed as the dominant SOI term in flat QDs with weak lateral confinement [35]. Instead, figure 3 shows that most of the $H_{\mathrm{BIA}}$ contribution comes from the cubic-in- $k b_{41}$ term (see green dotted line).

This term relies on intermediate $\mathrm{lh}$ states in order to mix hh $J_{z}=+3 / 2$ and $-3 / 2$ components (see equation (A.3) in the appendix), which implies that a simultaneous description of hh and lh states is necessary for realistic modeling.

By comparing the total relaxation rate coming from $H_{\mathrm{L}}+H_{\mathrm{BIA}}$ with that coming from $H_{\mathrm{L}}$ and $H_{\mathrm{BIA}}$, it is clear that the total rate is not just the sum of the two independent mechanisms. For example, at $\hbar \omega_{\perp}=30 \mathrm{meV}$, adding $H_{\mathrm{BIA}}$ to $H_{\mathrm{L}}$ enhances $1 / T_{1}^{\mathrm{h}}$ about an order of magnitude, even though the contribution coming from $H_{\mathrm{BIA}}$ alone is about 100 times smaller than that coming from $H_{\mathrm{L}}$. This can be understood by means of a perturbative reasoning: neither $H_{\mathrm{L}}$, equation (4), nor $H_{b_{41}}$ - the most relevant term of $H_{\mathrm{BIA}}$-, equation (A.3), contribute to hh-lh mixing at first order. $H_{\mathrm{L}}$ contributes at second order, due to terms involving non-zero products like $H_{\mathrm{L}}(1,2) H_{\mathrm{L}}(2,4)$, while $H_{b_{41}}$ does not. It contributes at third order, due to non-zero products like $H_{b_{41}}(1,2) H_{b_{41}}(2,3) H_{b_{41}}(3,4)$. However, the sum of the two Hamiltonians allows $H_{b_{41}}$ to contribute at second order. Thus, the effect of $H_{b_{41}}$ is clearly non-additive because it is enhanced by $H_{\mathrm{L}}$. Simultaneously accounting for both SOI terms is then required for quantitative estimates.

For a qualitative understanding of the geometry dependence of $1 / T_{1}^{\mathrm{h}}$, we rewrite the hole states, equation (14), as $\left|\Psi_{m}^{\mathrm{h}}\right\rangle=\sum_{J_{z}} c_{J_{z}}^{m}\left|\phi_{J_{z}}^{m}\right\rangle\left|3 / 2, J_{z}\right\rangle$, where $\left|\phi_{J_{z}}^{m}\right\rangle=\sum_{r} c_{J_{z}, r}^{m}|r\rangle$ is the envelope function associated with the periodic Bloch function $\left|3 / 2, J_{z}\right\rangle$. If we restrict to the diagonal components of $H_{\mathrm{h}-\mathrm{ph}}$, the matrix element determining the relaxation rate becomes

$$
\left\langle\Psi_{\mathrm{f}}^{\mathrm{h}}\left|\mathcal{H}_{\mathrm{h}-\mathrm{ph}}^{\lambda q}\right| \Psi_{\mathrm{i}}^{\mathrm{h}}\right\rangle \approx \sum_{J_{z}}\left(c_{J_{z}}^{\mathrm{f}}\right)^{*} c_{J_{z}}^{\mathrm{i}}\left\langle\phi_{J_{z}}^{\mathrm{f}}\left|\mathcal{H}_{\mathrm{h}-\mathrm{ph}}^{\lambda q}\right| \phi_{J_{z}}^{\mathrm{i}}\right\rangle .
$$

When the QD is oblated or spherical, the low-energy states are essentially hh states. Thus, the initial (final) state of the spin transition is mostly a pure spin-up (spin-down) hh state. Considering that $\left|c_{3 / 2}^{\mathrm{i}}\right| \gg\left|c_{3 / 2}^{\mathrm{f}}\right|\left(\left|c_{-3 / 2}^{\mathrm{f}}\right| \gg\left|c_{-3 / 2}^{\mathrm{i}}\right|\right)$, one can obtain an approximate expression

$$
\frac{1}{T_{1}^{\mathrm{h}}} \propto\left|\left\langle\Psi_{\mathrm{f}}^{\mathrm{h}}\left|\mathcal{H}_{\mathrm{h}-\mathrm{ph}}^{\lambda q}\right| \Psi_{\mathrm{i}}^{\mathrm{h}}\right\rangle\right|^{2} \propto\left|c_{3 / 2}^{\mathrm{f}}\right|^{2}\left|\left\langle\phi_{3 / 2}^{\mathrm{f}}\left|\mathcal{H}_{\mathrm{h}-\mathrm{ph}}^{\lambda q}\right| \phi_{3 / 2}^{\mathrm{i}}\right\rangle\right|^{2} .
$$

In other words, the relaxation rate is proportional to the spin admixture of the ground state through the squared coefficient of the minor hh component (here spin-up, $J_{z}=3 / 2$ ), and proportional to the efficiency of the hole-phonon coupling through the rightmost matrix element.

The geometry dependence of $1 / T_{1}^{\mathrm{h}}$ for a given SOI mechanism simply reflects the spin admixture variation. This can be seen in figure $3(\mathrm{~b})$, which shows that, for $H_{\mathrm{L}}$ and $H_{\mathrm{BIA}}$, $\left|c_{3 / 2}^{\mathrm{f}}\right|^{2}$ has the same qualitative dependence on the geometry as the corresponding $1 / T_{1}^{\mathrm{h}}$ values in figure 3(a). This allows us to interpret the observed maximum as a function of $\omega_{\perp}$. 


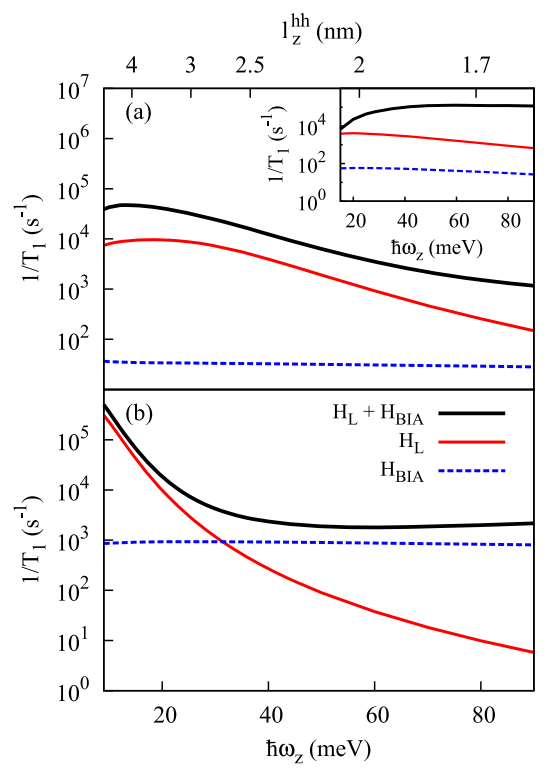

Figure 4. Hole spin relaxation as a function of the vertical confinement. Different SOI terms are considered. (a) Strong lateral confinement, $\hbar \omega_{\perp}=20 \mathrm{meV}$. (b) Weak lateral confinement, $\hbar \omega_{\perp}=5 \mathrm{meV}$. The inset in (a) is the corresponding result for GaAs. The system is the same as that of figures 2(b) and (c).

For $H_{\mathrm{L}}$, the spin admixture between the hh spin-up and spin-down components takes place necessarily through the intermediate lh components (see equation (4)). The weight of the minor hh component is then related to the strength of the off-diagonal terms of $H_{\mathrm{L}}(R$ and $S)$ and to the energy splitting between the hh and the lh states. For small $\omega_{\perp}$ values, the main effect of increasing the lateral confinement is to enhance the coupling terms, which are proportional to $k_{x}$ and $k_{y}$ (see equations (7) and (8)). As a result, the weight of the minor hh component $\left|c_{3 / 2}^{\mathrm{f}}\right|$ increases, hence $1 / T_{1}^{\mathrm{h}}$ increases. For larger $\omega_{\perp}$ values, however, when the lateral and vertical confinements become comparable, the main effect of increasing the lateral confinement is to bring $\mathrm{lh}$ states closer to hh ones [44, 45]. When this happens, the lh states stop acting as intermediate states for the admixture between hh components and start participating in the admixture themselves. This is at the expense of reducing the weight of the minor hh component, hence $1 / T_{1}^{\mathrm{h}}$ decreases.

Next, we analyze the mechanisms involved in the spin relaxation with varying vertical confinement. Figure 4 shows $1 / T_{1}^{\mathrm{h}}$ for the same systems as in figures 2 (b) and (c), but calculating the hole states with the diagonal terms of $H_{\mathrm{L}}$ plus hh-lh coupling (red solid line) or full $H_{\mathrm{BIA}}$ Hamiltonian (blue dashed line). Panel (a) corresponds to strong lateral confinement. The total rate has a maximum at $\hbar \omega_{z}=14 \mathrm{meV}$, whose origin is analogous to that described above for varying lateral confinement. Beyond the maximum, the total rate $\left(H_{\mathrm{L}}+H_{\mathrm{BIA}}\right)$ decreases with the vertical confinement strength, in agreement with previous studies [30]. However, we also find that the decrease eventually saturates. This is evident for 


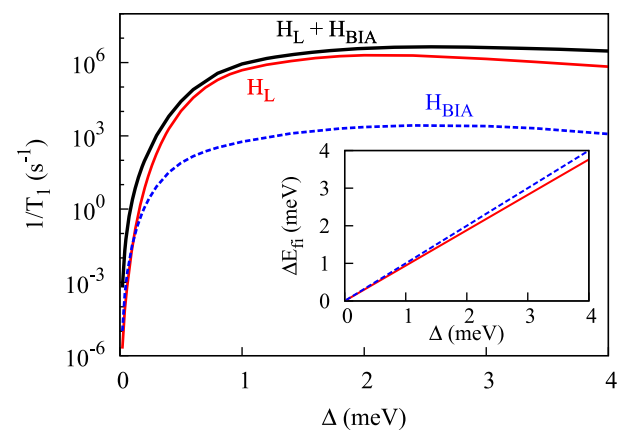

Figure 5. Hole spin relaxation as a function of the spin splitting energy in a QD with $\hbar \omega_{\perp}=20 \mathrm{meV}$ and $\hbar \omega_{z}=50 \mathrm{meV}$. Same legend as in figure 3 is used. The inset compares the energy splitting between the Kramer doublet for hh-lh coupling and Dresselhaus SOI.

InAs QDs with weak lateral confinement, as shown in figure 4(b), or GaAs QDs with strong lateral confinement, as in figure 4(a) inset. The origin of this saturation is the contribution of $H_{\mathrm{BIA}}$, which provides a lower bound to $1 / T_{1}^{\mathrm{h}}$. In particular, $H_{b_{41}}$ introduces off-diagonal coupling terms which are quadratic in $k_{z}$ (see operator $L_{41}$ in the appendix), instead of the linear $k_{z}$ terms of $H_{\mathrm{L}}$ (see $S$ operator in equation (4)). Since the uncoupled hh and lh energies are also quadratic in $k_{z}$, a perturbational analysis easily shows that the two contributions compensate each other. For strong $\omega_{z}$, when lateral confinement is negligible, the cancelation is exact and the relaxation rate does not depend on the vertical confinement.

The magnitude of the spin splitting also influences the dominant mechanism of spin admixture. This is illustrated in figure 5, which considers a self-assembled InAs QD with varying spin splitting energy. For large $\Delta, H_{\mathrm{L}}$ has a dominant contribution to the relaxation rate, but $H_{\mathrm{BIA}}$ becomes equally important for small enough $\Delta$. The relative enhancement of the role of $H_{\mathrm{BIA}}$ originates in its zero-field spin splitting, which leads to larger energy difference between the Kramers pair $\left(\Delta E_{\mathrm{fi}}\right)$ than with $\Delta$ alone, as shown in the figure inset. When $\Delta \rightarrow 0$ and the phonon wavelength increases beyond the QD size, the extra energy coming from the zero-field spin splitting of $H_{\mathrm{BIA}}$ provides a significant contribution to preserve the hole-phonon coupling efficiency.

\subsection{Light hole spin relaxation}

When the aspect ratio increases and the QD shape becomes prolate, the hole ground state evolves from the eminent hh character discussed so far to lh character, as noted, e.g. in colloidal nanorods [42-45]. Here we investigate how the change of the ground state affects the relaxation between the two highest hole states. Figure 6(a) shows the hole energy levels in a QD with $\hbar \omega_{\perp}=40 \mathrm{meV}$ as a function of $\hbar \omega_{z}$. In the limit of strong and weak vertical confinement, the two highest states are essentially hh and lh doublets, $J_{z}= \pm 3 / 2$ and $\pm 1 / 2$, respectively. In the intermediate regime, $\hbar \omega_{z}=9-17 \mathrm{meV}$, the two doublets cross. This gives rise to pronounced changes in the relaxation rate, as shown in figure 6(b). 


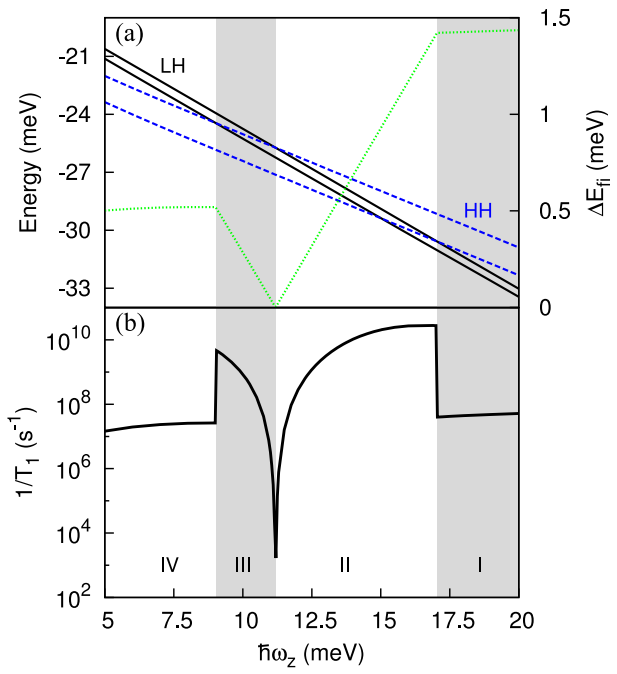

Figure 6. Hole energy levels (a) and spin relaxation rate (b) around the hh-lh crossing region. $\hbar \omega_{\perp}=40 \mathrm{meV}$ and $\Delta=2 \mathrm{meV}$. In (a), solid and dashed lines are used for states with dominant $\mathrm{lh}$ and hh character, respectively. The dotted line gives the energy splitting. Shades are used to distinguish the regions with different kinds of states involved in the transition.

The changes can be understood as follows. In region I of the figure, the two highest states are the hh doublet. The relaxation is between states with opposite spin and roughly constant energy splitting (see $\Delta E_{\mathrm{fi}}$, dotted line in figure 6(a)). At $\hbar \omega_{z}=17 \mathrm{meV}$, when we enter region II, the excited hh state crosses with the first lh state. Now the relaxation is between an $\mathrm{lh}$ $\left(J_{z}=-1 / 2\right)$ and an hh $\left(J_{z}=-3 / 2\right)$. Since lh have mixed spin-up and spin-down projections, there is no need for spin flip. Then, the $s^{\lambda}$ terms of the strain Hamiltonian, $H_{\mathrm{dp}}$, provide direct coupling with hh and the resulting transition is much faster. This explains the abrupt increase of $1 / T_{1}^{\mathrm{h}}$. However, the energy splitting between the hh and lh becomes smaller with decreasing $\omega_{z}$ because of their different masses. As a result, at $\hbar \omega_{z}=11 \mathrm{meV}$, the lh replaces the hh as the ground state. Near the degeneracy point, $\hbar \omega_{z}=11.2 \mathrm{meV}, \Delta E_{\mathrm{fi}}$ is so small that hole-phonon coupling becomes inefficient and the relaxation is strongly suppressed, but it increases again in region III for the same reasons as in region II. Finally, at $\hbar \omega_{z}=9 \mathrm{meV}$, the excited lh state crosses with the highest hh state. We thus enter region IV, where the transition takes place between two 1 h states with orthogonal Bloch functions, $|3 / 2, \pm 1 / 2\rangle . J_{z}$ admixture mechanisms are necessary and the relaxation becomes slow. As a matter of fact, the spin relaxation timescale for transitions between lh states is similar to that between hh states, in spite of the fact that their Bloch functions contain mixed spins.

\subsection{Mechanism of hole-phonon coupling}

Electron-acoustic phonon coupling in QDs is known to occur mainly through deformation potential interaction when the energy splitting is large $\left(\Delta E_{\mathrm{fi}}>0.1 \mathrm{meV}\right)$ and through 


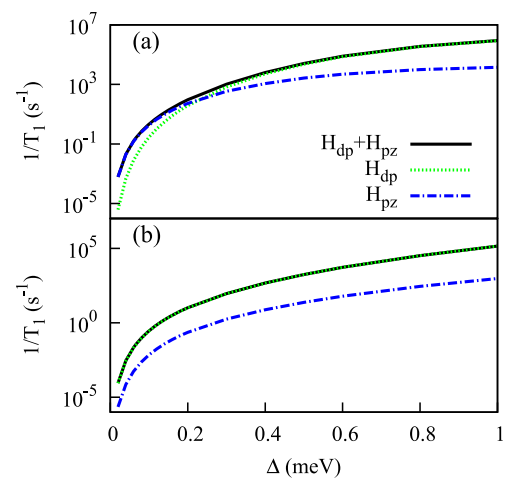

Figure 7. Hole spin relaxation as a function of the spin splitting energy. (a) Transition between hh states in a QD with $\hbar \omega_{\perp}=20$ and $\hbar \omega_{z}=50 \mathrm{meV}$. (b) Transition between lh states in a QD with $\hbar \omega_{\perp}=40$ and $\hbar \omega_{z}=5 \mathrm{meV}$.

piezoelectric potential when it is small [51]. In principle, for holes, the situation may differ because the deformation potential Hamiltonian, equation (17), is formally different from that of electrons. To investigate this point, in this section we compare the role of the two kinds of carrier-phonon coupling mechanism for holes subject to varying effective magnetic fields.

Figure 7(a) shows the spin relaxation rate in an oblate (quasi-2D) QD, where the highest states are hh, while figure 7(b) shows that in a prolate (quasi-1D) QD, where the highest states are $\mathrm{lh}$. For the spin transition within the hh doublet, panel (a), the behavior is analogous to that of electrons. Deformation potential interaction (dotted line) provides the largest contribution to $1 / T_{1}^{\mathrm{h}}$ except for very small $\Delta$, when piezoelectric interaction (dasheddotted line) takes over. This is because all the terms of $\mathcal{H}_{\mathrm{dp}}$ are proportional to the phonon momentum $\sqrt{q}$ (see equations (24)-(26)) while the piezoelectric potential is proportional to $1 / \sqrt{q}$ (see equation (23)). With decreasing $\Delta$, both mechanisms become inefficient, because for long phonon wavelength $\mathrm{e}^{\mathrm{iqr}} \approx 1$. Then, in equation (28), the matrix element $G_{r, r^{\prime}}(\mathbf{q}) \approx\left\langle r^{\prime} \mid r\right\rangle$, i.e. it tends to the overlap between the envelope components of the initial and final states. For $h$, these components have different symmetries, so the coupling vanishes. For example, in axially symmetric structures, the $J_{z}=+3 / 2$ component of the initial state has a well-defined azimuthal angular momentum $m_{z}=0$, which couples through the $s^{\lambda}$ operator of $H_{\mathrm{dp}}$ with the $J_{z}=+1 / 2$ component of the final state, for which $m_{z}=-2 .^{3}$

The situation for $\mathrm{lh}$ is quite different. As shown in figure 7(b), now deformation potential interaction is the dominant relaxation channel even for small $\Delta$. The underlying reason is that, in contrast to the hh case, the off-diagonal terms of $\mathcal{H}_{\mathrm{dp}}$ couple envelope components with the same symmetry. For example, the $J_{z}=+3 / 2$ and $1 / 2$ components of the initial and final state

3 In axially symmetric systems, the envelope functions of the Luttinger spinor have well-defined $z$-component of the orbital angular momentum, $m_{z}=F_{z}-J_{z}$. In oblate QDs, the highest doublet has $F_{z}= \pm 3 / 2$, and in prolate QDs it has $F_{z}= \pm 1 / 2$. See e.g. [45]. 
now have both $m_{z}=-1$, and hence are not orthogonal. As a consequence, $G_{r, r^{\prime}}(\mathbf{q})$ does not vanish when $q \rightarrow 0$.

\section{Summary}

We have investigated hole spin relaxation in InAs and GaAs QDs using 3D four-band $k \cdot p$ Hamiltonians. We have shown that the hole spin lifetime has a non-monotonic dependence on the lateral and vertical confinement strength. This is due to the interplay between the energy splitting of hh and lh, which is set by their different masses, and the anisotropic hh-lh coupling terms. The resulting geometry dependence of hole spin relaxation is qualitatively different from that of electrons.

hh-lh coupling and Dresselhaus SOI have been found to have comparable contributions to the spin admixture of hole states in self-assembled QDs, with the former becoming dominant for prolate structures, such as nanorods, and the latter for strongly oblate ones, such as gated QDs. The cubic-in- $k$ Dresselhaus term leads to an upper bound of $T_{1}^{\mathrm{h}}$ with increasing vertical confinement, which is missed when considering hh-lh coupling only.

We have also investigated the spin relaxation involving states with dominant $\mathrm{lh}$ character. Transitions between $\mathrm{lh}$ and hh states are very fast because lh have strong spin admixture. Instead, transitions between $\mathrm{lh}$ states with different Bloch angular momentum $J_{z}$ are as slow as the transitions between hh states with opposite spin. There is, however, a difference in the dominating hole-phonon interaction mechanism. At small energy splittings, the relaxation is mainly due to deformation potential interaction, unlike for hh transitions, where it is due to piezoelectric interaction.

\section{Acknowledgments}

Support from MICINN project CTQ2011-27324, UJI-Bancaixa project P1-1B2011-01, the Ramon y Cajal program (JIC) and UJI fellowship (CS) is acknowledged.

\section{Appendix. Dresselhaus Hamiltonian for holes}

In this appendix, we write the explicit matrix forms of the different $H_{\mathrm{BIA}}$ terms in Cartesian coordinates. Separating the different invariants in equation (9) we obtain

$$
H_{\mathrm{BIA}}=H_{C_{k}}+H_{b_{41}}+H_{b_{42}}+H_{b_{51}}+H_{b_{52}}
$$

where

$$
H_{C_{k}}=C_{k}\left(\begin{array}{cccc}
0 & -\frac{k_{-}}{2} & k_{z} & -\frac{\sqrt{3} k_{-}}{2} \\
-\frac{k_{+}}{2} & 0 & \frac{\sqrt{3} k_{+}}{2} & -k_{z} \\
k_{z} & \frac{\sqrt{3} k_{-}}{2} & 0 & -\frac{k_{-}}{2} \\
-\frac{\sqrt{3} k_{+}}{2} & -k_{z} & -\frac{k_{+}}{2} & 0
\end{array}\right)
$$


with $k_{ \pm}=k_{x} \pm \mathrm{i} k_{y}$ :

$$
H_{b_{41}}=b_{41}\left(\begin{array}{cccc}
\frac{3}{2} P_{41} & \frac{\sqrt{3}}{2} L_{41} & 0 & 0 \\
\frac{\sqrt{3}}{2} L_{41}^{\dagger} & \frac{1}{2} P_{41} & L_{41} & 0 \\
0 & L_{41}^{\dagger} & -\frac{1}{2} P_{41} & \frac{\sqrt{3}}{2} L_{41} \\
0 & 0 & \frac{\sqrt{3}}{2} L_{41}^{\dagger} & -\frac{3}{2} P_{41}
\end{array}\right),
$$

where $P_{41}=\left(k_{x}^{2}-k_{y}^{2}\right) k_{z}$ and $L_{41}=\mathrm{i} k_{-} k_{x} k_{y}-k_{+} k_{z}^{2}$ :

$$
H_{b_{42}}=b_{42}\left(\begin{array}{cccc}
\frac{27}{8} P_{41} & \frac{7 \sqrt{3}}{8} L_{41} & 0 & -\frac{3}{4} L_{42} \\
\frac{7 \sqrt{3}}{8} L_{41}^{\dagger} & \frac{1}{8} P_{41} & \frac{5}{2} L_{41} & 0 \\
0 & \frac{5}{2} L_{41}^{\dagger} & -\frac{1}{8} P_{41} & \frac{7 \sqrt{3}}{8} L_{41} \\
-\frac{3}{4} L_{42}^{\dagger} & 0 & \frac{7 \sqrt{3}}{8} L_{41}^{\dagger} & -\frac{27}{8} P_{41}
\end{array}\right),
$$

where $L_{42}=\mathrm{i} k_{+} k_{x} k_{y}+k_{-} k_{z}^{2}$ :

$$
H_{b_{51}}=b_{51}\left(\begin{array}{cccc}
0 & -\frac{\sqrt{3}}{4} K_{+} & \frac{\sqrt{3}}{2} K_{z} & -\frac{3}{4} K_{-} \\
-\frac{\sqrt{3}}{4} K_{-} & 0 & \frac{3}{4} K_{+} & -\frac{\sqrt{3}}{2} K_{z} \\
\frac{\sqrt{3}}{2} K_{z} & \frac{3}{4} K_{-} & 0 & -\frac{\sqrt{3}}{4} K_{+} \\
-\frac{3}{4} K_{+} & -\frac{\sqrt{3}}{2} K_{z} & -\frac{\sqrt{3}}{4} K_{-} & 0
\end{array}\right),
$$

where $K_{+}=K_{x}+\mathrm{i} K_{y}, \quad K_{-}=K_{x}-\mathrm{i} K_{y}, \quad K_{x}=k_{x}\left(k_{y}^{2}+k_{z}^{2}\right), \quad K_{y}=k_{y}\left(k_{x}^{2}+k_{z}^{2}\right) \quad$ and $\quad K_{z}=$ $k_{z}\left(k_{x}^{2}+k_{y}^{2}\right)$ :

$$
H_{b 52}=b_{52}\left(\begin{array}{cccc}
0 & -\frac{\sqrt{3}}{4} M_{+} & \frac{\sqrt{3}}{2} k_{z}^{3} & -\frac{3}{4} M_{-} \\
-\frac{\sqrt{3}}{4} M_{-} & 0 & \frac{3}{4} M_{+} & -\frac{\sqrt{3}}{2} k_{z}^{3} \\
\frac{\sqrt{3}}{2} k_{z}^{3} & \frac{3}{4} M_{-} & 0 & -\frac{\sqrt{3}}{4} M_{+} \\
-\frac{3}{4} M_{+} & -\frac{\sqrt{3}}{2} k_{z}^{3} & -\frac{\sqrt{3}}{4} M_{-} & 0
\end{array}\right),
$$

where $M_{+}=k_{x}^{3}+\mathrm{i} k_{y}^{3}$ and $M_{-}=k_{x}^{3}-\mathrm{i} k_{y}^{3}$.

\section{References}

[1] Fischer J, Trif M, Coish W A and Loss D 2009 Solid State Commun. 1491443

[2] Gerardot B D, Brunner D, Dalgarno P A, Öhberg P, Seidl S, Kroner M, Karrai K, Stoltz N G, Petroff P M and Warburton R J 2008 Nature 451441

[3] Brunner D, Gerardot B D, Dalgarno P A, Wüst G, Karrai K, Stoltz N G, Petroff P M and Warburton R J 2009 Science 32570

[4] Fallahi P, Yilmaz S T and Imamoglu A 2010 Phys. Rev. Lett. 105257402 
[5] Chekhovich E A, Krysa A B, Skolnick M S and Tartakovskii A I 2011 Phys. Rev. Lett. 106027402

[6] Eble B, Testelin C, Desfonds P, Bernardot F, Balocchi A, Amand T, Miard A, Lemaitre A, Marie X and Chamarro M 2009 Phys. Rev. Lett. 102144601

[7] Testelin C, Bernardot F, Eble B and Chamarro M 2009 Phys. Rev. B 79195440

[8] De Greve K et al 2011 Nature Phys. 7872

[9] Godden T M, Quilter J H, Ramsay A J, Wu Y, Brereton P, Boyle S J, Luxmoore I J, Puebla-Nunez J, Fox A M and Skolnick M S 2012 Phys. Rev. Lett. 108017402

[10] Greilich A, Carter S G, Kim D, Bracker A S and Gammon D 2011 Nature Photon. 5703

[11] Szumniak P, Bednarek S, Partoens B and Peeters F M 2012 Phys. Rev. Lett. 109107201

[12] Hsieh C Y, Cheriton R, Korkusinski M and Hawrylak P 2009 Phys. Rev. B 80235320

[13] Budich J C, Rothe D G, Hankiewicz E M and Trauzettel B 2012 Phys. Rev. B 85205425

[14] Roloff R, Eissfeller T, Vogl P and Pötz W 2010 New J. Phys. 12093012

[15] Liao Y H, Climente J I and Cheng S J 2011 Phys. Rev. B 83165317

[16] Kurtze H, Yakovlev D R, Reuter D, Wieck A D and Bayer M 2012 Phys. Rev. B 85195303

[17] Kowalik K, Krebs O, Lemaitre A, Gaj J A and Voisin P 2008 Phys. Rev. B 77161305

[18] Huxter V M, Kim J, Lo S S, Lee A, Sreekumari Nair P and Scholes G D 2010 Chem. Phys. Lett. 491187

[19] Lundstrom T, Schoenfeld W, Lee H and Petroff P M 1999 Science 2862312

[20] Reischle M, Beirne G J, Rossbach R, Jetter M and Michler P 2008 Phys. Rev. Lett. 101146402

[21] Li S D, Fu Y J and Cheng C 2012 Opt. Express 2019850

[22] Tsitsishvili E and Kalt H 2010 Phys. Rev. B 82195315

[23] Gündogdu K, Hall K C, Koerperick E J, Pryor C E, Flatté M E and Boggess T F 2005 Appl. Phys. Lett. 86113111

[24] Hall K C, Koerperick E J, Boggess T F, Shchekin O B and Deppe D G 2007 Appl. Phys. Lett. 90053109

[25] Laurent S, Eble B, Krebs O, Lemaitre A, Urbaszek B, Marie X, Amand T and Voisin P 2005 Phys. Rev. Lett. 94147401

[26] Heiss D, Schaeck S, Huebl H, Bichler M, Abstreiter G, Finley J J, Bulaev D V and Loss D 2007 Phys. Rev. B $\mathbf{7 6} 241306$

[27] Fras F, Eble B, Desfonds P, Bernardot F, Testelin C, Chamarro M, Miard A and Lemaitre A 2012 Phys. Rev. B 86045306

[28] Winkler R 2003 Spin-Orbit Coupling Effects in Two-Dimensional Electron and Hole Systems (Berlin: Springer)

[29] Woods L M, Reinecke T L and Kotlyar R 2004 Phys. Rev. B 69125330

[30] Lü C, Cheng J L and Wu M W 2005 Phys. Rev. B 71075308

[31] Bulaev D V and Loss D 2005 Phys. Rev. Lett. 95076805

[32] Trif M, Simon P and Loss D 2009 Phys. Rev. Lett. 103106601

[33] Wei H, Gong M, Guo G C and He L 2012 Phys. Rev. B 85045317

[34] Roszak K, Axt V M, Kuhn T and Machnikowski P 2007 Phys. Rev. B 76195324

[35] Tsitsishvili E, Baltz R V and Kalt H 2005 Phys. Rev. B 72155333

[36] He J, Zhong H and Scholes G D 2010 Phys. Rev. Lett. 105046601

[37] Luttinger J M 1956 Phys. Rev. 1021030

[38] Voon L C L Y and Willatzen M 2009 The k · p Method (Berlin: Springer) p 97

[39] Uenoyama T and Sham L J 1990 Phys. Rev. B 427114

[40] Planelles J, Climente J I and Segarra C 2012 J. Phys. Chem. C 11625143

[41] Vurgaftman I, Meyer J R and Ram-Mohan L R 2001 J. Appl. Phys. 895815

[42] Hu J, Li L S, Yang W, Manna L, Wang L W and Alivisatos A P 2001 Science 2922060

[43] Katz D, Wizansky T, Millo O, Rothenberg E, Mokari T and Banin U 2002 Phys. Rev. Lett. 89086801

[44] Voon L C L Y, Melnik R, Lassen B and Willatzen M 2004 Nano Lett. 4289

[45] Planelles J, Rajadell F and Climente J I 2010 J. Phys. Chem. C 1148337

[46] Blokland J H, Wijnen F J P, Christianen P C M, Zeitler U, Maan J C, Kailuweit P, Reuter D and Wieck A D 2007 Phys. Rev. B 75233305 
[47] Warburton R J, Miller B T, Dürr C S, Bödefeld C, Karrai K, Kotthaus J P, Medeiros-Ribeiro G, Petroff P M and Huant S 1998 Phys. Rev. B 5816221

[48] Schinner G J, Repp J, Schubert E, Kotthaus J P, Rai A K, Reuter D, Wieck A D, Govorov A O and Holleitner A W 2012 Single exciton emission from gate-defined quantum dots arXiv:1204.3199

[49] Madelung O 2004 Semiconductors: Data Handbook (Berlin: Springer)

[50] Khaetskii A V and Nazarov Y V 2001 Phys. Rev. B 64125316

[51] Climente J I, Bertoni A, Goldoni G and Molinari E 2006 Phys. Rev. B 74035313 

Hole spin relaxation in InAS/GaAs quantum dot molecules / C Segarra, J. I. Climente, F. Rajadell , J .Planelles

Journal of Physics: Condensed Matter, Volume 27, Number 41, p. 227 - 234

DOI: http://dx.doi.org/10.1088/0953-8984/27/41/415301

\section{Abstract:}

We calculate the spin-orbit induced hole spin relaxation between Zeeman sublevels of vertically stacked InAs quantum dots. The widely used Luttinger-Kohn Hamiltonian, which considers coupling of heavy- and light-holes, reveals that hole spin lifetimes ( $T$ 1) of molecular states significantly exceed those of single quantum dot states. However, this effect can be overcome when cubic Dresselhaus spin-orbit interaction is strong. Misalignment of the dots along the stacking direction is also found to be an important source of spin relaxation

Enlace al Repositori UJI (versión preprint): http://hdl.handle.net/10234/158626 


\title{
Electrons, holes, and excitons in GaAs polytype quantum dots
}

\author{
Juan I. Climente, Carlos Segarra, Fernando Rajadell, and Josep Planelles ${ }^{\text {a) }}$ \\ Departament de Química Física i Analítica, Universitat Jaume I, E-12080 Castelló, Spain \\ (Received 26 January 2016; accepted 20 March 2016; published online 31 March 2016)
}

\begin{abstract}
Single and multi-band $k \cdot p$ Hamiltonians for GaAs crystal phase quantum dots are used to assess ongoing experimental activity on the role of such factors as quantum confinement, spontaneous polarization, valence band mixing, and exciton Coulomb interaction. Spontaneous polarization is found to be a dominating term. Together with the control of dot thickness [Vainorius et al., Nano Lett. 15, 2652 (2015)], it enables wide exciton wavelength and lifetime tunability. Several new phenomena are predicted for small diameter dots [Loitsch et al., Adv. Mater. 27, 2195 (2015)], including non-heavy hole ground state, strong hole spin admixture, and a type-II to type-I exciton transition, which can be used to improve the absorption strength and reduce the radiative lifetime of GaAs polytypes. (C) 2016 AIP Publishing LLC. [http://dx.doi.org/10.1063/1.4945112]
\end{abstract}

\section{INTRODUCTION}

Semiconductor quantum dots (QDs) have been widely studied since the 1990s because of their appealing electronic and photonic properties. However, standard fabrication methods involve a degree of dispersity which limits exact reproducibility within an ensemble of dots, from run-to-run and from lab-to-lab. ${ }^{1-3}$ For example, in Stranski-Krastanov growth of InAs/GaAs QDs, the most widely employed technique to produce optically active III-V QDs, random diffusion of substrate material (GaAs) into the deposited material (InAs) leads to an ensemble of QDs with inhomogeneous composition, strain fields, and shapes. ${ }^{3}$ This translates into an inhomogeneous distribution of energy levels, which poses a challenge for the scalability of many technological applications demonstrated at a single-dot level. ${ }^{4,5}$

Crystal phase (polytype) QDs ${ }^{6}$ are likely to mitigate this problem. These structures exploit recent synthetic advances enabling control on the polytypical crystal structure of III-V nanowires, whereby one can grow alternating segments of wurtzite (WZ) grown along the [0001] direction and zincblende (ZB) grown along [111]. ${ }^{7}$ Because $\mathrm{WZ}$ and ZB phases have slightly different energy gaps at the $\Gamma$ point, band offsets are formed and carriers confined in one of the phases. ${ }^{8}$ One can then form QDs embedded in the wire, which turn out to have defect-free crystal structure, sharp interfaces, negligible strain and tapering, well defined shape, and homogeneous composition. Prospects have become especially promising with two studies published in the last months for GaAs polytype QDs. On the one hand, Vainorius et al. have reported exact control on the QD thickness, from bilayers to tens of $\mathrm{nm} .{ }^{9}$ On the other hand, Loitsch et al. have reported control of the wire diameter from typical values $(\sim 100 \mathrm{~nm})$ down to $7 \mathrm{~nm} .{ }^{10}$ Together, these studies pave the way towards full control of the QD confinement and, consequently, of the energy structure.

Progress in the synthesis of GaAs polytype QDs, however, has not been paralleled by theoretical understanding of the ensuing electronic and optoelectronic properties. As a

a)josep.planelles@uji.es. URL: http://quimicaquantica.uji.es/ result, several open questions remain which need to be clarified in order to eventually attain predictive design. To name a few: (i) the role of spontaneous polarization in WZ GaAs is not clear. The majority of experimental works simply neglect it, ${ }^{8-12}$ but recent theoretical ${ }^{13}$ and experimental ${ }^{14}$ studies point to a value of $P_{s p}=0.0023 \mathrm{C} / \mathrm{m}^{2}$, which Jahn et al. deemed influential at a single-particle level. ${ }^{15}$ One then wonders if it is really important for Coulomb-bound excitons. (ii) The role of valence band (VB) coupling is also poorly understood. It is generally assumed that the hole ground state is a heavy hole $(\mathrm{HH}){ }^{9-12}$ This is consistent with polarization measurements of large diameter nanowires. ${ }^{16}$ However, radial confinement enhances valence band mixing. ${ }^{17}$ Therefore, the validity should be tested at least in the small diameter regime enabled by the work of Loitsch et al. ${ }^{10}$ (iii) The influence of electron-hole Coulomb interaction needs better assessment. Because WZ and ZB interfaces form a type-II band-alignment in $\mathrm{GaAs},{ }^{8}$ previous simulations of optical transition energies in GaAs polytypes either tend to neglect it ${ }^{9,15}$ or take it as a constant. ${ }^{10}$ However, the band offsets are so small that both electron and hole wave functions are expected to penetrate into each other's phase, leading to non-vanishing electron-hole overlap. ${ }^{12}$ What is more, strong Coulomb interactions could eventually overcome the band offsets and change the excitons from type-II to type-I. This is a possibility worth exploring.

In this work, we use a $\mathrm{k} \cdot \mathrm{p}$ model to study carriers confined in polytype QDs, which takes into account all the factors described above: spontaneous polarization, electron-hole Coulomb interaction, and valence band coupling of holes. The latter is included by building a 6-band Hamiltonian for ZB/WZ polytypes. We study polytype QDs within the confinement ranges made possible by the works of Vainorius ${ }^{9}$ and Loitsch, ${ }^{10}$ and evaluate the influence of the abovementioned factors on the energy structure of electrons, holes, and excitons. The results are discussed in view of the existing experiments.

\section{II. $\boldsymbol{k} \cdot \boldsymbol{p}$ HAMILTONIANS FOR WZ/ZB QDs}

In order to model polytypes, we use a $k \cdot p$ Hamiltonian spanned on the same Bloch functions in both crystal 
structures, the differences showing up in the parameters only. In this section, we describe such Hamiltonians for conduction band (CB) electrons, VB holes, and excitons.

\section{A. Electrons}

Low-energy electrons in $\mathrm{ZB}$ GaAs belong to the $\Gamma_{6}$ band, which is well separated from the valence band and the rest of CBs. This justifies the widely spread use of singleband models in the literature. In WZ GaAs, however, $\Gamma_{8 c}$ and $\Gamma_{7 c}$ bands are close to each other, and some band mixing can be expected. ${ }^{18,19}$ Lacking effective mass parameters describing such a coupling, we model WZ electrons with a single-band Hamiltonian of hybrid character: $\Gamma_{8 c}$ masses but optically bright, like the $\Gamma_{7 c}$ band. This picture is consistent with the observations of different recent experiments ${ }^{12,20,21}$ and suffices to assess the role of the physical factors we investigate. The polytype Hamiltonian then reads

$$
H_{e}=-\frac{\hbar^{2}}{2} \sum_{i=x, y, z} k_{i} \frac{1}{m_{i}^{*}} k_{i}+V_{c}^{c b}+q V_{s p} .
$$

Here, $m_{i}^{*}$ is the effective mass along the $i$ direction, which depends on the crystal phase, $k_{i}=-\nabla_{i}, V_{c}^{c b}$ is the 3D confinement potential arising from the conduction band-offset potential between $\mathrm{ZB}$ and $\mathrm{WZ}$ phases, $q$ is the electron charge, and $V_{s p}$ is the electrostatic potential due to the spontaneous polarization $P_{s p}$

The calculation of strain in polytype QDs deserves a short discussion. The initial strain in a heterostructure is given by the lattice mismatch. For a QD of a given material buried in a matrix of a different material with the same crystalline structure, it is zero in the matrix and $\epsilon_{i i}^{0}=\frac{a_{i}^{(m)}-a_{i}^{(Q D)}}{a_{i}^{(m)}}$ in the QD, where $a_{i}^{j}$ is the lattice constant in the direction $i$ for the medium $j{ }^{22}$ However, this expression cannot be employed in polytypes because QD and matrix have different crystalline structures. In our case, since we deal with $\mathrm{ZB}(111) / \mathrm{WZ}(0001)$ interfaces, we may reason as follows. The ZB unit cell contains 9 anions and 9 cations, while the WZ unit cell has the same basis but different height and only 6 pairs of ions (see, e.g., Fig. 1 in Ref. 23). Then, three WZ unit cells contain the same number of ions as two $\mathrm{ZB}$ unit cells. If the $\mathrm{ZB}$ and $\mathrm{WZ}$ materials are the same $(\mathrm{GaAs}$ in or case) and under the assumption that the lattices are ideal, the basis surface of both unit cells is the same, and so is the height of three WZ unit cells vs. two ZB ones. Therefore, the strain is ideally zero. This is consistent with theoretical calculations ${ }^{24}$ and experimental findings $\mathrm{s}^{9,10,24}$ pointing at negligible strain, as real lattice constants show but small deviations from ideal ones. One can then safely disregard it.

Since the strain is weak, so is the piezoelectric potential and its influence on the energy spectrum. By contrast, the spontaneous polarization potential $V_{s p}$ can have a significant influence. There is no spontaneous polarization in the $\mathrm{ZB}$ phase for symmetry reasons, but it is present in WZ, where $P_{s p}$ originates from the "eclipsed" dihedral conformation of layers $N$ and $N+2$, yielding a non-ideal tetrahedral coordination and associated electric dipoles. Current estimates for
GaAs are of $P_{s p} \approx 0.0023 \mathrm{C} \mathrm{m}^{-2},{ }^{13,14}$ about one order of magnitude weaker than in nitride materials. Since the change in $P_{s p}$ is large at the $\mathrm{ZB} / \mathrm{WZ}$ interface, it gives rise to an abrupt change in the built-in electric field, from zero in $\mathrm{ZB}$ up to an approximate constant value $F$ in WZ given by $F=P_{s p} /$ $\varepsilon$, with $\varepsilon$ the dielectric constant, and back again to zero in $\mathrm{ZB}$ (see, e.g., Fig. 4 in Ref. 14). Then, the QD acts like a capacitor, with effective negative and positive charges accumulating at the ZB/WZ and WZ/ZB interfaces, and an almost linear potential in between (see, e.g., CB profile in the insets of Fig. 2).

\section{B. Holes}

\section{Multi-band Hamiltonian}

To study the effect of VB mixing, we use a multi-band k.p Hamiltonian. In order to compare [111]-grown ZB and [0001]-grown WZ structures systematically, we write the six-band Hamiltonian employing for both phases the same basis functions ${ }^{23,25}$

$$
\begin{aligned}
\left|u_{1}\right\rangle & =-\frac{1}{\sqrt{2}}|(X+i Y) \uparrow\rangle & \left|u_{4}\right\rangle & =\frac{1}{\sqrt{2}}|(X-i Y) \downarrow\rangle \\
\left|u_{2}\right\rangle & =\frac{1}{\sqrt{2}}|(X-i Y) \uparrow\rangle & \left|u_{5}\right\rangle & =-\frac{1}{\sqrt{2}}|(X+i Y) \downarrow\rangle \\
\left|u_{3}\right\rangle & =|Z \uparrow\rangle & \left|u_{6}\right\rangle & =|Z \downarrow\rangle .
\end{aligned}
$$

For [0001] WZ, the resulting Hamiltonian in this basis reads $^{26}$

$$
H_{6 B}=\left[\begin{array}{cccccc}
F & -K^{*} & -H^{*} & 0 & 0 & 0 \\
-K & G & H & 0 & 0 & \sqrt{2} \Delta_{3} \\
-H & H^{*} & \lambda & 0 & \sqrt{2} \Delta_{3} & 0 \\
0 & 0 & 0 & F & -K & H \\
0 & 0 & \sqrt{2} \Delta_{3} & -K^{*} & G & -H^{*} \\
0 & \sqrt{2} \Delta_{3} & 0 & H^{*} & -H & \lambda
\end{array}\right],
$$

where

$$
\begin{aligned}
F & =\Delta_{1}+\Delta_{2}+\lambda+\theta, \\
G & =\Delta_{1}-\Delta_{2}+\lambda+\theta, \\
\lambda & =\frac{\hbar^{2}}{2 m_{e}}\left[A_{1} k_{z}^{2}+A_{2} k_{\perp}^{2}\right], \\
\theta & =\frac{\hbar^{2}}{2 m_{e}}\left[A_{3} k_{z}^{2}+A_{4} k_{\perp}^{2}\right], \\
K & =\frac{\hbar^{2}}{2 m_{e}} A_{5} k_{+}^{2}+\Delta K, \\
H & =\frac{\hbar^{2}}{2 m_{e}} A_{6} k_{+} k_{z}+\Delta H .
\end{aligned}
$$

Here, $m_{e}$ is the free electron mass, $A_{i}$ is the effective mass parameters, $k_{\perp}=k_{x}^{2}+k_{y}^{2}, k_{ \pm}=k_{x} \pm i k_{y}, \Delta_{1}$ is the crystal field splitting, $\Delta_{2}$ and $\Delta_{3}$ are the spin-orbit matrix elements, and $\Delta K=\Delta H=0$.

For [111] ZB, according to Bir-Pikus, ${ }^{27}$ the $[001] \mathrm{ZB}$ Hamiltonian-spanned on the basis of Eq. (2) - is first rotated $45^{\circ}$ along the $z$ axis, and then $54.7^{\circ}$ along the new $y^{\prime}$ 
axis. The resulting $z^{\prime}$ axis points along the [111] direction, while $x^{\prime}$ and $y^{\prime}$ do so along the $[11 \overline{2}]$ and $[\overline{1} 10]$ directions. The Hamiltonian obtained is formally identical to Eq. (3), but now

$$
\begin{aligned}
\Delta K & =2 \sqrt{2} \frac{\hbar^{2}}{2 m_{e}} A_{z} k_{-} k_{z}, \\
\Delta H & =\frac{\hbar^{2}}{2 m_{e}} A_{z} k_{-}^{2} .
\end{aligned}
$$

Additionally, the following relations emerge, which reduce the number of independent mass parameters to three, as expected for $\mathrm{ZB}$ :

$$
\begin{aligned}
& \Delta_{1}=0, \\
& \Delta_{2}=\Delta_{3}=\Delta / 3, \\
& A_{1}=-\gamma_{1}-4 \gamma_{3}, \\
& A_{2}=-\gamma_{1}+2 \gamma_{3}, \\
& A_{3}=6 \gamma_{3}, \\
& A_{4}=-3 \gamma_{3}, \\
& A_{5}=-\gamma_{2}-2 \gamma_{3}, \\
& A_{6}=-\sqrt{2}\left(2 \gamma_{2}+\gamma_{3}\right), \\
& A_{z}=\gamma_{2}-\gamma_{3},
\end{aligned}
$$

where $\gamma_{1}, \gamma_{2}$, and $\gamma_{3}$ are the Luttinger mass parameters.

It is worth noting that $H_{6 B}$-with $\mathrm{ZB}$ parameters, Eqs. (5) and (6) - actually shows all diagonal elements overstabilized by an amount $\Delta / 3$. This is due to the term $\frac{1}{3} \Delta(\sigma \cdot \mathbf{J})$ in the sum of invariants defining $H_{6 B},{ }^{27}$ which is needed to yield the Hamiltonian extradiagonal elements $H_{26}, H_{35}, H_{53}$, and $H_{62}$. We recover the zero origin at the top of the $\mathrm{HH}$ band by subtracting $\Delta / 3$ to all diagonal elements of $H_{6 B}$ in the $\mathrm{ZB}$ region.

The above considerations prompt us to obtain a Hamiltonian which is valid for both ZB and WZ regions, and hence open the possibility of dealing with polytypes. Since the parameters in the two phases are different, we should employ a variable mass Hamiltonian. ${ }^{28-31}$ The use of bulk multiband Hamiltonian with different parameters in each phase leads to an abrupt change at the interface. The resulting matrix functions, though, must be considered to vary slowly on the scale of the lattice constant but rapidly on the scale of the envelope changes. Furthermore, the Hamiltonian must be modified to ensure Hermiticity. In the one-band model, this was overcome by the use of the Ben Daniel-Duke kinetic term. ${ }^{32}$ The extension to multiband Hamiltonians was initially carried out by means the use of a symmetrization rule, ${ }^{33}$ the operator ordering with respect to the band parameters reflecting the boundary conditions at the abrupt interface. We have checked that starting from a conventionally symmetrized [001] ZB Hamiltonian spanned on the above basis set, Eq. (2), and following the above-mentioned rotation prescription, we end up with symmetrized form of the [111] ZB Hamiltonian. All the same, an envelope function theory for nanostructures accounting for abrupt interfaces was later developed, ${ }^{34-37}$ leading to a nonsymmetrized Hamiltonian. ${ }^{38}$ Therefore, we implement here a nonsymmetrized or Burt-Foreman form of the Hamiltonian. Namely,

$H_{6 B}^{B F}=\left[\begin{array}{cccccc}F-\rho & \kappa & \xi^{*} & 0 & 0 & 0 \\ \kappa^{*} & G+\rho & -\xi & 0 & 0 & \sqrt{2} \Delta_{3} \\ \eta & -\eta^{*} & \lambda & 0 & \sqrt{2} \Delta_{3} & 0 \\ 0 & 0 & 0 & F+\rho & \kappa^{*} & -\xi \\ 0 & 0 & \sqrt{2} \Delta_{3} & \kappa & G-\rho & \xi^{*} \\ 0 & \sqrt{2} \Delta_{3} & 0 & -\eta^{*} & \eta & \lambda\end{array}\right]$,

where

$$
\begin{aligned}
F & =\Delta_{1}+\Delta_{2}+\lambda+\theta, \\
G & =\Delta_{1}-\Delta_{2}+\lambda+\theta, \\
\lambda & =\frac{\hbar^{2}}{2 m_{e}}\left[k_{z} A_{1} k_{z}+k_{x} A_{2} k_{x}+k_{y} A_{2} k_{y}\right], \\
\theta & =\frac{\hbar^{2}}{2 m_{e}}\left[k_{z} A_{3} k_{z}+k_{x} A_{4} k_{x}+k_{y} A_{4} k_{y}\right], \\
\kappa & =\frac{\hbar^{2}}{2 m_{e}}\left[-k_{x} A_{5} k_{x}+k_{y} A_{5} k_{y}+i\left(k_{x} A_{5} k_{y}+k_{y} A_{5} k_{x}\right)\right]+\Delta \kappa, \\
\eta & =\frac{\hbar^{2}}{2 m_{e}}\left[-k_{z} A_{6}^{(+)} k_{+}-k_{+} A_{6}^{(-)} k_{z}\right]+\Delta \eta, \\
\xi & =\frac{\hbar^{2}}{2 m_{e}}\left[-k_{z} A_{6}^{(-)} k_{+}-k_{+} A_{6}^{(+)} k_{z}\right]+\Delta \xi, \\
\rho & =\frac{\hbar^{2}}{2 m_{e}}\left[i k_{y}\left(A_{5}^{(+)}-A_{5}^{(-)}\right) k_{x}-i k_{x}\left(A_{5}^{(+)}-A_{5}^{(-)}\right) k_{y}\right], \\
\Delta \xi & =\frac{\hbar^{2}}{2 m_{e}}\left[-\left(k_{x}-i k_{y}\right) A_{z}\left(k_{x}-i k_{y}\right)\right], \\
\Delta \eta & =\Delta \xi \\
\Delta \kappa & =-2 \sqrt{2} \frac{\hbar^{2}}{2 m_{e}}\left[\left(k_{x}+i k_{y}\right) A_{z}^{(+)} k_{z}+k_{z} A_{z}^{(-)}\left(k_{x}+i k_{y}\right)\right],
\end{aligned}
$$

with $A_{5}=A_{5}^{(+)}+A_{5}^{(-)}, A_{6}=A_{6}^{(+)}+A_{6}^{(-)}$and $A_{z}=A_{z}^{(+)}+A_{z}^{(-)}$. In the $\mathrm{WZ}$ region, $A_{z}^{(+)}=A_{z}^{(-)}=0$. In the $\mathrm{ZB}$ region, Eqs. (6) still hold.

The practical hindrance for the use of this Hamiltonian, especially for studying polytypes, is the lack of available $A_{i}^{( \pm)}$ coefficients. At this regard, Veprek et al. ${ }^{29}$ analyzed the spurious solution problem affecting the $k \cdot p$ envelope function method, which is related to the lack of ellipticity that the different sets of parameters confer to the Hamiltonian. They concluded by recommending the use of a complete asymmetric operator ordering $\left(A_{i}^{(+)}=A_{i}, A_{i}^{(-)}=0\right)$ for several ZB and WZ materials. ${ }^{29,30,39}$ We have checked that this also applies to GaAs.

We are now in a condition to write the complete Hamiltonian for holes in polytype QDs

$$
H_{h}^{6 B}=H_{6 B}^{B F}+\left(V_{c}^{v b}-q V_{s p}-\frac{\Delta}{3} Y_{Z B}\right) \mathbb{I}_{6 \times 6}
$$

$V_{c}^{v b}$ and $V_{s p}$ are the confining and spontaneous polarization potentials, respectively, which we obtain as described for electrons. $Y_{Z B}$ is a heaviside function, $Y_{Z B}=0$ in the WZ phase and $Y_{Z B}=1$ in the $\mathrm{ZB}$ one. 


\section{Single-band Hamiltonian}

The use of single-band models for the hole ground state in GaAs polytypes is justified under certain conditions. In $\mathrm{ZB}$, the degeneracy between heavy and light hole bands can be lifted by quantum confinement. In WZ, as can be seen in Eq. (3), the uppermost band $(F)$ is split from the others $(G, \lambda)$ by the spin-orbit $\left(\Delta_{2}\right)$ and crystal field $\left(\Delta_{1}\right)$ splittings, so degeneracy is lifted even at the $\Gamma$ point. Thus, in order to get the single-band Hamiltonian, we decouple the diagonal elements corresponding to the $F$ (heavy hole) band from the rest of the matrix in Eq. (9). This yields

$$
H_{h}=\Delta_{1}+\Delta_{2}+\sum_{i=x, y, z} \frac{\hbar^{2}}{2} k_{i} \frac{1}{m_{i}^{*}} k_{i}+V_{c}^{v b}-q V_{s p}-\frac{\Delta}{3} Y_{Z B},
$$

where the parameters $\Delta_{1}, \Delta_{2}$, and $m_{i}^{*}$ take different values in each crystal phase. In particular, for WZ, $m_{z}=1 /\left(A_{1}+A_{3}\right)$ and $m_{\perp}=1 /\left(A_{2}+A_{4}\right)$. For ZB, $m_{z}=-1 /\left(\gamma_{1}-2 \gamma_{3}\right)$ and $m_{\perp}=-1 /\left(\gamma_{1}+\gamma_{3}\right), \Delta_{1}=0$, and $\Delta_{2}=\Delta / 3$.

\section{Excitons}

We calculate neutral excitons using single-band Hamiltonians for both electron and hole

$$
H_{X}=H_{e}+H_{h}+V_{e h},
$$

where $V_{e h}$ is the electron-hole Coulomb interaction, which is obtained by integrating the Poisson equation in a dielectrically inhomogeneous environment.

\section{System and parameters}

We take ZB GaAs material parameters from Ref. 41. As for WZ GaAs, we take electron effective masses from Ref. 18 , VB ones from Ref. 42, and the spontaneous polarization $P_{s p}=0.0023 \mathrm{C} \mathrm{m}^{-2}$ from Ref. 13. Lacking more precise information for WZ, we use a dielectric constant $\varepsilon=13.18$ for both phases. $^{43}$

To define $V_{c}^{c b}$ and $V_{c}^{v b}$, we consider either ZB QDs embedded in WZ nanowires, see Fig. 1(a), or WZ QDs embedded in ZB nanowires, see Fig. 1(b). The corresponding band offset values, represented in the figure, are taken from Ref. 8. The nanowires are assumed to be surrounded by an insulating material with $V_{c}^{c b}=-V_{c}^{v b}=5 \mathrm{eV}$ and $\varepsilon=4$.

We use Comsol 4.2 to solve numerically the Hamiltonians described above. $V_{s p}$ is obtained by calculating the polarization charge density $\rho=-\nabla \cdot P_{s p}$ and solving the Poisson equation, $\nabla \cdot[\varepsilon \nabla V]=\rho$. Next, Hamiltonians $H_{e}$, $H_{h}^{6 B}$, and $H_{h}$ are integrated using finite elements. As for $H_{X}$, converged interacting electron and hole states are obtained by using an iterative Schrödinger-Poisson scheme.

\section{RESULTS}

\section{A. Electrons}

We start by investigating the ground state of a single electron in a ZB QD, like that in Fig. 1(a). The QD is hexagonal, with a typical radius of the circumscribed circle, $R=50 \mathrm{~nm}$, and variable thickness $L$. The results are plotted (a)
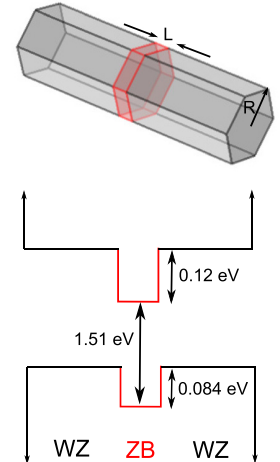

(b)
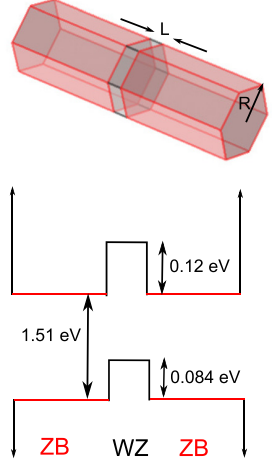

FIG. 1. (a) Sketch of ZB QD embedded in WZ wire and corresponding band-offset profile. (b) Same but for WZ QD embedded in ZB wire.

in Fig. 2, where we compare calculations with the expected spontaneous polarization of GaAs, $P_{s p}=2.3 \times 10^{-3} \mathrm{C} \mathrm{m}^{-2}$ (solid line), and calculations with an artificially weakened polarization, $P_{s p}=2.3 \times 10^{-4} \mathrm{C} \mathrm{m}^{-2}$ (dashed line). It is clear from the figure that, except for thin dots $(L<5 \mathrm{~nm}), P_{s p}$ plays a critical role in determining the electron energy. Under full polarization, the energy shows a linear dependence with $L$, determined by the capacitor-like built-in electric field. By contrast, under weakened polarization, the linear regime is preceded by a quadratic one (up to $L \lesssim 10 \mathrm{~nm}$ ), which is determined by quantum confinement. The magnitude of the energy stabilization is also very different. In fact, for $P_{s p}=2.3 \times 10^{-3} \mathrm{C} \mathrm{m}^{-2}$ and large $L$, the electric field leads to energies well below the CB bottom of bulk ZB.

These results are qualitatively similar to those obtained by Jahn and co-workers using a simpler 1D model with zincblende masses ${ }^{15}$ and confirm that the spontaneous polarization in GaAs polytypes cannot be neglected, at least at a single particle level. As we shall see below, in Section III C, the same is true for excitons in spite of the electron-hole attraction.

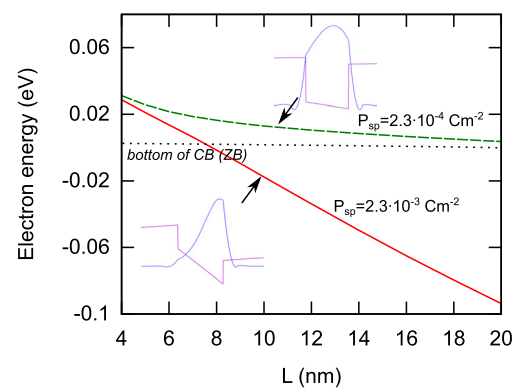

FIG. 2. Energy of the electron ground state in a ZB QD with realistic (solid line) and weakened (dashed line) spontaneous polarization, as a function of the dot thickness. Note the strong influence. The insets show the wave functions and band profiles for $L=10 \mathrm{~nm}$. 
The insets in Fig. 2 show the electron wave function and band profile for the full and weakened polarization values. Notice that $P_{s p}$ pushes the electron towards the $\mathrm{ZB} / \mathrm{WZ}$ interface and induces substantial spreading into the WZ phase.

The precise control of the thickness in GaAs polytype QD, recently achieved by Vainorius et al., ${ }^{9}$ suggests such structures could be used to build perfectly symmetric pairs of QDs. In principle, this could enable the formation of QD molecules with homonuclear character, unlike in selfassembled InAs/GaAs structures where the inherent structural asymmetries can only be overcome with external fields. ${ }^{44}$ Symmetric molecules can be of interest for applications like optical qubits ${ }^{45}$ or the development of superlattices with maximized coherent tunneling for solar cell devices. ${ }^{46}$ However, the results of Fig. 2 suggest that the strong influence of $P_{s p}$ can introduce significant asymmetries in the band profile of symmetric molecules. This is confirmed in Fig. 3, where one can see that for two identical ZB QDs separated by a thin WZ barrier, the electron wave function localizes mostly in one of the dots. This effect is already noticeable if the system has weakened $P_{s p}$ (upper plot), and it becomes dramatic for full $P_{s p}$ (lower plot), when tunneling is almost nearly suppressed.

\section{B. Holes}

The energetics of holes in WZ QDs is qualitatively similar to that of electrons in ZB dots. In this section, we focus on the role of VB mixing instead. In particular, we assess the validity of the usual assumption that the ground state has a well defined, single-band, $\mathrm{HH}$ character. $^{9-12}$

The eigenfunctions of Hamiltonian $H_{h}^{6 B}$ are sixcomponent spinors of the form: $\Psi_{h}^{6 B}=\sum_{i=1}^{6} f_{i}(\mathbf{r})\left|u_{i}\right\rangle$, where $f_{i}(\mathbf{r})$ is the envelope function associated with the $\left|u_{i}\right\rangle$ Bloch function. The weight of an individual component is computed as $\left|f_{i}\right|^{2}$. As can be seen in Eq. (2), HH character corresponds to Bloch functions $\left|u_{1}\right\rangle$ (spin up) and $\left|u_{4}\right\rangle$ (spin down), i.e., the $F$ band of Hamiltonian $H_{6 B}^{B F}$. To disentangle spin up and down components, a small Zeeman-like term is included in Eq. (9), $\Delta_{z}=B \mu_{B} g \mathbb{J}_{z}$, where $B=1 \mathrm{~T}$ is the longitudinal magnetic field, $\mu_{B}$ is the Bohr magneton, $g=4 / 3$ is

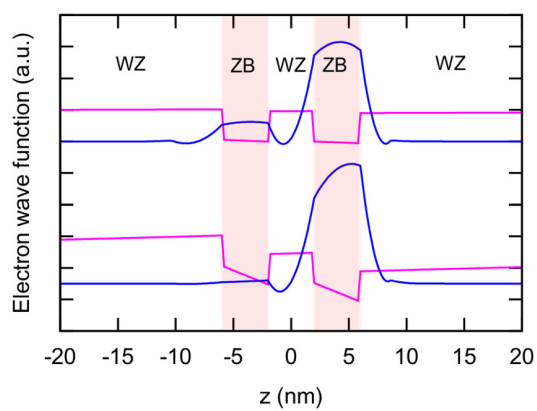

FIG. 3. Electron wave function and band profile in a ZB-WZ-ZB molecule with weakened and full $P_{s p}$. Both $\mathrm{ZB}$ QDs and the WZ barrier have thickness $L=4 \mathrm{~nm}$. the hole $g$-factor, and $\mathbb{J}_{z}$ is the angular momentum $z$-component diagonal matrix (with elements $\pm 3 / 2, \pm 1 / 2$ ).

We first estimate the total $\mathrm{HH}$ character from $\left(\left|f_{1}\right|^{2}+\left|f_{4}\right|^{2}\right)$. Fig. 4(a) shows the normalized HH weight for the ground state of WZ QDs. The QDs structure is that of Fig. 1(b), with the radius $R$ and thickness $L$ varying within a parameter space enabled by state-of-the-art fabrication, which includes the regime of quantum confinement in the radial direction. ${ }^{9,10}$ One can see that the ground state has almost exclusive $\mathrm{HH}$ character except for very thin radii, $R<5 \mathrm{~nm}$. In this region, the ground state rapidly switches from mainly $F$ band (HH) to mainly $\lambda$ band character, as shown in Fig. 4(a) inset. The origin of the ground state change can be understood as follows. In the bulk limit, the $F$ band of wurtzite is stabilized with respect to $G$ and $\lambda$ bands by the crystal field and spin-orbit splittings. However, the radial mass of $F$-band holes in WZ is $m_{\perp}^{F}=1 /\left(A_{2}+A_{4}\right)=-0.13$, much lighter than that of $\lambda$-band holes, $m_{\perp}^{\lambda}=1 / A_{2}=-0.617$. Therefore, with increasing radial confinement, the latter become more stable. It is worth noting that $\lambda$ holes are very light in the [0001] direction, $m_{z}^{\lambda}=1 / A_{1}=-0.05$. As a consequence, their kinetic energy exceeds the small band offset of the WZ/ ZB interface, and the wave function tends to localize outside the QD, as shown in Fig. 4(b). This result is a consequence of the radial confinement, and it is also found when the spontaneous polarization is neglected (not shown).

The change of ground state character we report here should be observable in the narrowest wires synthesized by Loitsch et al. ${ }^{10,11}$ Since the symmetry of the $\lambda$ band Bloch functions $\left(\left|u_{3}\right\rangle\right.$ and $\left|u_{6}\right\rangle$ in Eq. (2)) is different from that of $\mathrm{HHs}$, it should be seen in experiments as a change in the polarization of interband optical transitions.

Next, we analyze the $\mathrm{HH}$ spin admixture by representing the ratio between the weight of spin up and down $\mathrm{HH}$, $\left|f_{1}\right|^{2} /\left|f_{4}\right|^{2}$. This is done for QDs in the presence and absence of $P_{s p}$, Figs. 4(c) and 4(d), respectively. A remarkable observation is that moderate radial or vertical confinement leads to significant spin mixing between the Zeeman split levels. This is because the off-diagonal elements of Hamiltonian (7) scale with $k_{\perp}$ and $k_{z}$. Note that, in the presence of $P_{s p}$, the spin mixing takes place even for large $L$, because the vertical electrostatic confinement does not vanish with the dot thickness. Interestingly, the confinement leads to admixture between spin up and down $F$-band holes but coupling with $G$ and $\lambda$ bands remains negligible (recall Fig. 4(a) inset).

The spin mixing of HHs has influence on the magnetic properties of WZ/ZB QDs. For example, the values of the effective $g$-factors are often determined as $g=\left(E_{+}(B)-E_{-}\right.$ $(B)) / \mu_{B} B$, where $E_{ \pm}(B)$ is the energy of opposite spin projections under a magnetic field $B$. We have calculated the $g$ factor at $B=1 \mathrm{~T}$ switching on and off the spin-orbit term $\Delta$ in Hamiltonian (7). This leads to $g$ factors with enabled $\left(g^{s o}\right)$ and suppressed $\left(g^{0}\right)$ spin mixing. In Fig. 5, we plot the ratio $g^{s o} / g^{0}$ in two instances: QDs with (a) and without (b) spontaneous polarization. One can see that for strong confinement, band mixing can enhance $g$ factor values up to a factor of $2-3$. Unlike in other QD systems, where the confinement symmetry plays a critical role in determining the spin admixture strength, ${ }^{47}$ the mixing in polytypes is robust against 

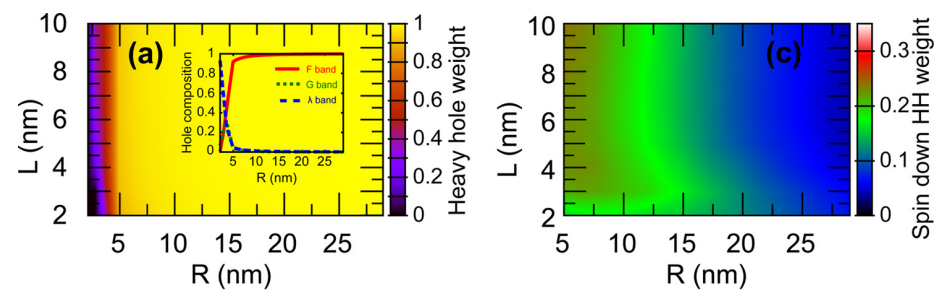

FIG. 4. (a) Weight of the HH ( $F$ band) component in the hole ground state, $\left(\left|f_{1}\right|^{2}+\left|f_{4}\right|^{2}\right) / \sum\left|f_{i}\right|^{2}$, as a function of dot radius and thickness in a WZ QD The inset shows the weight of each subband for $L=4 \mathrm{~nm}$. The ground state changes from $F$ band (HH) to $\lambda$ band for radii under $5 \mathrm{~nm}$. (b) Confining potential (red) and $\lambda$ hole envelope wave function (green) along the $z$ axis for a QD with $L=4 \mathrm{~nm}$ and $R=2.5 \mathrm{~nm}$. The $\lambda$ hole is not confined owing to its small mass. (c) and (d) Weight of spin down $\mathrm{HH}$ (c) and (d) $|f|^{2} / \sum|f|^{2}$, with and withonent $P$ $\left|f_{4}\right|^{2} / \sum\left|f_{i}\right|^{2}$, with and without $P_{s,}$ respectively.

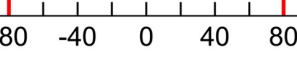

$\mathrm{z}(\mathrm{nm})$

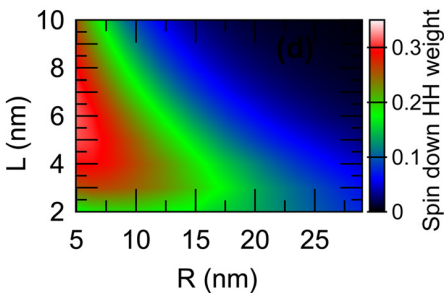

symmetry changes, as similar numbers are obtained if one replaces hexagonal wires by $\operatorname{triangular}^{48}$ (Figs. 5(c) and 5(d)) or cylindrical (Figs. 5(e) and 5(f)) ones.

Recent experiments with GaAs polytype QDs revealed strong dispersion of the measured excitonic $g$-factors depending on the confinement strength. ${ }^{12}$ As Fig. 5 shows, due to the VB mixing, the hole $g$-factor value can fluctuate substantially for different QD dimensions. This may partially explain the experimental observation.

One concludes from this section that the $\mathrm{HH}$ description of the ground state is valid except for small diameter wires, when a $\lambda$-band ground state is formed. However, in the presence of magnetic fields, one should be aware that VB mixing can strongly couple spin up and down $\mathrm{HH}$ states. We stress that such a spin mixing is mediated by excited (light-hole like) $G$ and $\lambda$ states, although they barely couple to the ground state themselves. In fact, as we show in the Appendix, the

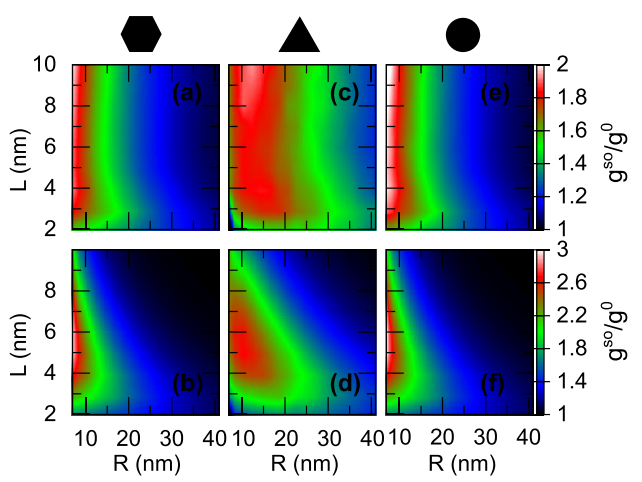

FIG. 5. Ratio of hole $g$-factors calculated with and without VB mixing, $g^{s o} /$ $g^{0}$. (a) and (b) Hexagonal QDs in the presence and absence of $P_{s p}$, respecively. (c) and (d) Same but for triangular QDs. (e) and (f) Same but for cyindrical QDs. For the sake of comparison, the nominal radius refers to the circumscribed circle of the triangle. For hexagons and cylinders, the actual radius is scaled so as to preserve the same area as the triangle. mixing cannot be described with effective two-band Hamiltonians. It is an intrinsic many-band coupling effect.

\section{Excitons}

In what follows, we investigate the influence of confinement and spontaneous polarization on the properties of the ground state exciton. In order to compare with available experiments, we restrict to radii $R \geq 5 \mathrm{~nm}$, where the singleband HH description is valid. The exciton state is thus calculated with Eq. (11), which fully accounts for electron-hole Coulomb interaction.

Figure 6 shows the exciton energy in WZ QDs embedded in ZB wires, panels (a) and (b), and ZB QDs embedded in WZ wires, panels (d) and (e). The left column corresponds to full spontaneous polarization, $P_{s p}=2.3 \times 10^{-3} \mathrm{C} \mathrm{m}^{-2}$, and the right one to artificially weakened polarization, $P_{s p}=2.3 \times 10^{-4} \mathrm{C} \mathrm{m}^{-2}$. ${ }^{49}$ One can see that for the realistic value of $P_{s p}$, the exciton energy has a very strong dependence on both the QD radius and thickness. The wavelength tunability is actually remarkable, as the energy can be tuned by over $700 \mathrm{meV}$, well above and below the bulk band gap $(1.51 \mathrm{eV})$, from $1.65 \mathrm{eV}$ (visible) to $1.0 \mathrm{eV}$ (near infrared). For weak $P_{s p}$, instead, the tunability is reduced. Radial quantum confinement still plays a role, enabling exciton emission up to $1.65 \mathrm{eV}$ for the narrowest wires. By contrast, the influence of the dot thickness is largely suppressed, as in the single-particle case we saw in Fig. 2. Consequently, the lowerbound exciton emission is only $1.41 \mathrm{eV}$, roughly the indirect band gap between the bottom of the $\mathrm{ZB} \mathrm{CB}$ and the top of the WZ VB, see Fig. 1, which is the smallest possible energy allowed by quantum confinement alone.

It is worth noting that for large thicknesses, the electronhole overlap decreases. This effect is especially pronounced in the presence of full $P_{s p}$, as shown in Figs. 6(c) and 6(f), where it can be seen that the exciton electron and hole wave functions localize at opposite interfaces. For this reason, the exciton becomes gradually dark, and optical experiments may not be able to observe low energy states. 


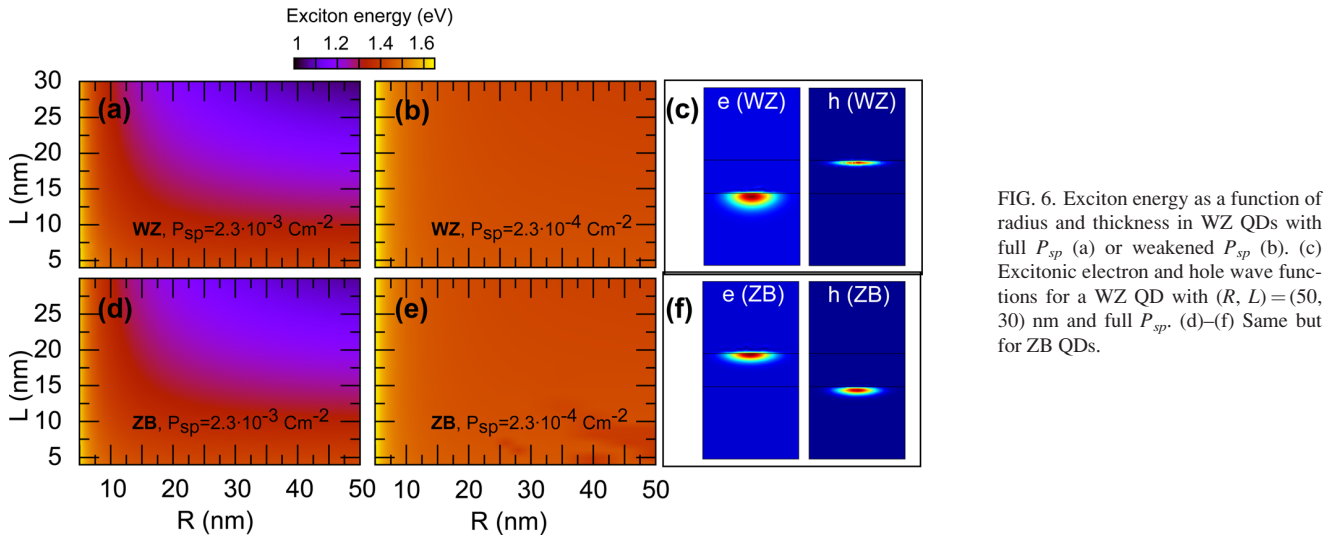

To better compare the optical properties of WZ QDs and ZB QDs, in Fig. 7, we plot the exciton energy as a function of the dot thickness. Two representative cases are considered. In Fig. 7(a), we study typical QDs, with large radius, $R=50 \mathrm{~nm}$ and full $P_{s p}$. In this case, the thickness dependence is linear for both $\mathrm{WZ}$ and $\mathrm{ZB}$, owing to the large built-in electric field coming from $P_{s p}$, as already noted for electrons in Fig. 2. It follows that the spontaneous polarization prevails over Coulomb interactions in GaAs polytypes. This validates similar theoretical predictions obtained for ZB QDs at a single-particle level, ${ }^{15}$ which here we extend to WZ QDs. Besides, we find that excitons in WZ QDs (green line) have lower energy than those in ZB QDs (red line), regardless of $L$. This is due to the smaller kinetic energy of the confined carrier, as for holes in WZ $m_{z}=0.89$, while for electrons in ZB $m_{z}=0.067$. For the same reason, holes leak out of the QD to a lesser extent than electrons. Consequently, the electron-hole overlap-proportional to the size of dots in Fig. 7-is also weaker for WZ QDs.

Interestingly, the behavior described above changes drastically when one switches to QDs with strong radial confinement. This can be seen in Fig. 7(b), which corresponds to QDs with $R=5 \mathrm{~nm}$. First, the thickness dependence becomes quadratic in spite of $P_{s p}$. This is because the radial confinement provides enough energy for the carriers to escape from the electrostatic potential wells. The resulting wave functions are no longer localized near the WZ/ZB interface, but rather all over the QD, see Fig. 7(c). Hence, they become sensitive to the quantum confinement in the growth direction. Second, ZB becomes the lowest emitting structure for thin dots $(L<15 \mathrm{~nm})$. The origin of this inversion can be inferred from Fig. 7(c) as well. The electron in the ZB QD can compensate for the strong radial confinement by penetrating into the WZ region, but the hole in the WZ QD cannot. This is again due to the relative masses $m_{z}$ of the confined carrier. Third, the electron-hole overlap is enhanced with respect to that of large diameter QDs, for both WZ and ZB (compare the size of the circles in Figs. 7(a) and 7(b)). This is also connected with the confined carrier delocalizing all over the QD and penetrating into the wire crystal phase, which reduces the separation from the outer carrier. In other words, the radial confinement induces a gradual transition
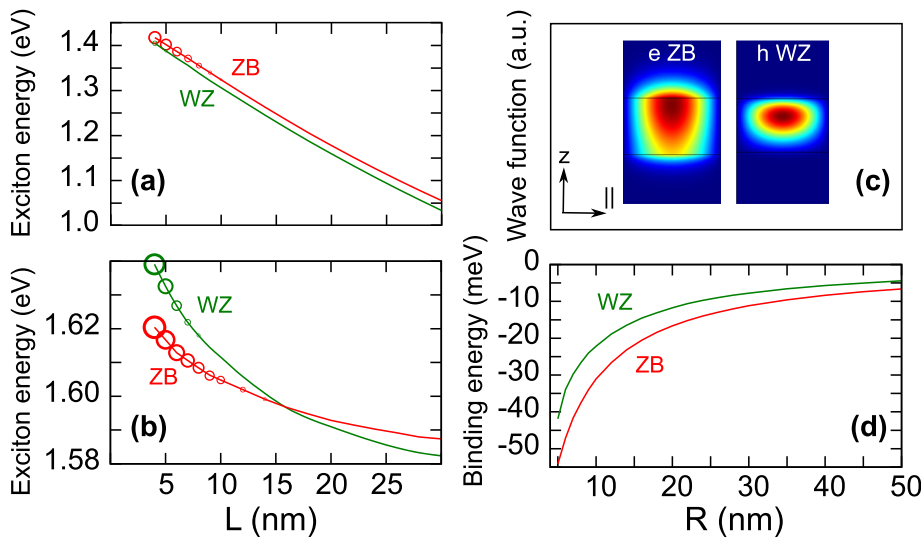

FIG. 7. (a) Exciton energy vs thickness in WZ (green) and ZB (red) QDs with $R=50 \mathrm{~nm}$. (b) Same but with $R=5 \mathrm{~nm}$. Note the qualitative change in behavior between the two radii. The size of the circles is proportional to the electron-hole envelope function overlap. (c) Wave function of exciton's electron in a ZB QD (left) and hole in a WZ QD (right), both with $(R$ $L)=(5,5)$ nm (d) Exciton Coulo energy vs QD radius, with $L=4 \mathrm{~nm}$. 
from the usual type-II behavior of GaAs polytype QDs to a type-I one. One then concludes that reverse reaction growth ${ }^{10}$ can be used for structural designs which improve the absorption strength and reduce the radiative lifetime of GaAs polytypes, as previously proposed for other (spontaneous polarization free) materials. ${ }^{50}$

We note that the type-II to type-I transition is partially stimulated by Coulomb interaction. As shown in Fig. 7(d), the exciton binding energy scales up with radial confinement. For small radii, it reaches several tens of $\mathrm{meV}$, the same order of magnitude as the $\mathrm{ZB} / \mathrm{WZ}$ band offsets. As a result, it helps bring electron and hole closer together in the growth direction.

Before closing this section, we briefly discuss the relation of the results in Fig. 7 with those of available experiments. Vainorius and co-workers recently measured the pholuminescence of $\mathrm{WZ}$ and $\mathrm{ZB}$ QDs with variable thickness and large radii, $R \approx 45-60 \mathrm{~nm} .{ }^{9}$ They found that $\mathrm{WZ}$ emission is redshifted with respect to $\mathrm{ZB}$ one by a few tens of meV, in agreement with our Fig. 7(a). However, in their experiments, the emission energy was less sensitive to $L$. From $L=5 \mathrm{~nm}$ to $30 \mathrm{~nm}$, the exciton emission in ZB QDs redshifted by about $60 \mathrm{meV}$, one order of magnitude less than we predict. They noted that a similar redshift could be obtained in theory if one considers quantum confinement only. We have confirmed this with our model, by considering excitons under weakened polarization (not shown). ${ }^{51}$ The question then arises of whether the experimental system had, for some reason, suppressed spontaneous polarization at the $\mathrm{ZB} / \mathrm{WZ}$ GaAs interface. Lacking more precise information about the system, at present we can only speculate for possible reasons. In some InAs polytypes, interface charges originating in spontaneous polarization were found to be concealed by dopant carriers. ${ }^{52}$ Even in undoped wires, unintentional doping or migrating surface charges may play such a role. ${ }^{53}$ Certainly, GaAs has a wider band gap and stronger spontaneous polarization than InAs ${ }^{13}$ but, having uncapped wires, the authors of Ref. 9 mentioned that surface charges were possibly present in their samples, inducing fluctuations of tens of meV among QDs of the same thickness but different diameter. Therefore, dopant compensation of $P_{s p}$ charges may be taking place. New experiments with capped GaAs/ AlGaAs wires could study the linear or quadratic dependence on $L$ to confirm the presence of spontaneous polarization. An alternative explanation could be related to the emission brightness. In the presence of full $P_{s p}$, we expect the ground state electron-hole overlap to decrease with $L$, see Fig. 7(a). For thick QDs, the overlap is so small that the ground state should be optically dark. If this is the case in Ref. 9, the measured photoluminescence may be arising not from the ground state but from higher energy exciton states, whose brightness is increasingly larger. This would be consistent with the fact that the experiments did not observe any systematic effect of the QD thickness on the photoluminescence intensity, ${ }^{54}$ even though one would expect fading intensity as the ground state becomes darker. At this regard, we note that, even for weakened $P_{s p}=2.3 \times 10^{-4} \mathrm{C} \mathrm{m}^{-2}$, when the calculated redshift is similar to the experimental one, we find an order of magnitude decrease in the ground state electron-hole overlap with $L$, which should be visible experimentally.

As for the radius dependence, the experiments of Loitsch et al. ${ }^{10,11}$ reported exciton emission up to $1.610 \mathrm{eV}$ for $\mathrm{WZ}$ QDs with $R \approx 6.5 \mathrm{~nm}$ and random thickness, which is blueshifted by $100 \mathrm{meV}$ with respect to bulk GaAs. According to our estimates in Fig. 7(b), for thin dots, one could reach even stronger blueshifts. In addition, the experiments measured excitonic lifetimes ranging between 0.5 and $0.8 \mathrm{~ns}$, roughly one order of magnitude shorter than the values observed in large radius QDs (3-8 ns). ${ }^{8}$ This was taken as being indicative of a type-II to type-I transition, which is actually consistent with our prediction for increasing radial confinement. For a more quantitative comparison, we consider that the exciton lifetime $(\tau)$ relates to the electron-hole overlap $S_{e h}$ as $\tau \propto 1 / S_{c h}^{2}$. We take a WZ QD with $R=7 \mathrm{~nm}$ and another with $R=50 \mathrm{~nm}$. As can be seen in Fig. 7, the overlap is sensitive to the exact dot thickness $L$. Experimentally, we only know that in Refs. 10 and $11 \mathrm{~L}$ takes unknown values from the twin plane limit up to $\sim 10 \mathrm{~nm}^{55}$ Assuming an average $L=5 \mathrm{~nm}$, the calculated exciton lifetime is 3.5 times shorter in the $R=7 \mathrm{~nm}$ QD. The ratio increases to 30 if one considers $R=5 \mathrm{~nm}$ instead, because the stronger radial confinement further promotes the type-I behavior. It also increases to 10 assuming QDs with $L=10 \mathrm{~nm}$, because the spontaneous polarization enhances the type-II character, especially in the QD with weak radial confinement. All these values are close to the experimental range.

New experiments systematically comparing ZB and WZ dots with small diameter would be useful to confirm the other new phenomena we predict in this regime, namely, the change of the VB forming the hole ground state under $R=5 \mathrm{~nm}$ ( $\lambda$ band), and the fact that ZB QDs emit at lower energies than WZ ones for short $L$.

\section{CONCLUSION}

We have developed a $\mathrm{k} \cdot \mathrm{p}$ model to investigate WZ/ZB polytype QDs, including 3D confinement, spontaneous polarization effects, VB coupling through a Burt-Foreman six-band Hamiltonian, and electron-hole Coulomb interaction for excitons. When applied to GaAs QDs, we find a number of relevant observations:

(i) Contrary to what is often assumed, the spontaneous polarization in GaAs is not negligible; it should dominate the electronic structure for QDs with thickness above $\sim 5 \mathrm{~nm}$.

(ii) The hole ground state has nearly pure $\mathrm{HH}$ character except for the narrowest wires, $R<5 \mathrm{~nm}$, when it switches to $\lambda$ band

(iii) When subject to a magnetic field, the $\mathrm{HH}$ ground states experience a strong spin mixing, mediated by excited valence bands.

(iv) The strong radial confinement brings about a transition from indirect (type-II) to direct (type-I) excitons and partially masks spontaneous polarization effects. Besides, ZB QDs start emitting at lower energies than WZ QDs. 
Further experiments are called for to confirm the above points, which should help improve current understanding of the behavior and opportunities of these promising structures.

\section{ACKNOWLEDGMENTS}

We are grateful to P. Caroff, M. E. Pistol, and B. Loitsch for useful discussions. Support from UJI Project No. P1-1B2014-24, MINECO Project No. CTQ2014-60178-P, and a FPU grant (C.S.) is acknowledged.

\section{APPENDIX: THE EFFECTIVE HAMILTONIAN}

The spin mixing observed in Section II B basically involves the energetically close $\mathrm{HH}$ up $\left|u_{1}\right\rangle$ and down $\left|u_{4}\right\rangle$ states. It originates from the spin-orbit interaction term $\Delta_{s o}=\sqrt{2} \Delta_{3}$, as no mixing occurs if we set $\Delta_{s o}=0$, due to the resulting block form of Hamiltonian (3), thus preventing the interaction between spin up and spin down states. Also, it is the result of a complex multiband interaction that cannot be reduced to an effective two band model. We can show it by reordering and splitting the basis vectors as follows, $\left\{\left\{\left|u_{1}\right\rangle,\left|u_{4}\right\rangle\right\},\left\{\left|u_{2}\right\rangle,\left|u_{3}\right\rangle,\left|u_{5}\right\rangle,\left|u_{6}\right\rangle\right\}\right\}$, that turns the Hamiltonian (3) into:

$\left[\begin{array}{cc|cccc}F & 0 & -K^{*} & -H^{*} & 0 & 0 \\ 0 & F^{\prime} & 0 & 0 & -K & H \\ \hline-K & 0 & G & H & 0 & \Delta_{s o} \\ -H & 0 & H^{*} & \lambda & \Delta_{s o} & 0 \\ 0 & -K^{*} & 0 & \Delta_{s o} & G^{\prime} & -H^{*} \\ 0 & H^{*} & \Delta_{s o} & 0 & -H & \lambda^{\prime}\end{array}\right] \equiv\left[\begin{array}{c|c}H^{A A} & H^{A B} \\ \hline & \\ H^{B A} & H^{B B}\end{array}\right]$

where $X=F, G, \lambda$ differs from $X^{\prime}$ in a small $\kappa \mu_{B} B \mathbb{J}_{z}$ Zeeman term.

The $2 \times 2$ effective Hamiltonian $H^{\text {eff }}=H^{A A}$ $+H^{A B}\left(I^{B B} E-H^{B B}\right)^{-1} H^{B A}$, with $I^{B B}$ the $4 \times 4$ identity matrix, is usually approximated by setting $\left(H^{B B}\right)_{i j} \approx E_{i}^{B B} \delta_{i j}$ that allows an straightforward calculation of the inverse involved in the effective Hamiltonian, so that

$$
\left(H^{e f f}\right)_{i j}=\left(H^{A A}\right)_{i j}-\sum_{k \in B} \frac{H_{i k}^{A B} H_{k j}^{B A}}{E_{k}^{B B}-E} .
$$

However, the particular form of $H^{A B}$ and $H^{B A}$ leads to zero extradiagonal elements for this approximate effective Hamiltonian.

A more elaborate, but still simple, approximate effective Hamiltonian is obtained by setting $H \approx 0, G \approx G^{\prime}$, and $\lambda \approx \lambda^{\prime}$ in $H^{B B}$, thus yielding a twofold (cross-like) diagonal matrix. Its inverse $M=\left(I^{B B} E-H^{B B}\right)^{-1}$ is still a twofold cross-like diagonal matrix, and the product $H^{A B} M H^{B A}$ is diagonal again.

Similar results are obtained by employing the Lowdin perturbation theory to account for the action of $\left\{\left|u_{2}\right\rangle,\left|u_{3}\right\rangle,\left|u_{5}\right\rangle,\left|u_{6}\right\rangle\right\}$ on the Hamiltonian expanded in the $\left\{\left|u_{1}\right\rangle,\left|u_{4}\right\rangle\right\}$ basis set.

A multi-band Hamiltonian is needed to enable strong interaction between two states corresponding to the basis $i$ and $j$ despite $H_{i j}=0$. One of the simpler Hamiltonians illustrating this point would involve the basis set $\left\{\left|u_{1}\right\rangle,\left|u_{2}\right\rangle,\left|u_{3}\right\rangle\right\}$

$$
\left[\begin{array}{ccc}
F & 0 & \Delta_{1} \\
0 & F^{\prime} & \Delta_{2} \\
\Delta_{1} & \delta_{2} & X
\end{array}\right]
$$

$\left|u_{1}\right\rangle-\left|u_{3}\right\rangle$ mixing occurs at first order, $c_{13}^{(1)}=\frac{H_{31}}{\Delta E_{13}}$, while $\left|u_{1}\right\rangle-\left|u_{2}\right\rangle \quad$ interaction holds at second order, $c_{12}^{(2)}=\frac{H_{23} H_{31}}{\Delta E_{12} \Delta E_{13}}=c_{13}^{(1)} \frac{H_{23}}{\Delta E_{12}}=\frac{\Delta_{2}}{F-F^{\prime}}$. However, for a small Zeeman splitting, $\left|F-F^{\prime}\right| \ll \Delta_{2}$. Then, $c_{13}^{(1)} \ll c_{12}^{(2)}$.

${ }^{1}$ D. M. Talapin and Y. Yin, J. Mater. Chem. 21, 11454 (2011).

${ }^{2}$ W. J. Baumgardner, Z. Quan, J. Fang, and T. Hanrath, Nanoscale 4, 3625 (2012).

${ }^{3}$ J. J. Finley, M. Sabathil, P. Vogl, G. Abstreiter, R. Oulton, A. I. Tartakovskii, D. J. Mowbray, M. S. Skolnick, S. L. Liew, A. G. Cullis, and M. Hopkinson, Phys. Rev. B 70, 201308(R) (2004).

${ }^{4}$ S. E. Economou, J. I. Climente, A. Badolato, A. S. Bracker, D. Gammon, and M. F. Doty, Phys. Rev. B 86, 085319 (2012).

${ }^{5}$ H. Kim, S. M. Thon, P. M. Petroff, and D. Bouwmeester, Appl. Phys. Lett. 'H. Kim, S. M. Thon,
95, 243107 (2009).

${ }^{6}$ N. Akopian, G. Patriarche, L. Liu, J.-C. Harmand, and V. Zwiller, Nano Lett. 10, 1198 (2010).

${ }^{7}$ P. Caroff, J. Bolinsson, and J. Johansson, IEEE J. Sel. Top. Quantum Electron. 17, 829 (2011).

${ }^{8}$ D. Spirkoska, J. Arbiol, A. Gustafsson, S. Conesa-Boj, F. Glas, I. Zardo, M. Heigoldt, M. H. Gass, A. L. Bleloch, S. Estrade, M. Kaniber, J. Rossler, F. Peiro, J. R. Morante, G. Abstreiter, L. Samuelson, and A. Fontcuberta i Morral, Phys. Rev. B 80, 245325 (2009).

${ }^{9}$ N. Vainorius, S. Lehmann, D. Jacobsson, L. Samuelson, K. A. Dick, and M.-E. Pistol, Nano Lett. 15, 2652 (2015).

${ }_{10}^{10}$ M.-E. Pistol, Nano Let. 15, 2552 (2015). B. Loitsch, D. Rudolph, S. Morkötter, M. Döblinger, G. Grimaldi, L.
Hanschke, S. Matich, E. Parzinger, U. Wurstbauer, G. Abstreiter, J. J. Hanschke, S. Matich, E. Parzinger, U. Wurstbauer, G.
Finley, and G. Kolbmüller, Adv. Mater. 27, 2195 (2015).

Finley, and G. Kolbmüller, Adv. Mater. 27, 2195 (2015).
${ }^{11}$ B. Loitsch, J. Winnerl, G. Grimaldi, J. Wierzbowski, D. Rudolph, S. ${ }^{11}$ B. Loitsch, J. Winnerl, G. Grimaldi, J. Wierzbowski, D. Rudolph, S.
Morkötter, M. Döblinger, G. Abstreiter, G. Kolbmüller, and J. J. Finley, Nano Lett. 15, 7544 (2015).

${ }^{12}$ P. Corfdir, B. V. Hattem, E. Uccelli, S. Conesa-Boj, P. Lefebvre, A. Fontcuberta i Morral, and R. T. Phillips, Nano Lett. 13, 5303 (2013).

${ }^{13}$ A. Belabbes, J. Furthmüller, and F. Bechstedt, Phys. Rev. B 87, 035305 (2013).

${ }^{14}$ B. Bauer, J. Hubmann, M. Lohr, E. Reiger, D. Bougeard, and J. Zweckb, Appl. Phys. Lett. 104, 211902 (2014)

Appl. Phys. Lett. 104, 211902 (2014).
${ }^{15}$ U. Jahn, J. Lähnemann, C. Pfüller, O. Brandt, S. Breuer, B. Jenichen, M. ${ }^{15}$ U. Jahn, J. Lähnemann, C. Pfüller, O. Brandt, S. Breuer, B. Jenichen, M.
Ramsteiner, L. Geelhaar, and H. Riechert, Phys. Rev. B 85, 045323 Ramstei

${ }^{16}$ D. Spirkoska, Al. L. Efros, W. R. L. Lambrecht, T. Cheiwchanchamnangij, A. Fontcuberta i Morral, and G. Abstreiter, Phys. Rev. B 85, 045309 (2012).

${ }^{17}$ Al. L. Efros and W. R. L. Lambrecht, Phys. Rev. B 89, 035304 (2014). ${ }^{18}$ A. De and C. E. Pryor, Phys. Rev. B 81, 155210 (2010).

${ }^{19} \mathrm{~F}$. Bechstedt and A. Belabbes, J. Phys.: Condens. Matter 25, 273201 (2013).

${ }^{20} \mathrm{G}$. Signorello, E. Lörtscher, P. A. Khomyakov, S. Karg, D. L. Dheeraj, B. Gotsmann, H. Weman, and H. Riel, Nat. Commun. 5, 3655 (2014)

${ }^{21}$ Aotsmann, H. Weman, and H. Riel, Nat. Commun. 5, 3655 (2014).
. Corfdir, M. Heiss, S. Conesa-Boj, E. Uccelli, A. ${ }^{21}$ A. M. Graham, P. Corfdir, M. Heiss, S. Conesa-Boj, E. Uccelli, A.
Fontcuberta i Morral, and R. T. Phillips, Phys. Rev. B 87, 125304 Fontcub

${ }^{22}$ F. Rajadell, M. Royo, and J. Planelles, J. Appl. Phys. 111, 014303 (2012)
(2013). (see supplementary material).

${ }^{23}$ P. E. Faria Junior and G. M. Sipahi, J. Appl. Phys. 112, 103716 (2012).

${ }^{24}$ J. Lähnemann, O. Brandt, U. Jahn, C. Pfüller, C. Roder, P. Dogan, F. Grosse, A. Belabbes, F. Bechstedt, A. Trampert, and L. Geelhaar, Phys. Rev. B 86, 081302(R) (2012).

${ }^{25}$ S.-H. Park and S.-L. Chuang, J. Appl. Phys. 87, 353 (2000).

${ }^{26}$ S. L. Chr and S.-L. Chuang, J. Appl. Phys 87, 353 (2000).

${ }^{27}$ G. L. Bir and G. E. Pikus, Symmetry and Strain-Induced Effects in ${ }^{27}$ G. L. Bir and G. E. Pikus, Symmetry
Semiconductors (Wiley, New York, 1974).

Semiconductors (Wiley, New York, 1974).
${ }^{28} \mathrm{~F}$. Mireles and S. Ulloa, Phys. Rev. B 62, 2562 (2000).

${ }^{28}$ F. Mireles and S. Ulloa, Phys. Rev. B 62, $2562(2000)$.
${ }^{29}$ R. G. Veprek, S. Steiger, and B. Witzigmann, Phys. Rev. B 76, 165320 (2007). 
${ }^{30}$ R. G. Veprek, S. Steiger, and B. Witzigmann, Opt. Quantum Electron. 40, 1169 (2008).

${ }^{31}$ M. F. Schubert, Phys. Rev. B 81, 035303 (2010).

${ }^{32}$ D. J. Ben Daniel and C. B. Duke, Phys. Rev. 152, 683 (1966).

${ }^{33}$ R. Eppenga, M. F. H. Schurmans, and S. Colak. Phys. Rev B 36, 1554 (1987)

${ }^{34}$ M. G. Burt, J. Phys.: Condens. Matter 4, 6651 (1992); 11, 53 (1999).

${ }^{35}$ B. A. Foreman, Phys. Rev. Lett. 80, 3823 (1998); Phys. Rev. B 48, 4964(R) (1993); 56, R12748 (1997).

${ }^{36}$ P. N. Stavrinou and R. van Dalen, Phys. Rev. B 55, 15456 (1997).

${ }^{77}$ E. P. Pokatilov, V. A. Fonoberov, V. M. Fomin, and J. T. Devreese, Phys. Rev. B 64, 245328 (2001)

${ }^{38}$ Further research has been developed to improve the asymmetric or BurtForeman Hamiltonian, which brought about additional interface terms, see e.g. B. A. Foreman, Phys. Rev. B 72, 165345 (2005); J. Phys.: Condens. Matter 18, 1335 (2006); Phys. Rev. B 75, 235331 (2007). In Hamiltonian (7), we disregard them. In fact, we have checked that even using a symmetrized Hamiltonian the band coupling we observe is qualitatively consisttrized Hamiltonian the band coupling we observe is qualitatively consis-
ent with that of Fig. 4 . ent with that of Fig.

${ }^{39}$ X. Zhou, F. Bertazzi, M. Goano, G. Ghione, and E. Bellotti, J. Appl. Phys. 116, 033709 (2014)

${ }^{4}$ One could improve $H_{h}$, Eq. (10) by including the influence of decoupled bands on the HH effective mass. This can be done, e.g., by fitting independently the WZ and ZB one-band mass parameters from a series of 6band vs. one-band calculations. For the QDs we simulate, we have checked this leads to minor corrections. Since our study is of qualitative character, we have disregarded them.

${ }^{41}$ I. Vurgaftman, J. R. Meyer, and L. R. Ram-Mohan, J. Appl. Phys. 89 , $5815(2001)$.
${ }^{42}$ T. Cheiwchanchamnangij and W. R. L. Lambrecht, Phys. Rev. B 84 035203 (2011).

${ }^{43}$ S. Adachi, J. Appl. Phys. 58, R1 (1985)

${ }^{44}$ A. S. Bracker, M. Scheibner, M. F. Doty, E. A. Stinaff, I. V. Ponomarev,

J. C. Kim, L. J. Whitman, T. L. Reinecke, and D. Gammon, Appl. Phys. J. C. Kim, L. J. Whitman,
Lett. 89, 233110 (2006)

${ }^{45}$ M. Bayer, P. Hawrylak, K. Hinzer, S. Fafard, M. Korkusinski, Z. R.

M. Bayer, P. Hawrylak, K. Hinzer, S. Fafard, M. Korkusin
Wasilewski, O. Stern, and A. Forchel, Science 291, 451 (2001).

${ }^{66}$ S. Tomic, T. S. Jones, and N. M. Harrison, Appl. Phys. Lett. 93, 263105 (2008).

${ }^{47}$ J. Planelles, F. Rajadell, and J. I. Climente, Phys. Rev. B 92, 041302(R) (2015)

${ }^{48}$ J. Zou, M. Paladugu, H. Wang, G. J. Auchterloine, Y.-N. Guo, Y. Kim, Q. Gao, H. J. Joyce, H. H. Tan, and C. Jagadish, Small 3, 389 (2007).

${ }^{49}$ Using weakened $P_{s p}$ instead of null $P_{s p}$ allows us to calculate single particle sues confind in the wire phe, which form near the WZ/ZB intcle face. These states would oherwise spread all over the wire and poor starting point for the exciton self-consistent calculation.

N. Akopian, V. Zwiller, and J-C. Harmand, Nano Lett. 10, 4055 (2010).

Unlike the calculations of Ref. 9, we consider Coulomb interaction and different masses for each crystal phase.

C. Thelander, P. Caroff, S. Plissard, A. W. Dey, and K. Dick, Nano Lett. 11, 2424 (2011)

M. Hjort, S. Lehmann, J. Knutsson, A. A. Zakharov, Y. A. Du, S. Sakong, R. Timm, G. Nylund, E. Lundgren, P. Kratzer, K. A. Dick, and A. Mikkelsen, ACS Nano 8, 12346 (2014).

${ }^{54}$ M. E. Pistol, private communication (2015)

${ }^{55} \mathrm{~B}$. Loitsch, private communication (2015). 


\title{
Piezoelectric Control of the Exciton Wave Function in Colloidal CdSe/ CdS Nanocrystals
}

\author{
Carlos Segarra, ${ }^{\dagger}$ Juan I. Climente, ${ }^{\dagger}$ Anatolii Polovitsyn, ${ }^{\dagger}$ Fernando Rajadell, ${ }^{\dagger}$ Iwan Moreels, ${ }^{\ddagger}$
} and Josep Planelles $*^{\dagger}$

†Departament de Química Física i Analítica, Universitat Jaume I, E-12080 Castelló de la Plana, Spain

${ }^{*}$ Istituto Italiano di Tecnologia, Via Morego 30, IT-16163 Genova, Italy

(S) Supporting Information

ABSTRACT: Using multiband $k \cdot p$ calculations, we show that strain-engineered piezoelectricity is a powerful tool to modulate the electron-hole spatial separation in a wide class of wurtzite $\mathrm{CdSe} / \mathrm{CdS}$ nanocrystals. The inherent anisotropy of the hexagonal crystal structure leads to anisotropic strain and, consequently, to a pronounced piezoelectric field along the $c$ axis, which can be amplified or quenched through a proper design of the core-shell structure. The use of large cores and thick shells promotes a gradual departure from quantum confined nanocrystals to a regime dominated by piezoelectric confinement. This allows excitons to evolve from the usual type-I and quasi-type-II behavior to a type-II behavior in dot-in-dots, dot-in-rods, rod-in-rods, and dot-in-plates. Piezoelectric fields explain experimental observations for giant-shell nanocrystals, whose time-resolved photoluminescence reveals long exciton lifetimes for large cores, contrary to the expectations of standard quantum confinement models. They also explain the large differences in exciton lifetimes reported for different classes of $\mathrm{CdSe} / \mathrm{CdS}$ nanocrystals.

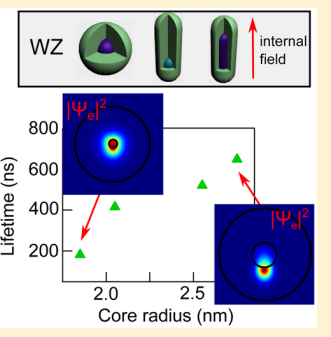

he spatial separation between electrons and holes in colloidal semiconductor nanocrystals (NCs) is a critical parameter that impacts the exciton emission lifetime, ${ }^{1}$ Auger recombination rate, ${ }^{2-4}$ electron-hole exchange interaction, charge separation time, ${ }^{6}$ and other properties of interest for optoelectronic devices such as LEDs, lasers, photovoltaic cells, or photocatalysts. ${ }^{7}$ Control of the electron-hole separation is usually achieved by means of band gap engineering in core/ shell hetero-NCs. An additional control mechanism was proposed for $\mathrm{CdTe} / \mathrm{ZnSe} \mathrm{NCs}$, which exploited the epitaxial strain arising from the large lattice mismatch between the two materials $(13.4 \%){ }^{8}$ The growth of a thick compressive shell around the core shifts the core band edges via the deformation potential, leading to a gradual transition from (unstrained) type-I band alignment to a (fully strained) type-II one. Subsequently, the influence of strain on the band structure and electron-hole wave functions was investigated in other core/shell structures including $\mathrm{CdSe} / \mathrm{CdTe}(6.7 \%$ lattice mismatch), ${ }^{6,9} \mathrm{ZnSe} / \mathrm{ZnTe}(7 \%),{ }^{10,11}$ and $\mathrm{CdS} / \mathrm{ZnS}(7 \%) .{ }^{12-14}$

In materials with a smaller lattice mismatch, strain-driven localization of carriers is less efficient, as the band edges are shifted only moderately. This is unfortunate because weakly strained NCs are less prone to interfacial defects and, hence, are preferred for their higher photoluminescence quantum yields. ${ }^{15}$ This is the case of CdSe/CdS NCs, ${ }^{16}$ (4.4\% for zinc-blende (ZB), $4 \%$ and $3.8 \%$ along the $a$ and $c$ axes in wurtzite (WZ)), which are structures of particular interest owing to their monodispersity, reduced blinking, narrow emission line width and high quantum yield. ${ }^{17,18}$ Several types of core-shell CdSe/ $\mathrm{CdS}$ heterostructures have been synthesized in the past decade: dot-in-dots (DiDs), ${ }^{2,15,17-21}$ dot-in-rods (DiRs), ${ }^{22-25}$ dot-inplates (DiPs), ${ }^{26}$ rod-in-rods (RiRs), ${ }^{27,28}$ tetrapods, ${ }^{29,30}$ and octapods. ${ }^{31}$ Carrier localization in these systems is generally assumed to be set by quantum confinement. The smaller gap of CdSe favors localization of both electron and hole inside the core (type-I exciton), but as the core size decreases, the electron kinetic energy allows it to overcome the $\mathrm{CB}$ offset barrier, delocalizing over both core and shell (quasi-type-II exciton). ${ }^{2,5,24,32}$ Note, however, that the spatial separation between electron and hole is typically restricted to small core systems and is ultimately limited by Coulomb interaction, which binds the electron to the vicinity of the hole. ${ }^{32,33}$

Very recently, a study on $\mathrm{CdSe} / \mathrm{CdS}$ RiRs with giant core and shell reported extremely long exciton lifetimes (up to 4400 $\mathrm{ns}) .^{28}$ This is 1 order of magnitude longer than any reported values for giant-shell CdSe/CdS $\mathrm{DiDs}^{5,18}$ and two longer than those of DiR or core-only $\mathrm{NCs},{ }^{24,28}$ which reflects a truly typeII behavior with well separated electrons and holes. It was shown that such exotic properties followed from the straininduced piezoelectric (PZ) charges arising at the $\mathrm{CdSe} / \mathrm{CdS}$ interfaces along the WZ $c$ axis. Strain-induced PZ fields have been shown to be important in several epitaxial structures, including $\mathrm{CdSe} / \mathrm{CdS}$ superlattices and $\mathrm{III}-\mathrm{V}$ quantum dots. ${ }^{34-36}$ This raises the question of how disruptive they can be in colloidal structures, if they are only important in large RiRs due to the anisotropic shape and weak longitudinal

Received: March 18, 2016

Accepted: May 25, 2016 


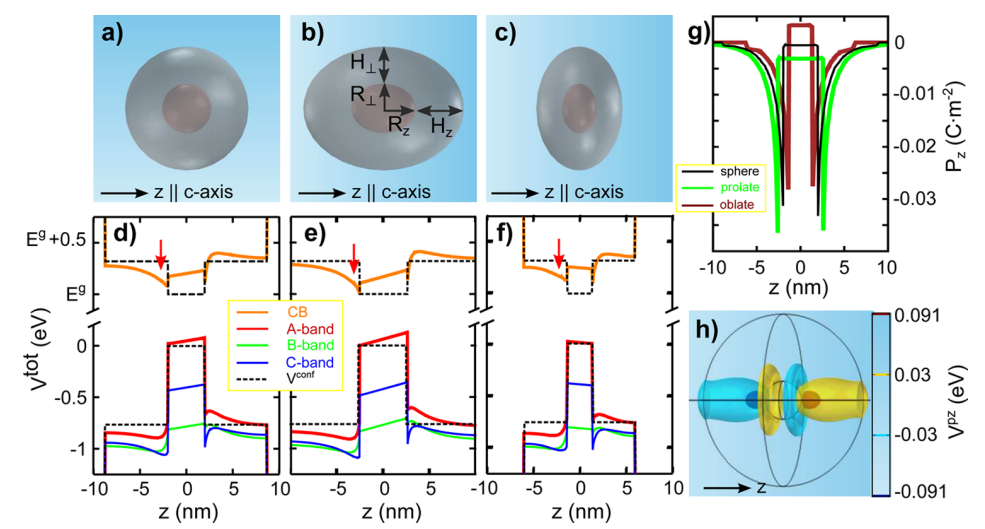

Figure 1. (a) - (c) schematic of spherical, prolate, and oblate DiDs. (d) -(f) CB and VB confining and total potential for a spherical $\mathrm{DiD}$ with core radius $R=2 \mathrm{~nm}$ and shell thickness $H=7 \mathrm{~nm}(\mathrm{~d})$, prolate $\mathrm{DiD}$ with aspect ratio $1.3: 1\left(R_{z}=1.3 R_{\perp}\right.$ and $\left.H_{z}=1.3 H_{\perp}\right)(\mathrm{e})$ and oblate $\mathrm{DiD}$ with aspect ratio 0.7:1 (f). (g) strain-induced polarization along the $c$-axis for the three kinds of $\mathrm{DiD}$. (h) isosurface representation of the piezoelectric potential for the spherical DiD. In (d) $-(\mathrm{f}), E^{g}$ is the bulk band gap.

confinement or if it is possible to use them as an efficient charge separation mechanism in other kinds of WZ NCs, with smaller core dimensions and different shapes. Potential signatures of $\mathrm{PZ}$ fields have been observed in $\mathrm{CdSe} / \mathrm{CdS}$ $\mathrm{DiDs}^{37,38}$ and DiRs, ${ }^{37}$ but it has been mostly overlooked because calculations for a few particular structures deemed it a minor effect. ${ }^{16,37,39}$ In this Letter, we show that with appropiate structural design, piezoelectricity indeed becomes a majo factor determining the electron-hole separation in most kinds of WZ CdSe/CdS NCs.

We consider excitons in CdSe/CdS NCs where both core and shell present WZ structure. A few theoretical considerations are useful for the discussion of the results. The excitonic electron and hole Hamiltonians read

$$
H_{j}=H_{j}^{\mathrm{kin}}+V_{j}^{\mathrm{conf}}+V_{j}^{\mathrm{str}}+V_{j}^{\mathrm{pz}}+V_{j}^{\mathrm{e}-\mathrm{h}}
$$

where $j=e$ or $h$ stands for electron or hole, $H_{j}^{\mathrm{kin}}$ is the kinetic energy term, $V_{j}^{\text {conf }}$ the confining potential defined by the band offsets between bulk CdSe and CdS, $V_{j}^{\text {str }}$ the strain induced deformation potential, $V_{j}^{\mathrm{pz}}$ the strain induced $\mathrm{PZ}$ potential and $V_{j}^{\mathrm{e}-\mathrm{h}}$ is Coulomb attraction exerted upon carrier $j$ by the other carrier. Special attention will be paid to the $\mathrm{PZ}$ potential term. In WZ, strain shifts the atomic nuclei inducing a PZ polarization vector

$$
\mathbf{P}=\left(\begin{array}{c}
e_{15} \epsilon_{x z} \\
e_{15} \epsilon_{y z} \\
e_{31}\left(\epsilon_{x x}+\epsilon_{y y}\right)+e_{33} \epsilon_{z z}
\end{array}\right)
$$

where $e_{r s}$ are $\mathrm{PZ}$ coefficients and $\epsilon_{i j}$ strain tensor components. Notice that the polarization along the $c$-axis, $P_{z}$, is particularly important because it involves diagonal strain components, which are larger than the off-diagonal (shear) ones. Because $e_{33}$ $\approx-2 e_{31}$, sizable polarization $P_{z}$ is expected when strain is anisotropic, that is, $\left(\epsilon_{x x}+\epsilon_{y y}\right) / 2 \neq \epsilon_{z z}$. The polarization is different in core and shell materials, as they experience differen strain forces and have different piezoelectric coefficients. As consequence, PZ charges $\rho(\mathbf{r})=-\nabla \mathbf{P}$ arise near the interface. Again, these are especially important along the $c$ axis. The PZ charges give rise to a $\mathrm{PZ}$ field according to the Poisson equation, $\nabla \epsilon(\mathbf{r}) \nabla \phi^{\mathrm{pz}}(\mathbf{r})=-4 \pi \rho(\mathbf{r})$, and the $\mathrm{PZ}$ potential is finally obtained as $V^{\mathrm{pz}}= \pm q \phi^{\mathrm{pz}}$, where plus and minus sign apply to $j=e$ and $j=h$, respectively, and $q$ is the electron charge.

We start by investigating spheroidal DiDs like those illustrated in Figure $1 \mathrm{a}-\mathrm{c}$. Consider first a fully spherical DiD. Figure $1(\mathrm{~d})$ shows the $\mathrm{CB}$ and VB potential profiles. Dashed lines represent the confinement potential $V^{\text {conf }}$, whereas solid lines represent the total single-particle potential, including strain-induced deformation potential and PZ terms, $V^{\text {tot }}=V^{\text {conf }}$ $+V^{\text {str }}+V^{\mathrm{pz}}$. By inspecting the $\mathrm{CB}$ potential, orange line, one can see that the inclusion of $V^{\mathrm{str}}+V^{\mathrm{pz}}$ has three important effects. First, the core potential is shallower. This is a consequence of $V^{\text {str }}$ (see Figure $S 1$ in the Supporting Information (SI) or ref 16). Second, the core bottom develops a built-in PZ field of $15 \mathrm{mV} / \mathrm{nm}$. This is a consequence of the $\mathrm{PZ}$ term, $V^{\mathrm{pz}}$ (see Figure S1 in SI or ref 16). Third, and most important, because the $\mathrm{CB}$ potential is shallow, the positive $\mathrm{PZ}$ charges accumulating at the bottom $\mathrm{CdSe} / \mathrm{CdS}$ interface form a potential well in the shell where electrons can be trapped (red arrow in Figure 1d).

For holes the situation is different. We have three subbands: A-, B- and C-band. All three subbands are shifted upward by $V^{\text {str. }}$. Contrary to electrons, this now results in a slightly deeper confinement. The effect of $V^{\mathrm{pz}}$ in the core is the same as for electrons, but owing to the different charge sign, holes will be pushed to the upper $\mathrm{CdSe} / \mathrm{CdS}$ interface, thus favoring electron-hole separation. On the other hand, because the Aband confinement potential $V^{\text {conf }}$ is very deep (see dashed lines), the negative PZ charges accumulating at the top $\mathrm{CdSe}$ / $\mathrm{CdS}$ interface do not suffice to localize the hole ground state outside the core.

Considering a prolate $\mathrm{DiD}$ instead of spherical, the $\mathrm{PZ}$ field increases (up to $23 \mathrm{mV} / \mathrm{nm}$ in Figure 1e). Conversely, for an oblate DiD the field is reduced and eventually the sign is even reversed, see Figure 1f.

The presence of a significant PZ field in the spherically symmetric $\mathrm{DiD}$ implies that even for structures with isotropic confinement, the inherent anisotropy of the WZ lattice leads 
Piezoelectric control of the exciton wave function in colloidal

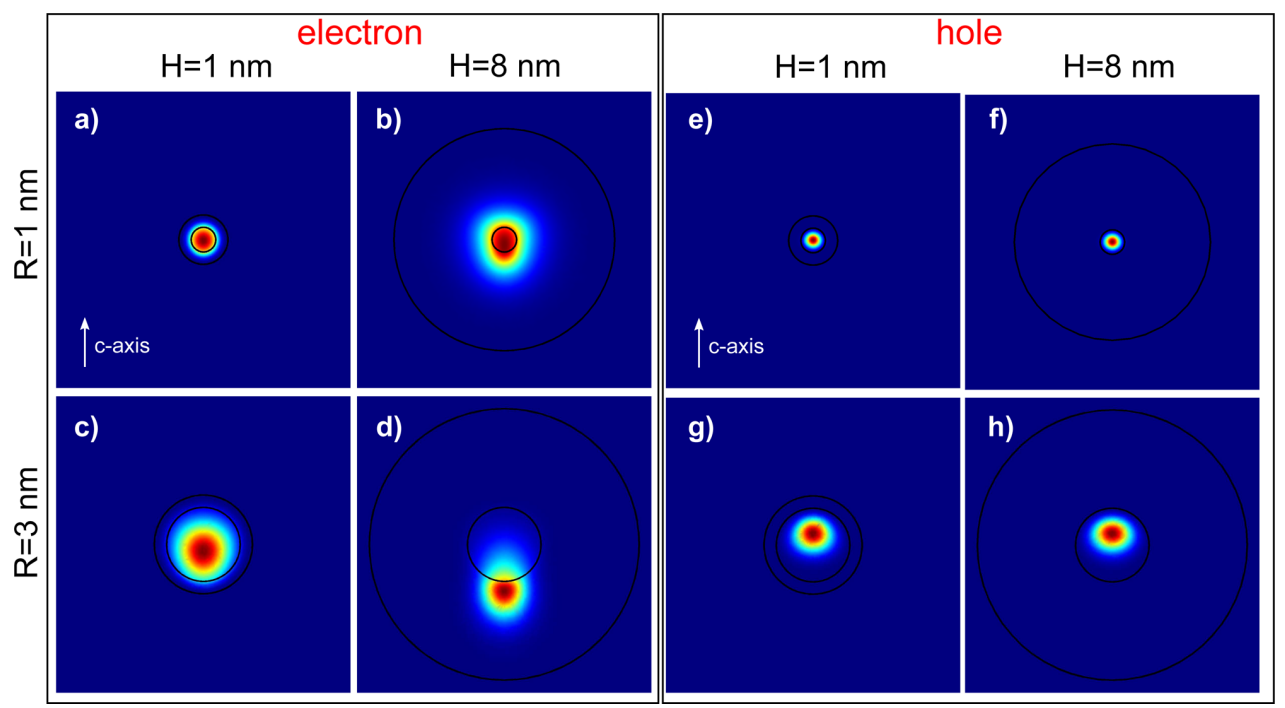

Figure 2. (a)-(d) Excitonic electron charge density in spherical DiDs with different core radius $R$ and shell thickness $H$. (e-h) Same for excitonic hole. The electron moves into the shell for large cores and thick shells, panel d.

pronounced polarization near the $\mathrm{CdSe} / \mathrm{CdS}$ interface, see black line in Figure $1 \mathrm{~g}$ and notice the different weight of $\epsilon_{z z}$ and $\epsilon_{\perp}=\left(\epsilon_{x x}+\epsilon_{y y}\right) / 2$ in Figure S2 of the SI. This leads to a mostly dipolar PZ potential, as shown in Figure $1 \mathrm{~h}$. The strain anisotropy can be conveniently manipulated by using anisotropic geometries. Prolate structures favor $\left|\epsilon_{z z}\right|>\left|\epsilon_{\perp}\right|$ in the core, whereas oblate ones do the opposite, see Figure S2 in SI. This translates into more negative (positive) $P_{z}$ values, see green (brown) line in Figure 1g. We then conclude that the shape of core/shell structures can be used to enhance, cancel or reverse PZ fields.

The next question is whether the magnitude of the attainable PZ fields is enough to influence excitonic wave functions in realistic DiDs. In Figure 2, we compare the exciton electron $(\mathrm{a}-\mathrm{d})$ and hole $(\mathrm{e}-\mathrm{h})$ charge densities for spherical DiDs with different dimensions. For small cores and thin shells $(R=1 \mathrm{~nm}$, $H=1 \mathrm{~nm}$ ), both electron (Figure $2 \mathrm{a}$ ) and hole (Figure 2e) are centered in the core. Growing a giant shell $(H=8 \mathrm{~nm})$ instead increases the core compression. As a result, $V^{\text {str }}$ reduces the $\mathrm{CB}$ confinement barrier (recall Figure 1d) and the electron wave function starts leaking into the shell in spite of the Coulomb attraction, Figure $2 \mathrm{~b}$. The hole, by contrast, remains in the core because of the high confinement barrier, Figure $2 \mathrm{f}$.

If the core is large enough $(R=3 \mathrm{~nm})$ but the shell is thin $(H$ $=1 \mathrm{~nm}$ ), electron and hole are pushed toward opposite sides of the core along the $c$-axis by the PZ field, Figure $2 c, g$, but the resulting overlap is still substantial. The most remarkable effect of piezoelectricity takes place when a large core is surrounded by a giant shell. As can be seen in Figure $2 \mathrm{~d}$, in this case the electron escapes from the core and localizes in the shell near the $\mathrm{CdSe} / \mathrm{CdS}$ interface. The electron is actually taking advantage of the potential minimum originated in the positive PZ interface charge, red arrow in Figure 1d. Because the hole remains in the core, Figure $2 \mathrm{~h}$, the spatial separation between the two carriers becomes large. In other words, by using large
CdSe cores and growing giant CdS shells around, a gradual transition from type-I to type-II exciton driven by $\mathrm{PZ}$ is feasible. For a comprehensive view of the effect of $\mathrm{PZ}$ on the exciton wave function of $\mathrm{DiDs}$, we compute the electron-hole overlap integral squared, $S_{\mathrm{eh}}^{2}=\left\langle\Psi_{\mathrm{e}} \mid \Psi_{\mathrm{h}}\right\rangle^{2}$, which is directly proportional to the radiative exciton decay rate. ${ }^{1}$ In Figure 3, we compare the overlap (a) excluding and (b) including strain and PZ effects $\left(V^{\text {str }}\right.$ and $\left.V^{p z}\right)$. The first case corresponds to DiDs governed by quantum confinement and Coulomb interactions only, which is the scenario assumed so far in the literature. The results are
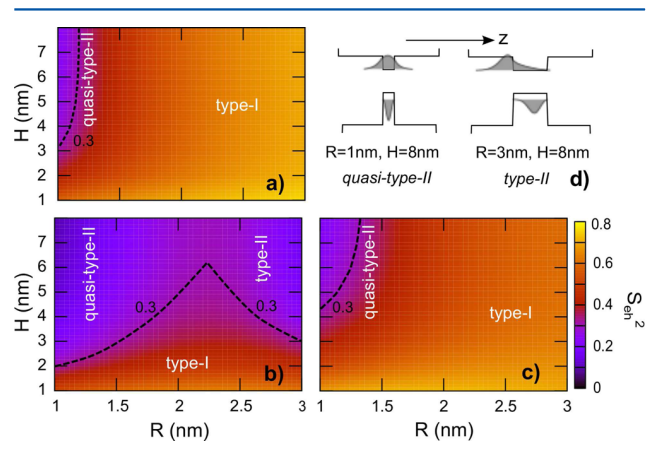

Figure 3. Electron-hole overlap squared for excitons confined in spherical WZ DiDs without (a) and with (b) strain and piezoelectric effects. The inclusion of strain and PZ reduces overlaps (compare the (dashed) isoline for $S_{e h}^{2}=0.3$ in the two panels) and introduces a typeII regime for large cores and thick shells. (c) Electron (top) and hole (bottom) charge densities along the $c$ axis for two DiDs of panel (b), evidencing quasi-type-II $(R=1 \mathrm{~nm})$ and type-II $(R=3 \mathrm{~nm})$ exciton character. (d) Same as (a) and (b) but for strained ZB DiDs. The absence of $\mathrm{PZ}$ in this case translates into absence of type-II regime. 
essentially the same as described, for example, in Figure if of ref 2. For core radii $R=1-1.5 \mathrm{~nm}$, increasing the shell thickness $H$ leads into a quasi-type-II regime, where the electron leaks into the shell due to the high kinetic energy in the core. For larger $R$, the electron stays inside the core no matter how thick the shell, and a type-I exciton is obtained with strong overlap values, similar to core-only samples.

The behavior changes drastically when strain and $\mathrm{PZ}$ effects are taken into account, Figure $3 \mathrm{~b}$. For thick shells, the quasitype-II regime extends toward larger core radii $(R \lesssim 2 \mathrm{~nm})$. This is a consequence of strain making $C B$ confinement shallower (see also $S_{\text {eh }}^{2}$ for $V^{\text {str }} \neq 0$ and $V^{\mathrm{pz}}=0$ in Figure.S4 of SI). Moreover, with further increasing core radius $(R \gtrsim 2.5$ $\mathrm{nm}$ ), instead of retrieving a type-I behavior the overlap decreases again. This is a consequence of the formation of PZ induced type-II excitons, as shown in Figure 2d,h. We stress that the nature of the reduced $S_{\text {eh }}$ values is different on both sides of the figure. For small $R$ (quasi-type-II regime), it is driven by the strong core confinement. For large $R$ (type-II regime), it is driven by strain-induced PZ. The different localization of electron and hole charge densities in each case is clearly seen in Figure 3c. For comparison, in Figure 3d we also represent $S_{\mathrm{eh}}^{2}$ for $\mathrm{ZB}$ core/ZB shell CdSe/CdS DiDs. Strain is still present in such structures, but the dipolar $\mathrm{PZ}$ potential is quenched because of the cubic lattice symmetry. The resulting behavior is similar to that of unstrained WZ NCs, Figure 3a, and it is qualitatively different from that of realistic WZ DiDs, Figure $3 \mathrm{~b}$. We shall see below that this difference between WZ and $\mathrm{ZB}$ DiDs is fully consistent with experimental data.

The PZ control of the electron-hole overlap of WZ DiDs we report in Figure 3b, which should have important consequences on exciton lifetimes, electron-hole exchange integrals, and so forth, is robust against deviations from sphericity (Figure S5 in SI). Since the actual value of the CB offset is often discussed, ${ }^{32}$ we have also studied the effect of changing from $0.32 \mathrm{eV}$ (the value used in Figure 3) to a lower estimate of $0.20 \mathrm{eV}$. The results are qualitatively unchanged, but the overlap values in the type-II regime become manifestly lower than those in the quasitype-II one, see Figure S6 in SI. It is also worth noting that although the PZ field is very efficient in separating carriers, its influence on the energy is modest. The exciton energy with and without strain differs at most in few tens of millielectronvolts (see Figure S7 in SI and ref 16)

To test the above predictions, we synthesized two series of giant-shell WZ DiDs with variable CdSe core radii and CdS shell thickness of about 20 and $15 \mathrm{ML}$, respectively (Figure 4a, see table S2 in SI for structural and optical properties). The resulting PL peak position varies between 648 and $663 \mathrm{~nm}$ and the time-resolved PL traces show a nonexponential decay (see Figure $4 b, c$ for the $20 \mathrm{ML}$ shell thickness series or Figure S10 in SI for the $15 \mathrm{ML}$ one). Figure $4 \mathrm{c}$ shows that as the core size increases, we observe a slower decay. Note however that previous measurements ${ }^{18}$ suggested the opposite, and the series with $15 \mathrm{ML}$ shell thickness does not reveal a clear trend; hence, further work remains needed to firmly establish this behavior. Corresponding lifetimes for both 20 and 15 ML shell DiDs are plotted in Figure 4d using solid and open symbols, respectively. The values are calculated either from the time when the PL signal has decayed to $1 / e$ (dots) or $1 / e^{2}$ (triangles) of its initial value, or from a multiexponential fit (diamonds) to the decay trace (see SI for the analysis and summary of all components). Regardless of the core size dependence, the long lifetimes, especially for the large-core samples, are in clear contrast to
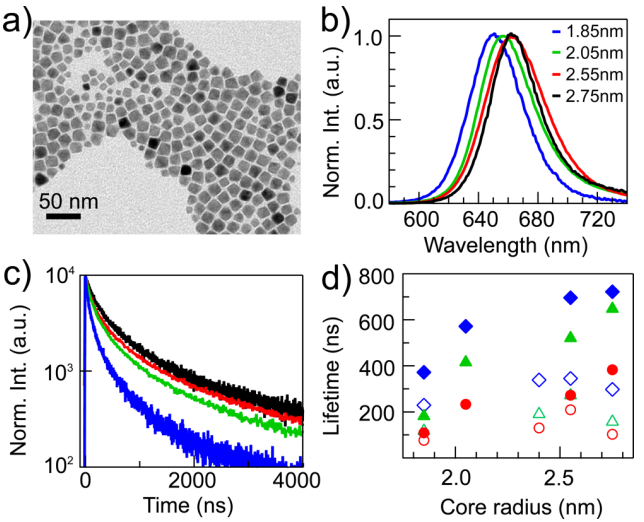

Figure 4. (a) Typical transmission electron microscope image of giantshell DiDs (core radius $2.05 \mathrm{~nm}, 20 \mathrm{ML}$ shell). (b) PL spectra for four different DiDs with $20 \mathrm{ML}$ shell. (c) Corresponding PL decay traces. (d) Resulting effective lifetimes determined from the 1/e decay time (dots), $1 / e^{2}$ decay time (triangles), and from a fit to the decay traces using a multiexponential function (diamonds, see SI for details). Solid (open) symbols are used for the $20 \mathrm{ML}(15 \mathrm{ML})$ shell thickness series. The thinner shell gives shorter lifetimes. In general, lifetimes largely exceed those of giant-shell ZB DiDs.

expectations from the usual confinement picture for $\mathrm{CdSe} /$ $\mathrm{CdS}$, where electron and hole states are expected to become localized into the core, approaching lifetimes of core-only CdSe NCs (ca. 15-20 ns). Such long lifetimes are indicative of a type-II regime, and they support the theory of PZ fields as an efficient mechanism of charge separation. Our values also exceed measurements of fluorescence lifetimes in $\mathrm{ZB} \mathrm{CdSe/}$ CdS DiDs with moderately large cores $(R \approx 1.5 \mathrm{~nm})$ and thick shells $(H \approx 5.5 \mathrm{~nm})$ which have yielded values of about $30 \mathrm{~ns}^{21}$ This is again consistent with the theoretical predictions of Figure 3, which showed that PZ in thick-shell WZ DiDs leads to reduced electron-hole overlap as compared to $\mathrm{ZB}$ ones. Moreover, the strong differences in PL lifetime between the two samples series studied here highlight the sensitivity of the final lifetime to the shell thickness, even when it is grown to a regime where strong quantum confinement no longer influences the optical properties. This provides extra confirmation on $\mathrm{PZ}$ playing a significant role in giant-shell NCs with WZ crystal structure.

Having confirmed the influence of PZ fields in DiDs, we next probe other WZ CdSe/CdS structures such as DiRs, DiPs and RiRs, which we model as ellipsoids with different degrees of anisotropy and different orientation of the $c$ axis. Let us consider first DiRs. Experiments available in the literature are generally consistent with a quantum confinement model, with a type-I band alignment and conduction band offsets between 0.1 and $0.3 \mathrm{eV}$. With decreasing core size, the excitons change from type-I to quasi-type-II behavior, ${ }^{24,32}$ yielding room temperature lifetimes between 8 and $40 \mathrm{~ns} .^{24,25,40}$ The absence of apparent $\mathrm{PZ}$ effects can be understood from two factors. First, typical cores for DiRs are small $(R \lesssim 2 \mathrm{~nm})$. The PZ dipole moment is then weak and, as noticed above (Figure 2 and Figure 3 ), $V^{\mathrm{pz}}$ has a minor influence. Second, the shell surrounding the core is thin on the lateral sides (usually $1 \mathrm{~nm}$ or less). As compared to the giant shells of DiDs, the thin lateral shell of DiRs allows the 
Piezoelectric control of the exciton wave function in colloidal
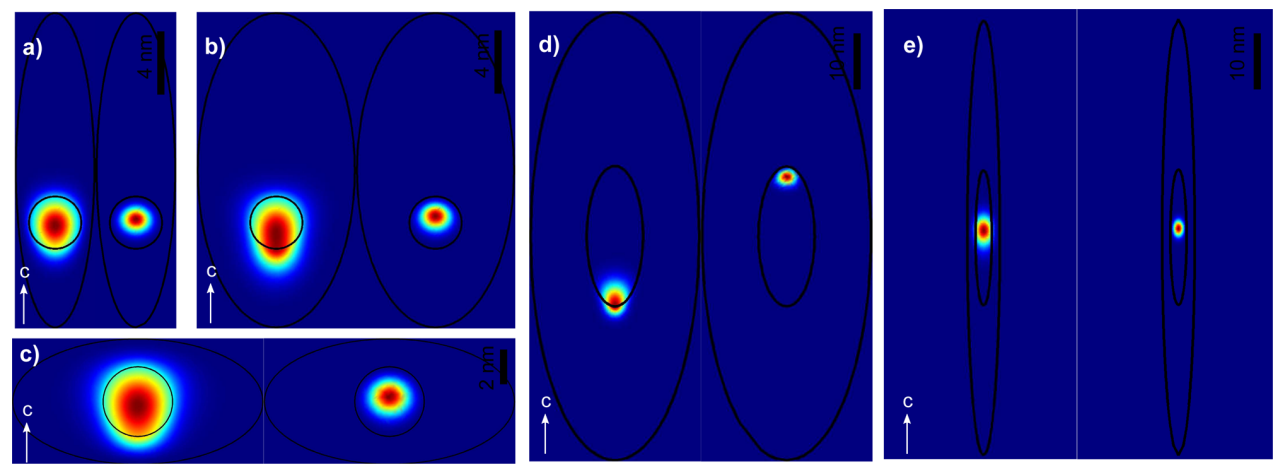

Figure 5. Excitonic charge density in different types of CdSe/CdS NCs. In each structure, the left (right) panel shows the electron (hole) density. (a) DiR with standard dimensions. (b) DiR with thicker lateral shell. The electron starts moving into the shell. (c) DiP with standard dimensions. (a) DiR with standard dimensions. (b) DiR with thicker lateral shell. The electron starts moving into the shell. (c) DiP with standard dimensions.
(d) RiR with large core and giant shell. The charge separation is extremely efficient. (h) RiR with thinner core and shell. The charge separation is suppressed.

core to dilate, relaxing the strain in orthogonal directions. Consequently, the strain near the CdSe/CdS interfaces along the $c$ axis, which is chiefly responsible for $P_{z}$ is reduced, see for example, Figure 4 in ref 41 . The overall result is that PZ effects in DiRs are weak. This is precisely what we see in Figure 5a, where we plot the exciton electron and hole charge densities in a DiR: the carriers are well confined inside the core in spite of the relatively large size we have assumed $(R=2 \mathrm{~nm})$. We note, however, that the picture would change if thicker shells could be grown, increasing the strain of the system. As shown in Figure $5 \mathrm{~b}$, keeping the same rod length as before but increasing $H_{\perp}$, the electron already starts moving toward the CdS shell, reducing the electron-hole overlap. We note that the enhancement of PZ fields with radial shell thickness explains recent experiments of Coropceanu et al., ${ }^{42}$ where the exciton lifetime of DiRs steadily increased from 20 to $60 \mathrm{~ns}$ with increasing shell thickness. For very thick shells, we predict a complete migration of the electron into the shell (Figure S8a in SI), so even longer lifetimes should be expected.

DiPs differ from DiRs mainly in that the shell is nearly twodimensional, with the $c$ axis pointing along the strong confinement direction. ${ }^{26}$ Yet, their behavior in terms of PZinduced electron delocalization (Figure $5 c$ ) is partly analogous to that of DiR: the thin shells along the $c$ axis weaken the strain. This in turn leads to weak PZ effects and carriers localized inside the core. As in the case of DiRs, electron-hole distance could be readily increased if thicker shells were grown (Figure $\mathrm{S} 8 \mathrm{~b})$.

The most favorable condition to maximize PZ effects is however achieved in recently synthesized RiRs. ${ }^{28}$ The giant shell grants strong strain. The prolate shape makes strain highly anisotropic. The long core allows for huge dipole moments, and longitudinal quantum confinement is too weak to compete. The result, as plotted in Figure 5d, is that electron and hole are separated toward opposite $\mathrm{CdSe} / \mathrm{CdS}$ interfaces along the $c$ axis, which explains the record exciton lifetimes measured in these structures. ${ }^{28}$ It is worth stressing that the giant shell plays a decisive role in RiRs too. RiRs with smaller core and shell width were previously synthesized by Sitt and co-workers, but long exciton lifetimes were not reported in such a case. ${ }^{27}$ We simulate RiRs with similar dimensions to theirs in Figure 5e. As can be seen, in spite of the high aspect ratio and the weak longitudinal confinement, charge separation is completely suppressed, with both electron and hole localizing inside the core. The underlying reason is again the weaker strain of the system, which results in a PZ field unable to compete against electron-hole Coulomb interaction.

An important conclusion from Figure 5 is that PZ successfully rationalizes the very different exciton lifetimes reported for different kinds of WZ CdSe/CdS NCs. In RiRs, ${ }^{28}$ lifetimes can be 1 order of magnitude longer than those in giant-shell DiDs (ref 18 and Figure 4), and these in turn are about 1 order of magnitude longer than those in DiRs. ${ }^{24}$ This result cannot be interpreted in terms of quantum confinement because all structures have voluminous shells, but it is perfectly consistent with the different degrees of PZ-induced electronhole separation we calculate.

To summarize, we have elucidated the conditions where strain-induced piezoelectricity becomes a practical mechanism for electron-hole spatial separation in several kinds of WZ $\mathrm{CdSe} / \mathrm{CdS}$ NCs. The PZ field requires anisotropic strain, which is present even in spherical heterostructures due to the anistropy of the WZ lattice. PZ charges thus accumulate on the CdSe/CdS interfaces, forming a dipole and a sizable built-in field along the $c$ axis, which enables directional charge separation. The magnitude of the PZ field can be enhanced using thick shells all around the core, which increase the strain in and around it, and using prolate cores, which reinforce the inherent strain anisotropy. The influence of PZ potential on the exciton wave function scales with the core size, as so does the PZ dipole moment, and on the shell thickness, as it provides space for the electron to escape from the core.

We have then shown that significant PZ effects are present not only in RiRs, as recently reported in ref 28 but also in giantshell DiDs and, to a lesser extent, in DiRs. In fact, the different strength of PZ fields in each kind of structure interprets the large variations of radiative lifetime reported in the literature. The present results show that, with appropiate design, band alignment can be engineered all the way from type-I or quasitype-II to a fully type-II one in any kind of CdSe/CdS NC, thus providing an efficient tool for tailoring electron-hole separation. 
ASSOCIATED CONTENT

S Supporting Information

The Supporting Information is available free of charge on the ACS Publications website at DOI: 10.1021/acs.jpclett.6b00622. Details of methods, supporting calculations of strain maps, band-edge structure, wave functions, electronhole overlap under different conditions, a list of the material parameters employed and further experimenta data. (PDF)

\section{AUTHOR INFORMATION}

\section{Corresponding Autho}

*E-mail: josep.planelles@uji.es.

Notes

The authors declare no competing financial interest.

\section{ACKNOWLEDGMENTS}

Support from MINECO project CTQ2014-60178-P, UJ project P1-1B2014-24 and a FPU grant (C.S.) is acknowledged. The present publication is further realized with the support of the Ministero degli Affari Esteri e della Cooperazione Internazionale (IONX-NC4SOL, I.M.).

\section{REFERENCES}

(1) Delerue, C.; Lannoo, M. Nanostructures: Theory and Modelling; Springer: Berlin, 2004.

(2) Garcia-Santamaria, F.; Chen, Y.; Vela, J.; Schaller, R. D.; Hollingsworth, J. A.; Klimov, V. I. Suppressed Auger Recombination in "Giant" Nanocrystals Boosts Optical Gain Performance. Nano Lett. 2009, 9, 3482-3488

(3) Cragg, G. E.; Efros, A. L. Suppression of Auger Processes in Confined Structures. Nano Lett. 2010, 10, 313-317.

(4) Climente, J. I.; Movilla, J. L.; Planelles, J. Auger Recombination Suppression in Nanocrystals with Asymmetric Electron-Hole Confinement. Small 2012, 8, 754-759.

(5) Brovelli, S.; Schaller, R. D.; Crooker, S. A.; Garcia-Santamaria, F. Chen, Y.; Viswanatha, R.; Hollingsworth, J. A.; Htoon, H.; Klimov, V.

I. Nano-engineered Electronhole Exchange Interaction Controls Exciton Dynamics in CoreShell Semiconductor Nanocrystals. Nat. Commun. 2011, 2, 280 .

(6) McDaniel, H.; Pelton, M.; Oh, N.; Shim, M. Effects of Lattice Strain and Band Offset on Electron Transfer Rates in Type-II Nanorod Heterostructures. J. Phys. Chem. Lett. 2012, 3, 1094-1098.

(7) Kovalenko, M.; Manna, L.; Cabot, A.; Hens, Z.; Talapin, D. V. Kagan, Ch. R.; Klimov, V. I.; Rogach, A. L.; Reiss, P.; Milliron, D. J.; et al. Prospects of Nanoscience with Nanocrystals. ACS Nano 2015, 9, $1012-1057$.

(8) Smith, A. M.; Mohs, A. M.; Nie, S. Tuning the Optical and Electronic Properties of Colloidal Nanocrystals by Lattice Strain. Nat Nanotechnol. 2009, 4, 56-63.

(9) Yang, S.; Prendergast, D.; Neaton, J. B. Strain-Induced Band Gap Modification in Coherent Core/Shell Nanostructures. Nano Lett. 2010, 10, 3156-3162.

(10) Fairclough, S. M.; Tyrrell, E. J.; Graham, D. M.; Lunt, P. J. B.; Hardman, S. J. O.; Pietzsch, A.; Hennies, F.; Moghal, J.; Flavell, W. R Watt, A. A. R.; Smith, J. M. Growth and Characterization of Strained and Alloyed Type-II ZnTe/ZnSe Core-Shell Nanocrystals. J. Phys Chem. C 2012, 116, 26898-26907.

(11) Yadav, S. K.; Sharma, V.; Ramprasad, R. Controlling Electronic Structure Through Epitaxial Strain in $\mathrm{ZnSe} / \mathrm{ZnTe}$ Nano-Heterostructures. J. Appl. Phys. 2015, 118, 015701.

(12) Khoo, K. H.; Arantes, J. T.; Chelikowsky, J. R.; Dalpian, G. M. First-Principles Calculations of Lattice-Strained Core-Shell Nanocrystals. Phys. Rev. B: Condens. Matter Mater. Phys. 2011, 84, 075311.
(13) Phadnis, C.; Sonawane, K. G.; Hazarika, A.; Mahamuni, S. Strain-Induced Hierarchy of Energy Levels in CdS/ZnS Nanocrystals. J. Phys. Chem. C 2015, 119, 24165-24173.

(14) Pahomi, T. E.; Cheche, T. O. Strain Influence on Optical Absorption of Giant Semiconductor Colloidal Quantum Dots. Chem. Phys. Lett. 2014, 612, 33-38.

(15) Cirillo, M.; Aubert, T.; Gomes, R.; Deun, R. V.; Emplit, P.; Biermann, A; Lange, H. Thomsen, C.; Brainis, E.; Hens, Z. Flash" Synthesis of CdSe/CdS Core-Shell Quantum Dots. Chem. Mater. 2014, 26, 1154-1160.

(16) Park, S. H.; Cho, Y. H. Characteristics of Built-in Polarization Potentials in Vertically and Laterally Arranged InGaN/GaN Quantum Dots. J. Appl. Phys. 2011, 109, 113103.

(17) Chen, O.; Zhao, J.; Chauhan, V. P.; Cui, J.; Wong, C.; Harris, D. K.; Wei, H.; Han, H. S.; Fukumura, D.; Jain, R. K.; Bawendi, M. G. Compact High-Quality CdSe/CdS Core/Shell Nanocrystals with Narrow Emission Linewidths and Suppressed Blinking. Nat. Mater. 2013, 12, 445-451.

(18) Christodoulou, S.; Vaccaro, G.; Pinchetti, V.; De Donato, F.; Grim, J. Q.; Casu, A.; Genovese, A.; Vicidomini, G.; Diaspro, A.; Brovelli, S.; et al. Synthesis of Highly Luminescent Wurtzite CdSe/ CdS Giant-shell Nanocrystals Using a Fast Continuous Injection Route. J. Mater. Chem. C 2014, 2, 3439-3447.

(19) Mahler, B.; Lequeux, N.; Dubertret, B. Ligand-Controlled Polytypism of Thick-Shell CdSe/CdS Nanocrystals. J. Am. Chem. Soc. 2010, 132, 953-959.

(20) van Embden, J.; Jasieniak, J.; Mulvaney, P. Mapping the Optical Properties of CdSe/CdS Heterostructure Nanocrystals: The Effects of Core Size and Shell Thickness. J. Am. Chem. Soc. 2009, 131, 1429914309.

(21) Qin, H.; Niu, Y.; Meng, R.; Lin, X.; Lai, R.; Fang, W.; Peng, X. Single-Dot Spectroscopy of Zinc-Blende CdSe/CdS Core/Shell Nanocrystals: Nonblinking and Correlation with Ensemble Measurements. J. Am. Chem. Soc. 2014, 136, 179-187.

(22) Carbone, L.; Nobile, C.; De Giorgi, M.; Della Sala, F.; Morello, G.; Pompa, P.; Hytch, M.; Snoeck, E.; Fiore, A.; Franchini, I. R.; et al. Synthesis and Micrometer-Scale Assembly of Colloidal CdSe/CdS Nanorods Prepared by a Seeded Growth Approach. Nano Lett. 2007, 7, 2942-2950.

(23) Sitt, A.; Hadar, I.; Banin, U. Band-Gap Engineering, Optoelectronic Properties and Applications of Colloidal Heterostructured Semiconductor Nanorods. Nano Today 2013, 8, 494-513.

(24) Raino, G.; Stoferle, T.; Moreels, I.; Gomes, R.; Kamal, J. S.; Hens, Z.; Mahrt, R. F. Probing the Wave Function Delocalization in CdSe/CdS Dot-in-Rod Nanocrystals by Time- and TemperatureResolved Spectroscopy. ACS Nano 2011, 5, 4031-4036.

(25) Wu, K.; Rodriguez-Cordoba, W. E.; Liu, Z.; Zhu, H.; Lian, T. Beyond Band Alignment: Hole Localization Driven Formation of Three Spatially Separated Long-Lived Exciton States in CdSe/CdS Nanorods. ACS Nano 2013, 7, 7173-7185.

(26) Cassette, E.; Mahler, B.; Guigner, J. M.; Patriarche, G.; Dubertret, B.; Pons, T. Colloidal CdSe/CdS Dot-in-Plate Nanocrystals with 2D-Polarized Emission. ACS Nano 2012, 6, 6741-6750.

(27) Sitt, A.; Salant, A.; Menagen, G.; Banin, U. Highly Emissive Nano Rod-in-Rod Heterostructures with Strong Linear Polarization. Nano Lett. 2011, 11, 2054-2060.

(28) Christodoulou, S.; Rajadell, F.; Casu, A.; Vaccaro, G.; Grim, J.; Genovese, A.; Manna, L.; Climente, J. I.; Meinardi, F.; Raino, G.; et al. Band Structure Engineering via Piezoelectric Fields in Strained Anisotropic CdSe/CdS Nanocrystals. Nat. Commun. 2015, 6, 7905.

(29) Talapin, D. V.; Nelson, J. H.; Shevchenko, E. V.; Aloni, S.; Sadtler, B.; Alivisatos, A. P. Seeded Growth of Highly Luminescent $\mathrm{CdSe} / \mathrm{CdS}$ Nanoheterostructures with Rod and Tetrapod Morphologies. Nano Lett. 2007, 7, 2951-2959.

(30) Fiore, A.; Mastria, R.; Lupo, M. G.; Lanzani, G.; Giannini, C.; Carlino, E.; Morello, G.; De Giorgi, M.; Li, Y.; Cingolani, R.; Manna, L. Tetrapod-Shaped Colloidal Nanocrystals of II-VI Semiconductors Prepared by Seeded Growth. J. Am. Chem. Soc. 2009, 131, 2274-2282. 
Piezoelectric control of the exciton wave function in colloidal CdSe/CdS nanocrystals

(31) Deka, S.; Miszta, K.; Dorfs, D.; Genovese, A.; Bertoni, G.; Manna, L. Octapod-Shaped Colloidal Nanocrystals of Cadmium Chalcogenides via "One-Pot" Cation Exchange and Seeded Growth. Chalcogenides via "One-Pot" Cation Exchange and Seeded Growth.
Nano Lett. 2010, 10, 3770-3776.

(32) Eshet, H.; Grünwald, M.; Rabani, E. The Electronic Structure of CdSe/CdS Core/Shell Seeded Nanorods: Type-I or Quasi-Type-II? Nano Lett. 2013, 13, 5880-5885.

(33) Shabaev, A.; Rodina, A. V.; Efros, A. L. Fine Structure of the Band-Edge Excitons and Trions in CdSe/CdS Core/Shell NanocrystBand-Edge Excitons and Trions in CdSe/CdS Core/Shell Nanoctst
als. Phys. Rev. B: Condens. Matter Mater. Phys. 2012, 86, 205311.

(34) Langbein, W.; Hetterich, M.; Grun, M.; Klingshirn, C.; Kalt, H. Picosecond Luminescence Dynamics in CdS/CdSe Stark Superlattices. Appl. Phys. Lett. 1994, 65, 2466-2468.

(35) Andreev, A. D.; O'Reilly, E. P. Theory of the Electronic Structure of GaN/AIN Hexagonal Quantum Dots. Phys. Rev. B: Condens. Matter Mater. Phys. 2000, 62, 15851-15870.

(36) Seguin, R.; Schliwa, A.; Rodt, S.; Potschke, K.; Pohl, U. W.; Bimberg, D. Size-dependent Fine-Structure Splitting in Self-Organized InAs/GaAs Quantum Dots. Phys. Rev. Lett. 2005, 95, 257402.

(37) Morello, G.; Della Sala, F.; Carbone, L.; Manna, L.; Maruccio, G.; Cingolani, R.; De Giorgi, M. Intrinsic Optical Nonlinearity in Colloidal Seeded Grown CdSe/CdS Nanostructures: Photoinduced Screening of the Internal Electric Field. Phys. Rev. B: Condens. Matter Screening of the Internal Electric Field. Phys. Rev. B: Condens. Matter
Mater. Phys. 2008, 78, 195313.

(38) Zhu, B. H.; Zhang, H. C.; Zhang, Z. Y.; Cui, Y. P.; Zhang, J. Y. Effect of Shell Thickness on Two-Photon Absorption and Refraction of Colloidal CdSe/CdS Core/Shell Nanocrystals. Appl. Phys. Lett. 2011, 99, 231903.

(39) Luo, Y.; Wang, L. W. Electronic Structures of the CdSe/CdS Core-Shell Nanorods. ACS Nano 2010, 4, 91-98.

(40) Muller, J.; Lupton, J. M.; Lagoudakis, P. G.; Schindler, F.; Koeppe, R.; Rogach, A. L.; Feldmann, J.; Talapin, D. V.; Weller, H. Wave Function Engineering in Elongated Semiconductor Nanocrystals with Heterogeneous Carrier Confinement. Nano Lett. 2005, 5, 20442049.

(41) Rajadell, F.; Royo, M.; Planelles, J. Strain in Free Standing CdSe/CdS Core-Shell Nanorods. J. Appl. Phys. 2012, 111, 014303.

(42) Coropceanu, I.; Rossinelli, A.; Caram, J. R.; Freyria, F. S.; Bawendi, M. G. Slow-Injection Growth of Seeded CdSe/CdS Nanorods with Unity Fluorescence Quantum Yield and Complete Shell to Core Energy Transfer. ACS Nano 2016, 10, 3295-3301. 


\section{Supplemental Material for "Piezoelectric control of the exciton wave function in colloidal $\mathrm{CdSe} / \mathrm{CdS}$ nanocrystals"}

Carlos Segarra, ${ }^{\dagger}$ Juan I. Climente, ${ }^{\dagger}$ Anatolii Polovitsyn,,${ }^{\dagger}$ Fernando Rajadell, ${ }^{\dagger}$ Iwan Moreels, $\stackrel{\ddagger}{\ddagger}$ and Josep Planelles ${ }^{*} \dagger$

Departament de Química Física i Analítica, Universitat Jaume I, E-12080, Castelló de la Plana, Spain, and Istituto Italiano di Tecnologia, Via Morego 30, IT-16163 Genova, Italy

E-mail: josep.planelles@uji.es

${ }^{*}$ To whom correspondence should be addressed

†Departament de Química Física i Analítica, Universitat Jaume I, E-12080, Castelló de la Plana, Spain

Istituto Italiano di Tecnologia, Via Morego 30, IT-16163 Genova, Italy 


\section{Theoretical method}

Strain maps are calculated in the continuous medium model by minimizing the elastic energy. The boundary conditions are zero normal stress for the free surface. ${ }^{1}$ The strain tensor elements $\varepsilon_{i j}(\mathbf{r})$, ensuing PZ polarization vector $\mathbf{P}$ and potential $V^{p z}$ are obtained using the multiphysics mode of Comsol 4.2 software. Electron $(j=e)$ and hole $(j=h)$ states of the ground-state exciton are calculated with Hamiltonian (1) of the main text. For electrons, $H_{e}^{k i n}$ is a $3 \mathrm{D}$ single-band (actually two uncoupled bands including spin) effective mass Hamiltonian. $H_{h}^{k i n}$ is a 3D six-band Hamiltonian for wurtzite including A-band, B-band and C-band with spin-orbit interaction. The same Hamiltonian is used for [111]-grown zinc-blende structures by employing due parameters. See Ref. ${ }^{2}$ for details of the Hamiltonian. The strain-induced deformation potential term, $V^{s t r}$ is isomorphic to the kinetic energy term and $V^{p z}$ is diagonal (see e.g. Ref. ${ }^{3}$ ) The Coulomb interaction term $V^{e-h}$ takes into account the dielectric mismatch with the dielectric surroundings of the NC. Interacting electron and hole states are obtained by iterative resolution of the Schrodinger-Poisson equation.

\section{Experimental method}

CdSe core nanocrystals where synthesized according to the procedure of Carbone et al. ${ }^{4}$ For all samples, the CdSe core diameter and concentration in solution were determined using the sizing curve of Jasieniak et al. ${ }^{5}$ The shell was grown using the procedure described in Ref. ${ }^{6}$ After synthesis, the nanocrystals were purified and finally dispersed in toluene.

Bright-field transmission electron microscopy was used to determine the final $\mathrm{NC}$ diameter (using ca. $50 \mathrm{NCs}$ ), from which the shell thickness could be calculated (see also table S2, note that the histogram reveals a minor contribution from sub-10 nm NCs which are likely CdS NCs that nucleated separately, these were not taken into account in the calculation of the final diameter). Steady-state and time-resolved luminescence spectra were measured using a FLS920 Edinburgh Instruments spectrofluorometer. We dispersed the DiDs in chloroform for all measurements, and 
excited the samples at $400 \mathrm{~nm}$ with a Xenon lamp to collect the PL spectra. The PL quantum efficiency was determined with an integrating sphere (2-measurement method $^{7}$ ), exciting the samples at $550 \mathrm{~nm}$. A $405 \mathrm{~nm}, 50 \mathrm{ps}$ pulsed laser was used to collect the PL decay via time-correlate single photon counting. The time between pulses was adjusted to $10 \mu$ s to ensure full decay between subsequent excitations. As the decay is nonexponential, we determined 3 different effective lifetimes to compare between the different sizes. The first focuses on the initial decay, and considers the time $\tau_{1}$ for the signal to decay to $1 / e$ of its maximal value. The second evaluates time delay $\tau_{2}$ until the decay reaches $1 / e^{2}$, yielding an effective $\tau_{2}=\Delta t / 2$. Finally, we fitted the traces to a sum of four exponentials, from which an area-weighted average lifetime $\tau_{3}$ can be determined (see table S3).

\section{Supporting calculations}

\section{Role of each potential term in the single-particle Hamiltonian}

As seen in Figure S1, for all DiD geometries (spherical, prolate and oblate), the band-offset confining potential $V^{\text {conf }}$ forms a confining well in the core region. By contrast, the strain-induced deformation potential $V^{s t r}$ forms a barrier. When considered together, $V^{s t r}$ greatly reduces the well depth one would expect with a confinement-only model. This stimulates electron leakage into the shell. The piezoelectric (PZ) potential $V^{p z}$ is similar to that reported by Park and Cho. ${ }^{3}$ Charges of opposite sign accumulate on each $\mathrm{CdSe} / \mathrm{CdS}$ interface forming a dipole and a nearly linear (capacitor-like) built-in field in the core. Notice that using prolate cores (ellipsoid is elongated along the $c$-axis) instead of spherical ones increases the field. In turn, using oblate cores reduces the field and can even revert the sign (that is the case we plot in the right column). As for the total potential $V^{\text {tot }}$, notice that in all cases a well is formed by the bottom CdSe/CdS interface where electrons can localize. This is because the positive PZ charges can compensate for the low conduction band offset of CdSe/CdS. 

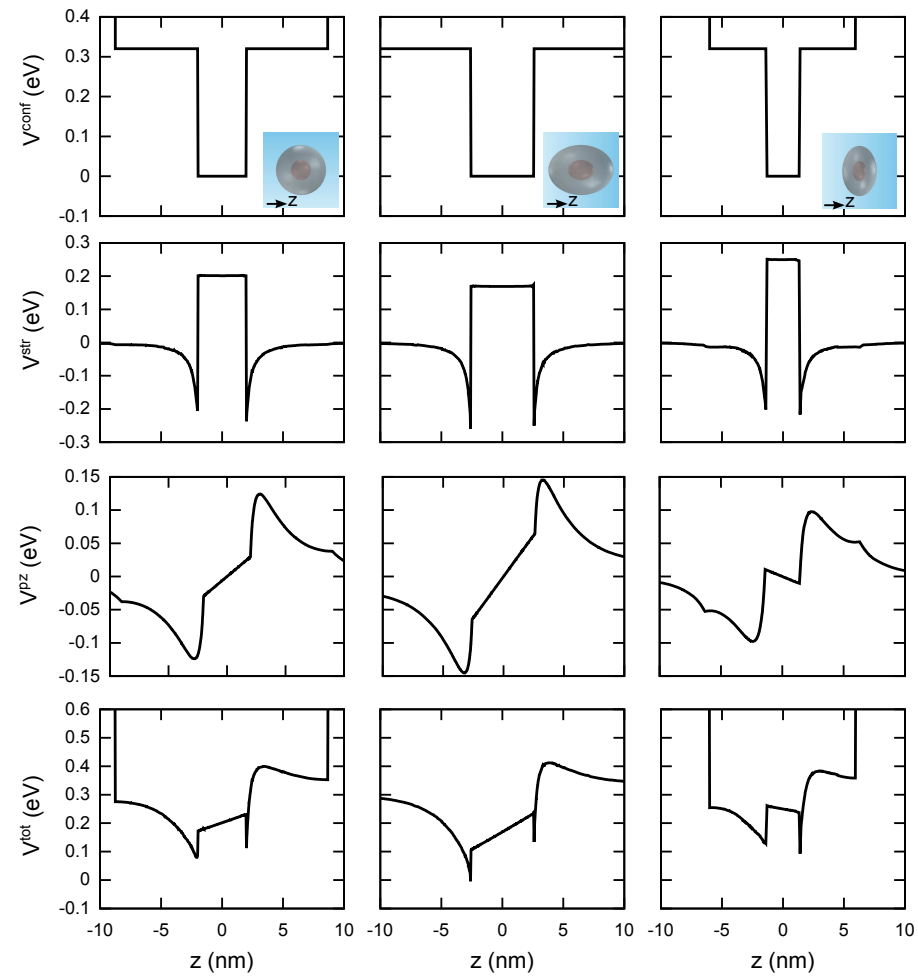

Figure S1: Top to bottom: $V^{\text {conf }}, V^{s t r}, V^{p z}$ and $V^{\text {tot }}=V^{\text {conf }}+V^{s t r}+V^{p z}$ along the $c$-axis of a dotin-dot (DiD). Left column: spherical geometry -core radius $R=2 \mathrm{~nm}$ and shell thickness $H=7$ nm-. Middle column: prolate geometry $-R_{\perp}=R, R_{z}=1.3 R, H_{\perp}=H$ and $H_{z}=1.3 H-$. Right column: oblate geometry $-R_{\perp}=R, R_{z}=0.7 R, H_{\perp}=H$ and $H_{z}=0.7 H-$.

\section{Effect of shape on the strain map}

In $\mathrm{CdSe} / \mathrm{CdS} \mathrm{NCs}$, the CdSe core is compressed because of its larger natural lattice constant (negative strain in the figure). In spherical cores, panels (a) and (d), the core strain is slightly anistropic as $\left|\varepsilon_{z z}\right| \gtrsim\left|\varepsilon_{\perp}\right|$. Since $P_{z}=e_{31}\left(\varepsilon_{x x}+\varepsilon_{y y}\right)+e_{33} \varepsilon_{z z}$ and $e_{33} \approx-2 e_{31}$, this leads to a small but finite negative PZ polarization $P_{z}$ inside the core, which can be seen in Fig.1(g) of the paper. In prolate cores, panels (b) and (e), $\varepsilon_{z z}$ becomes visibly more compressive (darker blue) and the strain anisotropy increases. This explains the more negative values of $P_{z}$ in Fig.1(g). The opposite occurs for oblate cores, panels (c) and (f). This explains the sign reversal of $P_{z}$ for oblate cores in Fig.1(g). 


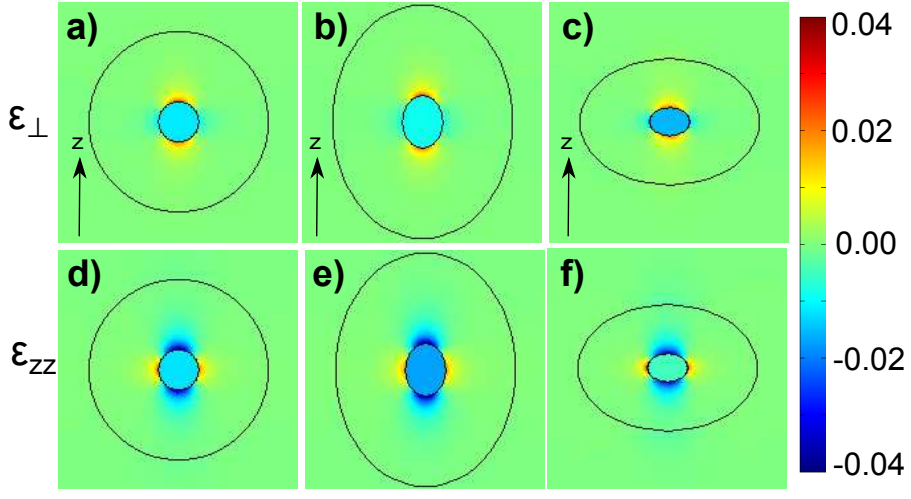

Figure S2: Diagonal strain along the $c$-axis $\left(\varepsilon_{z z}\right)$ and the orthogonal direction $\left(\varepsilon_{\perp}=1 / 2\left(\varepsilon_{x x}+\right.\right.$ $\left.\varepsilon_{y y}\right)$. Left column: spherical geometry. Middle column: prolate geometry. Right column: oblate geometry (same dimensions as in Figure S1).

The strain anisotropy becomes much more pronounced in the shell surrounding the core. Along the $c$ axis (vertical direction in the figure), $\varepsilon_{z z}$ remains compressive (negative) but $\varepsilon_{\perp}$ becomes tensile (positive). This explains the drastic change of $P_{z}$ at the interface (see Fig.1(g) of the main paper). Since PZ charges are proportional to the $P_{z}$ gradient, $\rho(\mathbf{r})=-\nabla \mathbf{P}$, significant PZ charges build up at the heterointerface.

\section{Influence of each potential term on the exciton's electron wave function}

To understand the origin of the electron localization in the shell reported in Fig.2(d) of the main paper, in Figure S3 we show the electron charge density calculated under different circumstances. When only the band-offset confinement is considered, Figure S3(a), the electron is well localized in the center of the core. Further adding the strain induced deformation potential, Figure S3(b), enhances leakage into the shell owing the overall shallower confining potential (recall Figure S1). It is the inclusion of the PZ field, Figure S3(c), that moves the electron to one of the core-shell interfaces. Adding Coulomb interaction with the hole -which remains in the core- moves the electron charge density partially back into the core, Figure S3(d). This is however a relatively minor effect because Coulomb interaction in a type-II system is weak. 

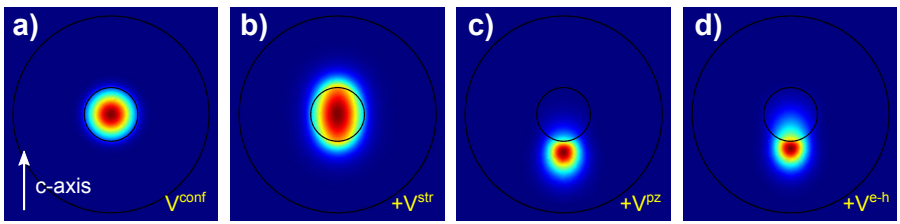

Figure S3: Electron charge density corresponding to the ground state exciton in a spherical DiD with $R=3 \mathrm{~nm}$ core and $H=8 \mathrm{~nm}$ shell, under different potential terms. (a) Confinement potential only, $H_{e}=H_{e}^{\text {kin }}+V_{e}^{\text {conf }}$. (b) Adding strain deformation potential, $H_{e}=H_{e}^{\text {kin }}+V_{e}^{\text {conf }}+V_{e}^{\text {str }}$. (c) Adding PZ potential, $H_{e}=H_{e}^{k i n}+V_{e}^{\text {conf }}+V_{e}^{s t r}+V_{e}^{p z}$. (d) Adding Coulomb interaction with hole in core, $H_{e}=H_{e}^{k i n}+V_{e}^{\text {conf }}+V_{e}^{s t r}+V_{e}^{p z}+V_{e}^{e-h}$.

\section{Exciton overlap and energy in dot-in-dots}

Figure S4 shows the electron-hole overlap squared, $S_{e h}^{2}=\left|\left\langle\Psi_{e} \mid \Psi_{h}\right\rangle\right|^{2}$, calculated for the ground state exciton in spherical DiDs. Unlike in Fig. 3 of the main text, we keep $V^{s t r}$ but set $V^{p z}=0$. One can see that the quasi-type-II (purple) region extends to larger core sizes than in the case neglecting strain (Fig.3(a)), owing to the shallower confinement. On the other hand, lacking PZ terms, there is no type-II exciton region for the largest core sizes, unlike in Fig. 3(b).

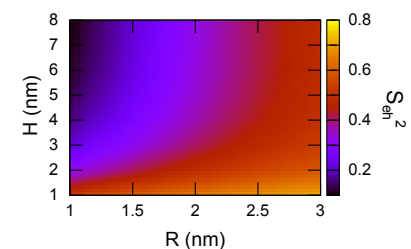

Figure S4: Electron-hole overlap squared, $\left|\left\langle\Psi_{e} \mid \Psi_{h}\right\rangle\right|^{2}$, in spherical DiDs with different core radius $R$ and shell thickness $H$. Defomation potential is considered but PZ potential is neglected.

Figure S5 illustrates the overlap for spherical, prolate and oblate DiDs considering the full Hamiltonian. Note that in all cases, small overlaps are expected for large cores $\left(R_{\perp}>2.5 \mathrm{~nm}\right)$. It follows that the $\mathrm{PZ}$ induced charge separation is generally robust against shape deformations. 


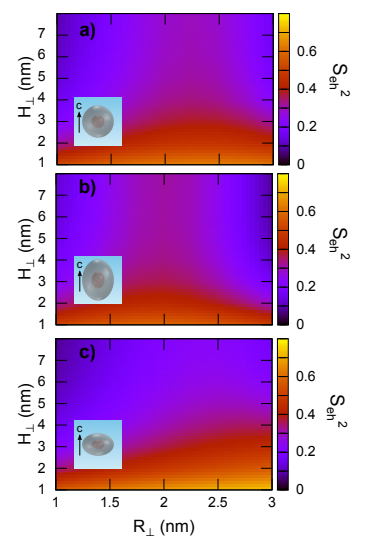

Figure S5: Electron-hole overlap squared, $\left|\left\langle\Psi_{e} \mid \Psi_{h}\right\rangle\right|^{2}$, in spherical (a), prolate (b) and oblate (c) DiDs with different lateral core radius $R_{\perp}$ and shell thickness $H_{\perp}$. For prolate dots, $R_{z}=1.3 R_{\perp}$. For oblate ones, $R_{z}=0.7 R_{\perp}$.

Figure S6 shows that the behavior reported in Fig. 3 of the paper is also robust against changes in the conduction band offset, a parameter whose exact value is often debated. Here we use a band offset of $0.20 \mathrm{eV}$ instead of the bulk value of $0.32 \mathrm{eV}$, to account for experiments suggesting a flatter core-shell alignment. ${ }^{8}$ The results are qualitatively consistent with Fig.3, but the lower band offset gives rise to a type-II regime (large $R$ and $H$ in panel (b)) with much more efficient charge separation than in the quasi-type-II one (small $R$ ). For $H=8 \mathrm{~nm}$, the overlap squared of $R=3 \mathrm{~nm}$ is 5 times smaller than that of $R=1 \mathrm{~nm}$.

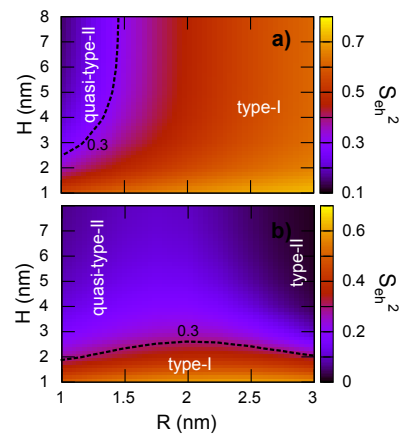

Figure S6: Same as Fig. 3 in the main text but considering a conduction band offset of $0.20 \mathrm{eV}$ instead of $0.32 \mathrm{eV}$. The dashed line is the $S_{e h}^{2}=0.3$ isoline. 
Figure S7 shows the exciton energy for spherical DiDs without (a) and with (b) strain and PZ effects. The results are similar, with a predominant influence of the core radius in both cases. The energy differences between the two cases are of few tens of meV at most, with a maximum of 80 meV for $R=1 \mathrm{~nm}$ and $H=8 \mathrm{~nm}$. One concludes that, while PZ has important effects on the exciton wave function, its influence on the energy is relatively weak.

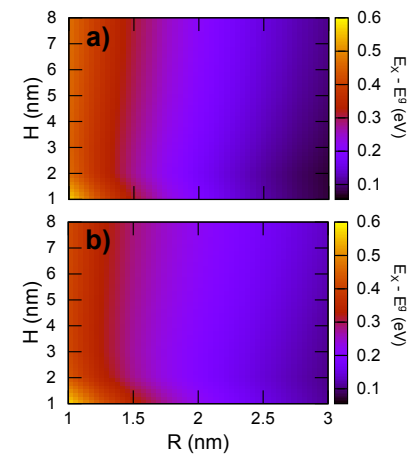

Figure S7: Exciton energy in spherical DiDs with $V^{s t r}=V^{p z}=0$ (a), and $V^{s t r} \neq 0, V^{p z} \neq 0$ (b). $E^{g}$ is the bulk band gap.

\section{Towards type-II dot-in-rods and dot-in-plates}

Typical dot-in-rods (DiRs) have weak strain and PZ effects because of the thin shell in the direction orthogonal to the $c$-axis, $H_{\perp} \lesssim 1 \mathrm{~nm}$. Figure S8(a) shows that electron migration into the shell becomes feasible if one combines large cores with considerably thicker shells $\left(H_{\perp}=5 \mathrm{~nm}\right.$ in the figure). Similarly, typical dot-in-plates (DiPs) have thin shells along the $c$ axis. Figure S8(b) shows that growing thicker shells again triggers electron migration.

\section{Material Parameters}

Below we summarize the material parameters used in the calculations. $m_{0}$ is the free electron mass and $\varepsilon_{0}$ the vacuum permitivitty. A relative dielectric constant of 3 and confining potential of $5 \mathrm{eV}$ is taken outside the NC to account for the dielectric environment. See Ref. ${ }^{2}$ for the Burt-Foreman 


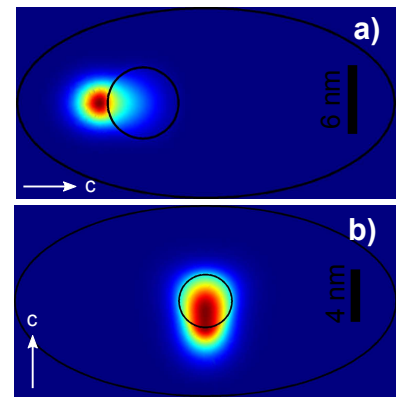

Figure S8: (a) Excitonic electron charge density in a DiR with large core and thick lateral shell (orthogonal to $c$ axis). As compared with the DiRs in Fig.5a,b of the main text, the electron is more delocalized. (b) Same but in a DiP with thick shell along $c$ axis. As compared with the DiP in Fig. $5 \mathrm{c}$ of the main text, the electron is more delocalized.

kinetic energy term of Hamiltonian (to avoid spurious solutions, the hole mass parameters we use follow the complete asymmetric operator ordering, i.e. $A_{i}^{(+)}=A_{i}$ and $A_{i}^{(-)}=0$ ), and see Ref. ${ }^{3}$ for the strain terms.

\begin{tabular}{|c|c|c|c|c|l|}
\hline Description & Symbol & CdSe value & CdS value & Units & Ref. \\
\hline Elastic modulus tensor & $C_{11}$ & $74.1 \cdot 10^{9}$ & $86.5 \cdot 10^{9}$ & $\mathrm{~Pa}$ & 9 \\
Elastic modulus tensor & $C_{12}$ & $45.2 \cdot 10^{9}$ & $54.0 \cdot 10^{9}$ & $\mathrm{~Pa}$ & 9 \\
Elastic modulus tensor & $C_{13}$ & $38.9 \cdot 10^{9}$ & $47.3 \cdot 10^{9}$ & $\mathrm{~Pa}$ & 9 \\
Elastic modulus tensor & $C_{33}$ & $84.3 \cdot 10^{9}$ & $94.4 \cdot 10^{9}$ & $\mathrm{~Pa}$ & 9 \\
Elastic modulus tensor & $C_{44}$ & $13.4 \cdot 10^{9}$ & $15.0 \cdot 10^{9}$ & $\mathrm{~Pa}$ & 9 \\
Piezoelectric constant & $e_{31}$ & -0.16 & -0.24 & $\mathrm{C} \cdot \mathrm{m}^{2}$ & 10 \\
Piezoelectric constant & $e_{33}$ & 0.347 & 0.44 & $\mathrm{C} \cdot \mathrm{m}^{2}$ & 10 \\
Piezoelectric constant & $e_{15}$ & -0.138 & -0.21 & $\mathrm{C} \cdot \mathrm{m}^{2}$ & 10 \\
Dielectric constant & $\varepsilon_{\perp}$ & 9.29 & 8.28 & $\varepsilon_{0}$ & 10 \\
Dielectric constant & $\varepsilon_{z}$ & 10.16 & 8.73 & $\varepsilon_{0}$ & 10 \\
Lattice constant $\| c$ axis & $c$ & 7.01 & 6.749 & $\AA$ & 10 \\
Lattice constant $\perp c$ axis & $a$ & 4.30 & 4.135 & $\AA$ & 10 \\
Conduction band offset & $c b o$ & 0.0 & 0.32 & $\mathrm{eV}$ & 11
\end{tabular}


Supplemental Material for "Piezoelectric control of the exciton wave function in colloidal CdSe/CdS nanocrystals"

\begin{tabular}{|c|c|c|c|c|l|} 
Valence band offset & $v b o$ & 0.0 & -0.40 & $\mathrm{eV}$ & 11 \\
Crystal field splitting & $\Delta_{1}$ & 0.039 & 0.027 & $\mathrm{eV}$ & 12 \\
Spin-orbit matrix element & $\Delta_{2}$ & 0.416 & 0.065 & $\mathrm{eV}$ & 12 \\
Spin-orbit matrix element & $\Delta_{3}$ & 0.416 & 0.065 & $\mathrm{eV}$ & 12 \\
Electron mass & $m^{*}$ & 0.11 & 0.21 & $m_{0}$ & 10 \\
Hole mass parameter & $A_{1}$ & -5.06 & -4.53 & $1 / m_{0}$ & 12 \\
Hole mass parameter & $A_{2}$ & -0.43 & -0.39 & $1 / m_{0}$ & 12 \\
Hole mass parameter & $A_{3}$ & 4.5 & 4.02 & $1 / m_{0}$ & 12 \\
Hole mass parameter & $A_{4}$ & -1.29 & -1.92 & $1 / m_{0}$ & 12 \\
Hole mass parameter & $A_{5}$ & -1.29 & -1.92 & $1 / m_{0}$ & 12 \\
Hole mass parameter & $A_{6}$ & -0.47 & -2.59 & $1 / m_{0}$ & 12 \\
CB Deformation pot. $\| c$ axis & $a_{c}^{z}$ & -1.76 & -4.5 & $\mathrm{eV}$ & 3 \\
CB Deformation pot. $\perp c$ axis & $a_{c}^{\perp}$ & -7.8 & -8.2 & $\mathrm{eV}$ & 3 \\
VB Deformation pot. & $D_{1}$ & -0.76 & -2.8 & $\mathrm{eV}$ & 13 \\
VB Deformation pot. & $D_{2}$ & -3.7 & -4.5 & $\mathrm{eV}$ & 13 \\
VB Deformation pot. & $D_{3}$ & -4.0 & -1.3 & $\mathrm{eV}$ & 13 \\
VB Deformation pot. & $D_{4}$ & 2.2 & 2.9 & $\mathrm{eV}$ & 13 \\
VB Deformation pot. & $D_{5}$ & -1.2 & 1.5 & $\mathrm{eV}$ & 13 \\
VB Deformation pot. & $D_{6}$ & -1.5 & -1.2 & $\mathrm{eV}$ & 13 \\
\hline & & & & \\
\hline
\end{tabular}

Table S1: Wurtzite CdSe and CdS parameters used in the calculations.

For calculations of zinc-blende phase, we take the material parameters given in Ref., ${ }^{9}$ except for the conduction deformation potential ${ }^{13}$ and the band-offsets. ${ }^{11}$ 


\section{Structural and optical data of experimental DID samples}

Below we provide a few representative transmission electron microscopy (TEM) images of DID samples under investigation, as well as the corresponding histograms of total (core plus shell) size distribution.
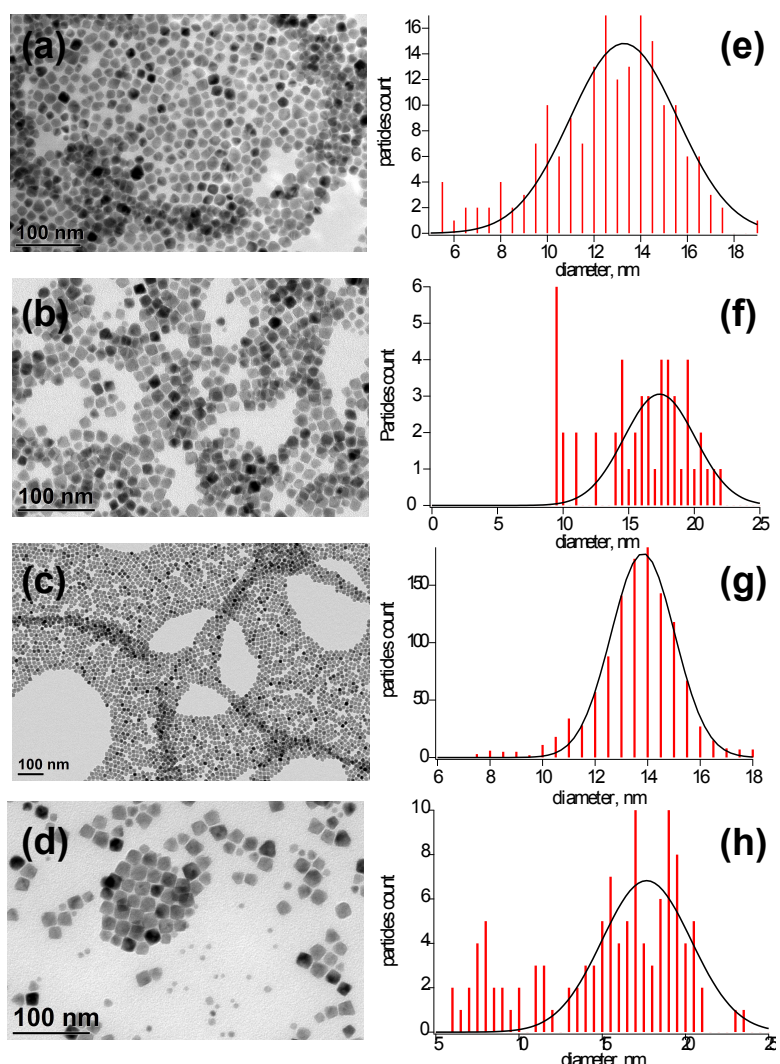

Figure S9: (a-d) TEM images of DiD samples 5, 1, 7 and 3 of table S1 (from top to bottom). (e-h) Corresponding histograms showing the final size distribution.

The complete structural data resulting from the TEM analysis is summarized in Table S2, along with the corresponding emission wavelength and measured quantum efficiency (see Methods). Samples 1-4 (5-8) have 20 ML (15 ML) nominal shell thickness.

Figure S10 shows PL spectra and corresponding decay traces for the 15ML shell thickness 
Supplemental Material for "Piezoelectric control of the exciton wave function in colloidal CdSe/CdS nanocrystals"

Table S2: Structural and optical data for the different DiD samples.

\begin{tabular}{|c|c|c|c|c|c|}
\hline Sample & $\begin{array}{c}\text { Core radius } \\
(\mathbf{n m})\end{array}$ & $\begin{array}{c}\text { Final radius } \\
(\mathbf{n m})\end{array}$ & $\begin{array}{c}\text { Shell thickness } \\
(\mathbf{n m})\end{array}$ & $\begin{array}{c}\text { Emission wavelength } \\
\mathbf{n m}\end{array}$ & $\begin{array}{c}\text { PL QE. } \\
\mathbf{\%}\end{array}$ \\
\hline 1 & 1.85 & 8.7 & 6.9 & 651 & 31 \\
2 & 2.05 & 7.95 & 5.9 & 657 & 58 \\
3 & 2.55 & 8.85 & 6.3 & 663 & 60 \\
4 & 2.75 & 9.5 & 6.8 & 663 & 43 \\
5 & 1.85 & 6.65 & 4.8 & 648 & 56 \\
6 & 2.40 & 6.1 & 3.7 & 656 & 54 \\
7 & 2.55 & 6.9 & 4.4 & 661 & 66 \\
8 & 2.75 & 8.0 & 5.3 & 664 & 51 \\
\hline
\end{tabular}

DiDs (samples 5 to 8 ). The figure is analogous to that of the 20ML shell thickness samples shown in Fig. 4(b,c) of the paper.
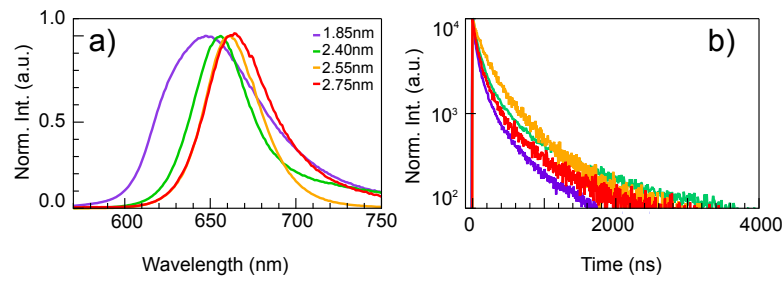

Figure S10: (a) PL spectra for DiD samples 5 to 8. (c) Corresponding PL decay traces. 


\section{Exciton lifetimes}

Table S3: An accurate fit to the PL decay traces requires a sum of four exponentials. The last decay component has a considerably longer lifetime, and can be attributed to delayed emission. ${ }^{14}$ The first three are used to calculate a weighted average lifetime. Weights $w_{i}$ are determined from the respective amplitudes and lifetimes: $w_{i}=A_{i} \tau_{i} / \sum_{k=1}^{3}\left(A_{k} \tau_{k}\right)$.

\begin{tabular}{|c|c|c|c|c|c|c|c|c|c|}
\hline Sample & $\begin{array}{c}\text { Core radius } \\
(\mathbf{n m})\end{array}$ & $\begin{array}{c}\tau_{\mathbf{1}} \\
(\mathbf{n s})\end{array}$ & $\mathbf{w}_{\mathbf{1}}$ & $\begin{array}{c}\tau_{\mathbf{2}} \\
(\mathbf{n s})\end{array}$ & $\mathbf{w}_{\mathbf{2}}$ & $\begin{array}{c}\tau_{\mathbf{3}} \\
(\mathbf{n s})\end{array}$ & $\mathbf{w}_{\mathbf{3}}$ & $\begin{array}{c}\tau_{\text {avg }} \\
(\mathbf{n s})\end{array}$ & $\begin{array}{c}\tau_{\text {delayed }} \\
(\mathbf{n s})\end{array}$ \\
\hline 1 & 1.85 & 24 & 0.04 & 113 & 0.35 & 539 & 0.61 & 378 & 3988 \\
2 & 2.05 & 49 & 0.04 & 189 & 0.29 & 773 & 0.67 & 572 & 4325 \\
3 & 2.55 & 62 & 0.06 & 246 & 0.27 & 936 & 0.67 & 696 & 4719 \\
4 & 2.75 & 88 & 0.05 & 288 & 0.29 & 961 & 0.66 & 722 & 4869 \\
5 & 1.85 & 31 & 0.12 & 106 & 0.41 & 388 & 0.47 & 229 & 1673 \\
6 & 2.40 & 33 & 0.07 & 125 & 0.40 & 545 & 0.53 & 339 & 3650 \\
7 & 2.55 & 33 & 0.01 & 160 & 0.39 & 473 & 0.60 & 345 & 1821 \\
8 & 2.75 & 41 & 0.11 & 139 & 0.42 & 505 & 0.46 & 298 & 2479 \\
\hline
\end{tabular}

\section{References}

1. Rajadell F.; Royo M.; Planelles J. Strain in Free Standing CdSe/CdS Core-Shell Nanorods. J. Appl. Phys. 2012, 111, 014303.

2. Climente J.I.; Segarra C.; Rajadell F.; Planelles J. Electrons, Holes, and Excitons in GaAs Polytype Quantum Dots. J. Appl. Phys. 2016, 119, 125705.

3. Park S.H.; Cho Y.H. Characteristics of Built-in Polarization Potentials in Vertically and Laterally Arranged InGaN/GaN Quantum Dots. J. Appl. Phys. 2011, 109, 113103.

4. Carbone L.; Nobile C.; De Giorgi M.; Della Sala F.; Morello G.; Pompa P.; Hytch M.; Snoeck E.; Fiore A.; Franchini I.R.; et al. Synthesis and Micrometer-Scale Assembly of Colloidal CdSe/CdS Nanorods Prepared by a Seeded Growth Approach. Nano Lett 2007, 7, 2942-2950.

5. Jaseniak J.; Smith L.; van Embden J.; Mulvaney P.; Califano M. Re-examination of the Size-Dependent Absorption Properties of CdSe Quantum Dots. J. Phys. Chem. C 2009, 113, 19468. 
Supplemental Material for "Piezoelectric control of the exciton wave function in colloidal CdSe/CdS nanocrystals"

6. Christodoulou S.; Vaccaro G.; Pinchetti V.; De Donato F.; Grim J.Q.; Casu A.; Genovese A.; Vicidomini G.; Diaspro A.; Brovelli S.; et al. Synthesis of Highly Luminescent Wurtzite CdSe/CdS Giant-shell Nanocrystals Using a Fast Continuous Injection Route. J. Mater. Chem. C 2014, 2, 3439-3447.

7. Leyre S.; Coutino-Gonzalez E.; Joos J.J.; Ryckaert J.; Meuret Y.; Poelman D.; Smet P.F.; Durinck G.; Hofkens J.; Deconinck G.; Hanselaer P. Absolute Determination of Photoluminescence Quantum Efficiency Using an Integrating Sphere Setup. Rev. Sci. Instrum. 2014, 85, 123115.

8. Eshet H.; Grünwald M.; Rabani E. The Electronic Structure of CdSe/CdS Core/Shell Seeded Nanorods: Type-I or Quasi-Type-II? Nano Lett. 2013, 13, 5880-5885 (and references therein).

9. Sadao, A. Handbook of Physical Properties of Semiconductors; Kluwert Academic Group, 2004.

10. Madelung, O. Semiconductors: Data Handbook; Springer, Berlin, 2004.

11. Wei S.H.; Zhang S. B.; Zunger A. First-principles Calculation of Band Offsets, Optical Bowings, and Defects in CdS, CdSe, CdTe, and Their Alloys. J. Appl. Phys. 2000, 87, 1304âĂŞ1311.

12. Jeon J.B.; Sirenko Y.M.; Kim K. W.; Littlejohn M.A.; Stroscio M.A. Valence Band Parameters of Wurtzite Materials. Solid State Communications 1996, 99, 423âĂŞ426.

13. Madelung O.; Rössler U.; Schulz M. II-VI and I-VII Compounds; Semimagnetic Compounds. Volume 41B; Springer Materials, 1999.

14. Rabouw F.T.; Kamp M.; van Dijk-Moes R.J.A.; Gamelin D.R.; Femius Koenderink A.; Meijerink, A.; Vanmaekelbergh D. Delayed Exciton Emission and Its Relation to Blinking in CdSe Quantum Dots. Nano Lett. 2015, 15, 7718-7725. 

PHYSICAL REVIEW B 93, 085312 (2016)

\title{
Edge states in dichalcogenide nanoribbons and triangular quantum dots
}

\author{
C. Segarra, ${ }^{1,2}$ J. Planelles, ${ }^{1}$ and S. E. Ulloa ${ }^{2, *}$ \\ ${ }^{1}$ Departament de Química Física i Analítica, Universitat Jaume I, Avenida de Vicent Sos Baynat, s/n, 12071 Castelló de la Plana, Spain \\ ${ }^{2}$ Department of Physics and Astronomy and Nanoscale and Quantum Phenomena Institute, Ohio University, Athens, Ohio 45701-2979, USA \\ (Received 30 October 2015; revised manuscript received 25 January 2016; published 23 February 2016)
}

\begin{abstract}
The electronic structure of monolayer $\mathrm{MoS}_{2}$ nanoribbons and quantum dots has been investigated by means of an effective $\mathbf{k} \cdot \mathbf{p}$ two-band model. Both systems with borders exhibit states spatially localized on the edges and with energies lying in the band gap. We show that the conduction- and valence-band curvatures determine the presence/absence of these states the origin of which has been related to the marginal topological properties
\end{abstract} of the $\mathrm{MoS}_{2}$ single-valley Hamiltonian.

DOI: 10.1103/PhysRevB.93.085312

\section{INTRODUCTION}

Since the discovery of graphene [1], atomically thin layered structures have attracted growing interest and several new two-dimensional (2D) materials have been prepared [2], including hexagonal $\mathrm{BN}$ and several transition metal dichalcogenides (TMDCs) [3,4]. There is a great variety of TMDCs, as many metal and chalcogen atoms can be combined to produce materials with properties that include metallic, semiconducting, and even superconducting behavior; the natural diversity of these materials with different properties makes them particularly promising for electronic and optical applications [5,6]. Unlike graphene, TMDCs such as $\mathrm{MoS}_{2}$ and $\mathrm{WS}_{2}$ have a finite band gap in the visible frequency range, which is indirect when in bulk (many layer) form, but becomes direct in the single 2D (trilayer) limit-where two S layers are separated by a layer of Mo or W metal atoms [7,8]. The direct gap in many of these single trilayer TMDCs makes them especially attractive candidates for optoelectronic and electronic applications [9-12], such as field-effect transistors [13-15], or photoaddressable sensors [5].

Although we know a great deal about the electronic states in single trilayers, it is important to gain a detailed understanding of the electronic structure of finite size systems such as nanoribbons and quantum dots, in order to fully and reliably tailor the properties of different TMDC materials and possible devices. Several works [16-21] have reported the existence of edge states in the gap of finite $\mathrm{MoS}_{2}$ systems under different conditions. More recently, consideration of polar discontinuity effects in these and stronger polar materials has predicted the appearance of charged metallic edge states in free-standing ribbons $[22,23]$. The presence of metallic (dispersive) edge states in TMDCs nanostructures is especially relevant as new device geometries and interfaces become available; they would be expected to strongly affect transport and optical properties of nanoribbons and 2D interfaces [24,25].

Edge or surface states also emerge in topological insulators, as has been intensely discussed in recent literature [26,27]. In those systems, it has been well established that the presence of edge states is a direct consequence of the principle of bulk-edge correspondence [26,28]: gapless states must be present at the domain wall separating two regions with different topological invariants. Although pristine graphene is

*ulloa@ohio.edu not a topological insulator due to its weak intrinsic spin-orbit interaction, the origin and character of edge states in gapped and bilayer graphene have been analyzed in terms of the topological properties of the Hamiltonians for individual valleys $[29,30]$. This analysis is made possible by the close analogy between graphene systems and 2D topological insulators. The details of this analogy and its limitations have been discussed in the literature, but allow one to understand the appearance and characteristics of symmetry allowed states at the edges of finite-size systems [31]. In light of the similar hexagonal structure of graphene and TMDCs, one may wonder if edge states in TMDCs single trilayers could be also analyzed in terms of the topological character imparted by the structure.

In this work, we use a two-band effective $\mathbf{k} \cdot \mathbf{p}$ model to investigate the electronic properties of $\mathrm{MoS}_{2}$ nanoribbons and small triangular crystallites ("quantum dots") as those appearing naturally in growth chambers. We find the generic appearance of midgap states with wave functions strongly localized near the edges of the structure, which can be clearly identified as edge states. Calculations for various sets of model parameters show that the appearance and characteristics of edge states are controlled by the curvature of the 2D "bulk" band structure. In particular, the sign of the band curvature parameters near the edge of the valence and conduction bands is found to be responsible for whether the edge states exist or not, and the relative magnitude of the effective masses determines the location of the states in the gap. As in graphene systems [31], all of these results can be understood as arising from the marginal topological properties of the $\mathrm{MoS}_{2}$ single-valley Hamiltonians. In particular, we demonstrate that the Chern number per inequivalent valley is nonvanishing in this structure, which suggests the system may sustain edge states (and yet the system is topological trivial, with overall vanishing Chern number).

We should comment that microscopic details such as bond saturation and/or reconstruction of edges in finite-size systems do affect the appearance and details of edge states. However, the lattice-symmetry "protection" that gives rise to the existence of edge states, as we discuss here, will strengthen the occurrence of such states under the effect of diverse microscopic details.

The remainder of the paper is organized as follows. Section II presents the Hamiltonian used to describe the $\mathrm{MoS}_{2}$ trilayers. Then, in Sec. III, we show and discuss typical numerical results for the two different systems under study: $\mathrm{MoS}_{2}$ nanoribbons (Sec. III A) and $\mathrm{MoS}_{2}$ quantum 
dots (Sec. III B), as defined by triangular crystallites. Finally, conclusions are given in Sec. IV.

\section{THEORETICAL MODEL}

As mentioned above, single trilayers of TMDC materials such as $\mathrm{MoS}_{2}$ are composed by a layer of Mo atoms sandwiched between two layers of S atoms. The metal atoms in this structure present trigonal prismatic coordination with the $\mathrm{S}$ atoms. The electronic structure of the single trilayer has a direct gap at two nonequivalent points $K$ and $K^{\prime}$ of the Brillouin zone. Several works have derived an effective $\mathbf{k} \cdot \mathbf{p}$ model in the vicinity of these points in order to study the low-energy physics of TMDC monolayers [32-35]. The proposed two-band Hamiltonian describing the valence and conduction bands up to second order in $k$ can be written as

$$
H=\left(\begin{array}{cc}
\varepsilon_{v}+\alpha k^{2} & \tau \gamma k_{-} \\
\tau \gamma k_{+} & \varepsilon_{c}+\beta k^{2}
\end{array}\right),
$$

where $k_{ \pm}=k_{x} \pm i \tau k_{y}$, and $\varepsilon_{c}=\Delta / 2$ and $\varepsilon_{v}=-\Delta / 2$ are the band-edge energies with $\Delta=1.9 \mathrm{eV}$ standing for the material band gap; $\mathbf{k}$ is the momentum relative to the $K / K^{\prime}$ points. The constants $\alpha, \beta$, and $\gamma$ are material parameters, while $\tau$ identifies the valley $K(\tau=1)$ or $K^{\prime}(\tau=-1)$

For the sake of simplicity, trigonal warping and other minor modifications present in the original model are neglected, although their inclusion would not qualitatively alter the main conclusions of the work presented here. Hamiltonian (1) takes into account the electron-hole symmetry breaking obtained from first-principles calculations by using unlike parameters $\alpha$ and $\beta$. Although different authors report different values of these parameters, some dependent on the details of the calculations, we employ here $\alpha=1.72 \mathrm{eV}^{2}, \beta=-0.13 \mathrm{eV} \AA^{2}$, and $\gamma=3.82 \mathrm{eVA}$, as fitted from density functional theory calculations [33], unless noted otherwise.

Notice that Eq. (1) ignores the spin degree of freedom for clarity of presentation. Consideration of spin-orbit coupling in these materials results in effectively producing two valence-band edges, as a spin-dependent gap appears, with corresponding spin-valley coupling in the valence band. The conduction band in $\mathrm{MoS}_{2}$ has a sizable but relatively weaker spin-orbit splitting $[36,37]$. Spin-orbit interactions will then result in a doubling of the states we discuss here. We revisit this issue in the discussion section below. We also notice that the edges of the nanostructures are defined by hard-wall boundary conditions in all simulations, and are assumed to result in no intervalley coupling — as expected of zigzag edges, although the full equivalence of these conditions would require further investigations, especially as detailed comparisons with experiments develop [21].

\section{NUMERICAL RESULTS AND DISCUSSION}

We study the electronic properties of two different types of 2D nanostructures: nanoribbons, where particles are confined in one direction, and quantum dots, where they are confined to triangular nanocrystallites. The calculations are carried out using COMSOL utilities over a fine grid (the finest default), and converged until the desired accuracy (typically $10^{-12}$ in the eigenvalues).

\section{A. Nanoribbons}

The nanoribbons are defined over a finite width along the direction $x$ in our calculations, while maintaining translational invariance along the $y$ direction. As such, the momentum $k_{y}$ is a good quantum number and the two-component spinor wave function of Hamiltonian (1) can be written in the form $\psi(x, y)=e^{i k_{y} y} \phi(x)$, where $\psi$ and $\phi$ have components over the $c, v$ basis. As a consequence, the eigenvalue equation of this 2D Hamiltonian turns into a set of two coupled second-order differential equations in one dimension that depend on the quantum number $k_{y}$. We solve numerically these equations for an $\mathrm{MoS}_{2}$ nanoribbon of 10-nm width, wide enough to allow decoupled states on both edges, as we will see. The results obtained are summarized in Fig. 1.

Figure 1(b) shows the calculated sub-band dispersion. Notice that the finite width of the ribbon has only slightly opened the gap, as the effective masses near the band edges, $m_{v}$ and $m_{c}$, are both $\approx 0.5$, and the size quantization is only a few meV. Most importantly, we find two states inside the band gap, with a nearly linear dispersion. The levels cross at $k_{y}=0$ and $E=0.816 \mathrm{eV}$, relatively close to the edge of the conduction band. These midgap states disperse upwards in energy, close to the conduction band for not large $k_{y}$ values $\left[k_{y} \approx \pm 0.05\left(2 \pi / a_{0}\right)\right.$, see Fig. 1(b)], and soon admix with the band states, becoming indistinguishable from them. For lower energies, however, the midgap states remain well defined and exhibit increasing edge localization, as we will see below.

In order to study the origin of these states, and dependence on band-structure features, we carry out the same calculations but for other sets of parameters than those in Ref. [33]. We only tune the $\alpha$ and $\beta$ values since $\gamma$ does not qualitatively affect the results. In Fig. 1(a) we exchange the signs of both $\alpha$ and $\beta$ ( $\alpha=$ $-1.72 \mathrm{eV}^{2}$ and $\beta=0.13 \mathrm{eV} \AA^{2}$ ) and observe that the states lying inside the gap disappear. In Fig. 1(c) we keep the signs unaltered to those in panel (b) but modify $\beta$ to have the same absolute value of $\alpha\left(\alpha=1.72 \mathrm{eV}^{2}\right.$ and $\left.\beta=-1.72 \mathrm{eV}^{2}\right)$.

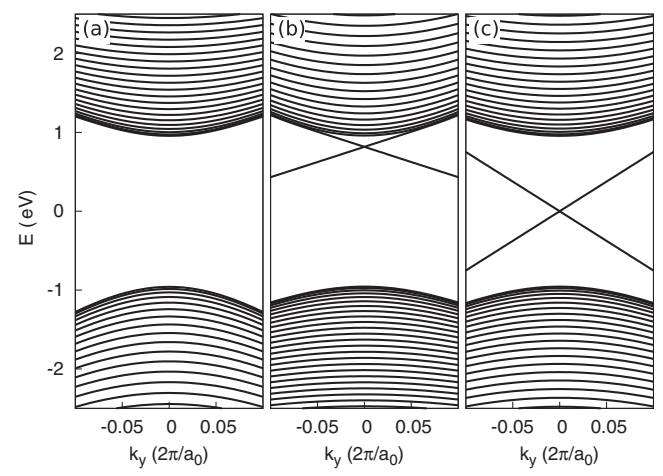

FIG. 1. Energy-band dispersion for $\mathrm{MoS}_{2}$ nanoribbons considering different values of $\alpha$ and $\beta$ : (a) $\alpha=-1.72 \mathrm{eV}^{2}$ and $\beta=$ $0.13 \mathrm{eV}^{2}$, (b) $\alpha=1.72 \mathrm{eV}^{2}$ and $\beta=-0.13 \mathrm{eV}^{2}$, and (c) $\alpha=$ $1.72 \mathrm{eV}^{2}$ and $\beta=-1.72 \mathrm{eV}^{2}$. The edges are parallel to the $y$ direction, and the wave vector $k_{y}$ is measured with respect to the $\mathrm{K}$ valley, where $a_{0}=3.193 \AA$ is the lattice constant. 

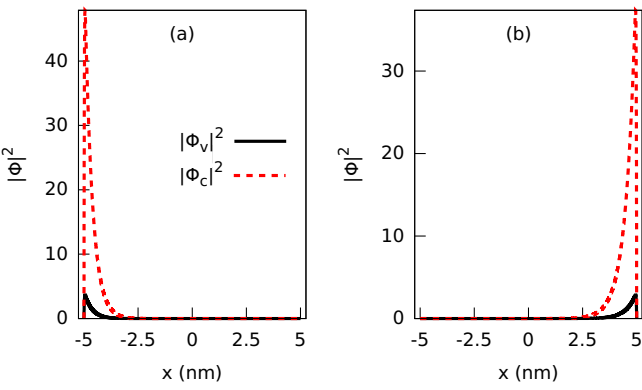

FIG. 2. Wave-function squared modulus $|\phi|^{2}$ of the two states at $k_{y}=0.01 \times 2 \pi / a_{0}$, with energies lying in the band gap (a) $E=0.778 \mathrm{eV}$ and (b) $E=0.855 \mathrm{eV}$. Black solid lines correspond to the valence-band component and red dashed lines correspond to the conduction band.

In this case, the two states inside the gap are still present but they have lower energies compared to Fig. 1(b). As expected from symmetry, the dispersion bands now cross at $k_{y}=0$ and $E=0$, since $\alpha=\beta$ confers electron-hole symmetry to the Hamiltonian.

By comparing the results in Fig. 1 for the three sets of parameters, it is clear that the presence or absence of states inside the gap is determined by the sign of both $\alpha$ and $\beta$ curvatures. Midgap states exist if $\alpha>0$ and $\beta<0$ and are absent if $\alpha<0$ and $\beta>0$ [38]. Furthermore, changes in the relative value of these two parameters affect the energy of the states inside the gap. When $|\alpha|>|\beta|$ the states are closer to the conduction band as in Fig. 1(b), and when $|\alpha|<|\beta|$ they become closer to the valence band.

One can qualitatively analyze this behavior in terms of the "bare" effective masses for valence and conduction bands, as determined by the $\alpha$ and $\beta$ coefficients. A negative $\beta$ (and corresponding negative mass $\simeq 1 / \beta$ ) in the conduction band is "inverted," and that symmetry is contained in the states even after the mixing due to $\gamma$. The inverse effective masses for the full Hamiltonian (1) near the edges are, however, given by $2\left(\beta+\gamma^{2} / \Delta\right) / \hbar^{2}$ for the conduction band, and by $2(\alpha-$ $\left.\gamma^{2} / \Delta\right) / \hbar^{2}$ for the valence band, and therefore dominated by the large value of $\gamma$

To further explore the nature of the states inside the gap, we analyze the wave functions in Fig. 2. As an example, we choose the states for $k_{y}=0.01 \times 2 \pi / a_{0}$ in Fig. 1(b), which are slightly away from the degeneracy point, and well away from the conduction band states. Figure 2(a) corresponds to the lower state at $E=0.778 \mathrm{eV}$ and Fig. 2(b) corresponds to the higher one at $E=0.855 \mathrm{eV}$. We clearly observe that both states are localized at opposite edges of the $\mathrm{MoS}_{2}$ nanoribbon - and have opposite dispersion, as expected of independent edge states. We see that the conduction-band component (red dashed line) is the dominant contribution to the wave function. Calculation of the relative weight of the two components yields $w\left(\phi_{c}\right)=93 \%$ and $w\left(\phi_{v}\right)=7 \%$ for the conduction- and valence-band components, respectively. These values can be directly obtained from the parameters $\alpha$ and $\beta$ using the expressions $w\left(\phi_{c}\right)=|\beta| /(|\alpha|+|\beta|)$ and $w\left(\phi_{v}\right)=|\alpha| /(|\alpha|+|\beta|)$. These expressions hold as long as the edge states are relatively far from the bulk bands. Notice also the asymmetry in the wave functions as seen, for instance, in the different maximum value of $\left|\phi_{c}\right|^{2}$, and their different $x$ extension. This asymmetry is due to the proximity of the conduction band. The higher-energy edge state is slightly more admixed with the bulk states and, thus, its wave-function results somewhat more delocalized. The asymmetry in the states continues to grow as $k_{y}$ increases further.

The results summarized in Figs. 1 and 2 can be related to those coming from the model proposed by Bernevig, Hughes, and Zhang [39], in connection with the observation of the quantum spin Hall effect (QSHE). In that work, the QSHE was predicted in $\mathrm{HgTe}$ quantum wells larger than a critical thickness, due to a band inversion in the low-energy effective Hamiltonian. For $\Delta<0$, bands are inverted and the system shows topological behavior. One consequence is that edge states will form when a transition between two distinct topological phases takes place, as predicted by the principle of bulk-edge correspondence [26]. In our system, Eq. (1), we have $\Delta>0$, which is apparently trivial, although the sign of the bare band curvatures $(\alpha>0$ and $\beta<0)$ yields also a situation with inverted bands. As such, the origin of the edge states here can be analyzed in terms of the topological character of the model in Eq. (1).

To explore this relationship further, Fig. 3 shows the energy spectrum as a function of $\Delta$, for a given set of $\alpha$ and $\beta$ parameters. The spectra shown are for $k_{y}=0$ and band curvatures $\alpha=1.72 \mathrm{eV}^{2}$ and $\beta=-0.13 \mathrm{eVA}^{2}$ in Fig. 3(a), and $\alpha=1.72 \mathrm{eV}^{2}$ and $\beta=-1.72 \mathrm{eV}^{2}$ in Fig. 3(b). Two red dashed lines in each panel show the limits of the band gap, for reference. In both cases shown, we see that a trivial situation develops, with no states in the gap, for negative $\Delta$ values. As $\Delta$ increases and changes to positive values, the conduction and valence bands seem to be similar, except for the appearance of a pair (for $k_{y}=0$ ) of degenerate edge states with energies clearly in the gap. Notice that the edge states separate from the conduction band for larger $\Delta$ values in Fig. 3(a), but remain

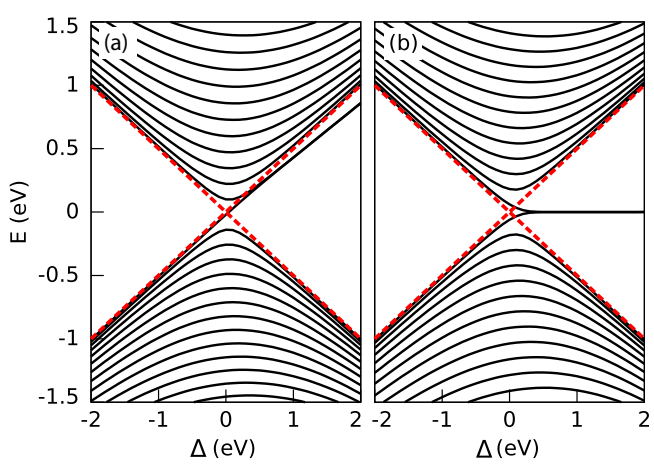

FIG. 3. Energy spectrum of a $\mathrm{MoS}_{2}$ nanoribbon as in Fig. 1, shown as a function of the band gap $\Delta$, for $k_{y}=0$. Two sets of parameters are considered: (a) $\alpha=1.72 \mathrm{eV}^{2}$ and $\beta=-0.13 \mathrm{eVA}^{2}$ and (b) $\alpha=1.72 \mathrm{eV}^{2}=-\beta$. Red dashed lines indicate the edges of the band gap. Midgap edge states appear for $\Delta>0$ in both cases. 
exactly equidistant from both bands for $|\alpha|=|\beta|$ in Fig. 3(b), as expected, appearing closer to the conduction band for more asymmetric $|\alpha|>|\beta|$ values.

Next, we look at these results with the help of the Chern number associated with the occupied band (topological invariant). For a two-level Hamiltonian written in the form $H(\mathbf{k})=\mathbf{g}(\mathbf{k}) \cdot \boldsymbol{\sigma}$, where $\boldsymbol{\sigma}$ is a vector with the Pauli matrices as components, the Chern number is given by [26]

$$
c=\frac{1}{4 \pi} \int d^{2} k\left(\partial_{k_{x}} \hat{\mathbf{g}} \times \partial_{k_{y}} \hat{\mathbf{g}}\right) \cdot \hat{\mathbf{g}},
$$

where $\hat{\mathbf{g}}=\mathbf{g} /|\mathbf{g}|$ and the integral is computed over the entire Brillouin zone. For the Hamiltonian in Eq. (1), one obtains $c=$ $\tau / 2[\operatorname{sgn}(\Delta)+\operatorname{sgn}(\alpha-\beta)]$, fully independent of the value of $\gamma$. That means that for $\Delta>0$ one obtains $c=0$ for $\alpha<\beta$, while $c=\tau$ for $\alpha>\beta$. A nonzero value of $c$ suggests that a topological invariant is present, and this goes along with the previous discussion based on band inversion arguments. It is important to note, however, that the contribution of the $K$ and $K^{\prime}$ valleys to the topological invariant has opposite signs, which produces an overall $c=0$. As a result, one can strictly state that multivalley materials such as graphene or $\mathrm{MoS}_{2}$ are topologically trivial.

In spite of the strict trivial topology of Eq. (1), a nonvanishing $c$ for a single valley can be associated with marginal topological properties, in analogy with topological insulators [31]. This analogy has, however, important limitations. Since $c$ per valley is not a well-defined topological invariant, $c \neq 0$ does not guarantee the existence of edge states at the boundaries with the vacuum. Furthermore, and perhaps most important, is the fact that if edge states are present they are not topologically protected against backscattering and can then be affected by any type of disorder and/or valley coupling. Nevertheless, it is the case that edge states in bilayer graphene have been shown to be robust under moderate disorder [29], and to exhibit pure valley currents, as indicated by the local valley Berry curvature and associated Chern number $[40,41]$.

We should also comment that although, for simplicity, we have suppressed the spin degree of freedom in these calculations its role can be easily established. The presence of spin clearly results in two edge states per border of the structure, instead of the single state presented above [see Fig. 1(b)]. As the spin-orbit interaction in the valence band is large (yet much smaller than the band gap, and diagonal, pinning the spin projection to each of the valleys), the two edge states on the same border but different spin projection appear slightly shifted in energy and with minimally different dispersion (not shown). This simple duplication of edge states with different spins and energies would of course be strongly affected if the edges couple valleys, something that will depend on the border terminations and corresponding boundary conditions $[18,21]$.

\section{B. $\mathrm{MoS}_{2}$ triangular quantum dots}

We next investigate the electronic properties of $\mathrm{MoS}_{2}$ quantum dots formed by finite-size flakes, using our Hamiltonian model and appropriate boundary conditions. Similar structures have also been studied by different approaches in the small-crystallite regime $[42,43]$. The flakes are equilateral (a)

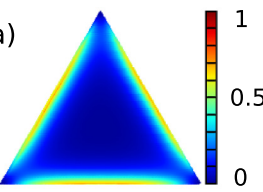

(c)

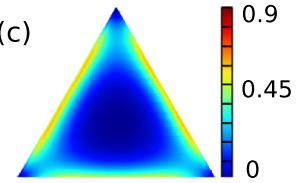

(e)

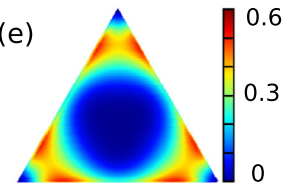

(g)

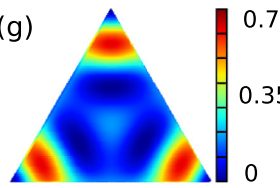

(i)

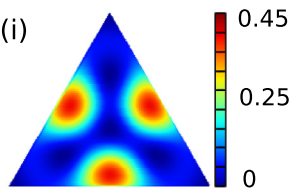

(k)

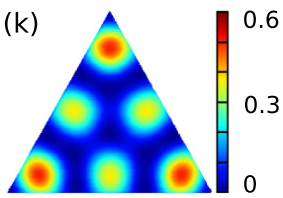

(b)

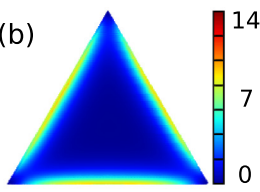

(d)

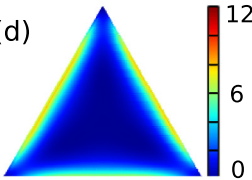

(f)

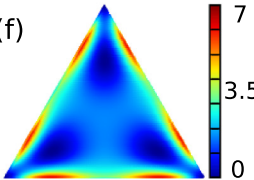

(h)

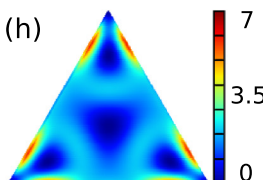

(j)

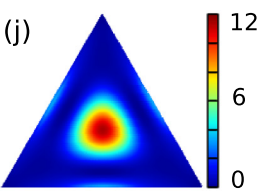

(I)

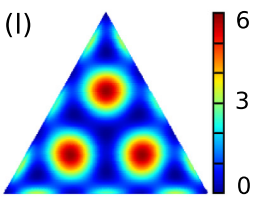

FIG. 4. Wave-function squared modulus of selected states with energies close to the conduction band. Left and right columns illustrate the valence-band $\left|\phi_{v}\right|^{2}$ and conduction-band $\left|\phi_{c}\right|^{2}$ components, respectively. Different states are arranged in rows with increasing energy: (a, b) $E=0.907 \mathrm{eV}$, (c, d) $E=0.962 \mathrm{eV}$, (e, f) $E=1.015 \mathrm{eV}$, (g, h) $E=1.022 \mathrm{eV}$, (i, j) $E=1.057 \mathrm{eV}$, and (k, l) $E=1.100 \mathrm{eV}$. As before, $\left|\phi_{c}\right|^{2}$ components are generally larger for these states, close to the conduction band.

triangles, as it is a commonly synthesized shape [44-47]. In this case, carriers are confined in the two directions of space and we must numerically solve the coupled differential equations in two variables in order to find eigenvalues and eigenfunctions of the Hamiltonian. In the results that follow, Fig. 4, the quantum dot side length is $10 \mathrm{~nm}$, and we employ the same parameters as in the previous section [see Fig. 1(b)]. 
The results obtained for this system show the presence of several states with energies in the gap. They can be seen as the result of the discretization of the edge states along each border, which are then hybridized near the corners of the flake. We illustrate this behavior in Fig. 4, where the squared modulus of the wave function for a selection of states with energy close to the conduction band $(E \approx 0.95 \mathrm{eV})$ is shown. We choose this range of energies because we know that for $\alpha=1.72 \mathrm{eVA}^{2}$ and $\beta=-0.13 \mathrm{eV \AA}^{2}$ [33] the edge states are closer to the conduction band. By gradually increasing energy, we also get to compare the clearly "bulk" and edge states in the flake. In Figs. 4(a)-4(d) we can see that the first two states are clearly edge states with wave functions localized near the triangle border, with similar appearance to that shown in Fig. 2 near each of the edges. The next two states in energy, Figs. 4(e)-4(h), also show wave functions mainly near the edges, but noticeably more delocalized than the previous two. This suggests that the edge states are partially admixed with the bulk conduction band, due to their energy proximity. Finally, Figs. 4(i)-4(1) show two conduction states with wave functions completely delocalized over the entire triangular quantum dot. A representation of the real and imaginary parts of the wave functions (not shown) allows one to see the wave-function nodes more easily, and see that their number increases with the energy of the states, an expected signature of quantization.

We should emphasize that we have also found the same pattern of edge states appearing for the curvature parameters as in the case of nanoribbons. As such, one can also invoke the marginal topological character of the Hamiltonian as the origin of edge states in these zero-dimensional nanostructures.

\section{CONCLUSIONS}

The low-energy electronic structure of monolayer $\mathrm{MoS}_{2}$ nanoribbons and quantum dots has been investigated using an effective two-band $\mathbf{k} \cdot \mathbf{p}$ model. We have shown that both systems present edge states with energies in the gap. Nanoribbons exhibit only one state per edge at a given value of the quantum number $k_{y}$, while in quantum dots, due to full confinement, the edge states appear distinctly away from the states that would form the sub-band continuum in a large triangular flake. As the energy of the edge states increases, for both nanoribbons and quantum dots, the edge states hybridize with the "bulk" and cease to be so well localized near the edges of the structure.
We have also found that the curvature of the bands, represented by parameters $\alpha$ and $\beta$, determines the presence $(\alpha>0$ and $\beta<0)$ or absence $(\alpha<0$ and $\beta>0)$ of edge states as well as their energy. This behavior is reminiscent of the marginal topological properties of materials such as $\mathrm{MoS}_{2}$, as the Chern number per valley is indeed nonzero, reflecting a finite Berry curvature in each valley. Similar results are of course expected for other TMDC nanostructures, as long as the relative band curvature parameter signs are similar to those presented here.

We should emphasize that first-principles calculations are typically used to determine continuum model parameters. As the former may depend on functionals and other details of the calculations, the latter may indeed vary among different implementations and/or authors. In fact, some fittings result in values of $\alpha$ and $\beta$ that are indeed substantially different, and for which the edge states we discuss here are not apparent [33]. It is also clear that tight-binding parametrizations may similarly allow for the presence of edge states, as explicitly seen in the literature [19]. These edge states, however, are found to be rather robust and to exist over a wide range of parameters. Similar conclusions have been reached in a recent preprint [48].

We draw the attention of the reader to the issue of atomic reconstructions and bondings in real crystallites. Such microscopic effects would surely modify the detailed edge state dispersions and characteristics of the states we have discussed here. Polarization and charge compensation may even result in interesting charged edge states, unlike those we have discussed $[22,23]$. It would be interesting to explore both theoretically and experimentally how truly robust these edge states are in nanoribbons and other natural structures with edges, and understand how the different effects compete. Exploring what observable consequences they have on the effective trapping of photoactivated carriers and excitons, or how they modulate the interaction between adsorbed/embedded impurity atoms, may provide further insights into the appearance of edge states in these systems.

\section{ACKNOWLEDGMENTS}

This work was supported by a Spanish FPU Gran (C.S.). Support from NSF Grant No. DMR-1508325 (S.E.U.) UJI Project No. P1.1B2014-24, and MINECO Project No. CTQ2014-60178-P (J.P.) is acknowledged.
[1] K. S. Novoselov, A. K. Geim, S. V. Morozov, D. Jiang, Y. Zhang, S. V. Dubonos, I. V. Grigorieva, and A. A. Firsov, Science 306, 666 (2004).

[2] K. S. Novoselov, D. Jiang, F. Schedin, T. J. Booth, V. V. Khotkevich, S. V. Morozov, and A. K. Geim, Proc. Natl. Acad. Sci. USA 102, 10451 (2005).

[3] P. Miró, M. Audiffred, and T. Heine, Chem. Soc. Rev. 43, 6537 (2014).

[4] S. Z. Butler, S. M. Hollen, L. Cao, Y. Cui, J. A. Gupta, H. R. Gutiérrez, T. F. Heinz, S. S. Hong, J. Huang, A. F. Ismach, E. Johnston-Halperin, M. Kuno, V. V. Plashnitsa, R. D. Robinson,
R. S. Ruoff, S. Salahuddin, J. Shan, L. Shi, M. G. Spencer, M. Terrones, W. Windl, and J. E. Goldberger, ACS Nano 7, 2898 (2013).

[5] Q. H. Wang, K. Kalantar-Zadeh, A. Kis, J. N. Coleman, and M. S. Strano, Nat. Nanotechnol. 7, 699 (2012).

[6] Z. M. Wang, $\mathrm{MoS}_{2}$ : Materials, Physics, and Devices (Springer, Switzerland, 2014)

[7] K. F. Mak, C. Lee, J. Hone, J. Shan, and T. F. Heinz, Phys. Rev. Lett. 105, 136805 (2010).

[8] A. Splendiani, L. Sun, Y. Zhang, T. Li, J. Kim, C. Y. Chim, G. Galli, and F. Wang, Nano Lett. 10, 1271 (2010). 
[9] L. Britnell, R. M. Ribeiro, A. Eckmann, R. Jalil, B. D. Belle, A. Mishchenko, Y.-J. Kim, R. V. Gorbachev, T. Georgiou, S. V. Morozov, A. N. Grigorenko, A. K. Geim, C. Casiraghi, A. H. Castro Neto, and K. S. Novoselov, Science 340, 1311 (2013).

[10] A. Pospischil, M. M. Furchi, and T. Mueller, Nat. Nanotechnol. 9, 257 (2014).

[11] J. S. Ross, P. Klement, A. M. Jones, N. J. Ghimire, J. Yan, D. G. Mandrus, T. Taniguchi, K. Watanabe, K. Kitamura, W. Yao, D. H. Cobden, and X. Xu, Nat. Nanotechnol. 9, 268 (2014).

[12] B. W. H. Baugher, H. O. H. Churchill, Y. Yang, and P. JarilloHerrero, Nat. Nanotechnol. 9, 262 (2014).

[13] B. Radisavljevic, A. Radenovic, J. Brivio, V. Giacometti, and A. Kis, Nat. Nanotechnol. 6, 147 (2011).

[14] D. Lembke and A. Kis, ACS Nano 6, 10070 (2012).

[15] H. Wang, L. Yu, Y.-H. Lee, Y. Shi, A. Hsu, M. L. Chin, L.-J. Li, M. Dubey, J. Kong, and T. Palacios, Nano Lett. 12, 4674 (2012).

[16] M. V. Bollinger, J. V. Lauritsen, K. W. Jacobsen, J. K. Nørskov, S. Helveg, and F. Besenbacher, Phys. Rev. Lett. 87, 196803 (2001).

[17] H. Pan and Y.-W. Zhang, J. Mater. Chem. 22, 7280 (2012).

[18] E. Erdogan, I. H. Popov, A. N. Enyashin, and G. Seifert, Eur. Phys. J. B 85, 33 (2012).

[19] S. Pavlović and F. M. Peeters, Phys. Rev. B 91, 155410 (2015).

[20] D. Davelou, G. Kopidakis, G. Kioseoglou, and I. N. Remediakis, Solid State Commun. 192, 42 (2014).

[21] C. G. Péterfalvi, A. Kormányos, and G. Burkard, Phys. Rev. B 92, 245443 (2015)

[22] F. Güller, A. M. Llois, J. Goniakowski, and C. Noguera, Phys. Rev. B 87, 205423 (2013).

[23] M. Gibertini and N. Marzari, Nano Lett. 15, 6229 (2015).

[24] Y. Gong, J. Lin, X. Wang, G. Shi, S. Lei, Z. Lin, X. Zou, G. Ye, R. Vajtai, B. I. Yakobson, H. Terrones, M. Terrones, B. K. Tay, J. Lou, S. T. Pantelides, Z. Liu, W. Zhou, and P. M. Ajayan, Nat. Mater. 13, 1135 (2014)

[25] K. Chen, X. Wan, J. Wen, W. Xie, Z. Kang, X. Zeng, H. Chen, and J.-B. Xu, ACS Nano 9, 9868 (2015).

[26] M. Z. Hasan and C. L. Kane, Rev. Mod. Phys. 82, 3045 (2010).

[27] Special topic on topological insulators, APL Mat. 3, issue 8 (2015)

[28] J. C. Y. Teo and C. L. Kane, Phys. Rev. B 82, 115120 (2010).

[29] J. Li, I. Martin, M. Büttiker, and A. F. Morpurgo, Nat. Phys. 7, 38 (2011).

[30] W. Yao, S. A. Yang, and Q. Niu, Phys. Rev. Lett. 102, 096801 (2009).
[31] J. Li, A. F. Morpurgo, M. Büttiker, and I. Martin, Phys. Rev. B 82, 245404 (2010).

[32] G. B. Liu, W. Y. Shan, Y. Yao, W. Yao, and D. Xiao, Phys. Rev. B 88, 085433 (2013).

[33] A. Kormányos, V. Zólyomi, N. D. Drummond, P. Rakyta, G. Burkard, and V. I. Fal'ko, Phys. Rev. B 88, 045416 (2013).

[34] H. Rostami, A. G. Moghaddam, and R. Asgari, Phys. Rev. B 88, 085440 (2013)

[35] A. Kormányos, G. Burkard, M. Gmitra, J. Fabian, V. Zólyomi, N. D. Drummond, and V. Fal'ko, 2D Mater. 2, 022001 (2015).

[36] K. Kośmider, J. W. González, and J. Fernández-Rossier, Phys. Rev. B 88, 245436 (2013).

[37] A. Kormányos, V. Zólyomi, N. D. Drummond, and G. Burkard, Phys. Rev. X 4, 011034 (2014).

[38] We do not consider parameters $\alpha$ and $\beta$ with the same sign since in these cases there is not a real gap separating the bands.

[39] B. A. Bernevig, T. L. Hughes, and S.-C. Zhang, Science 314, 1757 (2006).

[40] R. V. Gorbachev, J. C. W. Song, G. L. Yu, A. V. Kretinin, F. Withers, Y. Cao, A. Mishchenko, I. V. Grigorieva, K. S. Novoselov, L. S. Levitov, and A. K. Geim, Science 346, 448 (2014).

[41] Y. Shimazaki, M. Yamamoto, I. V. Borzenets, K. Watanabe, T. Taniguchi, and S. Tarucha, Nat. Phys. 11, 1032 (2015).

[42] H. Schweiger, P. Raybaud, G. Kresse, and H. Toulhoat, J. Catal. 207, 76 (2002).

[43] T. Li and G. Galli, J. Phys. Chem. C 111, 16192 (2007).

[44] X. Duan, C. Wang, J. C. Shaw, R. Cheng, Y. Chen, H. Li, X. Wu, Y. Tang, Q. Zhang, A. Pan, J. Jiang, R. Yu, Y. Huang, and X. Duan, Nat. Nanotechnol. 9, 1024 (2014).

[45] M. S. J. Marshall and M. R. Castell, Chem. Soc. Rev. 43, 2226 (2014).

[46] Z. Liu, M. Amani, S. Najmaei, Q. Xu, X. Zou, W. Zhou, T. Yu, C. Qiu, A. G. Birdwell, F. J. Crowne, R. Vajtai, B. I. Yakobson, Z. Xia, M. Dubey, P. M. Ajayan, and J. Lou, Nat. Commun. 5, 5246 (2014).

[47] W. Bao, N. J. Borys, C. Ko, J. Suh, W. Fan, A. Thron, Y Zhang, A. Buyanin, J. Zhang, S. Cabrini, P. D. Ashby, A. Weber-Bargioni, S. Tongay, S. Aloni, D. F. Ogletree, J. Wu, M. B. Salmeron, and P. J. Schuck, Nat. Commun. 6, 7993 (2015).

[48] A preprint submitted after ours describes the appearance of edge states in monolayer $\mathrm{MoS}_{2}$ using tight-binding and low-energy Hamiltonians. They reach very similar conclusions to ours, and emphasize the topological protection of the edge modes. See H. Rostami, R. Asgari, and F. Guinea, arXiv:1511.07003. 


\section{Bibliography}

[1] M. V. Kovalenko, L. Manna, A. Cabot, Z. Hens, D. V. Talapin, C. R. Kagan, V. I. Klimov, A. L. Rogach, P. Reiss, D. J. Milliron, P. GuyotSionnnest, G. Konstantatos, W. J. Parak, T. Hyeon, B. A. Korgel, C. B. Murray, and W. Heiss, Prospects of nanoscience with nanocrystals, ACS Nano 9, 1012 (2015).

[2] M. A. Kastner, Artificial atoms, Phys. Today 46, 24 (1993).

[3] D. J. Norris, Measurement and assignment of the size-dependent optical spectrum in cadmium selenide (CdSe) quantum dots, Ph.D. thesis, Massachusetts Institute of Technology (1995).

[4] P. V. Kamat, Quantum dot solar cells. Semiconductor nanocrystals as light harvesters, J. Phys. Chem. C 112, 18737 (2008).

[5] W. R. Algar, A. J. Tavares, and U. J. Krull, Beyond labels: A review of the application of quantum dots as integrated components of assays, bioprobes, and biosensors utilizing optical transduction, Anal. Chim. Acta 673, 1 (2010).

[6] D. D. Awschalom, D. Loss, and N. Samarth, eds., Semiconductor spintronics and quantum computation (Springer, Berlin, 2002).

[7] V. Wood and V. Bulović, Colloidal quantum dot light-emitting devices, Nano Rev. 1, 5202 (2010),

[8] T.-H. Kim, K.-S. Cho, E. K. Lee, S. J. Lee, J. Chae, J. W. Kim, D. H. Kim, J.-Y. Kwon, G. Amaratunga, S. Y. Lee, B. L. Choi, Y. Kuk, J. M. Kim, and K. Kim, Full-colour quantum dot displays fabricated by transfer printing, Nat. Photonics 5, 176 (2011).

[9] E. Jang, S. Jun, H. Jang, J. Lim, B. Kim, and Y. Kim, Whitelight-emitting diodes with quantum dot color converters for display backlights, Adv. Mater. 22, 3076 (2010).

[10] L. Jacak, P. Hawrylak, and A. Wójs, Quantum dots (Springer, Berlin, 1998). 
[11] D. Bimberg, M. Grundmann, and N. N. Ledentsov, Quantum dot heterostructures (Wiley, Chichester, U.K., 1999).

[12] M. A. Reed, R. T. Bate, K. Bradshaw, W. M. Duncan, W. R. Frensley, J. W. Lee, and H. D. Shih, Spatial quantization in GaAs-AlGaAs multiple quantum dots, J. Vac. Sci. Technol. B 4, 358 (1986).

[13] B. J. van Wees, L. P. Kouwenhoven, C. J. P. M. Harmans, J. G. Williamson, C. E. Timmering, M. E. I. Broekaart, C. T. Foxon, and J. J. Harris, Observation of zero-dimensional states in a onedimensional electron interferometer, Phys. Rev. Lett. 62, 2523 (1989).

[14] U. Meirav, M. A. Kastner, and S. J. Wind, Single-electron charging and periodic conductance resonances in GaAs nanostructures, Phys. Rev. Lett. 65, 771 (1990).

[15] C. G. Smith, M. Pepper, H. Ahmed, J. E. F. Frost, D. G. Hasko, D. C. Peacock, D. A. Ritchie, and G. A. C. Jones, The transition from oneto zero-dimensional ballistic transport, J. Phys. C Solid State 21, 893 (1988).

[16] L. Goldstein, F. Glas, J. Y. Marzin, M. N. Charasse, and G. Le Roux, Growth by molecular beam epitaxy and characterization of InAs/GaAs strained-layer superlattices, Appl. Phys. Lett. 47, 1099 (1985).

[17] P. M. Petroff and S. P. DenBaars, MBE and MOCVD growth and properties of self-assembling quantum dot arrays in III-V semiconductor structures, Superlattices Microst. 15, 15 (1994).

[18] C. B. Murray, C. R. Kagan, and M. G. Bawendi, Synthesis and characterization of monodisperse nanocrystals and close-packed nanocrystal assembles, Annu. Rev. Mater. Sci. 30, 545 (2000).

[19] A. I. Ekimov, A. L. Efros, and A. A. Onushchenko, Quantum size effect in semiconductor microcrystals, Solid State Commun. 56, 921 (1985).

[20] D. Spirkoska, J. Arbiol, A. Gustafsson, S. Conesa-Boj, F. Glas, I. Zardo, M. Heigoldt, M. H. Gass, A. L. Bleloch, S. Estrade, M. Kaniber, J. Rossler, F. Peiro, J. R. Morante, G. Abstreiter, L. Samuelson, and A. Fontcuberta i Morral, Structural and optical properties of high quality zinc-blende/wurtzite GaAs nanowire heterostructures, Phys. Rev. B 80, 245325 (2009).

[21] N. Akopian, G. Patriarche, L. Liu, J.-C. Harmand, and V. Zwiller, Crystal phase quantum dots, Nano Lett. 10, 1198 (2010).

[22] K. S. Novoselov, A. K. Geim, S. V. Morozov, D. Jiang, Y. Zhang, S. V. Dubonos, I. V. Grigorieva, and A. A. Firsov, Electric field effect in atomically thin carbon films, Science 306, 666 (2004). 
[23] A. D. Güçlü, P. Potasz, M. Korkusinski, and P. Hawrylak, Graphene quantum dots (Springer, Berlin, 2014).

[24] P. Recher and B. Trauzettel, Quantum dots and spin qubits in graphene, Nanotechnology 21, 302001 (2010).

[25] J. Shen, Y. Zhu, X. Yang, and C. Li, Graphene quantum dots: emergent nanolights for bioimaging, sensors, catalysis and photovoltaic devices, Chem. Commun. 48, 3686 (2012).

[26] X.-X. Song, D. Liu, V. Mosallanejad, J. You, T.-Y. Han, D.-T. Chen, H.-O. Li, G. Cao, M. Xiao, G.-C. Guo, and G.-P. Guo, A gate defined quantum dot on the two-dimensional transition metal dichalcogenide semiconductor $W_{S} e_{2}$, Nanoscale 7, 16867 (2015).

[27] W. Qiao, S. Yan, X. Song, X. Zhang, Y. Sun, X. Chen, W. Zhong, and Y. Du, Monolayer $\mathrm{MoS}_{2}$ quantum dots as catalysts for efficient hydrogen evolution, RSC Adv. 5, 97696 (2015).

[28] A. Srivastava, M. Sidler, A. V. Allain, D. S. Lembke, A. Kis, and A. Imamoğlu, Optically active quantum dots in monolayer $W S e_{2}$, Nat. Nanotechnol. 10, 491 (2015).

[29] E. C. Le Ru, P. Howe, T. S. Jones, and R. Murray, Strain-engineered InAs/GaAs quantum dots for long-wavelength emission, Phys. Rev. B 67, 165303 (2003).

[30] J. Zhang, Y. Huo, A. Rastelli, M. Zopf, B. Höfer, Y. Chen, F. Ding, and O. G. Schmidt, Single photons on-demand from light-hole excitons in strain-engineered quantum dots, Nano Lett. 15, 422 (2015).

[31] L. C. Lew Yan Voon and M. Willatzen, The $k \cdot p$ method (Springer, Berlin, 2009).

[32] G. L. Bir and G. E. Pikus, Symmetry and strain-induced effects in semiconductors (Wiley, New York, 1974).

[33] R. Winkler, Spin-orbit coupling effects in two-dimensional electron and hole systems (Springer, Berlin, 2003).

[34] P.-O. Löwdin, A note on the quantum-mechanical perturbation theory, J. Chem. Phys. 19, 1396 (1951).

[35] G. Bastard, Wave mechanics applied to semiconductor heterostructures (Les éditions de physique, Les Ulis, France, 1988).

[36] D. J. BenDaniel and C. B. Duke, Space-charge effects on electron tunneling, Phys. Rev. 152, 683 (1966).

[37] M. G. Burt, A new effective-mass equation for microstructures, Semicond. Sci. Technol. 3, 1224 (1988). 
[38] M. G. Burt, The justification for applying the effective-mass approximation to microstructures, J. Phys.: Condens. Matter 4, 6651 (1992).

[39] M. G. Burt, Fundamentals of envelope function theory for electronic states and photonic modes in nanostructures, J. Phys.: Condens. Matter 11, 53 (1999).

[40] B. A. Foreman, Effective-mass Hamiltonian and boundary conditions for the valence bands of semiconductor microstructures, Phys. Rev. B 48, 4964 (1993).

[41] B. A. Foreman, Elimination of spurious solutions from eight-band $k \cdot p$ theory, Phys. Rev. B 56, R12748 (1997).

[42] A. De and C. E. Pryor, Predicted band structures of III-V semiconductors in the wurtzite phase, Phys. Rev. B 81, 155210 (2010).

[43] S. L. Chuang and C. S. Chang, k.p method for strained wurtzite semiconductors, Phys. Rev. B 54, 2491 (1996).

[44] Y. M. Sirenko, J.-B. Jeon, K. W. Kim, M. A. Littlejohn, and M. A. Stroscio, Envelope-function formalism for valence bands in wurtzite quantum wells, Phys. Rev. B 53, 1997 (1996).

[45] M. F. Schubert, Interband tunnel junctions for wurtzite III-nitride semiconductors based on heterointerface polarization charges, Phys. Rev. B 81, 035303 (2010).

[46] P. E. Faria Junior and G. M. Sipahi, Band structure calculations of InP wurtzite/zinc-blende quantum wells, J. Appl. Phys. 112, 103716 (2012).

[47] S.-H. Park and S.-L. Chuang, Comparison of zinc-blende and wurtzite GaN semiconductors with spontaneous polarization and piezoelectric field effects, J. Appl. Phys. 87, 353 (2000).

[48] J. M. Luttinger, Quantum theory of cyclotron resonance in semiconductors: general theory, Phys. Rev. 102, 1030 (1956).

[49] J. I. Climente, J. Planelles, and W. Jaskólski, Magneto-optical transitions in nanoscopic rings, Phys. Rev. B 68, 075307 (2003)

[50] J. Planelles and W. Jaskólski, $k \cdot p$ Hamiltonians for quantum dots in a magnetic field, J. Phys.: Condens. Matter 15, L67 (2003).

[51] J. Planelles and J. I. Climente, Magnetic field implementation in multiband $k \cdot p$ hamiltonians of holes in semiconductor heterostructures, J. Phys.: Condens. Matter 25, 485801 (2013).

[52] C. E. Pryor and M. E. Flatté, Landé g factors and orbital momentum quenching in semiconductor quantum dots, Phys. Rev. Lett. 96, 026804 (2006). 
[53] J. van Bree, A. Y. Silov, P. M. Koenraad, M. E. Flatté, and C. E. Pryor, $g$ factors and diamagnetic coefficients of electrons, holes, and excitons in InAs/InP quantum dots, Phys. Rev. B 85, 165323 (2012).

[54] R. Hanson, L. P. Kouwenhoven, J. R. Petta, S. Tarucha, and L. M. K. Vandersypen, Spins in few-electron quantum dots, Rev. Mod. Phys. 79, 1217 (2007).

[55] J. Fabian, A. Matos-Abiague, C. Ertler, P. Stano, and I. Žutić, Semiconductor spintronics, Acta Phys. Slovaca 57, 565 (2007).

[56] M. W. Wu, J. H. Jiang, and M. Q. Weng, Spin dynamics in semiconductors, Phys. Rep. 493, 61 (2010).

[57] G. Dresselhaus, Spin-orbit coupling effects in zinc blende structures, Phys. Rev. 100, 580 (1955).

[58] Y. A. Bychkov and E. I. Rashba, Oscillatory effects and the magnetic susceptibility of carriers in inversion layers, J. Phys. C: Solid State Phys. 17, 6039 (1984),

[59] L. D. Landau and E. M. Lifshitz, Theory of elasticity (Pergamon, Oxford, 1986).

[60] K. H. Yoo, J. D. Albrecht, and L. R. Ram-Mohan, Strain in layered zinc blende and wurtzite semiconductor structures grown along arbitrary crystallographic directions, Am. J. Phys. 78, 589 (2010).

[61] J. F. Nye, Physical properties of crystals: Their representation by tensors and matrices (Oxford, New York, 1985).

[62] S. Schulz, M. A. Caro, E. P. O'Reilly, and O. Marquardt, Symmetryadapted calculations of strain and polarization fields in (111)-oriented zinc-blende quantum dots, Phys. Rev. B 84, 125312 (2011)

[63] J. Bardeen and W. Shockley, Deformation potentials and mobilities in non-polar crystals, Phys. Rev. 80, 72 (1950).

[64] C. Herring and E. Vogt, Transport and deformation-potential theory for many-valley semiconductors with anisotropic scattering, Phys. Rev. 101, 944 (1956).

[65] T. Chakraborty, Quantum dots: A survey of the properties of artificial atoms (Elsevier, Amsterdam, 1999).

[66] Y. Aharonov and D. Bohm, Significance of electromagnetic potentials in the quantum theory, Phys. Rev. 115, 485 (1959).

[67] R. G. Chambers, Shift of an electron interference pattern by enclosed magnetic flux, Phys. Rev. Lett. 5, 3 (1960).

[68] H. Batelaan and A. Tonomura, The Aharonov-Bohm effects: Variations on a subtle theme, Phys. Today 62, 38 (2009). 
[69] V. M. Fomin, ed., Physics of quantum rings (Springer, Berlin, 2014).

[70] I. Vurgaftman and J. R. Meyer, Band parameters for nitrogencontaining semiconductors, J. Appl. Phys. 94, 3675 (2003).

[71] S. Nakamura, S. Pearton, and G. Fasol, The blue laser diode (Springer, Berlin, 2000).

[72] S. Nakamura, T. Mukai, and M. Senoh, Candela-class highbrightness InGaN/AlGaN double-heterostructure blue-light-emitting diodes, Appl. Phys. Lett. 64, 1687 (1994).

[73] P. Lefebvre and B. Gayral, Optical properties of GaN/AlN quantum dots, C. R. Phys. 9, 816 (2008).

[74] D. Lagarde, A. Balocchi, H. Carrère, P. Renucci, T. Amand, X. Marie, S. Founta, and H. Mariette, Room-temperature optical orientation of the exciton spin in cubic GaN/AlN quantum dots, Phys. Rev. B 77, 041304 (2008),

[75] Y.-H. Liao, J. I. Climente, and S.-J. Cheng, Dominant channels of exciton spin relaxation in photoexcited self-assembled (In, Ga)As quantum dots, Phys. Rev. B 83, 165317 (2011).

[76] H. Tong and M. W. Wu, Theory of excitons in cubic III-V semiconductor GaAs, InAs and GaN quantum dots: Fine structure and spin relaxation, Phys. Rev. B 83, 235323 (2011).

[77] N. O. Lipari and M. Altarelli, A new method in the theory of indirect excitons in semiconductors, Solid State Commun. 18, 951 (1976).

[78] U. Ekenberg and M. Altarelli, Subbands and Landau levels in the twodimensional hole gas at the GaAs-Al $G a_{1-x} A s$ interface, Phys. Rev. B 32, 3712 (1985).

[79] E. Martinez-Guerrero, C. Adelmann, F. Chabuel, J. Simon, N. T. Pelekanos, G. Mula, B. Daudin, G. Feuillet, and H. Mariette, Selfassembled zinc blende GaN quantum dots grown by molecular-beam epitaxy, Appl. Phys. Lett. 77, 809 (2000).

[80] J. Hu, L.-S. Li, W. Yang, L. Manna, L.-W. Wang, and A. P. Alivisatos, Linearly polarized emission from colloidal semiconductor quantum rods, Science 292, 2060 (2001).

[81] J. Noborisaka, J. Motohisa, S. Hara, and T. Fukui, Fabrication and characterization of freestanding GaAs/AlGaAs core-shell nanowires and AlGaAs nanotubes by using selective-area metalorganic vapor phase epitaxy, Appl. Phys. Lett. 87, 093109 (2005).

[82] A. Fontcuberta i Morral, D. Spirkoska, J. Arbiol, M. Heigoldt, J. R. Morante, and G. Abstreiter, Prismatic quantum heterostructures syn- 
thesized on molecular-beam epitaxy GaAs nanowires, Small 4, 899 (2008)

[83] M. Keplinger, T. Mårtensson, J. Stangl, E. Wintersberger, B. Mandl, D. Kriegner, V. Holý, G. Bauer, K. Deppert, and L. Samuelson, Structural investigations of core-shell nanowires using grazing incidence X-ray diffraction, Nano Lett. 9, 1877 (2009),

[84] R. E. Algra, M. Hocevar, M. A. Verheijen, I. Zardo, G. G. W. Immink, W. J. P. van Enckevort, G. Abstreiter, L. P. Kouwenhoven, E. Vlieg, and E. P. A. M. Bakkers, Crystal structure transfer in core/shell nanowires, Nano Lett. 11, 1690 (2011).

[85] D. Rudolph, S. Funk, M. Döblinger, S. Morkötter, S. Hertenberger, L. Schweickert, J. Becker, S. Matich, M. Bichler, D. Spirkoska, I. Zardo, J. J. Finley, G. Abstreiter, and G. Koblmüller, Spontaneous alloy composition ordering in GaAs-AlGaAs core-shell nanowires, Nano Lett. 13, 1522 (2013).

[86] L. J. Lauhon, M. S. Gudiksen, D. Wang, and C. M. Lieber, Epitaxial core-shell and core-multishell nanowire heterostructures, Nature 420, 57 (2002).

[87] M. Heigoldt, J. Arbiol, D. Spirkoska, J. M. Rebled, S. Conesa-Boj, G. Abstreiter, F. Peiró, J. R. Morante, and A. Fontcuberta i Morral, Long range epitaxial growth of prismatic heterostructures on the facets of catalyst-free GaAs nanowires, J. Mater. Chem. 19, 840 (2009).

[88] J. Hasen, L. N. Pfeiffer, A. Pinczuk, S. He, K. W. West, and B. S. Dennis, Metamorphosis of a quantum wire into quantum dots, Nature 390, 54 (1997).

[89] C. Fasth, A. Fuhrer, M. T. Björk, and L. Samuelson, Tunable double quantum dots in InAs nanowires defined by local gate electrodes, Nano Lett. 5, 1487 (2005).

[90] B. M. Wong, F. Léonard, Q. Li, and G. T. Wang, Nanoscale effects on heterojunction electron gases in GaN/AlGaN core/shell nanowires, Nano Lett. 11, 3074 (2011).

[91] A. Bertoni, M. Royo, F. Mahawish, and G. Goldoni, Electron and hole gas in modulation-doped $G a A s / A l_{1-x} G a_{x} A s$ radial heterojunctions, Phys. Rev. B 84, 205323 (2011).

[92] A. Ballester, J. Planelles, and A. Bertoni, Multi-particle states of semiconductor hexagonal rings: Artificial benzene, J. Appl. Phys. 112, 104317 (2012).

[93] T. Chakraborty and P. Pietiläinen, Electron-electron interaction and 
the persistent current in a quantum ring, Phys. Rev. B 50, 8460 (1994).

[94] A. Lorke, R. Johannes Luyken, A. O. Govorov, J. P. Kotthaus, J. M. Garcia, and P. M. Petroff, Spectroscopy of nanoscopic semiconductor rings, Phys. Rev. Lett. 84, 2223 (2000).

[95] M. Jung, J. S. Lee, W. Song, Y. H. Kim, S. D. Lee, N. Kim, J. Park, M.-S. Choi, S. Katsumoto, H. Lee, and J. Kim, Quantum interference in radial heterostructure nanowires, Nano Lett. 8, 3189 (2008).

[96] Ö. Gül, N. Demarina, C. Blömers, T. Rieger, H. Lüth, M. I. Lepsa, D. Grützmacher, and Th. Schäpers, Flux periodic magnetoconductance oscillations in GaAs/InAs core/shell nanowires, Phys. Rev. B 89, 045417 (2014).

[97] Ö. Gül, H. Y. Günel, H. Lüth, T. Rieger, T. Wenz, F. Haas, M. Lepsa, G. Panaitov, D. Grützmacher, and Th. Schäpers, Giant magnetoconductance oscillations in hybrid superconductor-semiconductor core/shell nanowire devices, Nano Lett. 14, 6269 (2014).

[98] A. Szabo and N. S. Ostlund, Modern quantum chemistry (Dover, New York, 1996).

[99] K. Niemelä, P. Pietiläinen, P. Hyvönen, and T. Chakraborty, Fractional oscillations of electronic states in a quantum ring, Europhys. Lett. 36, 533 (1996)

[100] U. F. Keyser, C. Fühner, S. Borck, R. J. Haug, M. Bichler, G. Abstreiter, and W. Wegscheider, Kondo effect in a few-electron quantum ring, Phys. Rev. Lett. 90, 196601 (2003).

[101] A. Emperador, F. Pederiva, and E. Lipparini, Spin- and localizationinduced fractional Aharonov-Bohm effect, Phys. Rev. B 68, 115312 (2003).

[102] L. E. Lipparini, Modern many-particle physics (World Scientific, Singapore, 2008).

[103] F. V. Kusmartsev, J. F. Weisz, R. Kishore, and M. Takahashi, Strong correlations versus U-center pairing and fractional Aharonov-Bohm effect, Phys. Rev. B 49, 16234 (1994).

[104] J. P. Perdew and Y. Wang, Accurate and simple analytic representation of the electron-gas correlation energy, Phys. Rev. B 45, 13244 (1992).

[105] S. Das Sarma, J. Fabian, X. Hu, and I. Žutić, Spin electronics and spin computation, Solid State Commun. 119, 207 (2001). 
[106] S. Datta and B. Das, Electronic analog of the electro-optic modulator, Appl. Phys. Lett. 56, 665 (1990).

[107] D. Loss and D. P. DiVincenzo, Quantum computation with quantum dots, Phys. Rev. A 57, 120 (1998).

[108] D. P. DiVincenzo, Topics in quantum computers, in Mesoscopic electron transport, edited by L. L. Sohn, L. P. Kouwenhoven, and G. Schön (Springer, 1997) pp. 657-677.

[109] L. Chirolli and G. Burkard, Decoherence in solid-state qubits, Adv. Phys. 57, 225 (2008).

[110] J. Fischer, M. Trif, W. A. Coish, and D. Loss, Spin interactions, relaxation and decoherence in quantum dots, Solid State Commun. 149, 1443 (2009).

[111] A. V. Khaetskii and Y. V. Nazarov, Spin relaxation in semiconductor quantum dots, Phys. Rev. B 61, 12639 (2000).

[112] M. I. Dyakonov, ed., Spin physics in semiconductors (Springer, Berlin, 2008).

[113] C. Lü, J. L. Cheng, and M. W. Wu, Hole spin relaxation in semiconductor quantum dots, Phys. Rev. B 71, 075308 (2005).

[114] A. V. Khaetskii and Y. V. Nazarov, Spin-flip transitions between Zeeman sublevels in semiconductor quantum dots, Phys. Rev. B 64, 125316 (2001).

[115] C. F. Destefani and S. E. Ulloa, Oscillatory spin relaxation rates in quantum dots, Phys. Rev. B 72, 115326 (2005).

[116] S. Takahashi, R. S. Deacon, K. Yoshida, A. Oiwa, K. Shibata, K. Hirakawa, Y. Tokura, and S. Tarucha, Large anisotropy of the spin-orbit interaction in a single InAs self-assembled quantum dot, Phys. Rev. Lett. 104, 246801 (2010).

[117] S. Avetisyan, P. Pietiläinen, and T. Chakraborty, Strong enhancement of Rashba spin-orbit coupling with increasing anisotropy in the FockDarwin states of a quantum dot, Phys. Rev. B 85, 153301 (2012).

[118] L. M. Woods, T. L. Reinecke, and R. Kotlyar, Hole spin relaxation in quantum dots, Phys. Rev. B 69, 125330 (2004).

[119] D. V. Bulaev and D. Loss, Spin relaxation and decoherence of holes in quantum dots, Phys. Rev. Lett. 95, 076805 (2005).

[120] E. Tsitsishvili, R. v. Baltz, and H. Kalt, Exciton-spin relaxation in quantum dots due to spin-orbit interaction, Phys. Rev. B 72, 155333 (2005). 
[121] K. Roszak, V. M. Axt, T. Kuhn, and P. Machnikowski, Exciton spin decay in quantum dots to bright and dark states, Phys. Rev. B 76, 195324 (2007),

[122] J. M. Luttinger and W. Kohn, Motion of electrons and holes in perturbed periodic fields, Phys. Rev. 97, 869 (1955).

[123] D. Heiss, S. Schaeck, H. Huebl, M. Bichler, G. Abstreiter, J. J. Finley, D. V. Bulaev, and D. Loss, Observation of extremely slow hole spin relaxation in self-assembled quantum dots, Phys. Rev. B 76, 241306 (2007).

[124] V. N. Golovach, A. Khaetskii, and D. Loss, Phonon-induced decay of the electron spin in quantum dots, Phys. Rev. Lett. 93, 016601 (2004).

[125] P. Stano and J. Fabian, Orbital and spin relaxation in single and coupled quantum dots, Phys. Rev. B 74, 045320 (2006).

[126] P. Scarlino, E. Kawakami, P. Stano, M. Shafiei, C. Reichl, W. Wegscheider, and L. M. K. Vandersypen, Spin-relaxation anisotropy in a GaAs quantum dot, Phys. Rev. Lett. 113, 256802 (2014).

[127] O. Olendski and T. V. Shahbazyan, Theory of anisotropic spin relaxation in quantum dots, Phys. Rev. B 75, 041306 (2007).

[128] S. Amasha, K. MacLean, I. P. Radu, D. M. Zumbühl, M. A. Kastner, M. P. Hanson, and A. C. Gossard, Electrical control of spin relaxation in a quantum dot, Phys. Rev. Lett. 100, 046803 (2008).

[129] S. Prabhakar, R. Melnik, and L. L. Bonilla, Electrical control of phonon-mediated spin relaxation rate in semiconductor quantum dots: Rashba versus Dresselhaus spin-orbit coupling, Phys. Rev. B 87, 235202 (2013).

[130] T. Mano, M. Abbarchi, T. Kuroda, B. McSkimming, A. Ohtake, K. Mitsuishi, and K. Sakoda, Self-assembly of symmetric GaAs quantum dots on (111)A substrates: Suppression of fine-structure splitting, Appl. Phys. Express 3, 065203 (2010)

[131] I. Vurgaftman and J. R. Meyer, Spin-relaxation suppression by compensation of bulk and structural inversion asymmetries in [111]oriented quantum wells, J. Appl. Phys. 97, 053707 (2005).

[132] K. Biermann, A. Hernández-Mínguez, R. Hey, and P. V. Santos, Electrically tunable electron spin lifetimes in GaAs(111)B quantum wells, J. Appl. Phys. 112, 083913 (2012).

[133] A. Balocchi, T. Amand, G. Wang, B. L. Liu, P. Renucci, Q. H. Duong, and X. Marie, Electric field dependence of the spin relaxation 
anisotropy in (111) GaAs/AlGaAs quantum wells, New J. Phys. 15, 095016 (2013).

[134] I. Žutić, J. Fabian, and S. Das Sarma, Spintronics: Fundamentals and applications, Rev. Mod. Phys. 76, 323 (2004).

[135] H. J. Krenner, M. Sabathil, E. C. Clark, A. Kress, D. Schuh, M. Bichler, G. Abstreiter, and J. J. Finley, Direct observation of controlled coupling in an individual quantum dot molecule, Phys. Rev. Lett. 94, $057402(2005)$.

[136] G. Ortner, M. Bayer, Y. Lyanda-Geller, T. L. Reinecke, A. Kress, J. P. Reithmaier, and A. Forchel, Control of vertically coupled InGaAs/GaAs quantum dots with electric fields, Phys. Rev. Lett. 94, 157401 (2005).

[137] J. R. Petta, A. C. Johnson, J. M. Taylor, E. A. Laird, A. Yacoby, M. D. Lukin, C. M. Marcus, M. P. Hanson, and A. C. Gossard, Coherent manipulation of coupled electron spins in semiconductor quantum dots, Science 309, 2180 (2005).

[138] E. A. Stinaff, M. Scheibner, A. S. Bracker, I. V. Ponomarev, V. L. Korenev, M. E. Ware, M. F. Doty, T. L. Reinecke, and D. Gammon, Optical signatures of coupled quantum dots, Science 311, 636 (2006).

[139] A. N. Vamivakas, C.-Y. Lu, C. Matthiesen, Y. Zhao, S. Fält, A. Badolato, and M. Atatüre, Observation of spin-dependent quantum jumps via quantum dot resonance fluorescence, Nature 467, 297 $(2010)$

[140] S. E. Economou, J. I. Climente, A. Badolato, A. S. Bracker, D. Gammon, and M. F. Doty, Scalable qubit architecture based on holes in quantum dot molecules, Phys. Rev. B 86, 085319 (2012).

[141] M. F. Doty, J. I. Climente, A. Greilich, M. Yakes, A. S. Bracker, and D. Gammon, Hole-spin mixing in InAs quantum dot molecules, Phys. Rev. B 81, 035308 (2010).

[142] O. Voskoboynikov, C. P. Lee, and O. Tretyak, Spin-orbit splitting in semiconductor quantum dots with a parabolic confinement potential, Phys. Rev. B 63, 165306 (2001).

[143] C. F. Destefani, S. E. Ulloa, and G. E. Marques, Spin-orbit coupling and intrinsic spin mixing in quantum dots, Phys. Rev. B 69, 125302 (2004),

[144] D. V. Bulaev and D. Loss, Spin relaxation and anticrossing in quantum dots: Rashba versus Dresselhaus spin-orbit coupling, Phys. Rev. B 71, 205324 (2005). 
[145] M. P. Nowak, B. Szafran, F. M. Peeters, B. Partoens, and W. J. Pasek, Tuning of the spin-orbit interaction in a quantum dot by an in-plane magnetic field, Phys. Rev. B 83, 245324 (2011).

[146] T. K. Johal, R. Rinaldi, A. Passaseo, R. Cingolani, A. Vasanelli, R. Ferreira, and G. Bastard, Imaging of the electronic states of self-assembled $\operatorname{In}_{x} \mathrm{Ga}_{1-x}$ As quantum dots by scanning tunneling spectroscopy, Phys. Rev. B 66, 075336 (2002).

[147] M. Müller, A. Cerezo, G. D. W. Smith, L. Chang, and S. S. A. Gerstl, Atomic scale characterization of buried $\operatorname{In}_{x} G a_{1-x}$ As quantum dots using pulsed laser atom probe tomography, Appl. Phys. Lett. 92, 233115 (2008).

[148] A. D. Giddings, J. G. Keizer, M. Hara, G. J. Hamhuis, H. Yuasa, H. Fukuzawa, and P. M. Koenraad, Composition profiling of InAs quantum dots and wetting layers by atom probe tomography and crosssectional scanning tunneling microscopy, Phys. Rev. B 83, 205308 (2011).

[149] B. Mahler, P. Spinicelli, S. Buil, X. Quelin, J.-P. Hermier, and B. Dubertret, Towards non-blinking colloidal quantum dots, Nat. Mater. 7, 659 (2008)

[150] Y. Chen, J. Vela, H. Htoon, J. L. Casson, D. J. Werder, D. A. Bussian, V. I. Klimov, and J. A. Hollingsworth, "Giant" multishell CdSe nanocrystal quantum dots with suppressed blinking, J. Am. Chem. Soc. 130, 5026 (2008).

[151] F. Zhang, V. H. Crespi, and P. Zhang, Prediction that uniaxial tension along $\langle 111\rangle$ produces a direct band gap in germanium, Phys. Rev. Lett. 102, 156401 (2009).

[152] A. M. Smith, A. M. Mohs, and S. Nie, Tuning the optical and electronic properties of colloidal nanocrystals by lattice strain, Nat. Nanotechnol. 4, 56 (2009).

[153] Y. H. Huo, B. J. Witek, S. Kumar, J. R. Cardenas, J. X. Zhang, N. Akopian, R. Singh, E. Zallo, R. Grifone, D. Kriegner, R. Trotta, F. Ding, J. Stangl, V. Zwiller, G. Bester, A. Rastelli, and O. G. Schmidt, A light-hole exciton in a quantum dot, Nat. Phys. 10, 46 (2013).

[154] C. Delerue and M. Lannoo, Nanostructures: Theory and modelling (Springer, Berlin, 2004).

[155] S. Christodoulou, F. Rajadell, A. Casu, G. Vaccaro, J. Q. Grim, A. Genovese, L. Manna, J. I. Climente, F. Meinardi, G. Rainò, T. Stöferle, R. F. Mahrt, J. Planelles, S. Brovelli, and I. Moreels, Band 
structure engineering via piezoelectric fields in strained anisotropic CdSe/CdS nanocrystals, Nat. Commun. 6, 7905 (2015).

[156] S. Brovelli, R. D. Schaller, S. A. Crooker, F. García-Santamaría, Y. Chen, R. Viswanatha, J. A. Hollingsworth, H. Htoon, and V. I. Klimov, Nano-engineered electron-hole exchange interaction controls exciton dynamics in core-shell semiconductor nanocrystals, Nat. Commun. 2, 280 (2011).

[157] M. Grundmann, O. Stier, and D. Bimberg, InAs/GaAs pyramidal quantum dots: Strain distribution, optical phonons, and electronic structure, Phys. Rev. B 52, 11969 (1995).

[158] J. Simon, N. T. Pelekanos, C. Adelmann, E. Martinez-Guerrero, R. André, B. Daudin, L. S. Dang, and H. Mariette, Direct comparison of recombination dynamics in cubic and hexagonal GaN/AlN quantum dots, Phys. Rev. B 68, 035312 (2003).

[159] N. Vainorius, S. Lehmann, D. Jacobsson, L. Samuelson, K. A. Dick, and M.-E. Pistol, Confinement in thickness-controlled GaAs polytype nanodots, Nano Lett. 15, 2652 (2015).

[160] B. Loitsch, J. Winnerl, G. Grimaldi, J. Wierzbowski, D. Rudolph, S. Morkötter, M. Döblinger, G. Abstreiter, G. Koblmüller, and J. J. Finley, Crystal phase quantum dots in the ultrathin core of GaAsAlGaAs core-shell nanowires, Nano Lett. 15, 7544 (2015),

[161] F. García-Santamaría, Y. Chen, J. Vela, R. D. Schaller, J. A. Hollingsworth, and V. I. Klimov, Suppressed Auger recombination in "giant" nanocrystals boosts optical gain performance, Nano Lett. 9, $3482(2009)$.

[162] J. I. Climente, J. L. Movilla, and J. Planelles, Auger recombination suppression in nanocrystals with asymmetric electron-hole confinement, Small 8, 754 (2012).

[163] H. McDaniel, M. Pelton, N. Oh, and M. Shim, Effects of lattice strain and band offset on electron transfer rates in type-II nanorod heterostructures, J. Phys. Chem. Lett. 3, 1094 (2012).

[164] S. M. Fairclough, E. J. Tyrrell, D. M. Graham, P. J. B. Lunt, S. J. O. Hardman, A. Pietzsch, F. Hennies, J. Moghal, W. R. Flavell, A. A. R. Watt, and J. M. Smith, Growth and characterization of strained and alloyed type-II ZnTe/ZnSe core-shell nanocrystals, J. Phys. Chem. C 116, 26898 (2012).

[165] O. Chen, J. Zhao, V. P. Chauhan, J. Cui, C. Wong, D. K. Harris, H. Wei, H.-S. Han, D. Fukumura, R. K. Jain, and M. G. Bawendi, Compact high-quality $C d S e-C d S$ core-shell nanocrystals with narrow 
emission linewidths and suppressed blinking, Nat. Mater. 12, 445 (2013).

[166] S. Christodoulou, G. Vaccaro, V. Pinchetti, F. De Donato, J. Q. Grim, A. Casu, A. Genovese, G. Vicidomini, A. Diaspro, S. Brovelli, L. Manna, and I. Moreels, Synthesis of highly luminescent wurtzite CdSe/CdS giant-shell nanocrystals using a fast continuous injection route, J. Mater. Chem. C 2, 3439 (2014).

[167] S. Assali, L. Gagliano, D. S. Oliveira, M. A. Verheijen, S. R. Plissard, L. F. Feiner, and E. P. A. M. Bakkers, Exploring crystal phase switching in GaP nanowires, Nano Lett. 15, 8062 (2015).

[168] K. A. Dick, C. Thelander, L. Samuelson, and P. Caroff, Crystal phase engineering in single InAs nanowires, Nano Lett. 10, 3494 (2010).

[169] M. Bouwes Bavinck, K. D. Jöns, M. Zieliński, G. Patriarche, J.-C. Harmand, N. Akopian, and V. Zwiller, Photon cascade from a single crystal phase nanowire quantum dot, Nano Lett. 16, 1081 (2016).

[170] B. Loitsch, D. Rudolph, S. Morkötter, M. Döblinger, G. Grimaldi, L. Hanschke, S. Matich, E. Parzinger, U. Wurstbauer, G. Abstreiter, J. J. Finley, and G. Koblmüller, Tunable quantum confinement in ultrathin, optically active semiconductor nanowires via reverse-reaction growth, Adv. Mater. 27, 2195 (2015).

[171] A. M. Graham, P. Corfdir, M. Heiss, S. Conesa-Boj, E. Uccelli, A. Fontcuberta i Morral, and R. T. Phillips, Exciton localization mechanisms in wurtzite/zinc-blende GaAs nanowires, Phys. Rev. B 87, 125304 (2013).

[172] P. Corfdir, B. Van Hattem, E. Uccelli, S. Conesa-Boj, P. Lefebvre, A. Fontcuberta i Morral, and R. T. Phillips, Three-dimensional magneto-photoluminescence as a probe of the electronic properties of crystal-phase quantum disks in GaAs nanowires, Nano Lett. 13, 5303 (2013).

[173] K. S. Novoselov, D. Jiang, F. Schedin, T. J. Booth, V. V. Khotkevich, S. V. Morozov, and A. K. Geim, Two-dimensional atomic crystals, Proc. Natl. Acad. Sci. U.S.A. 102, 10451 (2005).

[174] P. Miró, M. Audiffred, and T. Heine, An atlas of two-dimensional materials, Chem. Soc. Rev. 43, 6537 (2014).

[175] Z. M. Wang, ed., MoS $S_{2}$ : Materials, physics, and devices (Springer International, Switzerland, 2014).

[176] L. Britnell, R. M. Ribeiro, A. Eckmann, R. Jalil, B. D. Belle, A. Mishchenko, Y.-J. Kim, R. V. Gorbachev, T. Georgiou, S. V. Morozov, A. N. Grigorenko, A. K. Geim, C. Casiraghi, A. H. Castro Neto, 
and K. S. Novoselov, Strong light-matter interactions in heterostructures of atomically thin films, Science 340, 1311 (2013).

[177] A. Pospischil, M. M. Furchi, and T. Mueller, Solar-energy conversion and light emission in an atomic monolayer $p$-n diode, Nat. Nanotechnol. 9, 257 (2014).

[178] J. S. Ross, P. Klement, A. M. Jones, N. J. Ghimire, J. Yan, D. G. Mandrus, T. Taniguchi, K. Watanabe, K. Kitamura, W. Yao, D. H. Cobden, and $\mathrm{X} . \mathrm{Xu}$, Electrically tunable excitonic light-emitting diodes based on monolayer WSe 2 p-n junctions, Nat. Nanotechnol. 9, 268 (2014).

[179] M. V. Bollinger, J. V. Lauritsen, K. W. Jacobsen, J. K. Nørskov, S. Helveg, and F. Besenbacher, One-dimensional metallic edge states in $\mathrm{MoS}_{2}$, Phys. Rev. Lett. 87, 196803 (2001).

[180] D. Davelou, G. Kopidakis, G. Kioseoglou, and I. N. Remediakis, $\mathrm{MoS}_{2}$ nanostructures: Semiconductors with metallic edges, Solid State Commun. 192, 42 (2014).

[181] E. Erdogan, I. H. Popov, A. N. Enyashin, and G. Seifert, Transport properties of $\mathrm{MoS}_{2}$ nanoribbons: edge priority, Eur. Phys. J. B 85, 33 (2012).

[182] S. Pavlović and F. M. Peeters, Electronic properties of triangular and hexagonal MoS $S_{2}$ quantum dots, Phys. Rev. B 91, 155410 (2015).

[183] Special topic: topological insulators, APL Mat. 3, issue 8 (2015).

[184] S. Zhang, Topological field theory and the discovery of topological materials, Phys. Scr. T146, 014022 (2012),

[185] M. Z. Hasan and C. L. Kane, Colloquium: Topological insulators, Rev. Mod. Phys. 82, 3045 (2010)

[186] M. Z. Hasan and J. E. Moore, Three-dimensional topological insulators, Annu. Rev. Condens. Matter Phys. 2, 55 (2011).

[187] F. Güller, A. M. Llois, J. Goniakowski, and C. Noguera, Polarity effects in unsupported polar nanoribbons, Phys. Rev. B 87, 205423 (2013).

[188] M. Gibertini and N. Marzari, Emergence of one-dimensional wires of free carriers in transition-metal-dichalcogenide nanostructures, Nano Lett. 15, 6229 (2015).

[189] H. Rostami, A. G. Moghaddam, and R. Asgari, Effective lattice Hamiltonian for monolayer $\mathrm{MoS}_{2}$ : Tailoring electronic structure with perpendicular electric and magnetic fields, Phys. Rev. B 88, 085440 (2013). 
[190] G.-B. Liu, W.-Y. Shan, Y. Yao, W. Yao, and D. Xiao, Threeband tight-binding model for monolayers of group-VIB transition metal dichalcogenides, Phys. Rev. B 88, 085433 (2013).

[191] A. Kormányos, V. Zólyomi, N. D. Drummond, P. Rakyta, G. Burkard, and V. I. Fal'ko, Monolayer $M o S_{2}$ : Trigonal warping, the $\Gamma$ valley, and spin-orbit coupling effects, Phys. Rev. B 88, 045416 (2013).

[192] A. Kormányos, G. Burkard, M. Gmitra, J. Fabian, V. Zólyomi, N. D. Drummond, and V. Fal'ko, $k \cdot p$ theory for two-dimensional transition metal dichalcogenide semiconductors, 2D Mater. 2, 022001 (2015).

[193] B. A. Bernevig, T. L. Hughes, and S.-C. Zhang, Quantum spin Hall effect and topological phase transition in HgTe quantum wells, Science 314, 1757 (2006).

[194] J. Li, A. F. Morpurgo, M. Büttiker, and I. Martin, Marginality of bulk-edge correspondence for single-valley Hamiltonians, Phys. Rev. B 82, 245404 (2010).

[195] W. Yao, S. A. Yang, and Q. Niu, Edge states in graphene: From gapped flat-band to gapless chiral modes, Phys. Rev. Lett. 102, 096801 (2009).

[196] J. Li, I. Martin, M. Büttiker, and A. F. Morpurgo, Topological origin of subgap conductance in insulating bilayer graphene, Nat. Phys. 7, 38 (2011).

[197] Y. Shimazaki, M. Yamamoto, I. V. Borzenets, K. Watanabe, T. Taniguchi, and S. Tarucha, Generation and detection of pure valley current by electrically induced Berry curvature in bilayer graphene, Nat. Phys. 11, 1032 (2015).

[198] X. Duan, C. Wang, J. C. Shaw, R. Cheng, Y. Chen, H. Li, X. Wu, Y. Tang, Q. Zhang, A. Pan, J. Jiang, R. Yu, Y. Huang, and X. Duan, Lateral epitaxial growth of two-dimensional layered semiconductor heterojunctions., Nat. Nanotechnol. 9, 1024 (2014).

[199] M. S. J. Marshall and M. R. Castell, Scanning tunnelling microscopy of epitaxial nanostructures., Chem. Soc. Rev. 43, 2226 (2014)

[200] W. Bao, N. J. Borys, C. Ko, J. Suh, W. Fan, A. Thron, Y. Zhang, A. Buyanin, J. Zhang, S. Cabrini, P. D. Ashby, A. Weber-Bargioni, S. Tongay, S. Aloni, D. F. Ogletree, J. Wu, M. B. Salmeron, and P. J. Schuck, Visualizing nanoscale excitonic relaxation properties of disordered edges and grain boundaries in monolayer molybdenum disulfide, Nat. Commun. 6, 7993 (2015)

[201] R. G. Veprek, S. Steiger, and B. Witzigmann, Ellipticity and the 
spurious solution problem of $k \cdot p$ envelope equations, Phys. Rev. B 76, 165320 (2007).

[202] R. G. Veprek, S. Steiger, and B. Witzigmann, Operator ordering, ellipticity and spurious solutions in $k \cdot p$ calculations of III-nitride nanostructures, Opt. Quantum Electron. 40, 1169 (2008).

[203] M. A. Stroscio and M. Dutta, Phonons in nanostructures (Cambridge University Press, Cambridge, 2001).

[204] T. Uenoyama and L. J. Sham, Carrier relaxation and luminescence polarization in quantum wells, Phys. Rev. B 42, 7114 (1990). 